\title{
Manufacturing and Experimental Investigation of
}

\section{Copper Heat Pipes}

\author{
by \\ Feng Cai \\ B. Eng.
}

A thesis submitted to the Faculty of Graduate Studies and Research in partial fulfillment of the requirements for the degree of

\section{Master of Applied Science}

\author{
Ottawa-Carleton Institute for Mechanical and Aerospace Engineering \\ Department of Mechanical and Aerospace Engineering \\ Carleton University \\ Ottawa, Ontario
}

Canada

January 2005

(C) Feng Cai, 2005 


$\begin{array}{ll}\begin{array}{l}\text { Library and } \\ \text { Archives Canada }\end{array} & \begin{array}{l}\text { Bibliothèque et } \\ \text { Archives Canada }\end{array} \\ \begin{array}{l}\text { Published Heritage } \\ \text { Branch }\end{array} & \begin{array}{l}\text { Direction du } \\ \text { Patrimoine de l'édition }\end{array} \\ \begin{array}{l}\text { 395 Wellington Street } \\ \text { Ottawa ON K1A ON4 }\end{array} & \begin{array}{l}\text { 395, rue Wellington } \\ \text { Ottawa ON K1A ON4 } \\ \text { Canada }\end{array}\end{array}$

Your file Votre référence

ISBN: 0-494-06786-1

Ourfile Notre référence

ISBN: 0-494-06786-1

NOTICE:

The author has granted a nonexclusive license allowing Library and Archives Canada to reproduce, publish, archive, preserve, conserve, communicate to the public by telecommunication or on the Internet, loan, distribute and sell theses worldwide, for commercial or noncommercial purposes, in microform, paper, electronic and/or any other formats.

The author retains copyright ownership and moral rights in this thesis. Neither the thesis nor substantial extracts from it may be printed or otherwise reproduced without the author's permission.
AVIS:

L'auteur a accordé une licence non exclusive permettant à la Bibliothèque et Archives Canada de reproduire, publier, archiver, sauvegarder, conserver, transmettre au public par télécommunication ou par l'Internet, prêter, distribuer et vendre des thèses partout dans le monde, à des fins commerciales ou autres, sur support microforme, papier, électronique et/ou autres formats.

L'auteur conserve la propriété du droit d'auteur et des droits moraux qui protège cette thèse. $\mathrm{Ni}$ la thèse ni des extraits substantiels de celle-ci ne doivent être imprimés ou autrement reproduits sans son autorisation.
In compliance with the Canadian

Privacy Act some supporting forms may have been removed from this thesis.

While these forms may be included in the document page count, their removal does not represent any loss of content from the thesis.
Conformément à la loi canadienne sur la protection de la vie privée, quelques formulaires secondaires ont été enlevés de cette thèse.

Bien que ces formulaires aient inclus dans la pagination, il n'y aura aucun contenu manquant.

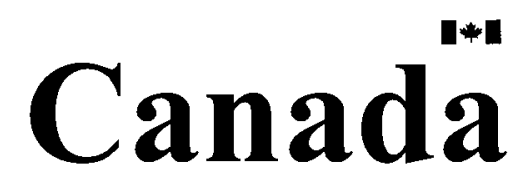


The undersigned recommend to the

Faculty of Graduate Studies and Research acceptance of the thesis

\title{
Manufacturing and Experimental Investigation of
}

\section{Copper Heat Pipes}

\author{
by \\ Feng Cai \\ B. Eng. \\ in partial fulfillment of the requirements \\ for the degree of Master of Applied Science
}

Dr. J. C. Beddoes

Chair, Department of Mechanical and Aerospace Engineering

Dr. J.E. Donald Gauthier, Co-Thesis Supervisor

Department of Mechanical and Aerospace Engineering, Carleton University

Dr. T. Kaya, Co-Thesis Supervisor

Department of Mechanical and Aerospace Engineering, Carleton University

January 2005 


\section{Abstract}

This thesis work is to perform copper heat pipe manufacturing, testing, and theoretical analyses. The details are presented in the corresponding chapters in this thesis.

A detailed heat pipe manufacturing process is introduced, including primary design consideration, material preparation and treatment, component assembly, evacuation and working fluid charging, and the final sealing and finishing process. Among these processes, the challenges related to the evacuation and fluid charging processes as well as corresponding impacts are analyzed. The corresponding solutions are discussed.

A series of experiments are conducted. The experiments involve six heat pipe configurations as well as copper tube configurations for comparison purposes. The performances are tested with difference cooling conditions combining different orientations. Detailed experimental analyses focussed on performance parameters such as the heat pipe transfer capacity and effective axial thermal conductivity, with regard to specific testing conditions. Some phenomena were observed, and the corresponding explanations are discussed.

Mathematical models are applied to analyze the heat pipe characteristics and performance. The models involve thermal network throughout the heat pipes, and the heat transfer capacity under various circumstances. The effective length model is proposed to explain the unexpected results.

This thesis also points out opportunities to improve the heat pipe manufacturing techniques. 


\section{Acknowledgements}

From the bottom of my heart, I thank Professor Tarik Kaya, my supervisor. It was his appreciation that led me to the opportunity with which I can do what I want and what I love. Today, I gratefully show the small piece of shell found on the beach; and thank the person who led me to this beach, and provided guidance to find the shell.

I would also like to thank Professor J.E. Donald Gauthier, who provided support for me to perform in Carleton University. Also, I appreciate his knowledge and patience with guiding me on the right track of studying.

I would like to thank Professor Xiao Huang who provided nickel wick material in our work. I will also thank Erin MacDonald who helped me with technical papers and related information; and thank Shane Storring who helped me with experimental hardware, with photo documentaries, and with wording in this thesis. I would like to thank the people working in the machine shop who provided assistance in the heat pipe manufacturing.

I would thank all of the people who have been helping me, directly and indirectly, or have been providing convenience in our work.

Here, I would thank the place where my career began, Shanghai Institute of Spacecraft Engineering, Chinese Academy of Space Technology. It is where I practiced professional career, laying the foundation for my future. Also, I would mention my Alma Mater, Hefei University of Technology, China.

Finally, I remain sanity to the love and support of my parents. Thank You.

Feng Cai

Carleton University, Ottawa, Canada 


\section{Table of Contents}

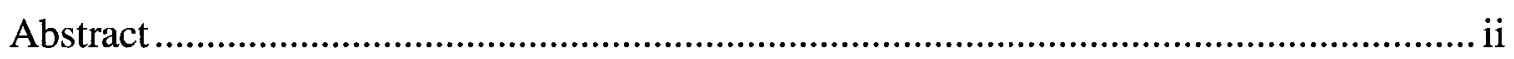

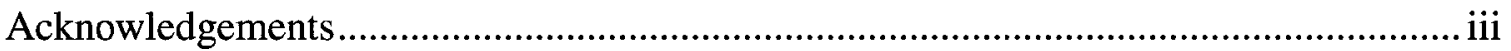

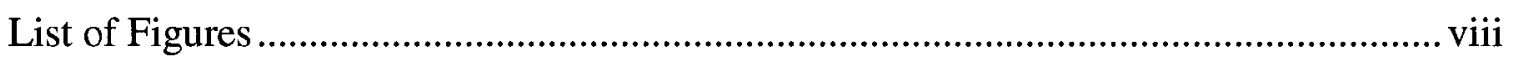

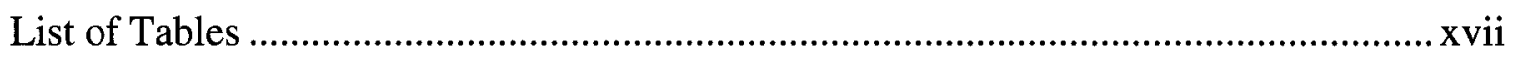

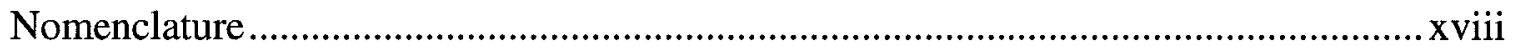

Chapter 1 Introduction ........................................................................................... 1

1.1 Introduction to Heat Pipe Technology .............................................................. 1

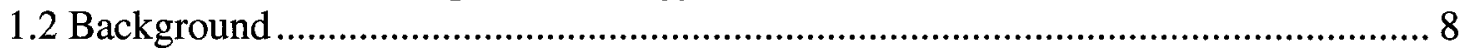

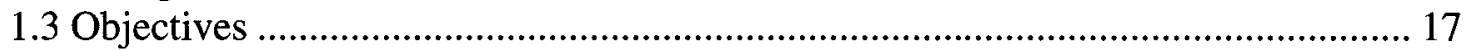

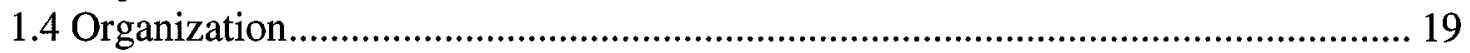

Chapter 2

Heat Pipe Manufacturing Process ............................................................................. 21

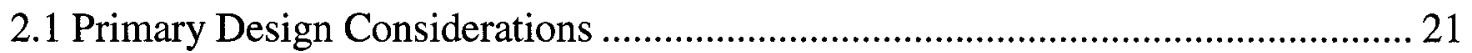

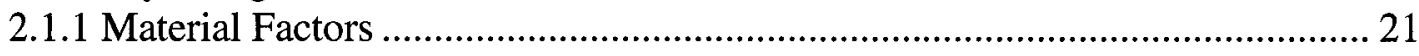

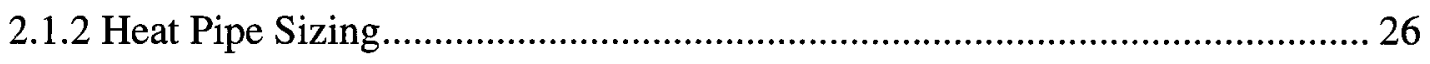

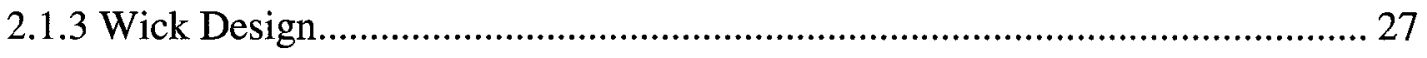

2.2 Heat Pipe Manufacturing Process.................................................................. 29

2.2.1 Material Preparation and Treatment ........................................................... 29

2.2.2 Component Assembly ..................................................................................... 33

2.2.3 Evacuation and Fluid Charging …………………........................................ 37

2.2.3.1 Heat Pipe Charging Device..................................................................... 39

2.2.3.2 Vacuum Process Gas Flow Analysis ..................................................... 47

2.2.3.3 Gas Load Analysis ................................................................................. 55

2.2.3.4 Fluid Charging Analysis ........................................................................... 63

2.2.4 Post Charge Sealing .................................................................................. 70

2.2.5 Finishing Process ………………............................................................ 71

2.3 Chapter Closure ............................................................................................ 72 
Chapter 3

Experimental Investigation of Heat Pipe Characteristics ............................................ 74

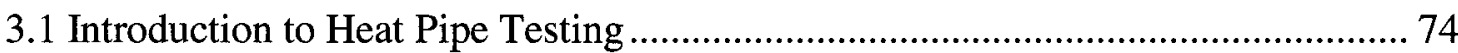

3.1.1 Summary of Heat Pipe Testing Approaches ............................................... 75

3.1.2 Introduction to Experimental Apparatus.................................................. 76

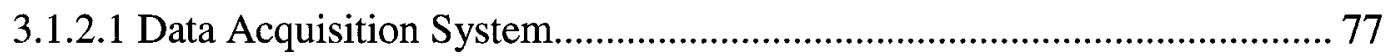

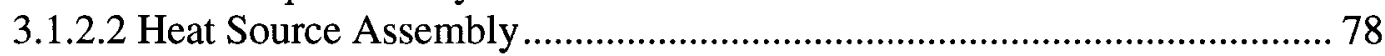

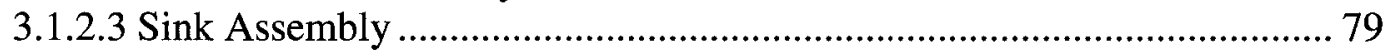

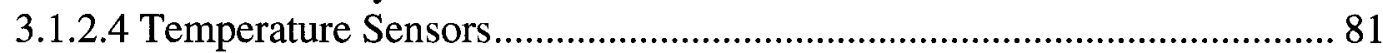

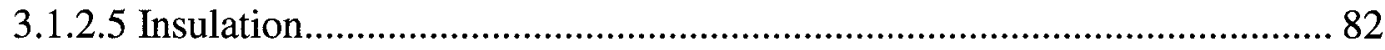

3.1.2.6 Power Supply and Control system .................................................. 82

\subsection{Experiment 1}

Investigations of Heat Source Characteristics ........................................................ 83

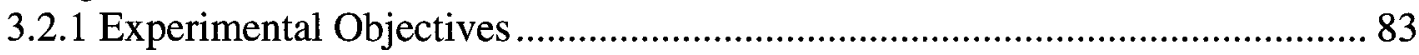

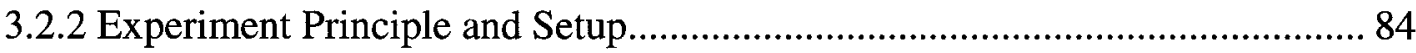

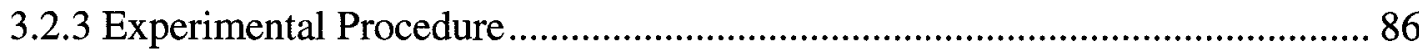

Section 3: Testing the Heater Assembly with Insulation............................ 89

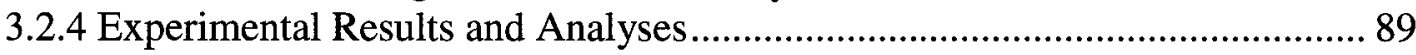

Conclusions of Heat Source Assembly Testing ................................................. 91

\subsection{Experiment 2}

Investigations of Non-Heat Pipe Tubes Characteristics ......................................... 92

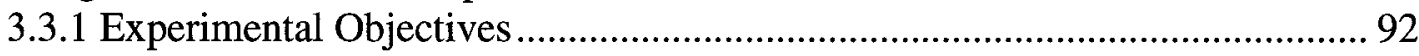

3.3.2 Experiment Principle and Setup............................................................... 93

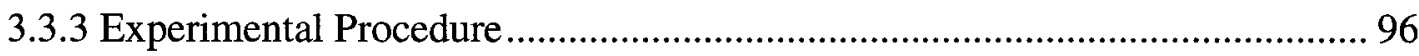

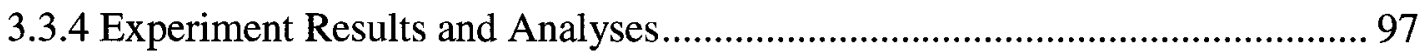

3.3.4.1 Typical Heat Transfer Temperature Characteristics ................................ 97

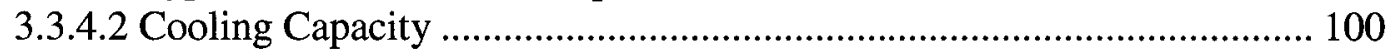

3.3.4.3 Axial Temperature Profile ................................................................ 104

3.3.4.4 Heating-Cooling Temperature Gradient Characteristics ....................... 107

3.3.4.5 Axial Thermal Resistance and Conductivity ..................................... 110

3.3.5 Conclusion of the non-heat pipe testing................................................. 114

\subsection{Experiment 3}

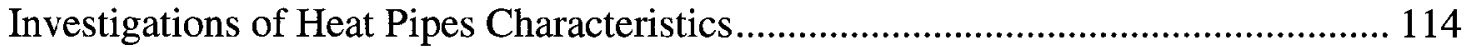

3.4.1 Experimental Objective ................................................................ 115

3.4.2 Experiment Principle and Apparatus ...................................................... 115 


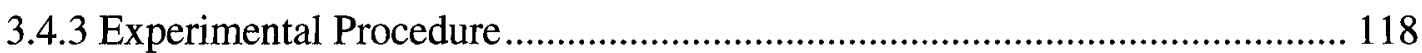

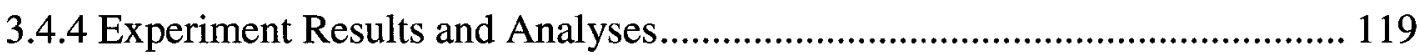

3.4.4.1 Typical Heat Pipe Temperature Characteristics ...................................... 119

3.4.4.2 Heater Cooling Capacity .......................................................................... 122

3.4.4.3 Axial Temperature Profile ................................................................... 127

3.4.4.3.1 Typical Heat Pipe Axial Temperature Profile .................................. 127

3.4.4.4 Evaporator-Condenser Temperature Gradient Characteristics ............... 144

3.4.4.4.1 Comparison of Heat Pipe Evaporator-condenser Temperature

Gradient................................................................................................ 145

3.4.4.4.4 Evaporator-Condenser Temperature Gradient on Amount of Fluid 158

3.4.4.5 Axial Thermal Resistance and Effective Thermal Conductivity ............. 163

3.4.5 Investigation of Nickel-Copper Heat Pipe ....................................................... 170

3.4.5.3 Axial Temperature Profile ...................................................................... 170

3.4.5.3.1 Heat Pipe Axial Temperature Profile regarding Heat Load.............. 170

3.4.5.3.2 Heat Pipe Axial Temperature Profile regarding Cooling Approach 175

3.4.5.3.3 Heat Pipe Axial Temperature Profile regarding Orientation ............ 177

3.4.5.4 Evaporator-Condenser Temperature Difference Characteristics ............. 179

3.4.5.5 Axial Thermal Resistance and Effective Conductivity............................. 180

3.4.6 Conclusion of Heat Pipe Testing .................................................................. 187

3.5 Experiment 4

Verification of Wind Effect on the Thermocouples on the Condenser ........................ 188

3.5.1 Experimental Objectives........................................................................... 189

3.5.2 Conclusion of Wind Effect Investigation ...................................................... 197

3.6 Discussion on the Heat Pipe Characteristics........................................................ 198

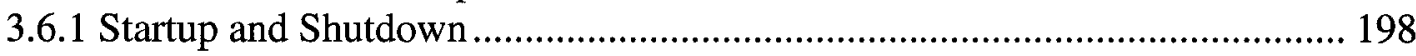

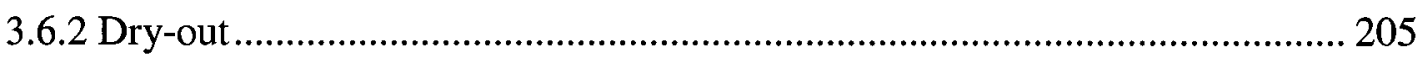

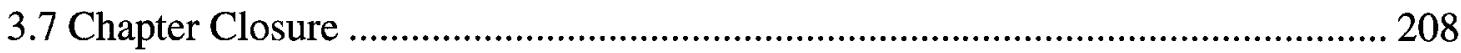

Chapter 4

Mathematical Modeling and Experimental Verification ................................................ 209

4.1 Thermal Network and Temperature Analyses ...................................................... 209

4.1.1 Heat Pipe Thermal Network …….......................................................... 209

4.1.2 Heat Pipe Thermal Network and Temperature Analyses ………………...... 212

4.2 Heat Transfer Capacity Analyses...................................................................... 222

4.2.1 Capillary Effect on Heat Transfer Capacity.................................................... 223

4.2.2 Vapour Flow Effect on Heat Transfer Capacity .......................................... 229

4.2.3 Flow Interface Effect on Heat Transfer Capacity …………………………... 229 
4.2.4 Boiling Effect on Heat Transfer Capacity ............................................. 230

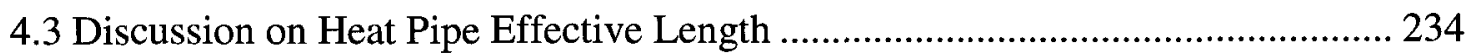

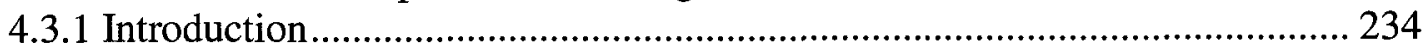

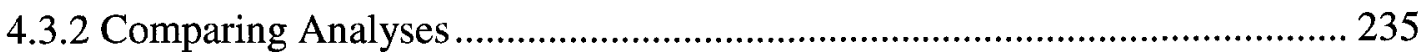

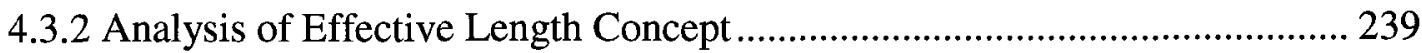

4.3.2.1 Definition of Effective Length......................................................... 240

4.3.2.2 Mechanism of Effective Length...................................................... 240

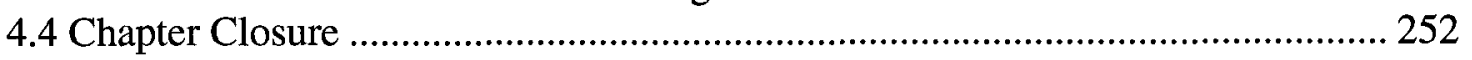

Chapter 5

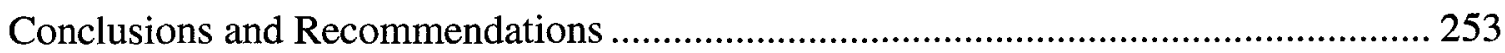

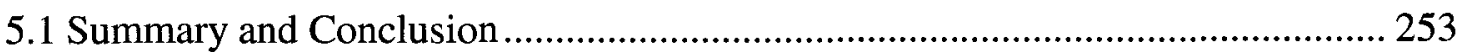

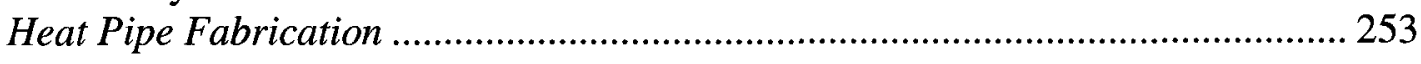

Evacuation and Fluid Charging Process Analyses ............................................. 254

Heat Pipe Performance Testing ..................................................................... 255

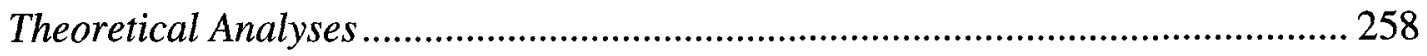

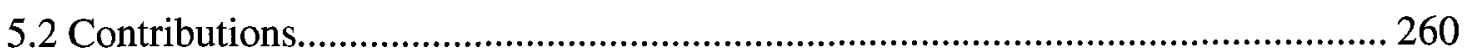

Heat Pipe Manufacturing at Canadian University ............................................. 260

Theoretical Analysis on Heat Pipe Evacuation Process...................................... 260

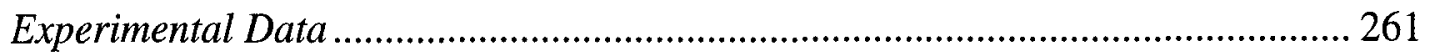

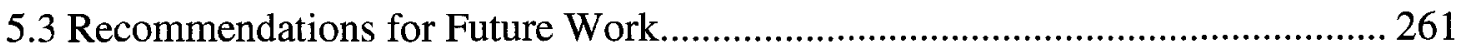

The Improvement of Vacuum and Charging System ............................................ 262

Improvement of Sealing Method ....................................................................... 263

Improvement of Testing Data Acquisition Function ........................................... 263

Power Supply System Stability Control ........................................................ 263

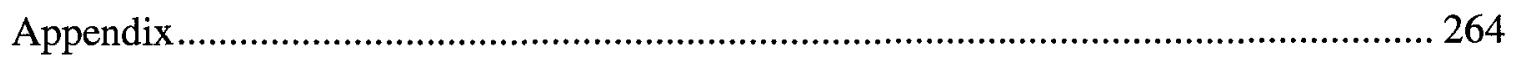

Appendix I: The Specifications of the Testing Elements ....................................... 264

Appendix II: The Experimental Result Recordings.............................................. 269

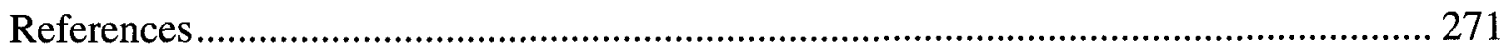




\section{List of Figures}

Figure 1.1 Typical heat pipe wick configurations and structures

Figure 1.2 Schematic diagram of a heat-pipe-cooled leading edge showing regions of net heat input

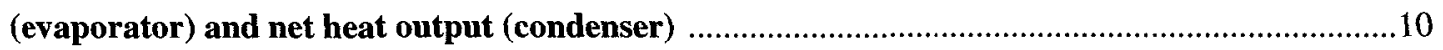

Figure 2.1 Flow chart for heat pipe wick design ........................................................................28

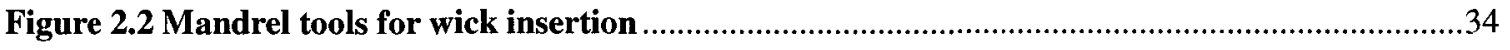

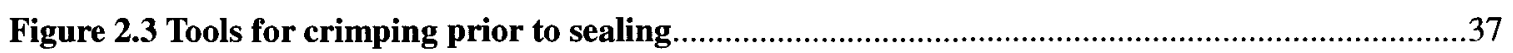

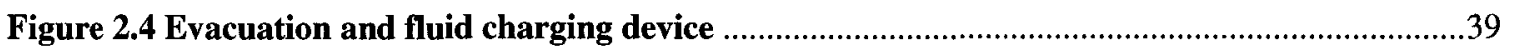

Figure 2.4a Schematics of the evacuation and fluid charging device ........................................40

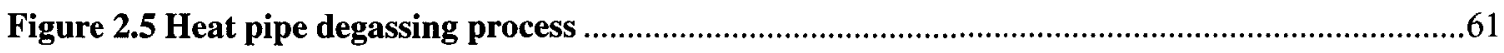

Figure 2.6 Charged fluid stays in the Tygon hose before applying the cooling bag ...........................69

Figure 2.7 Fluid was sucked into the heat pipe when applied the cooling bag................................69

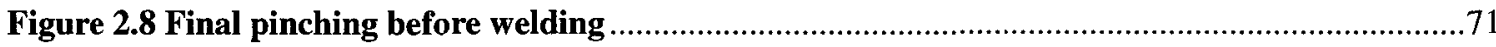

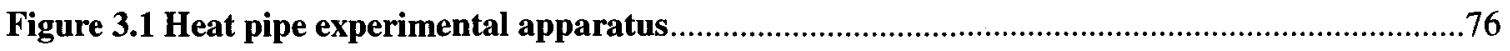

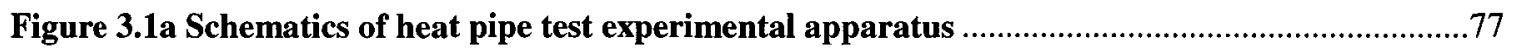

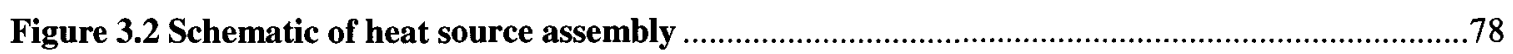

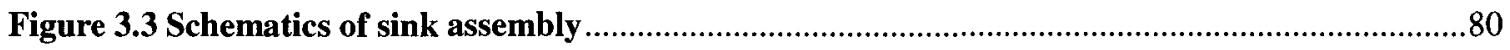

Figure 3.4 Schematics of the heat source assembly experimental setup .......................................85

Figure 3.5 Heat source assembly power -temperature profile (non-insulated) ...................................90

Figure 3.6 Heat source assembly power -temperature profile (insulated) ......................................90

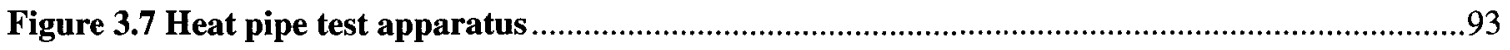

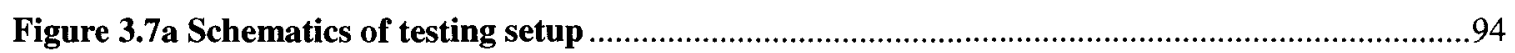

Figure3.8 Thermocouple locations along the test sample axial position ......................................95

Figure 3.10 Copper tube with mesh power-temperature profile (insulated) .....................................98

Figure 3.11 Copper tube with mesh \& water power-temperature profile (non-insulated) ...................99

Figure 3.12 Heater surface temperature comparison with non-heat pipe tubes (non-insulated, natural

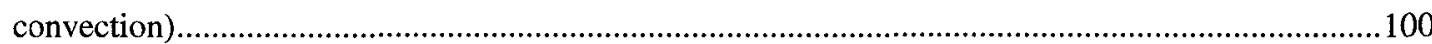

Figure 3.13 Heater surface temperature comparison with non-heat pipe (non-insulated, forced

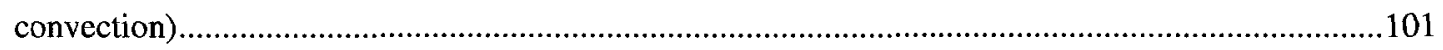

Figure 3.14 Heater surface temperature comparisons with non-heat pipe tubes .............................101

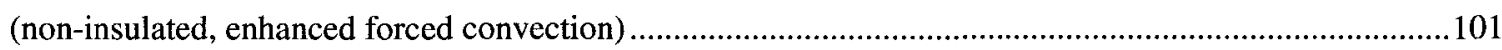

Figure 3.15 Heater surface temperature comparison with non-heat pipe tubes (insulated, natural convection) 
Figure 3.16 Heater surface temperature comparison with non-heat pipe tubes (insulated, forced convection).

Figure 3.17 Heater surface temperature comparison with non-heat pipe tubes (insulated, enhanced forced convection) 103

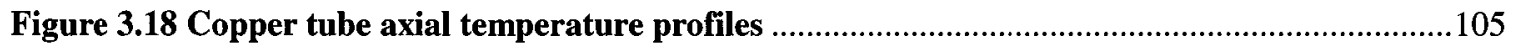

Figure 3.19 Copper tube with mesh axial temperature profile..........................................................105

Figure 3.20 Copper tube with mesh \& water axial temperature profile...........................................106

Figure 3.21 Copper tube heating-cooling temperature differences as function of heat power input 108

Figure 3.22 Copper tube with mesh heating-cooling temperature difference as function of heat power input 108

Figure 3.23 Copper tube with mesh \& water heating-cooling temperature difference as function of

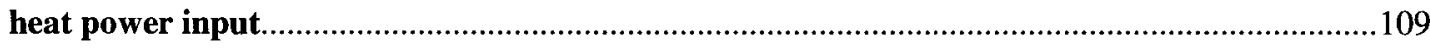

Figure 3.24 Copper tube axial thermal resistance as function of heat power input ...........................110

Figure 3.25 Copper tube with mesh axial thermal resistance as function of heat power input ..........111

Figure 3.26 Copper tube with mesh \& water axial thermal resistance as function of heat power input

Figure 3.27 Copper tube axial thermal conductivity as a function of heat power input .

Figure 3.28 Copper tube with mesh axial thermal conductivity as a function of heat power.............113

Figure 3.29 Copper tube with mesh \& water axial thermal conductivity versus heat load ................113

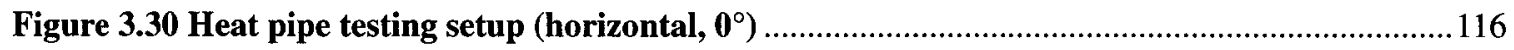

Figure 3.31 Heat pipe testing setup (vertical, against gravity, $+\mathbf{9 0}^{\circ}$ )...........................................116

Figure 3.32 Heat pipe testing setup (vertical, gravity assisted, $\mathbf{- 9 0 ^ { \circ }}$ ).............................................117

Figure 3.33 Schematics of thermocouple distribution along the heat pipe axial................................117

Figure 3.34 Typical heat pipe time-temperature profile (insulated, horizontal, natural convection) $[0,1$,

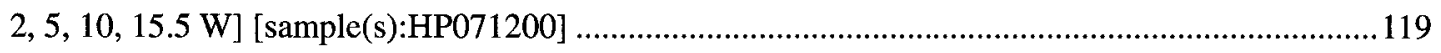

Figure 3.35 Typical heat pipe time-temperature profile (horizontal, enhanced forced convection) $[0,1,2$,

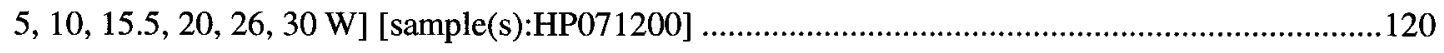

Figure 3.36 Typical heat pipe time-temperature profile (insulated, gravity assisted vertical, natural convection) $[0,1,2,5,10,15.5 \mathrm{~W}]$ [sample(s):HP071200] ...

Figure 3.37 Typical heat pipe time-temperature profile (insulated, against gravity vertical, natural convection) [0, 1, 2, $5 \mathrm{~W}$ ] [sample(s):HP071200] .

Figure 3.38 Heater surface temperature comparison with heat pipes (insulated, horizontal, natural convection) [sample(s):HP062300, HP052502, HP051701, HP071200, HP071402, HP071301] ......123

Figure 3.39 Heater surface temperature comparison with heat pipes (insulated, horizontal, enhanced forced convection) [sample(s):HP052502, HP051701, HP071200, HP071402, HP071301]

Figure 3.40 Heater surface temperature comparison with heat pipes (insulated, gravity assisted vertical, natural convection) [sample(s): HP052502, HP051701, HP071200, HP071402, HP071301]..... 125 
Figure 3.41 Heater surface temperature comparison with heat pipes (insulated, against gravity vertical, natural convection) [sample(s): HP052502, HP051701, HP071200, HP071402, HP071301] .126

Figure 3.42 Heat pipe axial temperature profile (insulated, horizontal, natural convection) [sample(s): HP071200].

Figure 3.43 Heat pipe axial temperature profile (insulated, horizontal, enhanced forced convective) [sample(s): 071200].

Figure 3.44 Heat pipe axial temperature profile (insulated, gravity assisted vertical, natural convection) [sample(s): 071200]

Figure 3.45 Heat pipe axial temperature profile (insulated, against gravity vertical, natural convection) [sample(s): HP071200]

Figure 3.46 Axial temperature profile comparison regarding cooling approach (insulated, horizontal) [sample(s): HP 052502]

Figure 3.47 Axial temperature profile comparison regarding orientation (insulated, natural convection) [sample(s): HP 051701]

Figure 3.48 Axial temperature profile regarding wick structure (insulated, horizontal, natural convection) [sample(s): HP051701, HP071200, HP071301].

Figure 3.49 Axial temperature profile regarding wick structure (insulated, horizontal, enhanced forced convection) [sample(s): HP051701, HP071200, HP071301].

Figure 3.50 Axial temperature profile regarding wick structure (insulated, gravity assisted vertical, natural convection) [sample(s): HP051701, HP071200, HP071301].....

Figure 3.51 Axial temperature profile regarding wick structure (insulated, against gravity vertical, natural convection) [sample(s): HP051701, HP071200, HP071301].

Figure 3.52 Axial temperature profile regarding working fluid (insulated, horizontal, natural convection) [sample(s): HP051701, HP071402]

Figure 3.53 Axial temperature profile regarding working fluid (insulated, horizon, enhanced forced convection) [sample(s): HP051701, HP071402]

Figure 3.54 Axial temperature profile regarding working fluid (insulated, gravity assisted vertical, natural convection) [sample(s): HP051701, HP071402] ...

Figure 3.55 Axial temperature profile regarding working fluid (insulated, against gravity vertical, natural convection) [sample(s): HP051701, HP071402] .

Figure 3.56 Axial temperature profile regarding amount of working fluid (insulated, horizontal, natural convection) [sample(s): HP051701, HP052502]

Figure 3.57 Axial temperature profile regarding amount of working fluid (insulated, horizontal, forced convection) [sample(s): HP051701, HP052502]

Figure 3.58 Axial temperature profile regarding amount of working fluid (insulated, horizon, enhanced forced convection) [sample(s): HP051701, HP052502]

Figure 3.59 Axial temperature profile regarding amount of working fluid (insulated, gravity assisted 
vertical, natural convection) [sample(s): HP051701, HP052502].....

Figure 3.60 Axial temperature profile regarding amount of working fluid (insulated, against gravity vertical, natural convection) [sample(s): HP051701, HP052502].

Figure 3.61 Comparison of evaporator-condenser temperature difference [sample: HP051701] ......145

Figure 3.62 Evaporator-condenser temperature difference comparison [sample: HP052502] ..........145

Figure 3.63 Evaporator-condenser temperature difference comparison [sample: HP071200] ...........147

Figure 3.64 Evaporator-condenser temperature difference comparison [sample: HP071402] ...........148

Figure 3.65 Evaporator-condenser temperature difference comparison [sample: HP071301] ...........148

Figure 3.66 Comparison of evaporator-condenser temperature difference (insulated, horizontal, natural convection) [sample(s): HP052502, HP0051701, HP07 1200, HP071402, HP071301].....

Figure 3.67 Comparison of evaporator-condenser temperature difference regarding wick mesh (insulated, horizontal, natural convection) [sample(s): HP051701, HP071200, HP071301].

Figure 3.68 Comparison of evaporator-condenser temperature difference regarding wick mesh (insulated, horizontal, enhanced forced convection) [sample(s): HP051701, HP071200, HP071301]

Figure 3.69 Comparison of evaporator-condenser temperature difference regarding wick mesh (insulated, gravity assisted vertical, natural convection) [sample(s): HP051701, HP071200, HP071301]

Figure 3.70 Comparison of evaporator-condenser temperature difference regarding wick mesh (insulated, gravity assisted vertical, enhanced forced convection) [sample(s): HP051701, HP071200, HP071301]

Figure 3.71 Comparison of evaporator-condenser temperature difference regarding wick mesh (insulated, against gravity vertical, natural convection) [sample(s): HP051701, HP071200, HP071301]

Figure 3.72 Comparison of evaporator-condenser temperature difference regarding wick mesh (insulated, against gravity vertical, enhanced forced convection) [sample(s): HP051701, HP071200, HP071301]

Figure 3.73 Comparison of evaporator-condenser temperature difference regarding working fluid (insulated, horizontal, natural convection) [sample(s): HP051701, HP071402]. .156

Figure 3.74 Comparison of evaporator-condenser temperature difference regarding amount of working fluid (insulated, horizontal, natural convection) [sample(s): HP052502, HP051701] .........159

Figure 3.75 Comparison of evaporator-condenser temperature difference regarding amount of working fluid (insulated, horizontal, forced convection) [sample(s): HP052502, HP051701] .........160

Figure 3.76 Comparison of evaporator-condenser temperature difference regarding amount of working fluid (insulated, horizontal, enhanced forced convection) [sample(s): HP052502, HP051701]

Figure 3.77 Comparison of evaporator-condenser temperature difference regarding amount of 
working fluid (insulated, gravity assisted vertical, natural convection) [sample(s): HP052502, HP051701]

Figure 3.78 Comparison of evaporator-condenser temperature difference regarding amount of working fluid (insulated, against gravity vertical, natural convection) [sample(s): HP052502,

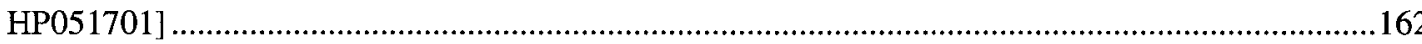

Figure 3.79 Axial thermal resistance under circumstances [sample(s); HP052502] ............................163

Figure 3.80 Comparison of effective axial thermal conductivity [sample(s): HP052502] ....................165

Figure 3.81 Comparison of effective axial thermal conductivity (insulated, horizontal, natural convection) [sample(s): HP052502, HP051701, HP071200, HP071402, HP071301]..........................166

Figure 3.82 Comparison of effective axial thermal conductivity (insulated, horizontal, forced convection) [sample(s): HP052502, HP051701, HP071200, HP071402, HP071301] 166

Figure 3.83 Comparison of effective axial thermal conductivity (insulated, horizontal, enhanced forced convective cooling) [sample(s): HP052502, HP051701, HP071200, HP071402, HP071301] .

Figure 3.84 Comparison of effective axial thermal conductivity (insulated, gravity assisted vertical, natural convection) [sample(s): HP052502, HP051701, HP071200, HP071402, HP071301]

Figure 3.85 Comparison of effective axial thermal conductivity (insulated, against gravity vertical, natural convection) [sample(s): HP052502, HP051701, HP071200, HP071402, HP071301]

Figure 3.86 Nickel foam heat pipe axial temperature profile (insulated, horizontal, natural convection) [sample(s): HP062400]

Figure 3.87 Nickel foam heat pipe axial temperature profile (insulated, horizontal, forced convection) [sample(s): HP062400]

Figure 3.88 Nickel foam heat pipe axial temperature profile (insulated, horizontal, enhanced convection) [sample(s): HP062400]

Figure 3.89 Nickel foam heat pipe axial temperature profile (insulated, gravity assisted vertical, natural convection) [sample(s): HP062400]

Figure 3.90 Nickel foam heat pipe axial temperature profile (insulated, against gravity vertical, natural convection) [sample(s): HP062400]

Figure 3.91 Nickel foam heat pipe axial temperature profile regarding cooling approach (insulated, horizontal) [sample(s): HP062400]

Figure 3.92 Nickel foam heat pipe axial temperature profile regarding cooling approach (insulated, gravity assisted vertical) [sample(s): HP062400]

Figure 3.93 Nickel foam heat pipe axial temperature profile regarding cooling approach (insulated, against gravity vertical) [sample(s): HP062400]

Figure 3.94 Nickel foam heat pipe axial temperature profile regarding orientation (insulated, natural convection) [sample(s): HP062400]

Figure 3.95 Nickel foam heat pipe axial temperature profile regarding orientation (insulated, forced convection) [sample(s): HP062400] 
Figure 3.96 Nickel foam heat pipe axial temperature profile regarding orientation (insulated, enhanced forced convection) [sample(s): HP062400]

Figure 3.97 Nickel foam heat pipe evaporator-condenser temperature difference (insulated) [sample(s): HP062400].

Figure 3.98 Nickel foam heat pipe axial thermal resistance (insulated) [sample(s): HP062400] ..........180

Figure 3.99 Nickel foam heat pipe effective axial thermal conductivity (insulated) [sample(s): HP062400].

Figure 3.100 Comparison of nickel foam heat pipe effective axial thermal conductivity with others (insulated, horizontal, natural convection) [sample(s): HP052502, HP071200, HP062400, HP071402, HP071301]

Figure 3.101 Comparison of nickel foam heat pipe effective axial thermal conductivity with others (insulated, horizontal, forced convection) [sample(s): HP051701, HP071200, HP062400, HP071402, HP071301]

Figure 3.102 Comparison of nickel foam heat pipe effective axial thermal conductivity with others (insulated, horizontal, enhanced forced convection) [sample(s): HP051701, HP071200, HP062400, HP071402, HP071301].

Figure 3.103 Comparison of nickel foam heat pipe effective axial thermal conductivity with others (insulated, gravity assisted vertical, natural convection) [sample(s): HP052502, HP051701, HP071200, HP062400, HP071301]. 184

Figure 3.104 Comparison of nickel foam heat pipe effective axial thermal conductivity with others (insulated, gravity assisted vertical, forced convection) [sample(s): HP052502, HP071200, HP062400, HP071402, HP071301]. 184

Figure 3.105 Comparison of nickel foam heat pipe effective axial thermal conductivity with others (insulated, gravity assisted vertical, enhanced forced convection) [sample(s): HP052502, HP071200, HP071200, HP062400, HP071402, HP071301]...

Figure 3.106 Comparison of nickel foam heat pipe effective axial thermal conductivity with others (insulated, against gravity vertical, natural convection) [sample(s): HP052502, HP071200, HP071200, HP062400, HP071402, HP071301]

Figure 3.107 Comparison of nickel foam heat pipe effective axial thermal conductivity with others (insulated, against gravity vertical, forced convection) [sample(s): HP052502, HP071200, HP071200, HP062400, HP071402, HP071301]

Figure 3.108 Comparison of nickel foam heat pipe effective axial thermal conductivity with others (insulated, against gravity vertical, enhanced forced convection) [sample(s): HP052502, HP071200, HP071200, HP062400, HP071402, HP071301].

Figure 3.109 Setup and thermocouple distribution for the wind effect verification experiment, insulted vs. non-insulated .

Figure 3.110 Comparison of thermocouple measurements with, and without insulation (horizontal, 
forced convection) [sample(s): HP071301]

191

Figure 3.111 Comparison of thermocouple measurements with, and without insulation (horizontal, enhanced forced convection) [sample(s): HP071301]

Figure 3.112 The setup for the experiment of verifying wind effect on the condenser thermocouples with wholly enclosed condenser.....

Figure 3.113 Comparison of thermocouple measurements with, and without insulation under the condition of wholly enclosing the condenser (horizontal, forced convection) [sample(s): HP071301]

Figure 3.114 Comparison of thermocouple measurements with, and without insulation under the condition of wholly enclosing the condenser (horizontal, enhanced forced convection) [sample(s): HP071301] 194

Figure 3.115 Setup for the experiment with directly blowing condenser. 195

Figure 3.116 Comparison of thermocouple measurements with, and without insulation under the condition of directly blowing the condenser (horizontal, forced convection) [sample: HP071301] 196

Figure 3.117 Comparison of thermocouple measurements with, and without insulation under the condition of directly blowing the condenser (horizontal, enhanced convection) [sample: HP071301]

Figure 3.118 Copper water heat pipe startup (insulated, horizontal, natural convection) [sample(s): HP051701]

Figure 3.119 Copper water heat pipe startup (insulated, horizontal, natural convection) [sample(s):HP071200]

Figure 3.120 Nickel-copper water heat pipe startup (insulated, horizontal, natural convection) [sample(s):HP062400]

Figure 3.121 Copper acetone heat pipe startup (insulated, horizontal, natural convection) [sample(s):HP071402] 200

Figure 3.122 Copper water heat pipe shutdown (insulated, horizontal, natural convection) [sample(s):HP071200] 201

Figure 3.123 Nickel-copper water heat pipe shutdown (insulated, horizontal, natural convection) [sample(s):HP062400]

Figure 3.124 Copper acetone heat pipe shutdown (insulated, horizontal, natural convection) [sample(s):HP071402] 202

Figure 3.125 Copper water heat pipe setup (insulated, gravity assisted vertical, enhanced force convection) [sample(s):HP071200] 203

Figure 3.126 Copper acetone heat pipe dry-out (insulated, horizontal, forced convection) [sample(s):HP071402]

Figure 3.127 Copper acetone heat pipe dry-out axial temperature profile (insulated, horizontal, forced 
convection) [sample(s):HP071402]

Figure 3.128 Copper water heat pipe dry-out (insulated, horizontal, forced convection) [sample(s):HP071301]

Figure 3.129 Copper water heat pipe dry-out axial temperature profile (insulated, horizontal, forced convection) [sample(s):HP071301]

Figure 4.1 Schematics of heat pipe thermal resistance network. .210

Figure 4.2 Simplified equivalent heat pipe thermal resistance network. .211

Figure 4.3 Comparison of predicted and measured evaporator temperature (100 mesh, water, insulated, horizontal, natural convection) [sample(s):HP052502]

Figure 4.4 Comparison of predicted and measured evaporator temperatures (insulated, horizontal, natural convection) [sample(s):HP071200]

Figure 4.5 Comparison of predicted and measured evaporator temperatures (insulated, horizontal, natural convection) [sample(s):HP071402]

Figure 4.6 Comparison of predicted and measured evaporator temperatures (insulated, horizontal, natural convection) [sample(s):HP062400]

Figure 4.7 Comparison of predicted and measured evaporator temperatures (insulated, gravity assisted vertical, natural convection) [sample(s):HP052502]

Figure 4.8 Comparison of predicted and measured evaporator temperatures (insulated, against gravity vertical, natural convection) [sample(s):HP052502].

Figure 4.9 Comparison of predicted and measured evaporator temperatures (insulated, against gravity vertical, natural convection) [sample(s):HP062400]

218

Figure 4.10 Comparison of predicted and measured evaporator temperatures (insulated, horizontal, forced convection) [sample(s):HP052502]

Figure 4.11 Comparison of predicted and measured evaporator temperatures (insulated, gravity assisted vertical, enhanced forced convection) [sample(s):HP052502]

Figure 4.12 Comparison of predicted and measured evaporator temperatures (insulated, against gravity vertical, enhanced forced convection) [sample(s):HP052502]

Figure 4.13 Comparison of predicted and measured evaporator temperatures (100 copper mesh, acetone; insulated, horizontal, enhanced forced convection) [sample(s):HP071402] .....................221

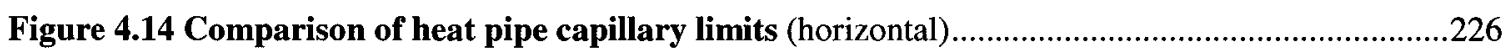

Figure 4.15 Comparison of heat pipe capillary limits (horizontal) ................................................2227

Figure 4.16 Comparison of heat pipe capillary limit (gravity assisted vertical) .............................228

Figure 4.17 Comparison of heat pipe capillary limit (gravity assisted vertical) ...............................228

Figure 4.18 Comparison of predicted heat transfer limits (100 copper mesh, water) .....................232

Figure 4.19 Comparison of predicted heat transfer limits (50 copper mesh, water) .......................232

Figure 4.20 comparison of predicted heart transfer limits $(100+50$ composite copper mesh, water $) .233$ 
Figure 4.21 Comparison of predicted heat transfer limits (100 copper mesh, acetone)

Figure 4.23 Comparison of evaporator temperature with correction (insulated, horizontal, enhanced forced convection) [sample(s):HP051701]

Figure 4.24 Comparison of evaporator temperature with correction (insulated, horizontal, forced convection) [sample(s):HP071200]

Figure 4.25 Capillary limit as a function of heat pipe effective length (against gravity vertical) [100 copper mesh water heat pipe].

Figure 4.26 Capillary limit as a function of heat pipe effective length (against gravity vertical) $[100$ copper mesh acetone heat pipe] .....

Figure 4.27 Capillary limit as a function of heat pipe effective length (against gravity vertical) [nickel foam water heat pipe].

Figure 4.28 Capillary limit as a function of heat pipe effective length (horizontal) [100copper mesh acetone heat pipe]

Figure 4.29 Simulation of heat pipe effective length change rate as functions of temperature and

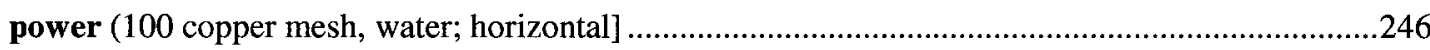

Figure 4.30 Simulation of heat pipe effective length change rate as functions of temperature and power [ 50 copper mesh water, horizontal]

Figure 4.31 Simulation of heat pipe effective length change rate as functions of temperature and power [100 copper mesh acetone, horizontal]

Figure 4.32 Simulation of heat pipe effective length change rate as functions of temperature and power [composite copper mesh water, horizontal]

Figure 4.33 Simulation of heat pipe effective length change rate as functions of temperature and power [nickel foam water, horizontal]. 248

Figure 4.34 Simulation of heat pipe effective length change rate as functions of temperature and power [100 copper mesh water, horizontal]

Figure 4.35 Simulation of heat pipe effective length change rate as functions of temperature and power [50 copper mesh water, horizontal]

Figure 4.36 Simulation of heat pipe effective length change rate as functions of temperature and power [100 copper mesh acetone, horizontal]

Figure 4.37 Simulation of heat pipe effective length change rate as functions of temperature and power [composite copper mesh water, horizontal]

Figure 4.38 Simulation of heat pipe effective length change rate as functions of temperature and power [nickel foam water, horizontal]. 


\section{List of Tables}

Table 2.1 Estimations of heat pipe configuration heat transfer capacity ........................ 28

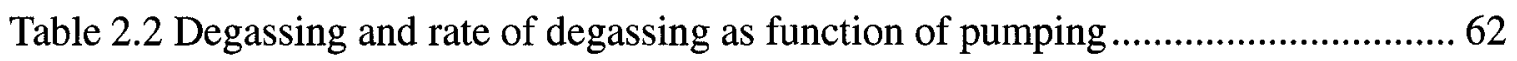

Table 4.1 Working fluid property change rate per unit temperature ............................ 244 


\section{Nomenclature}

A

$A_{D}$

$A_{E}$

$A_{\text {Per }}$

$A_{\text {piping }}$

$A_{v}$

$A_{w}$

$\dot{B}$

C

$C_{l}$

$C_{m p}$

$C_{\text {piping }}$

$C_{t r}$

$d_{w}$

$d_{\text {piping }}$

$d_{0}$

$d_{i}$

$d_{v}$

$h_{v}$

$f_{v}$

$f_{v} R e$

$f_{v} R_{\text {ev }}$

$F_{l}$

$F_{v}$

G

$J_{m}$

$k_{e . c}$

$k_{e . e}$ heat pipe cross-sectional area, based upon it external radius

degassing surface area $\left(\mathrm{cm}^{2}\right)$

effective evaporation area $\left(\mathrm{cm}^{2}\right)$

permeability area $\left(\mathrm{cm}^{2}\right)$

effective piping cross-sectional area $\left(\mathrm{cm}^{2}\right)$

vapour core cross-sectional area

wick cross sectional area

volume of gas impinging on unit area per unit time

conductance to gas flow

conductance to laminar gas flow

conductance to molecular gas flow

effective piping circumference

conductance to transition gas flow

wire diameter

effective piping diameter

pipe outer diameter

pipe internal diameter

vapour core diameter

vapour forced convection coefficient

drag coefficient for vapour flow

drag coefficient for circular vapour flow passage

drag coefficient for vapour flow in a circular passage

fractional coefficient for liquid flow

frictional coefficient for vapour flow

mass of gas incident on unit area surface per unit time

correction factor in molecular gas flow

effective thermal conductivity of liquid-saturated wick at condenser

effective thermal conductivity of liquid-saturated wick at evaporator 


\begin{tabular}{|c|c|}
\hline$k_{l}$ & thermal conductivity of liquid \\
\hline$k_{p}$ & thermal conductivity of pipe material \\
\hline$k_{v}$ & thermal conductivity of vapour \\
\hline$k_{w}$ & thermal conductivity of wick material \\
\hline$K_{m e s h}$ & wick permeability \\
\hline$K_{\text {Per }}$ & permeability coefficient \\
\hline$L_{a}$ & length of heat pipe adiabatic section \\
\hline$L_{c}$ & length of heat pipe condenser \\
\hline$L_{e}$ & length of heat pipe evaporator \\
\hline$L_{\text {eff }}$ & heat pipe effective length \\
\hline$L_{\text {eff.max }}$ & maximum heat pipe effective length \\
\hline$L_{p}$ & heat pipe length \\
\hline$L_{\text {piping }}$ & system effective piping length \\
\hline$M$ & molecular mass $(\mathrm{g} / \mathrm{mol})$ \\
\hline$n_{l . m}$ & layer number of the mesh \\
\hline$N_{m e s h}$ & mesh number, $1 / \mathrm{m}$ \\
\hline $\bar{p}$ & average pressure $\left(\mathrm{dyn} / \mathrm{cm}^{2}\right)$ \\
\hline$\Delta p$ & pressure difference $\left(\mathrm{dyn} / \mathrm{cm}^{2}\right)$ \\
\hline$p_{E}$ & evaporation pressure (Torr) \\
\hline$p$ & ambient pressure (Torr) \\
\hline$\Delta p$ & permeation pressure (Torr) \\
\hline$\Delta p$ & pressure variation after time, $\mathrm{t}$ \\
\hline$p_{u s}$ & ultimate pressure (Torr) \\
\hline $\bar{p}_{\min }$ & minimum average pressure \\
\hline $\bar{p}_{t r}$ & average transit pressure \\
\hline $\bar{p}_{t r, \max }$ & maximum average transit pressure \\
\hline $\bar{p}_{t r, \min }$ & minimum average transit pressure \\
\hline $\operatorname{Pr}$ & Prandtl number \\
\hline$P_{p m}$ & maximum available pump pressure \\
\hline
\end{tabular}


$Q_{b . \max }$

$Q_{c}$

$Q_{\text {c.max }}$

$Q_{\text {e.max }}$

$Q_{L}$

$Q_{L d}$

$Q_{L s}$

$Q_{g t}$

$Q_{E}$

$Q_{D}$

$Q_{P e r}$

$Q_{\text {s.max }}$

$r_{c}$

$r_{h . s}$

$r_{h . v}$

$r_{i}$

$r_{o}$

$R$

$R_{a}$

$R_{\text {te }}$

$R_{e}$

$\operatorname{Re}$

$R_{p . e}$

$R_{\text {p.e.axl }}$

$R_{v}$

$R_{v \cdot a}$

$R_{w . c}$

$\boldsymbol{R}_{\text {w.c.axl }}$

specific degassing rate

specific evaporation rate

heat flow rate

boiling limit on heat transfer rate

capillary transfer

capillary limit on heat transfer rate

entrainment limit on heat transfer rate

gas load relate to leakage

gas load relate to dynamic leak

gas load relate to static leak

rate of gas throughput

evaporation rate

degassing rate

gas permeation load

sonic limit on heat transfer rate

wick capillary radius

hydraulic radius of wick at vapour-wick interface

hydraulic radius for vapour flow

inside radius of pipe

outer radius of pipe

gas constant

thermal resistance in adiabatic section

thermal resistance in condensation section

thermal resistance in evaporation section

Reynolds number

thermal resistance of pipe wall at evaporator section

axial thermal resistance of pipe wall at evaporator section

thermal resistance for vapour flow

thermal resistance for vapour flow in adiabatic section

thermal resistance of heat pipe wick at condenser section

axial thermal resistance of heat pipe wick at condenser section 


\begin{tabular}{|c|c|}
\hline$R_{w . e}$ & thermal resistance of heat pipe wick at evaporator section \\
\hline$R_{\text {w.e.axl }}$ & axial thermal resistance of heat pipe wick at evaporator section \\
\hline$R_{p . a}$ & thermal resistance of pipe wall in adiabatic section \\
\hline$R_{w \cdot a}$ & thermal resistance of heat pipe wick in adiabatic section \\
\hline$R_{v, w . r a d}$ & radial thermal resistance between vapour and wick \\
\hline$R_{w, p . r a d}$ & radial thermal resistance between wick and pipe wall \\
\hline$R_{p . a m b . r a d}$ & radial thermal resistance between the pipe wall and ambient \\
\hline$R_{p . c}$ & thermal resistance of pipe wall at condenser section \\
\hline$R_{p . c . a x l}$ & axial thermal resistance of pipe wall at condenser section \\
\hline$R_{a}$ & general resistance in the adiabatic section \\
\hline$R_{p . e}$ & thermal resistance of pipe wall at evaporator section \\
\hline$R_{w . e}$ & thermal resistance of heat pipe wick at evaporator section \\
\hline$R_{w . c}$ & thermal resistance of heat pipe wick at condenser section \\
\hline$R_{p . c}$ & thermal resistance of pipe wall at condenser section \\
\hline$R_{\text {v. } a}$ & thermal resistance for vapour flow from evaporator to condenser \\
\hline$R_{H P . p}$ & thermal resistance based on pipe cross-sectional area \\
\hline$R_{w, r}$ & wick radial thermal resistance \\
\hline$R_{p . r}$ & pipe wall radial thermal resistance \\
\hline$R_{\text {tot.r.if }}$ & general radial thermal resistance at the front interface \\
\hline$R_{\text {tot.v. } a}$ & general radial thermal resistance in the vapour area \\
\hline$s_{f}$ & sticking coefficient \\
\hline$S$ & wire screen crimping factor, for copper wire screen mesh $\approx 1.05$ \\
\hline$S_{n}$ & nominal pumping speed (litre/s) \\
\hline$S_{\text {pump }}$ & pump port speed \\
\hline$t_{P e r}$ & permeation time \\
\hline$t_{p}$ & pipe wall thickness \\
\hline$t_{w}$ & wick thickness \\
\hline$t_{m e s h}$ & mesh thickness \\
\hline$T$ & temperature \\
\hline$T_{p . e}$ & evaporator pipe wall temperature \\
\hline$T_{p . c}$ & condenser pipe wall temperature \\
\hline
\end{tabular}




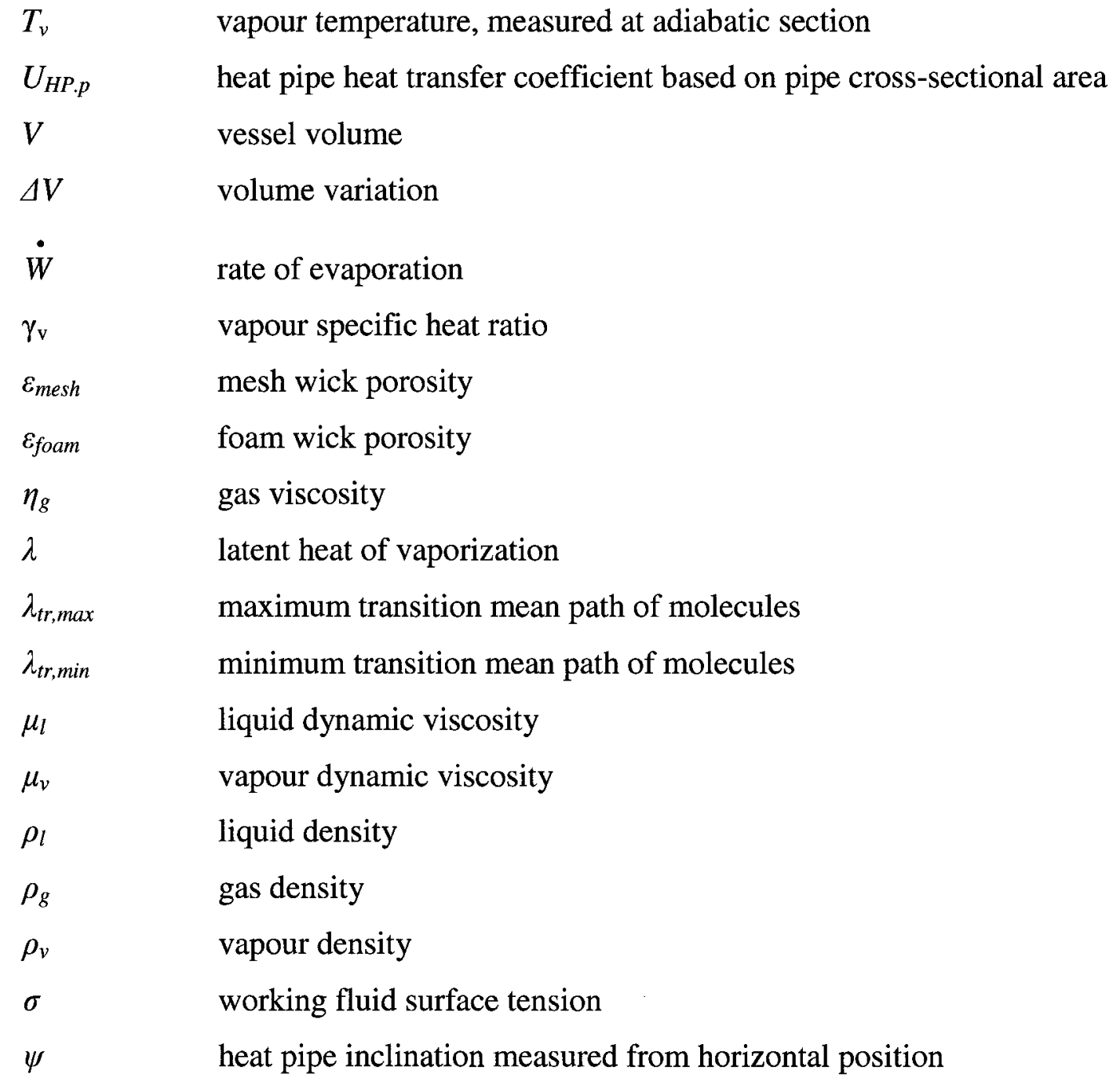




\section{Chapter 1 Introduction}

\subsection{Introduction to Heat Pipe Technology}

Heat pipes should be more accurately termed heat transfer pipes since they make use of a circulating liquid-vapour phase change to transfer heat rather than generate heat. The term "heat pipe" has been widely used, and evidently acceptable to preserve.

Heat transfer through phase change is a universal phenomenon, and can be observed in real life. A good example is the meteorological water circulation. First, heat is absorbed on the Earth's surface then liquid water evaporates into a vapour phase, and the warmed water vapour ascends into the troposphere. At a high altitude, the temperature is low enough for the water vapour to nucleate with the help of tiny solid particles, i.e., nucleate cores, such as dust. Hence, water transfers back to the liquid phase, forming liquid droplets. This is the so-called condensation process. During the condensation process, heat is dissipated and more and more vapour condenses, and the liquid droplets begin to steadily grow. When the water liquid droplets grow large enough they will drop back to the Earth's ground in the form of rain. This completes a liquid-vapour phase change circulation. In this circulation, two factors play driving roles. One is the temperature gradient between the Earth's surface and the high altitude troposphere. This factor provides buoyancy that drives the evaporating water vapour. The other one is the gravitational force that pulls the condensed water droplets back to the ground.

An early human application to transfer heat by using the liquid phase change is Perkins' boiler (Lock, 1992), which is the predecessor of the so-called thermosyphon. A problem with this specific heat transfer device is its dependence on inertial forces, e.g., 
gravitational or centrifugal forces. Without such inertial forces the thermosyphon cannot complete the phase change circulation and therefore ceases functioning.

To solve this problem, alternative approaches have been applied, the most commonly used is the capillary force. Capillary force occurs at the liquid-solid interface. It stems from the liquid surface tension and the solid geometric dimension. When the solid surface is not completely wetted the liquid would form a curved surface, thus forming a triple interface, i.e., solid, liquid, and gas. There are three forces to maintain the equilibrium state at the triple interface, i.e., the surface tension forces of the liquid and gas on the solid surface, and their body forces such as gravitational force. The balance among these forces determines the triple interface location. Classical thermodynamics deduces that liquid surface work is equal to the decrease of the liquid surfaces free energy, i.e., Helmholtz free energy (Myers, 1999). It also states that for a certain liquid at a certain temperature with a decreasing liquid surface area, the liquid surface will do more work to the external system. The variation of the surface free energy per unit surface area is defined as "surface tension". In a porous solid structure such as a wick with small pore dimensions, the solid-liquid interfaces have relatively small radii of curvature. Thus, the surface tension force would take a dominant role over the gravitational force. This force is termed "capillary force". The capillary force is proportional to the surface tension and inversely proportional to the pore radius. Based on this principle, the capillary force would drive the liquid without the help of inertial forces. In some cases, capillary forces may even go against the inertial forces depending on the liquid surface tension and the solid porous dimensions. A high liquid surface tension in a small dimensioned porous solid structure could drive the fluid against gravity. In a low 
pressure condition, e.g., vacuum, the liquid surface tension force would take a more advanced dominant position due to the elimination of gas. It is easier to permeate into the porous structure, and this is the so-called priming process as discussed by Dunn and Reay (1978).

The capillary force based phase change heat transfer approach generated the concept of heat pipe as mentioned at the beginning of this chapter. In 1938, the concept of capillary grooved liquid vaporizing tube was filed by U.S. Patent Office. In 1942, Gaugler first filed the heat pipe patent in the U.S.A. In 1963, Grover's patent was accepted by the U.S. Patent Office, even though seven other patents were cited as a result of the patent office search, including Gaugler's. In 1964, Grover filed the same patent in the U.K. (Dunn and Reay, 1978). Since then great progress has been made, and more types of heat pipes have been invented.

In the heat pipe development, the wick structure and materials are of major concern. In 1966, Levedahl filed the patent describing a heat pipe having axial grooves in the container wall. In 1969, Junkers filed a patent about a large number of arterial types of wicks which were suitable for heat pipes used in space. In 1971, Battelle Institute patented a parallel longitudinal capillary channel wick. In 1972, Brown et al filed sintered wick structure for heat pipe use. In 1973, the GE Company patented a radial holed wick for the heat pipe evaporation section. In 1975, Perkin-Elmer Corporation issued the patent of a high capability lobar arterial wick assembly (Dunn and Reay, 1978).

The main function of the wick in a heat pipe is to provide the capillary force to drive the condensed working fluid from condensation section (also referred to as the condenser) back to the evaporation section (also referred to as the evaporator), thus to 
complete the phase change circulation. The wick can also provide large areas for the evaporation and condensation sections to improve the evaporation and condensation processes. Also, wicks can assist in creating an even distribution of the evaporation temperature. Many wick structures and materials had been proposed and some of them have been used in practical applications. Peterson (1994) classified the wick structures into three categories: simple homogeneous, composite or non-homogenous, and advanced designs. The simple homogeneous wicks include wrapped screen mesh, sintered metal, and axially grooved structures, as shown in Figure 1.1 (a). To improve the flow and reduce flow resistance, the wicks with an open porous structure and a high permeability were required. Thus, the non-homogeneous wicks were invented. The existing nonhomogeneous wick structures include slab wick, pedestal artery structure, spiral artery structure, and tunnel artery structure, as shown in Figure 1.1 (b). To reduce the viscous shear force that occurs during the counter current liquid-vapour flow, advanced wick structure designs were applied to separate the liquid and vapour flow, these advanced designs include double wall artery structure, mono-groove structure, and channel wick structure, as shown in Figure 1.1 (c).

In parallel, several heat pipe manufacturing techniques have been developed. Euratom patented a groove manufacture technique for heat pipes in 1966 (UK 1125485) and a method for mesh forming and welding for heat pipes in 1969 (UK 1228103). In 1969, Metallgesellschaft Ag. patented a method for fabricating lithium/refractory heat pipes (UK 1194530). In 1970, the McDonnel Douglas Corporation patented a means for joining arteries in heat pipes having right angle bends (US 3620298). In the same year, Brown Boveri patented a method for porous wick manufacturing by means of vapour 
deposition (UK 1313525). In 1971, Q-Dot in the U.S.A. patented a technique for forming spirally grooved heat pipes using a special cutting tool (US 3753364), and in 1973 filed the same patent (UK 1433542) (Dunn and Reay, 1978). In 1975, the RCA Corporation obtained a Canadian patent for a method of making a heat pipe and wick (CA 960413). In 1979, Hydro-Quebec (Canada) was issued a patent regarding fabrication of ceramic heat pipes (CA 1066964).

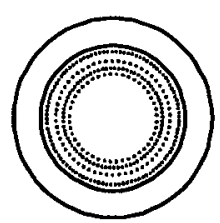

Wrapped screen

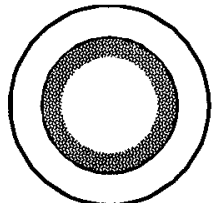

Sintered metal

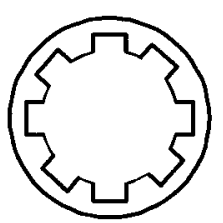

Axial Groove

(a) Simple homogeneous wick structures

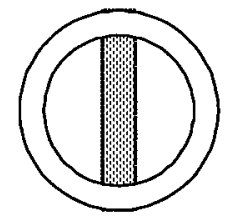

Slab

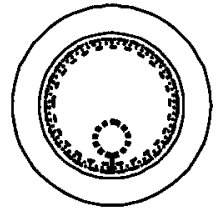

Pedestal artery

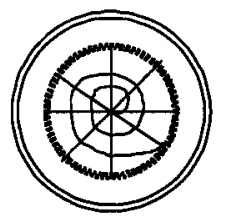

Spiral artery

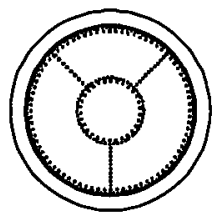

Tunnel artery

(b) Non- homogeneous wick structures

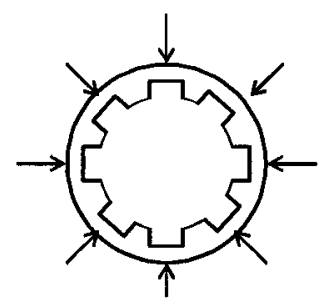

Axial groove (non-constant width groove)

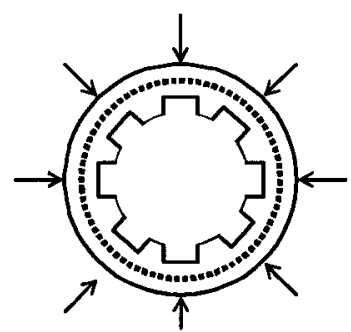

Double wall artery

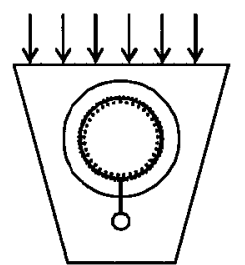

Mono-groove

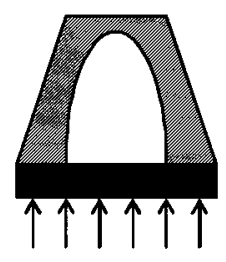

Channel wick

(c) Advance designed wick structures

Figure 1.1 Typical heat pipe wick configurations and structures. (Adapted from Peterson (1994)) 
Research on heat pipe manufacturing were introduced by Chi (1976), Dunn and Reay (1978), and Peterson (1994). Heat pipe manufacturing involves several steps of processes, namely the container tube machining, the materials and component cleaning, the wick forming and fitting in the container tube, the evaporation and fluid charging process, and the final sealing. With the increased maturity of manufacture techniques, heat pipe application has also entered into a wider application area.

A heat pipe can be divided into three sections in the axial direction; the evaporation, adiabatic, and condensation sections. The latent heat transfer mechanism makes the heat pipe distinct from heat transfer through a simple pipe, tube or solid rods. The heat transfer capacity of a heat pipe can be magnitudes greater than the best solid conductor with the same dimensions. With an appropriate length, a heat pipe can transfer heat with a very small axial temperature gradient compared with other solid counterparts having the same dimensions. The evaporation section can be adjusted by adjusting the condenser heat dissipating rate so a heat pipe can be used as an isothermal device. The capacity of transferring large heat fluxes through a relatively small area makes the heat pipe widely used in the thermal control of electronic components and systems. Once the heat pipe is started, its thermal response time is independent of heat pipe length. The only barrier to the fast response time is the conductive resistances at solid contact interfaces. This characteristic is useful in the temperature jump protection of electronics devices. Without moving parts, the heat pipe is free of mechanical vibration, and hence has a low maintenance requirement. Thus, it has a lower operation cost than other heat transfer devices. With the wick structure, the heat pipe is independent of inertial forces, including gravitational force and hence can work in all positions. This characteristic gives the heat 
pipe a unique feature for space applications. Also, the high ratio of heat transfer capacity to weight makes the heat pipe very competitive in the aeronautics industry. Heat pipe applications are also recorded in history. In 1965, high temperature heat pipes were used with a thermoionic converter for nuclear reactors, and were patented (US 3302042). In 1968, RCA filed a patent on heat pipe cooling of electronic tubes (UK 1183145). In 1970, the Q-Dot Corporation filed the patent on heat pipe air-to-air heat exchangers (UK 1255114). In 1970, heat pipes used to cool electronic components were filed for patent (US 3651865). Again, in 1970 TRW patented an isothermal furnace muffler (UK 1304771). In 1972, the GE Company patented cooling rotating machinery using rotating heat pipes (US 3788389) (Dunn and Reay, 1978).

Heat pipe applications can be classified by working temperature ranges. These ranges include: cryogenic, low temperature, medium temperature, high temperature, and high temperature with high melting materials (Ivanovskii et al, 1982). The cryogenic heat pipes are designed to operate in a temperature range from near $0 \mathrm{~K}\left(-273.14^{\circ} \mathrm{C}\right)$ to $200 \mathrm{~K}$ $\left(-73^{\circ} \mathrm{C}\right)$. Working fluids in this range include helium, argon, Krypton, nitrogen, oxygen, Freon, and ethane. This type of heat pipe has a very low heat transfer capacity due to the high viscosity, and high surface tension of the working fluid, and a small amount of vaporization. So this type of heat pipe has a very limited application region. The low temperature heat pipes are designed to work in the temperature range from $200 \mathrm{~K}\left(-73^{\circ} \mathrm{C}\right)$ to $500 \mathrm{~K}\left(227^{\circ} \mathrm{C}\right)$. Working fluids include Freon, ammonia, alcohol, acetone, water, and some organic compounds. Among these working fluids, water has very good thermophysical properties. This type of heat pipe can reach a higher axial heat transfer capacity than their cryogenic counterparts. These types of low temperature heat pipe have 
a wide application region. The medium temperature heat pipes are designed to work in the temperature range from $500 \mathrm{~K}\left(227^{\circ} \mathrm{C}\right)$ to $700 \mathrm{~K}\left(427^{\circ} \mathrm{C}\right)$. Working fluids include sulphur, mercury, alkali metals (caesium, rubidium), and some chemical compounds. This heat pipe shows a further increase in axial heat transfer capacity compared with the cryogenic and low temperatures heat pipes. The types of high temperature heat pipes are designed to operate at temperature above $750 \mathrm{~K}\left(477^{\circ} \mathrm{C}\right)$. Working fluids include potassium, sodium, lithium, lead, silver, indium, and other high metals with high melting temperatures. Among them, lithium is the most commonly used fluid, and can obtain a high axial heat flux of up to $1.5 \times 10^{8} \mathrm{~W} / \mathrm{m}^{2}$ or above. The high temperature heat pipes are usually used at the upper end of this temperature range; above $1300 \mathrm{~K}\left(1027^{\circ} \mathrm{C}\right)$. These heat pipes need to operate in a protected atmosphere, e.g., vacuum, inert gas. The greatest problem is the short working life due to the corrosion and high mass transfer (Ivanovskii et al, 1982).

\subsection{Background}

As introduced in the preceding section, as a high effective heat transfer device, the heat pipes have been applied in many areas. Because of the high ratio of heat transfer capacity to weight, the heat pipe takes a predominant position in the aeronautics and space applications.

In aeronautics applications, the nose and leading edge of wings can reach very high temperatures such as $1500 \mathrm{~K}\left(1227^{\circ} \mathrm{C}\right)$ with the flight speed approaching a high Mach number. Heat pipe techniques were applied to solve such problems and some experimental research was applied on this issue. In the early 1970's, several feasibility studies were performed to assess the application of heat pipes for cooling leading edges 
and nose caps of hypersonic vehicles. The NASA Langley Research Center (LaRC), through a contractual study, analytically verified the viability of heat pipes for cooling stagnation regions of hypersonic vehicles. In 1972, McDonnell Douglas Astronautics Co. (MDAC) compared four space shuttle wing leading-edge concepts: a passive carboncarbon concept, a passive coated columbium concept, an ablative concept, and a liquid metal/super-alloy heat pipe cooled concept. The heat pipe cooled concept was determined to be feasible and durable but was slightly heavier than the other candidate concepts. In 1973, MDAC fabricated a half-scale shuttle-type heat pipe cooled leading edge to verify feasibility of the concept. This model was tested by a series of radiant heat and aerothermal tests at NASA LaRC from 1977 to 1978 to verify heat pipe transient start-up, and steady state performances. In 1979, MDAC received a contract to optimize a heat pipe cooled wing leading edge for a single stage to orbit vehicle. Results of the follow up study indicated that the mass of a shuttle-type heat-pipe cooled leading edge could be reduced by over 40 percent if using of a more efficient structural design. In 1986, MDAC received a contract to design and fabricate a sodium/super-alloy heat pipe for an advanced shuttle-type vehicle. This advanced shuttle-type heat pipe was 6 feet long and was tested at MDAC by radiant heating and at Los Alamos National Laboratory (LANL) by induction heating (Glass et al, 1998). In a NASA research project, Camarda (1978) conducted the experimental investigation on the heat pipe cooled leading edge at Mach 7. In the experiments, a sodium filled Hastelly ${ }^{\circledR} \mathrm{X}$ heat pipe was involved. With this heat pipe, a leading edge cooling model was established, which had previously been tested in a radiant heating environment. It was subjected to steady state, earth entry aerothermal loads in the Langley 8-foot high temperature structure tunnel to verify performance in a 
realistic hypersonic environment. The experimental results indicated that heat pipes can accommodate very intense localized heating and effectively isothermal structural components. Specifically, results of the tests indicate that the use of heat pipes for leading edge cooling is a feasible method for lowering localized stagnation temperatures of hypersonic cruise vehicles and space transportation system vehicles sufficiently to allow the use of available and durable super-alloys for such applications (Camarda, 1978).

In another NASA research project, Glass et al (1998) conducted the fabrication and experimental investigation of the leading edge shaped heat pipe. A leading edge shaped heat pipe was successfully fabricated. In the test, the heat pipe did operate as a heat pipe over a portion of its length. However, designed temperatures and heat fluxes were not obtained due to premature failure of the heat pipe resulting from electrical discharge between the induction heating apparatus and the heat pipe.

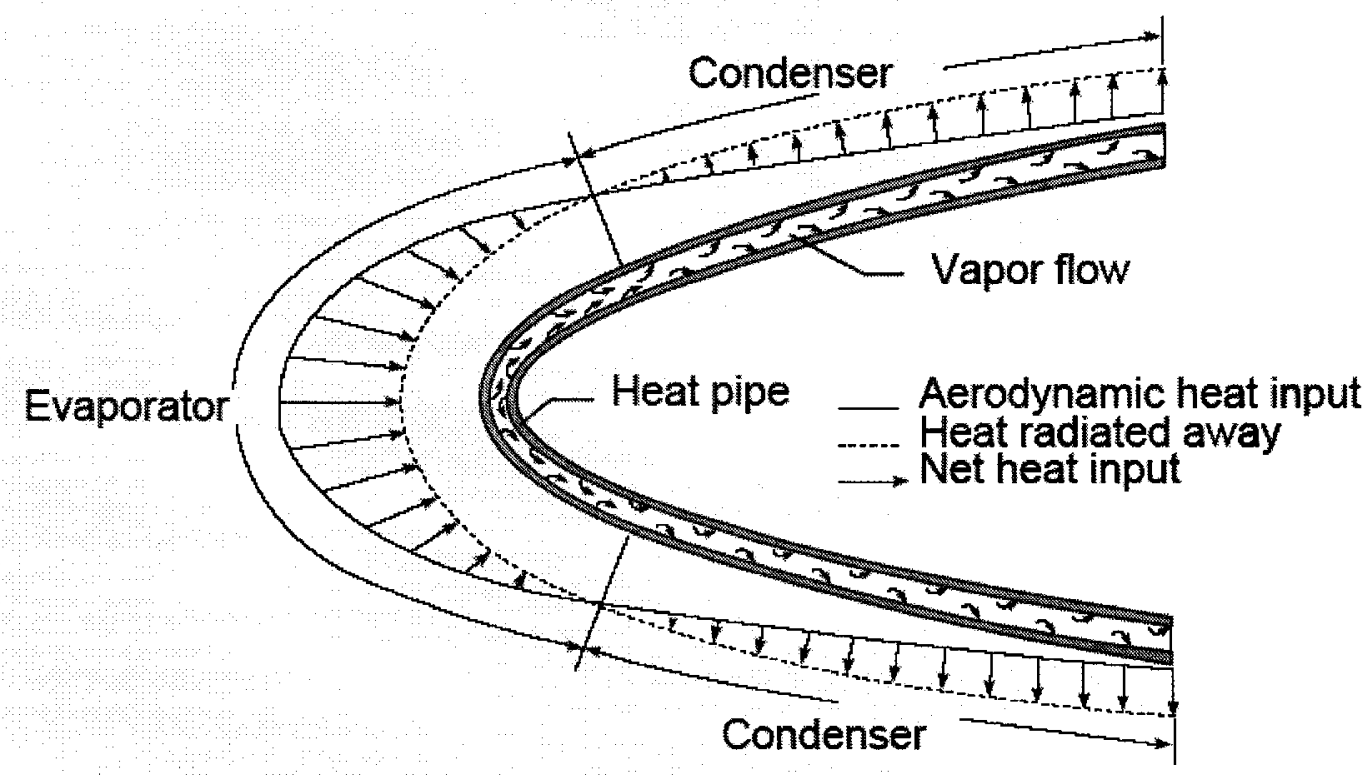

Figure 1.2 Schematic diagram of a heat-pipe-cooled leading edge showing regions of net heat input (evaporator) and net heat output (condenser) (Glass et al, 1998) 
As shown in Figure 1.2, the heat pipe had a "D-shaped" cross section and was fabricated from arc cast molybdenum rhenium. The artery was fabricated by wrapping three layers of $400 \times 400$ mesh Mo-5Re screen around a $0.125 "$ outer diameter steel mandrel which was held in place with a $0.003^{\prime \prime}$ diameter steel wire that spirally wrapped the length of the artery. The screen wick was fabricated by wrapping the first two layers of $400 \times 400$ mesh screen over a copper mandrel that had been machined to the wicks final shape. The assembly was then slid off the copper mandrel. Portions of the screen wick were removed in the nose portion of the wick. This allowed the screen wick to bend and still maintain a continuous artery through the nose section of the heat pipe. The nose section of the wick was formed by bending the wick assembly on the machined parts that form the nose section. An artery was included in the wick. Several issues were resolved during the fabrication of the heat-pipe container and wick with a sharp-leading-edge radius. After the heat pipe was assembled, $7.5 \mathrm{~g}$ lithium was charged into the heat pipe by distillation. The heat pipe was placed in a vacuum furnace at $1743^{\circ} \mathrm{C}$ for 53 hours. The heating device was designed to heat the entire width of the flat portion of the heat pipe for a chord length of $1.6^{\prime \prime}$ in the nose region. Initial tests heated the heat pipe to $927^{\circ} \mathrm{C}$, resulting in a heat flux of approximately $4.11 \times 10^{6} \mathrm{~W} / \mathrm{m}^{2}$. The heat pipe was tested in a vacuum chamber using induction heating and was started up from the frozen state several times. The pipe operated as a heat pipe over a portion of its length. However, the desired design temperatures and heat fluxes were not obtained due to premature failure of the heat pipe resulting from electrical discharge between the induction heating apparatus and the heat pipe (Glass et al, 1998). 
Another aeronautic application for heat pipes is in gas turbine cooling. As a thermal engine, gas turbines involve many aspects of heat transfer such as compressor inlet cooling and inter cooling, combustor wall cooling, guide vane and turbine blade cooling, turbine reheating, recuperation or regeneration. Much research about heat pipe for gas turbine applications has been conducted. Cao (2000) reported the experimental research on turbine blade cooling by micro/miniature heat pipes. In experimental studies, a set of high rotating speed test apparatus was used. The experiments mainly studied such aspects as operation and start-up characteristics of high temperature, radially rotating heat pipes. Effects of heat inputs, effects of cooling air flow rates, effects of dimensionless centrifugal forces, and effects of inner diameters of heat pipes were also studied. Heat pipes used were wickless with sodium and potassium as working fluids. And experimental comparisons were made between these two types of working fluids. The experimental results indicated that the combination of traditional air-cooling technology with radially rotating heat pipes is a feasible and effective approach to high temperature turbo engine blade cooling.

In space applications, heat pipes have also been widely used. The first flight of heat pipes took place in 1967. A stainless steel water heat pipe was applied on an Atlas-Agena vehicle and was launched into an earth orbit from Cape Kennedy (Dunn and Reay, 1978). In 1972, three different isothermal heat pipes, (i.e., axial grooved, spiral artery wick, and tunnel artery wick with ammonia working fluid) were used for thermal control in a large OAO-C (orbiting astronomical observatory). A gas-loaded ammonia heat pipe was also used on the OAO-C for cooling data process electronics (Chi, 1976). In 1973, the Hughes Aircraft Company and the NASA Langley Research Center conducted the experimental 
research on the heat pipe system for space shuttle traveling wave tube amplifier (TWTA). The heat pipe system consisted of a series of heat pipes with the container materials of stainless steel and aluminium. The wick structure was an axial groove, Refrasil (glass fibre) and Dynaloy, the fluids include DC-200 Hexafluorobenzene, $\mathrm{C}_{2} \mathrm{Cl}_{4}$, and Dow 4 were used. The system was tested at a $150 \mathrm{~W}$ heat load having a $3.7 \mathrm{~W} / \mathrm{in}^{2}\left(0.57 \mathrm{~W} / \mathrm{cm}^{2}\right)$ thermal density in a temperature range of $-20^{\circ} \mathrm{C}$ to $150^{\circ} \mathrm{C}$. The experimental results proved the heat pipe system was effective for the TWTA thermal control (Basiulis et al, 1973). In 1979, Ward et al reported the development of a heat performance thermal structural heat pipe. In that research, a new high performance axial-groove heat pipe had been developed. A trapezoidal axial-groove, octagon-exterior-shaped pipe was made and tested using an ammonia working fluid. The 0 -g heat transfer capacity exceeded the expected performance. However, asymmetric heating effects were shown to have a significant impact on the heat pipe performance at elevated power levels in a $1-\mathrm{g}$ environment. Savage et al (1979) reported the heat pipe experiment on ASTRO-8 (ii). In that experiment, four constant conductance artery, stainless-ammonia heat pipes were involved for investigation of 0 -g performance. One of the high power heat pipes experienced an early burn-out; and the other high power heat pipe showed higher evaporator temperatures than predicted. The heat pipe performance anomalies had been tentatively attributed to incomplete wick priming. The other two lower power heat pipe performed as predicted. In 1982, Basiulis reported design, fabrication, and tests of liquidmetal heat-pipes in a sandwich panel assembly. In this report, the fabrication and tests of an integral heat-pipe and sandwich panels were introduced. These tests synergistically 
combine the thermal efficiency of heat pipes and the structural efficiency of honeycomb sandwich panel construction.

Thermal issues relating to the high speed applications stem from the high heat flux and supersonic speeds. This requires that the heat pipes should operate in a high temperature range, e.g., 800 to $1500 \mathrm{~K}\left(527\right.$ to $\left.1227^{\circ} \mathrm{C}\right)$. They should also operate at a high heat flux of approximately $4 \times 10^{6} \mathrm{~W} / \mathrm{m}^{2}$. The corresponding approach usually applies liquid-metal based heat pipes. The thermal issues relating to the spacecraft cooling come from the compacted craft structure. Lack of convection is due to the weak gravity and natural air, the thermal contrast, and high heat density of the electronics. The essential focus is on the protection of the electronic instruments in the spacecraft. These characterize a static heat condition and relate to a conductance approach. This raises the need for transferring heat to radiative surface without power driven pumps. Heat pipe techniques are suitable approaches.

As the scale of circuit integration continues to increase, the chip heat fluxes grow up to $100 \mathrm{~W} / \mathrm{cm}^{2}\left(645 \mathrm{~W} / \mathrm{in}^{2}\right)$. Hence, the heat pipe approach to electronics cooling has been spreading into civil applications. Peterson reviewed the development in this application, and stated that the thermal control of electronic components has one principal objective, to maintain relatively constant component temperature equal to or below the manufacturer's maximum specified service temperature. Also, Peterson classified the heat pipe application in the electronics cooling into indirect and direct heat pipe approaches (Peterson, 1994).

The indirect heat pipe control is to mount the heating source components on the evaporator portion of the heat pipe and the heat is rejected through the heat pipe to a 
coolant, e.g., gas or liquid. This is done by either free or forced convection at the condenser. In 1982 and 1984, a device called "heat kicker" was reported. It is a small copper water heat pipe with aluminium fins attached to one end and the heat block attached at the other end. That is a typical indirect heat pipe approach to electronics cooling. Since then various indirect heat pipe cooling approaches on electronics have been evaluated. In 1984, a thermal switch for space applications was reported. Also, a heat pipe designed to rest underneath a standard dual-in-line package (DIP) was reported. Also, the concept of micro heat pipes was proposed. That is, a wickless heat pipe with a very small cross sectional area where the mean curvature of the vapour-liquid interface is a magnitude comparable to the reciprocal of the hydraulic radius of the flow channel. In 1988, a finned high power semiconductor was reported. Also, a flat plate heat pipe was reported. That is constructed from $1 \mathrm{~mm}$ copper sheet, 100 mesh copper screen, and water as the working fluid. In 1990, a combined analytical and experimental investigation was conducted on a micro heat pipe with a trapezoidal configuration. The tested heat pipes had lengths of $57 \mathrm{~mm}$ and cross-sectional areas of $1 \mathrm{~mm}^{2}$; they were constructed from both copper and sterling silver with ultra pure water as working fluid. The indirect heat pipe approach is a combination of the heat pipe and the electronic chips to be cooled. The manufacturing of heat pipe and electronic chips can be conducted separately, therefore makes it much easier to standardize, thus providing lower cost. However this approach is characterized as solid contact heat transport between the heat pipe evaporator external wall surface and the electronic chips surface. Thus, the thermal contact resistance becomes the bottleneck of the entire heat pipe thermal network. There is a limit on high power and high heat rejection applications required from electronic devices. 
The direct heat pipe approach, on the other hand, immerses the devices in a dielectric fluid, thus eliminating the contact thermal resistance outside the device (Peterson, 1994). This approach brought new problems. The first and the most important one is the critical heat flux; the maximum permissible level of the evaporator heat flux. Beyond this critical heat flux, the vapour would blanket the heat source, thus resulting in overheating, and eventually dry-out. The second one is the collapse of vapour bubbles that may cause vibration to the device. The third one is that the vapour bubble may decrease the electric breakdown voltage of the dielectric fluid (Peterson, 1994). Two approaches can be applied to solve these problems. One is to integrate the device as part of the wick structure to ensure the liquid fluid keeps contact with the device surface. In 1978, this concept was expended, and used to investigate the inclusion of electronic devices as an integral part of a heat pipe wick. This approach overcomes the problems of material performance and electrical compatibility and was a means to substantially reduce the junction temperature. In this experiment, several types of wicking materials including glass fibre bundles and powder wicks were investigated. Difficulties were encountered in applying the fibre bundle wick to microcircuits due to numerous lead wires. To solve this problem, a new wick consisting of eight layers of polyester cloth was used, and the working fluid was pentane. The experimental result illustrated that the total package resistance is a strong function of the percentage of working fluid present. The best performance occurred when 52 percent of the volume of the pore was filled with working fluid. At that level, the total package resistance was approximately $0.2 \mathrm{~W}$ over a heat flux range of $10-27 \mathrm{~W} / \mathrm{cm}^{2}$. The second approach to the vapour bubble impact on the device is the thin film evaporation technique. In 1980, a closed miniature thermosyphon 
was proposed using this technique. From 1986 to 1986, fundamental investigations of this device were conducted. The primary results indicated that an evaporating thin circular liquid film in the shape of an extended meniscus combined with condensation within a small volume acts as an effective heat spreader. The effects of both NCGs (noncondensable gases) and fluid flow in ultra thin film in the presence of evaporation were also investigated (Peterson, 1994).

\subsection{Objectives}

Heat pipe technology continues developing in the direction of high operation temperature and high power capacity. In addition, technologies tend to use micro machining techniques in the areas of microelectronics applications. In recent years, the design theories have been maturing, and new approaches have been proposed, e.g., new wick structures and new materials. New designs have also been conceived, e.g., wickless heat pipe, looped heat pipe, oscillation heat pipe, osmotic heat pipe. New wick manufacturing techniques have also been developed, e.g., sintering wick metals. However, all these heat pipe configurations must go through the evacuation and fluid charge process. These processes are critical in heat pipe manufacturing. The quality of evacuation and charging directly affects the heat pipe performance. There have been many published materials discussing this issue. Chi (1976), Dunn and Reay (1978), and Peterson (1994) mentioned the vacuum process in the heat pipe manufacturing procedure, and also provided schematics for such process. However, these published materials did not provide specific information about this process. There are also lacks of theoretical discussions and analyses on this process. This is especially true for the evacuation process factors 
affecting this process as well as the corresponding solutions. The existing approach to the heat pipe assembly evacuation is a trial and error process that causes great uncertainty.

Vacuum as well as the material surface process play key rules in the heat pipe manufacturing procedure. These factors affect the fluid wetting on the metal surface, affects the fluid priming in the wick structure. Schrader (1992), Marmur (1992), Myers (1999), Kaviany (1991), Carey (1992), Dunn and Reay (1978) conducted research on these issues and will be used to analyze the vacuum process in the following chapter on specific factors.

Heat pipes need wick structures to acquire the capillary force. As illustrated in the introductory section, the wick dimension is one of the important factors determining the capillarity of a wick structure. However, a high porosity wick structure in the heat pipe assembly would bring difficulty to the evacuation process.

This thesis work aims to conduct an investigation on heat pipe manufacturing, especially the evacuation process; and to apply theoretical analyses to the evacuation process. The main objectives for this thesis work can be summarized as follows:

1. Establish laboratory facilities for heat pipe fabrication and testing.

2. Conduct a survey of the existing heat pipe manufacturing procedures.

3. Develop a preliminary theoretical analysis approach to the heat pipe evacuation process.

4. According to the initial designs, make a series of heat pipes with different configurations.

5. Perform tests regarding the heat transfer capacity and temperature characteristics of heat pipes. 
6. Develop a mathematical model to verify the heat pipes performance by comparison of the calculated and measured results.

7. Summarize results and lay the foundation for future practices such as looped heat pipe manufacturing and developing, etc.

In this thesis work, copper was chosen, including container tubes and wire screen mesh because it has good thermal conductance characteristics. It is also easy to machine and is easily available. A piece of sintered nickel foam sheet was also utilized as a wick material.

\subsection{Organization}

This thesis presents studies of manufacturing and experimental investigation of conventional copper heat pipes. It is organized as follows:

Chapter 1 Introduction: introduces heat pipe concept and a brief development history. Provides the objectives, and presents the scope of the thesis.

Chapter 2 Heat Pipe Manufacturing Process: presents the primary design considerations, introduces the manufacturing process, and theoretically analyzes the evacuation process.

Chapter 3 Experimental Investigation of Heat Pipe Characteristics: provides details of the experimental investigation including a description of the test specimens, test apparatus, and experimental procedures. The results are presented and discussed.

Chapter 4 Mathematical Modeling and Experimental Verification: presents the mathematical analyses on the heat pipe thermal network, heat transfer capacities; compares theoretical predictions and experimental results. Also, establishes mathematical modeling to present the heat pipe effective length factor. 
Chapter 5 Conclusions and Recommendations: provides conclusions, points out some problems that occurred in the present work, recommends corresponding solutions, and discusses future work. 


\section{Chapter 2}

\section{Heat Pipe Manufacturing Process}

To conduct the study on heat pipes as well as their performance, we carried out series of heat pipe manufacture in the laboratory. With these heat pipes, a series of experimental investigations on the performance of the heat pipe were conducted. This chapter mainly introduces the heat pipe manufacturing process.

\subsection{Primary Design Considerations}

Heat pipe designs are discussed in detail in many published articles. Besides the general rules of design, specific design concerns should be considered due to various configurations. In our application, the purpose is to analyze the heat pipe manufacturing process, and verify the corresponding effects by theoretical analyses. To conduct this, detailed specifications for the heat pipes to be fabricated and tested are required. The following sections introduce primary considerations on designs for heat pipe testing samples in our applications.

\subsubsection{Material Factors}

Material is a major factor affecting heat pipe performance. For a specific case, the material selection is the first concern in the design. Materials in heat pipes mainly include the working fluid, wick, and container.

The working fluid is a major factor determining heat pipe characteristics and must be first considered. Working fluid in the heat pipe serves as a thermal media transferring 
heat through phase changes. Requirements for working fluids mainly include its chemical and physical properties. First, the fluid must possess a very good chemical stability; it should not decompose within the heat pipe working temperature range. Also, the working fluid should not react with the container and wick materials under any circumstances. For the physical properties, the working fluid should possess high latent heat, high thermal conductivity, high surface tension and low viscosity. Many working fluid options are suitable for our case, e.g., water, acetone, ethanol, and methanol. These liquids have chemical stability under $150^{\circ} \mathrm{C}$. Compared to other chemical liquids, water has a high latent heat and surface tension, and is not toxic. In our case, water is the first choice while the final decision needs to consider its compatibility with container and wick materials. Acetone has a lower latent heat than water but also has a lower boiling point than water. This gives the heat pipe with acetone working fluid a lower start-up temperature. It is therefore more suitable for electronic devices.

Wicks in heat pipes provide the function of pumping the working fluid to the evaporation section. The main requirement for wicks is high pumping capacity. This capacity relates to three factors; the amount of the fluid to be pumped, the geometric dimensions of the wick, and time. These three factors are affected by other conditions such as fluid physical properties and temperature. This section mainly analyzes the wickrelated factors affecting the pumping capacity. The pumping capacity is a synergistic action resulting from the wick capillarity and permeability. Capillarity is determined by the physical and geometric properties. Physical factors relate to the material, the surface finish, and the compatibility of the wick and the working fluid. The geometric factors include the capillary channel shape and size. To provide a capillary function, the wick 
must possess micro channels through which liquid flows. The shape and size of the channels must ensure that the fluid inside is not able to form independent droplets by surface tension. Instead, the fluid is pulled throughout the channels by surface tension. To realize such a mechanism, the channels must have a size small enough to hinder the fluid forming droplets in the channel. Being unable to form droplet, the fluid can not reach a pressure balance at the surface, it is then pulled by surface tension. As long as this unbalance exists, the moving fluid will continue to experience this wick pumping mechanism. Based on this analysis, the required channel size is determined by the fluid surface tension and the liquid viscosity. At a given temperature, the liquid has a higher surface tension would form larger droplet, thus allowing a large wick channel size. For a lower surface tension liquid, a smaller size channel is required to hinder the liquid forming droplets. However, the liquid surface tension and viscosity vary with working conditions such as temperature. With an increasing working temperature, the liquid surface tension and viscosity decrease. Thus, a smaller size of the wick channels is required to maintain the pumping function. However, with a decreasing wick channel size, the permeability decreases. So there is an optimum size for a wick in a given heat pipe configuration. There are many existing wick materials such as wire screen mesh and sintered metal foam. To select the right wick material, we need to consider the choice of the working fluid. Besides capillarity, wicks also require a good thermal conductivity, good heat stability, and good manufacturability. In this thesis, we primarily focus on copper material wicks. To make comparisons, a nickel based wick was also used in some cases. 
A heat pipe container acts as an isolating, heat transferring, and protecting medium. The container material must possess vacuum tightness to maintain the vacuum condition in the heat pipe. As will be discussed later in this chapter, the main factors destroying a vacuum condition include leakage and gas permeation. Leakage usually occurs in three circumstances; the raw material defects (cracks), sealing defects in the manufacturing process, and damage during operation. Leakage is a macro concept, it mainly corresponds to vacuum failures in an atmospheric environment, and the leaked gases involve all composition in the air. Leakage is avoidable by applying the right raw materials, right process techniques, and working in normal conditions. The gas permeation rate is determined by the material and the types of gases. Theoretically, all materials would be penetrated by specific gases, e.g., helium or hydrogen, avoiding penetration is neither possible nor unnecessary. In heat pipe applications, the most common container materials are metals due to high thermal conductivities. For the majority of air molecules, the permeations to metals are very limited and can be ignored. The major concern is leakage, and this issue relates to the sealing techniques, as will be discussed later in this chapter. At this point of view, vacuum tightness is one of important criterion for the container material selection is. The degree of vacuum required in the heat pipe application is in a medium level, the majority of metals possess vacuum tight capacities for heat pipe applications in this level.

The second function of the heat pipe container is transferring heat. This requires that the container provide good thermal conductance. All metals possess high thermal conductivities compared with the non-metal counterparts, e.g., Teflon $\left(0.35 \mathrm{~W} / \mathrm{m}^{\circ} \mathrm{C}\right)$, glass $\left(0.78 \mathrm{~W} / \mathrm{m}^{\circ} \mathrm{C}\right)$, rubber $\left(0.15 \mathrm{~W} / \mathrm{m}^{\circ} \mathrm{C}\right)$; and a few materials possess higher thermal 
conductivities, e.g., (at $\left.100^{\circ} \mathrm{C}\right)$ silver $\left(415 \mathrm{~W} / \mathrm{m}^{\circ} \mathrm{C}\right)$, copper $\left(379 \mathrm{~W} / \mathrm{m}^{\circ} \mathrm{C}\right)$,, aluminium $\left(206 \mathrm{~W} / \mathrm{m}^{\circ} \mathrm{C}\right)$ nickel $\left(83 \mathrm{~W} / \mathrm{m}^{\circ} \mathrm{C}\right)$ and carbon steel $\left(36 \mathrm{~W} / \mathrm{m}^{\circ} \mathrm{C}\right)$.

Another function of the heat pipe container is protection of the internal wick. This requires that the container possess enough mechanical strength. In order to get a good thermal conductance, the heat pipe is usually attached to the heating surface by a strong pressing force. The container should be able to normally function under such mechanical forces. The heat pipe internal vapour pressure could be many times higher than the external atmospheric pressure. Therefore, the container should possess enough mechanical strength for such high pressure conditions. Heat pipes usually have long tube shapes. Such a shap, to a great extent, improves the capacity of the container to resist the internal high pressure. Existing metals used in heat pipe applications are stainless steel, aluminium, copper, and nickel alloys.

To function as a heat pipe, these three materials need to work in a compatible way. This relates to the liquid wettability, depending on the fluid physical properties and the metal surface finish as well as the working conditions, e.g., temperature, inner pressure. One important concern is the compatibility among the materials; relating to the chemical reaction issue. Chemical reaction incompatibility would cause two fatal results; corrosion and gas generation. Either of them results in a degradation and failure of the heat pipe. Details of this issue of degradation are discussed in many published materials.

The research of Dunn \& Reay (1978) indicates that water / copper, acetone / copper, methanol / copper are recommended based on past successful usage. A comprehensive durability test by Hughes Aircraft Co. also recommended water / copper, acetone / copper, and methanol / copper. However, they did not recommend water / 
nickel combinations. Chi (1976) investigated the fluid-solid compatibility, and confirmed the compatibility of water / copper, methanol / copper, and water / nickel.

All the research just mentioned confirmed the compatibility of water / copper, acetone / copper, and some of the research indicated the uncertainty in the compatibility of water / nickel and acetone / nickel.

Based on this consideration, material configurations are given as below:

1. Water and acetone as working fluids

2. Copper wire screen mesh and nickel foam as wicks

3. Copper tube as container.

\subsubsection{Heat Pipe Sizing}

To determine a diameter for a heat pipe, we need to consider three aspects; heat flux capacity, external heat transfer, and material availability. The final dimensions are determined by considering these aspects on either the container tube or the wick structure.

The first concern is the maximum axial heat flux that relates to the vapour core diameter. Equation (2.1) indicates the relationship between the vapour are diameter $d_{v}$ and the maximum axial heat flux $Q_{\max }(\mathrm{Chi}, 1976)$.

$$
d_{v}=\left(\frac{20 \cdot Q_{\max }}{\pi \cdot \rho_{v} \cdot \lambda \cdot \sqrt{\gamma_{v} \cdot R_{v} \cdot T_{v}}}\right)^{1 / 2}
$$

This equation indicates that there is a direct proportional relationship between the vapour core diameter and the maximum axial heat flux. To obtain a large axial heat flux, we need a big vapour core diameter, also corresponding to a big external diameter $d_{o}$. 
Another factor related to the diameter is the wick thickness. This will be discussed later in related contents.

Another concern is the heat pipe length, which is determined by the length of the inner wick. Detailed analysis will be conducted later based on these two factors, and considering the availability of the commercial copper tube. We selected two diameter configurations; 1/4" (6.35 mm), and 3/8" (9.525 mm) outer diameters. The details are available in Appendix I. For the $1 / 4^{\prime \prime}$ samples, soft and normal copper tubes were applied respectively; results will be introduced in Section 2.2.4.

Besides the factors related to the container; heat pipe dimensions are also determined by the wick. This will be discussed in the next section.

\subsubsection{Wick Design}

Wick selection is a major concern in heat pipe design. This topic relates to the aspects of capillary pressure, liquid permeability, vapour flow, and manufacturability.

The process of determining the wick dimension is a trial and error process. There are many options of the commercial copper wire screen mesh. We preliminarily selected 100 and 50 mesh, which means the mesh number is 100 and 50 per inch (3937 and 1968 mesh $/ \mathrm{m}$ ) respectively. Corresponding wire diameters are $0.11 \mathrm{~mm}$ and $0.23 \mathrm{~mm}$ respectively. The nickel foam wick has a thickness of $1.96 \mathrm{~mm}$ and a pore radius of 0.5 $\mathrm{mm}$.

Figure 2.1 illustrates the trial and error process of the wick design. The detailed calculation process will be illustrated in Chapter 4 . 


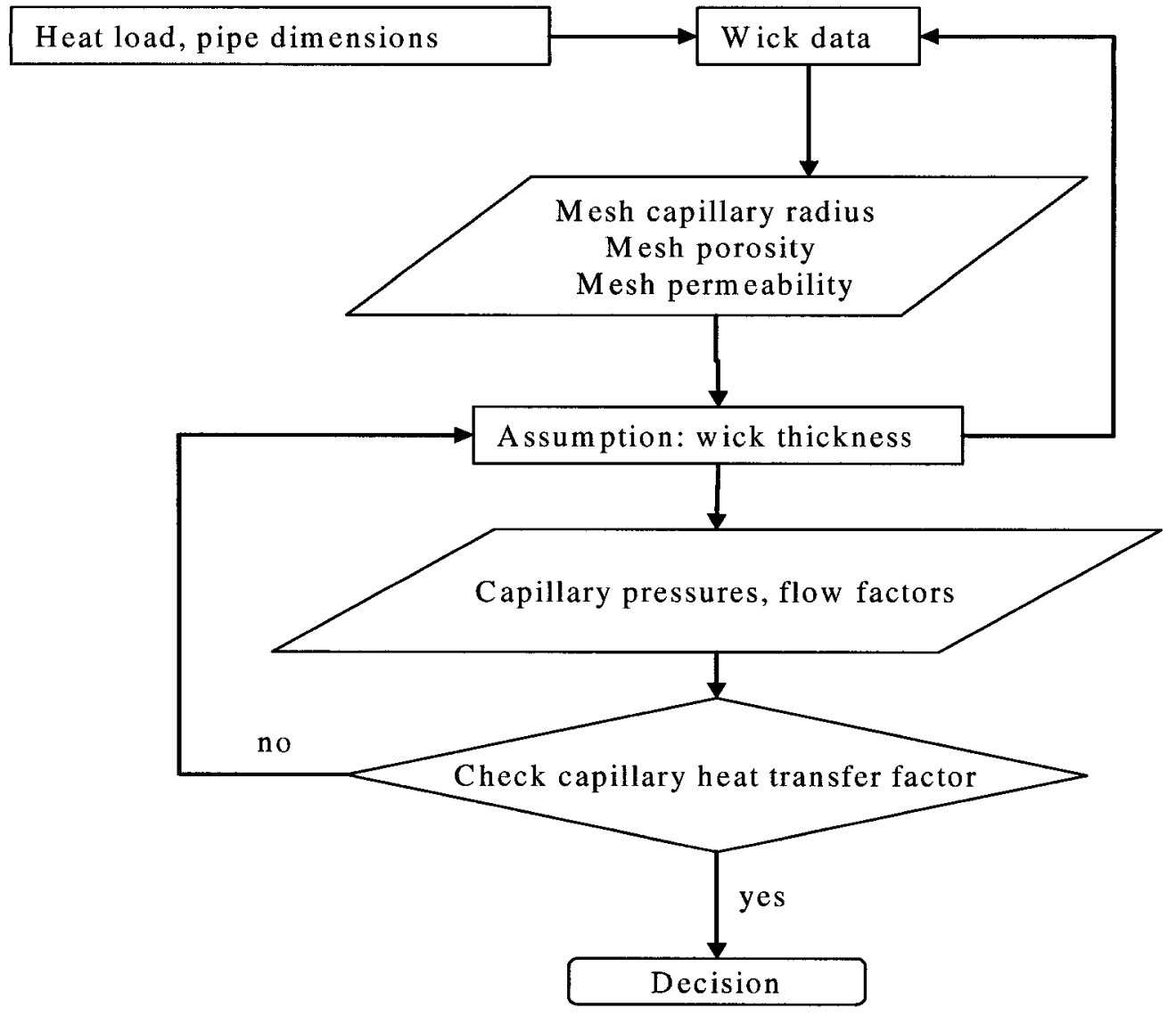

\section{Figure 2.1 Flow chart for heat pipe wick design}

The final calculation results of the wick dimensions are displayed in Appendix I. Also note that for different heat pipe configurations, preliminary calculations gave different dimensions. For simplicity in comparison, we took the same designed length for all $1 / 4^{\prime \prime}$ configurations. Table 2.1 shows the estimated heat transfer capacity based on the capillary limit for each configuration.

Table 2.1 Estimations of heat pipe configuration heat transfer capacity

\begin{tabular}{|c|c|c|c|}
\hline \multirow[t]{2}{*}{ Configuration } & \multirow[t]{2}{*}{ I.D. number } & \multicolumn{2}{|c|}{ Estimated capacity [W] } \\
\hline & & $\psi=0^{\circ}$ & $\psi=-90^{\circ}$ \\
\hline 100 copper mesh + water & $\begin{array}{l}\text { HP052502 } \\
\text { HP051701 }\end{array}$ & 48 & 135 \\
\hline 50 copper mesh + water & HP071200 & 125 & 592 \\
\hline $100+50$ copper mesh + water & HP071301 & 50 & 140 \\
\hline 100 copper mesh + acetone & HP071402 & 3.3 & 18.9 \\
\hline Nickel foam + water & HP062400 & 3740 & 9870 \\
\hline
\end{tabular}

Note: $\psi=0^{\circ}$, horizontal position. The evaporator and condenser locate at the same horizontal level.

$\psi=-90^{\circ}$, gravity assisted vertical position. The evaporator locates at the bottom; the condenser locates at the top position

$\Psi=+90^{\circ}$, against gravity vertical position. The evaporator locates at the bottom; the condenser locates at the top position.

These definitions are used throughout this thesis. Also, refer to Figures 3.30 to 3.32 


\subsection{Heat Pipe Manufacturing Process}

\subsubsection{Material Preparation and Treatment}

The material preparation includes selection, sizing and treatment of the container tube material, the wick structure and material, and the working fluid.

The first step is to cut the raw materials to a required size with a wheel tube cutter. This length is the sum of the calculated heat pipe working lengths, the margins for tube crimping at two ends, the margin spacing for the wick at two ends, and the margins for sealing at two ends. Margins for tube crimping depend on the size of the crimping tools. Margin spacing for the wick is to prevent the wick from being crimped flat at two ends; this length depends on the tube diameter. In this case, we gave the length of one tube radius, i.e., $3 \mathrm{~mm}$, to each end. After crimping, the deformed length at each end is actually about $5 \mathrm{~mm}$, but this deformed length only slightly affects the wick. The sealing margins depend on the valve connection space, crimping space and the sealing techniques. In our case, we selected a $35 \mathrm{~cm}$ margin for each heat pipe assembly. Wick cutting needs to be done carefully. The first cut determines the final size of the wick, so the operation must be accurate to $\pm 1 \mathrm{~mm}$. To do that, an accurate measurement must be applied, and clear marks must be traced on the wick material. To cut the wick material, we put the raw material on a flat hard wood board, and then cut the wick with a sharp knife against a steel ruler along the mark.

The second step is to machine the cut materials. The ends of the tubes require machining since the tube is cut by a wheel tube cutter, and therefore the end edges shrink in a radial direction. Specifically, recovery and refine the edge at the two ends with the help of a deburring tool. This will ensure the wick is easy to insert in later steps, and 
reduce the possibility of leaking after the sealing process. After this, the tube needs to be physically straightened. This process can be done with a pair of medium density fibreboards, each having a semicircular groove with the same radius as that of the copper tube. Put the tube in the two semicircular grooves, and then apply a pressing force with a clamp against the medium density fibre board. Moving the tube sections step by step and repeating the same process, the whole tube will be straightened. The wick material needs further refining. This process is mainly to remove the debris left in the cutting process. After this, the finished wick material needs to put between a pair of cleaned fibreboards with an applied force to keep it flat. Once these steps are completed, the tube and wick materials are ready for the next step; cleaning treatment.

The cleaning process is a very important step in the heat pipe manufacturing process. The cleaning quality not only affects heat pipe performance but also ensures the success of the heat pipe construction. There are four layers on the base metal surface; deformed hardened layer, oxide layer, absorbed gas layer, and the very top contaminant layer. Copper is known to be inert at normal temperatures and in dry environments. While in a humid environment, oxidization still occurs even at room temperature. The commercial copper products to some extent contain $\mathrm{CuO}$ on the surface. The oxides may form a wick plugging sludge after some time (Chi, 1976). Copper is a strong gas adsorbing metal, and can be used for gas adsorption in some applications, e.g., copper adsorption trap that will be discussed in Section 2.2.3. Also the commercial copper products have an absorbed gas layer on the surface. This layer of gas could be released or react with the working fluid, thus causing heat pipe degradation after some time, or even failure. Besides the gas releasing, the adsorbed gas would affect the heat pipe material 
wetting characteristic. Since adsorbed materials on a solid metal surface may cause its wetting characteristics to deviate significantly from those for a perfectly clean surface. The system whose operation is sensitive to liquid wetting characteristics, should be free from these absorbed substances (Carey, 1992). The contaminant layer is mainly composed of foreign matters that form the environment, e.g., dust, solid particles, oils, greases. Solid particles would plug the wick, thus decreasing the wick porosity and permeability. Dust can sometimes be trapped in the wick. Some dust would generate gases or react with the working fluid at the working temperature. Oils and greases can increase the fluid contact angle on the metal surface, thus worsening the fluid wettability on the metal surface. These contaminants would degrade the heat pipe performance or even cause failure. These layers must be removed from the materials prior to assembly. The contaminants are easily removed. The gas absorption on the metal surface can be classified into physical and chemical absorptions. The physical absorbed gases on the metal can be removed in the evacuation process and will be introduced in Section 2.2.3. The chemical absorbed gases need chemical treatments before the process of evacuation. Many methods of cleaning heat pipe materials are introduced in published materials. Dunn \& Reay (1978) introduced a pickling process for stainless steel materials, they suggest that "If the working fluid is a solvent, such as acetone, no extreme precautions are necessary to ensure good wetting, and an acid picking following by a rinse in the working fluid appears to be satisfactory." And "It is important, particularly when water is used as the working fluid, to avoid skin contact with the heat pipe components. Slight grease contamination can prevent wetting, and the use of surgical gloves for handling is advisable." (Dunn \& Reay, 1978) 
Chi (1976) introduced more detailed cleaning approaches for specific contaminants. For example, in the degreasing process, vapour, solvent treatment, or emulsifiable solvent treatment can be applied. In the solid particle removal process, alkaline bath treatment in spray, solvent circulation and brushing, mechanical agitation, or chemical passivation can be applied. In the deoxidizing process, acid bath treatment, chemical reduction, or hydrogen reduction can be applied.

Peterson (1994) introduced a more specific cleaning method for copper with water, acetone, or methanol configurations. He also recommended the use of solvents such as acetone or methanol as a working fluid that typically does not require complicated or involved cleaning processes. However, if water is to be used, care must be taken to ensure good wetting. One simple cleaning is to use a mild acidic solution as a cleaning agent in conjunction with vigorous brushing. This should be followed by several through flushing and rinsing cycles with water or the working fluid to be utilized. The specific method is given as below:

1) Soak and rinse the surfaces with an acetone solution to remove oil, grease, etc.

2) Soak in an alkaline solution of 50 percent phosphoric acid, 50 percent nitric acid solution at room temperature for 15 minutes.

3) Rinse with a pure working fluid and let soak at $50^{\circ} \mathrm{C}$ for 1 hour.

4) Repeat with a final rinse with a pure working fluid and then dry thoroughly with clean, dry air or nitrogen.

In our practical case, we adapted Peterson's approach, which is summarized below:

1) Clean the copper tube and wire screen mesh wick with clean compressed air. 
2) Soak the copper tube and wick in acetone at room temperature for 15 minutes.

3) Air dry the copper tube and wick at room temperature.

4) Soak the copper tube and wick in the solution of $120 \mathrm{cc}$ water $+20 \mathrm{cc}$ phosphoric acid $(86.6 \%)+20 \mathrm{cc}$ nitric $(68-70 \%)$, at room temperature for 20 minutes. Rinse with water.

5) Repeat Steps 1 to 3.

6) Assemble the treated materials and apply protection. (detailed procedure will be described in Section 2.2.2)

Note that, after the acid treatment, some blocked materials were found to float in form of film on the waste acid liquid surface. From the colour characteristics, this solid floating film is believed to contain copper oxides as well as other foreign matters. For the nickel foam wick, we applied the 10 percent nitric acid solution, as recommended by Dunn \& Reay (1978), and did not find any solids in the waste acid.

After completing the material treatment processes, we can turn to the next process; component assembly.

\subsubsection{Component Assembly}

This process is applied after the cleaning process. It is done at this stage to avoid being re-contaminated in a separate assembly process. After Step 5 as introduced in the preceding section, the cleaned copper tube and wick can be immediately applied to the assembly. First, hands and the entire tool workbench must be cleaned with alcohol. It may be necessary to use acetone for this step. Note that to avoid contaminating the components and to protect our hands, it is necessary to use surgical gloves. The main tool is an aluminium mandrel with the same outside diameter as that of the designed vapour 
core. Roll the copper wire mesh against the aluminium mandrel firmly, and then carefully try to insert the rolled copper mesh into the copper tube. Figure 2.2 shows the mandrels used in our applications.

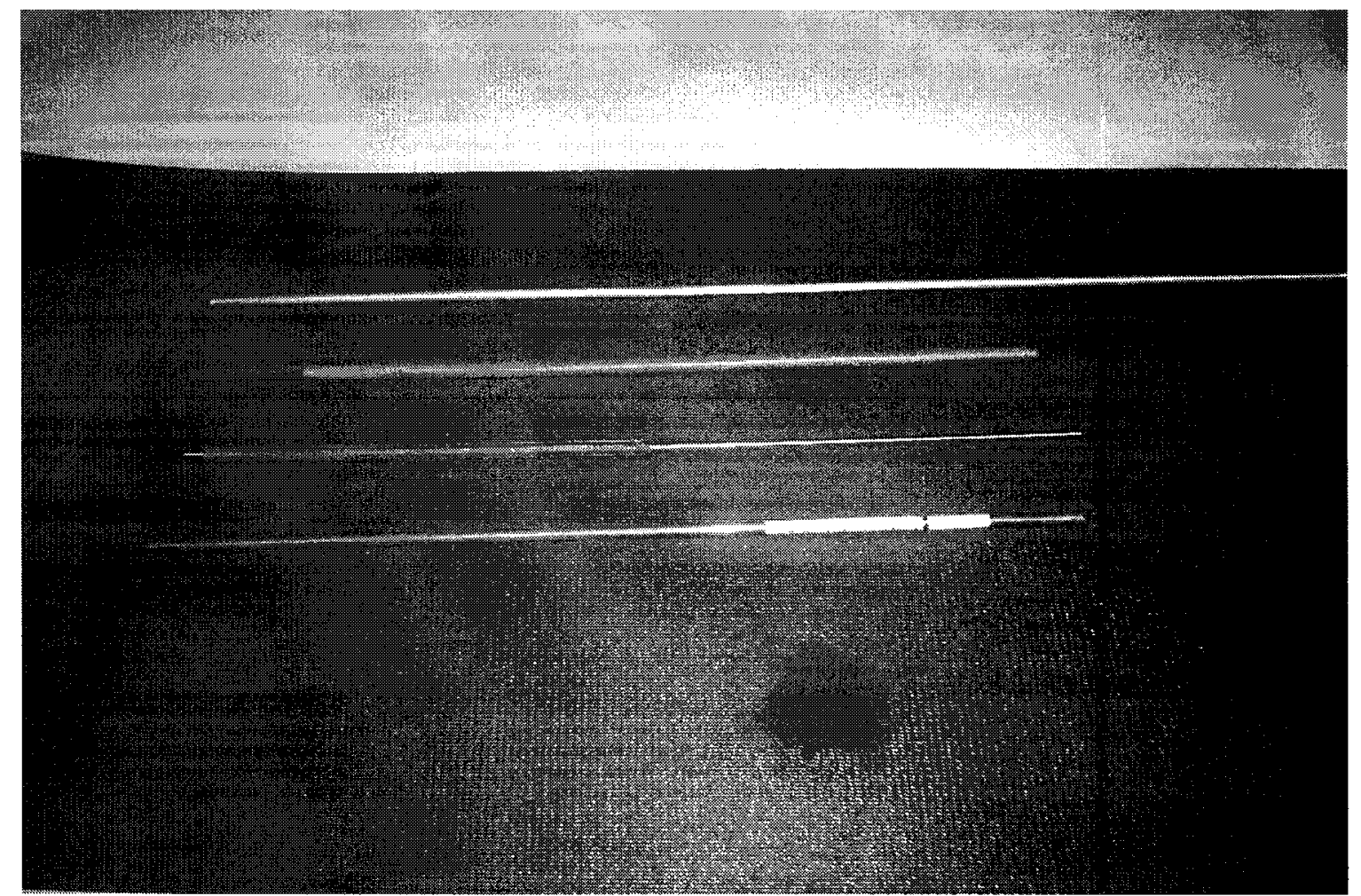

\section{Figure 2.2 Mandrel tools for wick insertion}

Care must be taken since after the acid treatment, the copper surfaces become dull, and this can make mesh insertion very difficult. A very tight roll of the mesh would be easier to insert, but would cause a big gap between the tube inner wall and the mesh roll, thus resulting in a high radial thermal resistance in the heat pipe. The mandrel plays a key role here, with an accurate mandrel diameter it is easy to get an appropriate mesh roll, and thus easy to operate the mesh insertion. When the entire mesh roll is inserted into the tube, apply a bigger aluminium tube with the same outer diameter as the copper tube internal diameter, and a same internal diameter as that of the mandrel outer diameter, thus further pushing the mesh roll to the required location in the copper tube. After this, we 
need to check the mesh roll condition, specifically, check if the mesh roll was deformed during the insertion. Ensure the mesh roll has a uniform internal diameter throughout the entire length. Also check for possible or debris left in the mesh roll inner surface.

After the acid treatment, the copper material surface becomes dull. This creates difficulty for wick insertion, while improving the working fluid wettability on the copper surface. Myers (1999) conducted research on the relationship between the surface condition and the liquid wettability concluded, "that is, if the 'true' contact angle of a liquid (an adhesive, say) is less then $90^{\circ}$ on the smooth surface, the angle will be even smaller on a rough surface. For a true contact angle $>90^{\circ}$, roughness will increase the apparent angle.... Practically, the preceding relationship indicates that if a liquid partially wets a surface, better wetting may be obtained if the surface is roughened in some way. Conversely, if wetting is not desired and a contact angle $>90^{\circ}$ can be attained, the situation can be further improved by roughening.” Also, “... the contact angle on a rough surface is lower than on a smooth surface of the same material...the liquid will spread over the rough surface spontaneously" (Carey, 1992). In our case, the working fluids are water and acetone. Both have an acute contact angle. According to Myers' (1999) conclusion, roughening the copper surface would improve the wettability of the working fluids, and thus improving heat pipe performance. Acid treatment can play the role of roughening the copper surface as well as removing the oxide, absorbed gases, and the contaminant layers. From this point of view, the acid treatment process also helps to improve the working fluid wettability on the copper surface, and thus improving the wick permeability. Therefore, it benefits the heat pipe performance with respect to hydrodynamics. 
Wick insertion was also attempted before the acid treatment. It was found to be easier than the one after acid treatment. However, penalty was paid for the post acid treatment. We applied the acid treatment process after assembly, since this process would remove amounts of contaminant with a high porous wick structure. It is very difficult to get rid of the acid removed contaminants; large amounts permanently stay in the wick structure. Due to this fact, the acid treatment process was used before assembly even though it made it difficult to insert the mesh roll.

After the assembly process, the heat pipe needed to be crimped at one end for sealing. Crimp one end of the assembly with the crimping tool, as shown in Figure 2.2, and then seal the ream to obtain a vacuum tight for the evacuation process that will be introduced in Section 2.2.3. After sealing, the assembly needs to be closed at the other end with a plastic cover, and then it needs to be put in a sealed plastic bag for protection until evacuation. Two techniques were tried for sealing; soldering and argon-arc welding. The soldering approach proved not to be applicable for a vacuum tight seal. The reason for the failure in soldering can be concluded that the solder applied was for regular water piping, not for vacuum tightening. Another reason for the failure is that the crimped copper tube had a rebound force that would destroy the soldered sealing. The third reason is that the copper and the solder are two different metals and therefore an interface between these metals would form. In the case of thermal shock, this interface could further develop and result in leaking. Also, these two metals have different thermal expansion coefficients, which can also lead to leakage. Soldering sealing for copper tubes are applied in the refrigerator industry. However, these seals are applied in different conditions. In the case of the refrigerator, the solder is silver-copper based, and the fluid 
charged is Freon, which would work in a high pressure condition rather than a vacuum. The argon-arc welding was proven effective in obtaining a vacuum tight seal in our case.

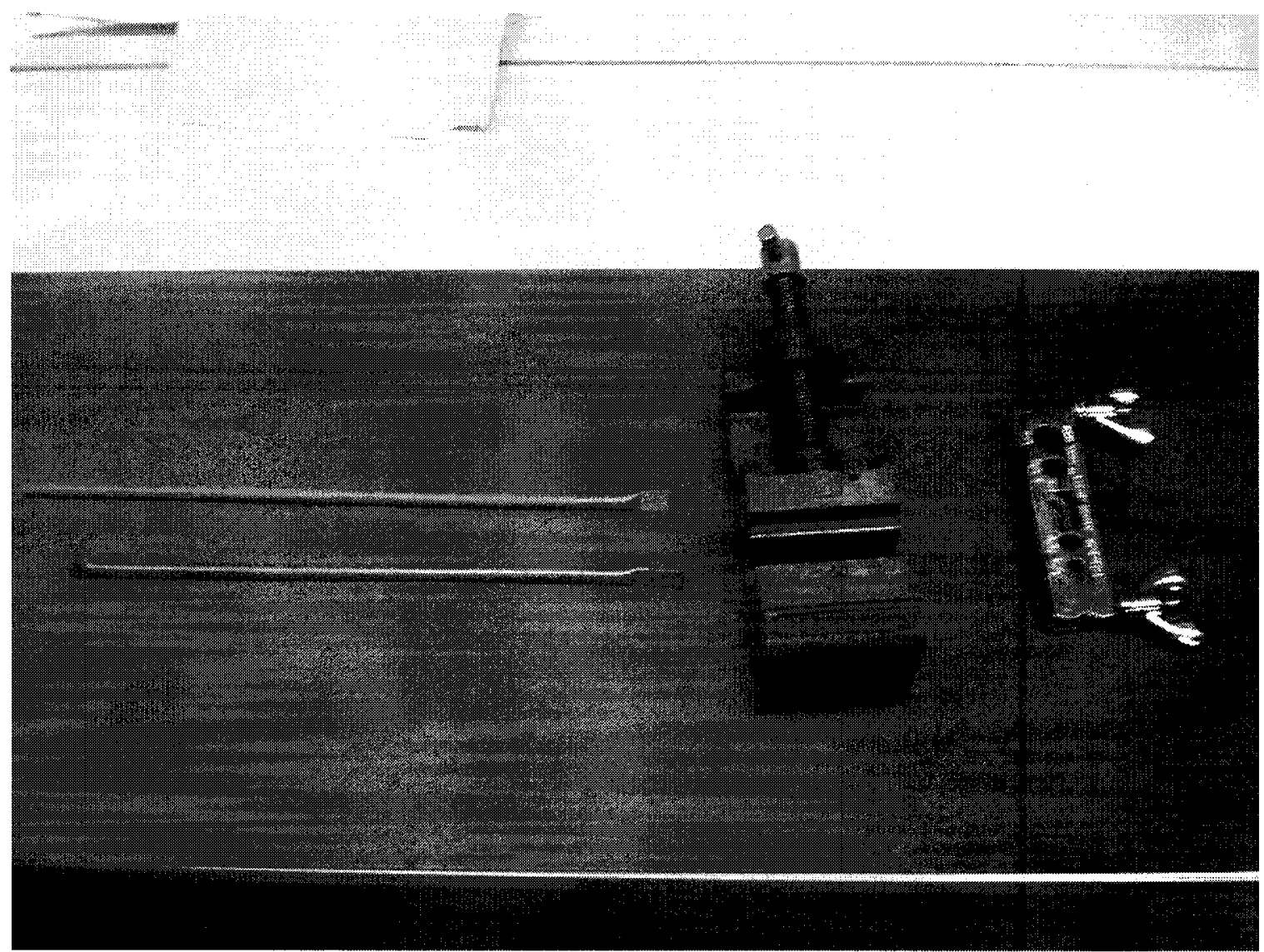

Figure 2.3 Tools for crimping prior to sealing

\subsubsection{Evacuation and Fluid Charging}

The evacuation and fluid charging process is the most important process in heat pipe manufacturing. These processes are introduced and discussed below.

The reason to evacuate the heat pipe assembly before charging the working fluid stems from the heat pipe working mechanism. The major reason relates to the working fluid evaporation. Gas law indicates that in a constant volume, vapour temperature is directly proportional to its pressure. At a low ambient pressure, a liquid needs a lower temperature to evaporate into a vapour phase. For example, at atmospheric pressure, i.e., 
760 Torr (1013.25 mbar), boiling temperature for water is $100^{\circ} \mathrm{C}$; for acetone it is $56^{\circ} \mathrm{C}$. When the ambient pressure decreases to 20 Torr (26.67 mbar), the boiling temperature for water is $20^{\circ} \mathrm{C}$. For acetone, to have the same boiling temperature as that of water, the corresponding ambient pressure is about 270 Torr (360 mbar). From this point of view, vacuum ensures startup at lower temperatures. Another reason for the vacuum is that the process can eliminate non-required gases and foreign materials from the assembly, thus ensuring the fluid is charged into the assembly. Also, it ensures the working fluid is not contaminated, and that the wick is clean. Schrader (1992) conducted experimental investigations of the vacuum effect on high- and medium-energy surfaces. In the experiments, water was purified by degassing, and it was found that the contact angle of water, purified in this manner, on copper film deposited in UHV (Ultra High Vacuum) was found to be $0^{\circ}$. Even though this result was obtained in an ultrahigh vacuum condition, and based on UHV deposited copper film, it can still indicate the fact that the vacuum process greatly improves the liquid wettability on the metal surface. Kaviany conducted detailed research on the mechanism of the vacuum to improve solid surface tension. He concluded that solid surface tension is larger in a vacuum than in the presence of any gas or vapour. This is due to the fact that the adsorbed foreign molecules reduce the intermolecular forces between the molecules of the solid (Kaviany, 1991). The significance of these results is that the vacuum process greatly helps a high surface tension liquid, e.g., water, with wetting in a small capillary radius metal wick structure. This could be impossible in an atmospheric environment where the metal wick capillary radii are too small. 


\subsubsection{Heat Pipe Charging Device}

The evacuation process is used to obtain a clean vacuum in the heat pipe assembly. That means this process has a cleaning function as well as getting a low pressure environment. The fluid charging process is to fill a certain amount of liquid working fluid into the cleaned vacuumed heat pipe assembly. These are opposite processes, and they are required to implement consecutively at one time.

Figure 2.4 and Figure 2.4a both illustrate the apparatus used for evacuation and charging. In Figure 2.4a, we can see that this evacuation and fluid charging system is composed of a vacuum pump, a trap, a piping system, a vacuum gauge, valves, and a fluid charging flask.

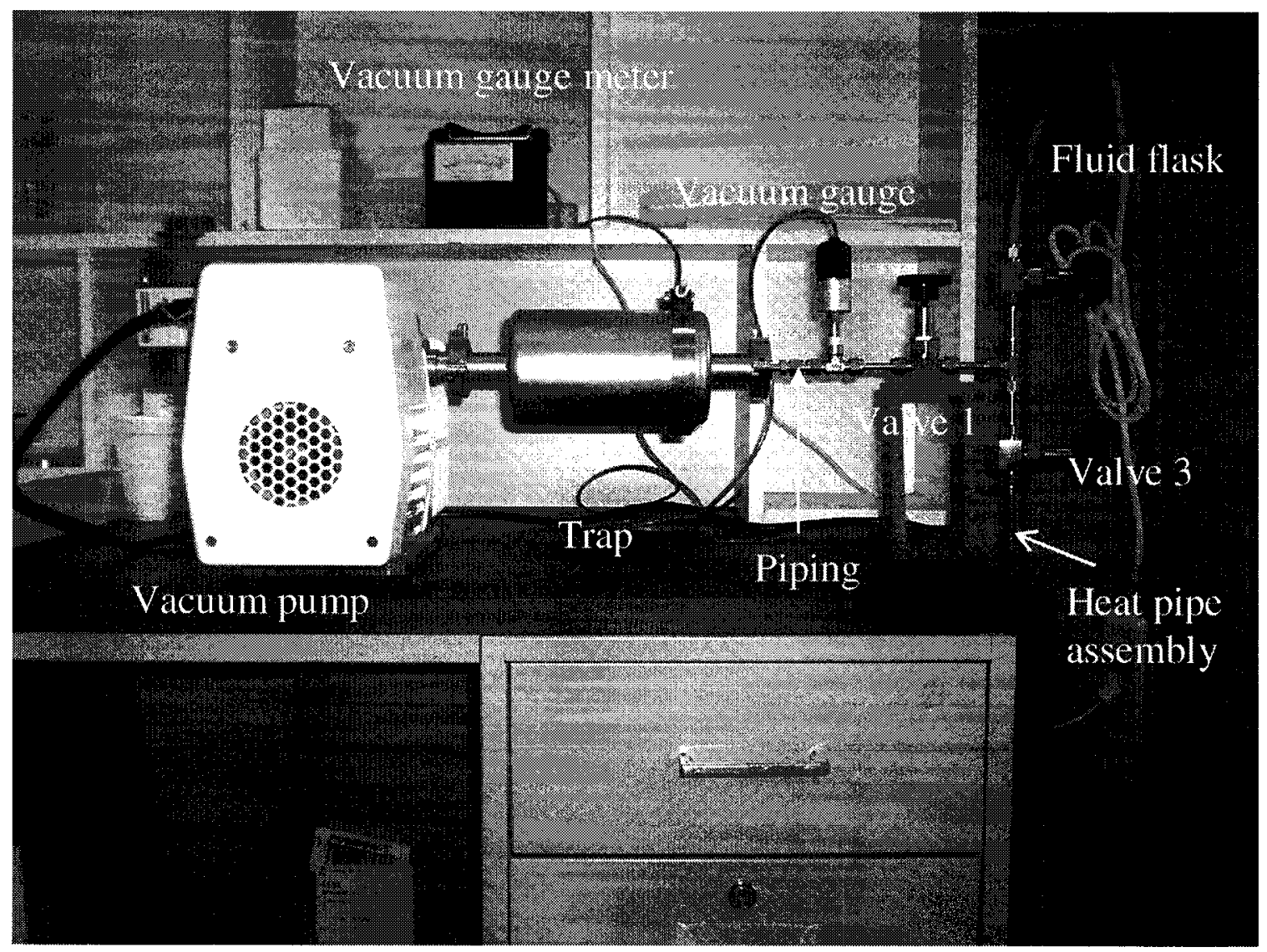

Figure 2.4 Evacuation and fluid charging device 


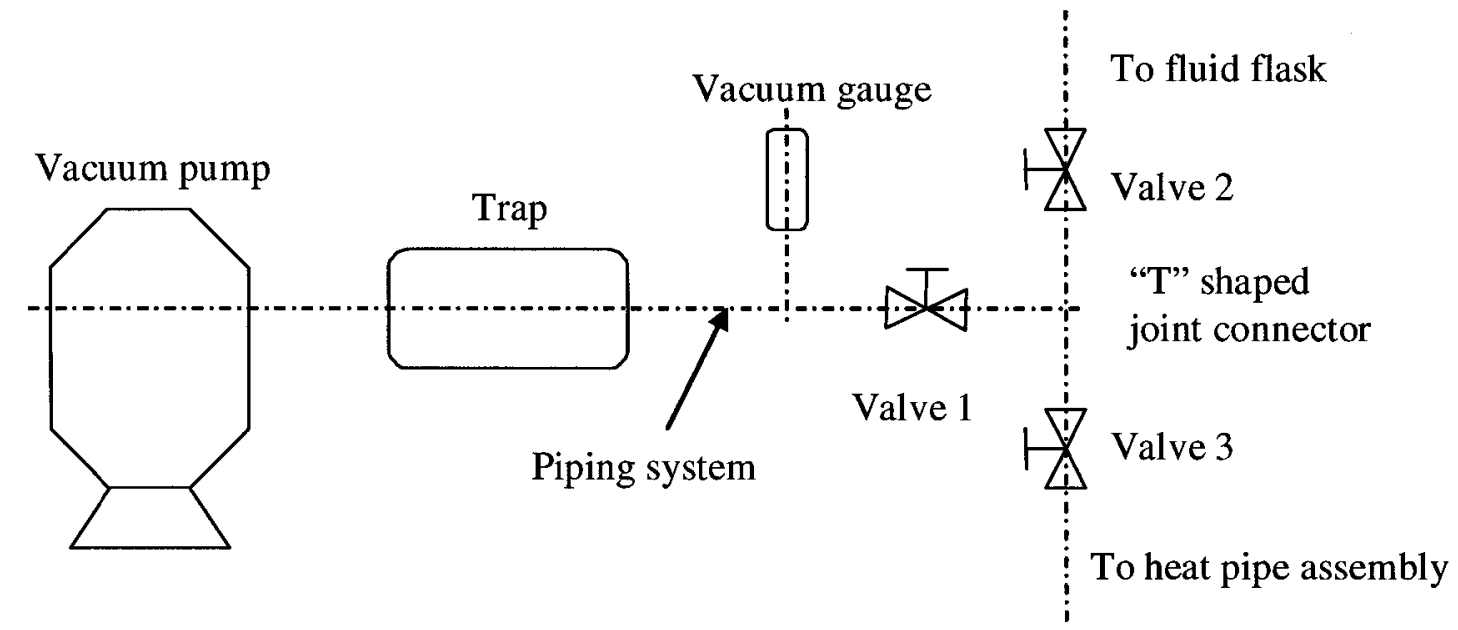

Figure 2.4a Schematics of the evacuation and fluid charging device

The vacuum pump used in the system is an ALCATEL Pascal Series 2005 SD twostage rotary vane vacuum pump. This pump has a nominal flow rate of $6.5 \mathrm{~m}^{3} / \mathrm{h}(1.8$ litre/s). The ultimate pressure with gas ballast closed is $1.5 \times 10^{-3}$ Torr. The ultimate pressure with gas ballast opened is $7.5 \times 10^{-3}$ Torr, and the maximum water vapour pumping capacity (ballast flow rate $1.1 \mathrm{~m}^{3} / \mathrm{h}$ ) is 25 Torr. The water vapour pumping capacity is $110 \mathrm{~g} / \mathrm{h}$. These pumps are designed for non-corrosive applications. It can be used for the production of TV tubes, the manufacture of electronic tubes, and is also suitable for heat pipe manufacturing.

The piping system consists of $1 / 4^{\prime \prime}$ stainless steel tubes connected with SWAGELOK stainless steel ferruled tube fittings. These stainless steel pipes have a low rate of gas emitting, and are suitable for this vacuum system.

Since the rotary vane vacuum pump is oil sealed, an adsorption trap is installed at the vacuum pump inlet fitting to prevent the mist of vacuum pump oil from diffusing into the vacuum system. The trap also absorbs relatively large amount condensable gases, e.g., water vapour mixed in the air, to prevent it from entering the vacuum pump directly and condense in the vacuum pump oil, and thus degrading the oil. The trap is a stainless 
steel container consisting of corrugated foil made of oxygen-free copper (OFHC copper) that is rolled together with a very large surface density. Unlike the cold traps, this trap, which can work at room temperature, absorbing the oil and water vapour, is free of operation. The importance is to keep the OFHC surface clean, i.e., free of grease. When the OFHC copper is contaminated, especially by grease, it needs refreshing by heating in a reducing gas atmosphere, e.g., hydrogen (Diels \& Jaeckel, 1966). Like all traps, this trap also has its drawback; large flow impedance (Diels \& Jaeckel, 1966). As shown in Figures 2.4 and $2.4 \mathrm{a}$, being installed serially in the main pipeline, the trap would act as a resistance to the piping conductance (defined as piping gas throughput capacity per unit pressure difference throughout the entire piping length), thus offsetting the vacuum pump's effectiveness. In our case, will be discussed later in this section, due to the heat pipe assembly can be treated as a long tube (large ratio of length to diameter) chamber, and with high porous wick inside, it would cause a high magnitude of gas flow resistance to the overall piping conductance compared with the trap. Under such circumstances, only increase the overall piping gas flow conductance will not provide a significant help to the heat pipe assembly in terms of gas eliminating. Thus, the trap resistance effect can be ignored.

The vacuum gauge in this system is a thermocouple principled vacuum measurement gauge that is made by Kurt J. Lesker Co.. The gauge measurement range is from atmospheric pressure to $10^{-3}$ Torr. As mentioned earlier in this section, the fluids to be charged are water and acetone. At room temperature, their vapour pressures are 20 Torr and 270 Torr respectively. Based on this, the expected vacuum degree is at $10^{-3}$ Torr level, so this vacuum measuring gauge is suitable in our case. The gauge measurement 
principle is based on the gas heat conductivity as a function of pressure. It is a total pressure measurement gauge. The advantages are its wide measuring range, remote pressure reading, and continuous pressure indication. However, the gauges pressure readings vary if the thermocouple is contaminated. Contaminates can be reduced by blackening treatment on the thermocouple surface. While on the other hand, being a protection, the blackening layer would act as insulation to the thermocouple, decreasing the thermocouple sensitivity to the temperature caused by its ambient pressure variance, and consequently affects the pressure measurement accuracy. The best way is to ensure the vacuum system is clean. This highlights the importance of the adsorption trap in a vacuum system. Another drawback of the thermocouple vacuum gauge is that its pressure reading depends on the nature of the gas. This requires a gauge calibration for a specific gas to be measured. In our case, the gauge was calibrated for air by the vender.

The three vacuum valves are SWAGELOK SS-4BK Bellows-Sealed Valves. Valve 1 controls the main pipeline to the vacuum chamber and fluid flask. In the evacuation process, Valve 1 is opened to connect the vacuum pump to the vacuum chamber, i.e., the heat pipe assembly. In the fluid charging process, Valve 1 is closed to stop the fluid from entering the vacuum system including the vacuum gauge, the trap, and the vacuum pump. Also, Valve 1 can be closed when replacing the assembly or refilling the fluid flask. Valve 2 is only used to control the fluid flask. During the entire evacuation process, Valve 2 is closed. During the fluid charging process, Valve 2 is allowed to be opened and must be closed immediately when the required amount of fluid is filled into the heat pipe assembly. Valve 3 controls the connection of the heat pipe assembly to the main vacuum pipeline. Valve 3 is required to be opened during the whole evacuation and fluid charging 
processes. After the fluid charging is complete, Valve 3 is required to be firmly closed before detachment from the system for the final sealing process. This requires that Valve 3 possess a good vacuum tight reliability in an atmospheric environment; this issue will be discussed in detail later.

In this case, the fluid charging flask experienced a few modifications. A general requirement for the fluid flask is to provide a required amount of fluid to the heat pipe assembly without any vacuum leakage. To meet this requirement and make the structure simple, a $1 / 4 "$ semitransparent Teflon hose be used as the fluid flask. The Teflon hose is connected to Valve 2 with a stainless steel ferrule ensuring a vacuum tight seal. The fluid to be charged can be pre-filled in the Teflon hose; while the other end of the Teflon hose is covered by a plastic cap to protect the fluid from ambient contaminants. This plastic cap is not required to provide a vacuum tight seal, thus, the fluid charging flask makes use of the atmospheric pressure to press the fluid into the heat pipe assembly. First, this flask has a very simple structure. It has only a Teflon hose attached to the valve with the ferruled tube fitting and covered by a plastic cap. No other extra container is connected, thus it reduces the possibility of leakage. Second, this flask combines the container and the ruler into one. Due to its semi-transparency, the amount of fluid can be controlled by reading the liquid surface change in the Teflon hose. Third, this flask structure makes use of the atmospheric pressure, thus making the operation easy. This flask structure also has a drawback; due to the fluid in the flask, it has a chance of contact the ambient air. This means that some air might be absorbed during the process. To reduce the air absorption possibility, the length of the Teflon hose was increased and pre-filled with much more fluid in the hose for each operation. In each operation, the hose is refilled with new fluid. 
The heat pipe assembly is connected to Valve 3 with the ferruled tube fitting. This connection must be vacuum tight and heat resistant since the assembly will be treated at a high temperatures.

Based on this apparatus, we successfully applied a heat pipe manufacturing process. The details of the procedure are summarized bellow:

1) Prepare a cooling bag in the freezer.

2) Pre-fill the flask with the required working fluid to a calculated length, corresponding to the fluid amount. Mark the amount of liquid to be charged by measuring the equivalent length on the hose.

3) Check the filled flask to make sure there are no air bubbles in the fluid.

4) Fix the filled flask to the system. Tighten the ferruled tube fitting, and close Valve 2.

5) Pre-vacuum the system. Start the vacuum pump with Valve 1 closed.

6) Fix Valve 3 to the heat pipe assembly with the ferrule tube fitting.

7) Weigh the heat pipe assembly with Valve 3 attached. Record the weight.

8) Fix the heat pipe with Valve 3 attached to the system with the ferrule tube fitting; close Valve 3.

9) Open Valve 1 to check the vacuum tightness of Valve 2 and Valve 3. If the gauge reading returns to its preceding position, it means the two valves have good vacuum tightness. Otherwise, one of the valves must contain a leakage. If this is the case, apply leak detection.

10) After eliminating the leak, open Valve 3 to vacuum the heat pipe assembly. When Valve 3 is open, the gauge reading will jump to a higher pressure. After a period 
of time, if the reading continuously displays a low pressure, it means the assembly has very good vacuum tightness. Otherwise, there could be more possible circumstances. One possible circumstance is that the assembly could have a big leak. Under such circumstances, the gauge reading will never turn to low pressure. Another possible circumstance is that the assembly could contain residual liquid from the cleaning process. Under such circumstances, if we apply heat to the assembly, and the gauge reading will slowly turn to a low pressure. The third possible circumstance is that the assembly could have both of the preceding circumstances. Under such circumstances, the gauge reading behaviour would be similar to the first circumstance. Detailed approaches to the leaking detecting system will be discussed later in this section.

11) Vacuum the assembly. When the gauge reading gets is near $2 \times 10^{-3} \mathrm{Torr}$, apply heating of about 400 to $500^{\circ} \mathrm{C}$ for about 1 minute. This time the gauge reading will again turn to a high pressure. At this point, stop heating and the gauge reading will turn back to the previous pressure or lower as the assembly cools down.

12) When the gauge reading reaches the stable low pressure, repeat Step 11 until the gauge reading no longer turns to high pressures. Hence, the assembly is evacuated to the required vacuum level. Record the gauge reading.

13) Check static vacuum. Close Valve 3, and detach the assembly with Valve 3 attached. Re-weigh to obtain the amount of residual liquid that was eliminated from the assembly. Then, leave the assembly with Valve 3 closed for a certain 
time period to check the leaking rate or the degassing rate at the room temperature. This time period can be a few hours to hundreds of hours.

14) Re-fix the assembly to the system to check the pressure loss. Start the vacuum pump with Valve 1 closed, when the gauge reading reaches stable-state at the preceding record, open Valve 3, and record the maximum value the gauge reads, comparing it to the preceding record obtained. The pressure loss multiplied by the assembly net internal volume and divided by the static time gives the rate of leaking. From this, evaluate the assembly including Valve 3 vacuum tightness. If the leaking rate is too high, the assembly needs further leaking detecting until the leaking rate is lower than the requirement criteria.

15) Fix the qualified assembly to the system, and start to charge the fluid. Open Valve 3 to vacuum the assembly to the required degree, i.e., lower than 10 milli-Torr. When the gauge reading stays at the lowest point, close Valve 1, and then open Valve 2 to let the fluid flow into the assembly through Valve 3. Meanwhile, watch the liquid surface changing in the Teflon hose. When the liquid surface reaches the mark, close Valve 2, and apply the cooling bag to the assembly for about two minutes.

16) Close Valve 3 , and detach the whole assembly with the closed Valve 3 . Weigh the whole assembly and subtract the record prior to the fluid charging to obtain the initial amount of charged fluid.

17) Crimp the pipe with the crimping tool at the calculated length, and then apply the narrow pincher to pinch the crimped flat section on the pipe. Then, tighten the 
wing-nuts to lock the pincher on the pinched pipe to prevent leaking during the following procedures.

18) Check the pinched assembly in hot water to verify its heat pipe functionality.

19) Send the pinched assembly to the machine shop for argon-arc welding. To operate the argon-arc welding, detach the attached Valve 3 by cutting from the flat section on the pipe, and then apply argon-arc welding to the cutting ream.

20) After argon-arc welding, repeat Step 18.

21) If the assembly works as a heat pipe in the preliminary check, loosen the wingnuts, and release the pincher. Weigh the finished heat pipe with Valve 3 attached, and record the results.

22) Weigh the detached Valve 3 and the cut copper tube then make a record.

23) Repeat Step 22. Comparing the result of Step 22, the residual fluid in Valve 3 and the cut copper can be obtained.

24) Use the record in Step 16, minus the result of Step 23, to find the net amount of fluid charged in the heat pipe.

25) The evacuation and charging processes are complete.

The practical conditions are more complex than the description in the procedure just given. Many factors would be involved in a specific operation of the evacuation and fluid charging, and these factors could bring effects during this process. Major factors are related to vacuum process, and will be discussed in the following sections.

\subsubsection{Vacuum Process Gas Flow Analysis}

Like all medium or higher vacuum processes, the gas pumping in our case also experienced viscous, transition, and molecular flows. The viscous range covers two 
phases; turbulent and laminar flows. The turbulent occurs at the initiation of the rough pumping process. At this phase, the gas flow has a high pressure and velocity. This flow can be described with Equation (2.2) (Berman, 1992).

$$
Q_{g t}=R e \cdot d_{\text {piping }} \cdot\left(\frac{\pi \cdot R \cdot T \cdot \eta_{g}}{4 \cdot M}\right)
$$

where, Reynolds number, $R e$, can be expressed as

$$
R e=\frac{d_{\text {piping }} \cdot v_{g} \cdot \rho_{g}}{\eta_{g}}
$$

for turbulent flow, $R e>2200$.

Substituting Equation (2.3) into Equation (2.2) yields

$$
Q_{g t}=\frac{\pi \cdot R \cdot T \cdot v_{g} \cdot \rho_{g} \cdot d_{p i p i n g}^{2}}{4 \cdot M}
$$

From Equation (2.2a) we can see that for a certain system, $Q_{g t}$ is directly proportional to the air density. At the initiation of pumping, the air has a high density, and thus the gas throughput is very high. In our case, due to the long piping (pipe length is 20 times greater than diameter); the gas flow conductance is very small, even with a big gas load. As long as there is no large leakage, the air density would decrease very fast, and then the gas throughput will reduce very quickly. So, the turbulent period is very short, and is estimated only a few seconds. A very large amount of gas is pumped in this short period of time. With a further decrease in gas density, Reynolds number will also decrease. When the Reynolds number gets lower than 1200 , the gas flow enters the laminar flow phase (Berman, 1992). For air at $23^{\circ} \mathrm{C}$, the conductance to laminar flow can be approximately expressed as, in ergs/s (Berman, 1992). 


$$
C_{l}=\left(\frac{\pi \cdot d_{\text {piping }}^{4}}{128 \cdot \eta_{g} \cdot L_{p i p i n g}}\right) \cdot \bar{p} \cdot \Delta p
$$

From Equation (2.4), we can see that the conductance to laminar flow is proportional to the pipeline diameter at the $4^{\text {th }}$ power, the average pressure, and two end pressure differences. It is inversely proportional to the pipe length and the gas viscosity. This relationship is termed Poiseuille's Law. In our case, the pipeline diameter is very small, and in the laminar flow phase. The average pressure is becoming lower, thus the conductance to laminar flow is very small compared with that of the turbulent flow. This laminar flow phase takes a longer time period than the turbulent flow. The time estimation for this phase is more complex, depending on the conditions. If the leakage is very small, and the gas emitting rate is also very small, this phase will take a very short time, about a few seconds. This will be analyzed later in this section in the transition flow section.

In this range, the pressure drop can be expressed as (for air at $23^{\circ} \mathrm{C}$ ),

$$
\Delta p=0.2 \cdot \frac{S_{p u m p} \cdot L_{\text {piping }}}{d_{\text {piping }}^{4}}
$$

From Equation (2.5), we can see that the axial pipeline pressure drop is proportional to the pump speed and the pipe length. It is inversely proportional to the pipeline diameter at the $4^{\text {th }}$ power. In our case, besides the small vacuum piping diameter, the vacuum gauge was located far from the assembly being measured. The pressure drop between the vacuum gauge and the assembly is relatively large, estimated at approximately 25 Torr.

With further decreases in pressure, the mean path of molecules increases when the mean path of molecules is of the same order of magnitude as the transverse dimensions of 
the pipe. Then, the flow enters the transition range, known as Knudsen's flow. Knudsen (1911) expressed the conductance to the transition flow in a long cylindrical pipe as

$$
C_{t r}=C_{l}+J_{m} \cdot C_{m p}
$$

Equation (2.6) is very difficult to use since the coefficients $J_{m}$ and $C_{m p}$ have complex forms, which are beyond the scope of this thesis, and are not discussed. Atta (1965) modified this equation and gave a more applicable form,

$$
C_{t r}=3.269 \times 10^{-2} \cdot\left(\frac{\bar{p} \cdot d_{\text {piping }}}{\eta_{g}}\right)+3.81 \cdot\left(\frac{T}{M}\right)^{1 / 2} \times \frac{\left[1+0.147 \cdot\left(\frac{M}{T}\right)^{1 / 2} \cdot\left(\frac{\bar{p} \cdot d_{p i p i n g}}{\eta_{g}}\right)\right]}{\left[1+0.181 \cdot\left(\frac{M}{T}\right)^{1 / 2} \cdot\left(\frac{\bar{p} \cdot d_{\text {piping }}}{\eta_{g}}\right)\right]}
$$

for long pipes, as (for air, at $23^{\circ} \mathrm{C}$ )

$$
C_{t r}=178.7 \cdot \bar{p} \cdot\left(\frac{d_{p i p i n g}^{4}}{L_{p i p i n g}}\right)+12.12 \cdot\left[\frac{\left(1+252.1 \cdot \bar{p} \cdot d_{p i p i n g}\right)}{\left(1+311.7 \cdot \bar{p} \cdot d_{p i p i n g}\right)}\right] \cdot\left(\frac{d_{p i p i n g}^{3}}{L_{\text {piping }}}\right)
$$

This relationship implies that the minimum conductance in the transition flow occurs when

$$
\bar{p}_{\text {min }} \cdot d_{\text {piping }}=5.47 \cdot\left(\frac{T}{M}\right)^{1 / 2} \cdot \eta_{g} \quad(\text { Torr } \mathrm{cm})
$$

For air at $23^{\circ} \mathrm{C}$, this gives

$$
\bar{p}_{\text {min }}=\frac{3.1 \times 10^{-3}}{d_{\text {piping }}} \text { (Torr) }
$$

Substituting $d_{\text {piping }}$ value into Equation (2.8a) gives the minimum average pressure for the transition range. It is found to be $6.51 \times 10^{-3}$ Torr.

Also, from Equation (2.8), we can get the minimum mean path of molecules as,

$$
\lambda_{\min }=1.59 \cdot d_{\text {piping }}
$$


Substituting $d_{\text {piping }}$ value into Equation $(2.8 \mathrm{~b})$ gives the minimum average mean path of molecules for the transition range. It is found to be $7.57 \mathrm{~mm}$.

From this relationship in Equation (2.7) we can deduce that the transition pressure in the transition flow occurs when,

$$
\bar{p}_{t r} \cdot d_{\text {piping }}=95.7 \cdot\left(\frac{T}{M}\right)^{1 / 2} \cdot \eta_{g}
$$

for air at $23^{\circ} \mathrm{C}$,

$$
\bar{p}_{t r}=\frac{5.43 \times 10^{-2}}{d_{\text {piping }}}
$$

substituting $d_{\text {piping }}$ value into Equation (2.9a) gives the transition average pressure for the transition range. It is found to be 0.114 Torr.

From this relationship in Equation (2.7), the maximum conductance in the transition flow occurs when

$$
\bar{p}_{t r, \max } \cdot d_{p i p i n g}=942 \cdot\left(\frac{T}{M}\right)^{1 / 2} \cdot \eta_{g}
$$

for air at $23^{\circ} \mathrm{C}$,

$$
\bar{p}_{t r \text { max }}=\frac{5.35 \times 10^{-1}}{d_{\text {piping }}} \text { (Torr) }
$$

substituting $d_{\text {piping }}$ value into Equation (2.10a) gives the maximum transition average pressure for the transition range as 1.12 Torr. Note that this pressure is the pressure in the transition phase from laminar range to the transition range. In practical observations, if there is no big leakage, the vacuum gauge reading passes this value very fast. It takes just a few seconds, which means the laminar flow range has a very short time period, as estimated before. 
From Equation (2.10), we can get the minimum mean path of molecules,

$$
\lambda_{\text {tr, } \max }=9.09 \times 10^{-3} \cdot d_{\text {piping }}
$$

substituting $d_{\text {piping }}$ value into Equation $(2.10 \mathrm{~b})$, the maximum average mean path of molecules for the transition range is calculated to be $0.04 \mathrm{~mm}$.

$$
\bar{p}_{t r \text { min }} \cdot d_{p i p i n g}=10 \cdot\left(\frac{T}{M}\right)^{1 / 2} \cdot \eta_{g}
$$

for air at $23^{\circ} \mathrm{C}$,

$$
\bar{p}_{t r, \min }=\frac{5.7 \times 10^{-3}}{d_{\text {piping }}}
$$

substituting $d_{\text {piping }}$ value into Equation $(2.11 \mathrm{a}$ ), we calculated the minimum transition average pressure for the transition range to be $1.2 \times 10^{-2}$ Torr. Also, this pressure is the transition pressure from transition flow range to the molecular flow range. From observation, the time period for the vacuum gauge reading from 1.12 Torr to $1.2 \times 10^{-2}$ Torr was a few minutes. This is the entire transition flow range time period. Also, from Equation (2.11), we can obtain the minimum transition mean path of molecules,

$$
\lambda_{t r \text {, min }}=9.09 \times 10^{-1} \cdot d_{\text {piping }}
$$

substituting $d_{\text {piping }}$ value into Equation $(2.11 \mathrm{~b})$, the minimum transition mean path of molecules for the transition range is estimated to be $4.33 \mathrm{~mm}$.

As the pressure is further decreased, the mean path of molecules will increase. When the mean free path of molecules is very large compared with the dimension of the space where the molecules move, the gas flow transits into molecular flow. In this range, the molecules move in a pipe in random straight lines striking the wall of the pipe at the end of each flight. Calculations take into account only those molecules well inside the 
pipe, far removed from the ends. Thus the effect of the entrance (exit) aperture to the pipe is not important (Berman, 1992). So, the molecular flow conductance of the pipe is independent of pressure. For a certain species of gas (M) and temperature (T), the molecular flow conductance depends on the geometry of the pipe (Berman, 1992).

The conductance can be expressed as, in $\mathrm{cm}^{3} / \mathrm{s}$,

$$
C_{m p}=\left(\frac{16}{3}\right) \cdot\left(\frac{R \cdot T}{2 \cdot \pi \cdot M}\right)^{1 / 2} \cdot \frac{A_{p i p i n g}^{2}}{C_{p i p i n g} \cdot L_{p i p i n g}}
$$

or, in litre/s

$$
C_{m p}=3.81 \cdot\left(\frac{T}{M}\right)^{1 / 2} \cdot \frac{d_{\text {piping }}^{3}}{L_{\text {piping }}}
$$

for air, at $23^{\circ} \mathrm{C}$, in litre/s,

$$
C_{m p}=12.1 \cdot \frac{d_{\text {piping }}^{3}}{L_{\text {piping }}}
$$

substituting the piping dimensions into Equation (2.12b) yields the conductance to the molecular flow in our vacuum system. It is found to be $3.27 \times 10^{-2}$ litre/s, which is just $1 / 55$ of the pump nominal pumping speed. Since then, the gauge reading changes very slowly, it usually takes a couple of hours to decrease the pressure. In our case, if we keep a constant temperature, from $1.2 \times 10^{-2}$ Torr to a lower pressure steady-state, e.g., $6 \times 10^{-3}$ Torr. Applying heat to the assembly can significantly reduce this time period. In some cases, this time was reduced to tens of minutes. This relates to many factors. One of these important factors is temperature. From Equation (2.12) we can see that for a given geometry, the conductance to molecular flow is proportional to the square root of the temperature. Rising the temperature to $300^{\circ} \mathrm{C}$, the conductance to the molecular flow in our vacuum system was $4.57 \times 10^{-2} \mathrm{litre} / \mathrm{s}$; an improvement of 40 percent. 
This temperature-related effect on the molecular flow was first discussed by Maxwell (1897), and subsequently studied by Knudsen (1910). It is termed "thermomolecular flow" or "thermal transpiration" (Berman, 1992). If two chambers, for instance, 1 and 2, are separated by a small orifice or porous plug containing gas at temperature $T_{1}$ and $T_{2}$ respectively, then gas transpiration will occur between the two chambers until an equilibrium state is established. The relation can be written as,

$$
p_{1} \cdot T_{2}^{1 / 2}=p_{2} \cdot T_{1}^{1 / 2}
$$

where, $p_{1}$ and $p_{2}$ represent pressures in chamber 1 and 2 respectively in the equilibrium state. Note that the temperatures in Equation (2.13) are in Kelvin.

This is of importance in analysis on the vacuum condition at low pressure and with porous plug. In our practical work, the problem is to evaluate the vacuum conditions in the wick structure. Detailed analysis on the porous structure vacuum condition is beyond the scope of this thesis. Here, we only applied the thermal transpiration theorem to estimate the pressure in the wick structure during the dynamic vacuum process. In our case, only one vacuum gauge was used. This gauge was located near the trap, having a distance from the heat pipe assembly, thus there was an uncertainty of indicating the pressure in the wick structure. Not knowing the vacuum conditions in the wick structure, we did not know whether the wick was ready for the fluid charging. In our work, we applied heating to the heat pipe assembly during the dynamic vacuum process. In the process, we measured the temperatures at the gauge and the assembly respectively, and recorded the stable process at the same time. For example, at the first heating the assembly temperature was estimated to be $400^{\circ} \mathrm{C}(673 \mathrm{~K})$, the temperature at the gauge was $50^{\circ} \mathrm{C}(323 \mathrm{~K})$; and the gauge reading was $3 \times 10^{-1}$ Torr. Substituting these three 
results into Equation (2.13), the current pressure in the wick at that time was obtained as $4.3 \times 10^{-1}$ Torr. After a few rounds of this process, the final result for the assembly temperature was $400^{\circ} \mathrm{C}(673 \mathrm{~K})$, the gauge temperature was $30^{\circ} \mathrm{C}(303 \mathrm{~K})$; and the gauge was reading $4 \times 10^{-3}$ Torr. The corresponding wick pressure was $5.96 \times 10^{-3}$ Torr. Note that in this application, the system was treated as an idealized thermal transpiration model. In fact, there was only one chamber in this model, i.e., the wick itself was modeled as a chamber rather than a porous plug, so the calculation result is optimistic. The thermal transpiration is based on a static vacuum condition, i.e., the transpiration is realized by the molecular motion; and the parameters are valid in an equilibrium state. The above application, the system was in a dynamic process not in an equilibrium state. Thus, the measured parameters were not valid for the thermal transpiration application. However this is the simplest approach to the wick pressure estimation by using only one vacuum gauge. The estimated results were verified by actual experiments.

\subsubsection{Gas Load Analysis}

The preceding section analyzes the gas flows in the vacuum process, which is a dynamic process. Finally, we need a static vacuum. This means that the heat pipe assembly still keeps a good vacuum condition after the final sealing. To explore this issue, the static vacuum checking was necessary.

For the static vacuum checks as described in Step 13 of Section 2.2.3.2, some assemblies kept a very good static vacuum. After a few days, they had almost no vacuum loss. However, others lost vacuum to some extent. They received hundreds of milli-Torrs of pressure when rechecked after a certain period of time. Therefore the source of vacuum loss was needed to be analyzed. 
Vacuum losses stem from sources of pressure increases, and this comes from gas load. In a vacuum environment, the gas load relates to the system leakage, the substance evaporation, material degassing, and the gas permeation. This can be expressed as (Berman, 1992),

$$
Q_{G}=Q_{L}+Q_{E}+Q_{D}+Q_{P e r}
$$

Leaks are the most common phenomenon in a vacuum system. In the dynamic vacuum process, leaking rate can be expressed as,

$$
Q_{L d}=p_{u s} \cdot S_{n}
$$

In the static vacuum condition, assuming there is only a leak gas load, and not any other gas loads, the leak rate can be expressed as,

$$
Q_{L s}=\frac{\Delta p \cdot V}{t}
$$

In our practical operation, for the 100 copper wire screen mesh wick configuration, the net volume is 0.004875 litres. If this assembly gets $2 \times 10^{-3}$ Torr vacuum loss after 60 hours static vacuum check, calculating with Equation (2.16), yields its leak rate as $4.514 \times 10^{-11}$ Torr $\cdot$ litre $/ s$.

There are many methods to detect a leak. In our work, because the system is not very complex, and the stainless steel ferrule fittings are reliable, we did not apply special leak detections. The only instrument we used was the vacuum gauge. By comparing the gauge readings before and after the static vacuum, and combining other gas load analyses, we obtained satisfactory results.

Substance evaporation is another major gas load in a vacuum system. The rate of evaporation can be expressed as, in $\mathrm{g} / \mathrm{cm}^{2} \mathrm{~s}$, 


$$
\dot{W}=G \cdot s_{f}
$$

or

$$
\dot{W}=5.834 \cdot p_{E} \cdot\left(\frac{M}{T}\right)^{1 / 2} s_{f}
$$

The specific evaporation rate of materials, assuming the sticking coefficient $s_{f}=1$, is, in $\operatorname{Torr} /\left(\mathrm{s} \cdot \mathrm{cm}^{2}\right)$,

$$
q_{E}=\dot{B} \cdot\left(p_{E}-p\right)
$$

or

$$
q_{E}=3.639 \cdot\left(\frac{T}{M}\right)^{1 / 2} \cdot\left(p_{E}-p\right)
$$

Evaporation rate is, in Torr $\cdot$ litre/s,

$$
Q_{E}=3.639 \cdot\left(\frac{T}{M}\right)^{1 / 2} \cdot\left(p_{E}-p\right) \cdot A_{E}
$$

Copper does not evaporate until the temperature reaches a level higher than $900^{\circ} \mathrm{C}$. In our case, the operating temperature is lower than $500^{\circ} \mathrm{C}$. Thus, copper evaporation does not need to be taken into account. The only material that can evaporate in the evacuation process is the residual liquid. The 100 mesh copper wire screen has a surface area of $155.6 \mathrm{~cm}^{2}$. Suppose 1 thousandth of the wick is wetted by water, i.e., the water evaporation area is $0.156 \mathrm{~cm}^{2}$. At $23^{\circ} \mathrm{C}$, the water evaporation pressure is 21 Torr. If the assembly has a 0.01 Torr vacuum inside, Equation (2.19) gives the water evaporation flow rate as 2.3 Torr litre/s, which is greater than the normal pumping speed. In fact, this process period is very short. As analyzed in the preceding section, when the gas flow enters the molecular range, the conductance is far lower than the pumping speed. On the 
other hand, at a low pressure environment, the water inside the assembly would evaporate at a high rate. During the evaporation process, the water temperature would quickly decrease. With the decreasing temperature, the water evaporation rate would slow down. Thus, the temperature becomes lower and lower, and the water evaporates slower and slower. This mutually related action consequently results in a lower temperature and lower pressure. When the temperature reaches the freezing point, the water begins to freeze, consequently the pressure further decreases. This looks as if it is a better vacuum. However, it is not. It is a false vacuum phenomenon. Due to the wick trapping effect, this false vacuum phenomenon is easier to happen to the porous wick structure. This false vacuum may possibly lead to future problems. If the assembly is sealed with a false vacuum, when the temperature rises, the water will re-evaporate at a very high flow rate, as analyzed above, destroying the "vacuum". There are many methods of solving this problem. One approach is warming the assembly to help the frozen liquid, melt and evaporate. In our applications, this method was applied, and was proven to be an effective method. Attention should be drawn to the warming temperature. It must be maintained at a mild level, i.e., just over the liquid current evaporation point; otherwise, it can cause copper materials to oxidize. Another approach is dry inert gas displacing or flashing. That is, to charge some inert gases such as nitrogen or argon into the wick at a certain vacuum degree. This method has been proven to be effective in porous structure material evacuation process. The main mechanism for this method is based on the surface tension difference between nitrogen and water as well as air. For instance, the surface tension for these substances are nitrogen $0.01 \mathrm{mN} / \mathrm{m}$ at $126 \mathrm{~K}\left(-147^{\circ} \mathrm{C}\right)$; water 58.91 $\mathrm{mN} / \mathrm{m}$ at $373 \mathrm{~K}\left(100^{\circ} \mathrm{C}\right)$; and oxygen $0.40 \mathrm{mN} / \mathrm{m}$ at $154 \mathrm{~K}\left(-119^{\circ} \mathrm{C}\right)($ Carey, 1992$)$. 
Although this data indicates values at different temperature conditions, it is not directly comparable. Note that among these three substances, nitrogen has the lowest surface tension value at the lowest temperature. As the surface tension decreases with rising temperature, the surface tension of nitrogen will still be the lowest among these three substances for the same temperature. Displacing the water vapour and oxygen, nitrogen in the pore with a low surface tension, and can be easily evacuated. Since the second method involves more devices components in the vacuum system, which could decrease system piping capacity and bring the chance of leaking, also increase cost. This approach was not applied in our case. Of course, if these two methods were combined it would be better to solve the false vacuum problems. The best way to solve the liquid-frozen false vacuum is to make the assembly as dry as possible, and to protect the dried assembly in a suitable environment.

Another type of quasi-evaporation problem is the effect due to the trapped contaminates in the wick. If a tiny non-metal particle were trapped in the wick, and finally were sealed in the heat pipe assembly then at a high working temperature, this particle could suddenly decompose. Consequently, it would release large amounts of gases, thus destroying the vacuum in the heat pipe. If the particle releases very little gas, the heat pipe would still function. The ways to eliminate such problems are to thoroughly clean the materials, especially the wick, to remove particles as much as possible; and apply heating to the assembly to make the particles decompose in advance. The second method similar to that is used in the degassing process.

Degassing is a process that the adsorbed gas is released from the metal or other materials surface. To realize degassing, the gas molecules need to be at a higher energy 
than the metal surface energy. Different metals have different surface energy, which correspond to a specific degassing rate. The degassing flow can be expressed as,

$$
Q_{D}=q_{D} \cdot A_{D}
$$

where, $q_{D}$ is specific degassing rate. For copper: $3.5 \times 10^{-8}$ Torr litre/s 1 hour; and $3.56 \times$ $10^{-10}$ Torr $\cdot$ litre/s 10 hour (Berman, 1992). For stainless steel: $9 \times 10^{-8}$ Torr $\cdot$ litre/s 1 hour; and $2 \times 10$ Torr $\cdot$ litre/s hour (Berman, 1992). And $A_{D}$ is degassing surface area $\left(\mathrm{cm}^{2}\right)$.

In our case, the surface area of the 100 mesh copper wire screen is $155.6 \mathrm{~cm}^{2}$; and the copper tube internal surface area is $44.86 \mathrm{~cm}^{2}$. The total degassing surface area is $200.4 \mathrm{~cm}^{2}$. Substituting these data in Equation (2.20) gives the degassing flow rate for the heat pipe assembly as $7.014 \times 10^{-6}$ Torr $\cdot$ litre $/ s 1$ hour, and $7.1 \times 10^{-8}$ Torr $\cdot$ litre/s 10 hour. These two results are far lower than the system pumping speed, and can be ignored in a dynamic vacuum process. However, for such a heat pipe assembly with a $4.875 \times 10^{-3}$ litre net internal volume, after a long time static vacuum, i.e., 100 hours, degassing related pressure rise could be at least 5.24 Torr, which is a significant value in our case. This comparison indicates that degassing can also cause a false vacuum in a dynamic process. There are normally two approaches to this problem; lengthen the vacuum process time and high temperature baking. The most common method is the method of heating the metal to be vacuumed. To implement this approach, first we must make sure there is no residual liquid left in the assembly. Otherwise, with liquid vapour the copper materials would oxidize at the high temperature during the heat degassing process. The suggested pre-degassing condition for copper is $500^{\circ} \mathrm{C}$ to $550^{\circ} \mathrm{C}$ for 8 hours in a high vacuum (Diele and Jaeckel 1966). For nickel, a temperature of $750^{\circ} \mathrm{C}$ to $950^{\circ} \mathrm{C}$ and 8 hours in high vacuum is sufficient (Diele and Jaeckel 1966). For higher or ultra high 
vacuum applications, a further degassing is needed, and the temperatures are required to be $1250^{\circ} \mathrm{C}$ for copper; and $1050^{\circ} \mathrm{C}$ for nickel (Diele and Jaeckel 1966). Such high temperature degassing is not necessary for our work, a medium vacuum application. In our work, the maximum temperature was $500^{\circ} \mathrm{C}$, and the baking time was about 1 minute. The vacuum was at a level of $10^{-3}$ Torr. Figure 2.5 shows the initial degassing process in a heat pipe evacuation. From the figure, we can see that when we applied the torch heating to the heat pipe assembly, the vacuum gauge reading moved from $6 \times 10^{-3}$ Torr to $7.5 \times 10^{-1}$ Torr. After a few such heating processes, the final vacuum reached was $4 \times 10^{-3}$ Torr.

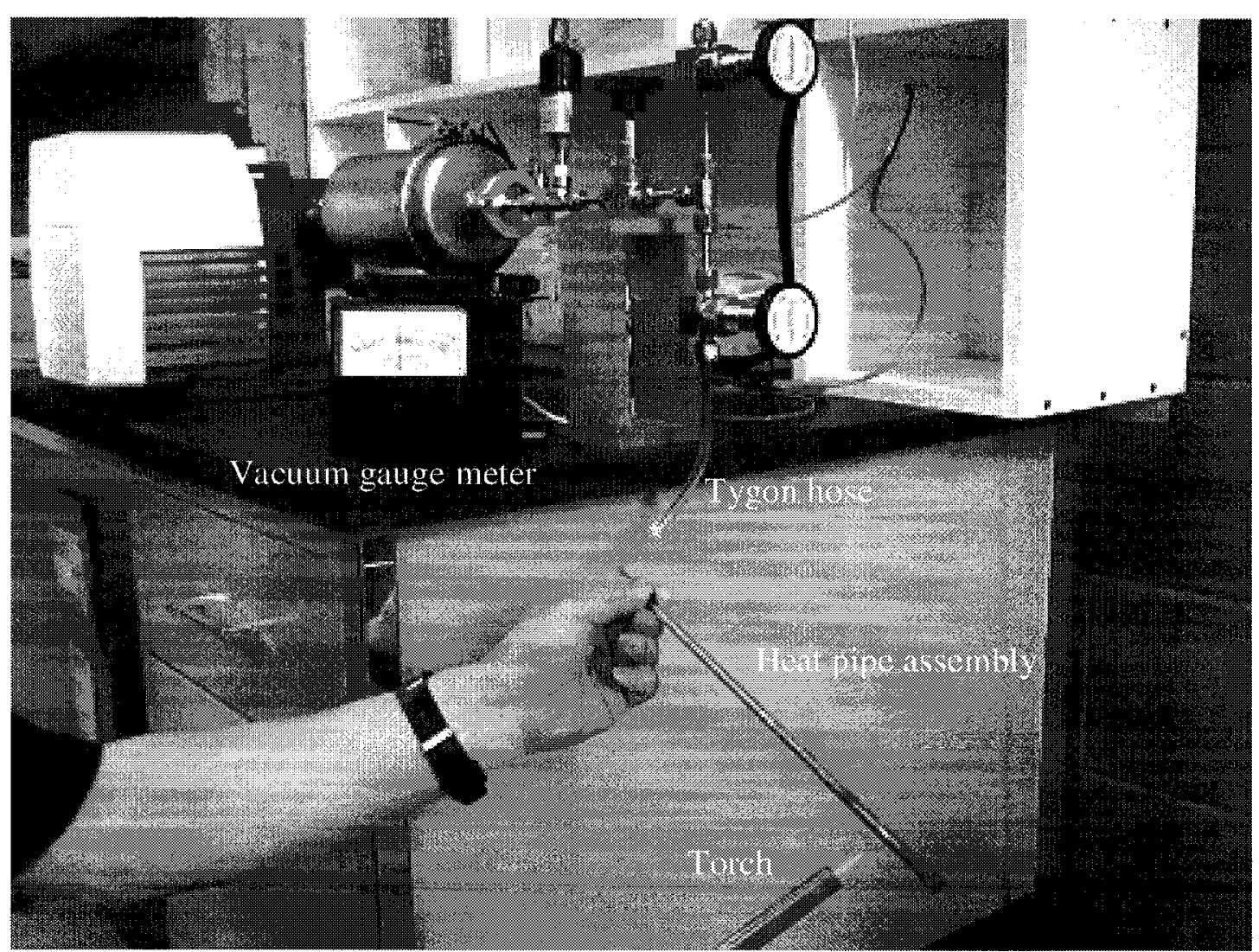

Figure 2.5 Heat pipe degassing process

Experimental results indicate that the degassing pressure and the rate of degassing for metals are functions of pumping time; and are inversely proportional to the pumping 
time. Table 2.2 shows the actual experiment records about the degassing pressure and the rate of degassing as function of pumping. From this table, we can see that the degassing rate decreases very fast with pumping time. Further increases in pumping time will not help degassing much. Besides, in our case the fluid charging required pressure of $5 \times 10^{-3}$ Torr is far higher than the degassing pressure for both metals. The more effective degassing acceleration is high temperature baking. Note that the temperature should be controlled within the required range due to the hydrogen permeation as will be discussed.

Table 2.2 Degassing and rate of degassing as function of pumping

\begin{tabular}{|c|c|c|c|c|c|c|c|c|c|}
\hline \multirow{2}{*}{$\begin{array}{c}\text { Impedance } \\
\text { [litre /s] }\end{array}$} & \multirow[t]{2}{*}{ Metal } & \multicolumn{8}{|c|}{ Time [min] } \\
\hline & & 5 & 10 & 15 & 20 & 25 & 30 & 60 & 120 \\
\hline \multirow[t]{6}{*}{0.07} & & \multicolumn{8}{|c|}{ Degassing pressure $\left[10^{-7}\right.$ Torr $]$} \\
\hline & $\mathrm{Cu}$ & 250 & 140 & 100 & 85 & 72 & 60 & 34 & 18 \\
\hline & $\mathrm{Ni}$ & 270 & 120 & 80 & 62 & 52 & 43 & 20 & 8 \\
\hline & & \multicolumn{8}{|c|}{ Rate of degassing $\left[10^{-9}\right.$ Torr litre $\left./ \mathrm{s} \mathrm{cm}^{2}\right]$} \\
\hline & $\mathrm{Cu}$ & 141 & 79 & 56.7 & 47.5 & 38 & 33.4 & $\overline{18.3}$ & 8.7 \\
\hline & $\mathrm{Ni}$ & 158 & 67.5 & 45 & 3.4 & 28.3 & 23.3 & 10 & 3 \\
\hline
\end{tabular}

(Source: Diels \& Jaeckel, 1966)

The gas permeation is a source of gas load. The related gas load is expressed as,

$$
Q_{P e r}=\frac{K_{P e r} \cdot A_{P e r} \cdot \Delta p}{t_{P e r}}
$$

This factor mainly affects vacuum performance for polymer type materials. For metals, this effect is very limited, and can be ignored in media and coarse vacuum conditions. The most common metal permeating gas is hydrogen. Hydrogen mainly has a strong permeation in chrome steel; and permeates stainless steel at a rate 10-100 lower than that in chrome steel (Norton, 1962). The permeation strength increases with the square root of the driving pressure (Norton, 1962). Rare gases do not permeate metals (Berman, 1992). Hydrogen permeation for copper is $1.67 \times 10^{-15}$ Torr litre $\mathrm{mm} / \mathrm{cm}^{2} \mathrm{~s}$ at $75^{\circ} \mathrm{C}$, and $1.75 \times 10^{-11}$ Torr liter $\mathrm{mm} / \mathrm{cm}^{2} \mathrm{~s}$ at $300^{\circ} \mathrm{C}$. For nickel it is $1.67 \times 10^{-15}$ Torr liter $\mathrm{mm} / \mathrm{cm}^{2} \mathrm{~s}$ 
at $20^{\circ} \mathrm{C}$, and $3.6 \times 10^{-6}$ Torr liter $\mathrm{mm} / \mathrm{cm}^{2} \mathrm{~s}$ at $300^{\circ} \mathrm{C}$ (Diels \& Jaeckel, 1966). From this comparison, we can see that the temperature strongly affects the hydrogen permeability, especially for nickel. In our case, the system is at a medium vacuum level. The values of hydrogen permeability for copper and nickel are very limited, and can be ignored. For polymer material vacuum, gas permeation cannot be ignored, especially helium permeation. In our work, we used a Tygon hose to connect the heat pipe assembly to Valve 3. In the dynamic vacuum process, we almost could not detect any difference in vacuum tightness performance between the stainless ferrule tube fitting and the Tygon hose. However, a great difference was shown in the static vacuum process between these two vacuum fitting materials. The stainless steel ferrule fitting did not show any pressure rise after a three-day period. The Tygon hose fitting experienced about 250 Torr in pressure rise after just a couple of hours of the static vacuum process.

Each of these sources of gas load could play a dominant role in a specific vacuum process or gas flow range. Based on the analyses on the gas load, it is helpful to recognize the source of problems that occurred in the actual experiments. While for a specific case, the problems could relate to more factors; to solve the problems we need to make specific analyses with respect to the specific circumstances.

\subsubsection{Fluid Charging Analysis}

The first question for fluid charging is the amount of fluid needed to charge into a specific heat pipe assembly. The volume of the fluid to be charged into a heat pipe can be obtained by the product of the wick volume and its porosity. The specific calculation process will be provided in Chapter 4 . To verify the theoretical calculation, we applied an experimental approach to the amount of fluid. First, a sample wick material was taken, 
and then its dimensions were recorded. Next, its volume was calculated, and its net weight was measured. The wick material was then placed in the working fluid. When it was saturated in the liquid, it was taken out from the liquid, and total weight was measured. A comparison of the weight difference before and after soaking the wick was taken. It was then required to obtain the net weight of the fluid absorbed by the wick. Dividing this net weight by the fluid density, the net volume of the fluid absorbed in the wick was obtained. Then, by dividing this net volume by the wick volume, the porosity of the wick was obtained. This process was applied respectively to the 100 and 50 copper mesh wire screens and the nickel foam for seven, nine and four times respectively, using pure water as the penetrating liquid. The average results of the copper mesh porosity for the 100 and 50 meshes was 0.6284 and 0.7047 respectively. Comparing with theoretical calculations, i.e., for 100 mesh is 0.629 , and for 50 mesh is 0.629 , the result of the 100 mesh was quite accurate; the result of the 50 mesh had a discrepancy of 12 percent. This could stem from manufacture tolerance and the experimental errors. With the same method, the average nickel foam wick porosity was obtained as 0.649 ; this result was consistent with another test done using alcohol as penetrating liquid.

In the wick porosity measurement, we found that a dry wick is very difficult to wet. This phenomenon also applied to the nickel foamed wick. This may be due to the material surface characteristic factor. As analyzed in Section 2.2.1 and Section 2.2.3, the surface of untreated metal materials has an adsorbed gas layer and contaminants. These would worsen the metal surface wetting characteristics. To address this issue, we treated the wick sample with acid. The wetting on the copper wire screen mesh looked better, but the nickel foam still had a light grey colour, which means the nickel was not 
completely wetted. This introduces hysteresis. Air has a lower surface tension than water and is therefore penetrates the nickel foam more easily. In reality, this was not consistent with the expectation. In this case, the copper wire screen mesh was an exception. Comparing these two results, the light grey colour was due to the large amount of micro air bubbles, which were trapped by the inkbottle shaped porous concavities in the nickel foam. The copper wire screen mesh has no such shaped concave wick structure. Therefore the existing air in the wick is easy to squeeze out by the penetrating water with a higher surface tension. In the nickel foam wick, the existing air in the concavities has no exit, and therefore it is easily trapped by the penetrating water. On the other hand, with a high surface tension, water has a large contact angle; this increases the barriers for the water to enter the concavities through the very small aperture. Furthermore, the water would compress the air enclosed in the concavities until the pressure equilibrium state is established. Consequently, the air is trapped in such a foam structured wick. We applied heating to the nickel foam sample and then put it into hot water. This time, the colour turned to dark grey after it was put into the hot water, the nickel foam was completely wetted. This is attributed to the heat that decreases the water surface tension, and thus decreasing the water contact angle, in turn improving water permeability in the foam structure. Having a small contact angle, water would "spread" rather than "fill" into the concave areas, thus the existing air in the concavities can easily exit, and will be not trapped in the foam structured wick. This fact supported the analysis about the wick trap effect on the air. A good comparison is the experiments done with alcohol. In that experiment, the alcohol penetrates the nickel foam very well at room temperature. One 
of the reasons for this is due to the good permeability of the alcohol with a lower surface tension.

Another question relating to the amount of fluid is the fluid charging rate. The fluid charging rate is the ratio of actual amount of charged fluid to the required amount of fluid for a heat pipe. The concept of over charge rate is here defined as the ratio of the extra amount of charged fluid to the required amount of fluid fully saturates the wick in a heat pipe. It was suggested to deliberately over charge the heat pipe from 10 to 20 percent (Peterson, 1994). In our practical application, we tried both normal and over charging. One example was the fine copper water with 58 percent overcharge. Other examples include a fine copper acetone heat pipe with 80 percent overcharge; and a 100 and 50 mesh composite copper wire screen water heat pipe with 30 percent overcharge. The experimental results indicate that the over charged heat pipes have a better performance, which will be discussed in Chapter 3, and Chapter 4. Note that higher overcharging rates do not increase heat pipe performance. Too much fluid in the heat pipe would reduce the vapour space, and enlarge the extra liquid volume, thus decreasing the vapour transfer heat capacity, and offsetting the heat pipe functionality. On the other hand, if the liquid is so much that it submerges the entire wick-vapour interface in the evaporation zone, the liquid would not evaporate until the entire extra liquid reaches the boiling point. In our cases, two heat pipe failures might be due to overcharging. There should be a critical overcharge rate for a specific heat pipe configuration at which point there is both enough extra fluid acting as buffer and enough inner space for the vapour. The opposite example is under charging. In our case, the nickel foam heat pipe was under charged by 8.69 percent, but in the experiments it performed normally. One of the explanations is that as 
long as the under charged water is enough to wet the entire length of the wick in the axial direction, it would more freely flow in the wick, thus leaving the wick partially dry in the radial direction. This partially radial dried wick has a lower radial thermal resistance that is a magnitude higher than the others in the heat pipe thermal network, as will be discussed in Chapter 4. The reduction of the wick radial thermal resistance would significantly improve the radial heat transfer, thus improving the entire heat pipe performance.

In the fluid charging process, another factor that should be considered is the gas adsorbed in the working fluid. Like metals, liquid working fluids also adsorb gas. Being charged in the heat pipe assembly under a vacuum condition, the adsorbed gases would be released from the liquid, thus the vacuum would be degraded. In cases of too much gases release, the vacuum would be destroyed, so it is necessary to purify and degas the fluid to be charged. Many published materials suggested that one should apply fluid purifying and degassing processes prior to fluid charging (Chi, 1976), (Peterson, 1994), and (Dunn \& Reay, 1978). These suggestions on purifying and degassing processes are based on similar principles as those introduced in Section 2.2.3.3 about the metal degassing process.

In our work, we used the purified water: distilled water and reverse osmosis (R.O.) water as working fluids instead of on-site purifying and degassing during the process of evacuation and charging. In the later testing periods, it was difficult to tell any differences between the heat pipe test samples with these two types of water. While, only with this result we can not suggest replace on-site fluid purifying and degassing techniques. The heat pipes have not experienced life testing, i.e., durability testing, yet. Conclusion will 
be hold on for a time. And further discussion of these issues will be included in Chapter 5.

Another concern in the fluid charging process relates to the fluid trapped in the heat pipe assembly. In our earlier trials, we found that after fluid charging, some assemblies were under charged due to part of the fluid being trapped inside the " $T$ " shaped joint connecting the three valves as illustrated in Figure 2.4. This could be due to two factors; the pipe pressure gradient, and the pipe capillarity. The pressure in the " $\mathrm{T}$ " shaped joint is lower than that in the wick section, thus the entered water would rather stay at the "T" shaped joint section. From other experiments, we also found that in an atmospheric environment the $1 / 4 "$ pipe has enough capillarity to hold water in a vertical position, and this also occurred to the fluid charging operations. To solve this problem, we applied a cooling bag to the assembly during fluid charging, as described in the procedure Step 15 in Section 2.2.3.1. This has proven to be a very effective and necessary step in the fluid charging process. Figures 2.6 and 2.7 show the "cooling suck" effect in the fluid charging process. Comparing these two figures, we find that before applying the cooling bag, there was much liquid water in the Tygon hose. Applying the cooling bag, the water was sucked into the heat pipe, the Tygon hose looked clear. After applied cooling, there was almost no residual water found in the "T" shaped joint connector, which means cooling "sucks" almost all the water into the heat pipe. 


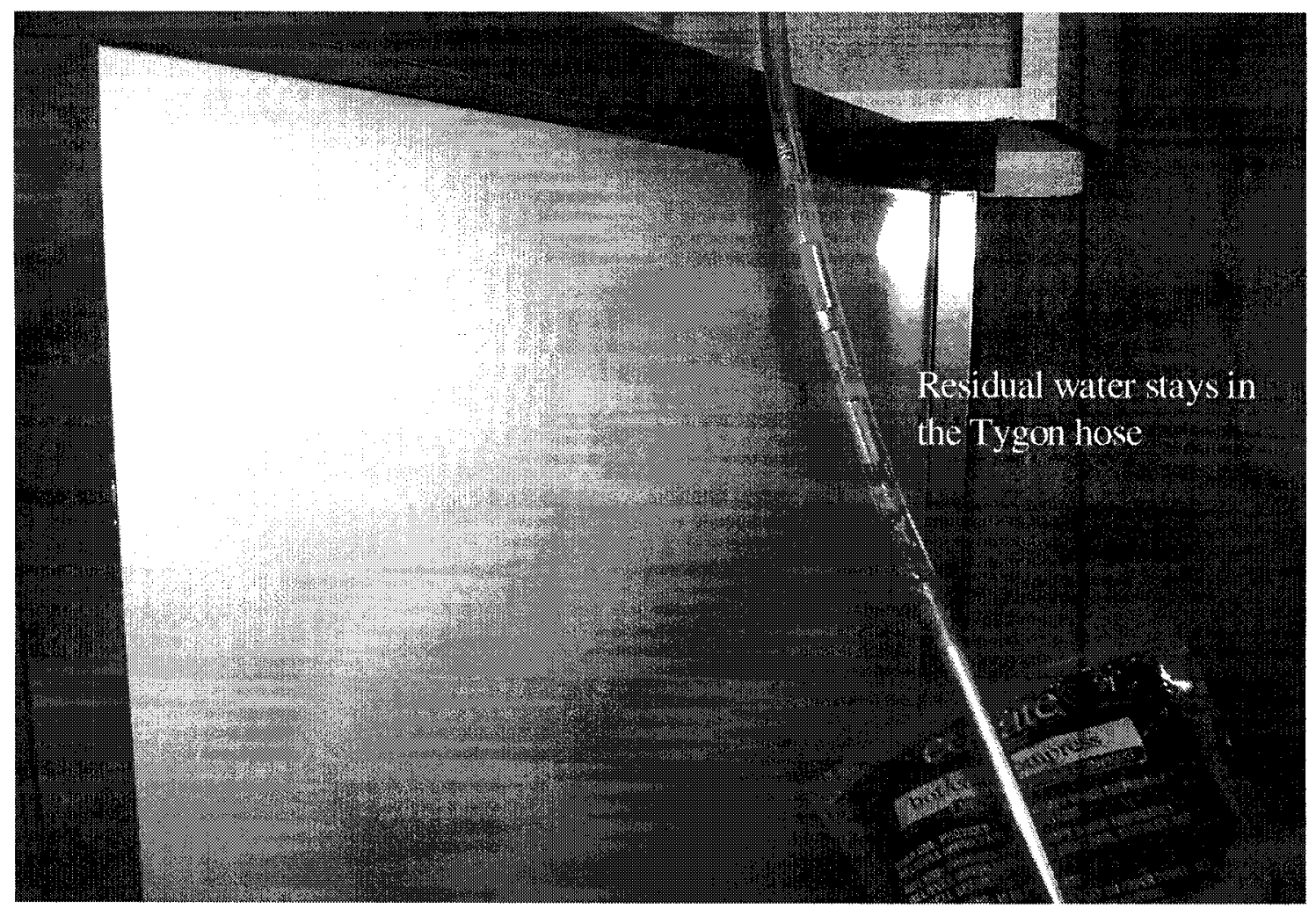

Figure 2.6 Charged fluid stays in the Tygon hose before applying the cooling bag

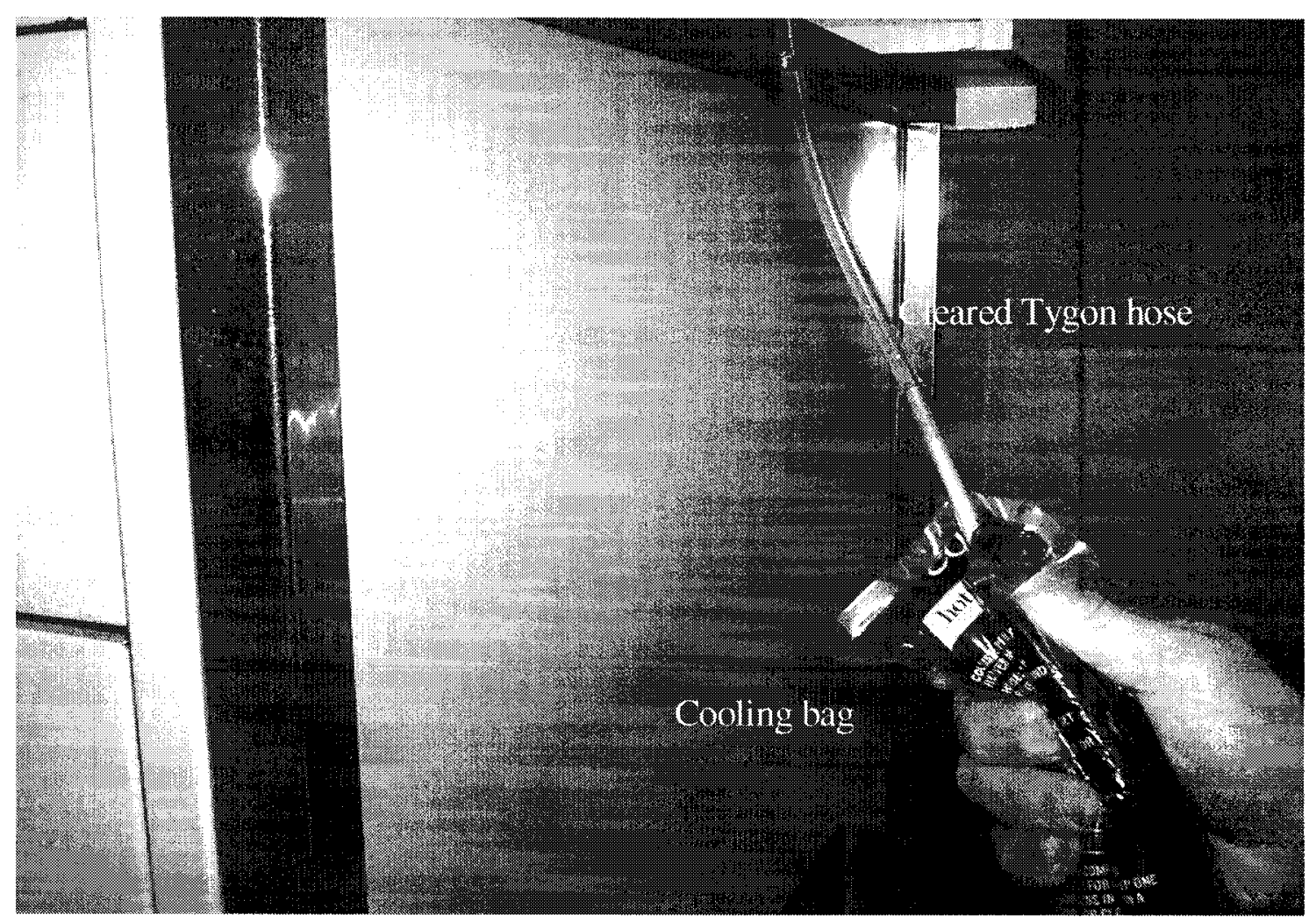

Figure 2.7 Fluid was sucked into the heat pipe when applied the cooling bag 


\subsubsection{Post Charge Sealing}

The sealing after the fluid charging is another challenge in the heat pipe manufacturing; this process determines the heat pipe success or failure. This section mainly discusses some concerns and related approaches in this process.

The major concern in this process is crimping. This process is taking place immediately after the heat pipe assembly fluid charging. Apply the crimping tools to the heat pipe to flatten it, and then apply the pinch-off tool to pinch the flat section to prepare for the final sealing. This process has two phases; flattening and final crimping. The flattening is applied in the clamp with a pair of 1 " wide flat tool steel plates to flatten the heat pipe on the section near the end of the attached Valve 3. In this phase, the pressing force should be appropriate. Too much pressure would destroy the container tube, resulting crack in the tube wall; while less pressure would leave space in the flatten section thus also destroying vacuum in the heat pipe. This space would contain air after pinch-off, and consequently the contained air would permeate into the heat pipe section after release the pinch-off tool, and result in the heat pipe failure. For instance, suppose the heat pipe inner pressure is $1 \times 10^{-3}$ Torr. Suppose this flat space has 1 thousandth the volume of the heat pipe, and contains air with an atmospheric pressure of 760 Torr, and then when an equilibrium state is reached, the new pressure will be 0.76 Torr. Thus, destroying the vacuum condition, and therefore resulting in a heat pipe failure.

To solve this dilemma, we applied appropriate forces to flatten the pipe, and applied a fully locked pinch-off tool, as shown in Figure 2.8. In the argon-arc welding process, after cutting off the pipe at the flat section, one should still leave the pinch-off tool locked and then apply heat to the heat pipe. When the heat pipe is hot the contained air inside the 
flat space would be repelled out, and then keep on finishing the welding. This approach was proven to be effective in later experiments. Another factor was the copper tube material. At the beginning, we used soft copper tube. It was found that compared with the normal copper tube, the soft copper tube was easy to melt out in the argon-arc welding process and thus resulting leak. Finally, we adapted the later one as the main heat pipe container tube material.

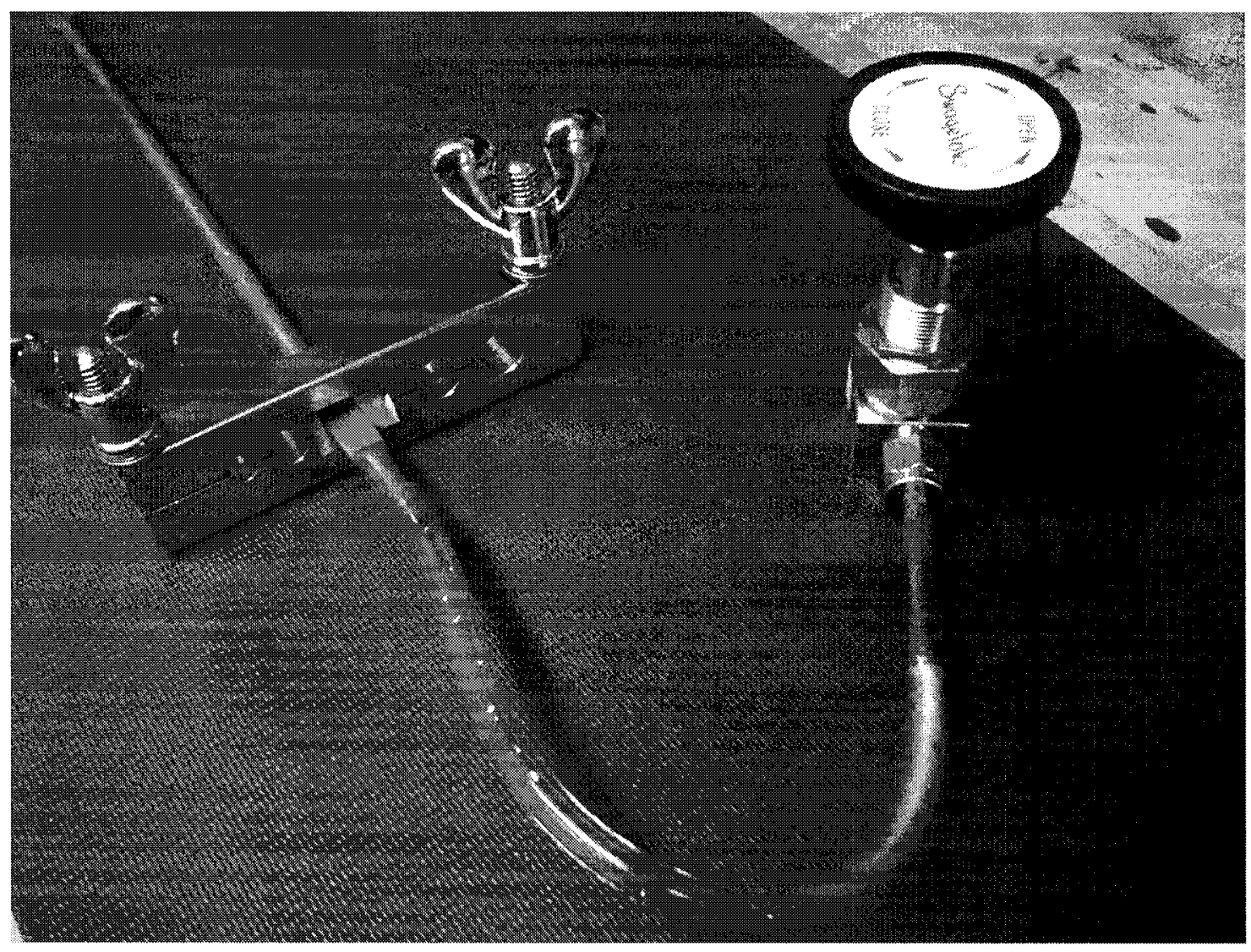

Figure 2.8 Final pinching before welding

\subsubsection{Finishing Process}

After post-charge sealing, the heat pipe needs a series of preliminary checks before the formal experimental testing. These preliminary checks include a welding check, heat pipe functioning test, and a capillary check. 
After argon-arc welding, keep the pinch-off tool locked and then check the welding ream with a magnifying glass. If any defects are found on the ream, the heat pipe needs to be re-worked with the argon-arc welding. After the welding check, a preliminary heat pipe function test is applied to check if the heat pipe possesses heat pipe functionality. This was done by using another copper tube with the same dimensions as the heat pipe to be tested, and then putting both into a hot water beaker. If by hand the heat pipe is hot much faster than the copper, then the heat pipe passes the preliminary test. Application of thermocouples to the heat pipe is then used to check the heat pipe and the copper tube with warm water. The same test is repeated with lower temperature water until the temperature is reached, at which point both perform similarly. After a specific amount of time, these tests can be re-applied to check if the heat pipes still possess heat pipe functionality.

The capillarity test is similar to the functional test. The main difference is that the pipes were positioned both vertically, and a heat source to the top ends was applied to check the temperature response on the bottom ends. In practical tests, the heat pipe responded much faster than the copper in an against gravity position. In our application, we used a torch as the heat source.

\subsection{Chapter Closure}

This chapter mainly introduces the heat pipe design considerations, and the detailed manufacturing process. In the design phase, the major factors were analyzed. In the manufacturing phase, several processes were introduced such as material treatment, evacuation and fluid charging, sealing, and finishing. In the evacuation and fluid charging 
processes, detailed analyses on the gas flow, the gas load, and fluid charging were discussed. This detailed introduction to the design and manufacturing process lays the foundation for experimental investigation into heat pipe characteristics, which will be covered in Chapter 3. 


\section{Chapter 3}

\section{Experimental Investigation of Heat Pipe Characteristics}

The purposes of heat pipe testing are acceptance checking or evaluation in mass production and performance investigation in research. Here the test program mainly focuses on the latter, heat pipe performance investigation and analyses. This chapter introduces detailed heat pipe testing process, and discuss some phenomena observed in the experiments.

\subsection{Introduction to Heat Pipe Testing}

As a heat transfer device, heat pipe performance is evaluated in terms of heat transfer capacity and efficiency. A typical heat pipe test involves laying a heat source upon the evaporator of the heat pipe, and applying cooling on its condenser, to investigate the heat pipe temperature characteristics, therefore to analyze the other characteristics. The directly measurable factor is the temperature. Other factors such as heat flux and pressure can be obtained through temperature analyses combining thermal physics and thermodynamics knowledge. From this point of view, it can be said that our heat pipe testing is temperature based. There is evidence that, to a great extent, temperature-based analyses are very practical and reliable. This will be further discussed in later sections. 
Besides temperature analyses, other tests are also carried out in the early and late stages of heat pipe manufacturing, e.g., wetting and capillarity of wick material, vacuum and leakage of the heat pipe assembly, and mechanical soundness of the container.

\subsubsection{Summary of Heat Pipe Testing Approaches}

Unlike the preliminary evaluation process as introduced in Chapter 2, which mainly verifies whether the product possesses the basic heat pipe characteristics, the heat pipe testing discussed in this chapter is essentially the heat pipe performance verification. This test acts as a working simulation of heat pipes, providing heat and cooling sources on the evaporator and condenser respectively, to verify the heat pipe performance.

The performance of a heat pipe involves two concerns "what" and "how". The former regards the absolute characteristics such as the heat transfer capacity, the range of normal working temperature, the temperature gradient between the evaporator and condenser. These are not time-related performance characteristics, i.e., they relate to steady-state operation. The latter relates the relative behaviours such as how fast a heat pipe can reach it steady-state working condition, how fast it responds to the external changes including power input and applied temperature. These are time-related performance, and are also called transient characteristics.

Temperature analysis is a major approach to heat pipe performance evaluation and the experimental investigation of a heat pipe. Selection of temperature measurement methods and tools is the first concern for the heat pipe experimental investigation. The following sections will introduce the experimental apparatus used in our work. 


\subsubsection{Introduction to Experimental Apparatus}

The elements for the heat pipe performance verification testing include a data acquisition and processing system, a heating source, a cooling source, a power supply and control system, insulation, and temperature sensors. These will be introduced respectively in the following sections. The detailed information about some of the testing elements is also provided the Appendix I. Figures 3.1 and 3.1a present the pictures of the experimental apparatus and the sketch of the test setup respectively.

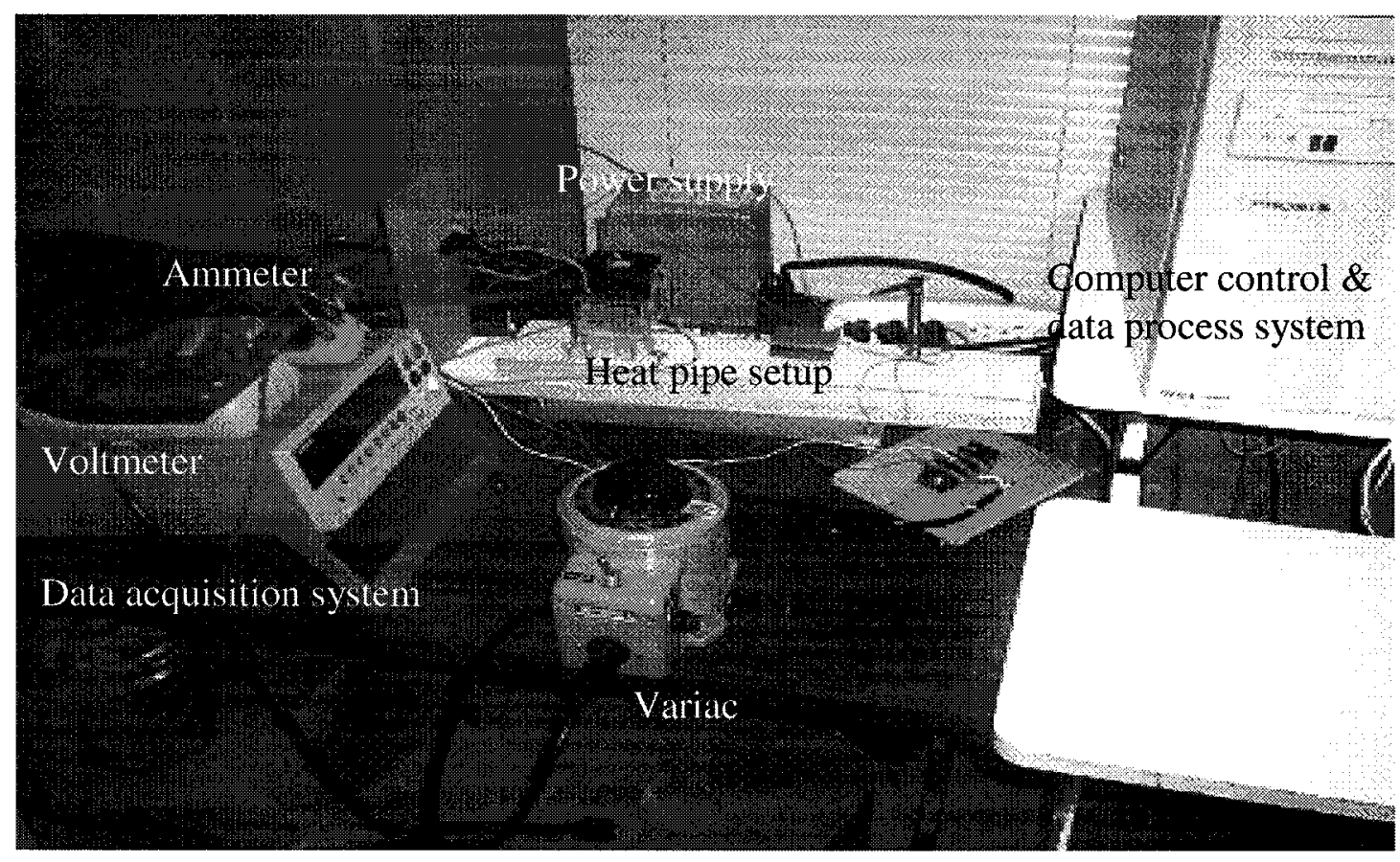

Figure 3.1 Heat pipe experimental apparatus 


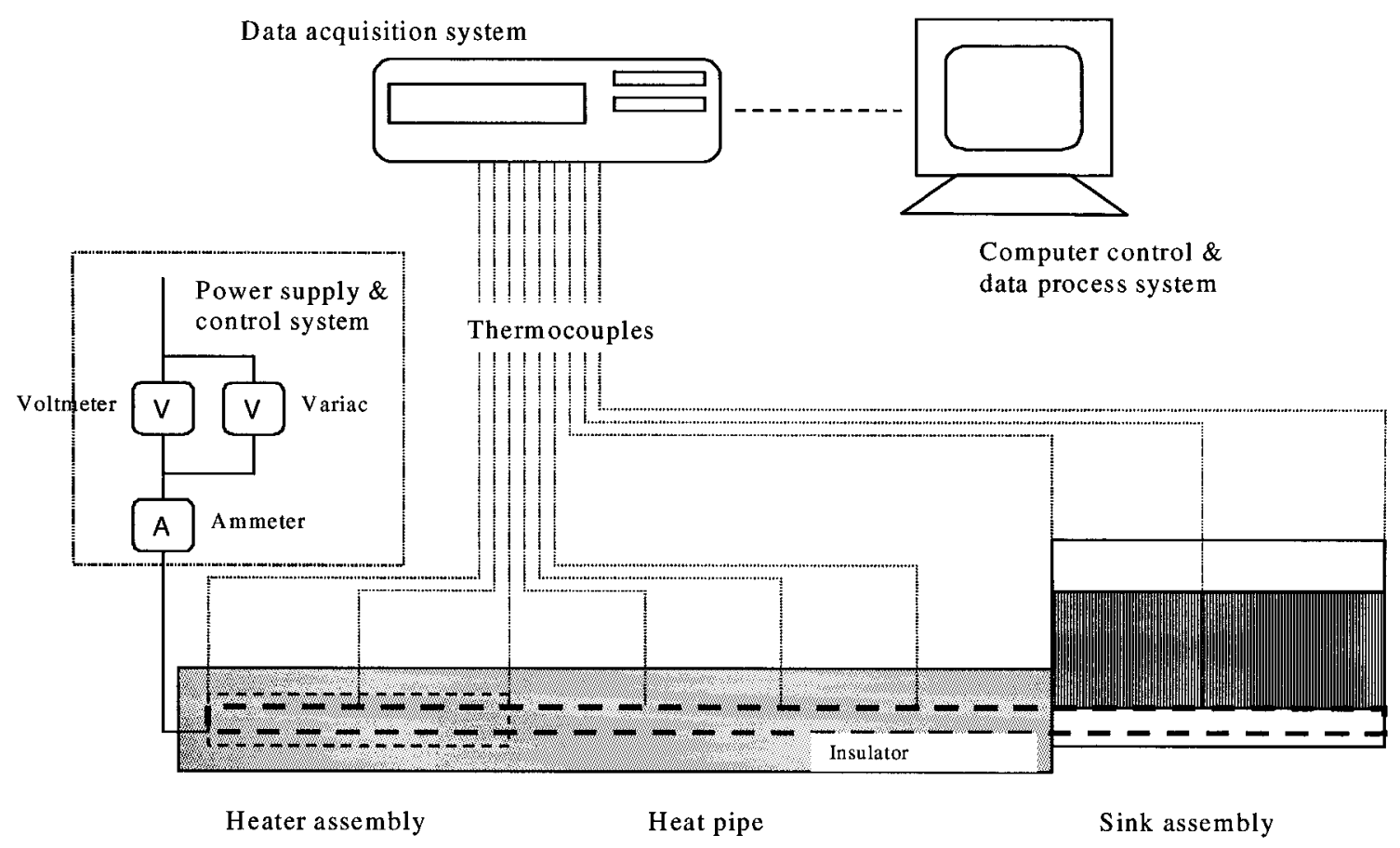

Figure 3.1a Schematics of heat pipe test experimental apparatus

\subsubsection{Data Acquisition System}

The data acquisition and processing system plays a critical role in these heat pipe performance tests. It implements temperature measurements during the process of the testing, transiently recording the values of each measurement, and saving these values as data in the form of a file. The file can then be transferred to the data process section, e.g., computer systems through I/O connection.

In this case, the data acquisition system is a KEITHLEY Model 2700 Multimeter/Switch System. This is a 61/2-digit high-performance multimeter/data acquisition system. It can measure voltage (DC and $\mathrm{AC}$ ), current (DC and $\mathrm{AC}$ ), resistance (2- and 4-wire), temperature (thermocouple, thermistor, and 4-wire RTD), frequency and period, and test continuity. In the testing, its temperature measurement function was employed. 


\subsubsection{Heat Source Assembly}

The heat source provides heat power input to the evaporator of the heat pipe; it also acts as a heat load simulator. Figure 3.2 illustrates the schematics of the heat source assembly. This is a special design for this testing. It is composed of two parts; a heating base, and a pressing board. The heating base is a $2 " \times 2$ " copper board with a thickness equal to the external diameter of the heat pipe, e.g., $3 / 8$ " or $1 / 4$ ". A corresponding semi-circular groove was machined on one side. The radius of the groove matches that of the heat pipe to be tested. On the either side of the groove, two threaded holes are set for tightening the pressing board with brass screws. On the other side of the copper board, an OMEGA KH 202/10 Kapton flexible heating film is permanently glued with OMEGA 101 epoxy adhesive. The Kapton flexible heating film is a Kapton with Teflon insulated electrical thermal heating film with an effective working area of $2 " \times 2 " \times 0.005$ "and can provide heating power up to $40 \mathrm{~W}$. With an even heat distribution the OMEGA 101 epoxy adhesive, is a special epoxy-based permanent adhesive suitable for heating elements. It possesses a relatively high thermal conductivity compared to regular glues.

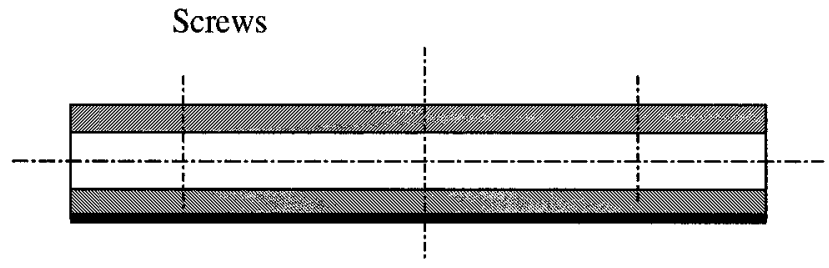

Thermal compound

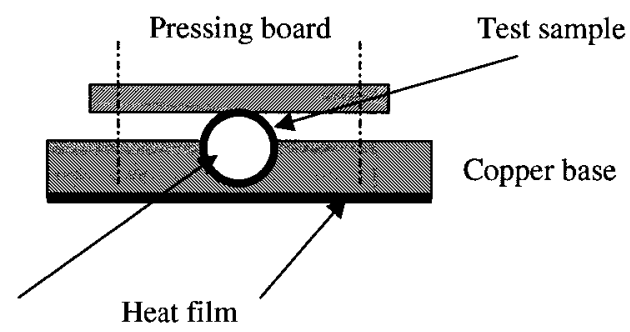

Figure 3.2 Schematic of heat source assembly

The other piece of the heater assembly is the pressing board. It is a piece of aluminium board with four screw holes. This pressing board is used for pressing the heat pipe evaporation section into the groove to ensure a good contact thermal conductance 
between the heat pipe evaporator and the groove surface on the copper heating base. To assist such approach, Wakefield Engineering 120 series thermal joint compound is applied between the contact surfaces. This thermal compound also has a good thermal conductivity, and helps ensure a uniform temperature distribution in the heat transfer interface. For specifications of each elements of the assembly, please refer to Appendix I. Two sets of such heater assemblies were applied in the testing applications. Each has a corresponding thickness of the copper base and radius of the groove regarding the heat pipe. This type of heater assembly is available for every heat pipe, possessing the same radius as that of the groove. This ensures that each heat pipes can be tested with the same heat source and in the same heat load condition, therefore making the tests comparable.

This design of heat source can work as a heating simulator providing heat in forms of heat conductance to the heat pipes or other tube-shaped test samples. It is suitable for the conductance application tests, such as electronic device cooling. Also, this design brings convenience to the experimental setup. The spaces between the copper base and the pressing board make the thermocouples can be fixed on the heating section of the test sample to be tested, along the axial direction. In this way, measure the axial temperature distribution.

\subsubsection{Sink Assembly}

The sink works as a cooling source, playing an opposite role to that of the heat source in the heat pipe performance testing. Being attached on the condenser of the heat pipe, the condenser dissipates the heat added to the evaporator by the heat source of the heat pipe. In a heat pipe system, a cooling sink and a heat source work with matched capacities. Figure 3.3 illustrates the schematics of the sink assembly. 


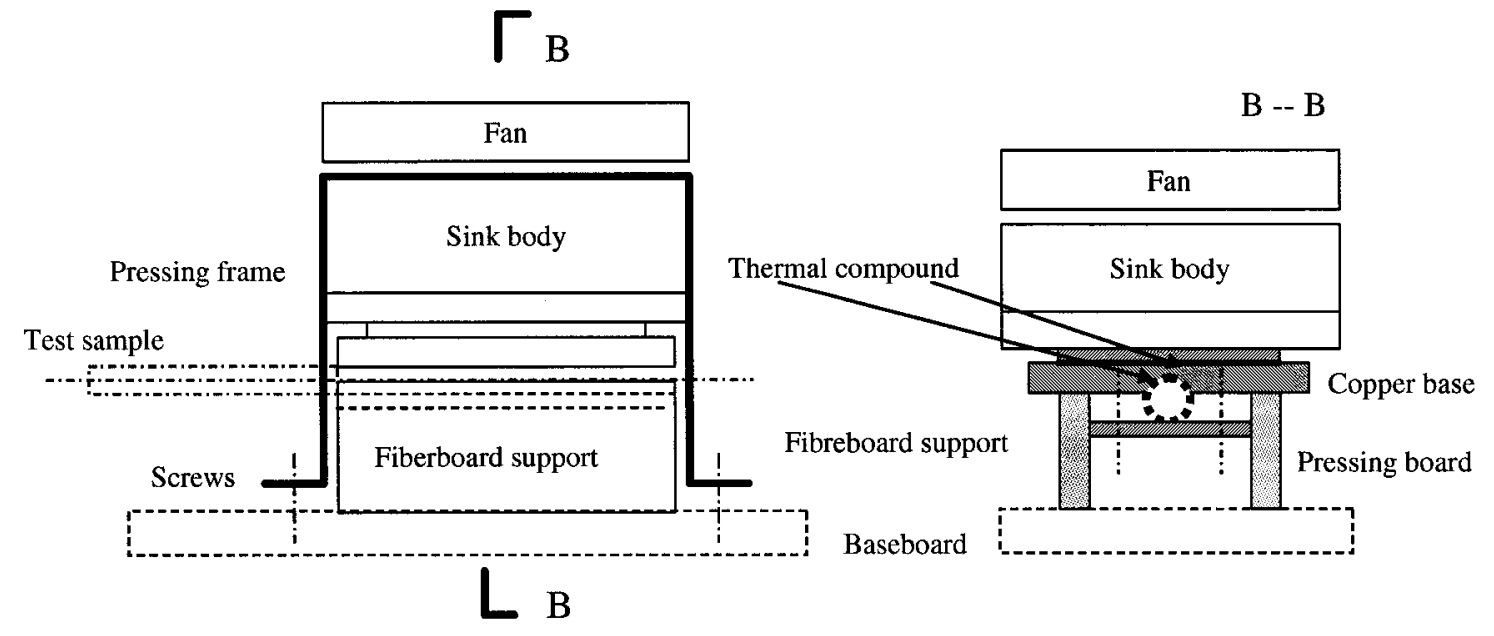

Figure 3.3 Schematics of sink assembly

This sink assembly is composed of five main parts; the finned sink body, the copper base, a pressing board, a DC fan, and a pair of fixing frames. The detailed specifications are provided in Appendix I.

The finned sink body is a compound material sink. The main body is aluminium; a $4 \mathrm{~cm}$ diameter round copper disc is permanently embedded in the bottom base of the main body to enhance thermal conductivity.

The copper base is similar to that of the heater assembly. The difference is that the one used in the sink assembly does not have the heating film attached on the side opposite the groove side. In the application, the copper base is attached with Wakefield Engineering 120 series thermal joint compound, and with an external pressing force applied by the fixing frames. These frames force the whole assembly against a pair of fibreboard supports on the base of the setup. The interface between the condenser and the sink copper base also takes the same approach as the one applied in the heater assembly.

Like the heater, this sink assembly is also available for every heat pipe having the same radius as that of the groove. This ensures that the heat pipes are tested with the 
same cooling source, i.e., in the same cooling condition, thus making the tests comparable.

Similar to the heat source assembly, the sink assembly dissipates heat through semicircular contact conductance. This also confines it to contact conductive heat transfer. Also, this design brings the same convenience as the one mentioned in Section 3.1.3.2 about the heat source assembly.

A DC fan can be attached on the top of the finned sink body, providing an impending blowing to the sink body. This fan can work at either low or high speed condition, thus the sink assembly can provide forced, and enhanced forced convections as well as natural convection, i.e., without fan, in the testing process. These terms will be used with the same meaning throughout the entire content in this thesis. The convection temperature is the room temperature. The fan was powered and controlled by a computer power supply.

\subsubsection{Temperature Sensors}

In our work, thermocouples were employed as temperature sensors. The thermocouples we used are OMEGA fine wire "T" type, copper-constantan. The wire diameter is 0.01 " $(0.254 \mathrm{~mm})$, wrapped with woven glass fibre insulator. The joint is a bare ball with diameter of $0.52 \mathrm{~mm}$.

Aluminum adhesive tape was employed to fix the thermocouples to the solid surface of the testing elements. This aluminium tape has an $80^{\circ} \mathrm{C}$ nominal working temperature, but in actual practice, it can work at higher temperature of up to $120^{\circ} \mathrm{C}$ with some special measures. 
The aluminium adhesive tape has a very thin glue film on one side, it would cause little affect to the thermal contact conductance between the thermocouple ball and the solid surface that will be measured. Thus the contact thermal resistance between the temperature measurement interfaces can be ignored in this case.

\subsubsection{Insulation}

Insulation, in this case, played the role of protection from environmental convection to the testing setup. The insulation materials applied in the testing include styrene foam and Nomex piping insulator. The styrene foam can work under $110^{\circ} \mathrm{C}$. It mainly works as an internal insulator, e.g., was used in the evaporation section, directly in contact with the heating element, protecting the Nomex piping insulator. The Nomex piping insulator, works as an external insulator, i.e., was used to wrap the evaporator attached with the heater, as well as the adiabatic section of the heat pipe. This layer protects the testing element from the environment, i.e., air convection to the setup.

The inner insulators, i.e., styrene foams, are naturally put against the heating elements and the high temperature parts, without any fixing configurations. The external insulators were wrapped against the internal insulators or the adiabatic section of the heat pipe, contacting the staff to be protected. Also they were fixed with adhesive tapes externally. Such insulation structure applied to the heat source is shown in Figure 3.4.

\subsubsection{Power Supply and Control system}

The heater is an electrical-resistance heating element. The power supply system is composed of a variac, a voltmeter and an ammeter. The resolution of the voltage meter and current meter are $0.1 \mathrm{~V}$ and $0.01 \mathrm{~A}$ respectively. Adjusting the variac sets the voltage 
for the power input. The input voltage value and the corresponding current value can be read by the voltmeter and the ammeter respectively. Multiplying these values gives the value of the input power, thus conducting the heating power supplying and controlling.

\subsection{Experiment 1}

\section{Investigations of Heat Source Characteristics}

The heat source plays an important role in the experiments. It provides heat power input to the evaporator of the heat pipe to be tested. Even though the main element properties are known from the venders, the whole assembly is designed, and manufactured domestically. To verify its performance it is necessary to make an investigation of the heating assembly.

\subsubsection{Experimental Objectives}

The objectives of these experiments are summarized as follows:

1. Calibrating the voltage-power relationship of the Kapton heating film.

2. Verifying the performance predicted in the design stage.

3. Investigating the maximum capacity of the assembly, e.g., maximum power under a certain temperature constrain, etc. Based on the results to determine the working condition limits, e.g., maximum power levels and corresponding temperatures, for the future testing. 
4. Calibrating the temperature sensor relative accuracy, e.g., relative accuracy of thermocouple measurement at various conditions. This provides a basis for further experiments.

5. Investigating the temperature distribution of the whole assembly to provide the basis for the future temperature measurements for other configurations.

6. Calibrating the whole assembly thermal balance. Thus providing a reference for other configurations.

7. Investigating the performance of the insulation, for instance, the maximum normal working temperature for the insulation materials, the thermal balance capacity of the insulation materials, and the effects of the insulation configurations.

8. Verifying the performance of the pressing board.

9. Verifying the performance the thermal compound between the interface of the heat pipe and the copper base.

10. Verifying the performance of the epoxy adhesive.

\subsubsection{Experiment Principle and Setup}

Figure 3.4 illustrates the schematic of the experiment setup with insulations. In the figure, the term "TC" stands for thermocouple, and the following digits represent the thermocouple number. 


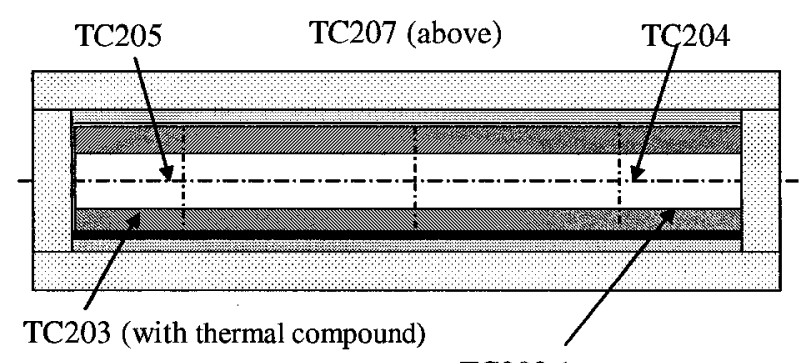

TC202 (Non-thermal compound)
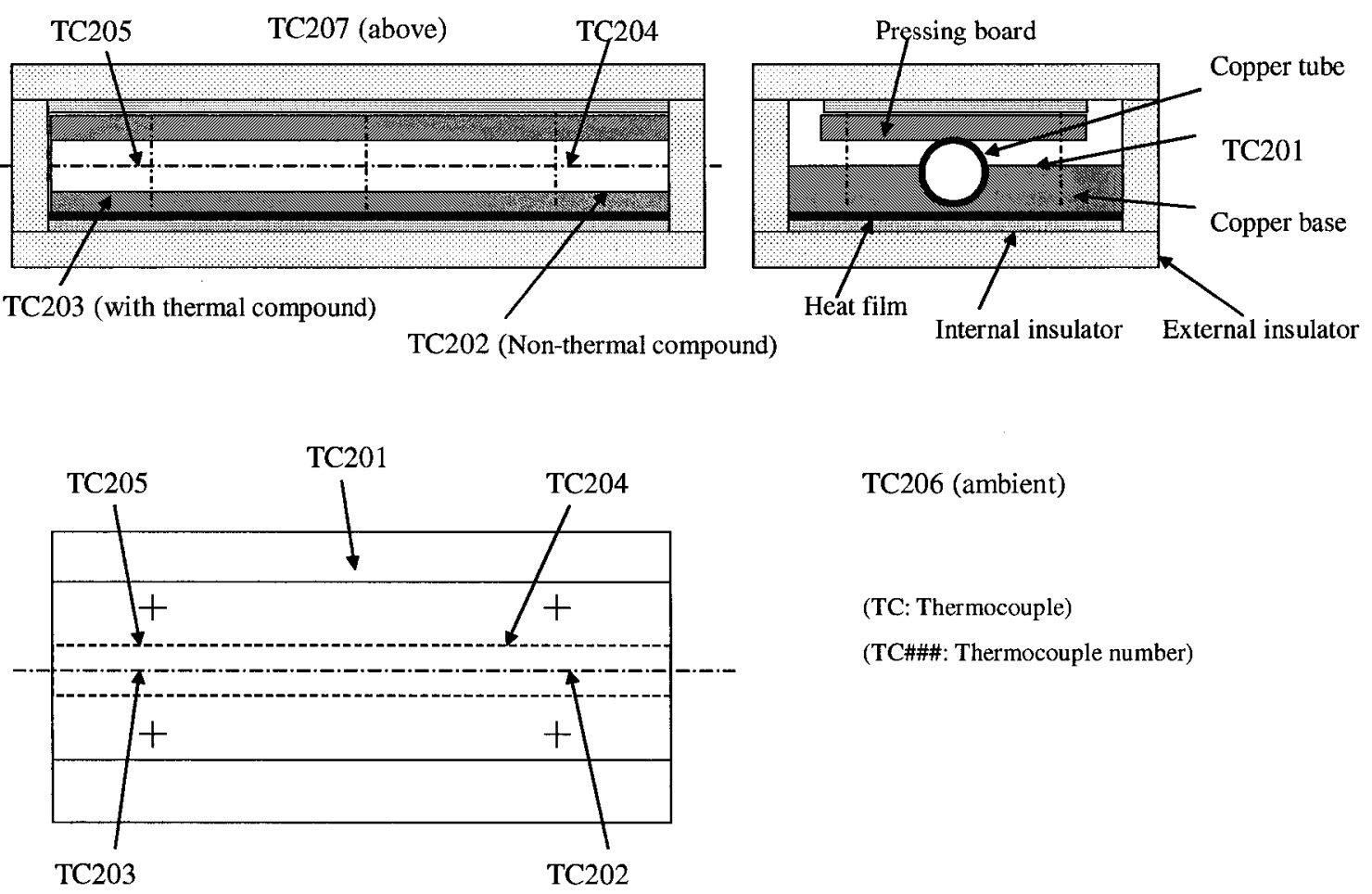

TC206 (ambient)

(TC: Thermocouple)

(TC\#\#: Thermocouple number)

\section{Figure 3.4 Schematics of the heat source assembly experimental setup}

A Nylon strip is applied to suspend the whole assembly, thus minimizing the possible heat conductance caused by any of the supports. From the figure, it can also be seen that a piece of copper tube is pressed, by the pressing board, into the groove in the copper base. The pressing board is fixed with four brass screws that are tightened. In the groove, thermal compound is applied to one half side, and two thermocouples are located between the tube and the groove surface at either thermal compound or non-thermal compound side respectively (TC203 and TC202), .The thermocouples are applied to measure the temperature and make comparisons. Two other thermocouples are located on the tube surface within the space between the pressing board and the copper base on either section of thermal compound and non-thermal compound respectively, (TC205 and TC204). These thermocouples are used to measure these sections' temperature thus to compare the effects of the heat transfer through the copper tube with, and without thermal 
compound. One thermocouple (TC201) is fixed at one side middle point of the copper, to measure the heater surface temperature. The other one (TC207) is suspended above the assembly to monitor the temperature of the natural heat flux above the assembly. One more (TC206) is fixed on the copper plate surface, and is located near the setup to measure the ambient temperature.

\subsubsection{Experimental Procedure}

\section{Section 1: Exploring the Relationship between the Setting Voltage Values and the Power Inputs for the Kapton Heating film}

This section explores the relationship between the voltage setting and the corresponding heating power. Based on the findings we know to which value to set the voltage to obtain the required heating power in the future tests. It starts with the measurement of the heating film electrical resistance, and based on this result we established the initial voltage-power relationship from Ohm's Law. Since electrical resistance is variable with the film working temperature, we need to determine the relationship of the voltage-power through trial and error, and finally obtain a series of values regardless of the temperature effect on the electrical resistance. This section consists of the following steps:

1. Measure the heating film electrical resistance. In this case, a multifunction meter was employed; the film wires were plugged, setting at the " $\Omega$ ", to read the resistance value in $\Omega$ directly. For this case, the film resistance value (R) is measured as $345 \Omega$ at room temperature. 
2. Determine the initial voltage value. Based on the result from Step 1, an initial setting voltage value at room temperature can be obtained through the derivation of Ohm's Law, i.e., $V=\sqrt{P \times R}$.

3. Verify the current value corresponding to the initial voltage value. Connect the wires of the heating film to the power line plugs, and install the variac, the voltmeter, and the ammeter. Set the voltage to the value obtained from Step 2, and then a current value can be read from the current meter. Compare this current value with the one predicted from the derivation of Ohm's Law, i.e., $I=V / R$. By monitoring the temperature changing on the copper base surface, with the increasing temperature, the current and voltage values could drift. Adjust the variac to maintain the voltage value near the initial value. When the temperature reaches the steady state, record the current and voltage values. Thus obtain the real values corresponding to the certain power input under the film corresponding normal working temperature.

4. Determine the relationship in value between the power input and the voltage value. Repeat Step 2, to predict a new voltage value for the new power input value. Then follow by repeating Step 3, to determine the real values of the voltage and current corresponding to the required power input under the film corresponding normal working temperature. Then repeat the preceding steps at higher power levels until the voltage constraint or the temperature constraint whichever is reached first. Obtaining a series of values of the voltage and current, thus determine the power-voltage relationship. 


\section{Section 2: Testing the Heater Assembly without Insulation}

This experiment is to investigate the heater assembly, under natural convection condition. It consists of the following steps:

1. Preparation. Suspend the assembly with the Nylon strips at a moderate height. Check all the connections, and make sure all the instruments are in working condition. Set the heat power supply to "off", and the variac to " $0 \mathrm{~V}$ ". Configure the data acquisition system, and assign the corresponding file name to the destination folder.

2. Establish a steady state. Start scanning with the data acquisition system, and meanwhile record the starting time and count number. Monitor the temperature curves from the 'inspection' screen until all the curves reach steady state, i.e., temperatures change within $\pm 0.1^{\circ} \mathrm{C} / 10$ minutes.

3. Apply power. Under a steady state, turn on the power switch, and adjust the variac to set the voltage to the value corresponding to the initial power according to the voltage-power relationship obtained from Section 1. Also record the starting time and count number. Repeat the process in Step 1 to obtain a new steady state under the power input level.

4. Increase power inputs. Repeat the preceding steps under higher power input levels, until the power limit or the temperature limit whichever is reached first.

5. Decrease power inputs. Repeat Steps 3 and 4 backward under lower power input levels, until the initial level is reached.

6. Shut down power input. When the steady state reaches the initial level, turn off the power switch to stop the power supply. Turn off the voltage meter and current 
meter; meanwhile record the time and count number. Waiting for the steady sate under such condition, and preparing for stopping process.

7. Shutdown. When a steady state is reached, it is time to shut down the experiment, stop scanning, save the file, and make a backup.

8. Closing. Turn off all the instruments whatever is allowed to be shut down, put all the setup in order or detach the assembly if the same experiment no longer needs to be done. Put all tools away.

\section{Section 3: Testing the Heater Assembly with Insulation}

This experiment is to investigate the heater assembly under an insulated condition, thus the environmental convection could be ignored. It follows the same steps as in Section 2. The difference is that in this test, a thermocouple was employed to monitor the temperature on the top surface of the insulator to evaluate the performance of the insulator, and provide a reference for future tests.

\subsubsection{Experimental Results and Analyses}

The experimental results about the voltage-power relationship of the Kapton heating film calibration are presented in Appendix II. 


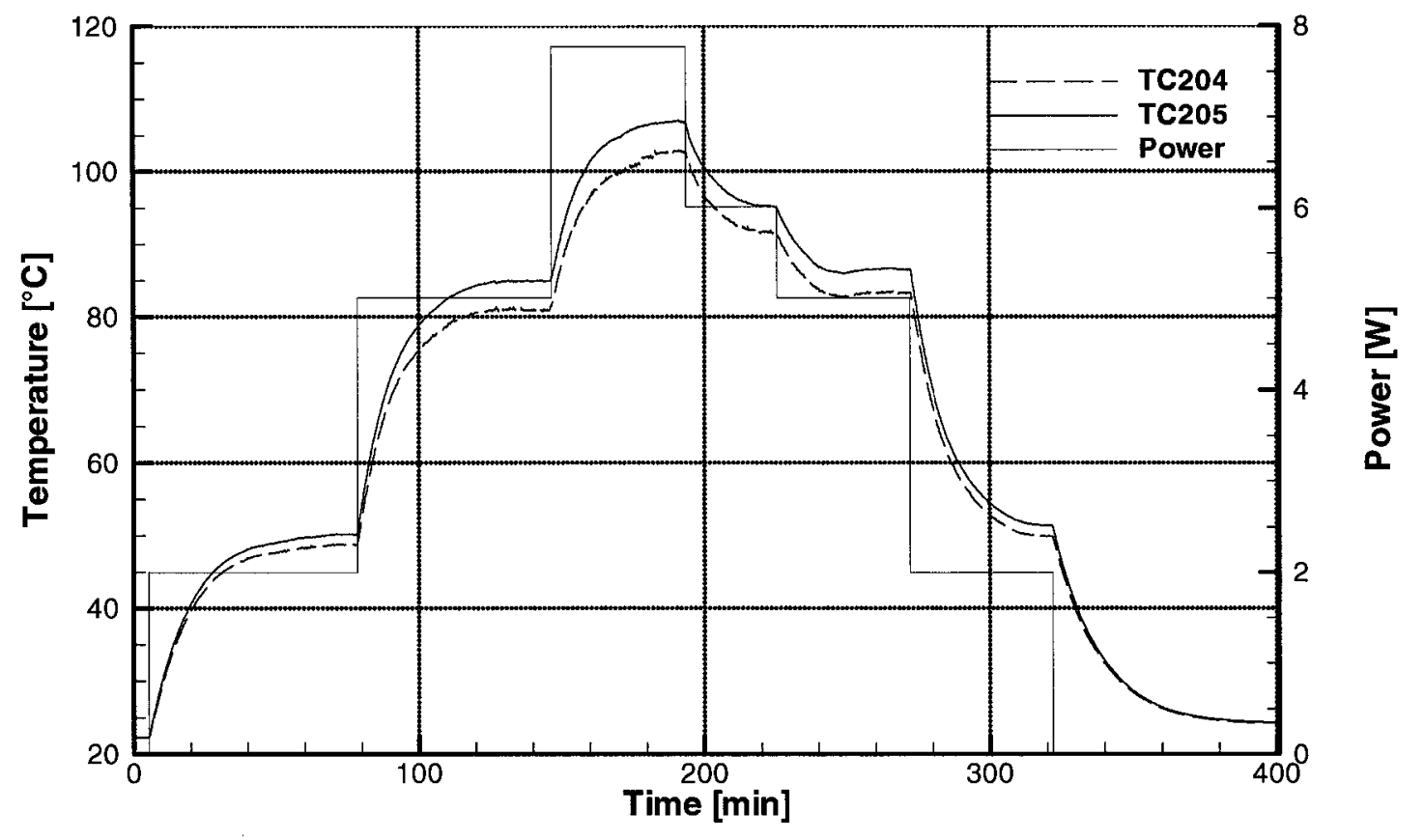

Figure 3.5 Heat source assembly power -temperature profile (non-insulated) [0, 2, 5, 7.5 W]

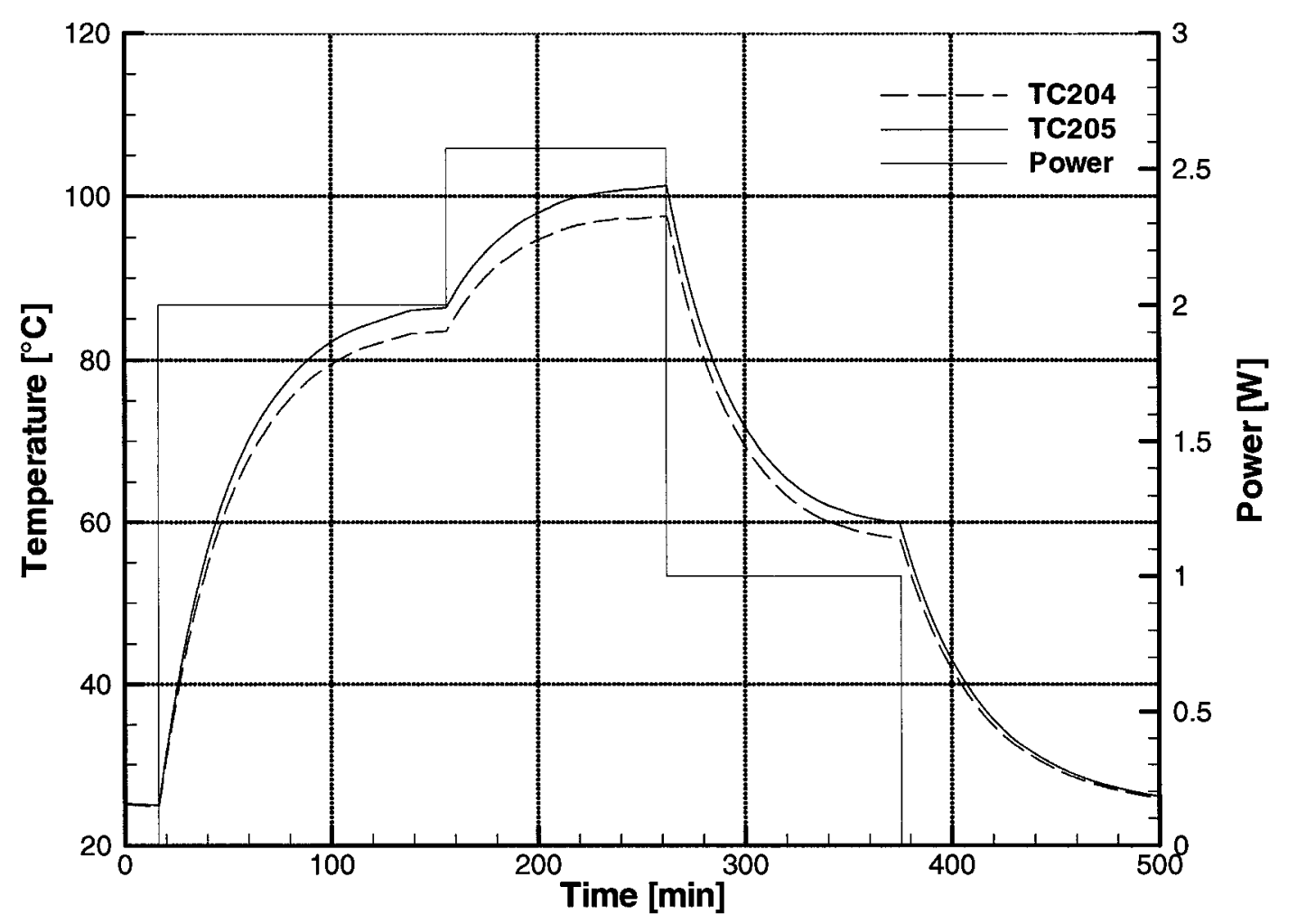

Figure 3.6 Heat source assembly power -temperature profile (insulated) [0, 2, 2.5 W] 
Figures 3.5 and 3.6 illustrate the record for the heat source assembly tests without, and with insulation respectively. The thermocouple positions in the figures are shown in Figure 3.4. From these figures it can be seen that under non-insulated condition, the heat source assembly can reach about $110^{\circ} \mathrm{C}$ at $8 \mathrm{~W}$. Under insulated condition, it reaches approximately $100^{\circ} \mathrm{C}$ at only $2.5 \mathrm{~W}$. This indicates that the insulation really acts well.

Analyzing the temperature curves in these figures, it can be found that between the two comparison groups, the temperatures of the thermal compound section (TC203 and TC205) are higher than those of the non-compound section (TC202 and TC204) respectively throughout the whole process. This supports the usefulness of the thermal compound application in the solid metal contact conductance.

\subsubsection{Conclusions of Heat Source Assembly Testing}

This experiment provides basic information on the heater assembly, and it can be concluded that:

1. The capacity of the heating film can reach $35 \mathrm{~W}$ at $115 \mathrm{~V}$.

2. The capacity of the heating film can reach $40 \mathrm{~W}$ at the voltage constraint of the heating film, $125 \mathrm{~V}$.

3. The OMEGA 101 epoxy adhesive can work under the temperature of $120^{\circ} \mathrm{C}$

4. The thermal joint compound can work under $120^{\circ} \mathrm{C}$

5. The insulators can work under $120^{\circ} \mathrm{C}$ with the styrene foam underneath

6. The aluminium adhesive tape can work under $120^{\circ} \mathrm{C}$, when overlap applied.

7. The assembly power limit is $8 \mathrm{~W}$ corresponding to $100^{\circ} \mathrm{C}$ temperature limitation under natural convection condition, in 220 minutes. With insulation, its power limit is $2.5 \mathrm{~W}$ corresponding to the same temperature limitation, in 180 minutes. 
There is a $5.5 \mathrm{~W}$ power difference and 40 minutes difference in the time to reach steady-state condition.

8. Thermal compound is necessary to use in this case, with solid metal contact conductance heat transfer. At the full power condition there is a $5^{\circ} \mathrm{C}$ difference between the areas of with, and without thermal compound, either in the thermal compound layer or on the tube surface. Such a result is consistent with our expectation.

9. This heat source assembly design is suitable for the tube heating test. It can work over a long time period, with full power. It also possesses a good repeatability.

\subsection{Experiment 2}

\section{Investigations of Non-Heat Pipe Tubes Characteristics}

This is a series of experiments with several configurations of copper tubes, e.g., regular copper tube, copper tube with copper wire mesh inserted, and copper tube with copper wire screen mesh inserted and water sealed inside, without vacuum. These tubes possess the same properties as those of the heat pipes to be tested. The results provide comparison information for future heat pipe testing

\subsubsection{Experimental Objectives}

The objectives of these experiments are summarized as follows: 
1. Verifying all the elements comprehensively, including the heat source assembly, the sink assembly, the power supply, the temperature sensors, the insulation, etc.

2. Investigating the temperature characteristics of the various configurations of the tubes.

3. Providing information for the future comparison analyses on the heat pipe performance.

\subsubsection{Experiment Principle and Setup}

Figure 3.7 shows the test apparatus used for the heat pipe tests, and Figure 3.7a illustrates the schematics of the experiment setup. The heat source and cooling sink assemblies are attached on either end of the tube. Both ends take the same pressing approach as described in Figure 3.7a. The thermal compound is applied through the full length of the groove between the tube and the groove surfaces.

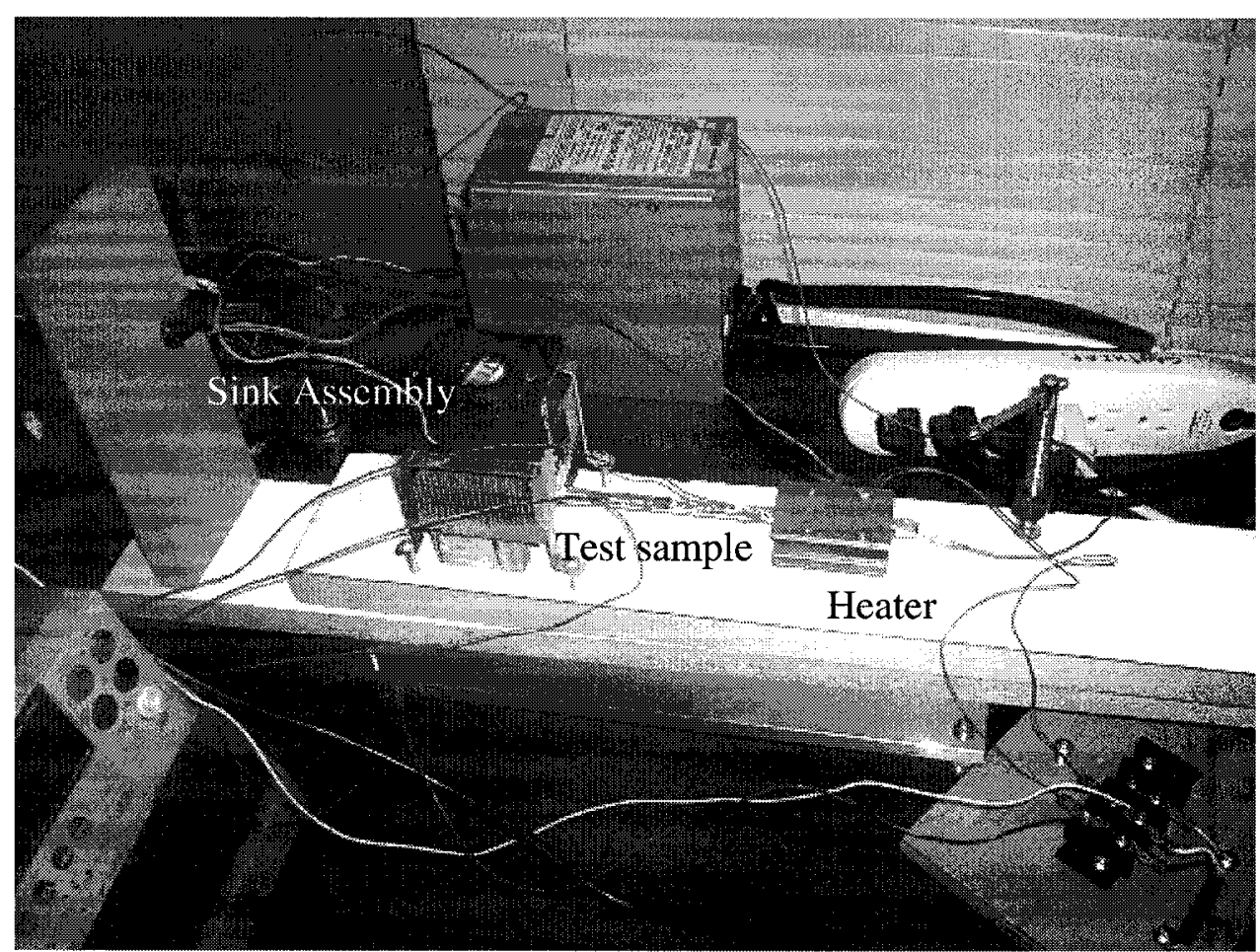

Figure 3.7 Heat pipe test apparatus 
A pair of aluminium strip frames fixes the whole sink assembly against the baseboard. These aluminium strip frames are pulled toward the baseboard by four screws. Underneath the sink copper base, a pair of fiberboard blocks is set at either side of the tube and the pressing board, supporting the whole assembly against the pressure from the aluminium strip frame. With a very low thermal conductivity the medium fiberboard blocks also play the role of insulator between the sink assembly and the baseboard. They act as shields against possible wind effect from the fan blowing through the sink fin slots.

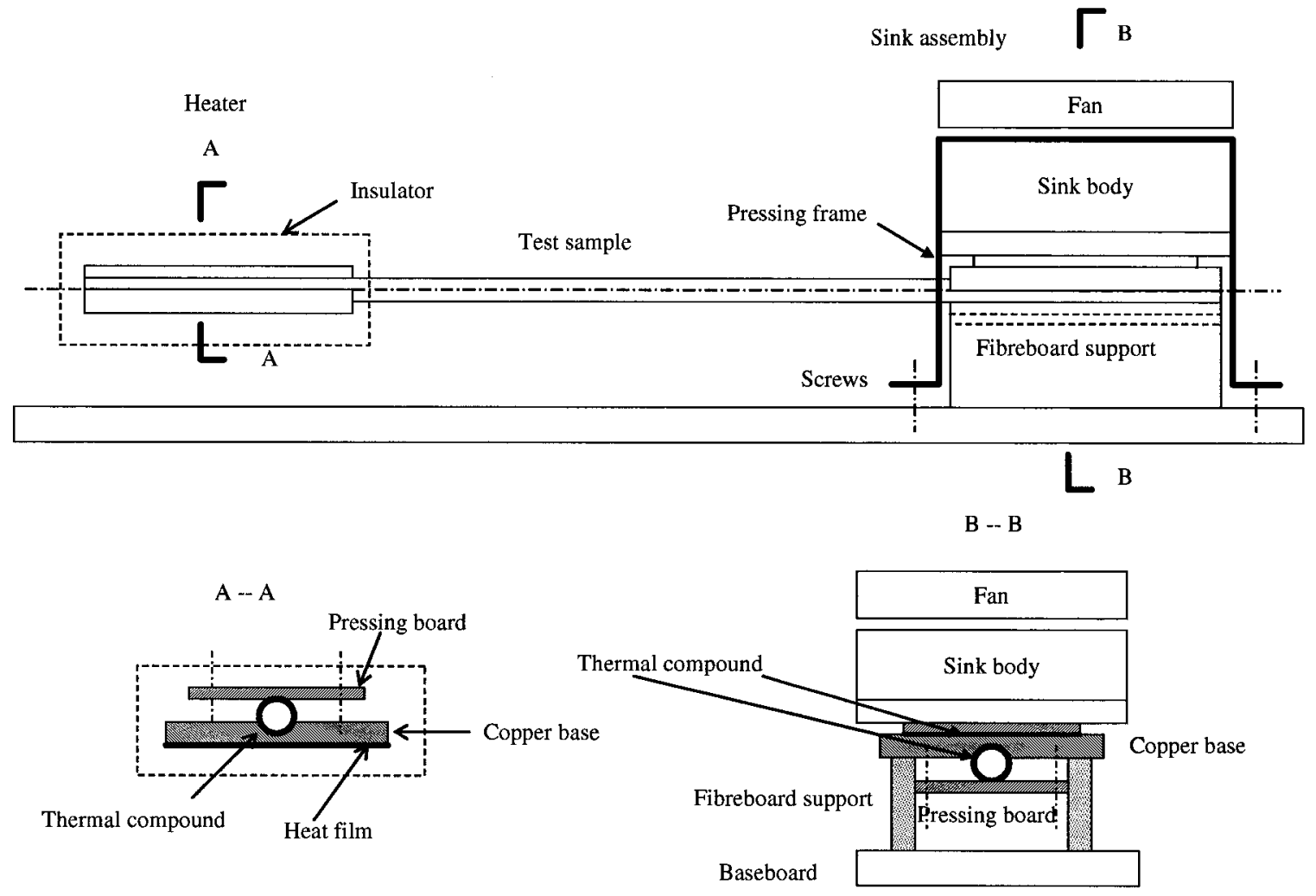

Figure 3.7a Schematics of testing setup 


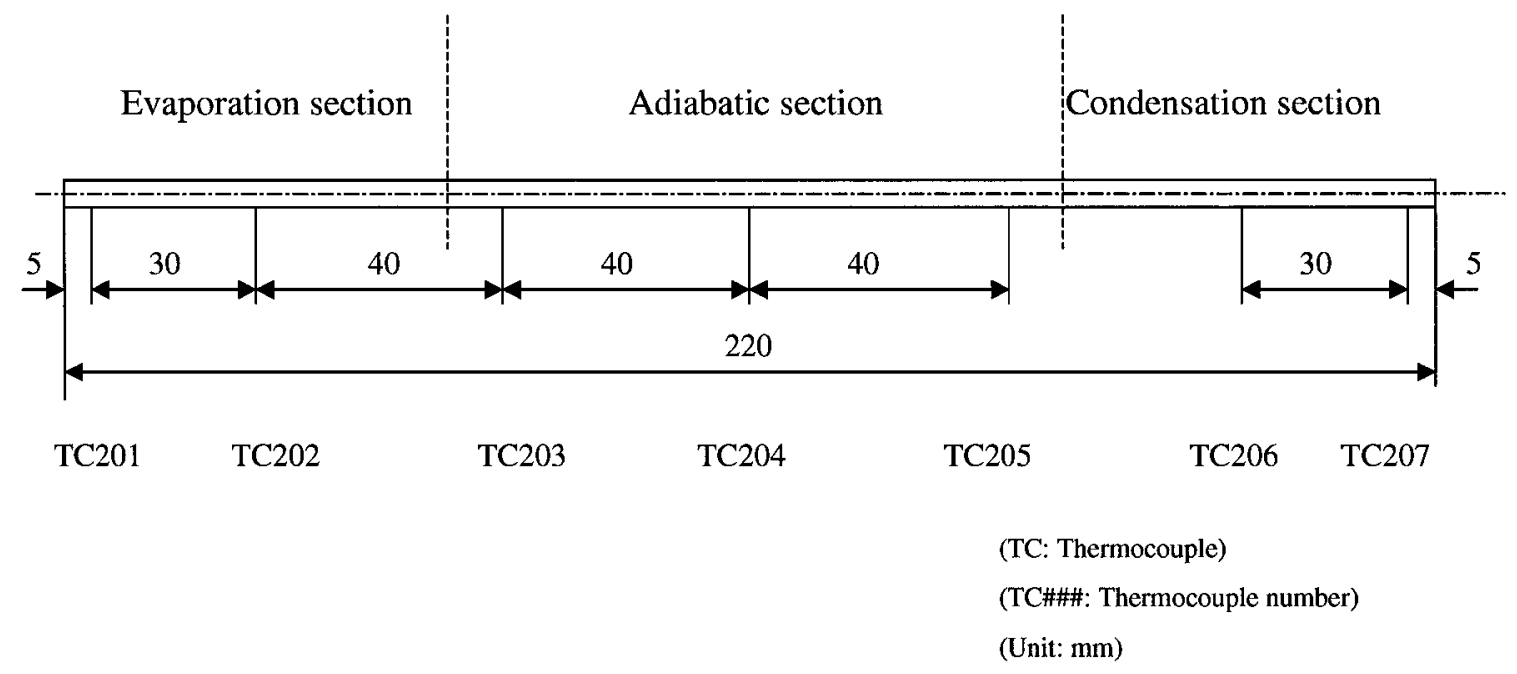

Figure3.8 Thermocouple locations along the test sample axial position

Figure 3.8 displays the thermocouple arrangement along the testing sample. All the thermocouples are fixed with the aluminium adhesive tape on the tube external surface. Two thermocouples (TC201 and TC202) located on the evaporation section measure the evaporator temperature at the end and middle point respectively. Similarly two thermocouples (TC206 and TC207) on the condensation section measure the condenser temperature at the middle and end point respectively. The other three thermocouples (TC203, TC204 and TC205) are evenly arranged along the adiabatic section surface to measure the temperature on this surface at the points of near the heat source, the middle and near the sink respectively.

Based on this setup, two configurations were tested: non-insulated and insulated. For each, three copper tube configurations were studied, i.e., regular copper tube, copper tube with copper wire mesh inserted, copper tube with copper wire mesh inserted and water sealed inside. These experiments are conducted only in the horizontal condition. 


\subsubsection{Experimental Procedure}

There were a total of three types of non-heat pipe copper tube configurations involved in the experimental investigation. These three configurations are copper tube, copper tube with copper mesh, and copper tube with copper mesh and water sealed inside. We tested each configuration with cooling conditions of natural convection, forced convection, and enhanced forced convection, under non-insulated and insulated conditions respectively. All the configurations were tested using the same procedure as described below:

1) Preparation. Fix the whole setup, check all the connections to make sure all are correctly connected either in hardware or software. Make sure all the instruments are in working condition. Set the heat power supply at "off", and the variac at "0 V". Configure the data acquisition system, and assign the corresponding file name to the destination folder.

2) Establish a steady state. Start scanning with the data acquisition system, and meanwhile record the starting time and count number, monitoring the temperature curves from the screen, and inspect from time to time through the inspection function of the system, until all the curves reach steady state, i.e., temperatures change within $\pm 0.1^{\circ} \mathrm{C}$ in about 10 minutes.

3) Apply power. Under a steady-state temperature, turn on the power switch, and adjust the variac to set the voltage to the value corresponding to the initial power according to the voltage-power relationship obtained from Experiment 1. Also record the starting time and count number. Repeat the process in Step 1 to obtain a new steady state under the power input level. 
4) Increase power inputs. Repeat the preceding steps under higher power input levels, until the power constraint or the temperature constraint whichever is reached first.

5) Decrease power inputs. Repeat Step 3 and 4 backward under lower power input levels, until the initial level is reached.

6) Shut down the power. When the steady state reach the initial level, turn off the power switch to stop power supply, turn off the voltmeter and ammeter, meanwhile record the time and count number, waiting for the steady sate under such condition, and preparing for stopping process.

7) Stopping. When a steady state arrives, it is the time to shut down the experiment, stop scanning, save the file, and make a backup.

8) Closing. Turn off all the instruments, put all the setup in order or detach the assembly if the same experiments no longer need to be done. Put all tools away.

\subsubsection{Experiment Results and Analyses}

From the experiments described above, results were collected and organized. Discussion of the results is provided below in the following topics.

\subsubsection{Typical Heat Transfer Temperature Characteristics}

Figures 3.9 to 3.11 illustrate the power stable temperature curves for the three non-heat pipe testing samples: copper tube, copper with copper wire screen mesh inserted, and copper tube with copper wire screen mesh inserted and with water charged, note that this test sample is not vacuumed. 


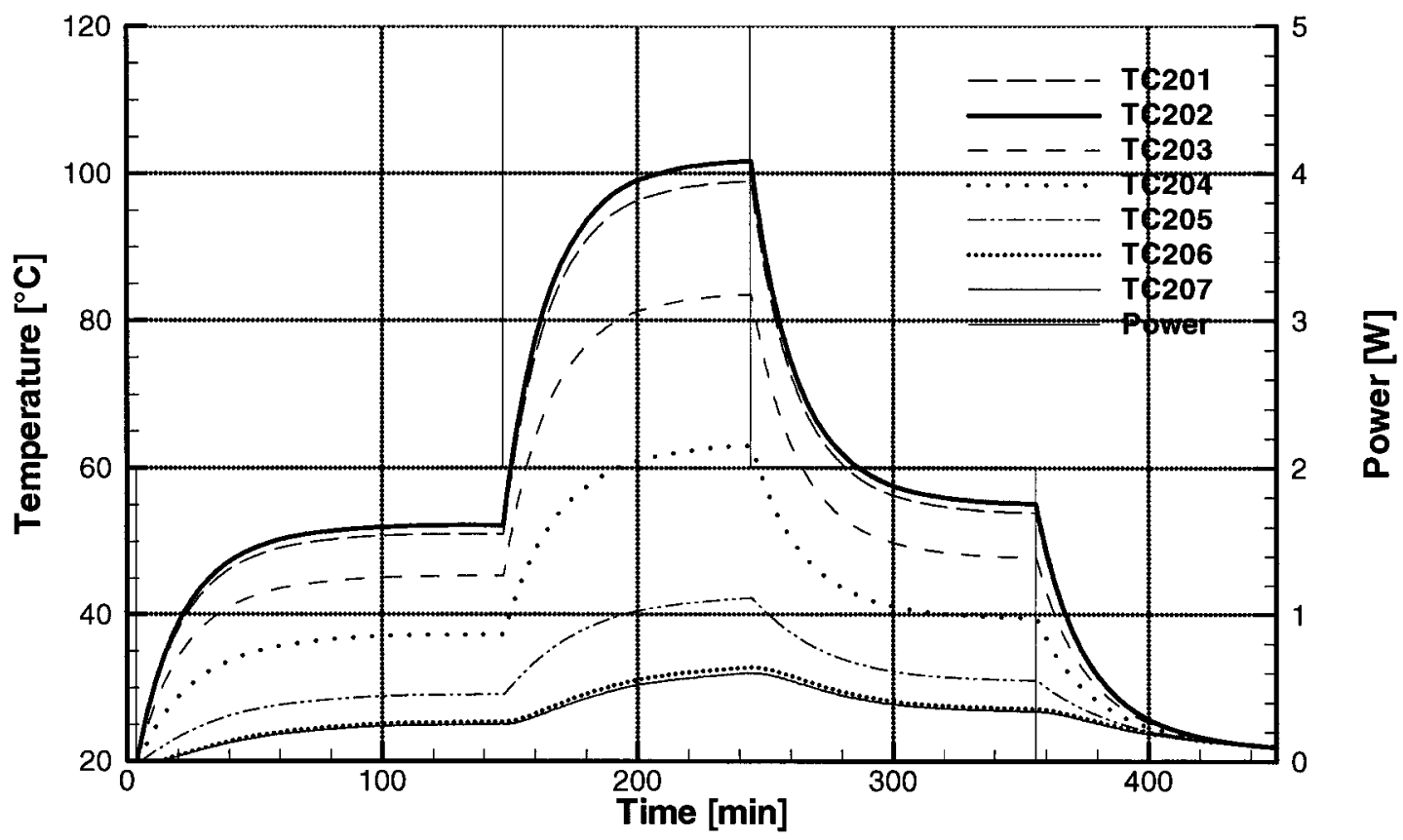

Figure 3.9 Copper tube power -temperature profile (insulated) $[0,2,5 \mathrm{~W}]$

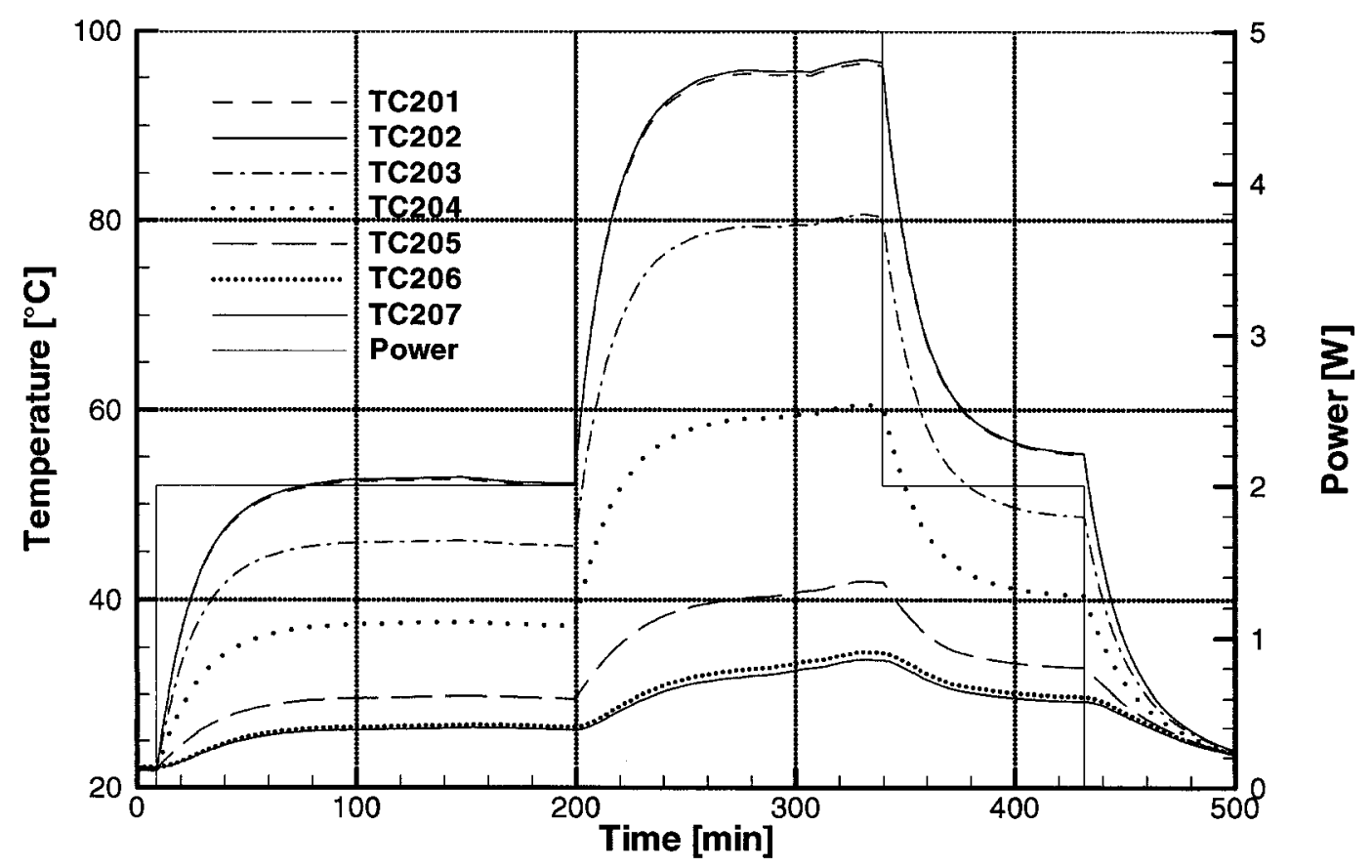

Figure 3.10 Copper tube with mesh power-temperature profile (insulated) $[0,2,5 \mathrm{~W}]$ 


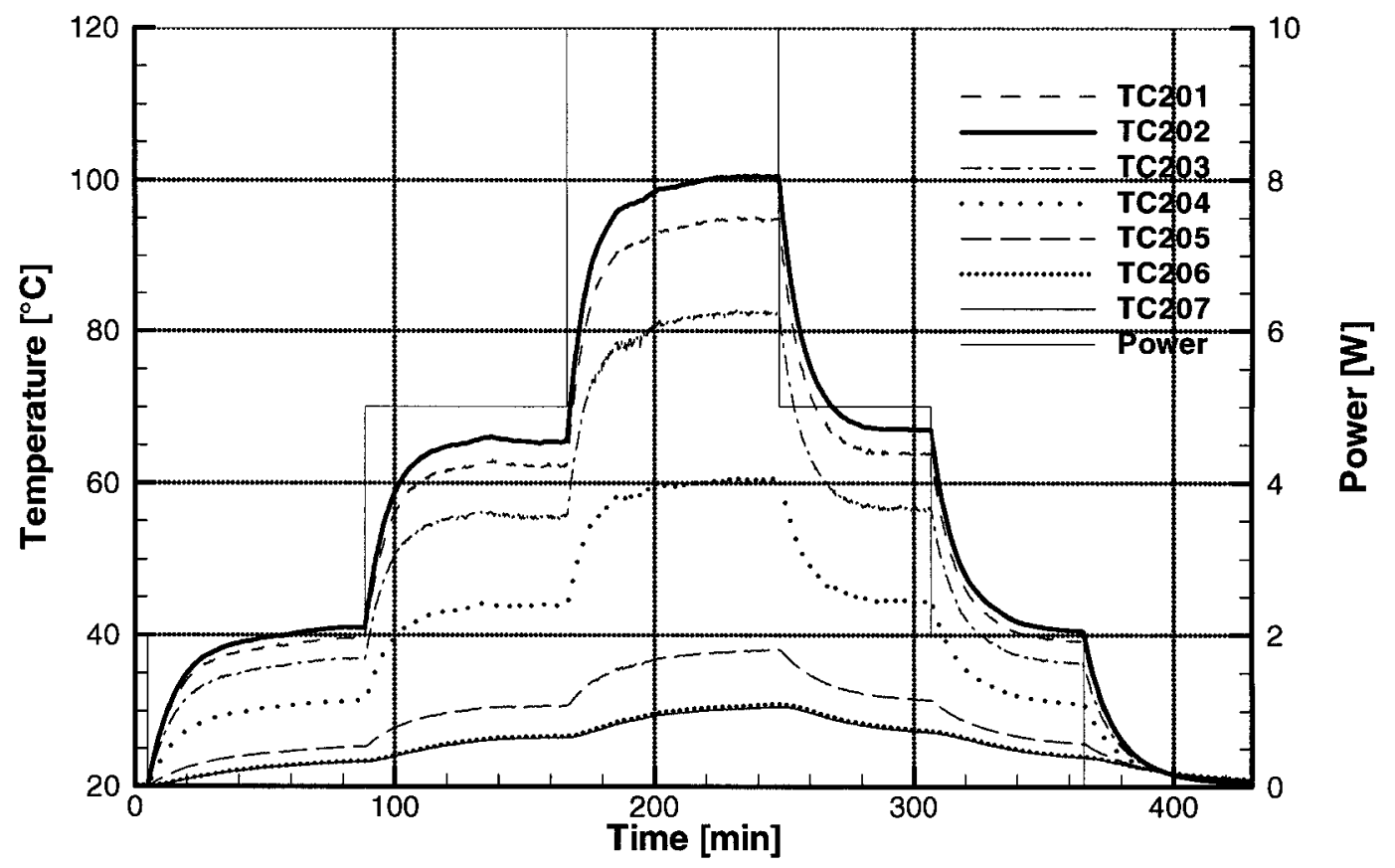

Figure 3.11 Copper tube with mesh \& water power-temperature profile (non-insulated) [2, $5,10 \mathrm{~W}]$

The above three figures are axial temperature characteristics curves obtained from the experiments. Each figure has seven curves, and can be divided into three groups. The top two curves are for the heating end, the middle three are for the adiabatic section, and the bottom two are for the cooling end. It can be seen that these three configurations have similar characteristics. The heating end temperature curves are very similar, the middle three temperature curves change with even gaps when the power varies, and the cooling end temperature curves almost converge throughout the whole process.

This is a typical conductance temperature characteristic. The temperatures distribute geometrically. This is determined by the conductance mechanism. That is, the conductance relies on molecular or atomic vibrations, without any lattice changes, without any phase change, and without any mass transfer. This process of heat transfer is different from that of heat pipes, which will be introduced in later sections. 


\subsubsection{Cooling Capacity}

This section mainly illustrates the results obtained from the corresponding experiments, and reports analyzes on the capacity of heat transfer for these non-heat pipe tubes. These results will also be employed in the following section for comparisons.

Figures 3.12 to 3.14 illustrate the comparisons of the heater surface temperature with different non-heat pipe copper tube configurations without insulation, and respectively under natural, forced, and enhanced forced convection cooling conditions.

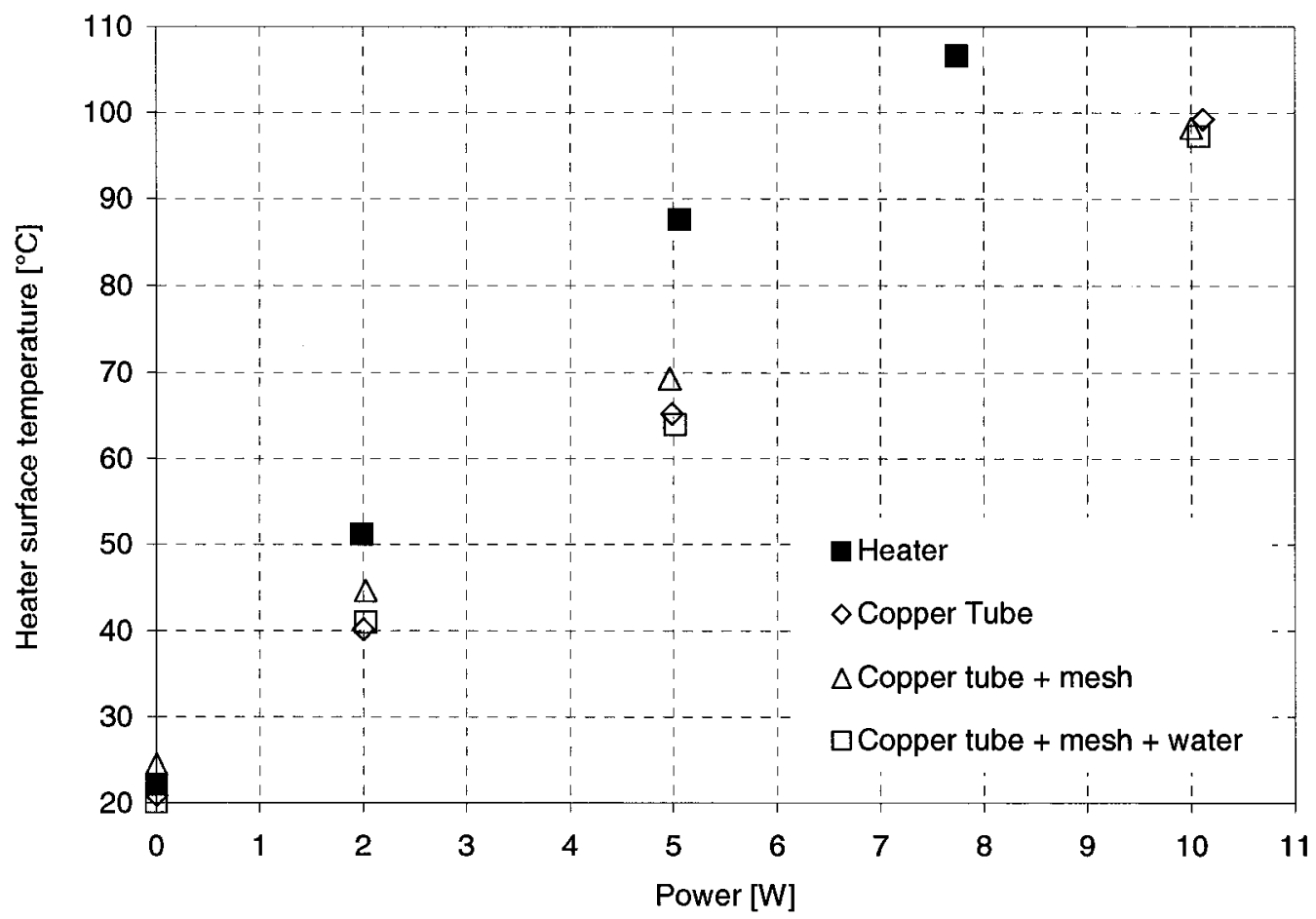

Figure 3.12 Heater surface temperature comparison with non-heat pipe tubes (noninsulated, natural convection) 


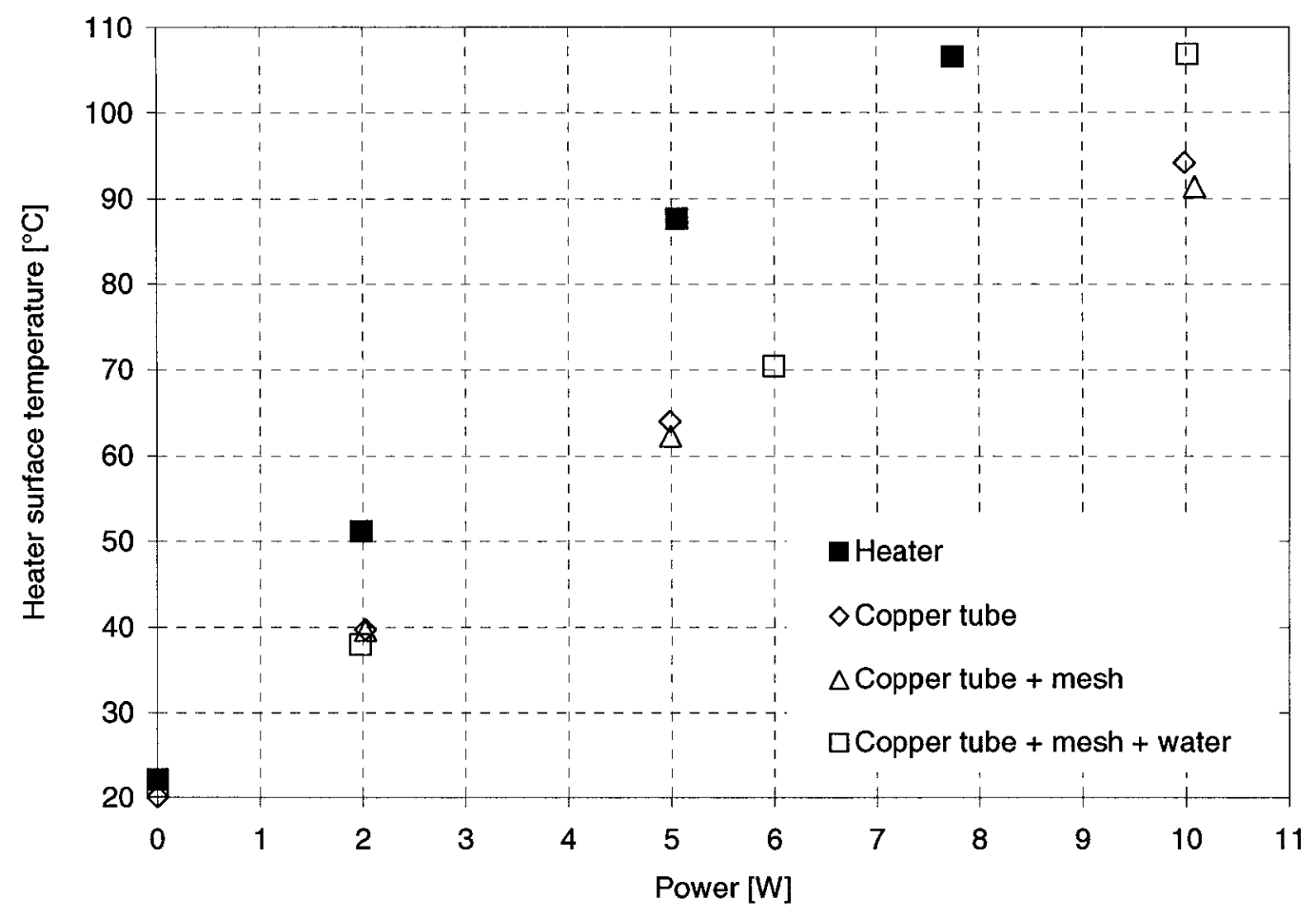

Figure 3.13 Heater surface temperature comparison with non-heat pipe (non-insulated, forced convection)

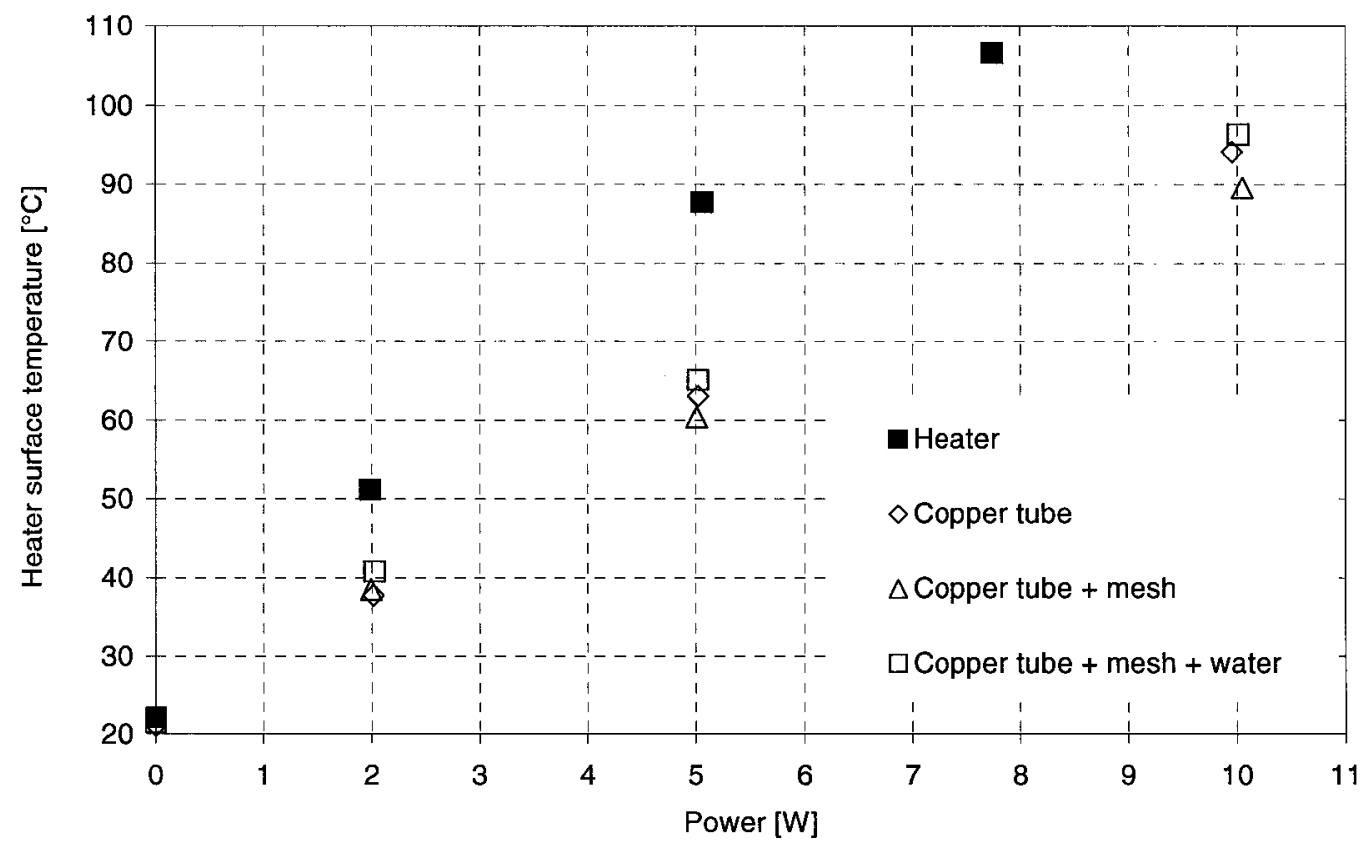

Figure 3.14 Heater surface temperature comparisons with non-heat pipe tubes (non-insulated, enhanced forced convection) 


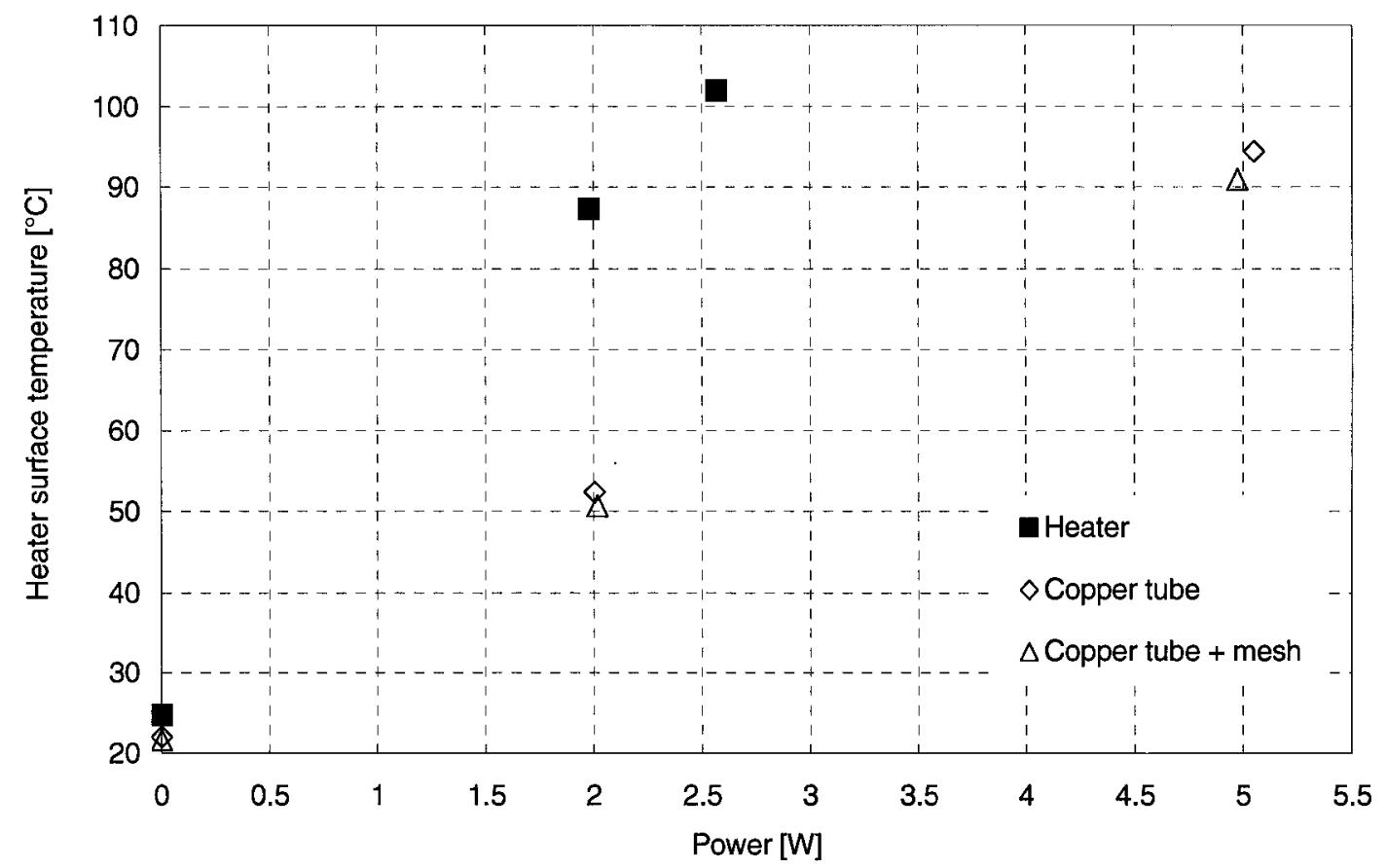

Figure 3.15 Heater surface temperature comparison with non-heat pipe tubes (insulated, natural convection)

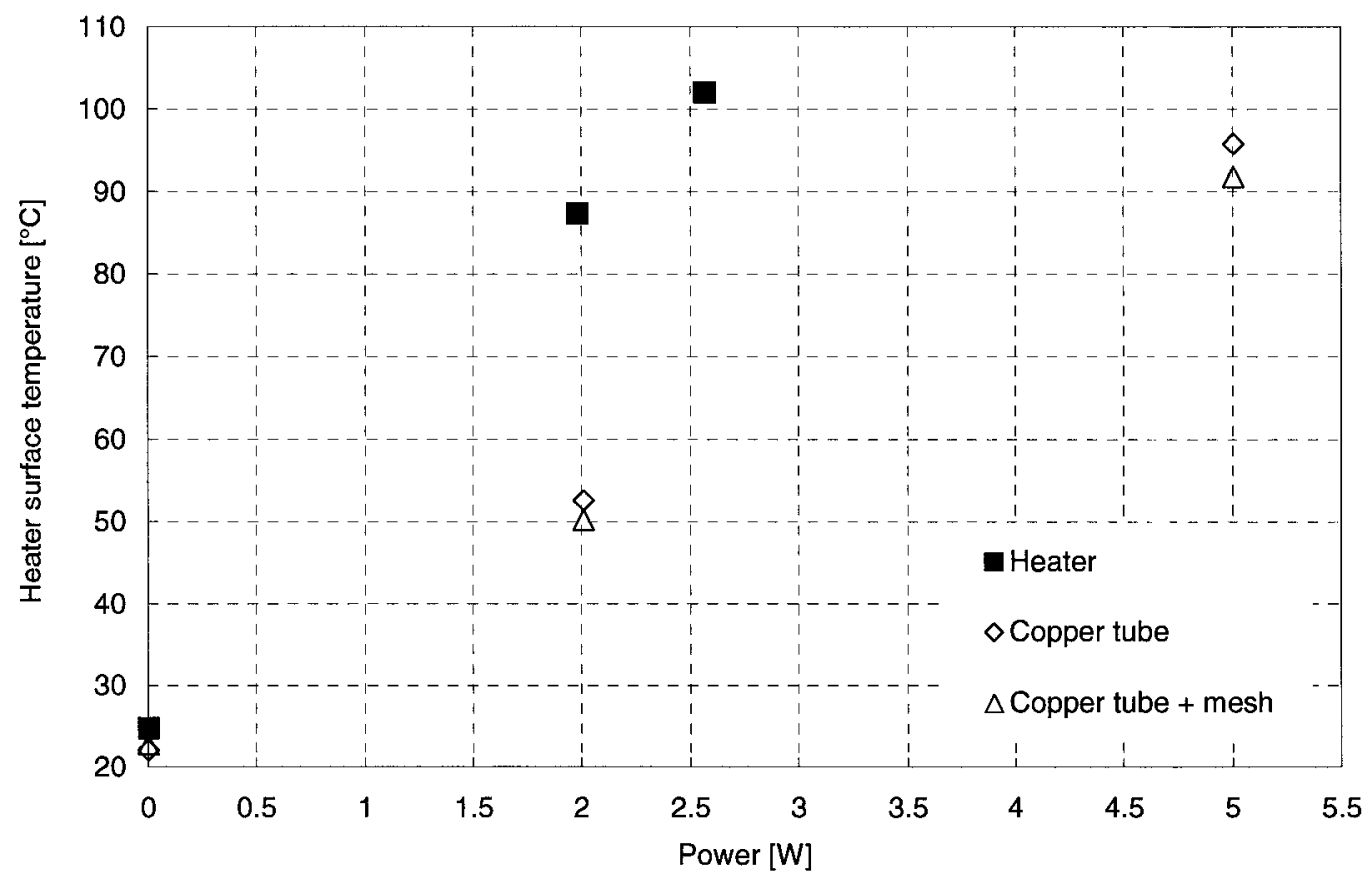

Figure 3.16 Heater surface temperature comparison with non-heat pipe tubes (insulated, forced convection) 


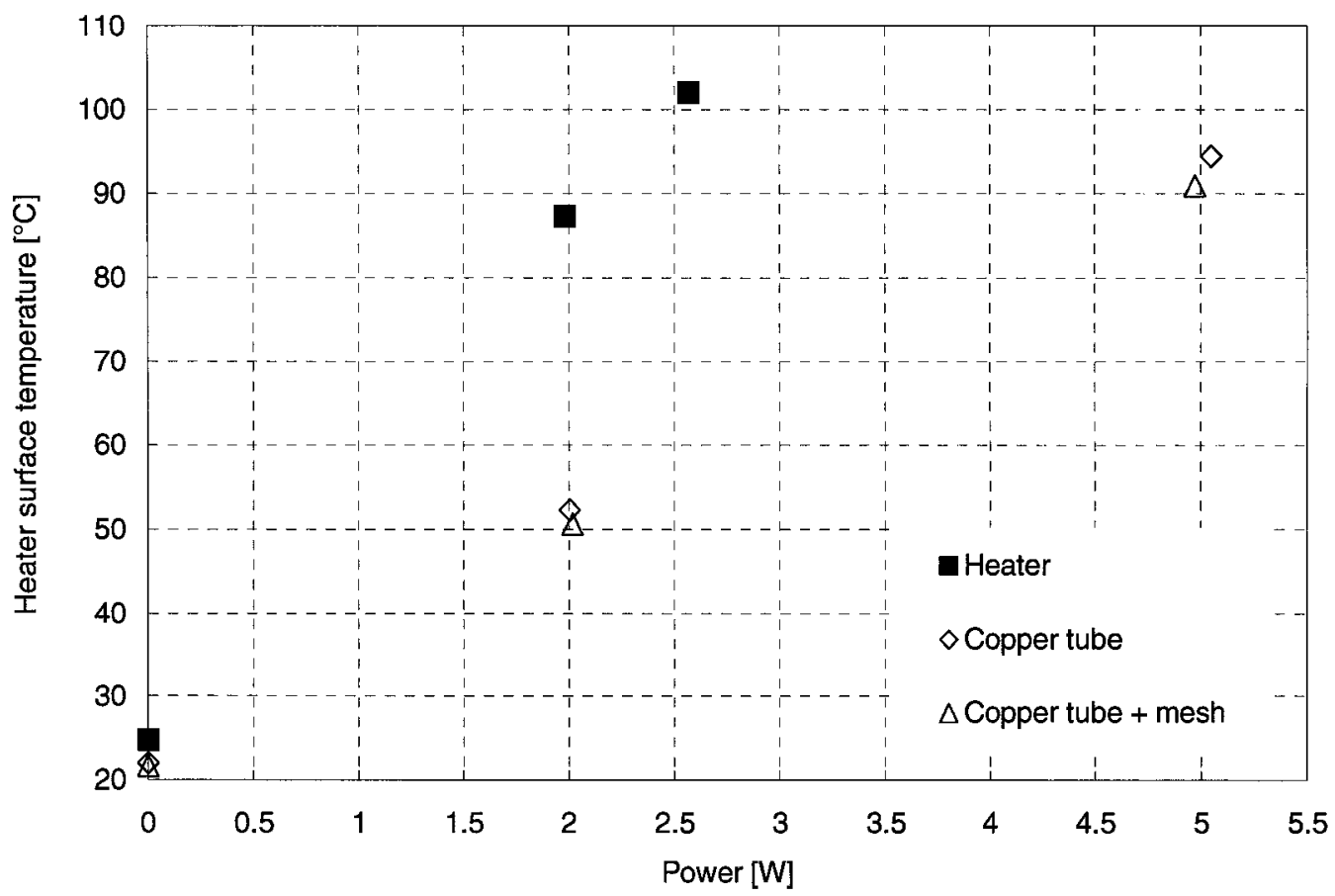

Figure 3.17 Heater surface temperature comparison with non-heat pipe tubes (insulated, enhanced forced convection)

Figures 3.15 to 3.17 illustrate the comparisons of the heater surface temperature with different non-heat pipe copper tube configurations with insulation, and respectively under natural, forced, and enhanced forced convection cooling conditions.

The above figures clearly illustrate the same fact that any copper tube configurations can extend the heater's power limit for the same temperature limit, or lower the temperature level for the same power input, either with or without insulations, to a considerable extent. Comparing these two groups of figures, it can be seen that in the non-insulated groups, the heater power limit is extended from 8 to $10 \mathrm{~W}$. Therefore, $2 \mathrm{~W}$ or 25 percent is gained. While in the insulated groups, the power limits are extended from about 2.5 to $5 \mathrm{~W}$. Therefore, $2.5 \mathrm{~W}$ or 100 percent is gained. The latter case illustrates better improvement. This can be explained by the fact that the non-insulated groups have not only copper tubes assistance with conductance heat transfer, but also have natural 
convective heat dissipation. Furthermore, having a higher surface temperature gradient, the heater itself possesses a good heat dissipating capacity. A considerable amount of heat is dissipated before reaching the copper tube. While the insulated groups rely almost only on the copper tubes heat transfer. Little heat can be dissipated through the insulations. Under such circumstances, the copper tubes play a significant role.

Comparing these two groups of figures, it can be concluded that there is little difference between natural, forced, and enhanced forced convective cooling. This is due to the fact that all three cooling approaches were implemented in the same environment, with a similar ambient temperature. Conductance performs in the forms of molecular or atomic vibrations. It is proportional to the temperature gradient along the heat transfer direction. Also, it is limited by the temperature difference along the heat transfer direction. Only increasing the convection speed on one end of the copper tube and without lowering the convection temperature will not help the overall heat transfer capacity. Since the solid copper tube has a limited conductance compared with the high speed convection, instead it acts as a bottleneck to the overall heat transfer network.

\subsubsection{Axial Temperature Profile}

The axial temperature distribution is commonly used to evaluate the temperature characteristics of a heat transfer device. This section investigates such characteristics on non-heat pipe samples. The results will be useful for comparison against future heat pipe tests. 


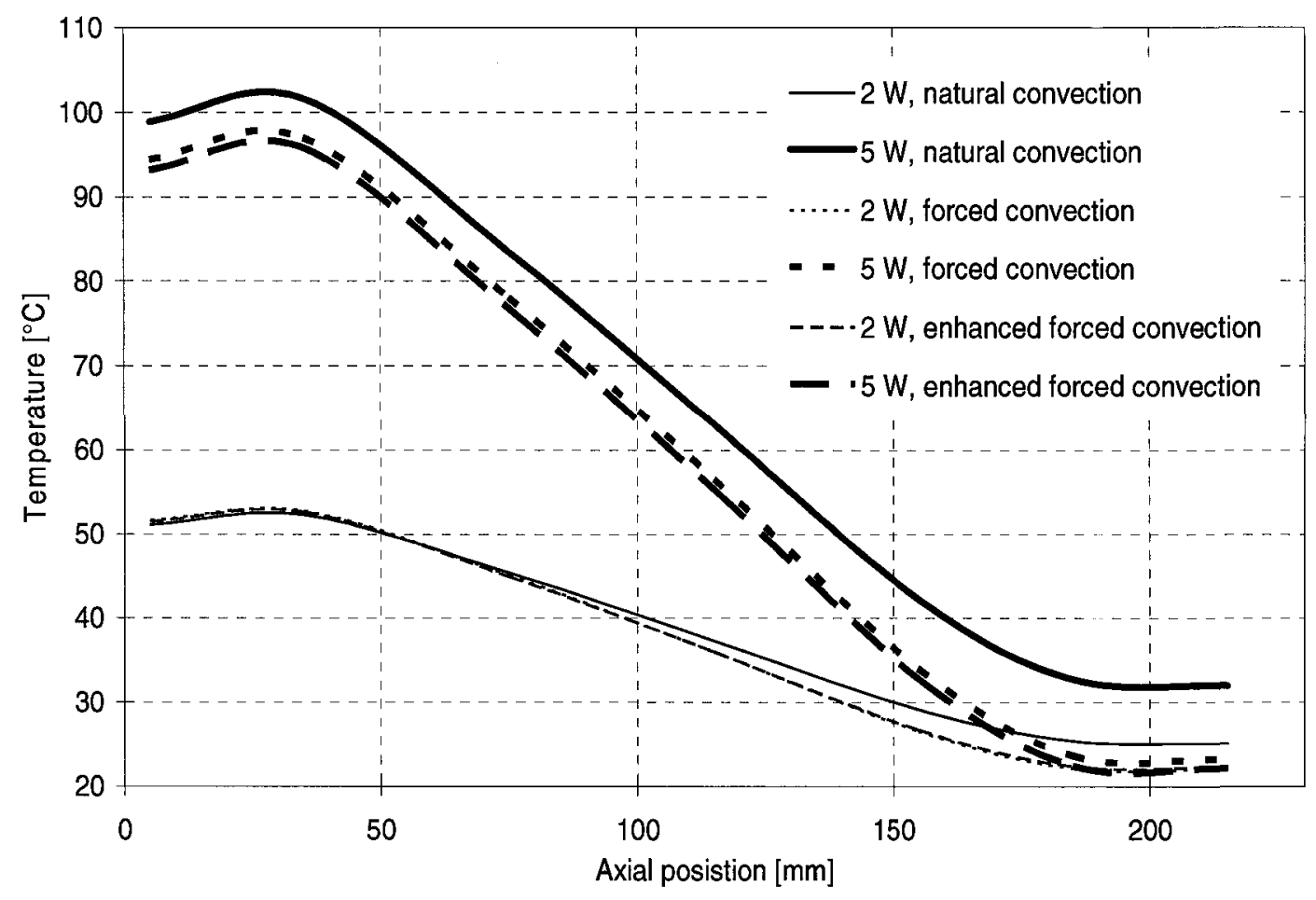

Figure 3.18 Copper tube axial temperature profiles

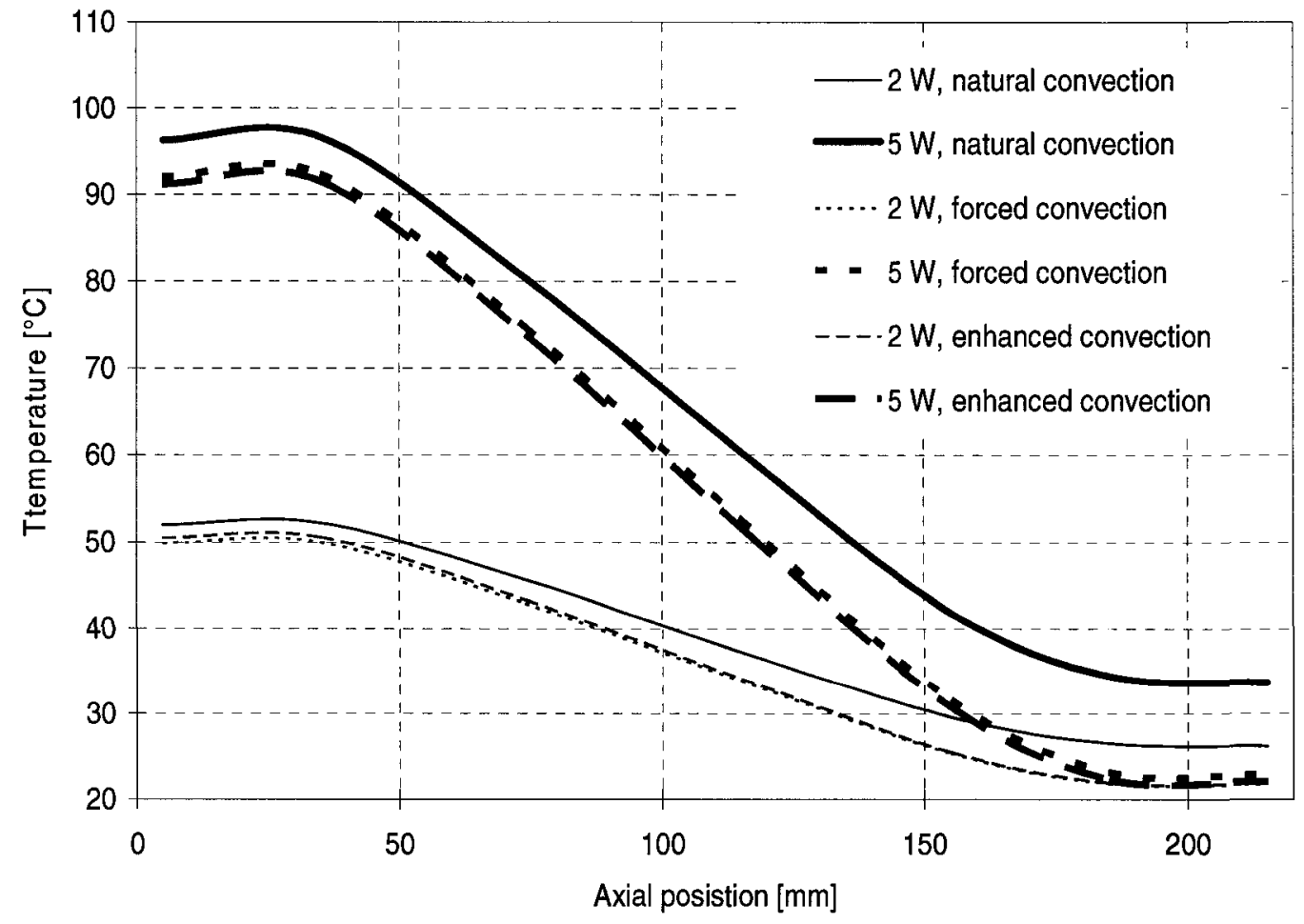

Figure 3.19 Copper tube with mesh axial temperature profile 


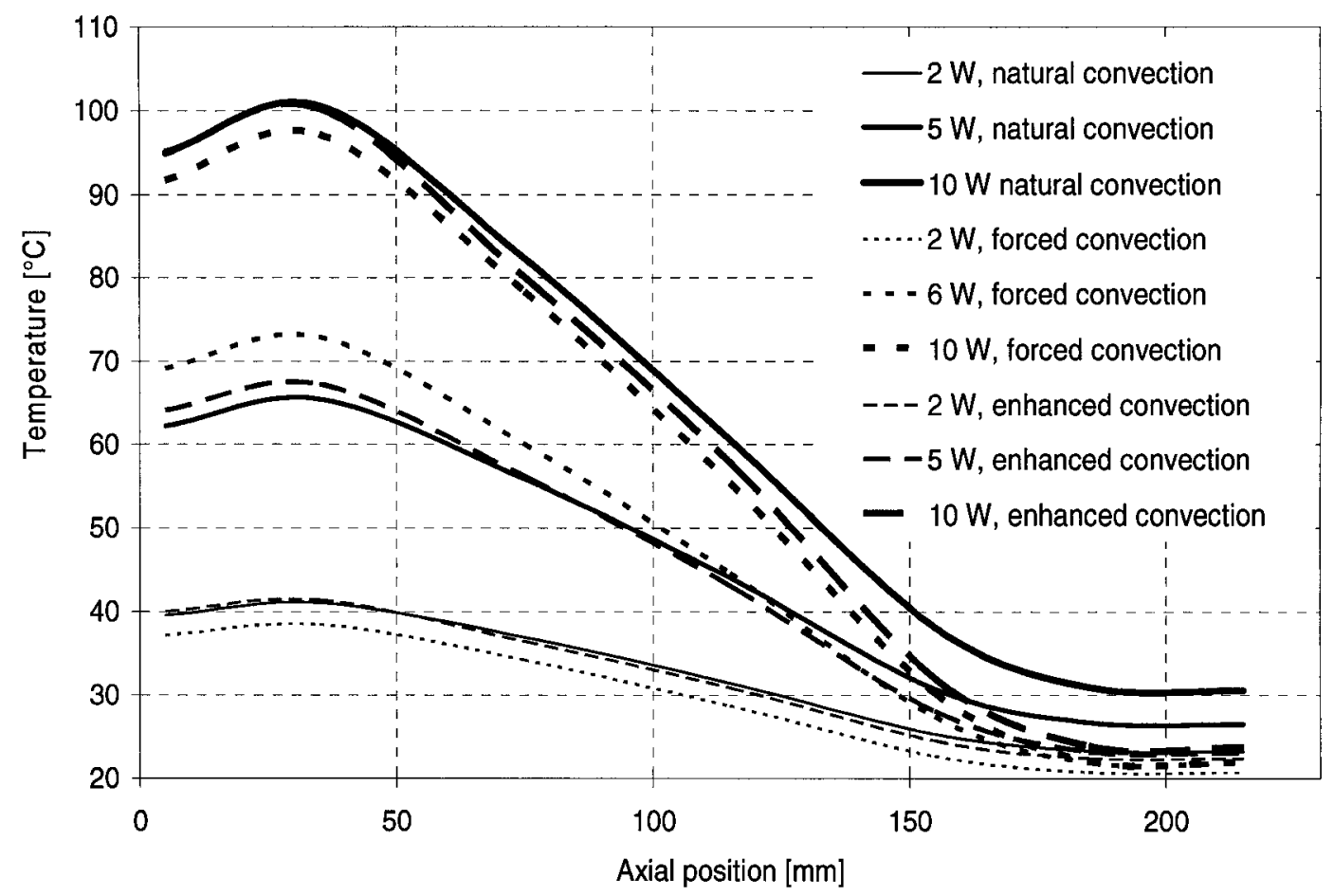

Figure 3.20 Copper tube with mesh \& water axial temperature profile

Figures 3.18 to 3.20 illustrate the copper tube axial temperature distribution at different power input levels. From the above figures, it is clear that there is a direct proportional relationship between the temperature and power input levels; the higher the power input, the higher the temperature contour. This is consistent with the preceding discussion of conductance mechanisms.

Another characteristic is that the shape of the curves is typically composed of three portions; two horizontal flat sections at either end, and a straight slope section in the middle section. This is also consistent with conductance characteristic. Temperature distribute directly and geometrically proportional to the temperature gradient. At the cooling sink, the temperature is even with either natural or convective cooling. This causes a flat temperature contour at this section. The middle slope part reflects the actual 
temperature gradient between the heating and cooling ends. However, it is more complex at the heating end. At a lower power input level, the curve at this part is nearly flat; this is due to a small temperature gradient of the heat source assembly. While at a higher power level, the contours take a curved shape. This reflects a bigger temperature gradient in the heat source assembly at a higher power level. Temperature gradient increases with_power level is due to the fact that the heat source has a better heat dissipation at its edges than the middle area.

From the above figures, it is also clear that there is a difference between natural, and forced convective cooling. This is especially apparent at a higher power level, or with a greater temperature gradient between either ends. There is little difference between normally and enhanced forced convective cooling, which is also consistent with the analysis about the conductance discussed in the preceding section. Temperature plays a main role in the conductance. Further increase in convection speed without temperature change would not improve the heat transfer capacity for a long solid pipe. The solid would be a bottleneck for a large convection.

We can also see that the case of copper tube with mesh and water does also take the similar characteristics. The slope of the middle section is constant, and the water inside without vacuum would not affect the temperature profile.

\subsubsection{Heating-Cooling Temperature Gradient Characteristics}

The heat conducted by the pipe is a function of the temperature gradient. This can be described by the temperature gradient between the heating and cooling ends, Figures 3.21 to 3.23 illustrate such characteristics. 


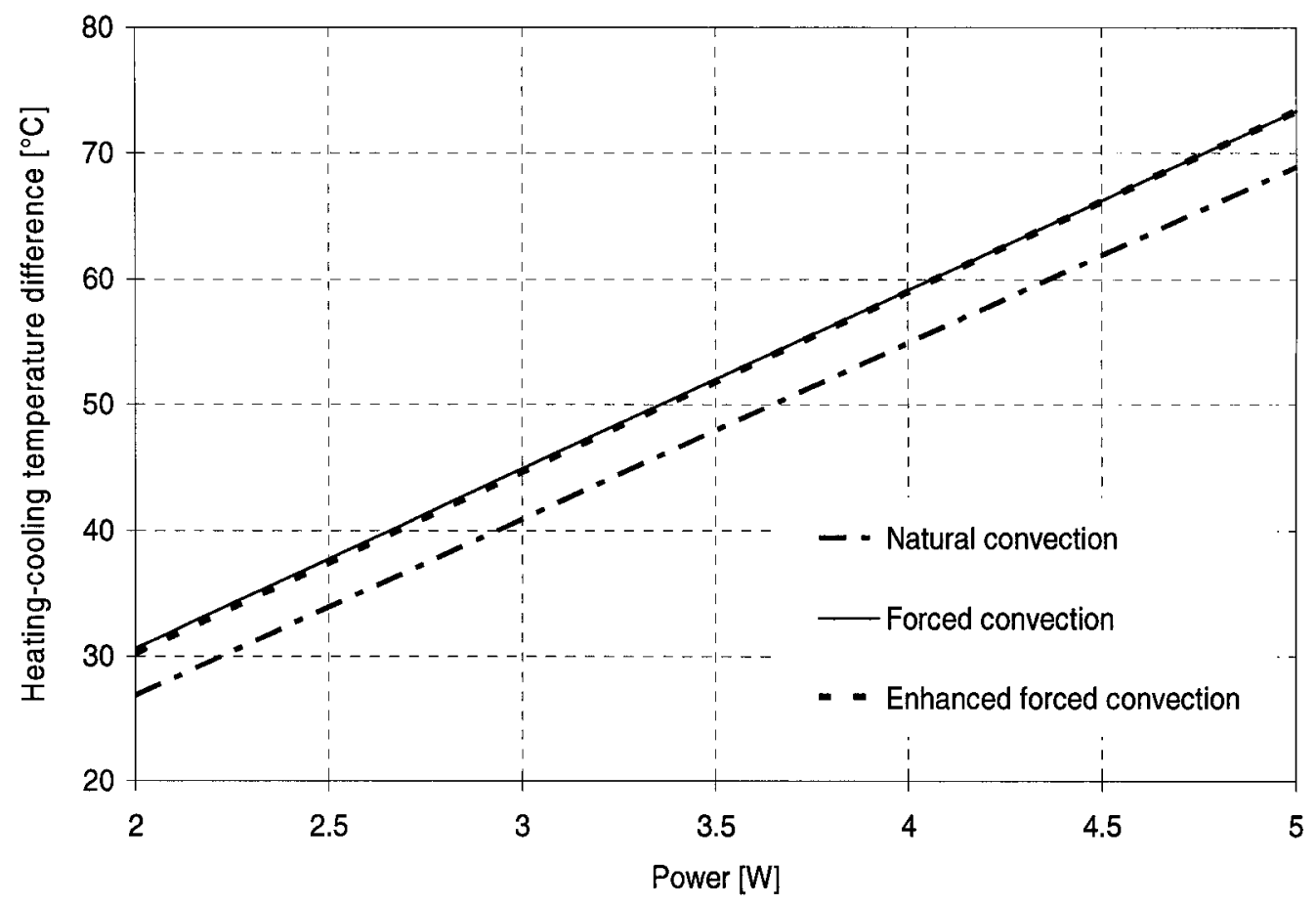

Figure 3.21 Copper tube heating-cooling temperature differences as function of heat power input

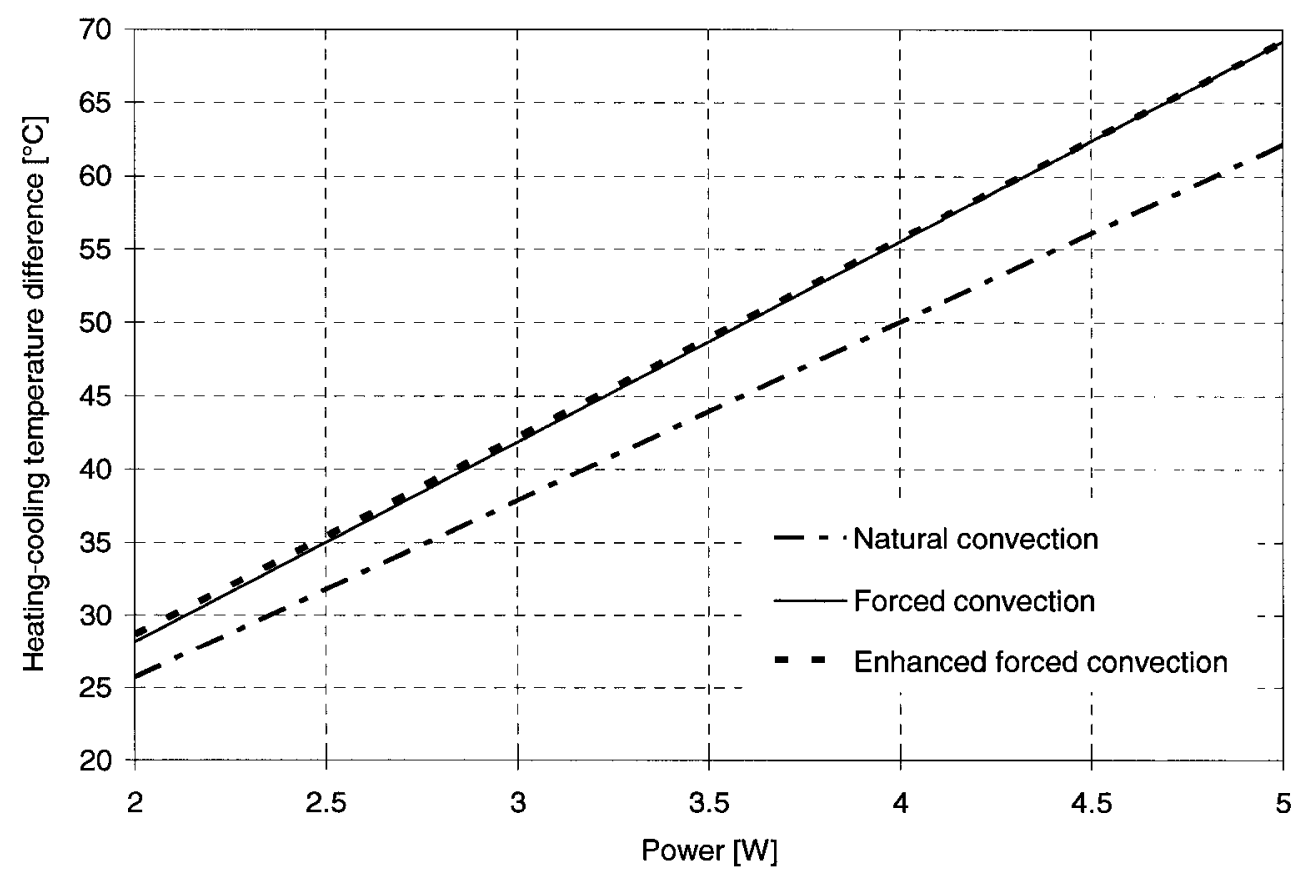

Figure 3.22 Copper tube with mesh heating-cooling temperature difference as function of heat power input 


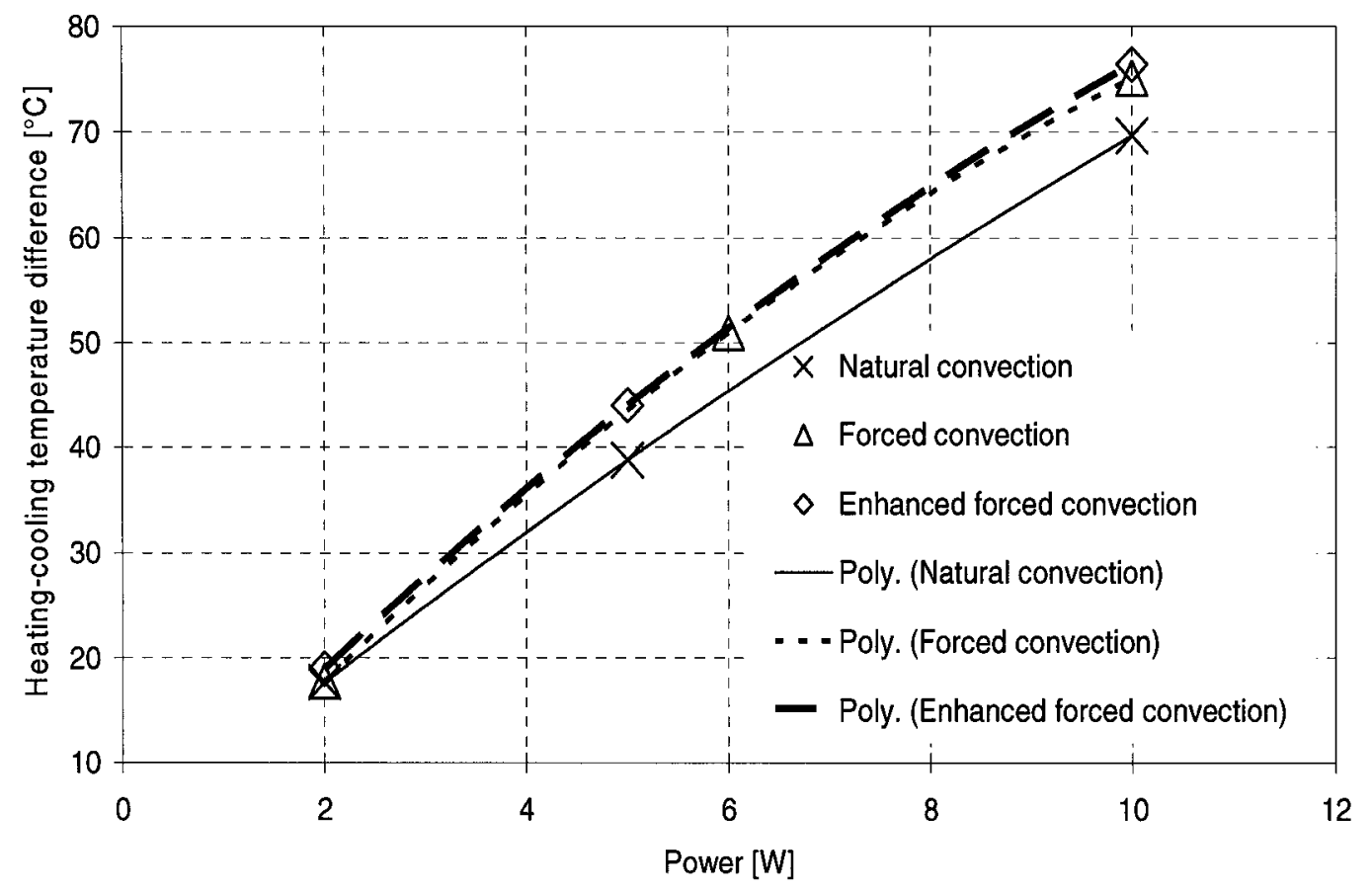

Figure 3.23 Copper tube with mesh \& water heating-cooling temperature difference as function of heat power input

From the above three figures, we can see a general tendency about the heatingcooling temperature difference, $\Delta \mathrm{T}(\mathrm{h}-\mathrm{c})$. It linearly changes with power in a direct proportion with a constant slope. This is consistent with the thermal conductance mechanism; the temperature profile associated with the geometry of the solid device. Also note that forced convection, either normal or enhanced forced convection causes greater $\Delta \mathrm{T}(\mathrm{h}-\mathrm{c})$ compared to the natural convection. This gap tends to increase with increasing power. Under convection conditions, the cooling end temperature is maintained constantly low, while with natural convection it is not. Thus, for the same heating temperature, the value of $\Delta T(h-c)$ comes out different. With a higher power load on the heating end, the heating temperature is also higher, thus causing a greater value in $\Delta \mathrm{T}(\mathrm{h}-\mathrm{c})$. 
It should be noticed that there are almost no difference between the normal, and enhanced forced convection about the $\Delta T(h-c)$. As discussed before, this is caused from the fact that further increases to the convection fluid speed without temperature change, would not help heat transfer through the solid tube, which could act as a bottleneck for the strong convection.

\subsubsection{Axial Thermal Resistance and Conductivity}

The axial thermal resistance is another important parameter to evaluate in a heat transfer device. For simple comparison, instead of resistance values, we take the ratio of the $\Delta T$ between heating and cooling to the heat transferred, $R=\Delta T / Q$. This is used to evaluate a testing sample. The following figures illustrate such relationship.

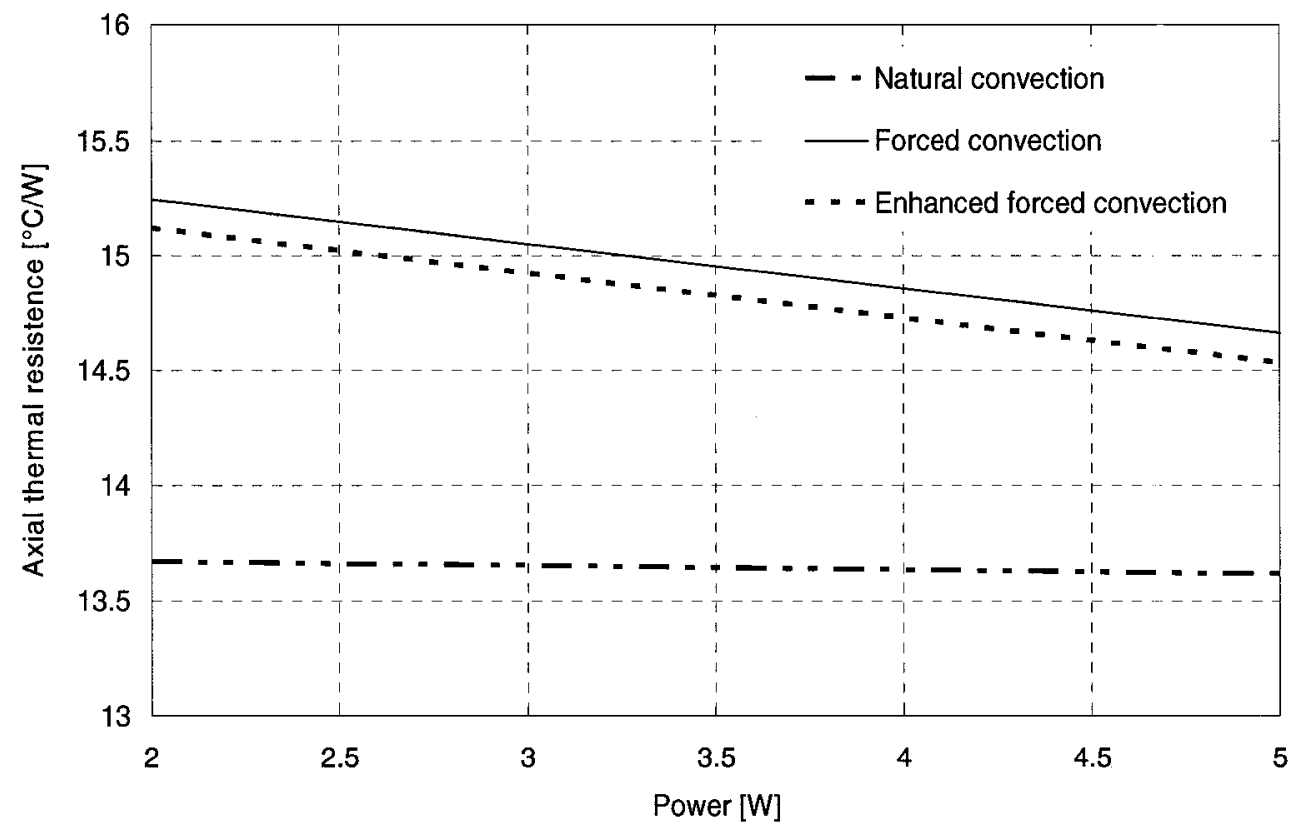

Figure 3.24 Copper tube axial thermal resistance as function of heat power input 


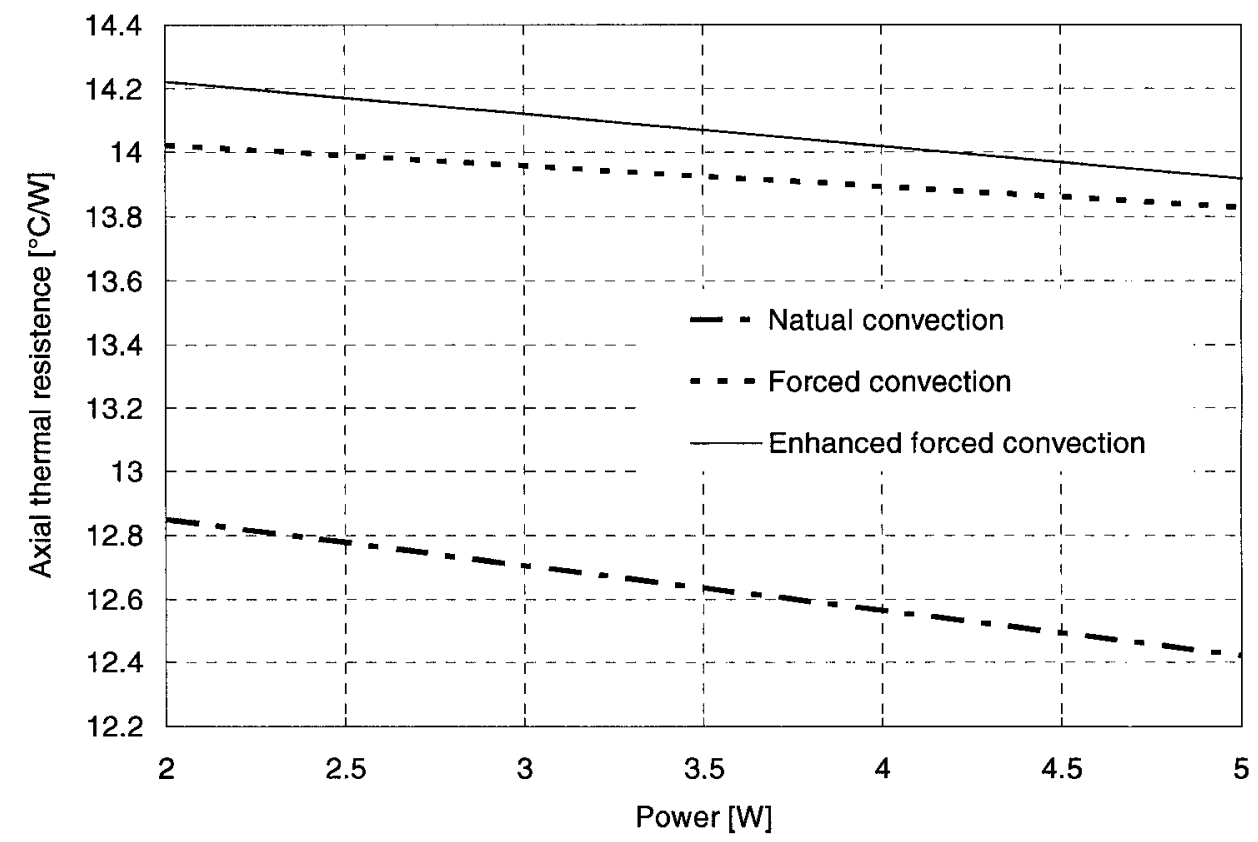

Figure 3.25 Copper tube with mesh axial thermal resistance as function of heat power input

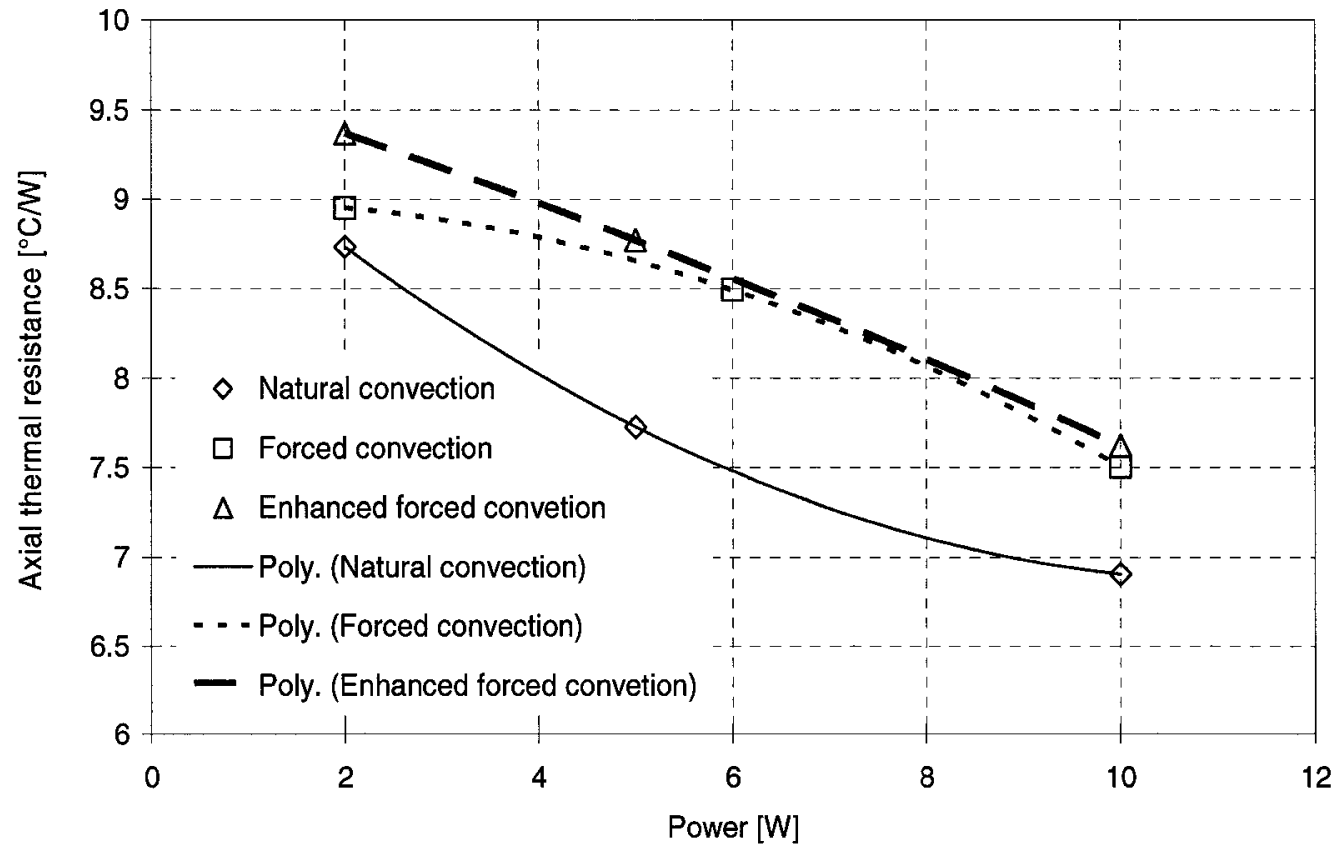

Figure 3.26 Copper tube with mesh \& water axial thermal resistance as function of heat power input 
The above three Figures 3.24 to 3.26 show that all thermal resistances are functions of power input. Forced convection causes higher thermal resistance than natural convection; and enhanced forced convection causes higher thermal resistance than normal forced convection. With increasing power, all the curves or scatter points tend to converge. All the curves tend to decline with increasing power input.

Figures 3.27 to 3.29 illustrate the axial thermal conductivity for these three testing samples. From these figures, it can be seen that the conductivity curves have opposite slope from those of thermal resistance. The axial thermal conductivity is defined as the ratio of heat transfer rate per unit cross-sectional area to the axial temperature gradient. This result is consistent with the definition of the thermal resistance relationship.

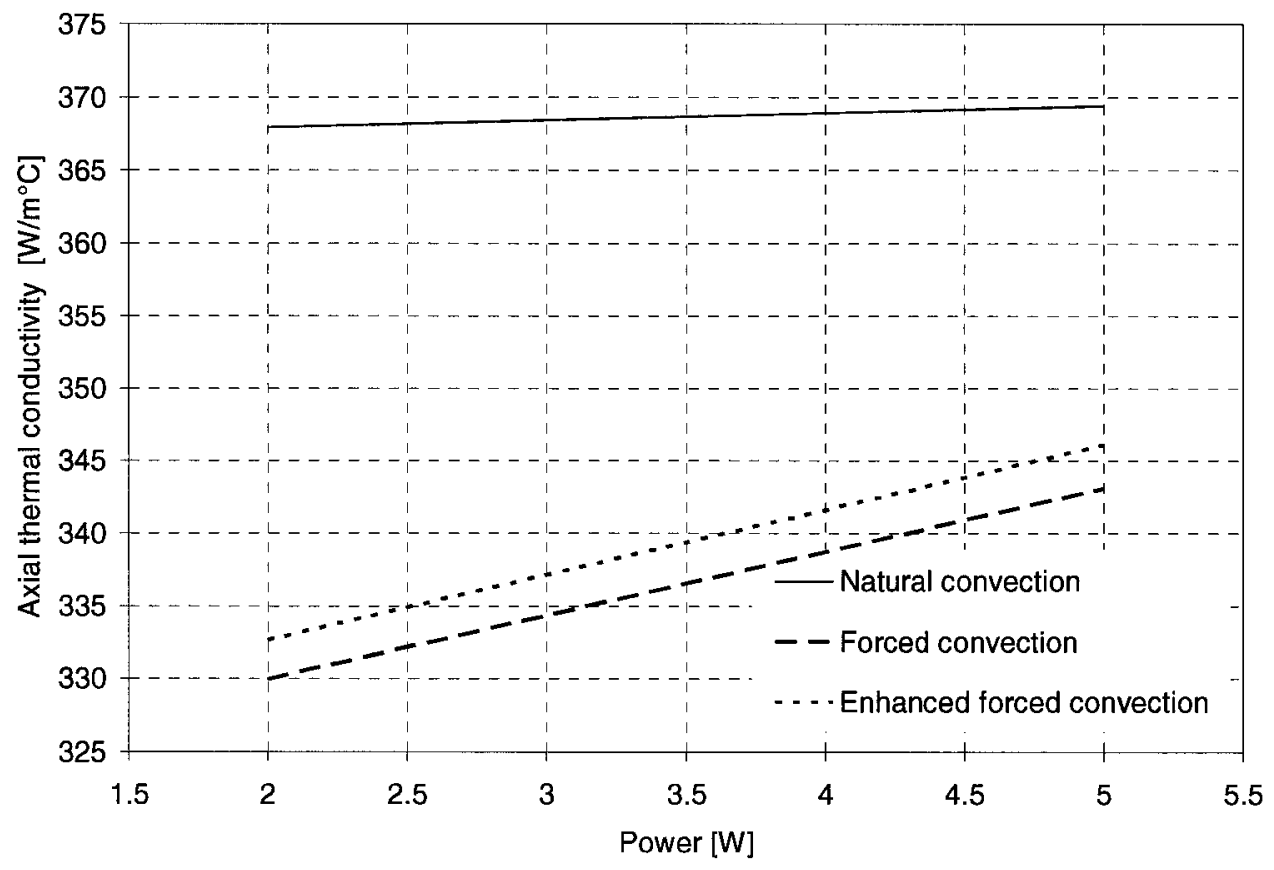

Figure 3.27 Copper tube axial thermal conductivity as a function of heat power input 


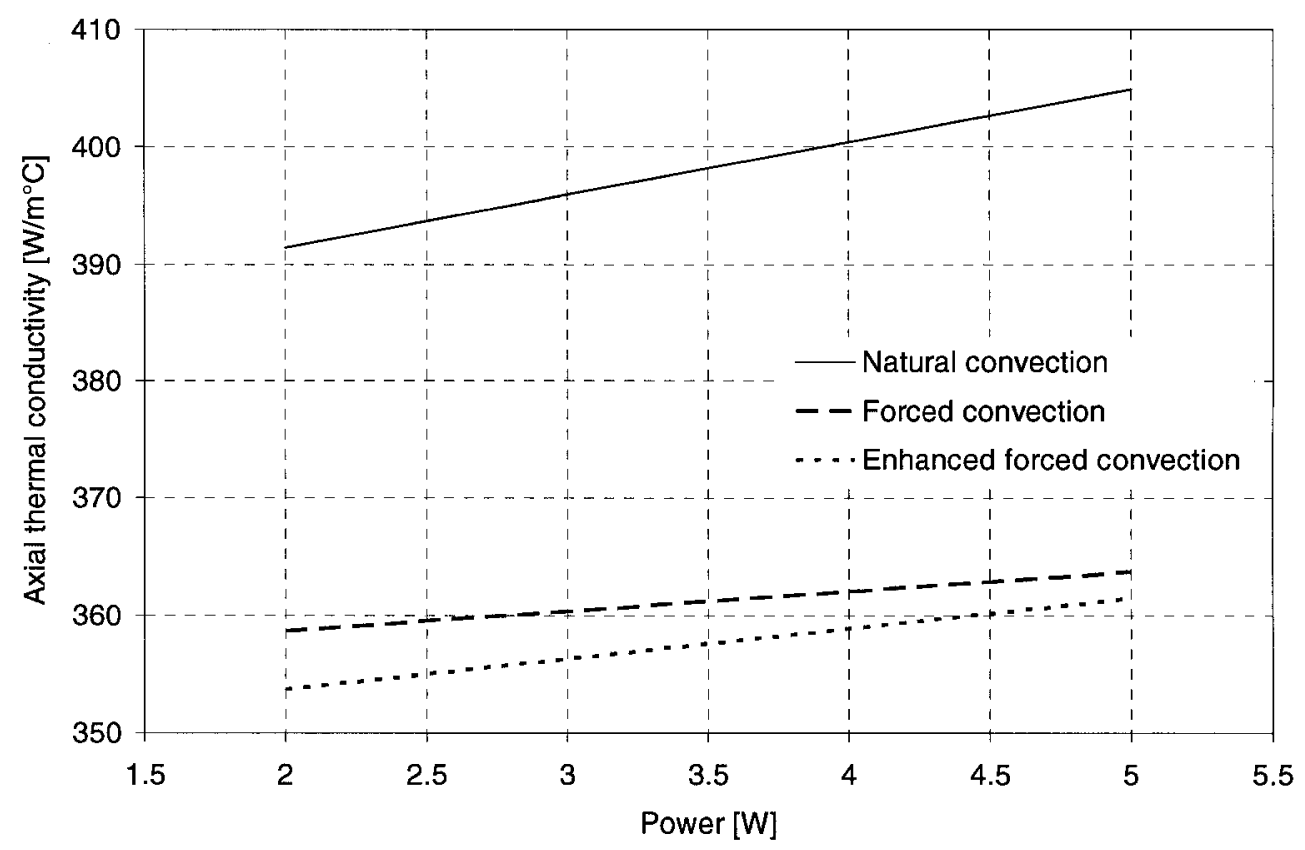

Figure 3.28 Copper tube with mesh axial thermal conductivity as a function of heat power

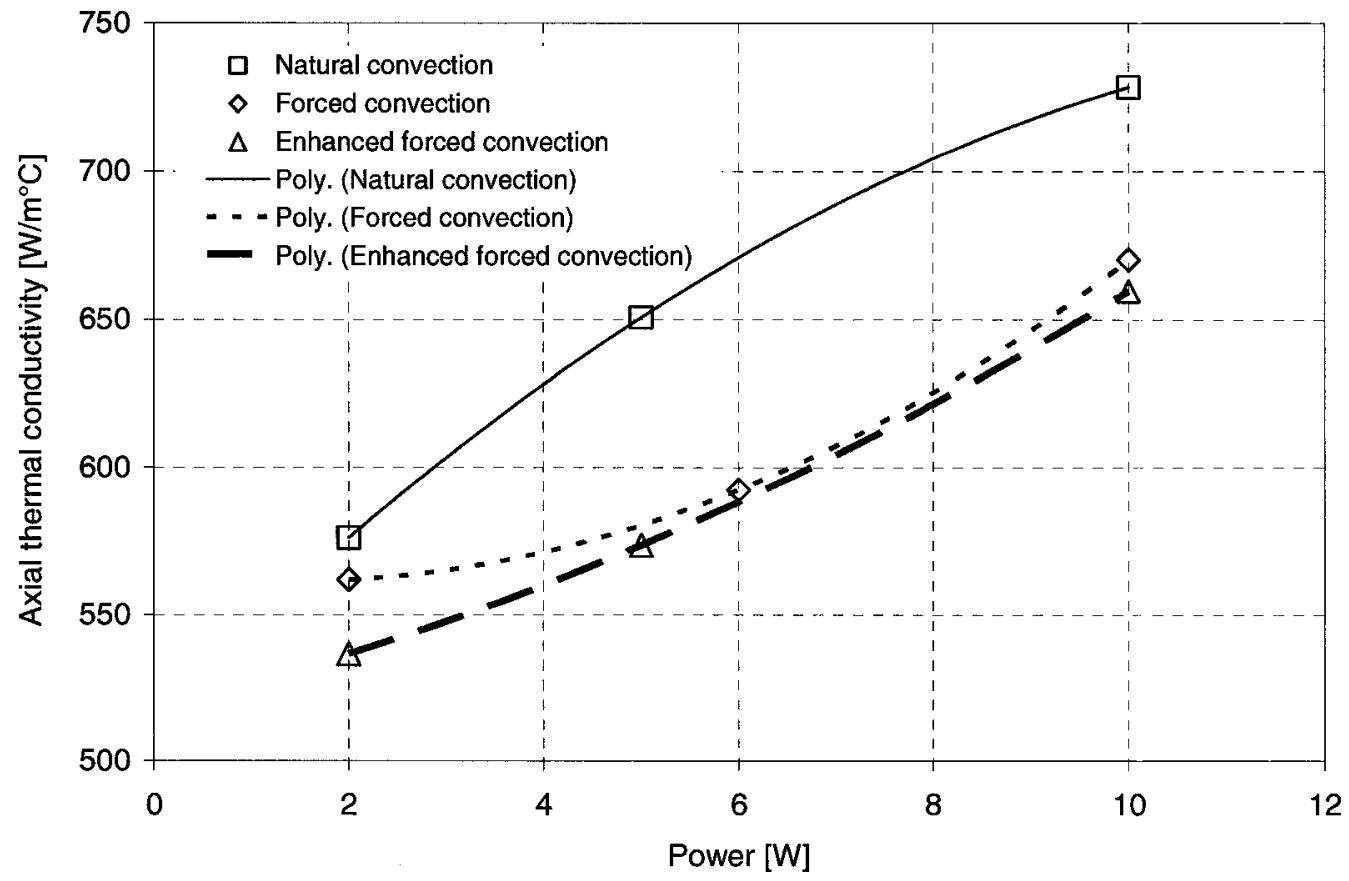

Figure 3.29 Copper tube with mesh \& water axial thermal conductivity versus heat load 


\subsubsection{Conclusion of the non-heat pipe testing}

1. The heater and sink assemblies are suitable for the tube transfer heat experiments.

2. The copper tube capacity to transfer heat is proportional to its solid cross-sectional area for the same heat-cooling distance.

3. Convective cooling affects the copper tube heat transfer. However, only enhancing the convection with the same ambient temperature would not help.

4. Axial temperature distribution is flat in the heating and cooling ends; the middle section takes a straight line with a constant slope.

5. The heating-cooling temperature gradient is directly proportional to the heat load on the heating end. The convection cooling methods have higher values than the natural approaches, and this difference increases with an increasing heat load.

6. The axial ratio of heating-cooling temperature dradient to the transferred heat is proportional to the heat load,

7. The temperature measurements are repeatable, and the temperature measurement approach is reliable.

\subsection{Experiment 3}

\section{Investigations of Heat Pipes Characteristics}

This is also a series of experiments with several configurations of heat pipes made in our laboratory, i.e., the wick structure, the wick material, the type of working fluid, the amount of charged work fluid, etc. Comparison analyses will be made based on the results obtained from these experiments, between different configurations and conditions. 


\subsubsection{Experimental Objective}

The objectives of these experiments are summarized as follows:

1. Verify the designs of the heat pipes.

2. Verify the technology of the heat pipe manufacturing.

3. Investigating the performance of the heat pipe that are designed and manufactured in our laboratory. These performance parameters include heat transfer capacity of the heat pipes, the heat transfer efficiency of the heat pipes, heat transfer limits of the heat pipes, the factors and the extent affecting the heat pipe performance.

4. Obtain results and analysis useful for future heat pipe applications as previously mentioned.

5. Identify any problems that may exist and provide feedback for the design and manufacture phase.

\subsubsection{Experiment Principle and Apparatus}

Detailed structures of the setup for the heat pipes are the same as those of the non-heat pipe applications, as described in Section of 3.3.2. The difference is that in the heat pipe experiments, the orientation factor affects the performance to some extent. Thus, the heat pipes to be tested are required can be fixed in certain orientations. A set of special rig was designed for such purpose. These include a heat pipe setup baseboard that can fix the heat pipe as well as the heat source and cooling sink assemblies. The baseboard can be fixed on a platform in three orientations; horizontal, vertical with gravity assisted, and vertical against gravity respectively. The platform was designed can be adjusted to meet the 
required horizontal level. Figures 3.30 and 3.32 illustrate the heat pipe setups in three orientations respectively.

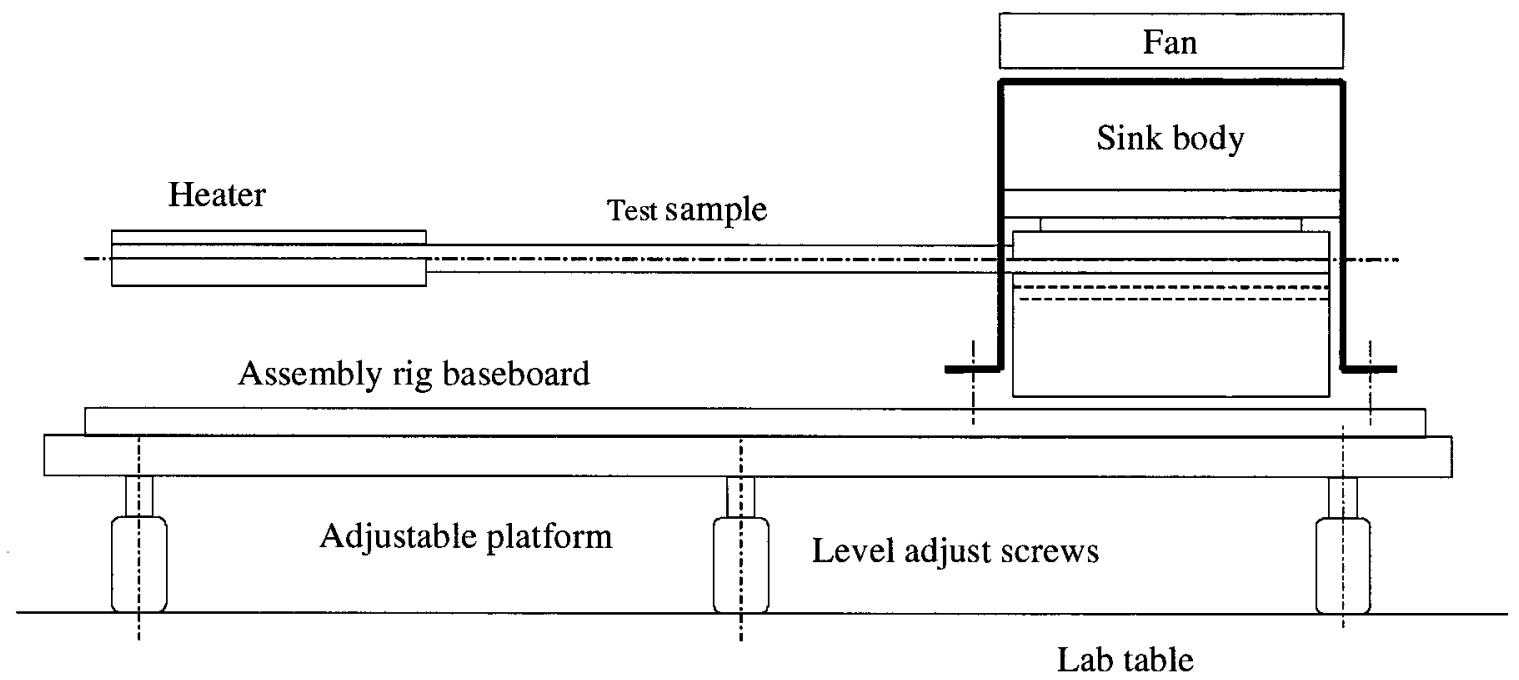

Figure 3.30 Heat pipe testing setup (horizontal, $0^{\circ}$ )

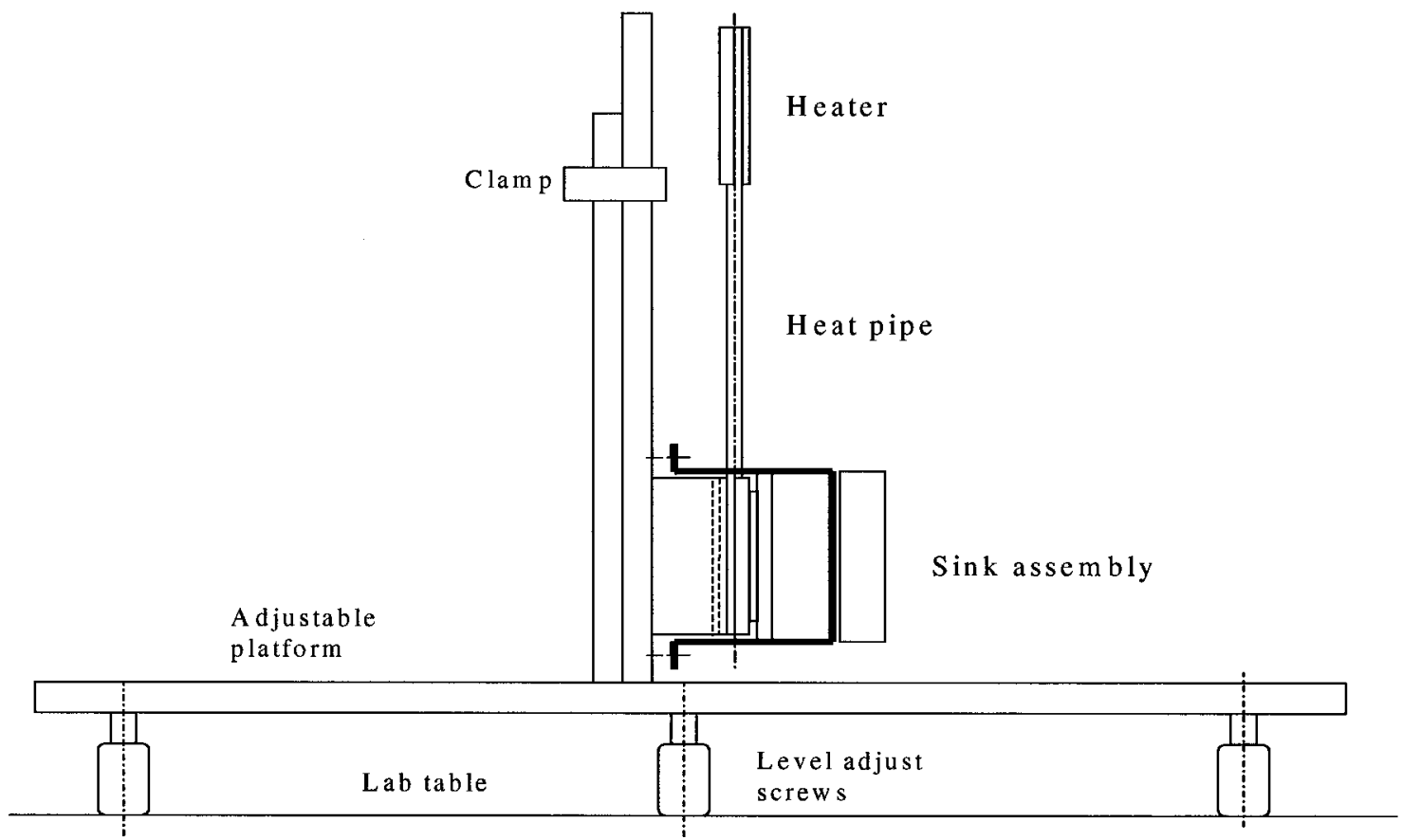

Figure 3.31 Heat pipe testing setup (vertical, against gravity, $+90^{\circ}$ ) 


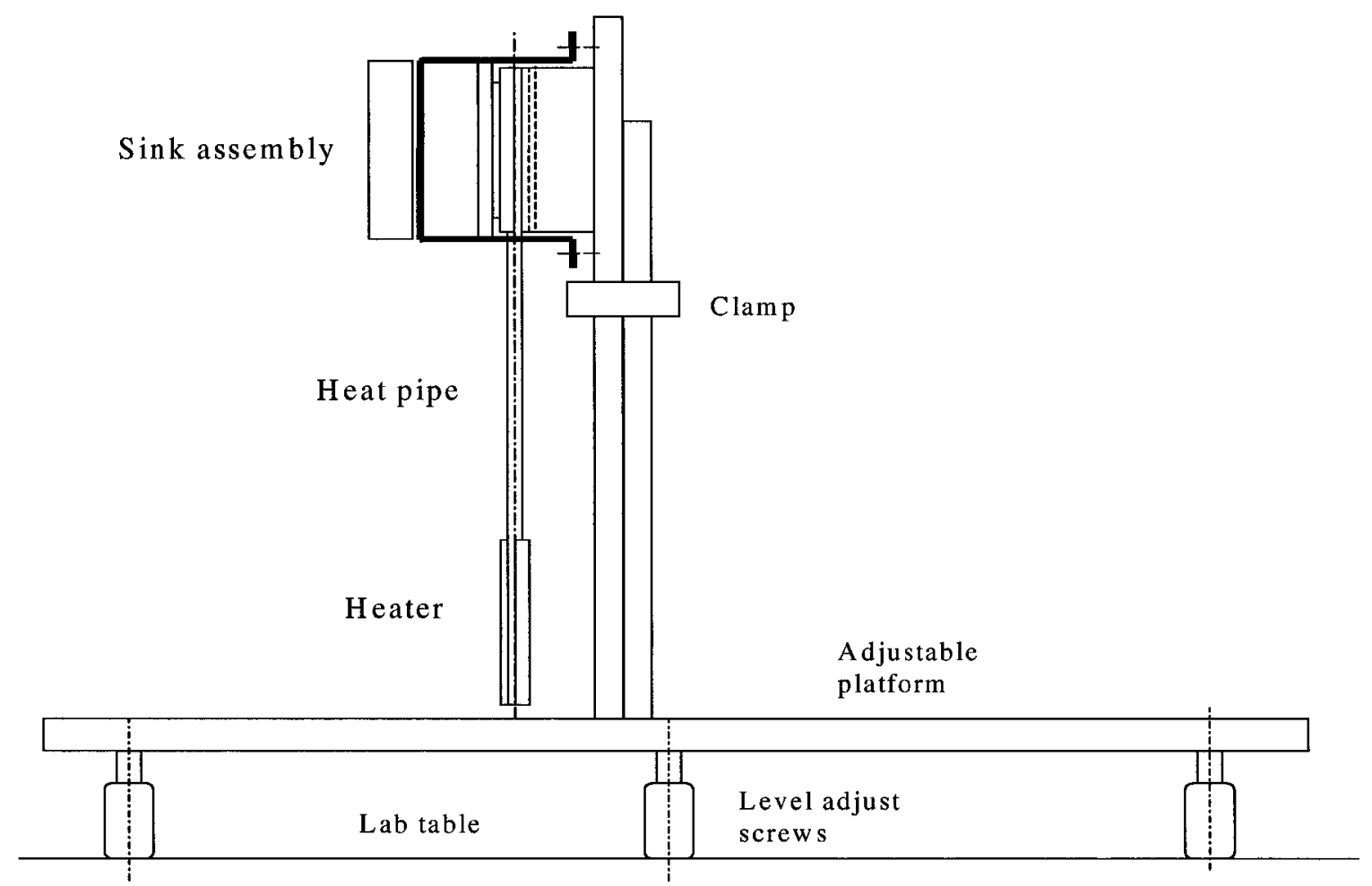

Figure 3.32 Heat pipe testing setup (vertical, gravity assisted, $-\mathbf{9 0}^{\circ}$ )

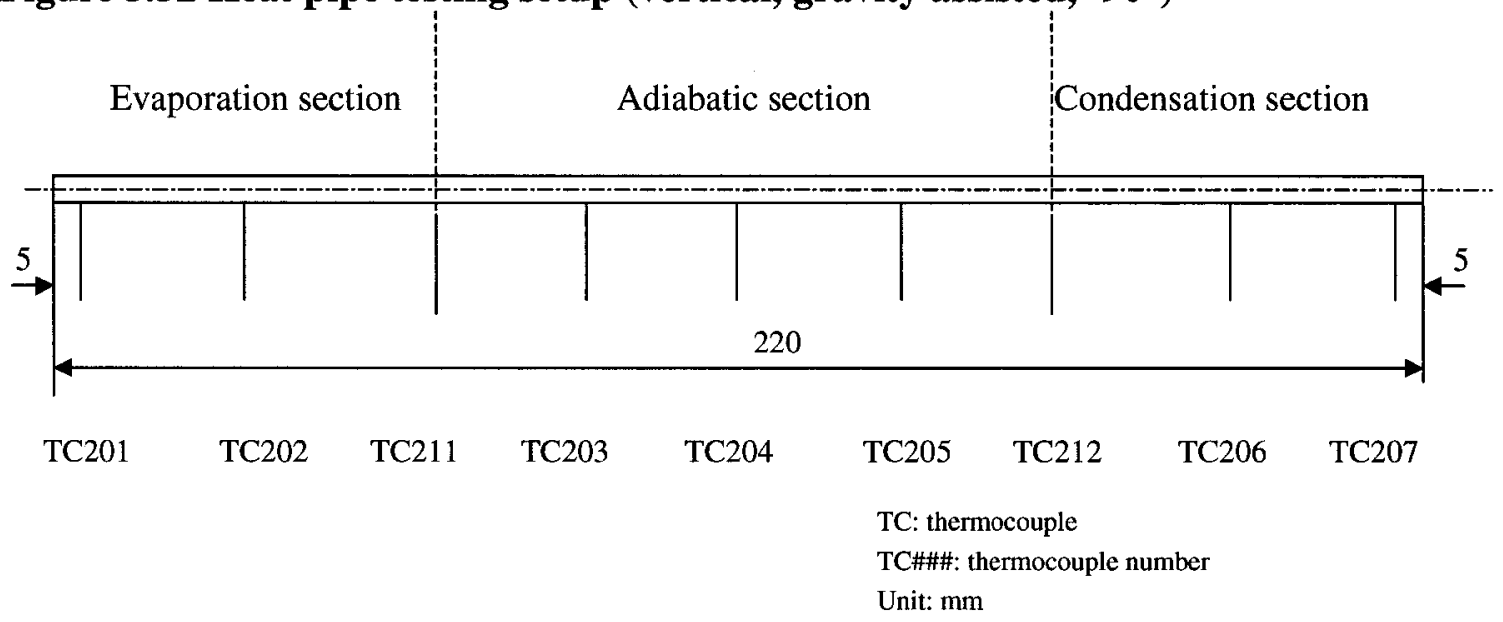

Figure 3.33 Schematics of thermocouple distribution along the heat pipe axial

Figure 3.33 displays the thermocouple arrangement along the testing samples. All the thermocouples are fixed with aluminium adhesive tape on the tube external surface. Three thermocouples (TC201, TC202 and TC211) are on the evaporation section to measure the evaporator temperature at the end, middle and near the adiabatic section point respectively. Similarly, three thermocouples (TC212, TC206 and TC207) are on the 
condensation section to measure the condenser temperature at the near the adiabatic section, and in the middle and end points respectively. The other three thermocouples (TC203, TC204 and TC205) are evenly arranged along the adiabatic section surface to measure the surface temperature of this section at the points near the heater, the middle, and near the sink respectively.

Based on this setup, two configurations, non-insulated and insulated, were applied to some heat pipes, and for each one, were applied in three orientations; horizontal, against gravity, and gravity assisted, as well as three cooling conditions, i.e., natural, forced convective, and enhanced forced convective cooling.

\subsubsection{Experimental Procedure}

As described in the preceding section, all the test samples experienced three orientations and three cooling conditions. Additionally some of the test samples experienced noninsulation as well as insulation configurations. Due to the orientation sensitivity, the heat pipe assembly is required to be fixed parallel to the baseboard within the tolerance. Furthermore, the whole rig is designed to be fixed at different orientation for specific experiments. This is done with the assistance of the adjustable platform described in the preceding section. After setting up the rig orientation, samples are ready for experiment. The other testing procedures are the same as those applied in the non-heat pipe experiments described in Section of 3.3.3. 


\subsubsection{Experiment Results and Analyses}

The results obtained in the above series of experiments will be discussed in the following sections. Since non-insulated tests are easily affected by environmental conditions, here we only present analyses based on data acquired from the insulated cases.

\subsubsection{Typical Heat Pipe Temperature Characteristics}

Six heat pipes were involved in the experiments including five copper meshes and a nickel foam heat pipe, Figure 3.34 illustrates the time-temperature profile of one of these heat pipes; a coarse mesh with a normally charged water heat pipe.

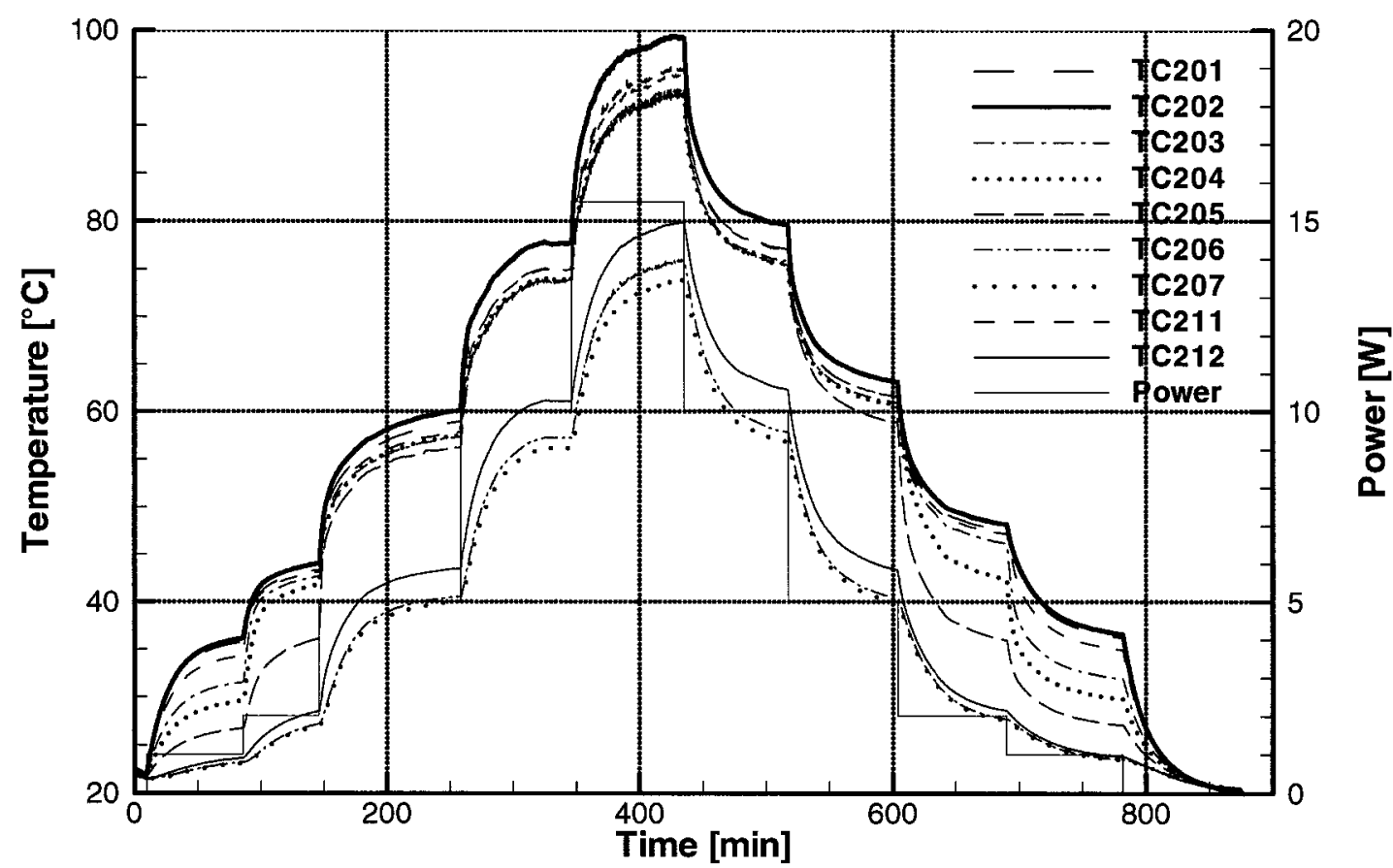

Figure 3.34 Typical heat pipe time-temperature profile (insulated, horizontal, natural convection) $[0,1,2,5,10,15.5 \mathrm{~W}]$ [sample(s):HP071200]

In the figure, there are three groups of curves, each indicating the temperatures of the three sections along the heat pipe axis; the evaporation, the adiabatic, and the 
condensation sections. Each group is composed of some single curves reflecting the measurements within each section. All curves reflect the process of temperature stabilization regarding the corresponding increase and decrease to power. In this case the whole process experienced such power levels, starting from $0 \mathrm{~W}$, then $1 \mathrm{~W}, 2 \mathrm{~W}, 5 \mathrm{~W}, 10$ $\mathrm{W}, 15.5 \mathrm{~W}$, until the limit of $15.5 \mathrm{~W}$ is reached, Power is then decreased with by the same intervals until the initial condition of $0 \mathrm{~W}$ is reached.

Figures 3.35 to 3.37 , display power stable temperature curves in the three situations; horizontal with natural convective cooling, horizontal with enhanced force convective cooling, gravity assisted vertical with natural convective cooling, and against gravity vertical with natural convective cooling respectively.

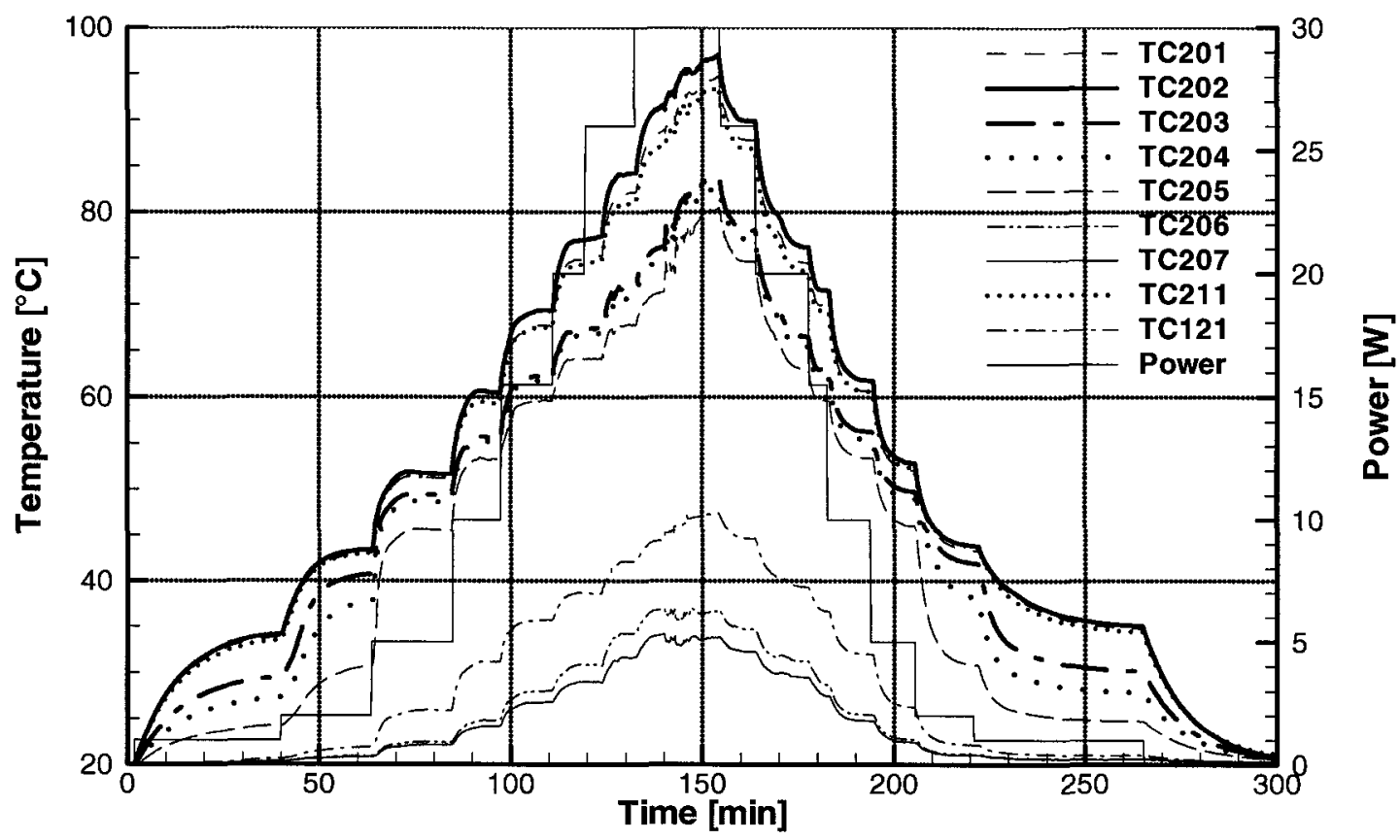

Figure 3.35 Typical heat pipe time-temperature profile (horizontal, enhanced forced convection) $[0,1,2,5,10,15.5,20,26,30 \mathrm{~W}]$ [sample(s):HP071200] 


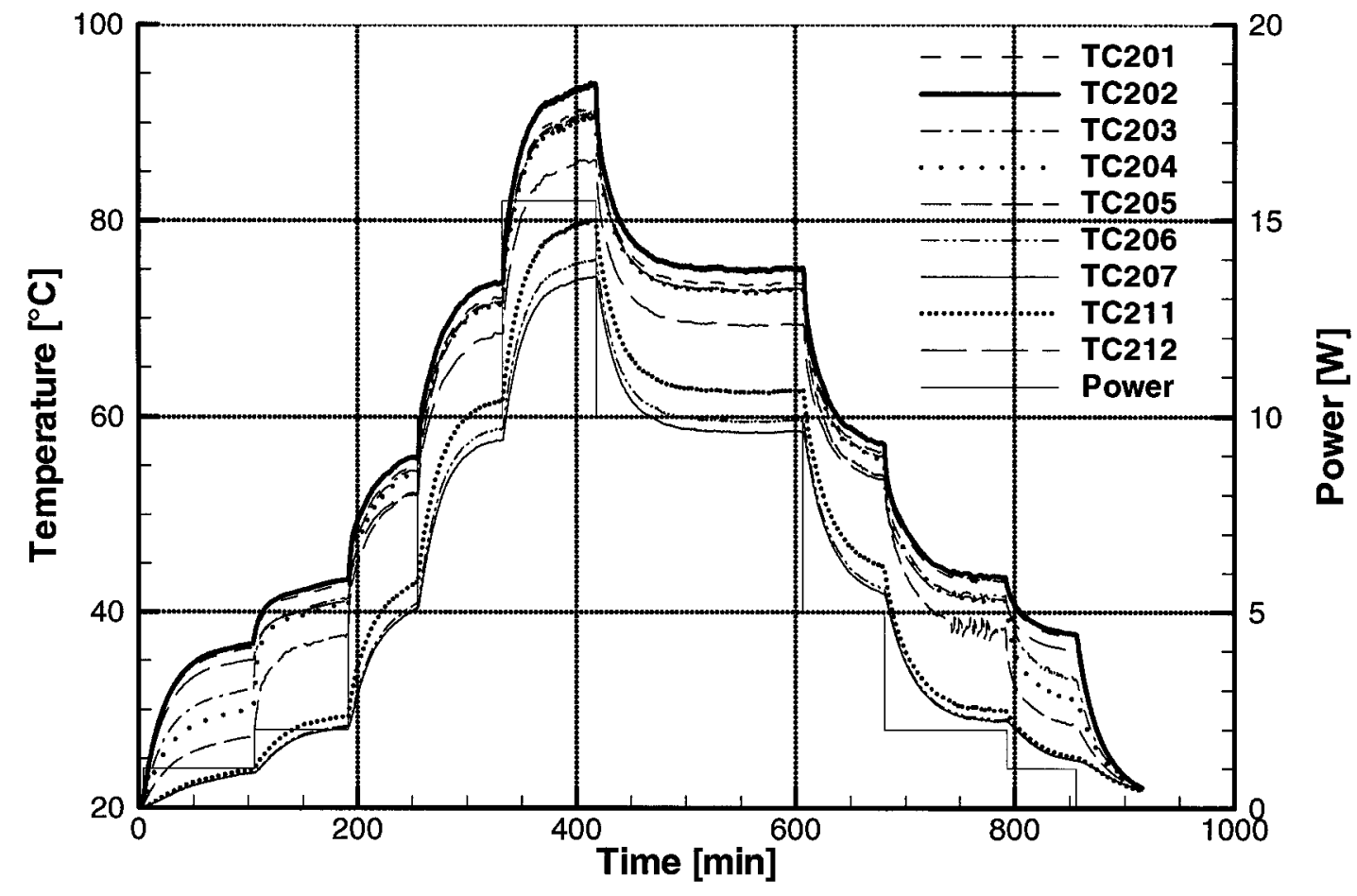

Figure 3.36 Typical heat pipe time-temperature profile (insulated, gravity assisted vertical, natural convection) $[0,1,2,5,10,15.5 \mathrm{~W}]$ [sample(s):HP071200]

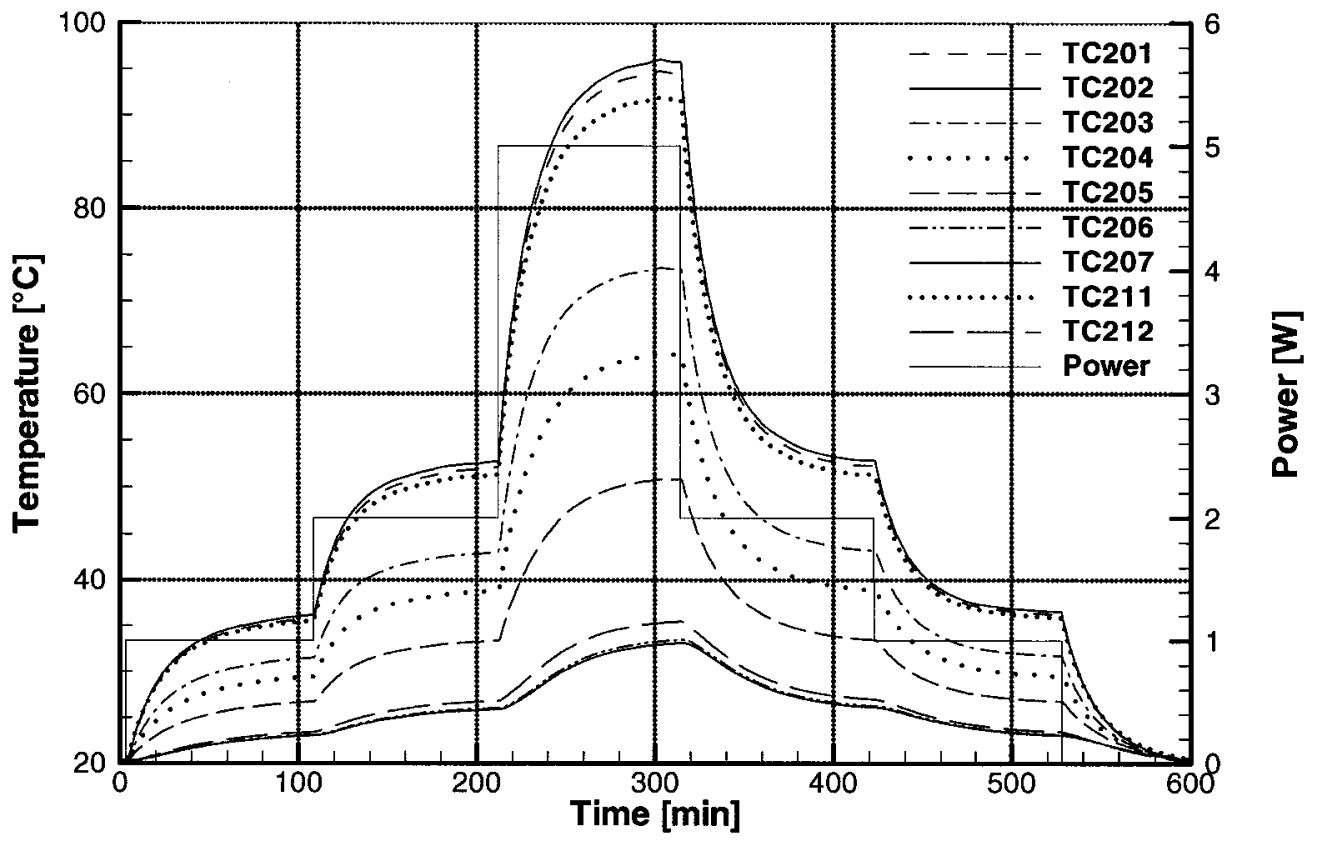

Figure 3.37 Typical heat pipe time-temperature profile (insulated, against gravity vertical, natural convection) $[0,1,2,5 \mathrm{~W}]$ [sample(s):HP071200] 
Among these four figures, all figures, except for the last one, show a similar tendency. With an increasing power input, the temperature distribution along the evaporator and condenser tend to diverge, while the adiabatic temperature curves tend to converge. Also, with increasing power input, inter-group (evaporator, adiabatic section and condenser) temperature curves tend to diverge. This temperature distribution profile distinguishes heat pipe from conductance device, e.g., copper tube, as illustrated in Section 3.3. The temperature diverging at either end is easy to understand with the knowledge of conductance as discussed in the experiments of copper tube heat transfer. The adiabatic temperature converging is due to the internal fluid phase change. It is the phase change that maintains the temperature during the heat transfer from the evaporator to the condenser through the fluid, consequently reducing the axial temperature difference. It is this adiabatic temperature convergence that narrows the evaporatorcondenser temperature gap, thus heat is transferred with a small temperature gradient.

The last figure, against gravity vertical case, shows a different temperature distribution profile. All the temperature curves tend to diverge with increasing power input. This indicates that this specific heat pipe in the condition of against gravity transfers heat relying upon a great axial temperature gradient rather than a phase change. Also, the evaporator temperature reaches about $100^{\circ} \mathrm{C}$ at only $5 \mathrm{~W}$.

\subsubsection{Heater Cooling Capacity}

One of the heat pipe applications is to dissipate heat from the heat source. The very direct assessment criterion is to compare the degree of temperature decrease from the heat source. The following figures present the records of the heater surface temperature with the heat pipes applied at certain orientations, horizontal, gravity assisted vertical, and 
against gravity vertical as well as certain cooling approaches on the condenser, natural, normal force, and enhanced forced convection.

The involved heat pipes have various configurations: fine, coarse, and composite mesh; water, and acetone working fluid; normally and over charged working fluid. All heat pipes and copper tubes involved possess the same geometric and physics properties, and are attached on the heater and sink in the same way. This help to evaluate their cooling capacity by comparing the heater surface temperature directly.

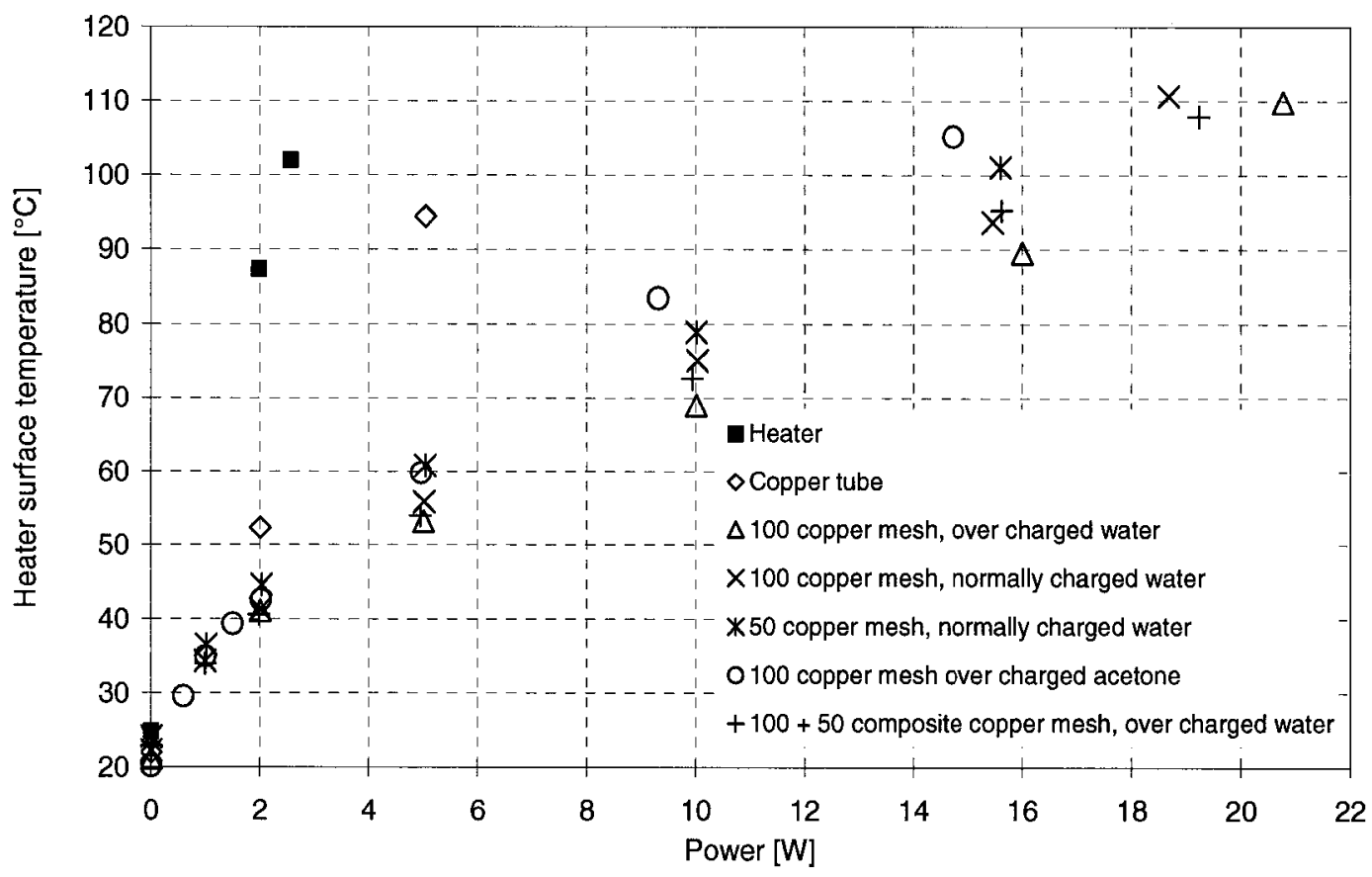

Figure 3.38 Heater surface temperature comparison with heat pipes (insulated, horizontal, natural convection) [sample(s):HP062300, HP052502, HP051701, HP071200, HP071402, HP071301]

Figure 3.38 displays the heater surface temperatures with copper and heat pipe assists in the condition of insulated heater, evaporator, and adiabatic section, horizontal orientation, with natural convection cooling on the condenser. The figure clearly shows that heat pipes decrease the heater surface temperature by 40 to $50^{\circ} \mathrm{C}$ at the same power input, of $2.5 \mathrm{~W}$. Extending the heater power capacity by about $13 \mathrm{~W}$ for the same 
temperature constraint of $100^{\circ} \mathrm{C}$; improves the cooling capacity by $10 \mathrm{~W}$ compared with the copper tube for the same temperature constraint.

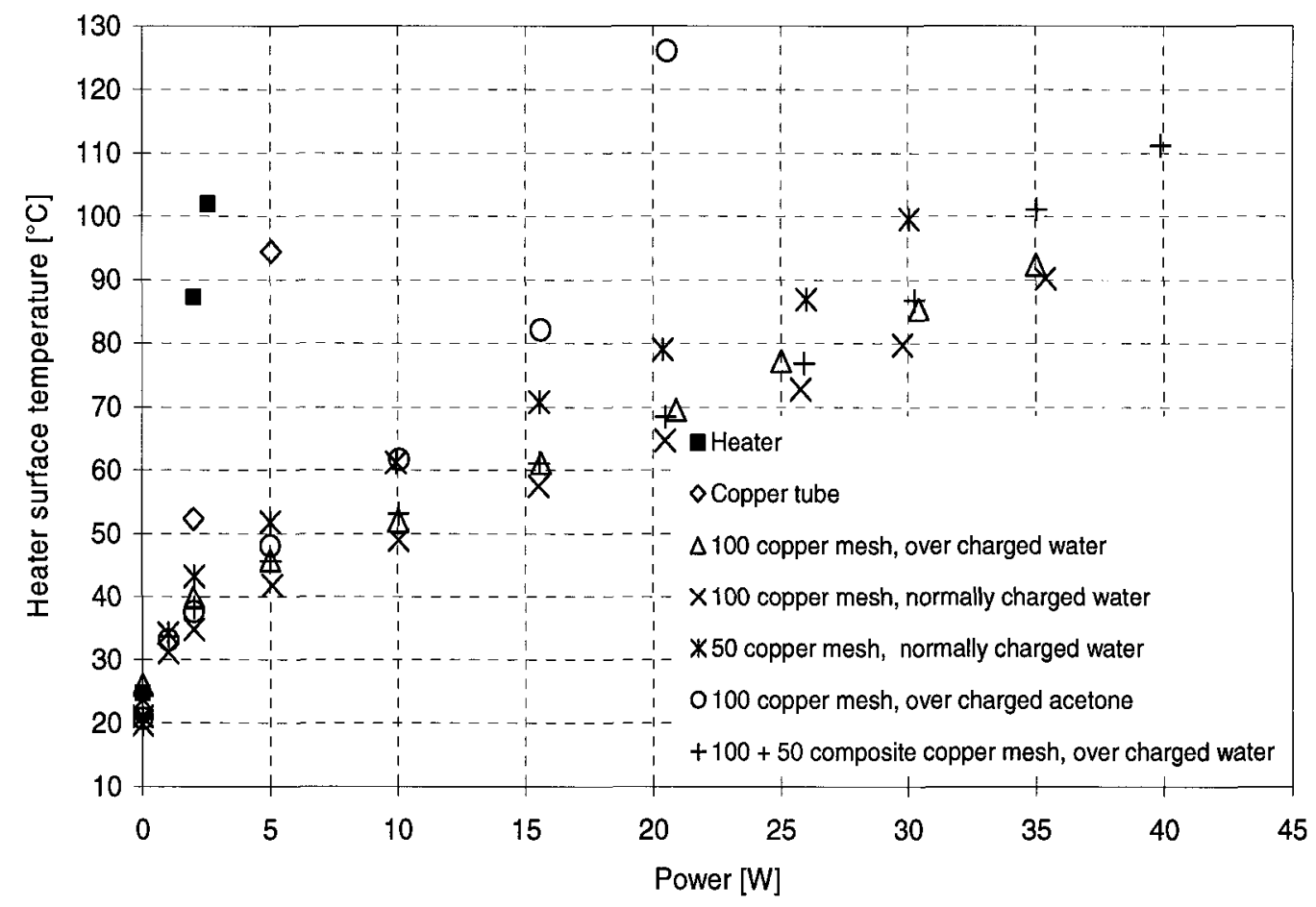

Figure 3.39 Heater surface temperature comparison with heat pipes (insulated,

horizontal, enhanced forced convection) [sample(s):HP052502, HP051701, HP071200, HP071402, HP071301]

Figure 3.39 displays the heater surface temperatures with copper and heat pipe assists with insulation on the heater, evaporator, and adiabatic sections, and at horizontal orientation, with enhanced forced convection cooling. The figure shows that heat pipes lower the heater surface temperature by 40 to $50^{\circ} \mathrm{C}$ at the same power input, $2.5 \mathrm{~W}$, similar to the case of natural convection cooling. While with enhanced convection cooling, heat pipes increase the heater power capacity by about $33 \mathrm{~W}$ for the same temperature constraint of $100^{\circ} \mathrm{C}$. A $20 \mathrm{~W}$ improvement is observed when compared to the natural convection case. This indicates that heat pipes maintain small adiabatic section temperature gradient at a certain heat load on the evaporator. Properly decreasing the condenser temperature would increase heat pipe cooling capacity. This is consistent 
with Fourier's Law. Note that the case of fine copper mesh acetone heat pipe is distinct from others. At about $15 \mathrm{~W}$, the heater surface temperature starts to jump. This is the socalled "dry out", and will be discussed in later sections.

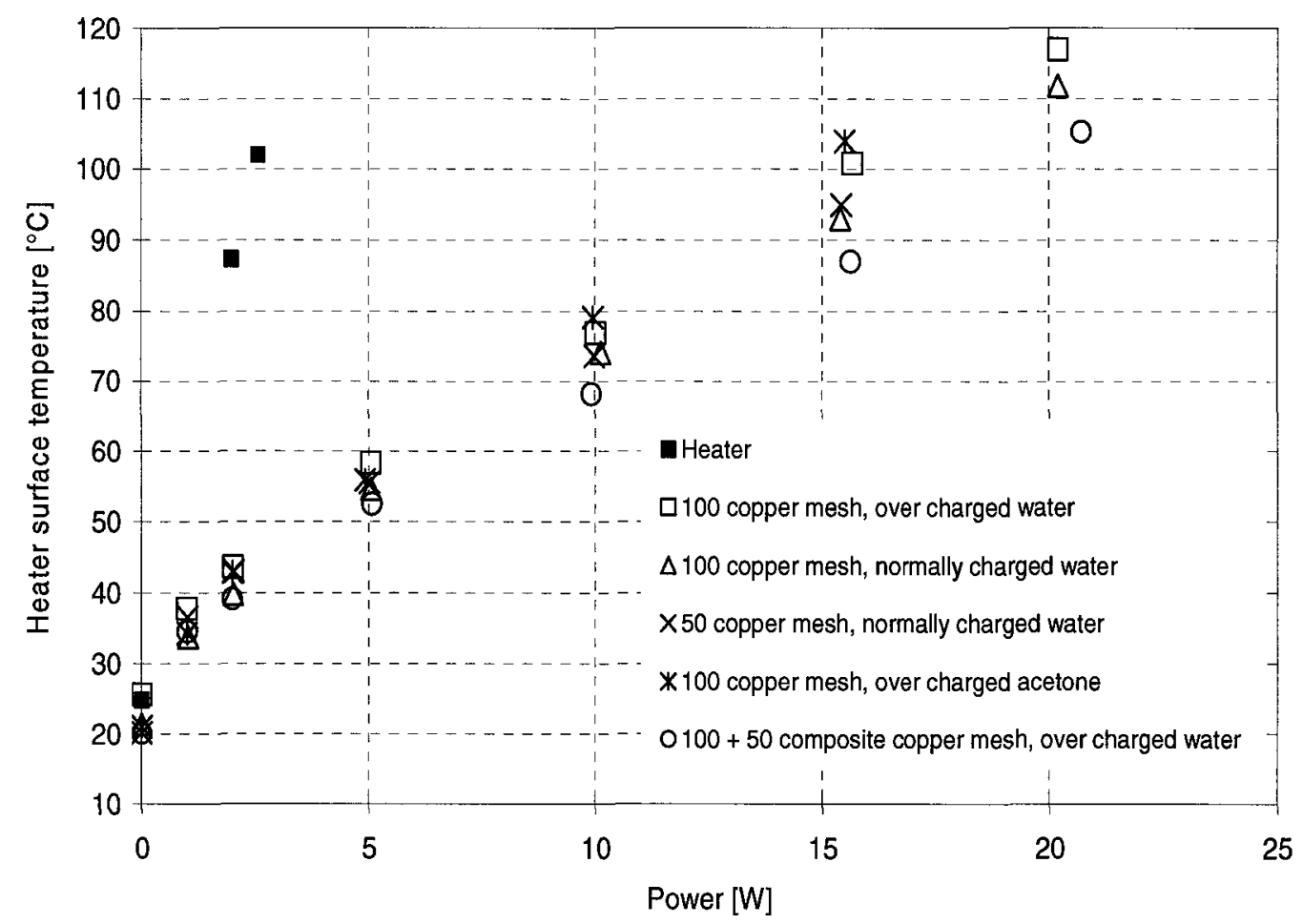

Figure 3.40 Heater surface temperature comparison with heat pipes (insulated, gravity assisted vertical, natural convection) [sample(s): HP052502, HP051701, HP071200, HP071402, HP071301]

Figure 3.40 displays the heater surface temperatures with heat pipe assist in the condition of insulated heater, evaporator, and adiabatic section, gravity assist vertical orientation, with natural convection cooling on the condenser. The figure shows general picture about the heat pipes cooling capacity with gravity assist vertical orientation in the condition of natural convection cooling on the condenser. This picture looks similar to the horizontal counterpart, but it can be seen that the over charged and normally charged heat pipes exchange positions at the heater surface temperature, especially in higher heat load. In the horizontal case, the over charged heat pipe (HP052502) keeps the heater to a lower 
temperature than the normally charged heat pipe (HP051701). While in the gravity assist vertical case, they exchange positions. The normally charged heat pipe keeps the heater to a lower temperature than the over charged heat pipe. This can be explained by the fact that the extra water stays in the evaporator when the heat pipe takes the gravity assist vertical orientation. This extra water works as a thermal buffer in the evaporator, and provides a thermal inertia to the evaporator, thus maintaining the heater at a slightly higher temperature than the case with normally charged heat pipe.

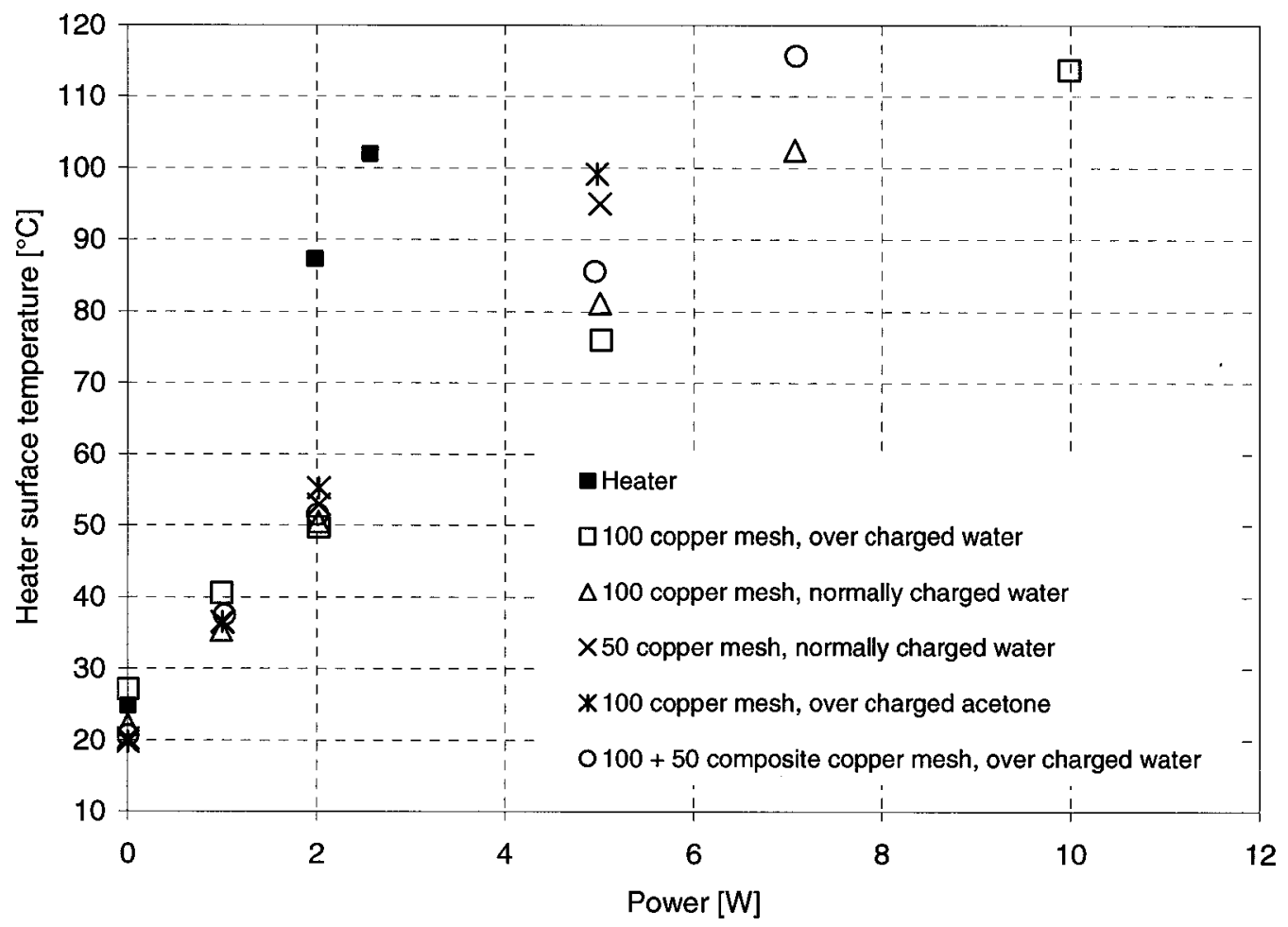

Figure 3.41 Heater surface temperature comparison with heat pipes (insulated, against gravity vertical, natural convection) [sample(s): HP052502, HP051701, HP071200, HP071402, HP071301]

Figure 3.41 displays the heater surface temperatures with heat pipe assist in the condition of insulated heater, evaporator, and adiabatic section, against gravity vertical orientation, with natural convection cooling on the condenser. The figure clearly shows that heat pipes with such condition decrease the heater surface temperature by 30 to $40^{\circ} \mathrm{C}$ 
for the same power input of $2.5 \mathrm{~W}$. It also increases the heater power capacity by approximately 3 to $5 \mathrm{~W}$ for the same temperature constraint of $100^{\circ} \mathrm{C}$. This is about a 10 W difference as compared to its horizontal counterpart. Also note that the acetone heat pipe configuration starts to "dry out" at only $7 \mathrm{~W}$. In this case, it dries out approximately $13 \mathrm{~W}$ earlier than the horizontal enhanced forced convection counterpart.

\subsubsection{Axial Temperature Profile}

The axial temperature profile is a main criterion for heat pipes. It distinguishes heat pipe from normal copper tubes in heat transfer characteristic. Unlike those of the copper tube discussed in an early section, the heat pipe axial temperature profile is more complex. This section will investigate this topic on certain aspects.

\subsection{Typical Heat Pipe Axial Temperature Profile}

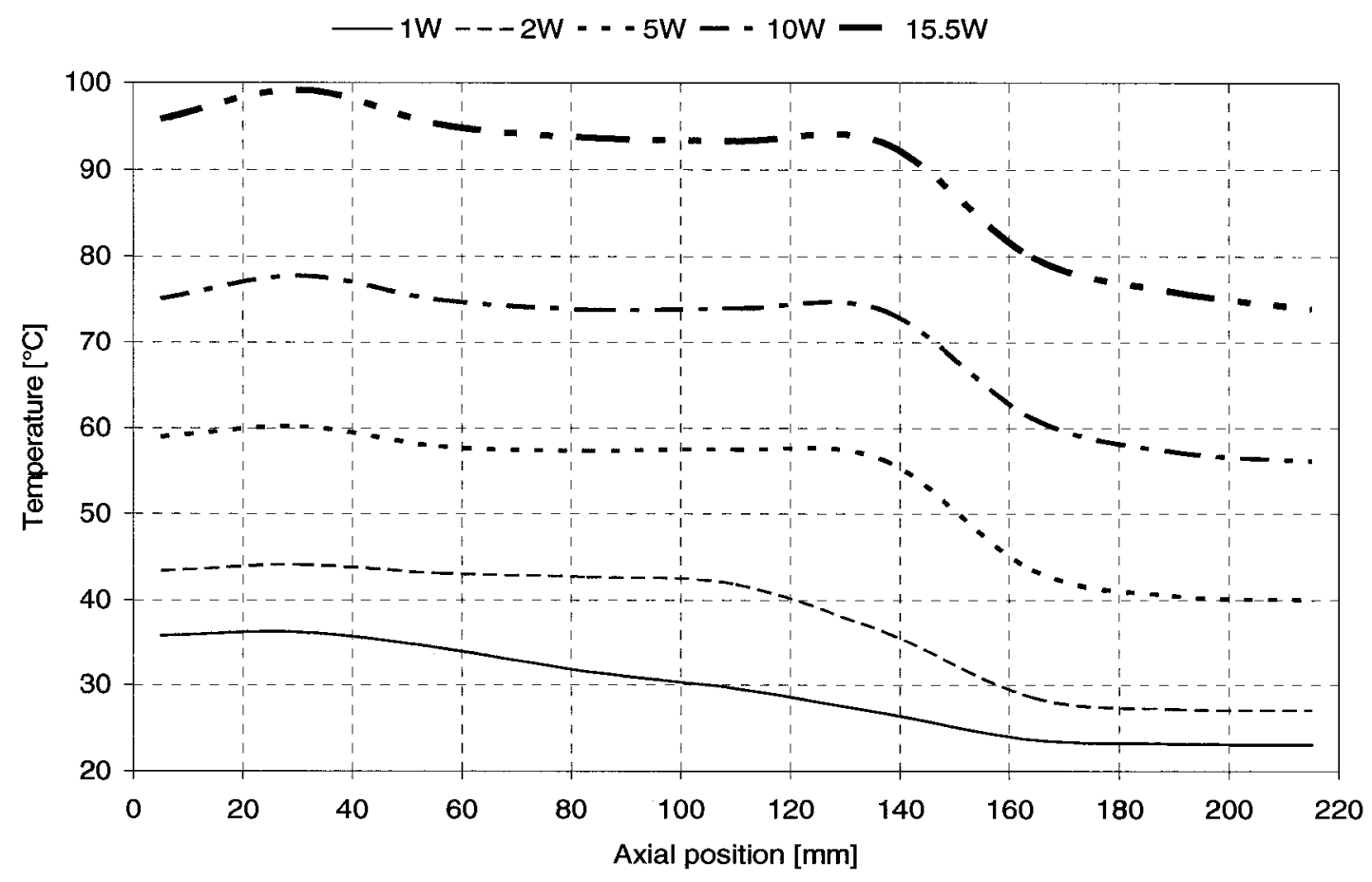

Figure 3.42 Heat pipe axial temperature profile (insulated, horizontal, natural convection) [sample(s): HP071200] 
Figure 3.42 is a typical heat pipe axial temperature distribution profile with the heat pipe in a horizontal orientation under natural convection cooling on the condenser. It demonstrates the temperature distribution along the heat pipe axial direction at different power input levels. The axial position can be divided into three sections, evaporation, $(0$ to $50 \mathrm{~mm}$ ), adiabatic (50 to $170 \mathrm{~mm}$ ), and condensation $(170$ to $220 \mathrm{~mm}$ ). From the figure it can be seen that the $1 \mathrm{~W}$ curve has only two flat sections at both end, and a constant slope straight line between these two sections. All other curves are composed of three flat sections, reflecting the temperatures on the evaporator, the adiabatic section, and the condenser respectively. The curves are evenly arranged in parallel proportionally to the power input level.

At $1 \mathrm{~W}$ level, the evaporator temperature reaches just approximately $35^{\circ} \mathrm{C}$. At such an evaporation temperature and a small evaporation-condensation temperature gradient, the internal water would not start to change phase. Also, the heat load is not enough to further rise the evaporator temperature, thus the heat pipe would not be activated. Instead, it just acts as a copper tube with copper mesh inserted and water charged. At the $2 \mathrm{~W}$ level, the evaporator temperature reaches about $43^{\circ} \mathrm{C}$. At this temperature the internal water starts to evaporate (refer to Chapter 2), and the heat load is enough to maintain such an evaporation temperature. Thus the heat pipe becomes active, and functions as a heat pipe. Note that, on the $2 \mathrm{~W}$ temperature curve, the evaporation-adiabatic temperature difference is very small, only a few degree Celsius, and the adiabatic flat starts from 50 $\mathrm{mm}$, and starts to decline at $100 \mathrm{~mm}$. It is $50 \mathrm{~mm}$ long, which is just half of the adiabatic section length. This is due to that fact that the water evaporates from the evaporator, and goes through the adiabatic section that is still in form of vapour. Phase change has not 
happened until the point is reached where the temperature is low enough for the vapour to start to condense. This point responds the one where the curve begins declining from the adiabatic section on the temperature curve. Compared to other higher power inputs, in the $2 \mathrm{~W}$ case the heat load is relatively low. The water and its vapour contains a small amount of heat, thus causes a small evaporation-adiabatic temperature gradient. This small amount of heat is easier to dissipate. Therefore before reaches the condenser, the vapour loses heat, and condensates to liquid, finishing phase change. The condensed water continues dissipating heat to the condenser in forms of liquid convection. Due to the fact that, at a lower power level, the water flowing speed is slow, thus the temperature gradient is relatively large compared with that of water or vapour at higher power levels. This is the main reason that the lower power axial temperature profile curves take slop adiabatic lines. In the higher power cases, the vapour contains more heat, thus causes a bigger evaporator-adiabatic temperature gradient.

Natural convection temperature on the condenser is passively determined by two factors; the ambient temperature and the heat flux come from the evaporator. In a given temperature environment, the condenser temperature is proportional to the heat load on the evaporator.

With increasing heat load, it can be seen in Figure 3.42 that the temperature curve in the evaporation section tends to become convex. Meanwhile, the temperature at the middle of the evaporator gets higher. This is due to a larger temperature difference between the heater or the evaporator and its surrounding area. Thus the boundary areas of the heater lose heat more than its centre point. This causes the middle temperature to 
become higher than others, making the temperature curve convex in the evaporation section.

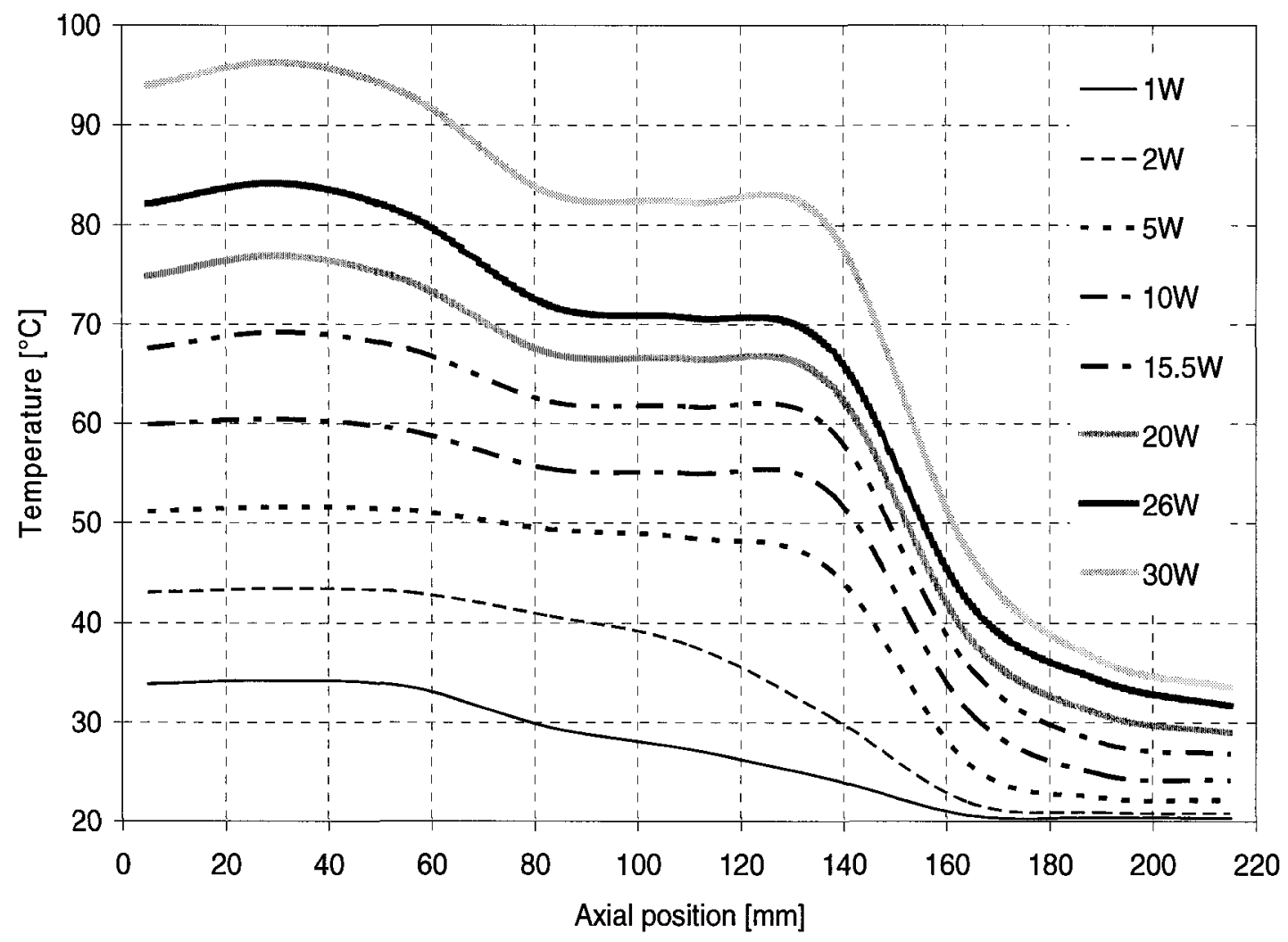

Figure 3.43 Heat pipe axial temperature profile (insulated, horizontal, enhanced forced convective) [sample(s): 071200]

Figure 3.43 displays the axial temperature profile of the same testing heat pipe as in Figure 3.42, i.e., horizontal with enhanced forced convection on the condenser. In this figure, it can be seen that the temperature curves over $5 \mathrm{~W}$ get a few degrees Celsius lower than the corresponding heat load case showed in the preceding figure. Also seen the fact that the $1 \mathrm{~W}$ curve is a little different in Figure 3.43 from it counterpart in Figure 3.42, The $1 \mathrm{~W}$ curve has two slopes straight section between the evaporator and condenser, and a very short adiabatic flat from 75 to $80 \mathrm{~mm}$. With an enhanced forced convection cooling on the condenser, a relatively greater evaporation-condensation 
temperature gradient causes a partial phase change in the internal water. This tiny phase change brings a very limited adiabatic flat on the temperature curve as shown in the figure. However, the heat load is too limited to maintain the vapour status, thus the vapour converts back to liquid. Hence it no longer acts as a heat pipe.

Figure 3.43 shows a very narrow condenser temperature range. This is caused by the enhanced forced convection on the condenser. In the conditions of forced, and enhanced forced convection the sink cooling capacity is stronger than the natural convection, thus the condenser temperature is confined to a narrow range. Therefore the evaporation-condensation temperature difference becomes larger.

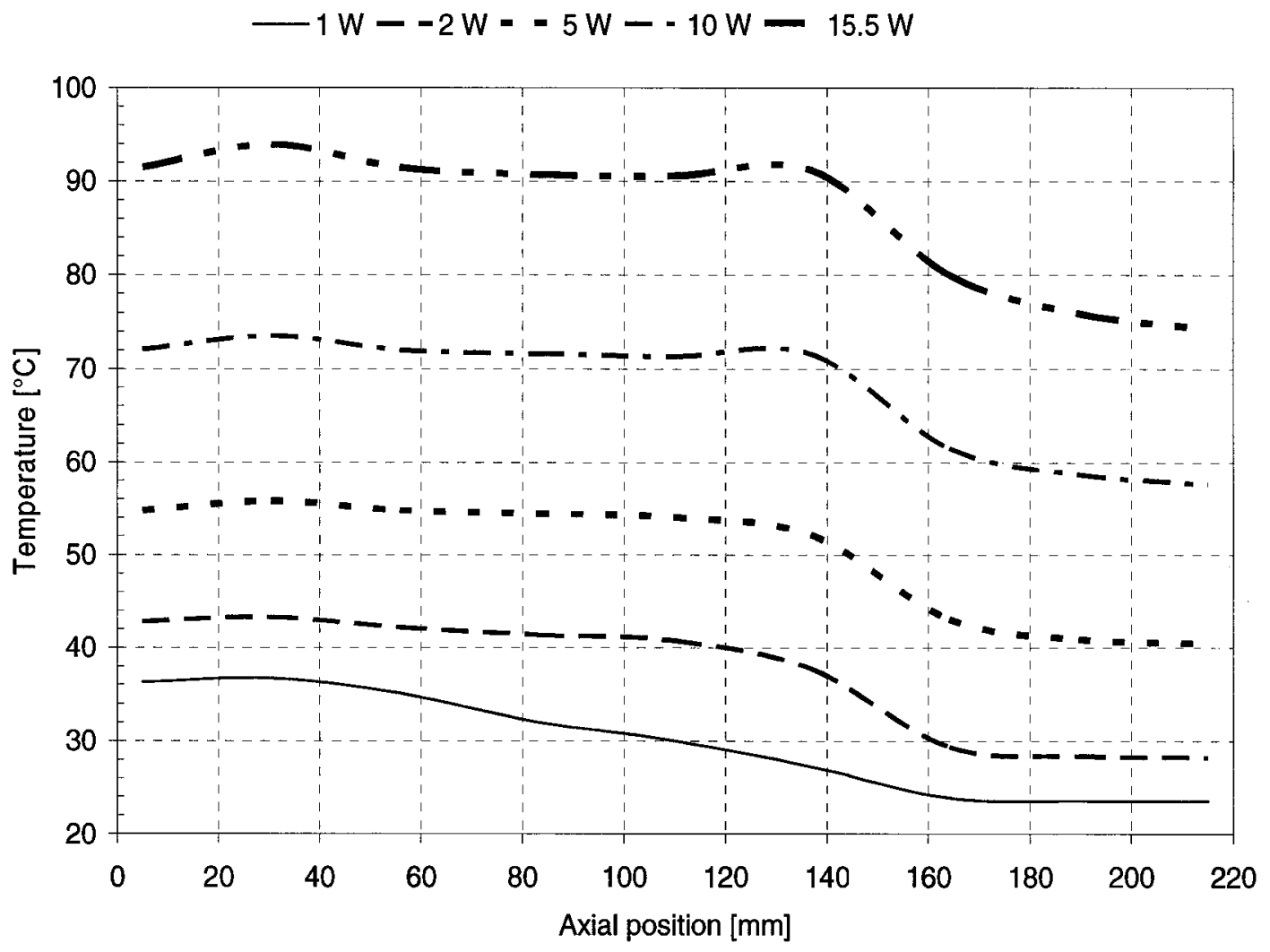

Figure 3.44 Heat pipe axial temperature profile (insulated, gravity assisted vertical, natural convection) [sample(s): 071200]

Figure 3.44 displays the axial temperature profile for the same heat pipe of the preceding figures in a gravity assisted vertical orientation, and natural convection cooling 
of the condenser. From this figure we can see that all temperatures take the same shapes as their horizontal counterpart described in Figure 3.42. The difference is that the later case causes a few degrees Celsius lower to the corresponding heat load. This is due to the gravity since it helps the liquid water turn back to the evaporator from the condenser, assisting heat transfer through the water phase change, and therefore heat dissipation.

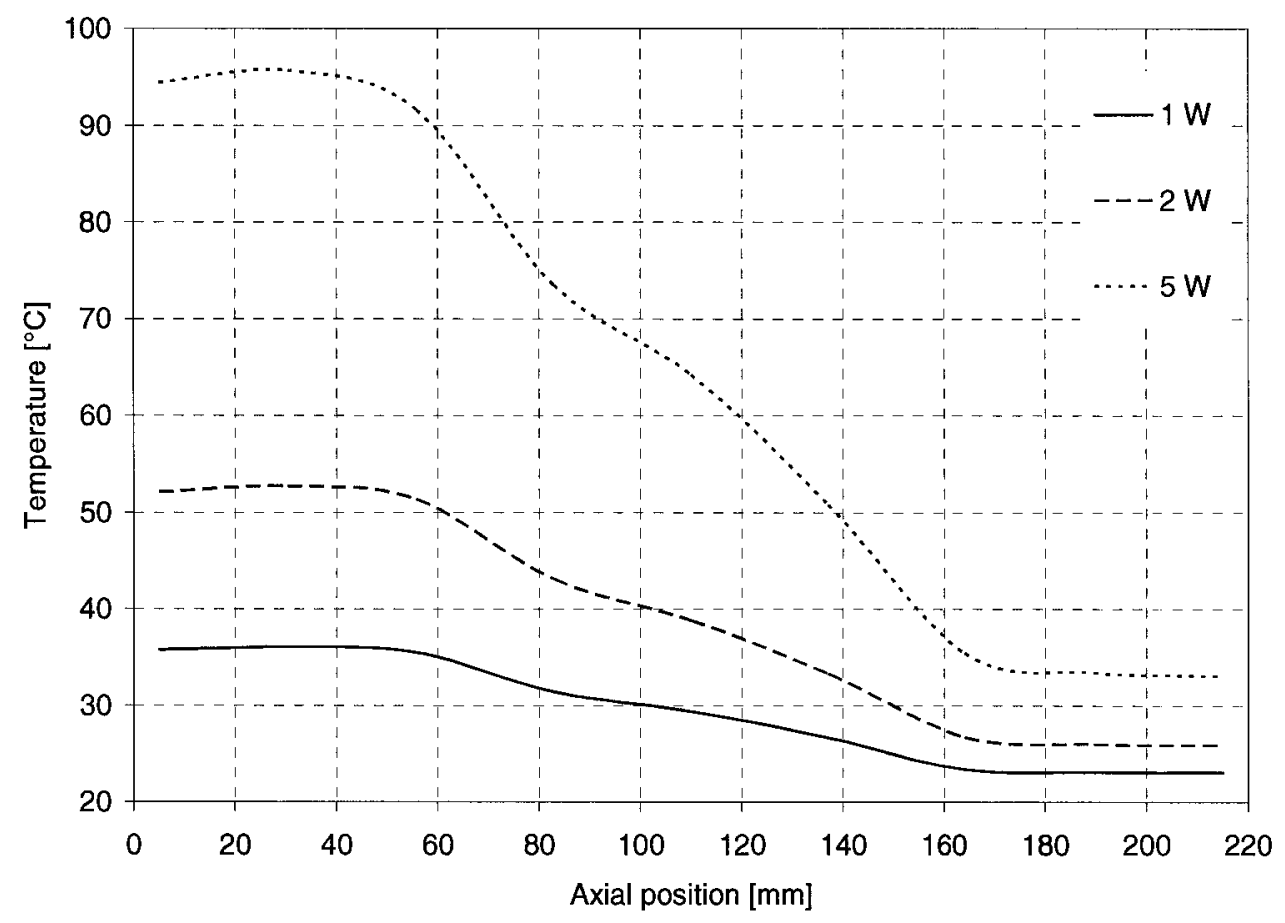

Figure 3.45 Heat pipe axial temperature profile (insulated, against gravity vertical, natural convection) [sample(s): HP071200]

Figure 3.45 displays the same heat pipe illustrated in the preceding figures. In this figure the heat pipe works in an against gravity vertical orientation with natural convection cooling on the condenser. Under such a condition, the heat load is limited to only $5 \mathrm{~W}$ for a $100^{\circ} \mathrm{C}$ temperature constraint.

It can be seen that the evaporator temperatures are higher than its horizontal and gravity assisted vertical counterparts with the increasing power input. The evaporationcondensation temperature differences are higher at the corresponding power level. The 
temperature curves have two flats at either end, but almost no adiabatic flats. This is due to the vertical orientation of against gravity. With such an orientation, the gravity offsets the capillary force, thus the water stagnates in the condenser. For instance, at $25^{\circ} \mathrm{C}$, for the heat pipe with 100 mesh copper wire screen and water charged, in an against gravity vertical orientation, the capillary force is $0.0059 \mathrm{~kg} / \mathrm{cm}^{2}$; the gravity with regard to a nominal effective length of $17 \mathrm{~cm}$ is $0.017 \mathrm{~kg} / \mathrm{cm}^{2}$. From this example it can be seen that the gravity is almost three times greater than the capillary force. This would halt the working fluid to reach the evaporator on the top of the heat pipe, thus ceasing heat pipe function.

Unlike the heat loading factors, cooling on the condenser affects the heat pipe axial temperature profile in a different way. The following figures illustrate such characteristics.

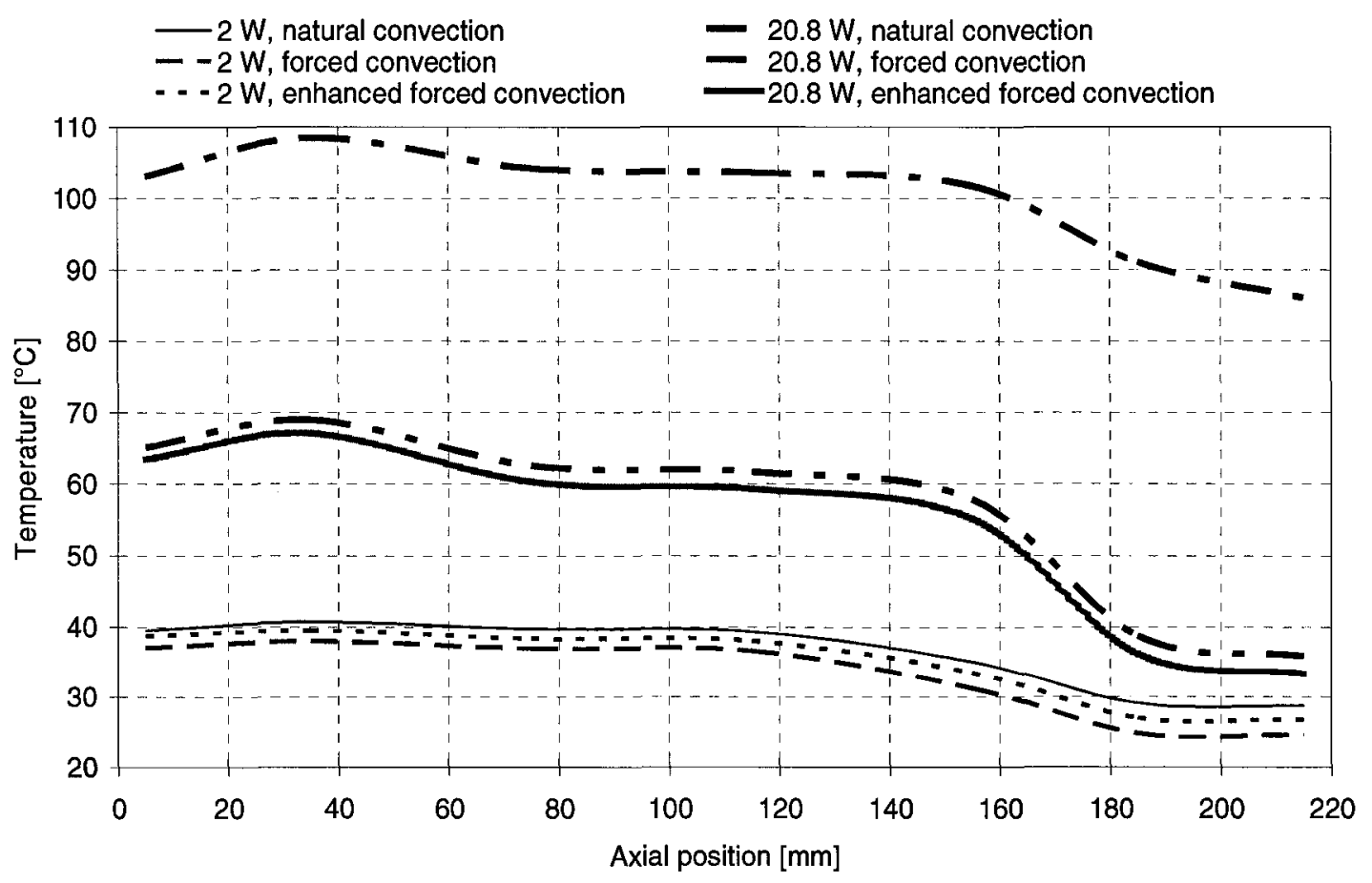

Figure 3.46 Axial temperature profile comparison regarding cooling approach (insulated, horizontal) [sample(s): HP 052502] 
Figure 3.46 displays axial temperature profile curves for the heat pipe with 100 mesh copper wire screen and over charged water. The heat pipe is in the horizontal orientation, and is tested at different cooling approaches; natural, forced, and enhanced convections. From the figure we can see there are three curves located very close in the low temperature area. They are $2 \mathrm{~W}$ with natural, forced, and enhanced convection cooling approaches. Compared with natural convection cooling, the normally and enhanced forced convection cooling are located very close at the media temperature with power input of $20 \mathrm{~W}$. One of the curves is located at the high temperature. These curves show that at low power levels, the heat pipe is not sensitive to cooling methods. At high power levels, the heat pipe shows obvious sensitiveness to the cooling approaches.

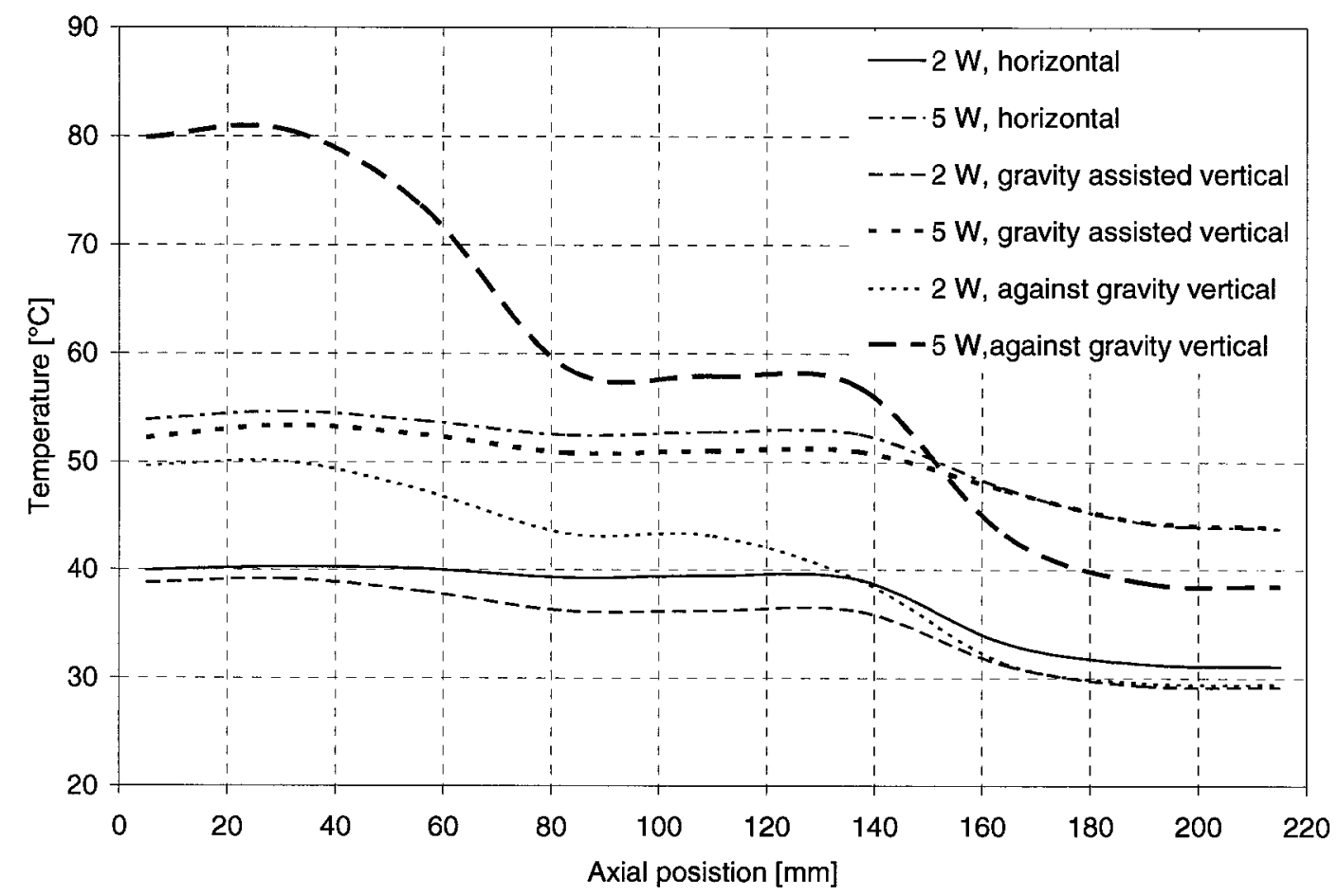

Figure 3.47 Axial temperature profile comparison regarding orientation (insulated, natural convection) [sample(s): HP 051701]

Figure 3.47 displays the axial temperature profile of the heat pipe with 100 mesh copper wire screen with normally charged water with respect to the orientations. From 
this figure we can see the four curves, horizontal, and gravity assisted vertical, move in parallel, and take a very small temperature gap of 1 or $2^{\circ} \mathrm{C}$ for both power level of 2 and $5 \mathrm{~W}$. Gravity assist brings a very small positive impact. However, the other two curves, against gravity vertical, are distinct from the others. They move not in parallel neither amongst themselves nor to others. At a certain power level, they have a higher evaporator temperature, but a lower condenser temperature, as well as a shorter adiabatic flat, compared to the others.

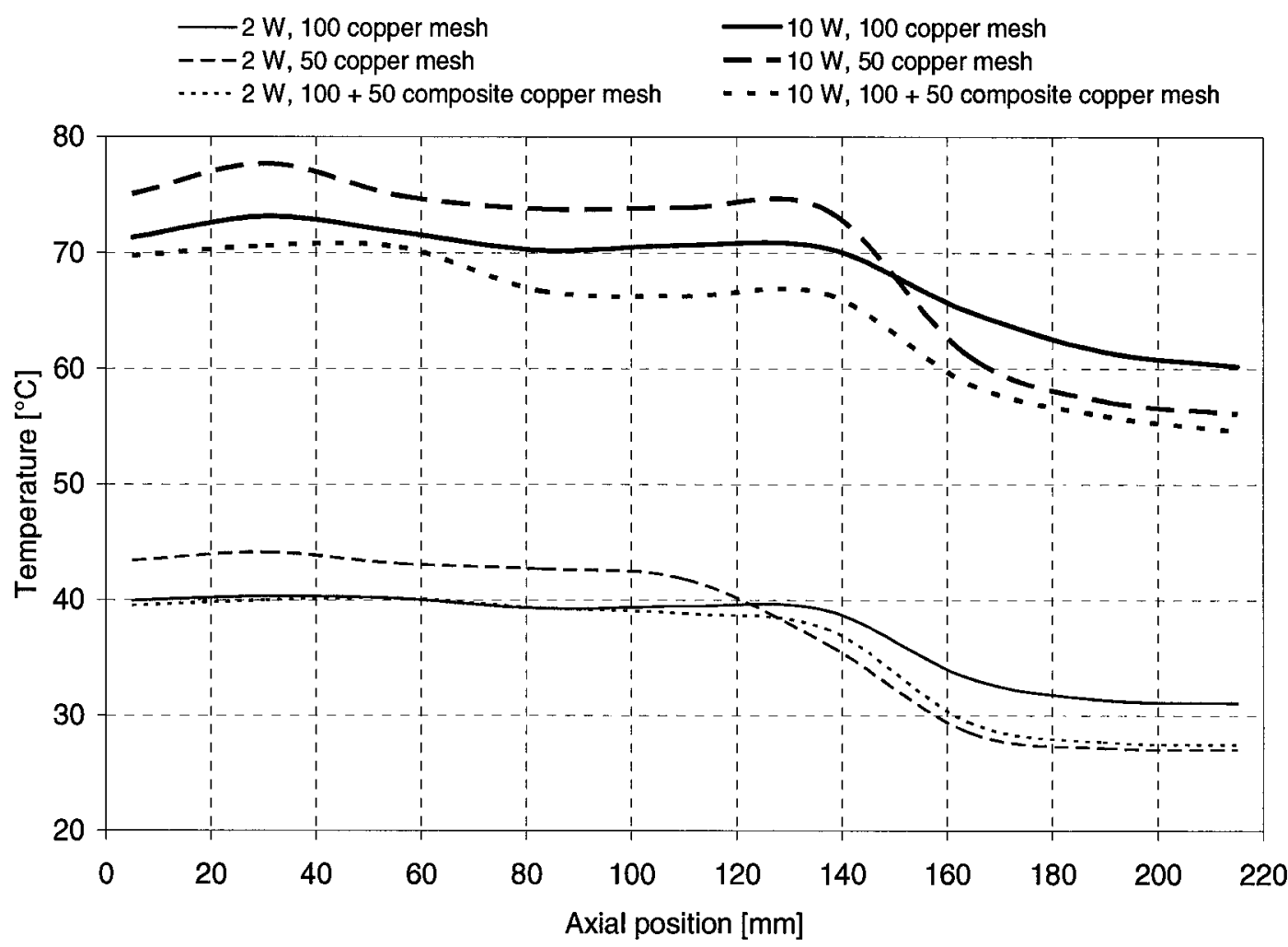

Figure 3.48 Axial temperature profile regarding wick structure (insulated, horizontal, natural convection) [sample(s): HP051701, HP071200, HP071301] 


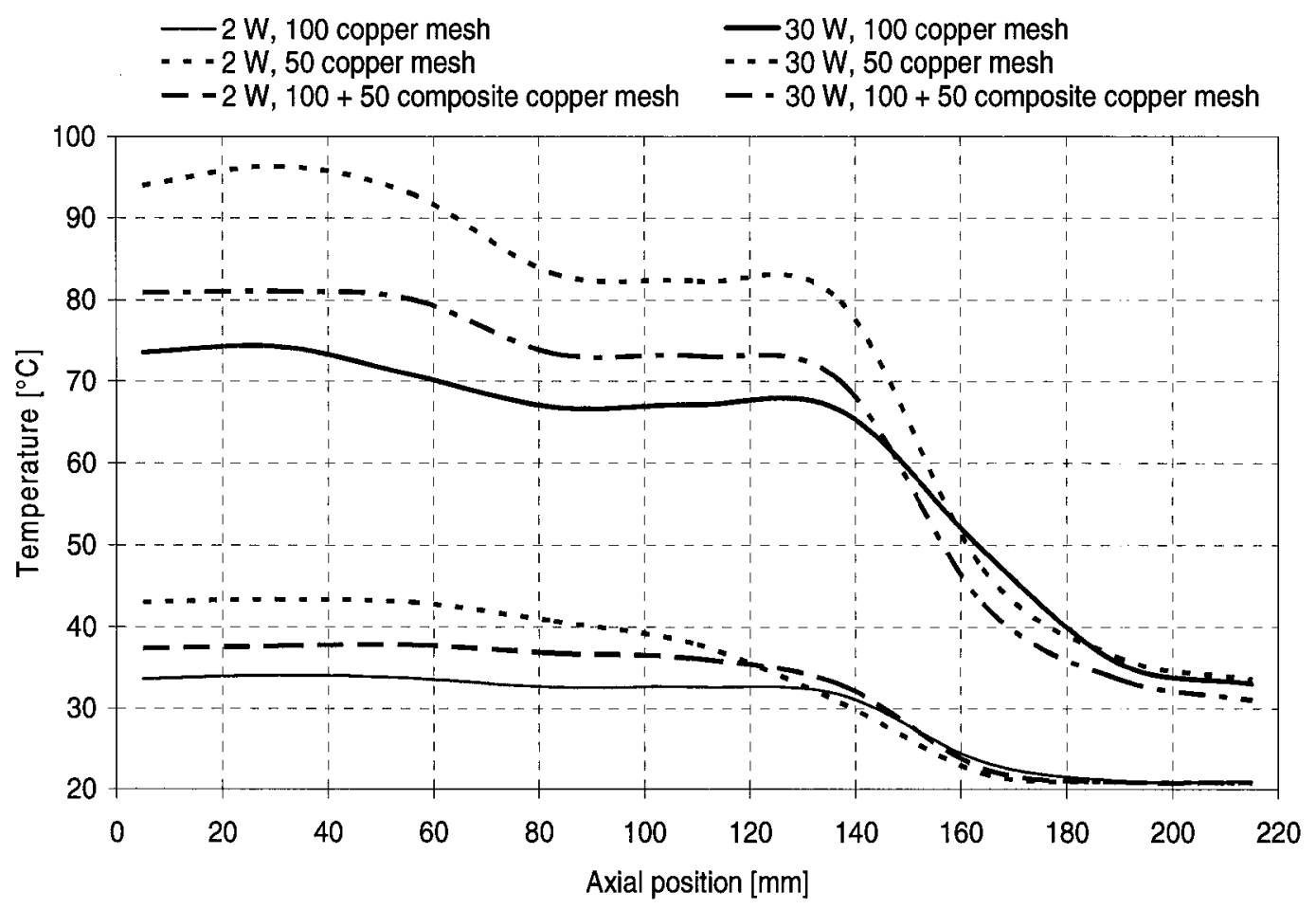

Figure 3.49 Axial temperature profile regarding wick structure (insulated, horizontal, enhanced forced convection) [sample(s): HP051701, HP071200, HP071301]

Figures 3.48 and 3.49 , illustrate the heat pipe axial temperature profile for various wick structures with horizontal orientation, and natural, enhanced convections respectively. The testing heat pipes are 100 (fine), 50 (coarse), and 100-50 composite meshes. From these two figures we can see that with natural convection cooling, the fine mesh heat pipe performs the best under all circumstances. The composite mesh performs the second best, and the coarse mesh sample performs the worst. However in the enhanced forced convection case, the fine and composite mesh test samples exchange positions and the coarse mesh test sample still performs the worst.

As is known, a fine mesh wick provides better capillary force but offset in the permeability; a coarse mesh wick has better permeability but pays a penalty in capillary force. To deal with this dilemma, a composite mesh gives an intermediate solution. The following figures illustrate results for the vertical cases. 


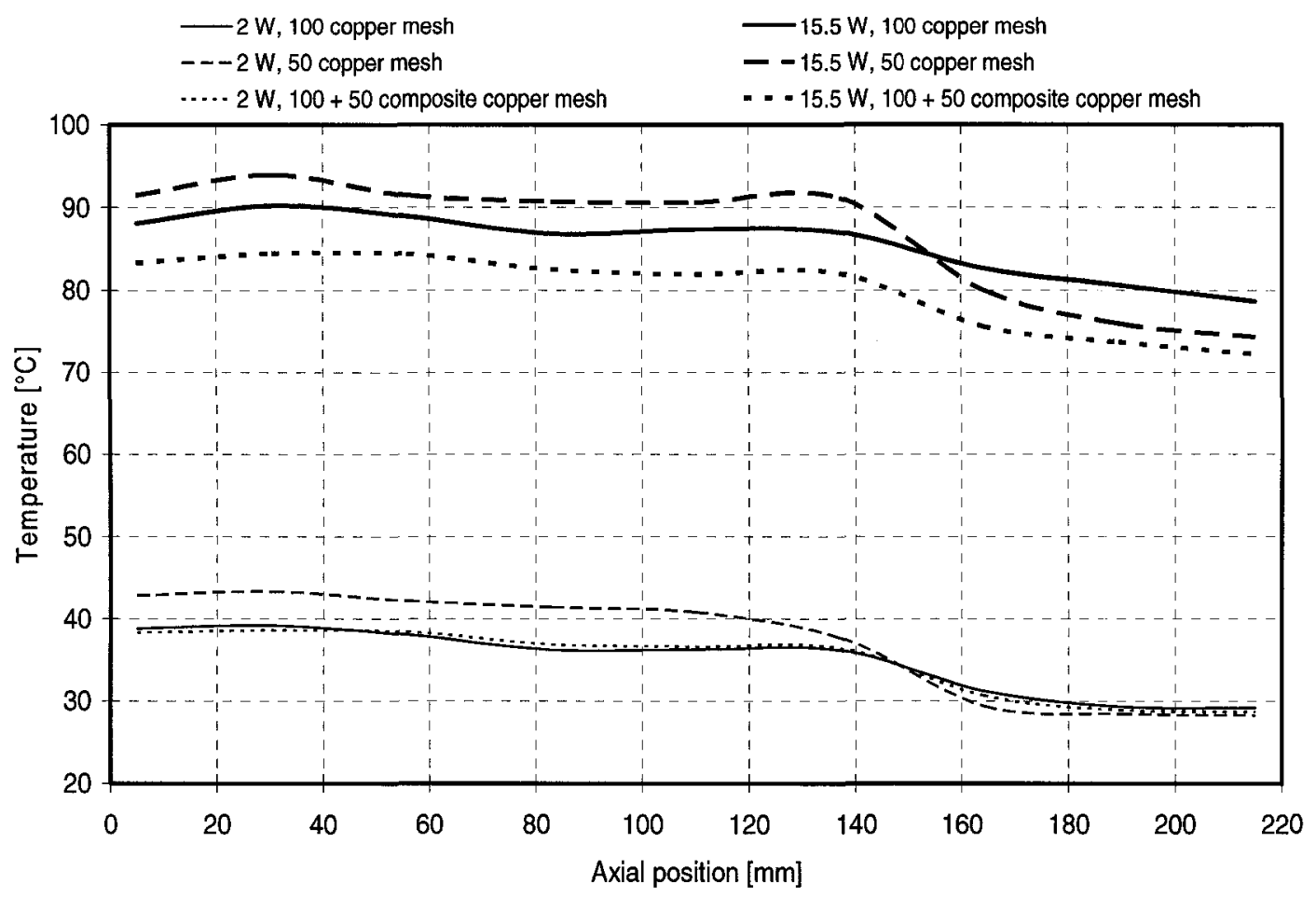

Figure 3.50 Axial temperature profile regarding wick structure (insulated, gravity assisted vertical, natural convection) [sample(s): HP051701, HP071200, HP071301]

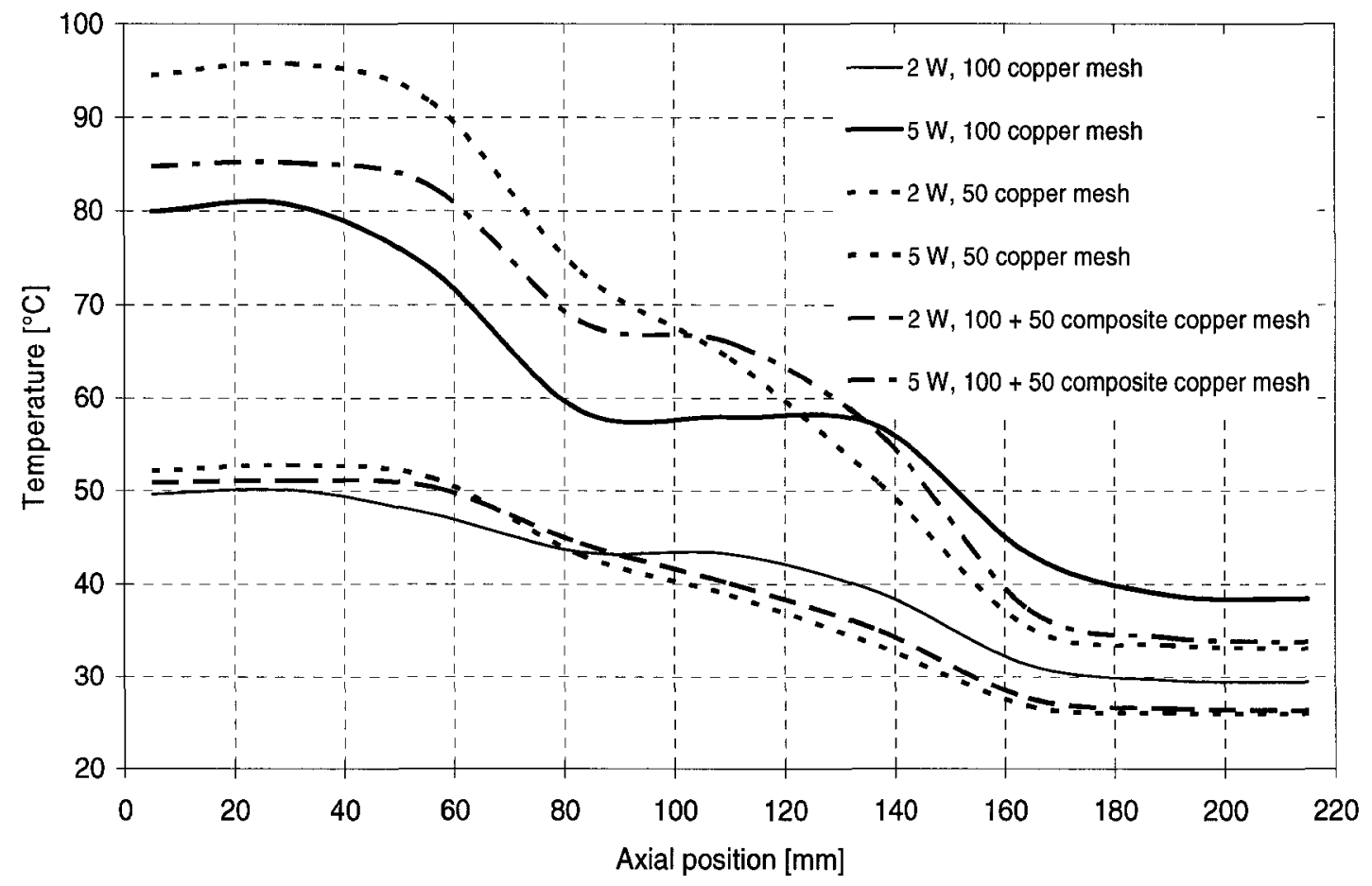

Figure 3.51 Axial temperature profile regarding wick structure (insulated, against gravity vertical, natural convection) [sample(s): HP051701, HP071200, HP071301] 
Figures 3.50 and 3.51 illustrate the three wick structure samples with natural convection at gravity assisted and against gravity vertical orientations respectively. In the gravity assisted case shown in Figure 3.50, the composite mesh sample shows a better performance than the fine mesh one at high heat load level. The coarse mesh sample still keeps the worst record at either low or high power levels. While in the against gravity case shown in Figure 3.51, the fine mesh sample takes over the best position again at either low or high power levels.

It can be seen that, from the wick structure related axial temperature profile comparison, there are some uncertainties between the fine and composite mesh test samples under circumstances. Many reasons should be responsible for this. A major concern is believed due to the fact that in the fabrication process, the two meshes are difficult to firmly and evenly combine, thus causing gaps between them, furthermore, the gap would result in great radial thermal resistance to the entire thermal network.

Figure 3.52 and 3.53 illustrate the axial temperature profile for different working fluid conditions. The samples involved are fine mesh water and acetone heat pipes, in horizontal orientation, and with natural and enhanced convections respectively. In both cases, the water sample shows a better performance, especially in the case of enhanced convection at high power level. In Figure 3.53, the acetone sample shows a strong tendency to dry out. Due to smaller latent heat comparing with water and lower surface tension. In a heat pipe, acetone is easily to rise in temperature, and is difficult to turn back to the evaporator. The same reason also leads to the similarities shown in Figures 3.54 and 3.55 . 


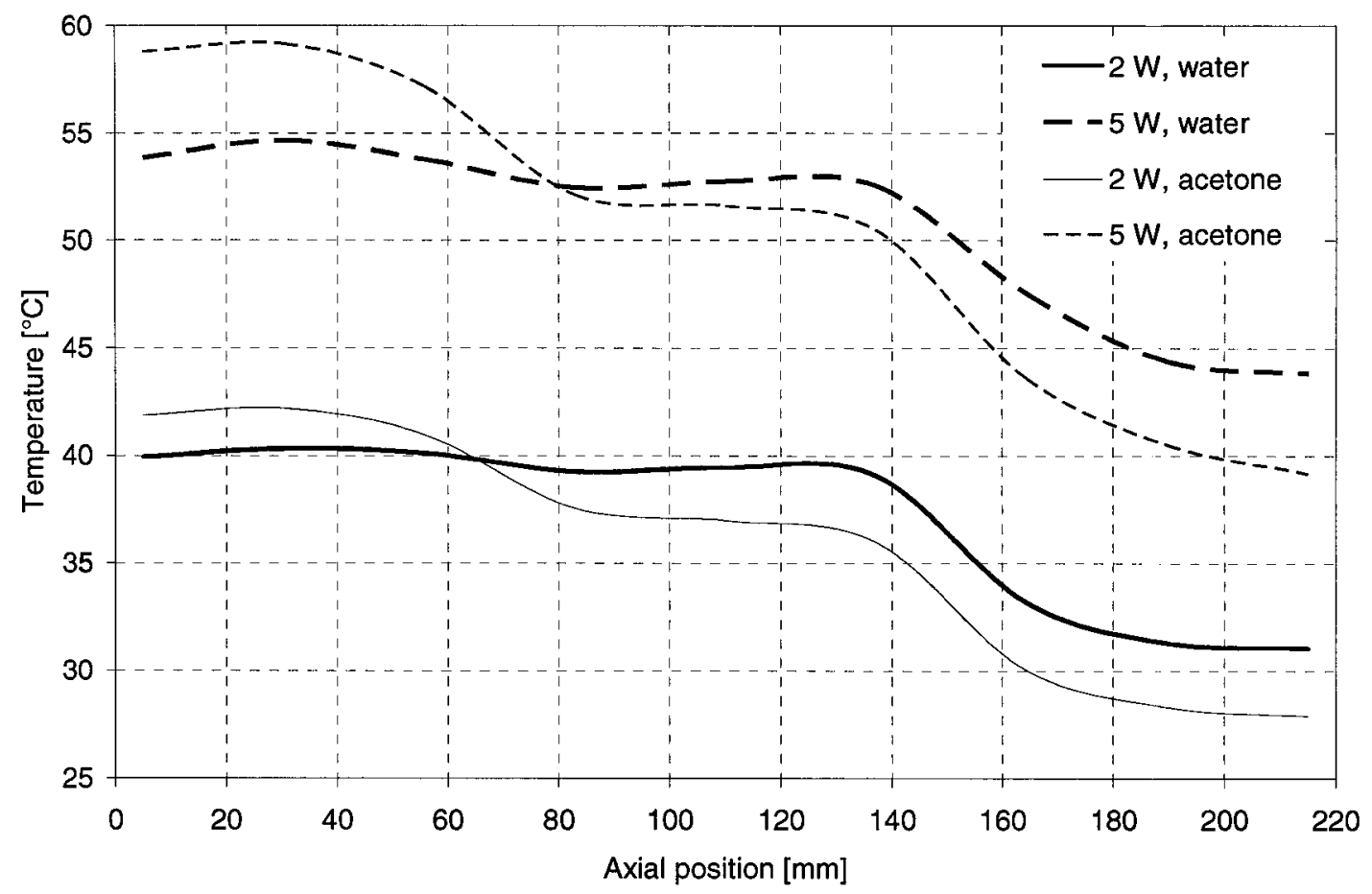

Figure 3.52 Axial temperature profile regarding working fluid (insulated, horizontal, natural convection) [sample(s): HP051701, HP071402]

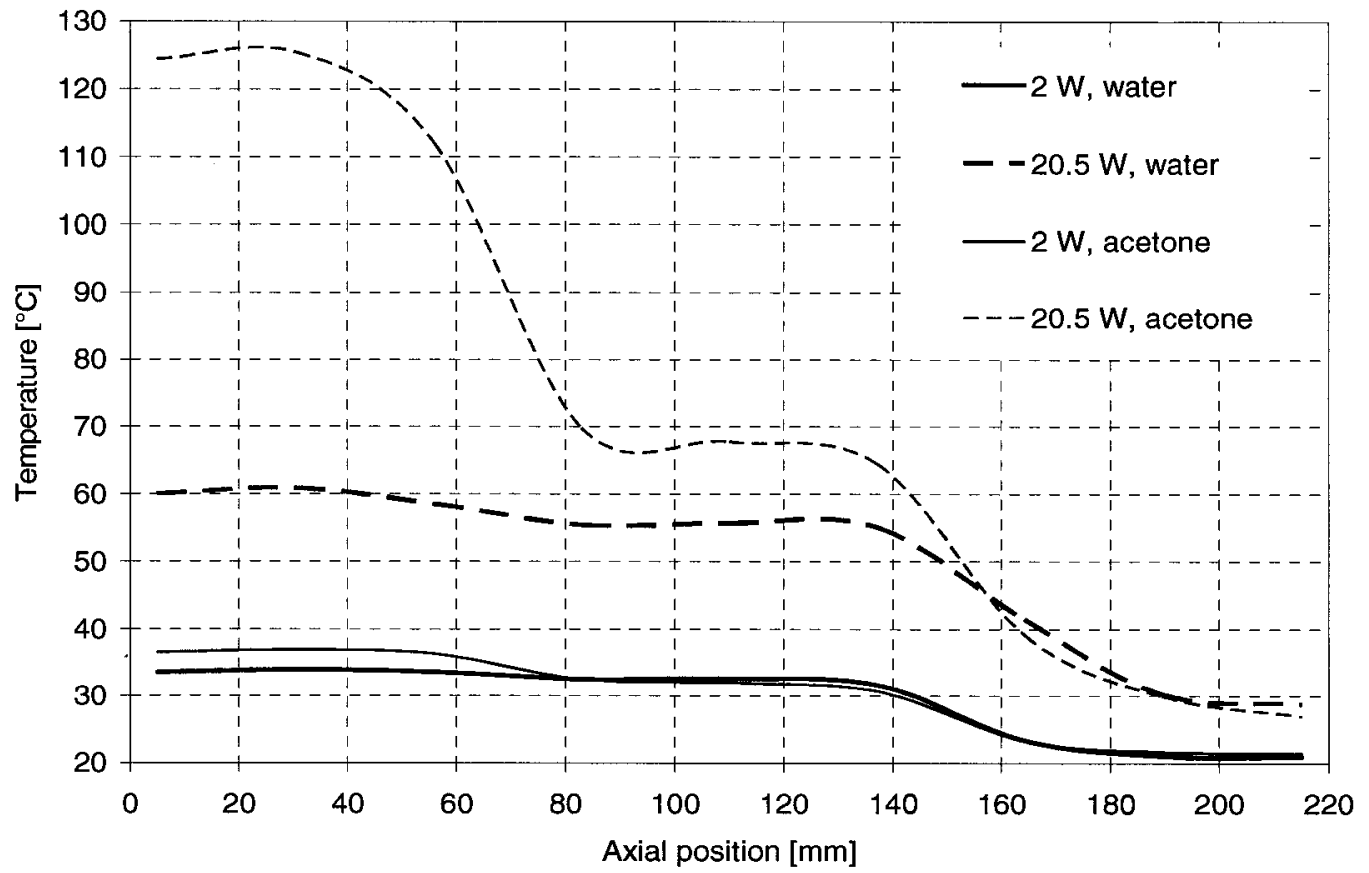

Figure 3.53 Axial temperature profile regarding working fluid (insulated, horizon, enhanced forced convection) [sample(s): HP051701, HP071402] 


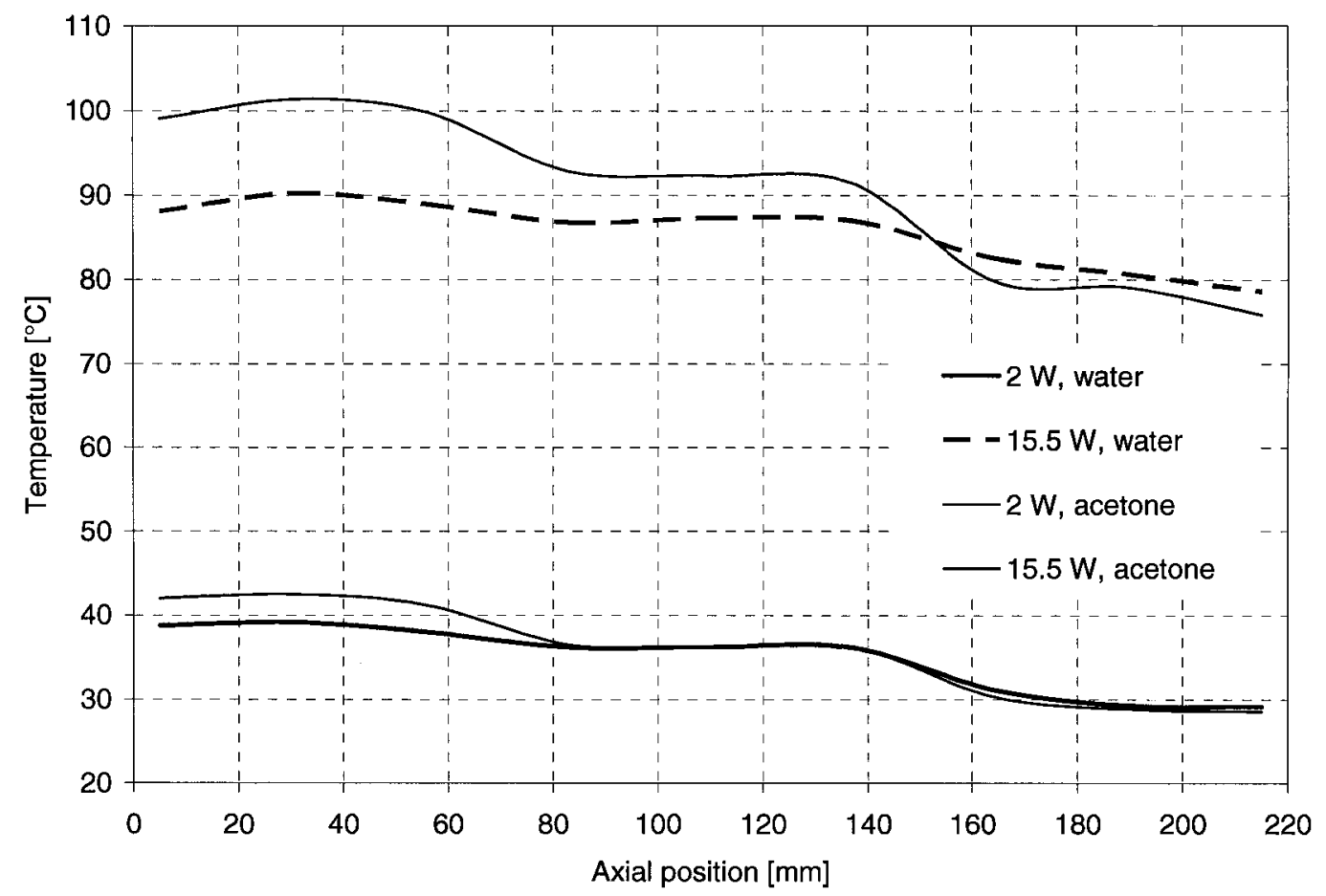

Figure 3.54 Axial temperature profile regarding working fluid (insulated, gravity assisted vertical, natural convection) [sample(s): HP051701, HP071402]

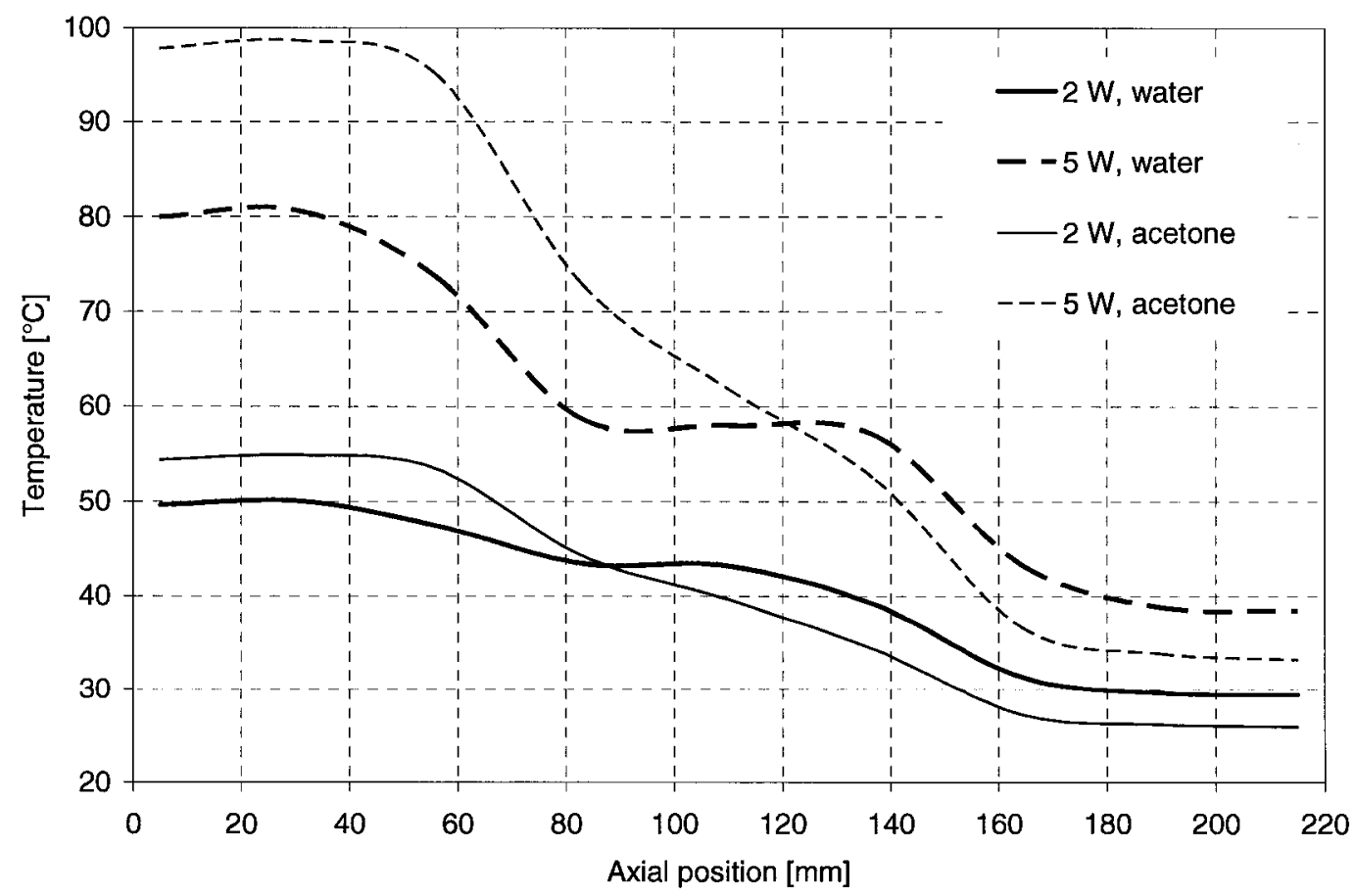

Figure 3.55 Axial temperature profile regarding working fluid (insulated, against gravity vertical, natural convection) [sample(s): HP051701, HP071402] 


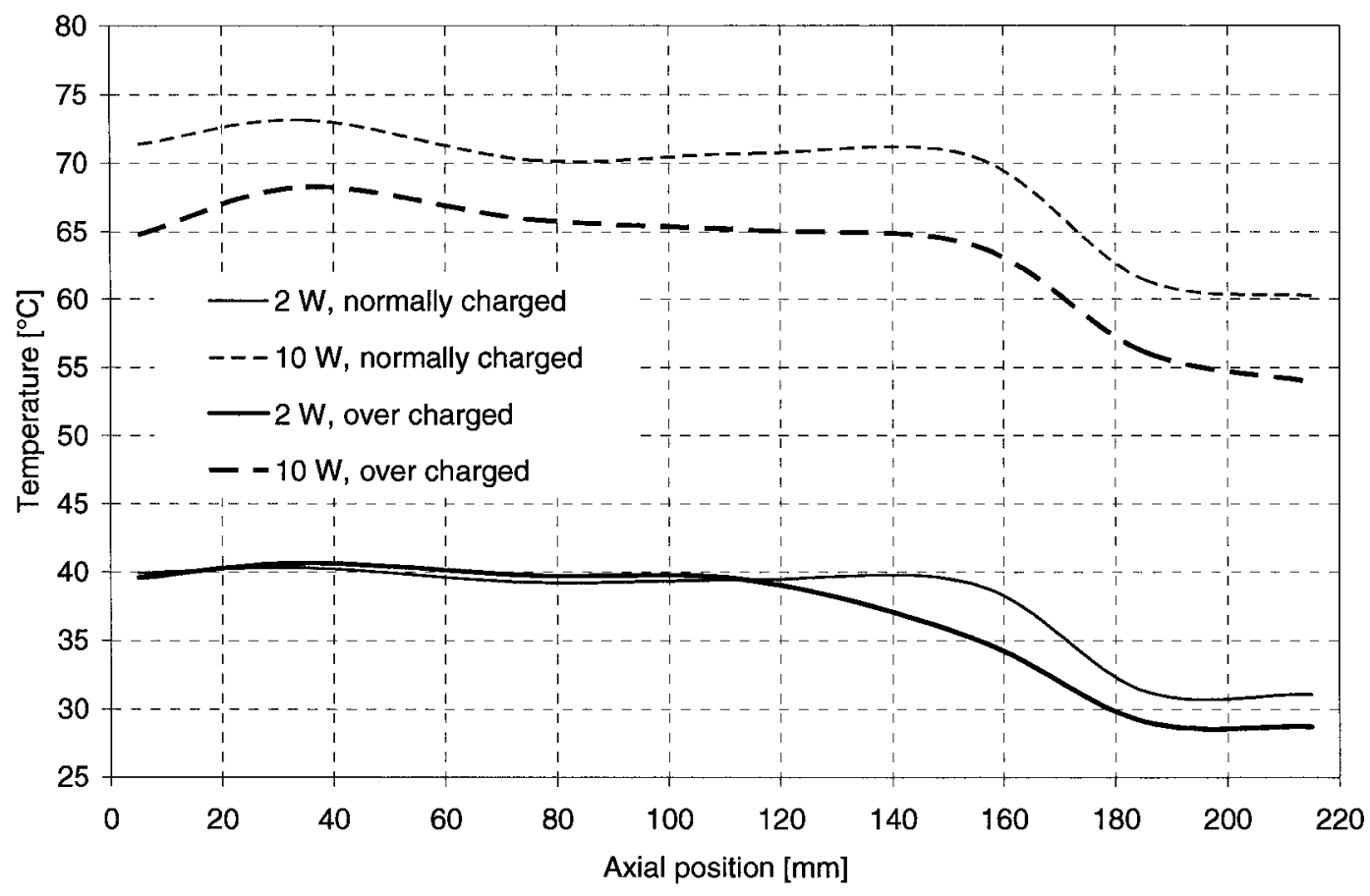

Figure 3.56 Axial temperature profile regarding amount of working fluid (insulated, horizontal, natural convection) [sample(s): HP051701, HP052502]

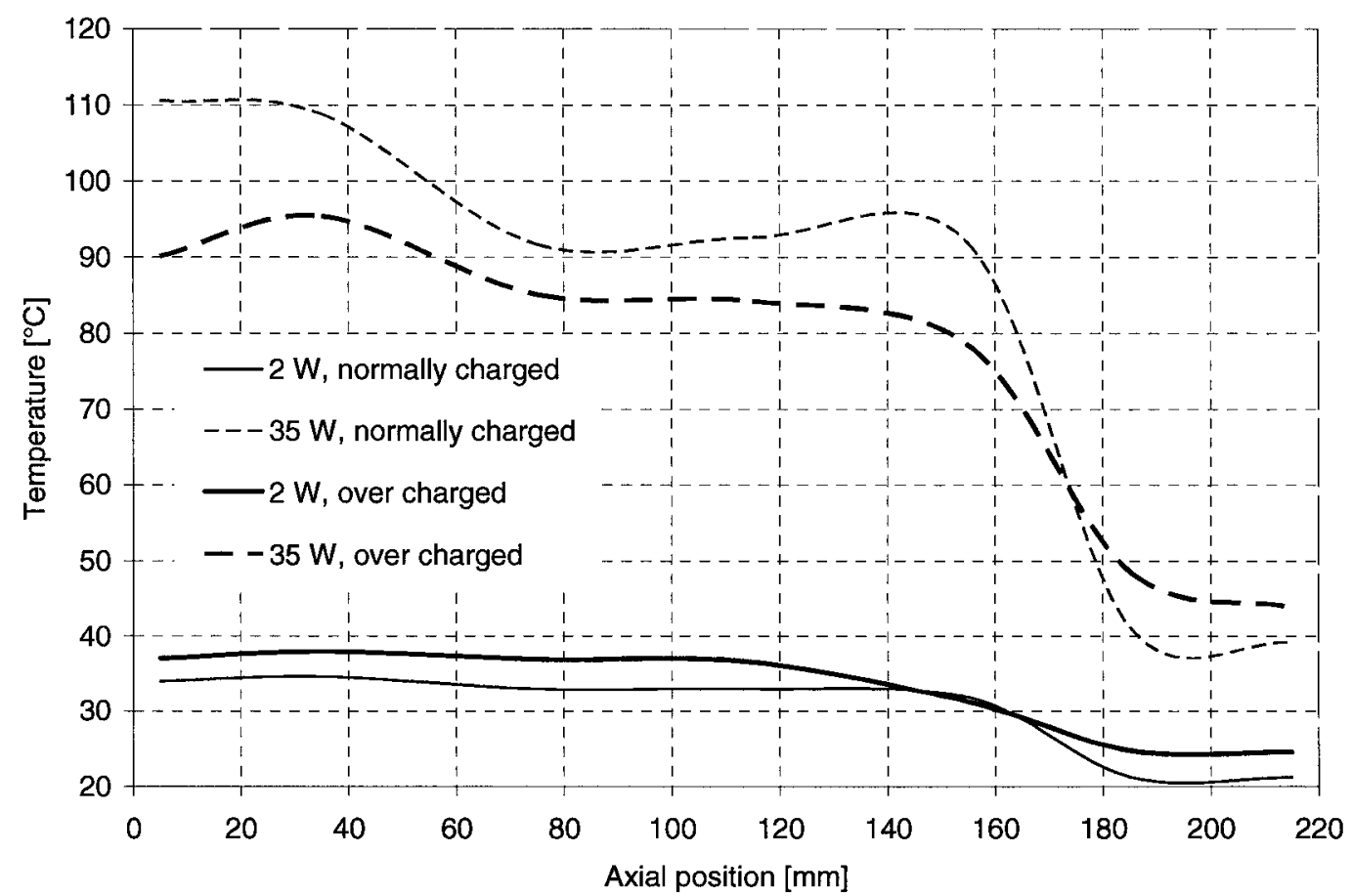

Figure 3.57 Axial temperature profile regarding amount of working fluid (insulated, horizontal, forced convection) [sample(s): HP051701, HP052502] 


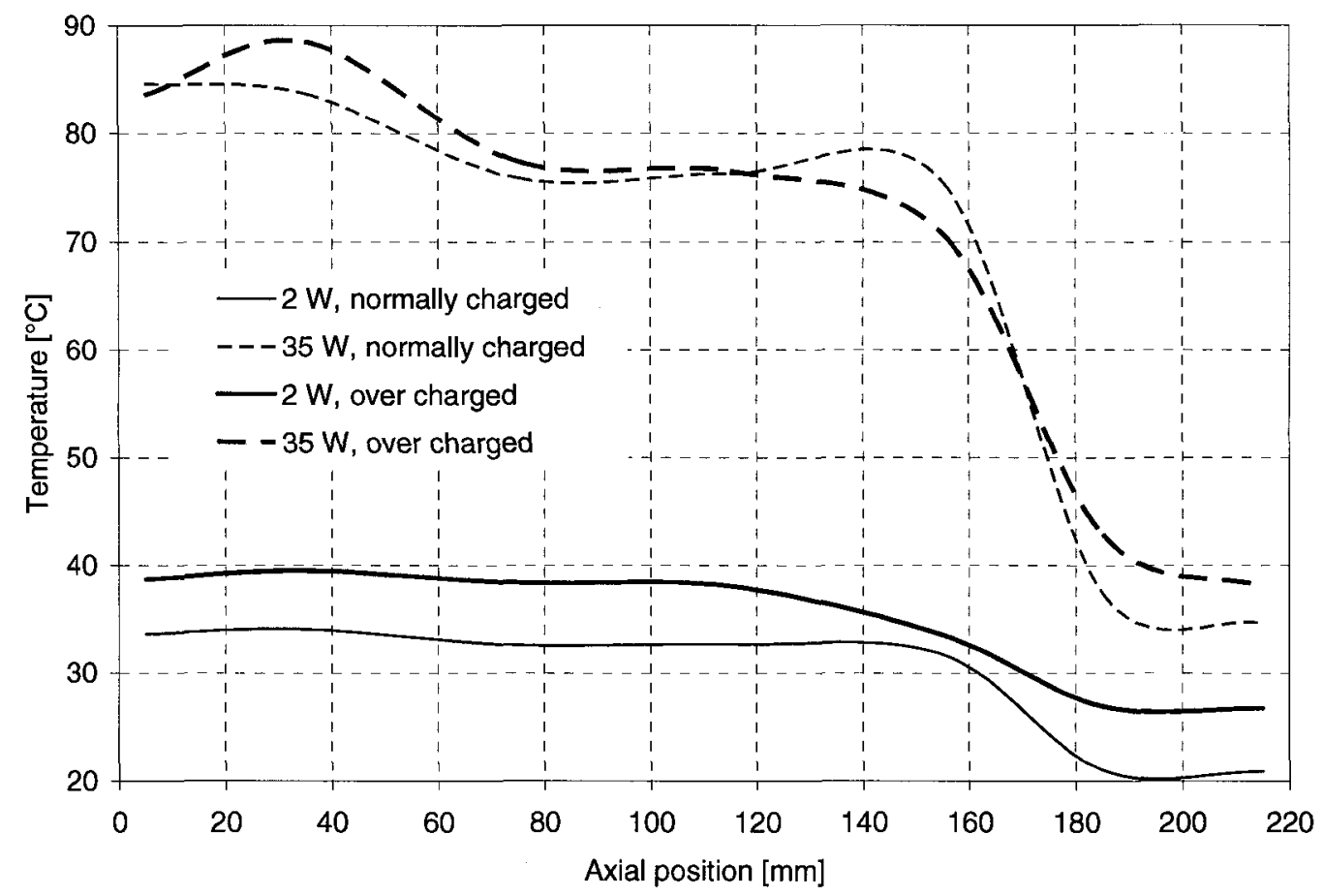

Figure 3.58 Axial temperature profile regarding amount of working fluid (insulated, horizon, enhanced forced convection) [sample(s): HP051701, HP052502]

Figures 3.56 to 3.58 illustrate the axial temperature profile when varying the amount of working fluid charged. The test conditions are at horizontal orientation with natural, forced and enhanced forced convections respectively. The testing samples are the heat pipes with 100 (fine) mesh copper wire screen wick structures and normally charged (109\%) as well as over charged (158\%) water.

These figures show that in all cases the normally charged sample performs better at low power levels, but at higher power levels, the over charged sample performs better. This is especially apparent in the condition of forced convection.

These results are consistent with prediction and the explanation that at a lower power level less water case is easier to evaporate, while at high power levels more water is helpful to maintain the wick saturation. Thus it ensures the phase-change circulation in the heat pipe. 


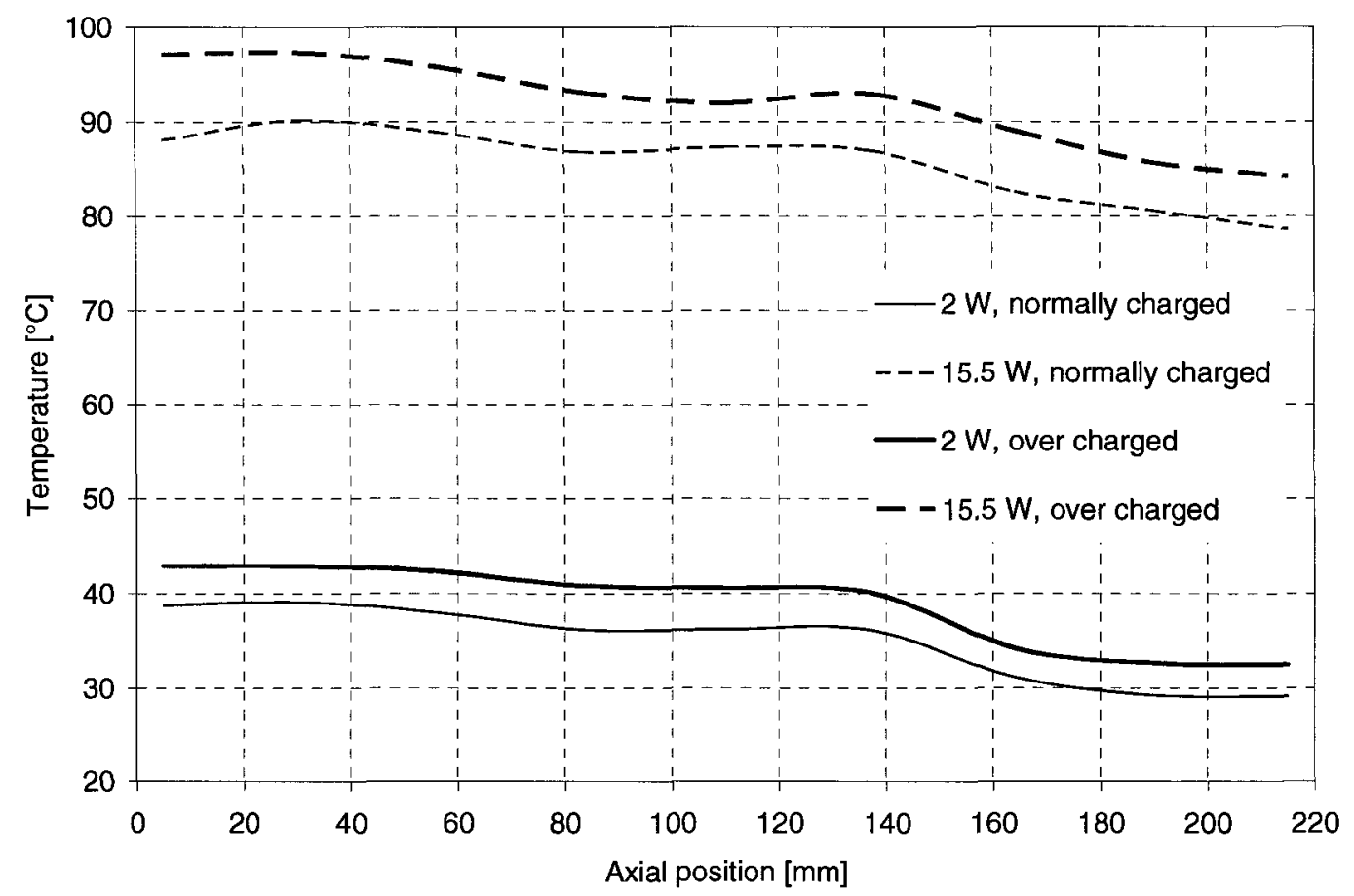

Figure 3.59 Axial temperature profile regarding amount of working fluid (insulated, gravity assisted vertical, natural convection) [sample(s): HP051701, HP052502]

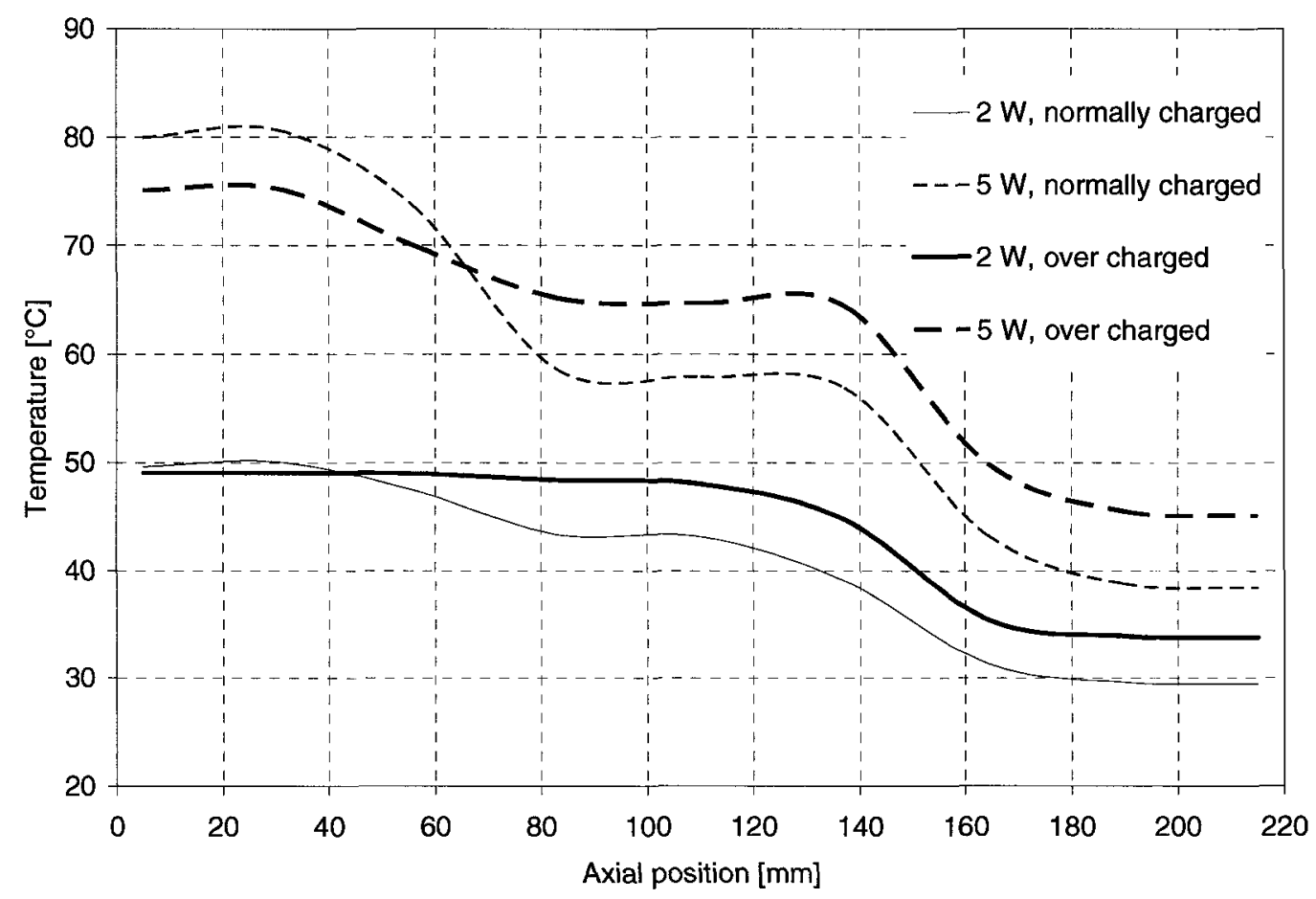

Figure 3.60 Axial temperature profile regarding amount of working fluid (insulated, against gravity vertical, natural convection) [sample(s): HP051701, HP052502] 
Figures 3.59 and 3.60 illustrate the axial temperature profile when varying the amount of working fluid charged in the heat pipe. The test conditions are natural convection at gravity assisted and against gravity orientations, respectively. The testing samples are the same as in preceding cases. Comparing these figures, and reviewing the horizontal natural convection case, we can see that in the gravity assisted case, the over charged sample performs worse then the normally charged sample at both power levels. However, in the case of against gravity with natural convection, the over charged sample does better then the other one at both power levels. It maintains a lower evaporator temperature although with a higher condenser temperature comparing to the normally charged sample. This result is consistent with the explanation for the case of copper tube with copper mesh inserted and water charged in Experiment 2. With the gravity assisted vertical orientation, the extra water in the over charged heat pipe would prefer to stay in the evaporator area. Thus, it acts as a thermal buffer, and hinders the temperature change in the evaporator. In the opposite orientation, the extra water stays in the condenser acting as a thermal buffer to maintain a low condenser temperature, in such a way preventing the evaporator-condenser temperature difference from rising. Therefore it keeps a relatively lower evaporator temperature as illustrated in Figure 3.60.

\subsubsection{Evaporator-Condenser Temperature Gradient Characteristics}

The evaporator-condenser temperature gradient is another characteristic to evaluate the heat pipe performance. This factor mainly reflects the heat pipe efficiency. A small value of this factor ensures great heat transfer efficiency. Unlike the copper tube configurations, this characteristic for the heat pipe configurations is very different under different circumstances. This section discusses this issue under different circumstances. 


\subsection{Comparison of Heat Pipe Evaporator-condenser Temperature Gradient}

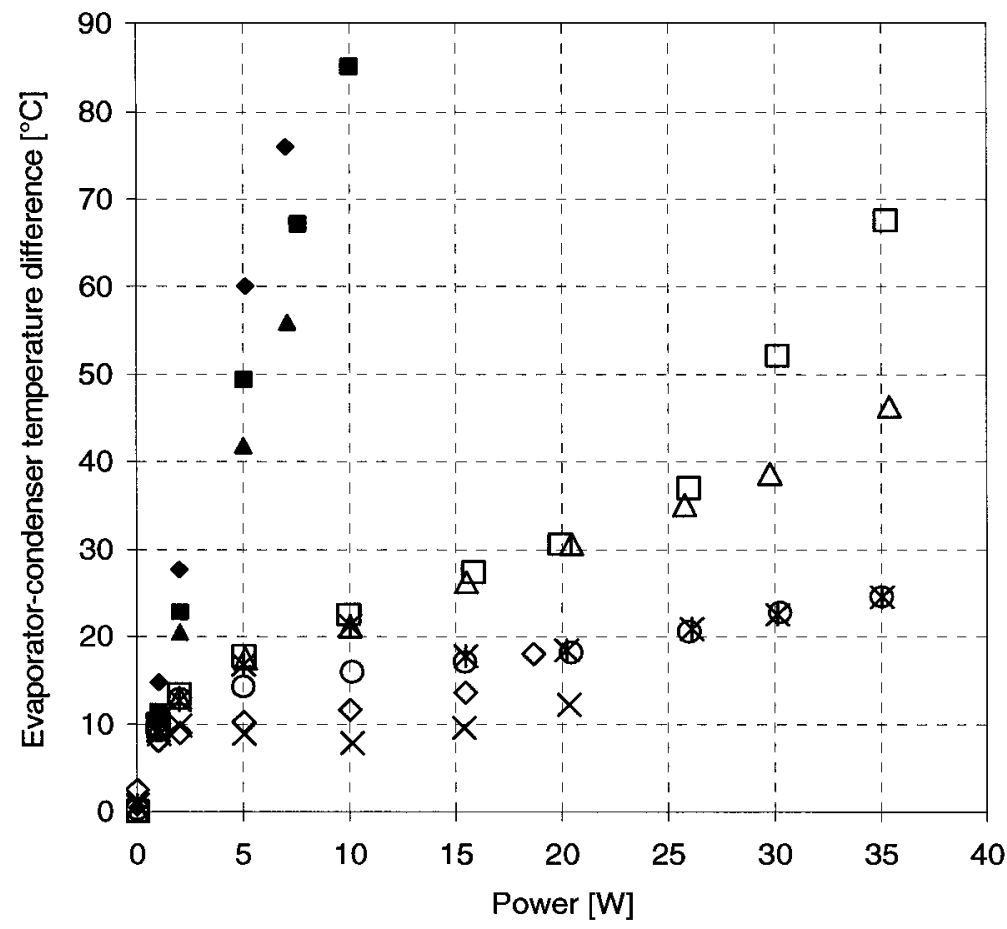

$\diamond$ Horizontal, natural convection

DHorizontal, forced convection

$\Delta$ Horizontal, enhanced forced convection

$X$ Gravity assisted vertical natural convection

* Gravity assisted vertical, forced convection

O Gravity assisted vertical, enhanced forced convection

$\Delta$ Against gravity vertical, natural convection

- Against gravity vertical, forced convection

Against gravity vertical enhanced forced convection

Figure 3.61 Comparison of evaporator-condenser temperature difference [sample: HP051701]

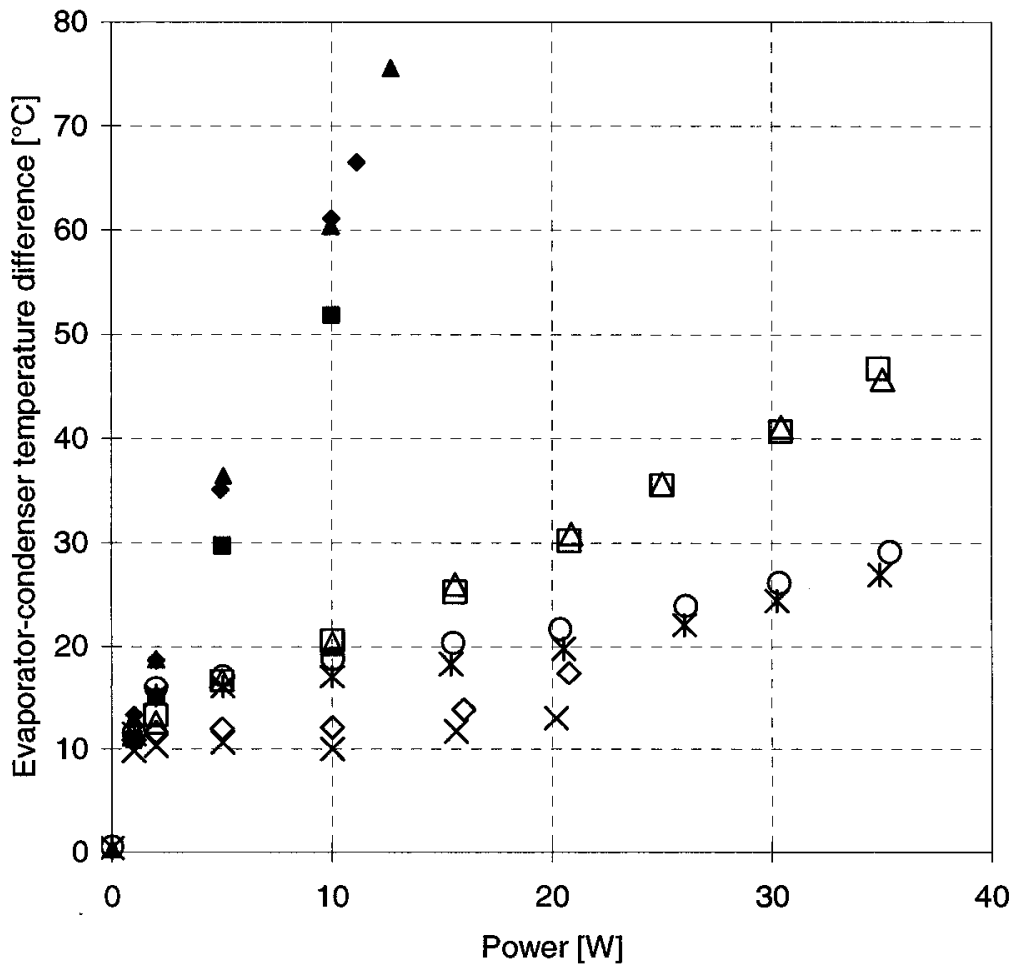

$\diamond$ Horizontal, natural convection

口Horizontal, forced convection

$\Delta$ Horizontal, enhanced forced convection

$\times$ Gravity assisted vertical natural convection

* Gravity assisted vertical, forced convection

O Gravity assisted vertical, enhanced forced convection

Against gravity vertical natural convection

- Against gravity vertical, forced convection

$\Delta$ Against gravity vertical, enhanced forced convection

Figure 3.62 Evaporator-condenser temperature difference comparison [sample: HP052502] 
Figures 3.61 and 3.62 illustrate the evaporator-condenser temperature difference comparison under different circumstances. The testing samples are fine copper mesh with normally and over charged water heat pipes. There are nine circumstances combined from the three orientations and three cooling approaches. Based on these configurations the figure can be divided into three groups according to either three orientations or three cooling approaches. In Figures 3.61 and 3.62, the trials of heat pipe working against gravity perform the worst with any cooling approach. This is due to the penalty of capillary force as explained earlier. The trials using gravity assist perform the best, especially when natural convection cooling is utilized. This can be due to the gravitational impact on the wick capillarity improvement. The second best one is the horizontal with natural convection cooling. It should be noted that in each orientation, natural convection has the smallest evaporator-condenser temperature difference, providing a small heat transfer capacity. While, the forced and enhanced forced convections caused large temperature gradient between the evaporator and the condenser, providing stronger capacities of transferring heat. These results are consistent with Fourier's Law.

Comparing these two figures, it can be seen that the normally charged heat pipe tends to dry out in forms of the evaporator-condenser temperature difference jumping with increasing power, as shown in Figures 3.61 and 3.62. This is also consistent with the explanation made in the preceding sections on the issues of cooling capacity and axial temperature distribution. 


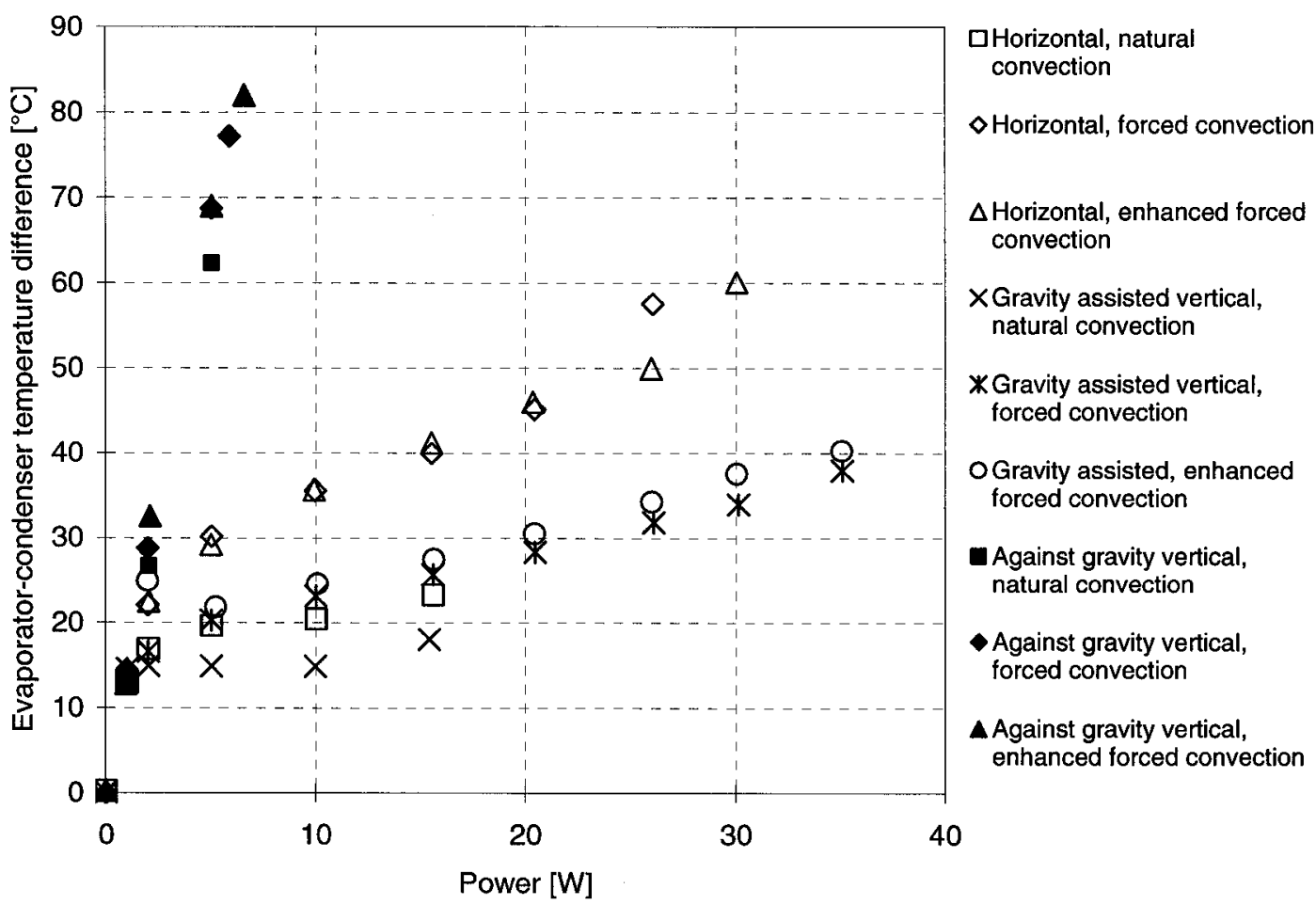

Figure 3.63 Evaporator-condenser temperature difference comparison [sample: HP071200]

Figure 3.63 illustrates the same topic with the testing sample of a heat pipe with 50 (coarse) mesh copper wire screen wick structure with normally charged water. This figure shows that this sample has a similar evaporator-condenser temperature difference tendency as the fine mesh counterparts illustrated in preceding figures. The difference is that the coarse mesh sample has about $10^{\circ} \mathrm{C}$ higher working temperature than the fine mesh counterparts.

Figure 3.64 relates to the fine copper mesh acetone sample on the same issue as in preceding figures. This figure shows that the acetone heat pipe performs between the fine mesh and coarse mesh counterparts. The exceptions are in the horizontal case with forced and enhanced forced convections. For these cases, dry-out occurred at $20 \mathrm{~W}$ in forms of the evaporator-condenser temperature difference keeps on increasing. The reason for this is explained in the section regarding the axial temperature profiles. 


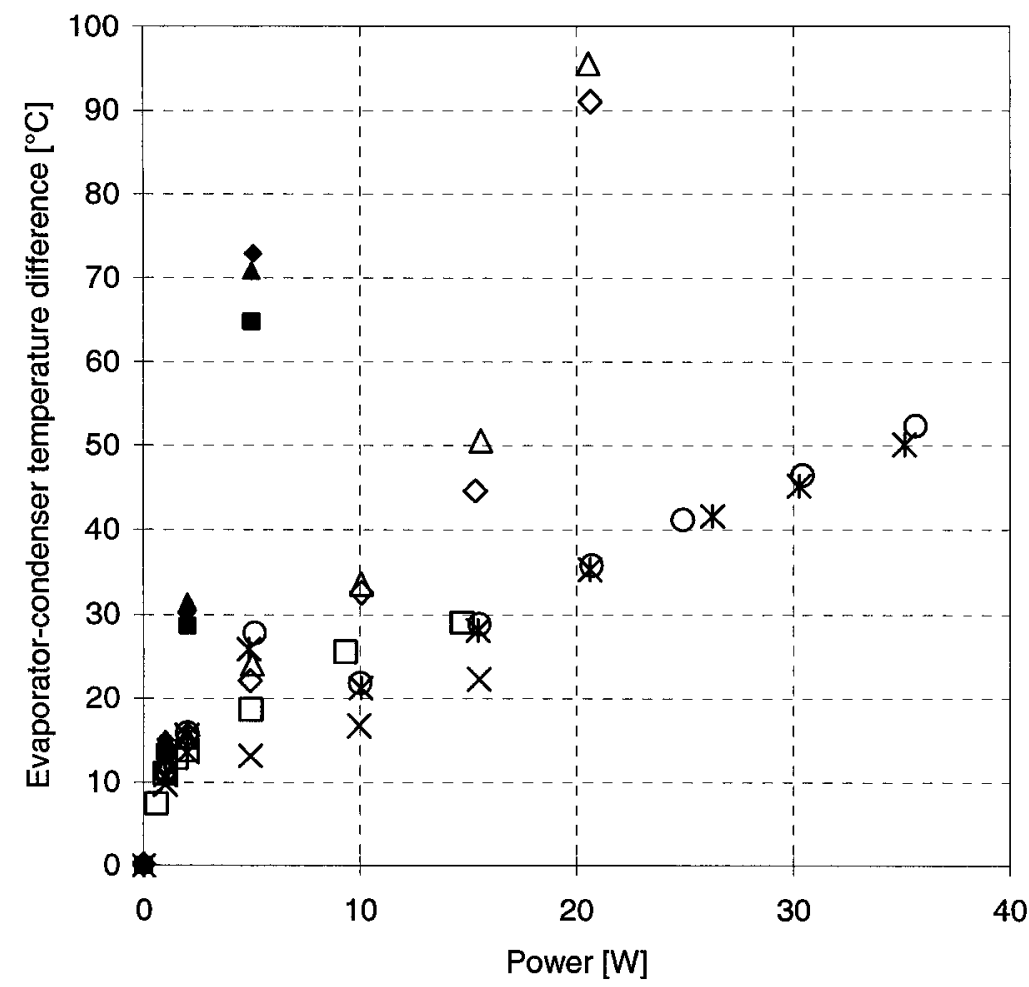

$\square$ Horizontal, natural convection

$\diamond$ Horizontal, forced convection

$\Delta$ Horizontal, enhanced forced convection

$\times$ Gravity assisted vertical, natural convection

* Gravity assisted vertical, forced convection

O Gravity assisted vertical, enhanced forced convection

Against gravity vertical, natural convection

- Against gravity vertical, forced convection

$\Delta$ Against gravity vertical enhanced forced convection

Figure 3.64 Evaporator-condenser temperature difference comparison [sample: HP071402]

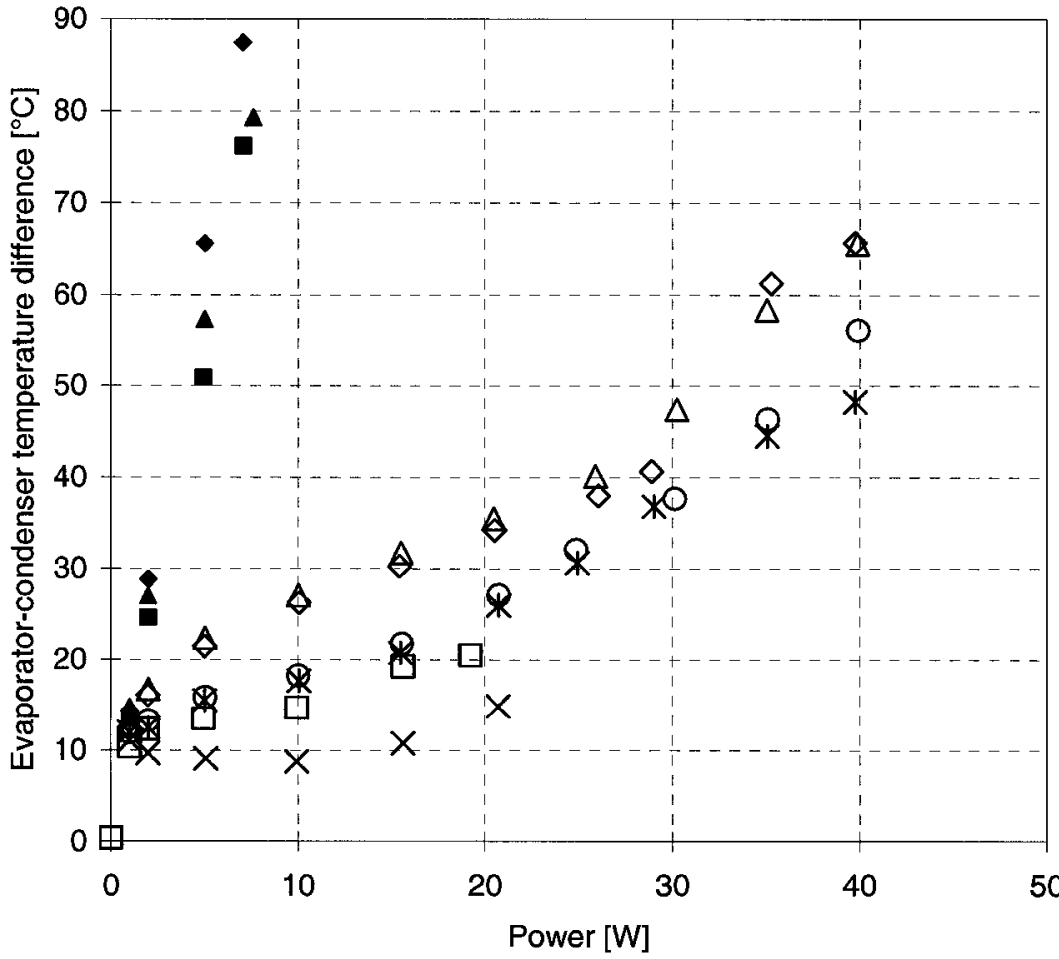

$\square$ Horizontal, natural convection

$\diamond$ Horizontal, forced convection

$\Delta$ Horizontal, enhanced forced convection

XGravity assisted vertical, natural convection

* Gravity assisted vertical, forced convection

O Gravity assisted vertical, enhanced forced convection

Against gravity vertical, natural convection

- Against gravity vertical, forced convection

$\Delta$ Against gravity vertical, enhanced forced convection

Figure 3.65 Evaporator-condenser temperature difference comparison [sample: HP071301] 
Figure 3.65 is about the composite mesh sample on the same issue. The figure shows that the composite mesh heat pipe performs no worse than others with gravity assist under all cooling approaches. It even does better at lower power levels, but worse at horizontal with forced and enhanced convections. These results are attributed to the wick better permeability but worse capillarity than the fine mesh.

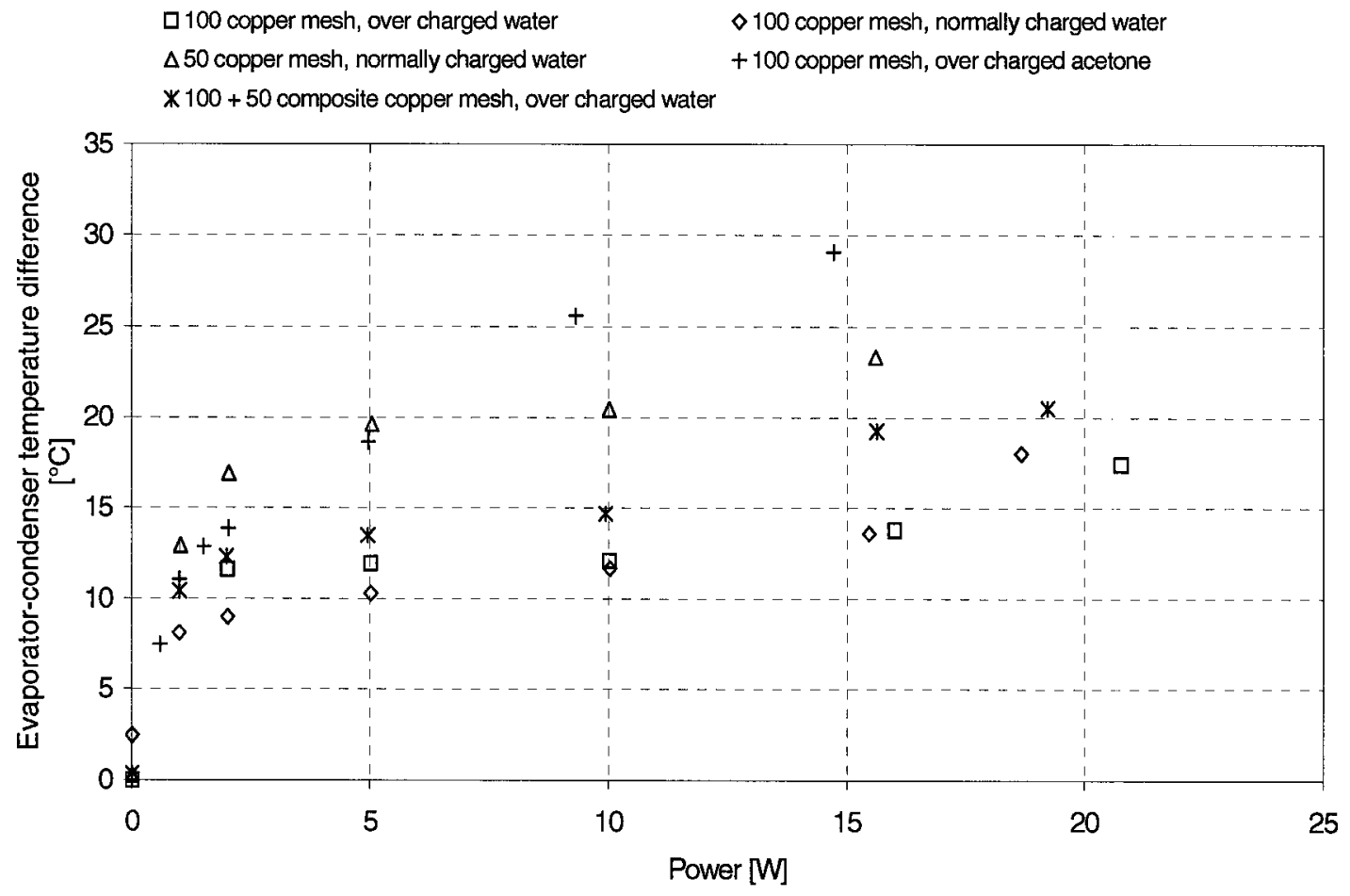

Figure 3.66 Comparison of evaporator-condenser temperature difference (insulated, horizontal, natural convection) [sample(s): HP052502, HP0051701, HP071200, HP071402, HP071301]

Figure 3.66 illustrates five test samples with respect to the issue of evaporatorcondenser temperature difference in horizontal orientation with natural convection cooling. In this figure, with increasing power, all the values increase sharply, and then turn to flat with the power level further increase. This tendency keeps on going until the power reaches a certain high value over which the evaporator-condenser temperature difference then begin to decline. Also in this figure, the samples with 100 (fine) copper mesh and water charged perform the best; while, the acetone sample performs the worst. 


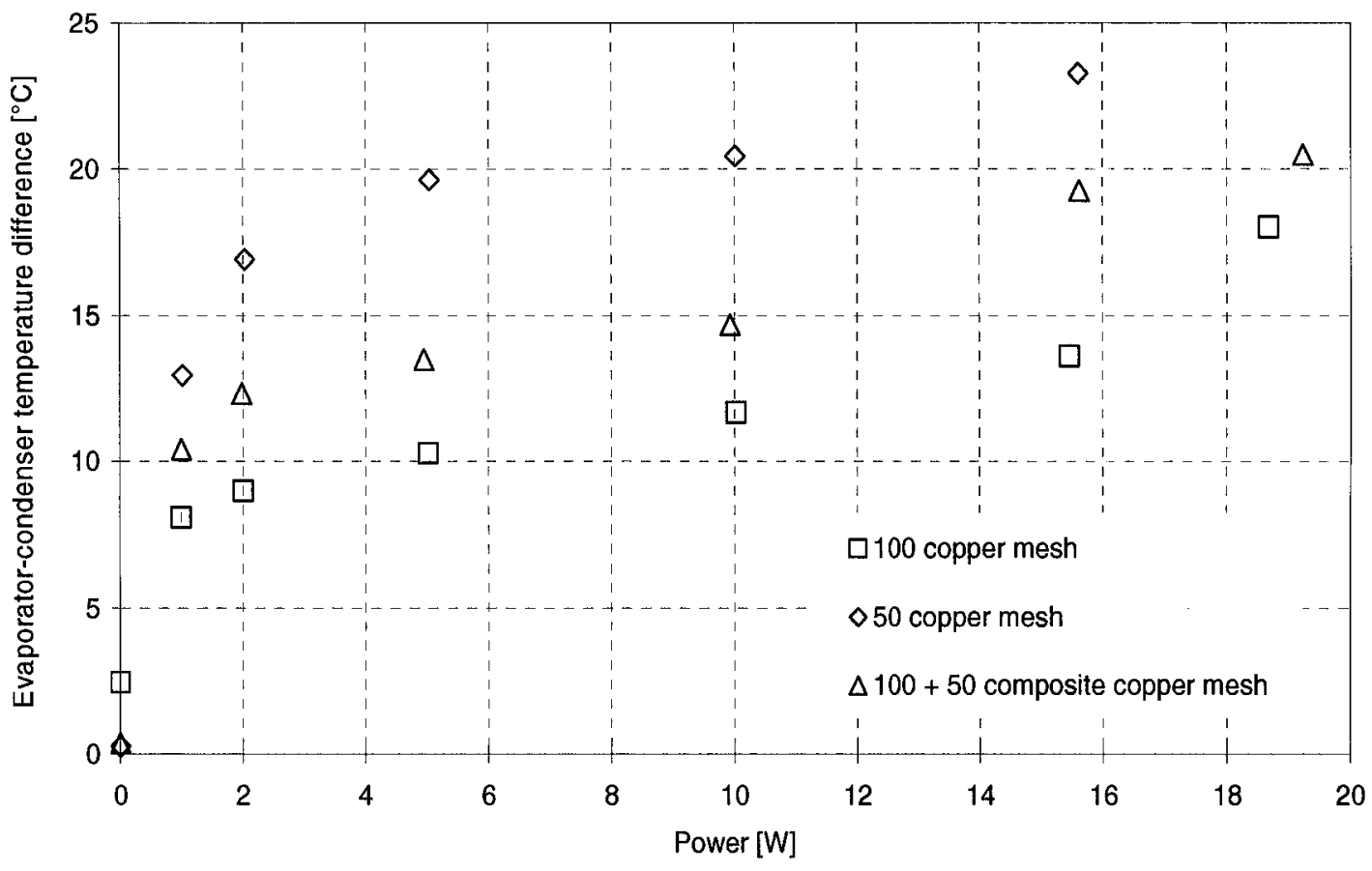

Figure 3.67 Comparison of evaporator-condenser temperature difference regarding wick mesh (insulated, horizontal, natural convection) [sample(s): HP051701, HP071200, HP071301]

Figure 3.67 is the comparison of evaporator-condenser temperature difference regarding wick structure at horizontal orientation with natural convection cooling. The testing samples are heat pipes with 100,50 and 100-50 composite meshes respectively. This figure shows that with respect to the evaporator-condenser temperature difference, among such three wicks, the heat pipe with a fine mesh has the smallest value, the coarse meshed heat pipe has the highest value, and the composite meshed heat pipe has a medium value. This result is consistent with predictions. The fine mesh has a better capillarity but worse permeability; the coarse mesh has a better permeability but a worse capillarity; while the composite mesh takes both advantages, has a better permeability than the fine mesh and a better capillarity than the coarse mesh. The factors affecting evaporator-condenser temperature difference are the wick capillarity and permeability. The capillarity ensures the phase change circulation, thus maintaining a small 
temperature change for a certain heat load. On the other hand, wick permeability mainly dominates the liquid flow status in the wick. In other words, it mainly affects the speed of the heat dissipation, i.e., heat transfer capacity. The results in Figure 3.68 exactly reflect this explanation.

Figure 3.68 illustrates the same comparison as that in Figure 3.69 in a horizontal orientation with enhanced forced convection. In this figure, all of the configurations move in a similar order as shown in the preceding figure. The difference is that with enhanced forced convection, all the values increase with an approximate constant ascending slope. With the increasing power input, the value of composite mesh sample tends to flow upwards, and the coarse mesh sample starts to jump, while the fine mesh sample still keep a relative stable state.

The enhanced forced convection provides stronger cooling capacity on the condenser. Consequently, this lowers the temperature in the condenser area. Compared to such a cooling capacity, the heat load is not enough to maintain the evaporatorcondenser temperature difference. Thus, the temperature difference values keep increasing with the heat load. On the other hand, a greater temperature difference causes a larger pressure difference between the evaporator and condenser. This requires higher wick capillarity to maintain the temperature difference. Among the three samples, the fine mesh possesses a better capillarity, thus maintaining a stable state in the evaporatorcondenser temperature difference. The other two samples are not able to maintain a stable state and tend to decline. 


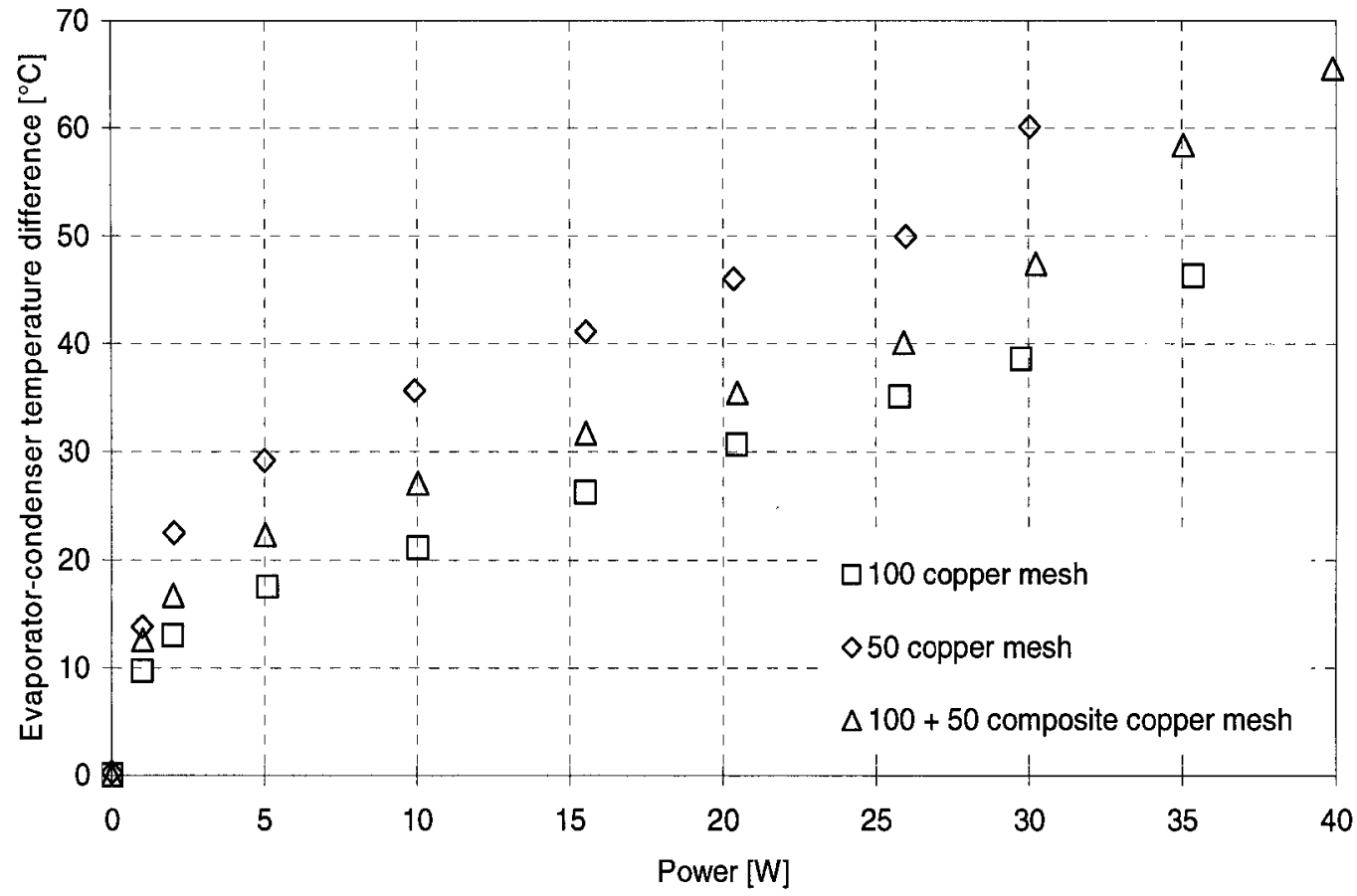

Figure 3.68 Comparison of evaporator-condenser temperature difference regarding wick mesh (insulated, horizontal, enhanced forced convection) [sample(s): HP051701, HP071200, HP071301]

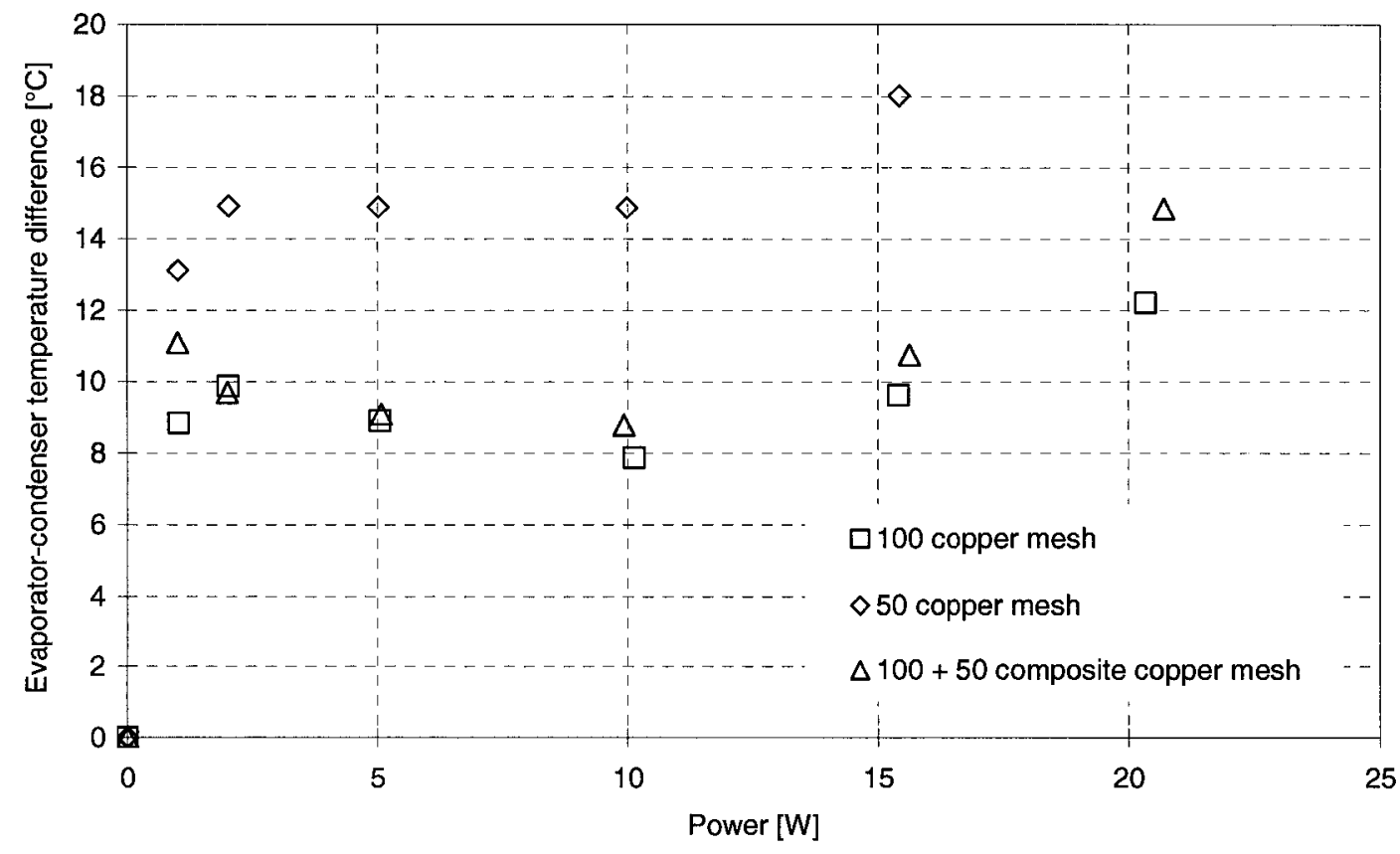

Figure 3.69 Comparison of evaporator-condenser temperature difference regarding wick mesh (insulated, gravity assisted vertical, natural convection) [sample(s): HP051701, HP071200, HP071301] 
Figure 3.69 illustrates the same comparison as those in Figures 3.67 and 3.68 in gravity assisted vertical orientation with natural convection cooling. This figure shows a different picture from the preceding cases. Compared with the one in the horizontal orientation, the case with gravity assisted takes concave shaped scatter curves. When the power is high enough, the curves start to decline, and turn back at a certain power input value, forming concave curves. As explained before, in gravity assisted vertical orientation, the gravitational force would help the water condensed in the condenser return to the evaporator. With increasing power input, the phase change begins to activate. Thus, the evaporator-condenser temperature difference declines. At appropriate heat loads, the rate of evaporation is not very high. Compared with such lower water evaporation, the returned water from the condenser is enough to compensate the water consumption in the evaporator. With the increasing power, the evaporation rate starts to accelerate, while the water return rate still keep constant due to the wick capillary limit. Thus the difference between rates of water evaporation consumption and returning from condenser increases with elevating power inputs. Consequently the evaporator-condenser temperature difference inclines, as can be seen in Figure 3.69. Also during the whole power range, the three configurations take the same order as the preceding cases. 


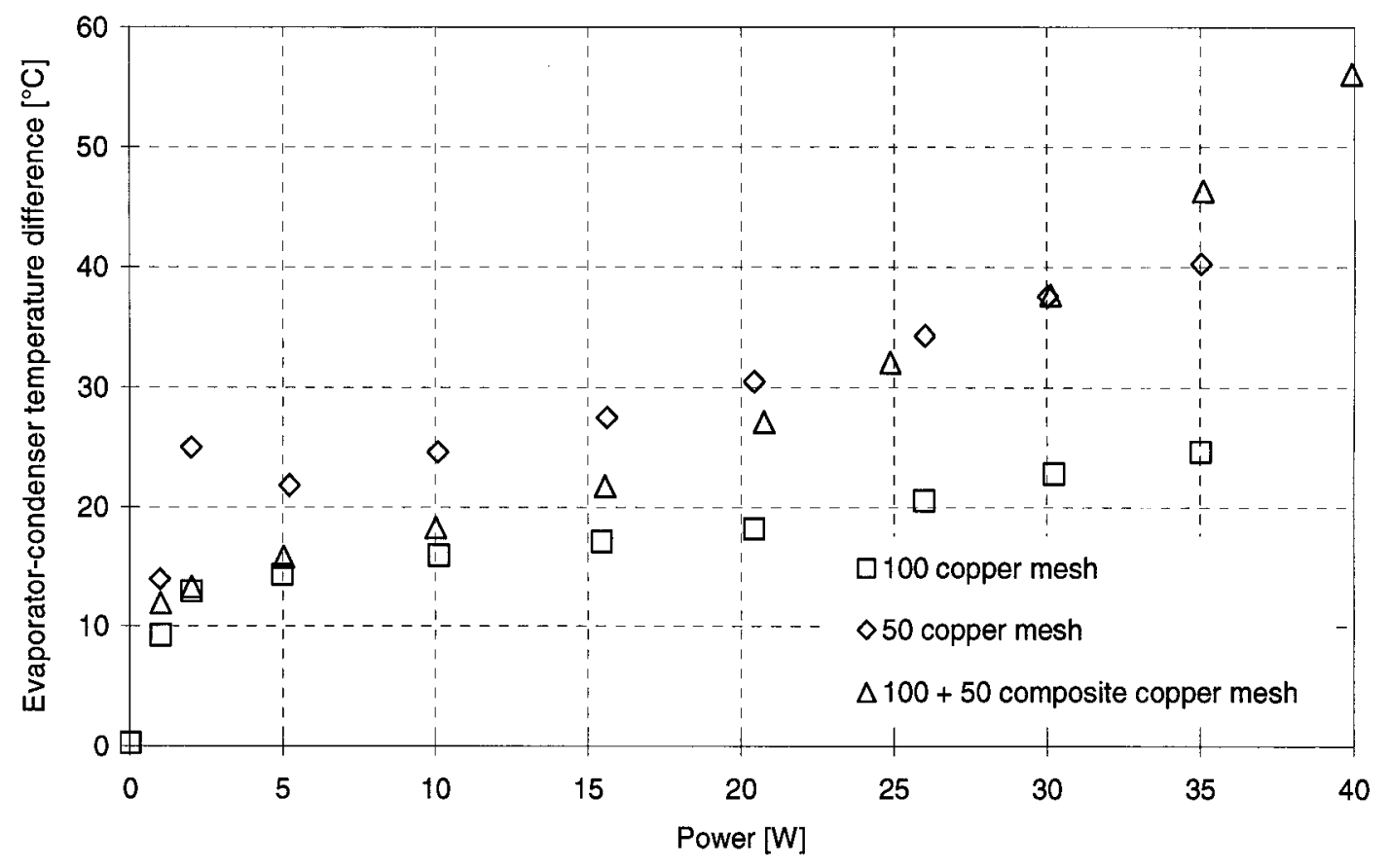

Figure 3.70 Comparison of evaporator-condenser temperature difference regarding wick mesh (insulated, gravity assisted vertical, enhanced forced convection) [sample(s): HP051701, HP071200, HP071301]

Figure 3.70 illustrates the same issue under enhanced forced convection. From this figure it is observed that the curves are flattened by the condenser with a strong cooling sink. Due to a better permeability, the coarse mesh sample has a high rate of feedback water and thus has a very short concave section in a low heat load range. With the increasing power, such permeability is offset by its worse capillarity. Thus, the value starts to increase. The composite mesh sample has a medium permeability and capillarity, thus its curve goes between the other two test samples. With the power input increasing and the condenser temperature maintained low, water tends to stay in the condenser. With a better capillarity, the composite mesh sample has a stronger capacity to hold the condensed water in the condenser then the coarse mesh sample. Thus, it is more difficult for the composite mesh sample to feedback the water to the evaporator to maintain a low evaporator-condenser temperature gradient. With the heat load increasing, the value of 
the temperature difference increases more than the coarse mesh sample. This observation is represented in the figure. After a specific power level, the curve of the composite mesh sample overtakes the coarse sample. With the best capillarity throughout the entire wick, the fine mesh sample possesses enough capillary force to drive back the condensed water from the condenser. However, there is also a tendency to hold water in its condenser. Thus the fine mesh sample still keeps a stable evaporator-condenser temperature gradient under such a condition.

Figures 3.71 and 3.72 illustrate the same issue as for the preceding cases at an against gravity vertical orientation, with natural and enhanced convection respectively. Due to the fact that the heat pipes tend to cease functioning under such conditions, their evaporator-condenser temperature gradient characteristics perform as those of copper tube configurations as described before. The figures above also show the similar tendency as the copper tube configurations.

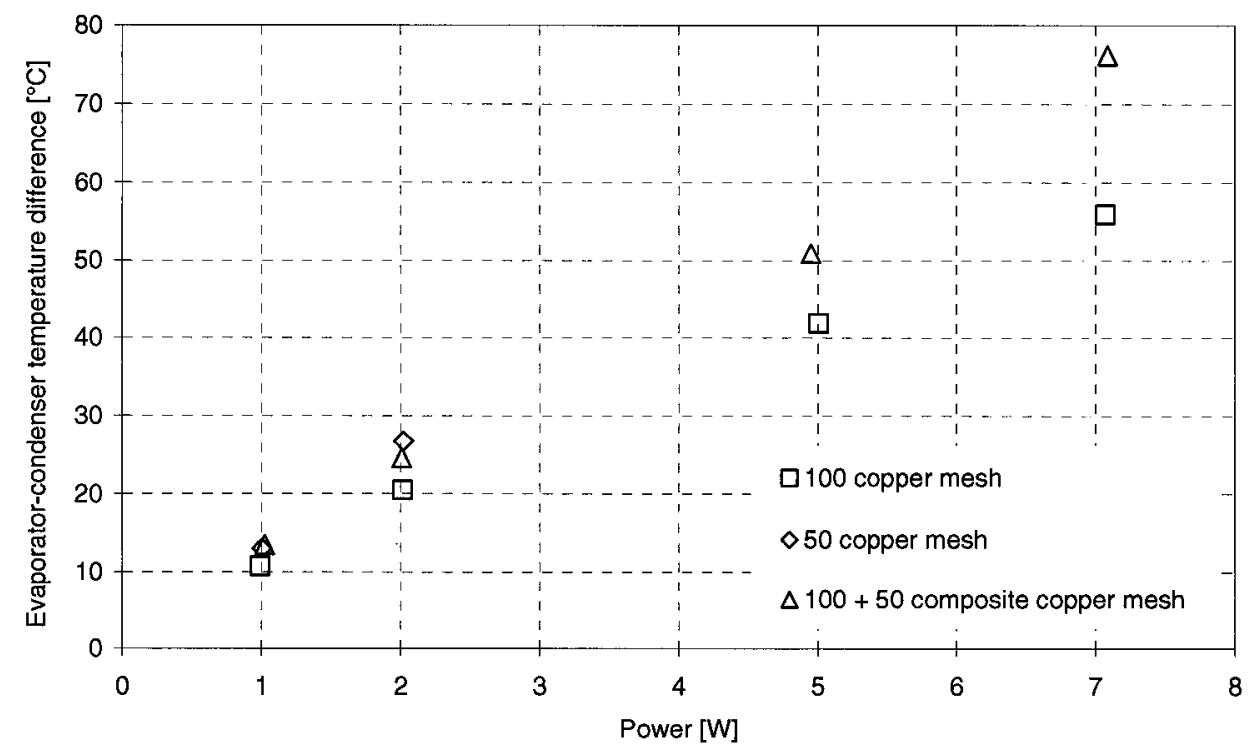

Figure 3.71 Comparison of evaporator-condenser temperature difference regarding wick mesh (insulated, against gravity vertical, natural convection) [sample(s): HP051701, HP071200, HP071301] 


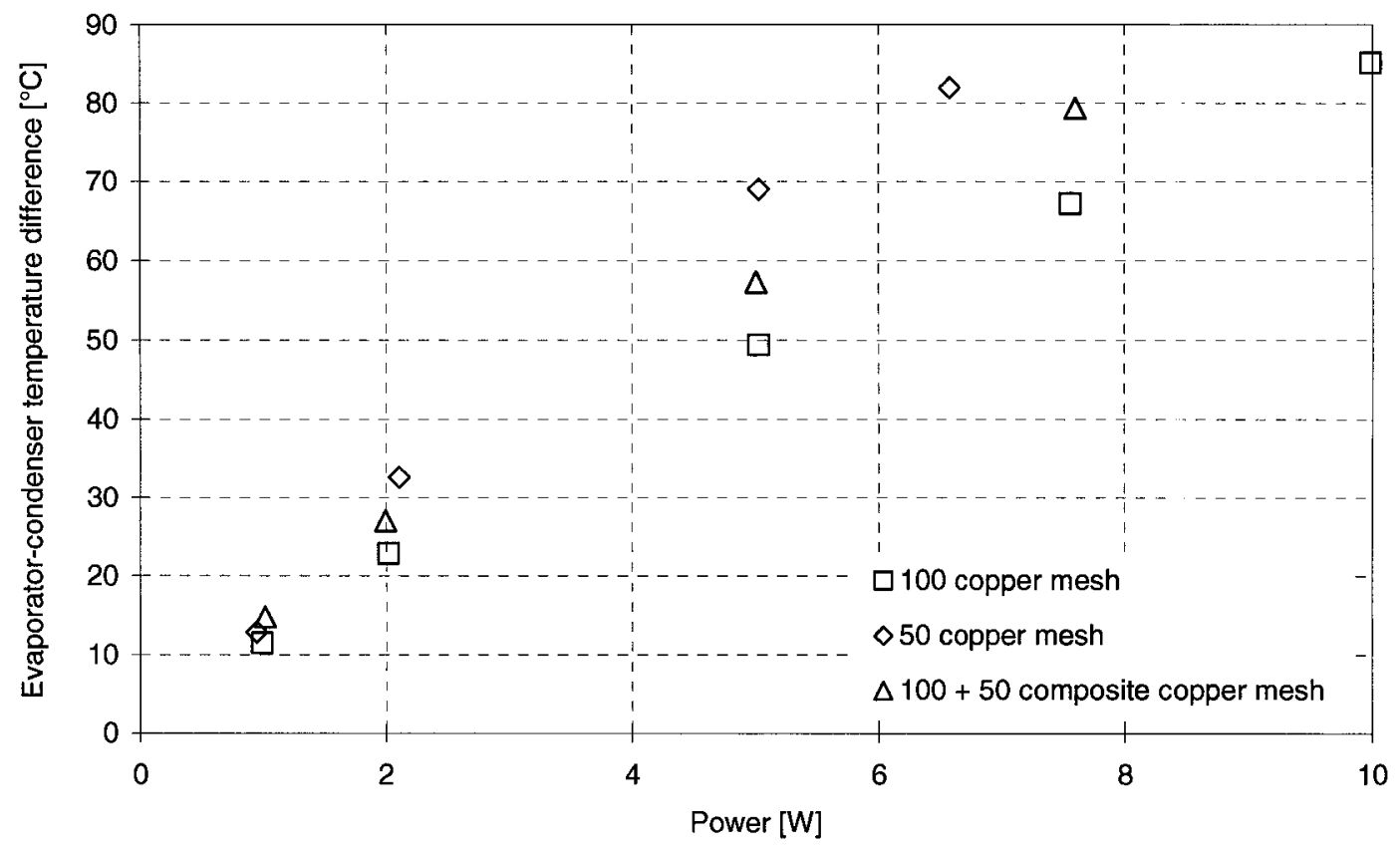

Figure 3.72 Comparison of evaporator-condenser temperature difference regarding wick mesh (insulated, against gravity vertical, enhanced forced convection) [sample(s): HP051701, HP071200, HP071301]

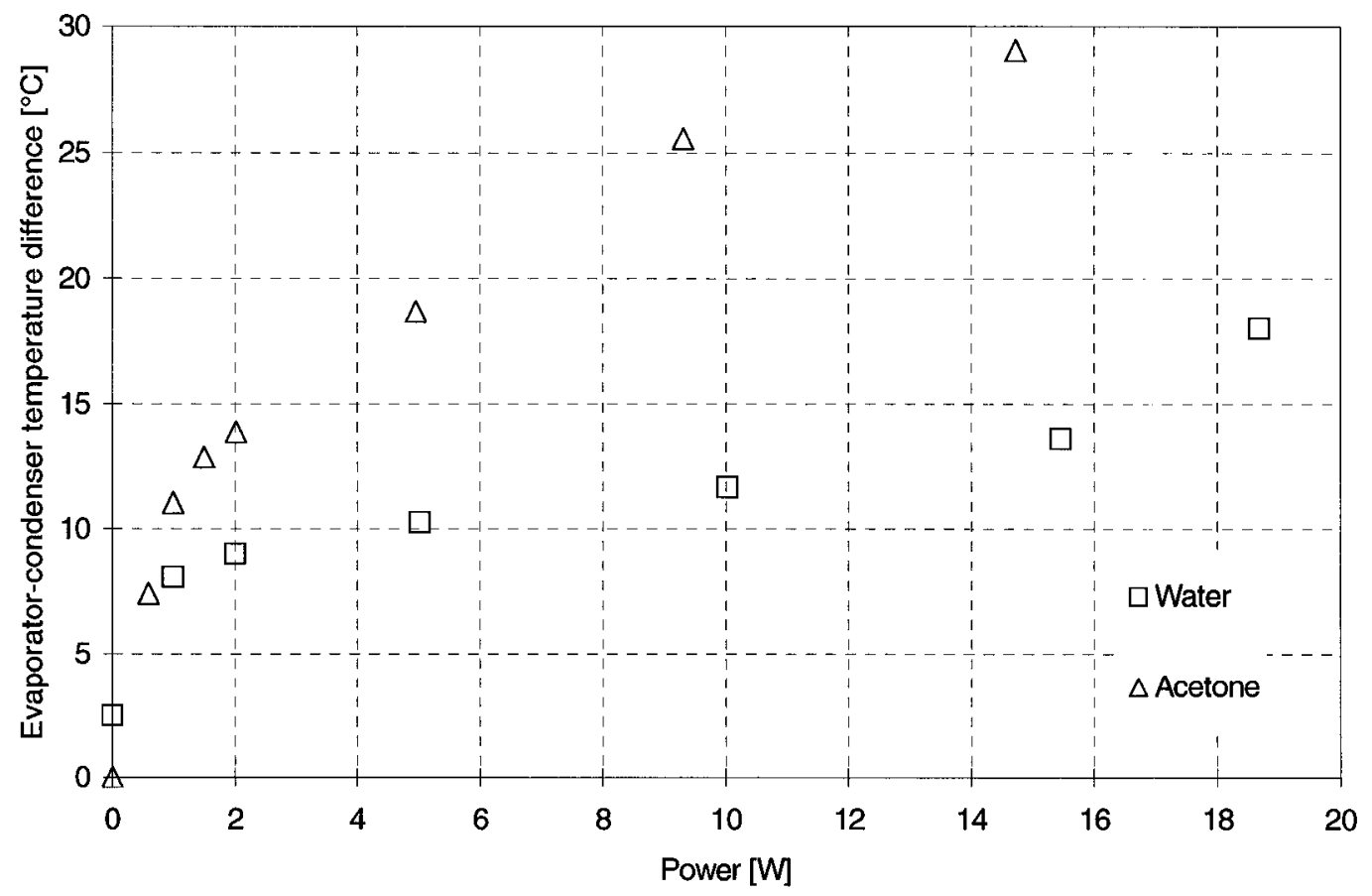

Figure 3.73 Comparison of evaporator-condenser temperature difference regarding working fluid (insulated, horizontal, natural convection) [sample(s): HP051701, HP071402] 
Figure 3.73 illustrates the comparison of evaporator-condenser temperature gradient between two working fluid samples. The testing samples are fine copper mesh water and acetone heat pipes. From the figure we can see that the water heat pipe yields a clear flat curve in the middle power range. While the acetone sample does not, instead, the scatter points form an ascending line with increasing power. These two samples curves diverge with increasing power.

Regarding the working fluid properties, there are many factors affecting the heat pipe evaporator-condenser temperature gradient. The dominant factor is the surface tension. In this case, the surface tension of water is $6.66 \times 10^{2} \mathrm{~N} / \mathrm{m}$, and for acetone is $1.86 \times 10^{2} \mathrm{~N} / \mathrm{m}$ at $60^{\circ} \mathrm{C}$, respectively. There is a $4.8 \times 10^{2} \mathrm{~N} / \mathrm{m}$ surface tension difference between these two working fluids, and a corresponding capillary pressure difference of $3.559 \times 10^{6} \mathrm{~N} / \mathrm{m}^{2}$ between the two working fluids with 100 mesh copper wire screen wick. This ensures that the heat pipe with water charged has a better wick wetting capacity, Thus, water is better to maintain phase change and therefore to maintain a relative constant smaller temperature gradient than the acetone sample.

The second factor is latent heat of working fluids. At $60^{\circ} \mathrm{C}$, latent heat for water is $2359 \mathrm{~kJ} / \mathrm{kg}$, while for acetone it is $517 \mathrm{~kJ} / \mathrm{kg}$. Such a difference leads to a result that water would intake more heat than acetone to evaporate. It also means that water would maintain a constant evaporation temperature, and release more heat than acetone to condense, and maintaining a constant condensation temperature. Consequently, it maintains a total smaller evaporator-condenser temperature gradient. Heat pipe fills working fluid by volume, considering the density difference, for the same volume, water 
possesses more mass than acetone; this would enhance the latent heat effect discussed above for the same size heat pipe.

The third factor is specific heat. For instance, at $100^{\circ} \mathrm{C}$ the specific heat for water is $2370 \mathrm{~kJ} / \mathrm{kgK}$, and for acetone is $470 \mathrm{~kJ} / \mathrm{kgK}$, thus water has higher thermal stability than acetone. At the same power level the water heat pipe has a smaller temperature change at either end than the acetone one, therefore has a total smaller temperature gradient between two ends than the acetone sample. Also the density difference would enhance such thermal inertia difference.

In Figure 3.73, the two curves have a different tendency. The water one has a relative flat section in the middle range of the power level, and starts to rise over a certain power level. The slope tends to be steeper with the increasing power. The acetone sample has a small convex section in the middle power range, and the slope tends to decline with the increasing power. This can also be due to thermal inertia. With a higher thermal inertia, water temperature changes more slowly with changing heat load. At higher power levels, it is also the high thermal inertia that maintains a large temperature gradient for the same cooling capacity.

\subsection{Evaporator-Condenser Temperature Gradient on Amount of Fluid}

The amount of working fluid is one of the main factors affecting the heat pipe performance. This section discusses the evaporator-condenser temperature gradient affected by the amount of working fluid charged. The testing samples are the heat pipes with 100 mesh copper wire screen with over charged (158\%) and normally charged (109\%) water respectively. 


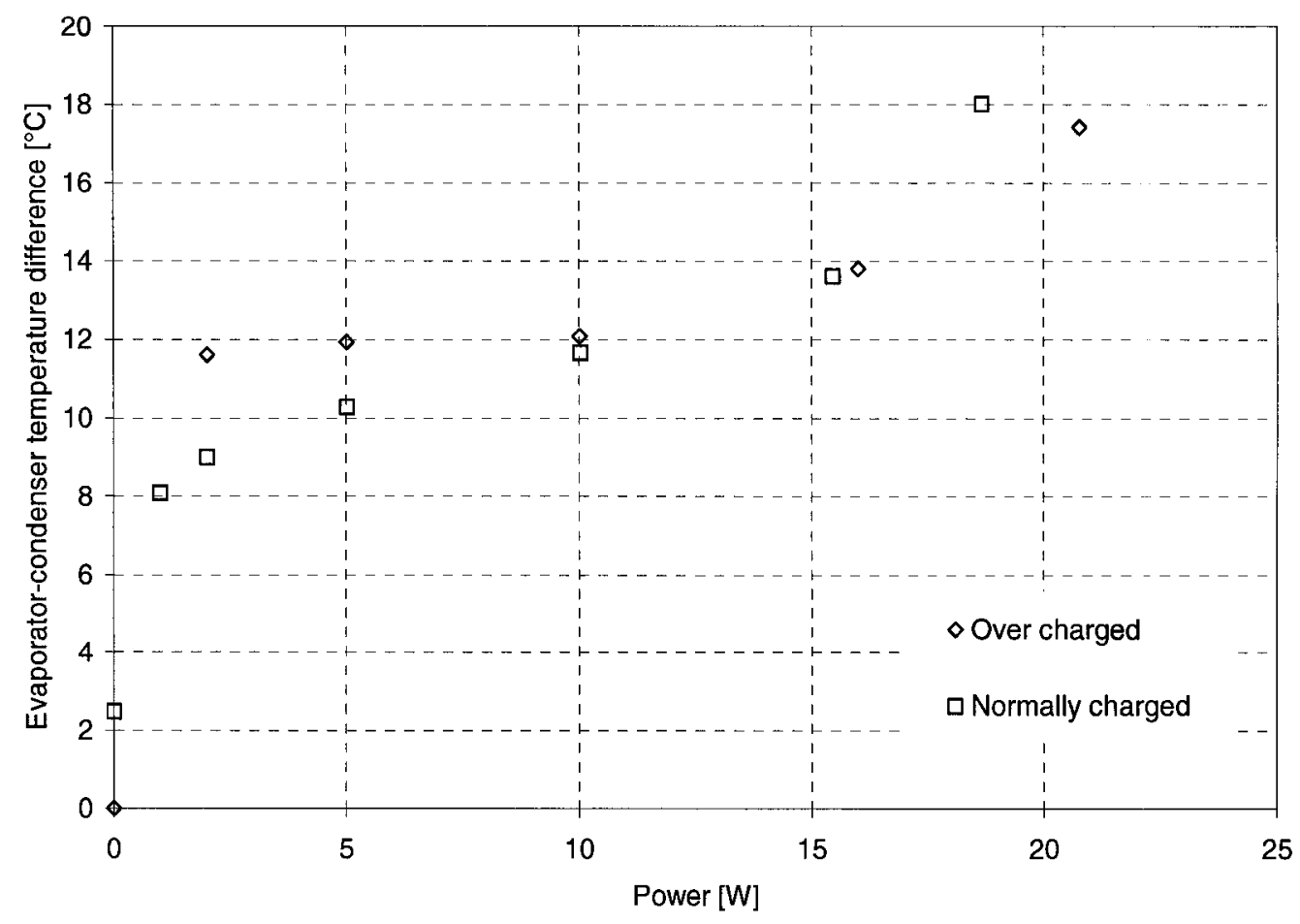

Figure 3.74 Comparison of evaporator-condenser temperature difference regarding amount of working fluid (insulated, horizontal, natural convection) [sample(s): HP052502, HP051701]

Figure 3.74 displays the comparison of evaporator-condenser temperature difference performances for the over charged and normally charged heat pipes, in horizontal orientation, with natural convection cooling. From the figure it can be seen that the normally charged sample performs better in the lower power range (below 10 W). In the middle power level, both samples perform similarly. By entering the higher power level, e.g., $18 \mathrm{~W}$, the normally charged sample tends to steeply rise, and the over charged sample rises with a mush smaller slope.

As discussed before, the over charged sample has extra amount of water. Such water has a large thermal inertia and needs a higher heat load for start-up. In a high power level the heat load is enough to activate the extra water and the entire heat pipe starts-up. It is also this thermal buffer that hinders the evaporator temperature change. 


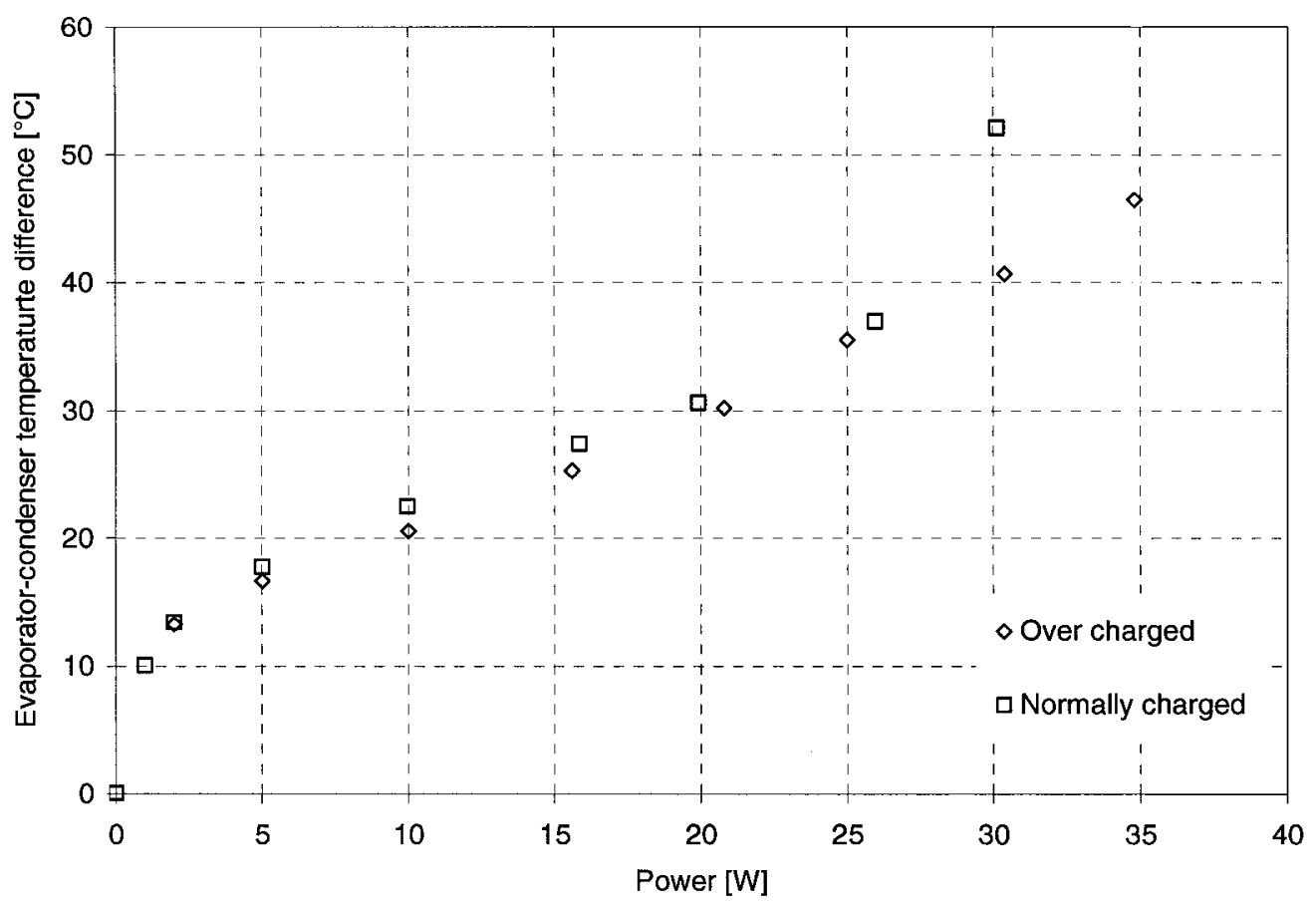

Figure 3.75 Comparison of evaporator-condenser temperature difference regarding amount of working fluid (insulated, horizontal, forced convection) [sample(s): HP052502, HP051701]

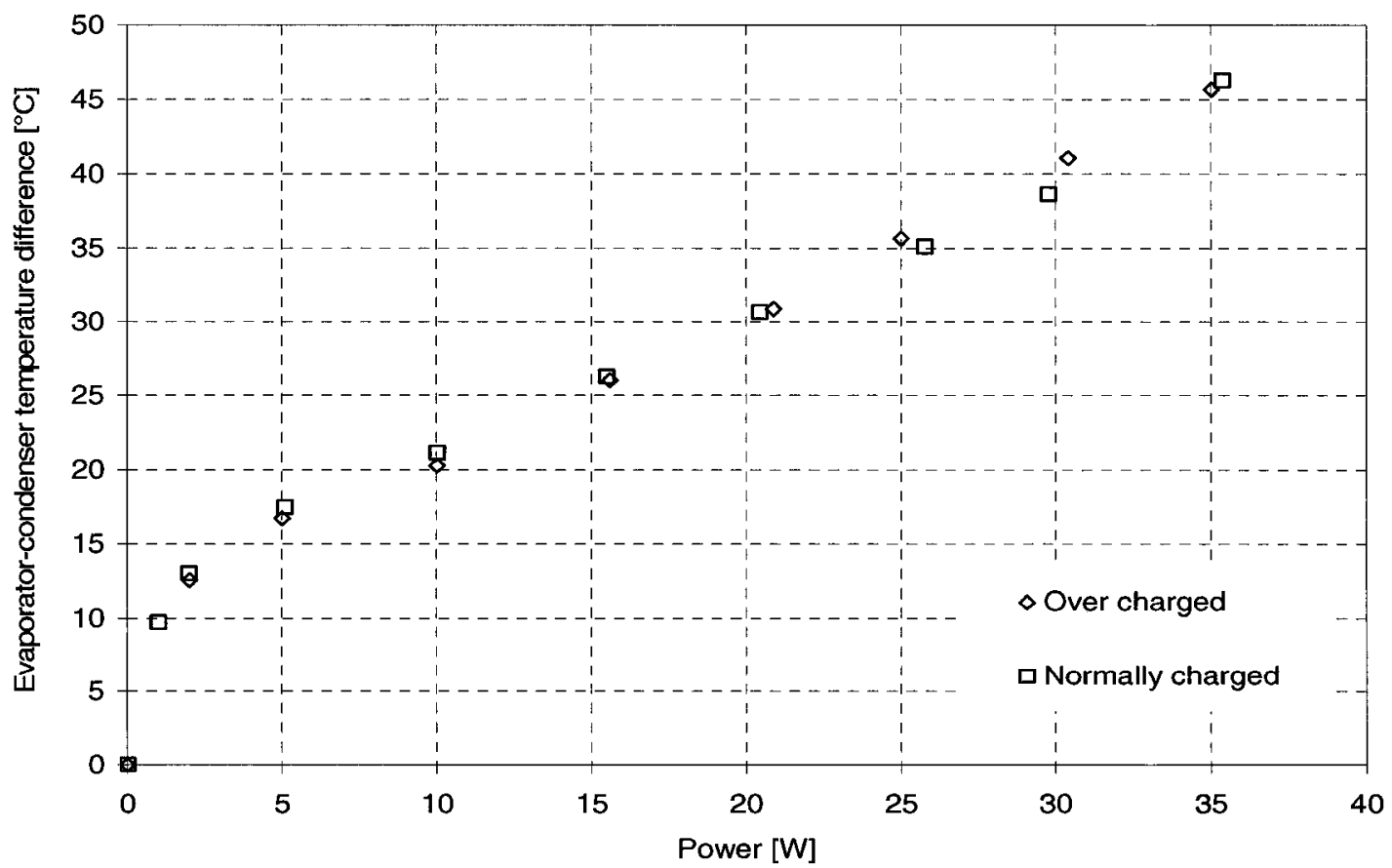

Figure 3.76 Comparison of evaporator-condenser temperature difference regarding amount of working fluid (insulated, horizontal, enhanced forced convection) [sample(s): HP052502, HP051701] 
Figures 3.75 and 3.76 display the forced convection and enhanced convection cases. From these figures it can be seen that both perform the same, the exception is the case under normal forced convection cooling. The normally charged sample starts to rise steeply entering the high power level, the over charged sample keep the same tendency.

Under both convection conditions, the condenser has a very high cooling capacity to dissipate large amounts of heat transferred by the water vapour. The vapour quickly condenses in the condenser end and maintains a relatively lower temperature. With such a low temperature and fine mesh, it retains all condensed water. Thus, there is no any extra water remaining in the evaporator for both samples and both samples have the same fluid condition throughout the entire heat pipe with the exception of the condenser end. This makes both samples perform the same. Under normally forced convection conditions, the cooling capacity is not strong enough to condense the vapour into water immediately, entering the high power level. The normally charged sample has no extra water to buffer the high heat load laid on the evaporator. The evaporator temperature starts to rise very quickly, thus the temperature difference between the two ends begin to rise. Under the enhanced forced convection condition, the cooling capacity is strong enough to condense the vapour into water very quickly. Thus there is enough liquid water to return to the evaporator to meet the heat load. Therefore under such conditions, both samples perform the same.

Figures 3.77 and 3.78 display the gravity assisted and against gravity vertical cases. It can be found that, in the gravity assisted vertical orientation; the normally charged sample performs better; while in the opposite orientation the over charged sample does better. This can be due to the water thermal inertia, and effective length decrease due to 
the extra water, for each orientation respectively. The effective length will be discussed in Chapter 4.

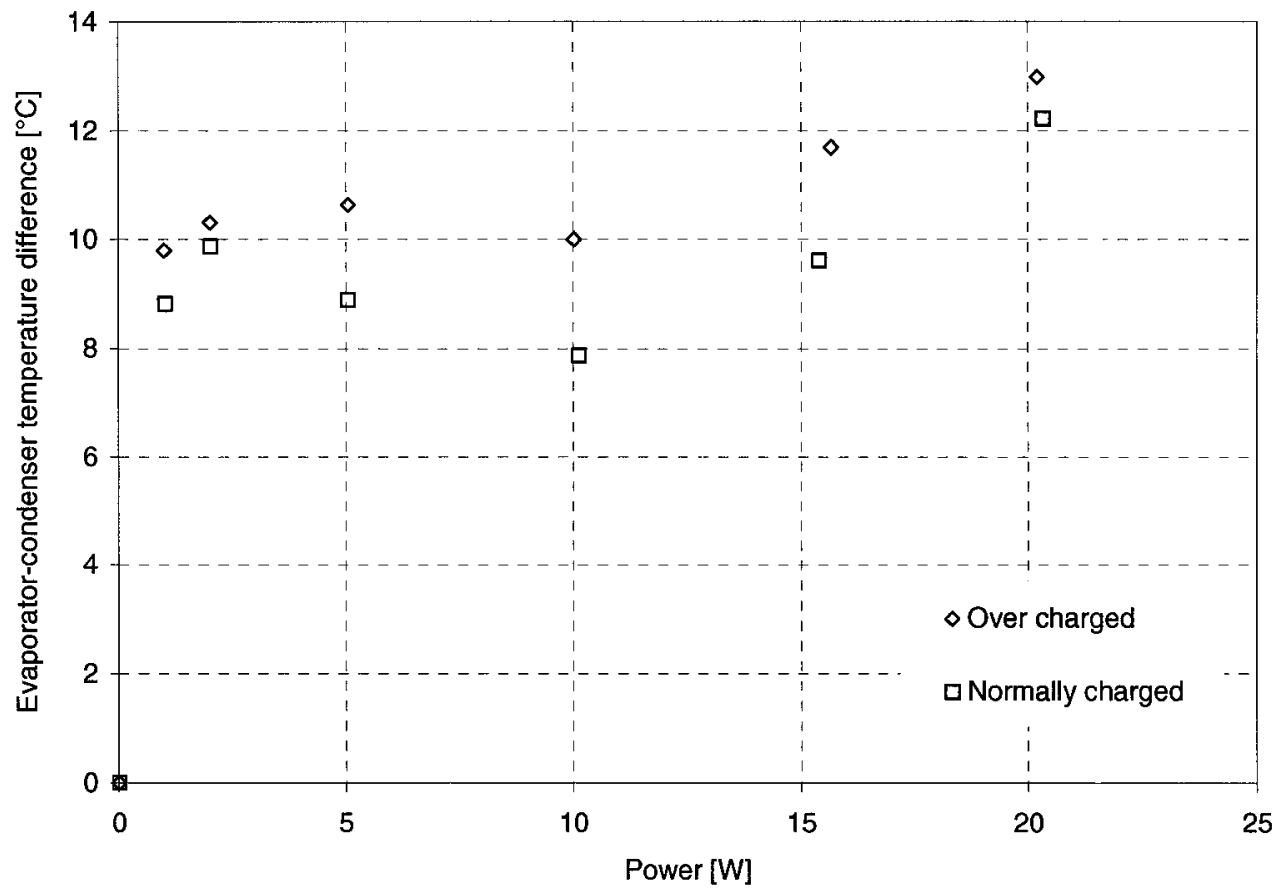

Figure 3.77 Comparison of evaporator-condenser temperature difference regarding amount of working fluid (insulated, gravity assisted vertical, natural convection) [sample(s): HP052502, HP051701]

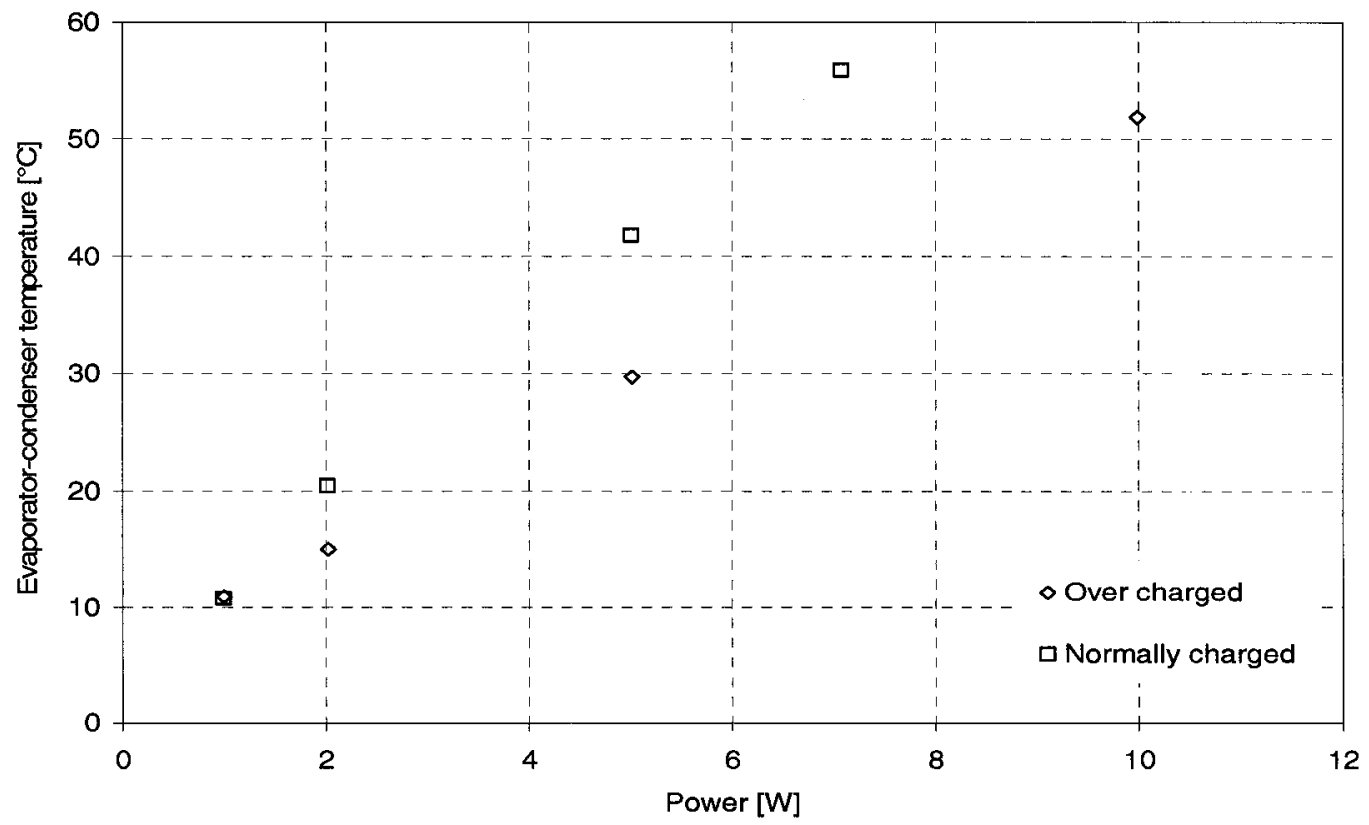

Figure 3.78 Comparison of evaporator-condenser temperature difference regarding amount of working fluid (insulated, against gravity vertical, natural convection) [sample(s): HP052502, HP051701] 


\subsubsection{Axial Thermal Resistance and Effective Thermal Conductivity}

The thermal resistance is an important concern for heat transfer devices, which is defined as the ratio of heating-cooling temperature difference to the heat transferred through the section between these two ends. It shows the temperature difference penalty paid per unit heat. A well performing heat transfer device possesses a small value of thermal resistance.

As a composite structure heat transfer device, heat pipe thermal resistance is more complex than any single material device. Due to the heat transferred in the axial direction, the axial thermal resistance is of interest. It is defined as the ratio of evaporator-condenser temperature difference to the heat transferred through the heat pipe.

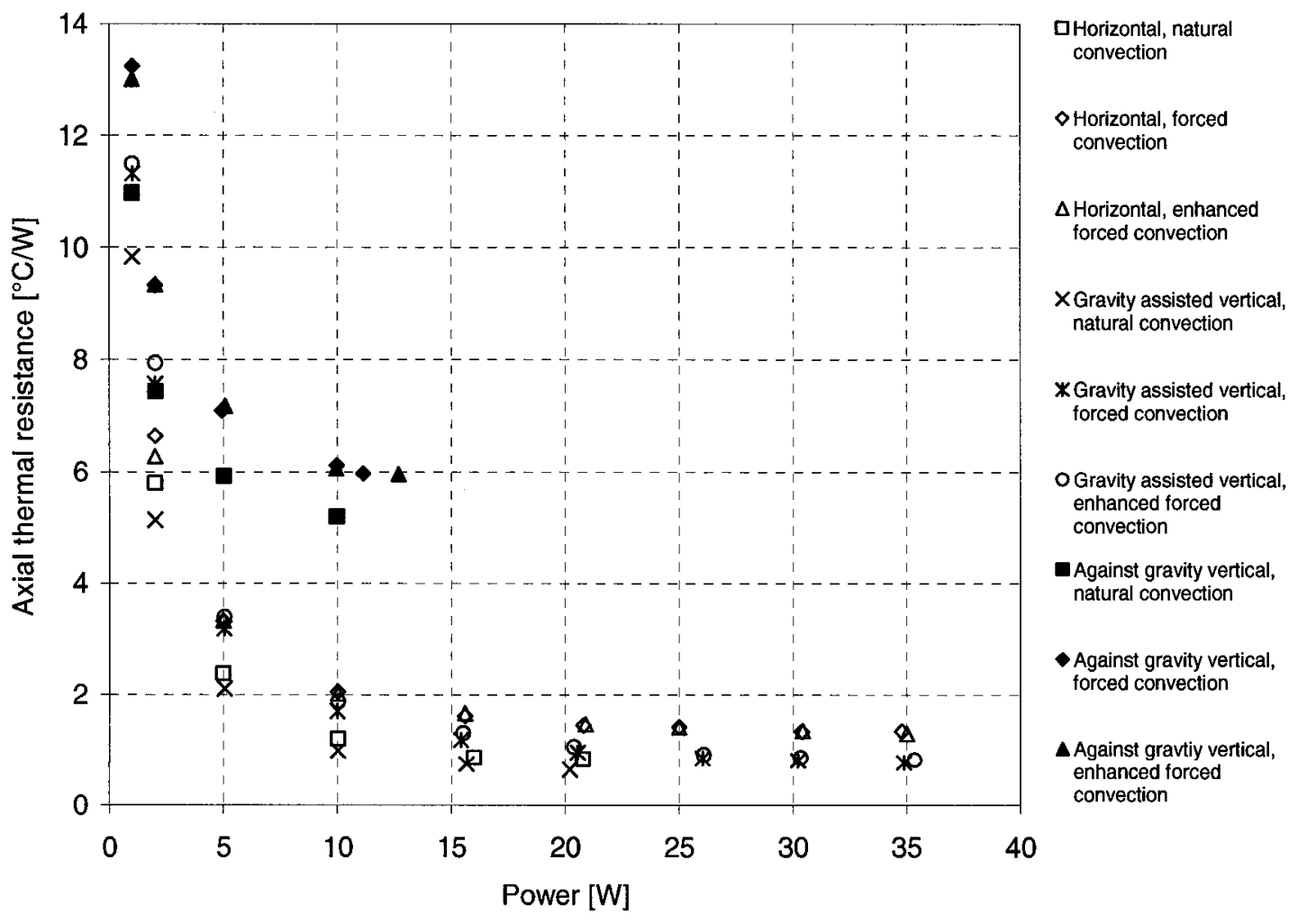

Figure 3.79 Axial thermal resistance under circumstances [sample(s): HP052502] 
Figure 3.79 illustrates the axial thermal resistances under different conditions. The testing sample here is the fine copper mesh over charged water heat pipe. From this figure, it can be seen that thermal resistance distribution varies with power. At the beginning, axial thermal resistance declines sharply with increasing power, and then turns to flat in the medium power levels. Also in the figure, it should be noted that the groups working at against gravity have very large axial thermal resistances.

There are also figures for comparison of axial thermal resistances for other testing samples and comparison of axial thermal resistance regarding different issues such as cooling approaches, orientations, wick structure, working fluid, and the amount of working fluid.

The concept of axial thermal resistance is about the entire heat transfer network. The value varies with different factors, e.g., dimension of the heat pipe. This concept is not a good criterion to compare heat pipe characteristics. To solve this problem, we need to involve the concept of effective axial thermal conductivity.

The so-called effective axial thermal conductivity can be defined as the ratio of heat transfer rate per unit cross-sectional area of the heat pipe to its axial temperature gradient. It eliminates the dimension factors and allows comparison between heat pipes with different dimensional configurations. Effective axial thermal conductivity stems from heat and temperature gradient. It is a better criterion to evaluate a heat pipe on both effectiveness and efficiency.

Figure 3.80 illustrates the axial thermal conductivities under different conditions. The testing sample here is the fine copper mesh over charged water heat pipe. 


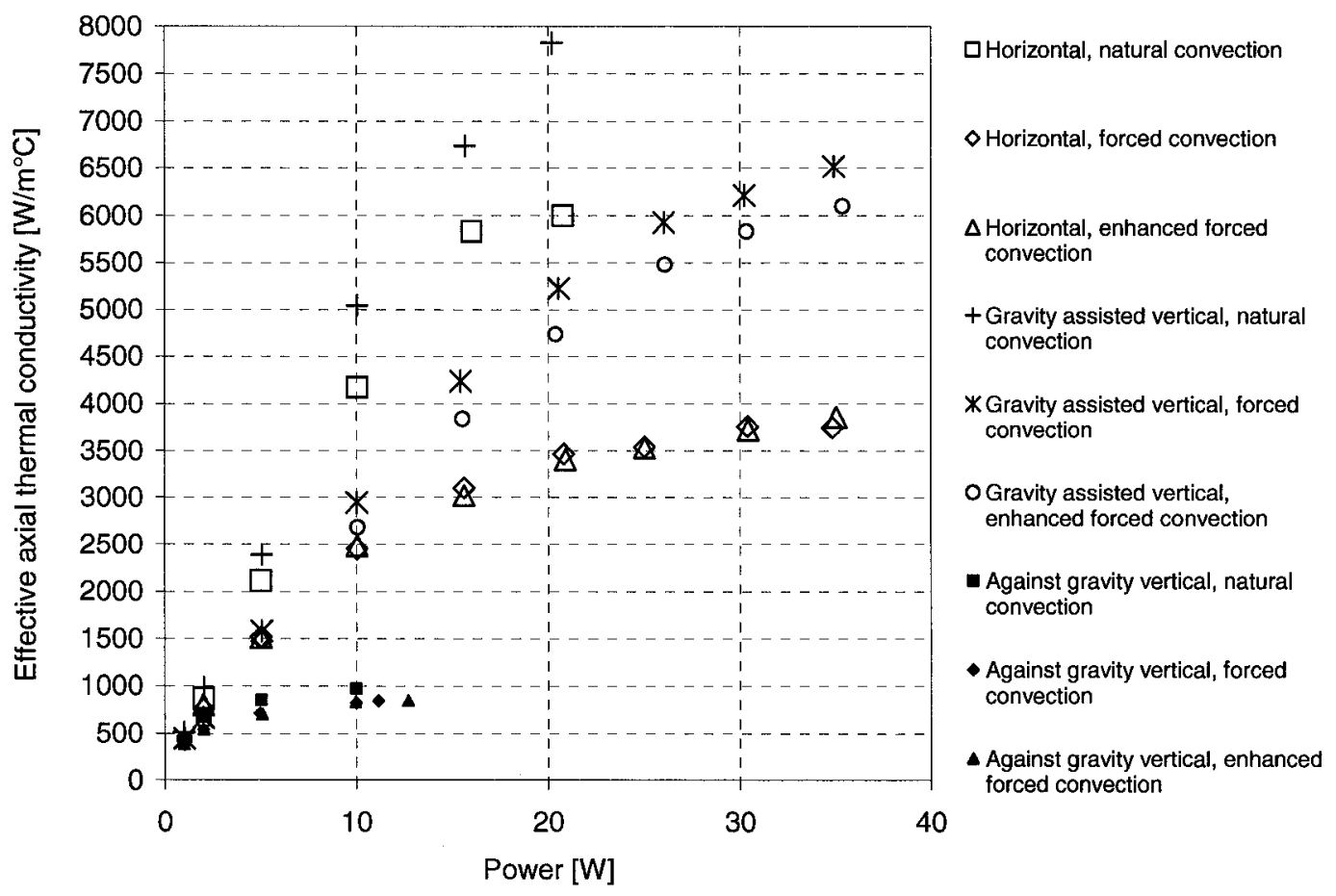

Figure 3.80 Comparison of effective axial thermal conductivity [sample(s): HP052502]

From the figure it can be seen that the values of the effective axial thermal conductivity vary in a very large range, from 500 to $8000 \mathrm{~W} / \mathrm{m}^{\circ} \mathrm{C}$. The lower values are much higher than that of copper tube configurations, i.e., $380 \mathrm{~W} / \mathrm{m}^{\circ} \mathrm{C}$. The high values are over 20 times that of copper tube configurations. The nine scatter point curves in the figure can be divided into three groups according to either orientations, i.e., horizontal, gravity assisted vertical, and against gravity vertical; or cooling approaches, i.e., natural, normal forced, and enhanced forced convections. The bottom three are at against gravity vertical orientation. The top two are with natural convection approaches at either horizontal or gravity assisted vertical orientations. The rest of the configurations are distributed between them.

Effective axial thermal conductivities on specific issues will be discussed in the following contents. 


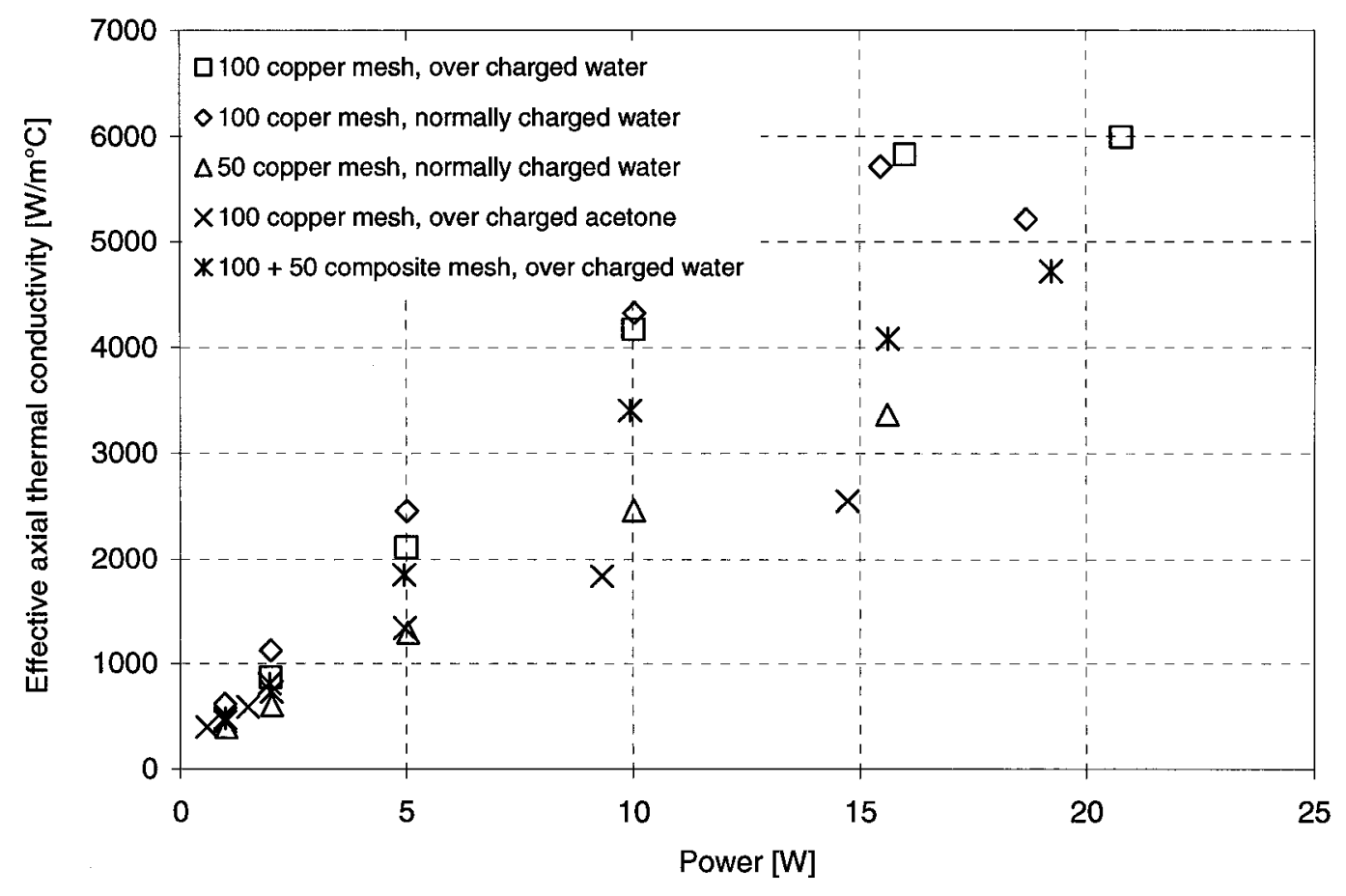

Figure 3.81 Comparison of effective axial thermal conductivity (insulated, horizontal, natural convection) [sample(s): HP052502, HP051701, HP071200, HP071402, HP071301]

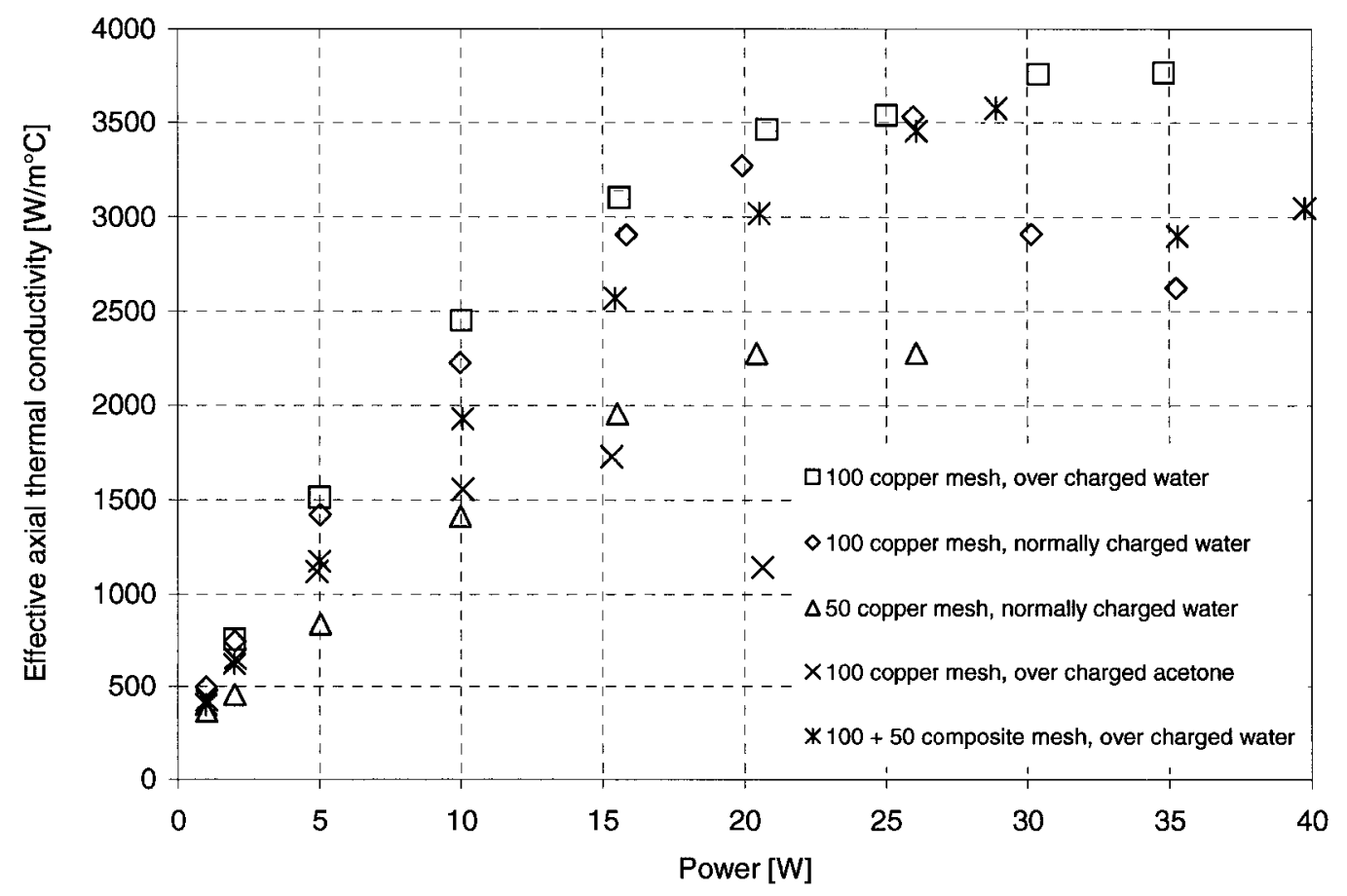

Figure 3.82 Comparison of effective axial thermal conductivity (insulated, horizontal, forced convection) [sample(s): HP052502, HP051701, HP071200, HP071402, HP071301] 


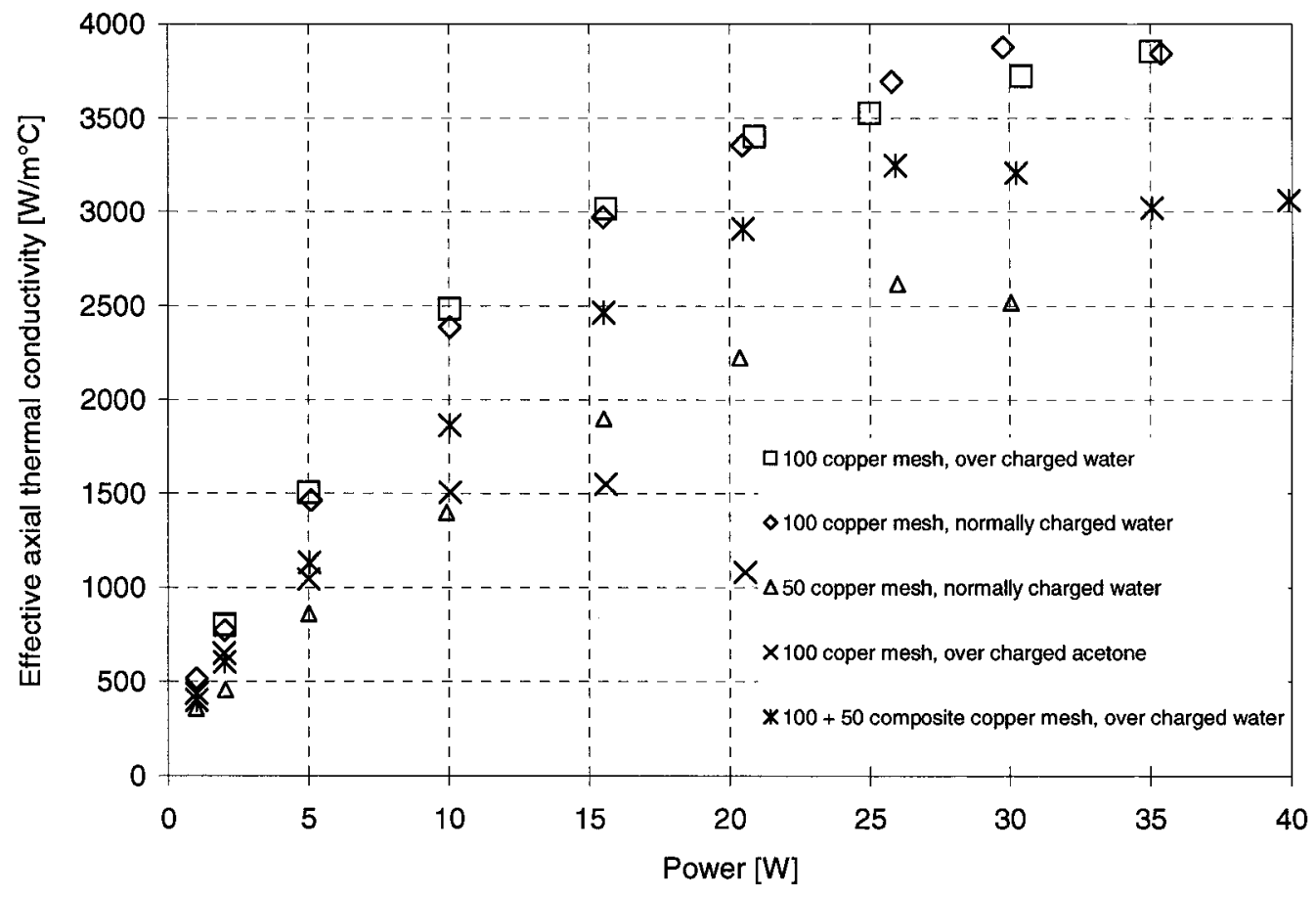

Figure 3.83 Comparison of effective axial thermal conductivity (insulated, horizontal, enhanced forced convective cooling) [sample(s): HP052502, HP051701, HP071200, HP071402, HP071301]

Figures 3.81 to 3.83 , illustrate the effective axial thermal conductivity of the five testing samples, at horizontal orientation, and with three cooling approaches respectively.

Cooling is one of the factors affecting the effective axial thermal conductivity. Comparing these three figures, it can be seen that all the five samples have the best effective axial thermal conductivities with natural convection cooling. This is due to a much smaller axial temperature gradient with natural convection than these with forced, or enhanced forced convections, even though the later samples have more capacity of heat transfer.

The second factor affecting the effective axial thermal conductivity is wick structure. From the three figures, it can be seen that with natural, forced, or enhanced forced convection, the fine mesh samples perform the best with the composite mesh second, and the coarse mesh sample the last. In the forced and enhanced forced 
convections cases, in the high power range, the coarse and composite mesh samples lose their stability in effective axial thermal conductivities, thus declining. These are consistent with the axial temperature gradient regarding wick mesh, as discussed in preceding sections on the same issue.

Like other characteristics, the heat pipe effective axial thermal conductivity is also affected by the working fluids. Comparing Figures 3.81 to 3.83 , the water samples, with any configuration, perform better than the acetone ones, and the difference change with increasing power. Note that with forced and enhanced forced convections, the effective axial thermal conductivity values of the acetone heat pipe start to drop when the power reaches a certain value, e.g., $15 \mathrm{~W}$. While for the water heat pipe, the effective axial thermal conductivity still keep the ascending tendency until $25 \mathrm{~W}$ or more. This is also consistent with the discussions in preceding sections on the related issues, i.e., axial temperature gradient with respective to working fluid.

Besides the working fluid, the amount of the working fluid is also a factor affecting the effective axial thermal conductivity. From Figures 3.81 to 3.83 , comparing the samples of over charged and normally charged water heat pipes, it can be seen that under all three cooling conditions, these two test samples do not have much differences in the value of effective axial thermal conductivity, but the over charged sample has a better thermal stability when entering high power level than the normally charged sample. These are also consistent with the axial temperature gradient characteristics regarding the working fluid amount, as discussed before. 


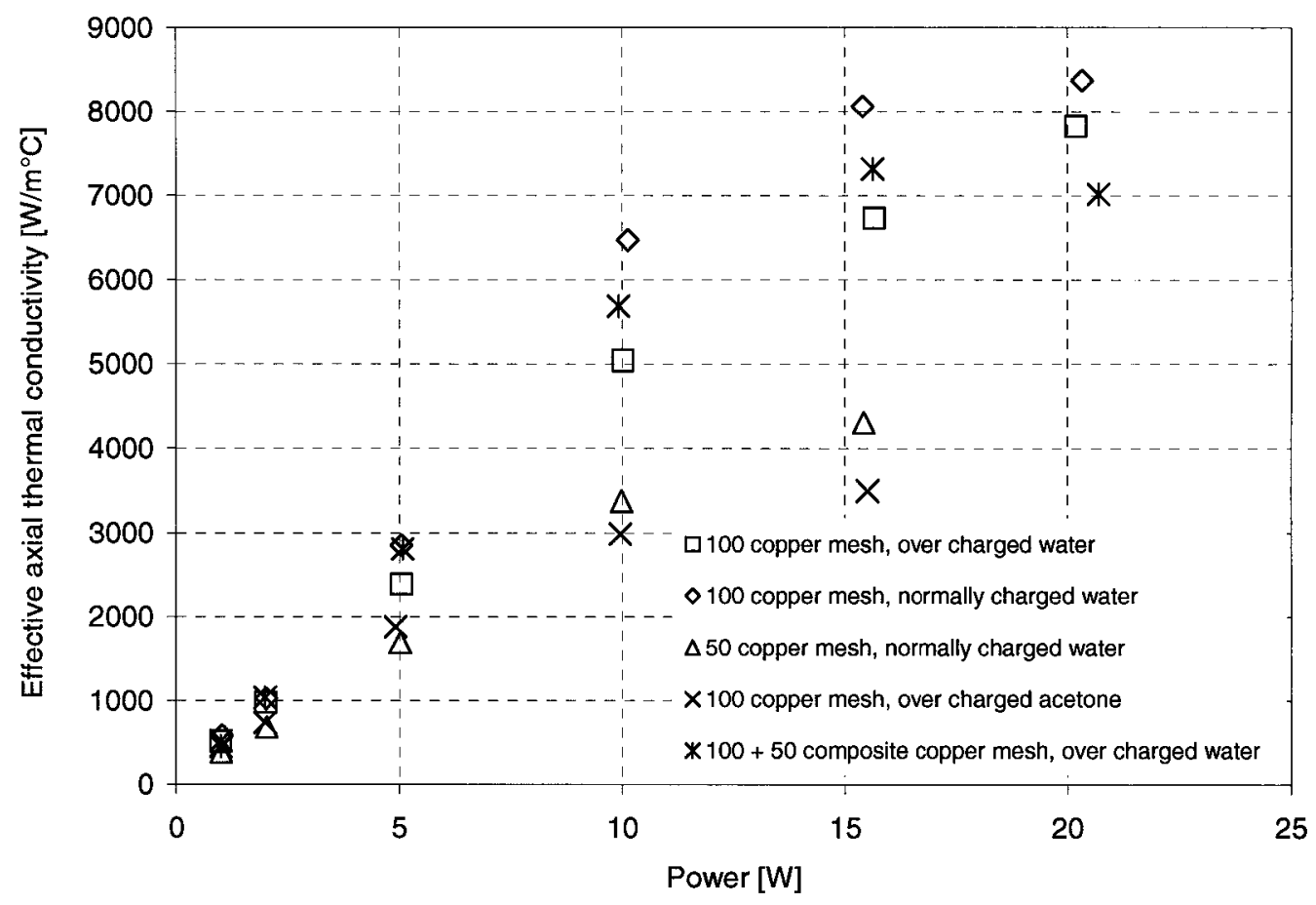

Figure 3.84 Comparison of effective axial thermal conductivity (insulated, gravity assisted vertical, natural convection) [sample(s): HP052502, HP051701, HP071200, HP071402, HP071301]

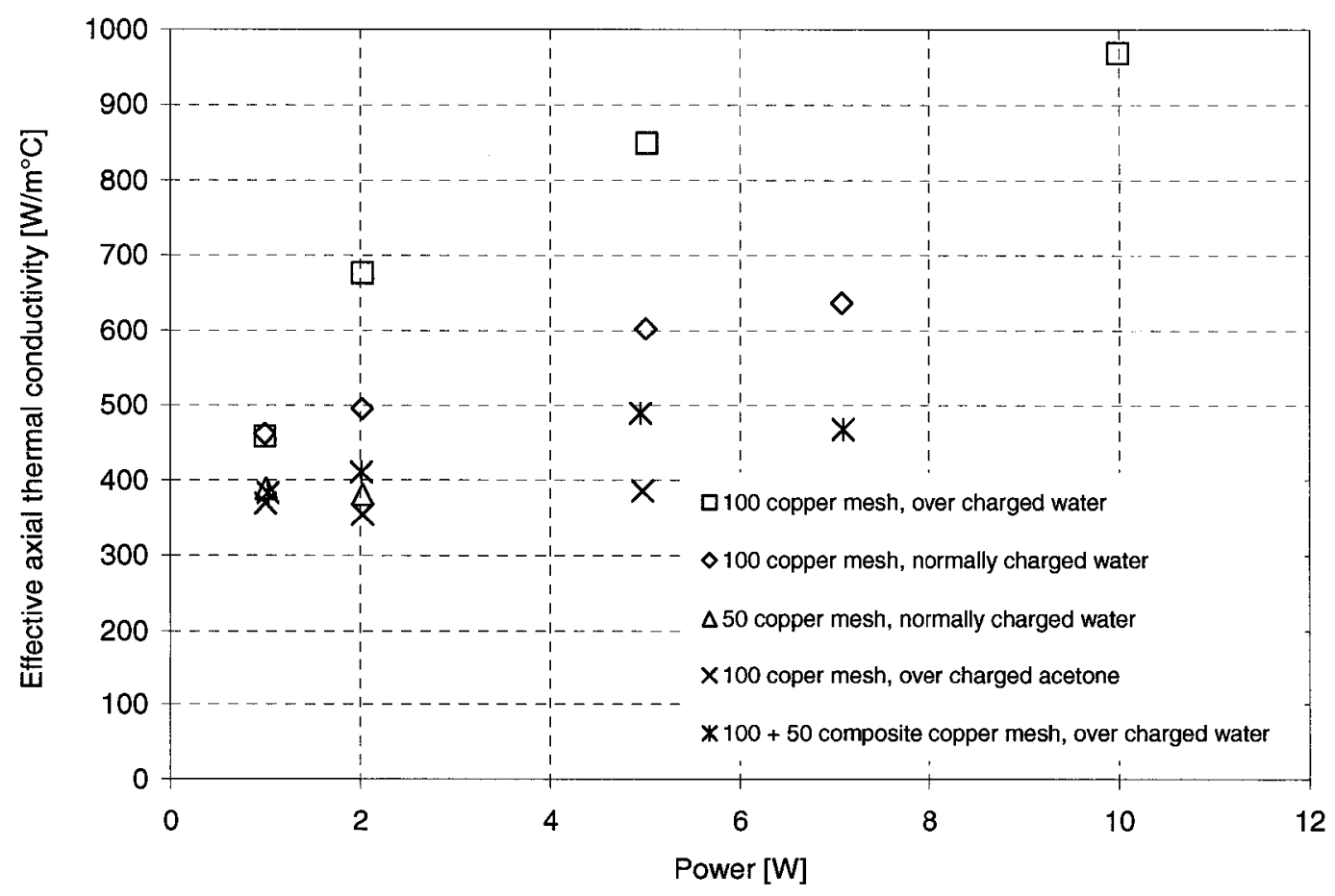

Figure 3.85 Comparison of effective axial thermal conductivity (insulated, against gravity vertical, natural convection) [sample(s): HP052502, HP051701, HP071200, HP071402, HP071301] 
Figures 3.84 and 3.85 illustrate the effective axial thermal conductivities for the five samples respectively; natural convection, gravity assisted and against gravity vertical orientations. Comparing with the horizontal orientation, at the same power level, the gravity assisted case improves the effective axial thermal conductivity for the fine mesh over charged water sample by about $2000 \mathrm{~W} / \mathrm{m}^{\circ} \mathrm{C}$ or 33 percent. The fine mesh normally charged water sample is changed by about $2200 \mathrm{~W} / \mathrm{m}^{\circ} \mathrm{C}$ or 38 percent. The composite mesh over charged water sample is changed by about $2000 \mathrm{~W} / \mathrm{m}^{\circ} \mathrm{C}$ or 40 percent. The coarse mesh normally charged water sample is changed by about $1000 \mathrm{~W} / \mathrm{m}^{\circ} \mathrm{C}$ or 30 percent. Finally, the acetone sample improves by over $1000 \mathrm{~W} / \mathrm{m}^{\circ} \mathrm{C}$ or 40 percent. The against gravity case decreases the effective axial thermal conductivity for the fine mesh, over charged water sample by approximately $3100 \mathrm{~W} / \mathrm{m}^{\circ} \mathrm{C}$ or 77.5 percent. The fine mesh normal-charged water sample is changed by approximately $1900 \mathrm{~W} / \mathrm{m}^{\circ} \mathrm{C}$ or 76 percent. The composite mesh over charged water sample is changed by $1300 \mathrm{~W} / \mathrm{m}^{\circ} \mathrm{C}$ or 72 percent. The coarse mesh over charged water sample is changed by $100 \mathrm{~W} / \mathrm{m}^{\circ} \mathrm{C}$ or 20 percent. Finally, the acetone is changed by approximately $900 \mathrm{~W} / \mathrm{m}^{\circ} \mathrm{C}$ or 70 percent.

\subsubsection{Investigation of Nickel-Copper Heat Pipe}

The same series of experiments were also applied to a heat pipe with a nickel foam wick and copper case. The working fluid was water. The detailed specific configuration is available in Appendix I. This section discusses the characteristics of the nickel foam heat pipe with regard to heat transfer efficiency.

\subsubsection{Axial Temperature Profile}

\subsection{Heat Pipe Axial Temperature Profile regarding Heat Load}




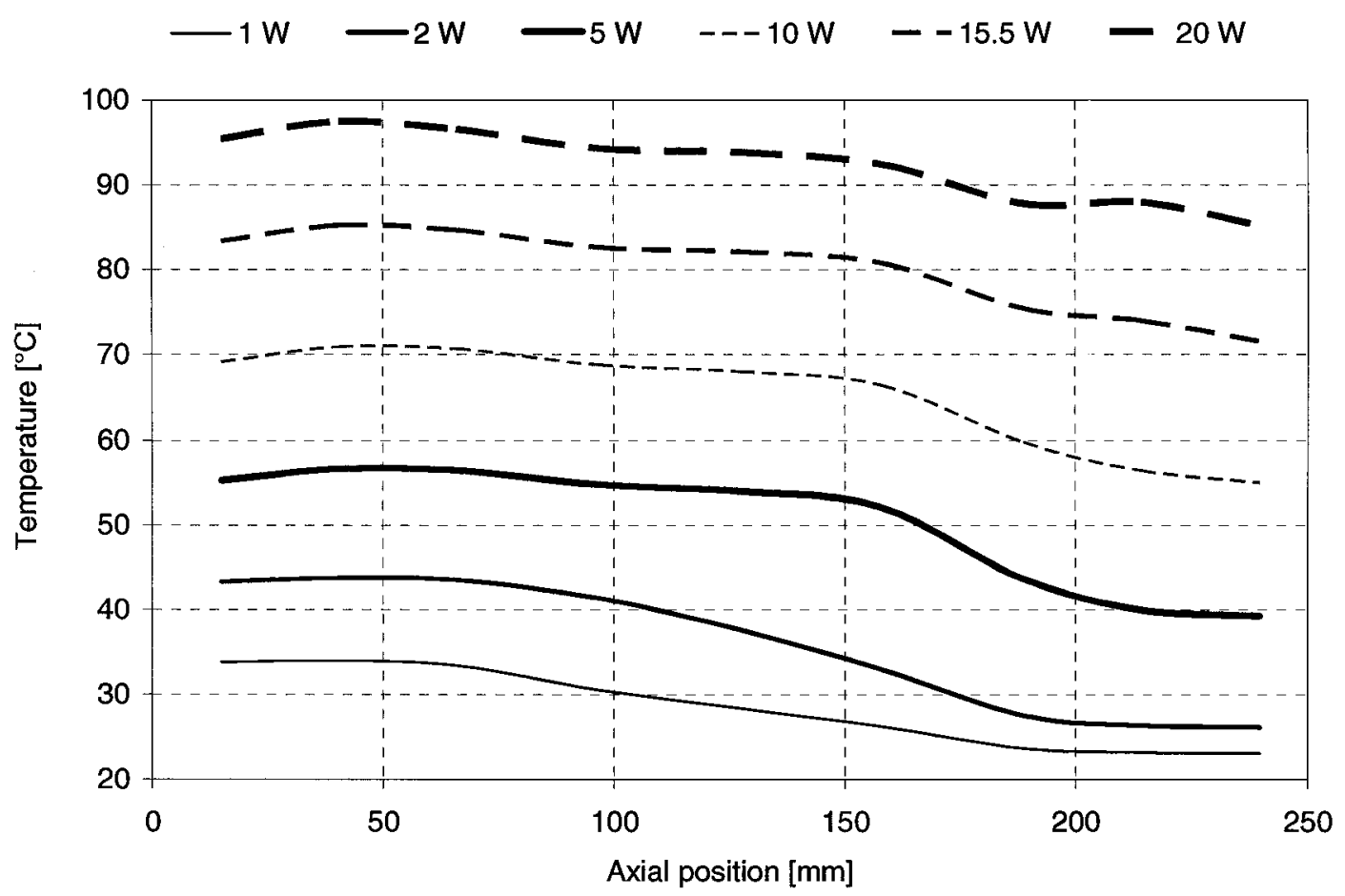

Figure 3.86 Nickel foam heat pipe axial temperature profile (insulated, horizontal, natural convection) [sample(s): HP062400]

Figure 3.86 illustrates the axial temperature profile for the nickel foam water heat pipe at a horizontal orientation with natural convection cooling. Compared with the copper mesh counterparts, the nickel wick heat pipe has similar curves. At lower power levels, the adiabatic section take straight line with a constant slope, performing as a solid metal tube. With increasing power, the adiabatic section tends to be flat.

From Figures 3.87 and 3.88, it can be seen that with forced and enhanced forced convection, the evaporator-adiabatic temperature difference tend to be large at either gravity assisted and against gravity vertical orientations. These are similar to its copper mesh wick counterparts on the same issues. 


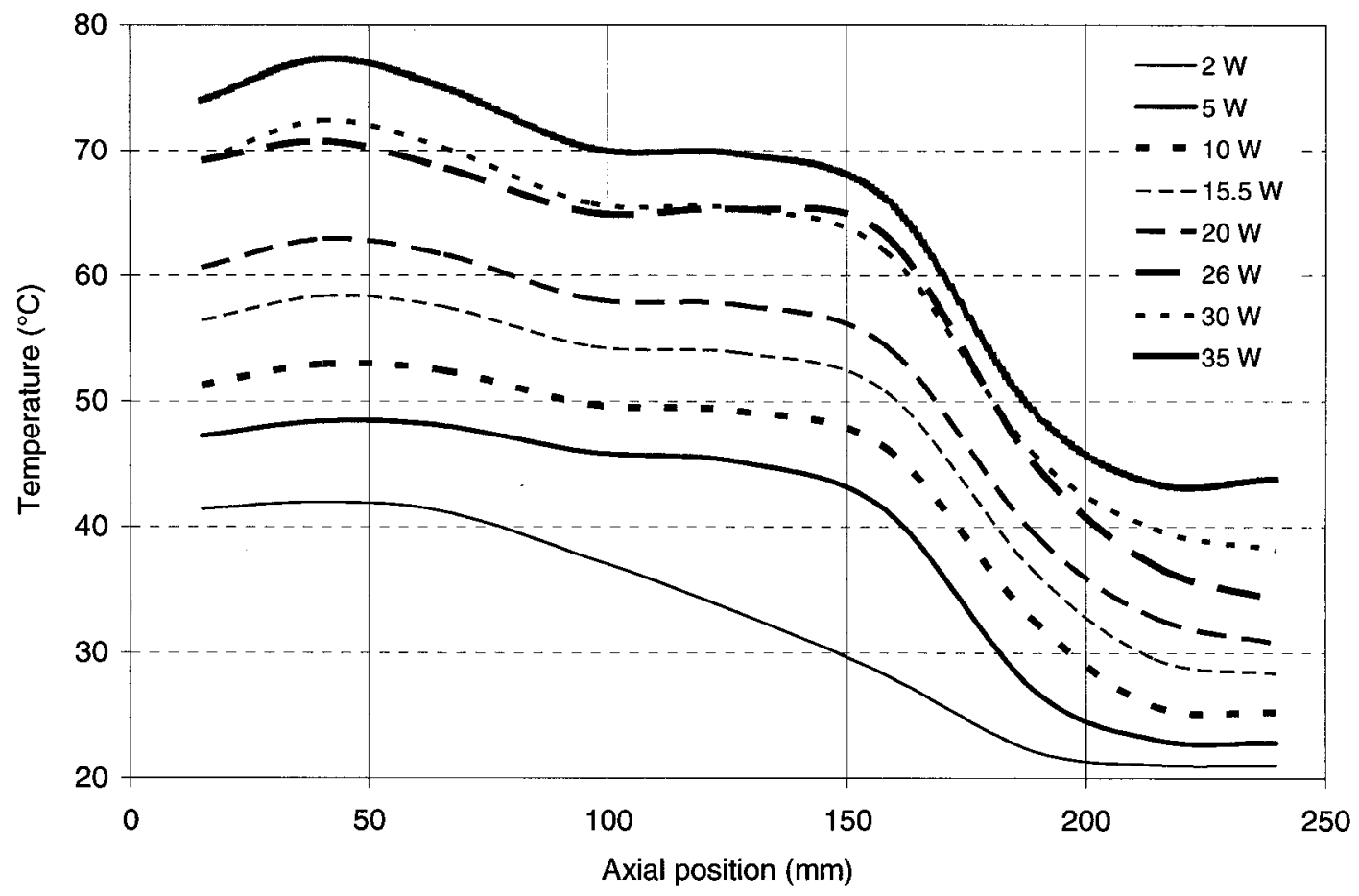

Figure 3.87 Nickel foam heat pipe axial temperature profile (insulated, horizontal, forced convection) [sample(s): HP062400]

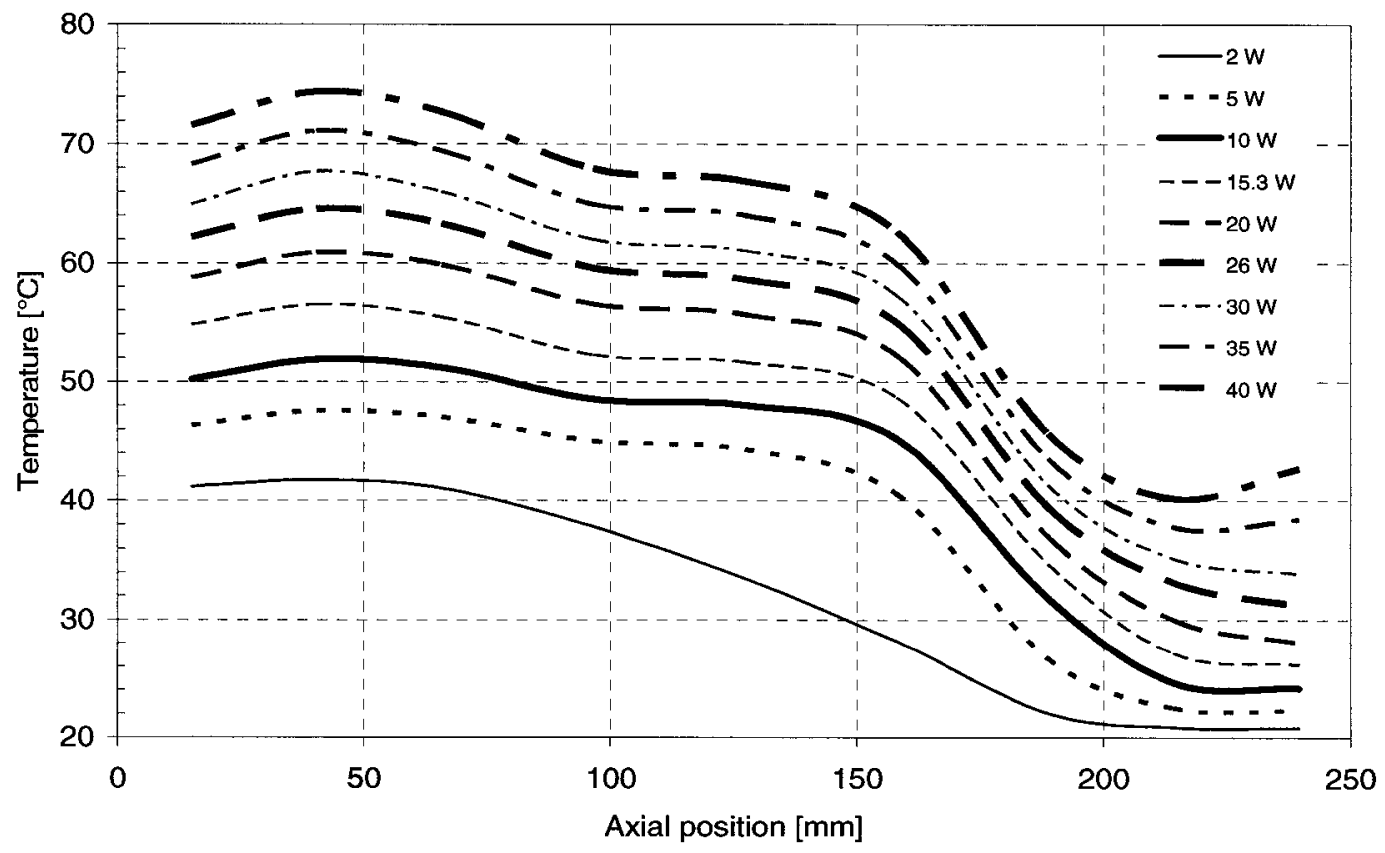

Figure 3.88 Nickel foam heat pipe axial temperature profile (insulated, horizontal, enhanced convection) [sample(s): HP062400] 


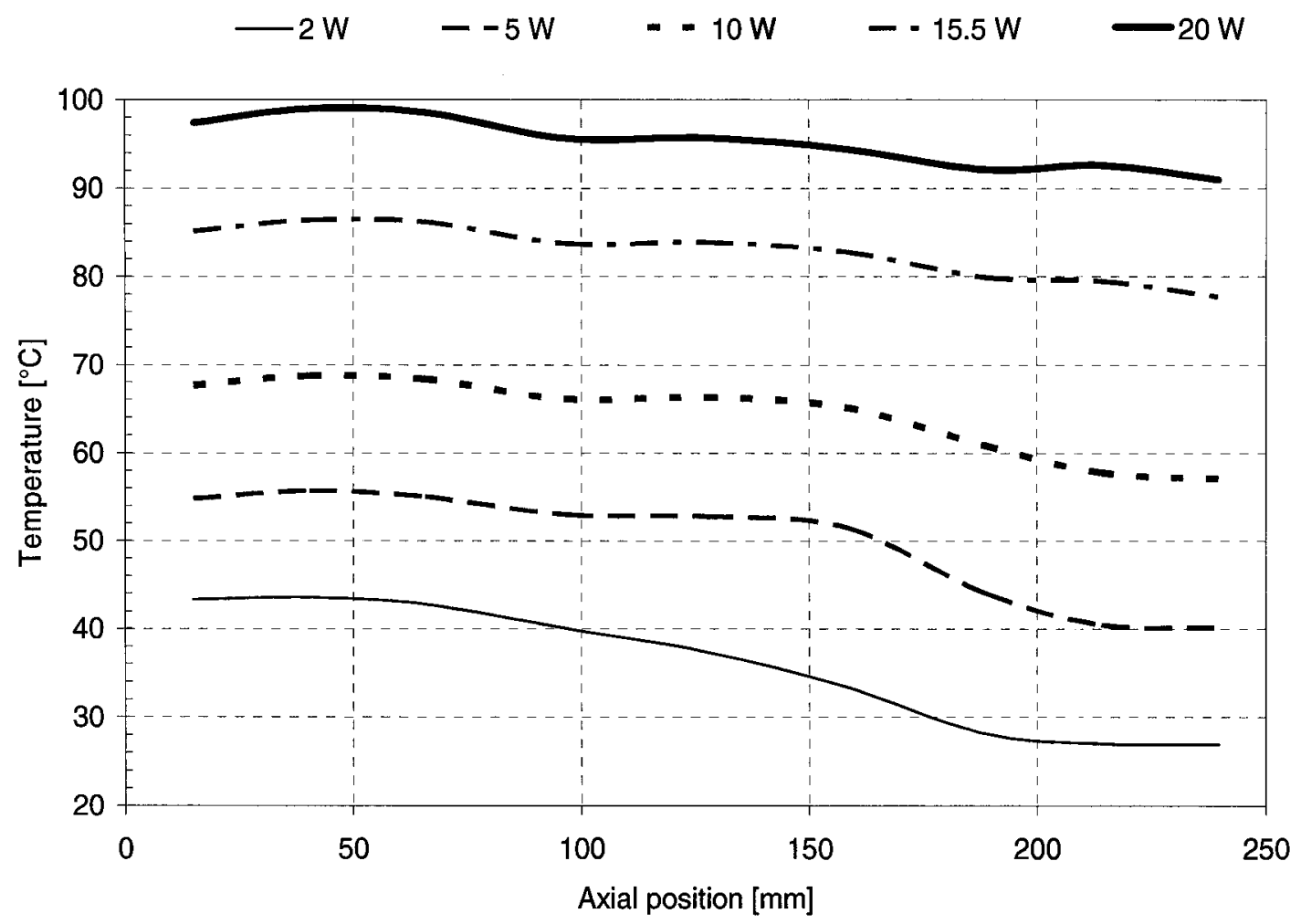

Figure 3.89 Nickel foam heat pipe axial temperature profile (insulated, gravity assisted vertical, natural convection) [sample(s): HP062400]

Figure 3.89 illustrates the axial temperature profile for the nickel sample at the gravity assisted vertical orientation, and natural convection cooling condition. Compared with its copper mesh wick counterparts, the nickel sample curves tend to be more flat with ascending power level. This indicates that the nickel sample would work more efficiently at higher power as compared with the copper mesh heat pipe with gravity assist. 


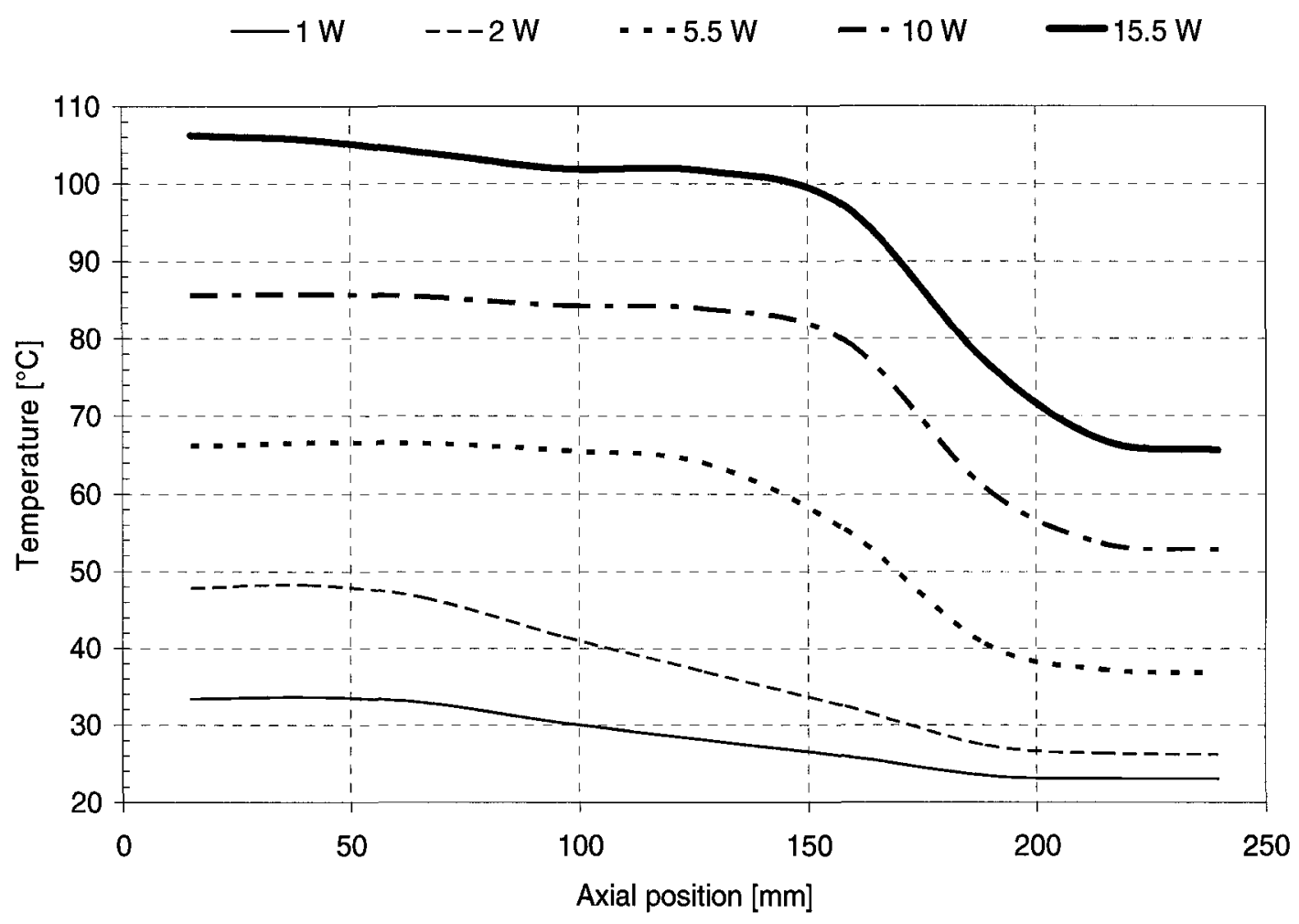

Figure 3.90 Nickel foam heat pipe axial temperature profile (insulated, against gravity vertical, natural convection) [sample(s): HP062400]

Figure 3.90 illustrates the axial temperature profile for the nickel sample at the against gravity vertical orientation, and with natural convection cooling. Comparing with its copper mesh wick counterparts, the nickel sample curves still keep the same shapes as these at the other two orientations, showing insensitiveness to orientation. The nickel sample characteristics distinguish it from its copper mesh wick counterparts. This is due to the nickel foam wick fine porous structure. This effect provides a stronger capillary force than that of the copper wire screen wick. It therefore ensures the wick be saturated at any orientations. 


\subsection{Heat Pipe Axial Temperature Profile regarding Cooling Approach}

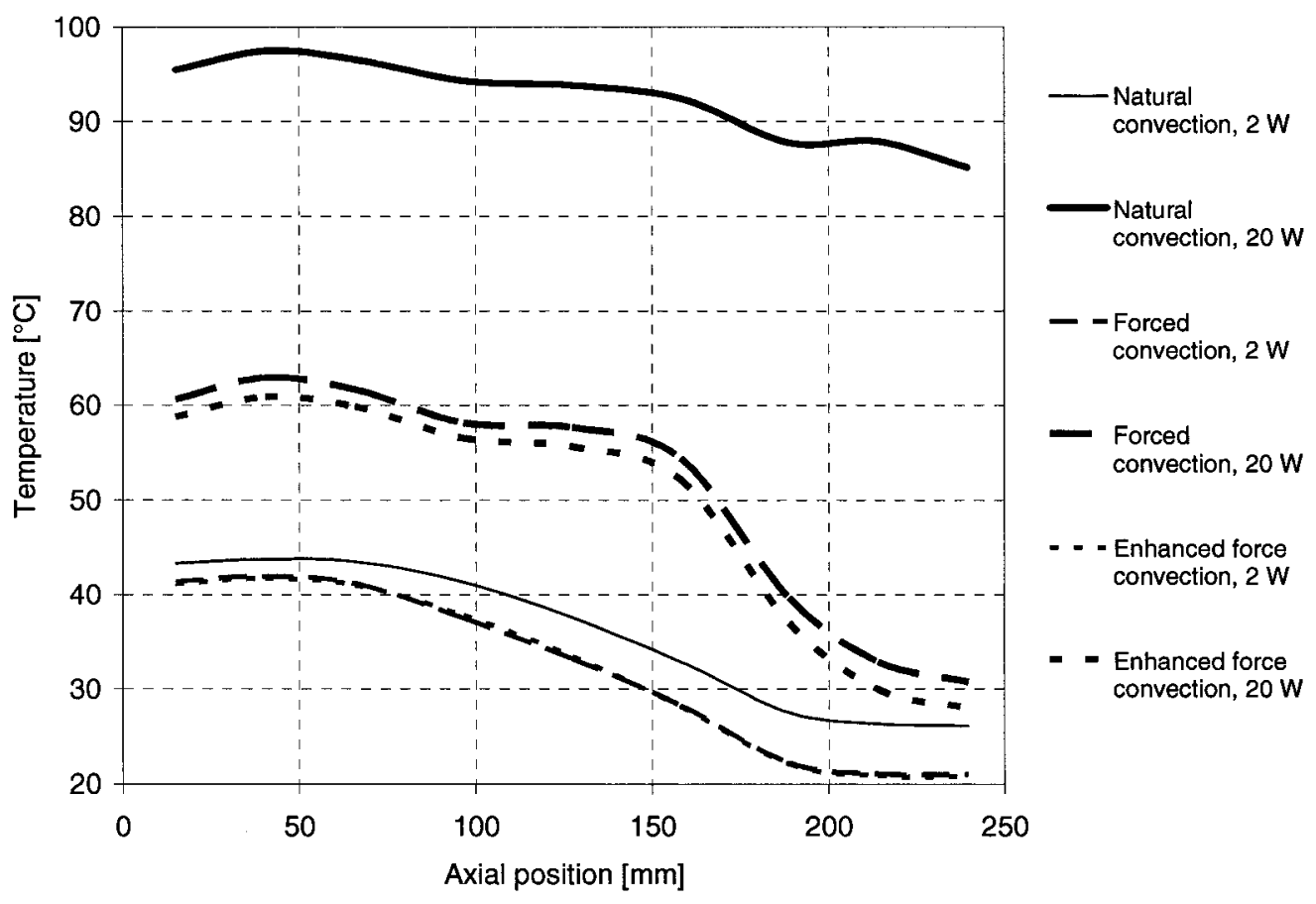

Figure 3.91 Nickel foam heat pipe axial temperature profile regarding cooling approach (insulated, horizontal) [sample(s): HP062400]

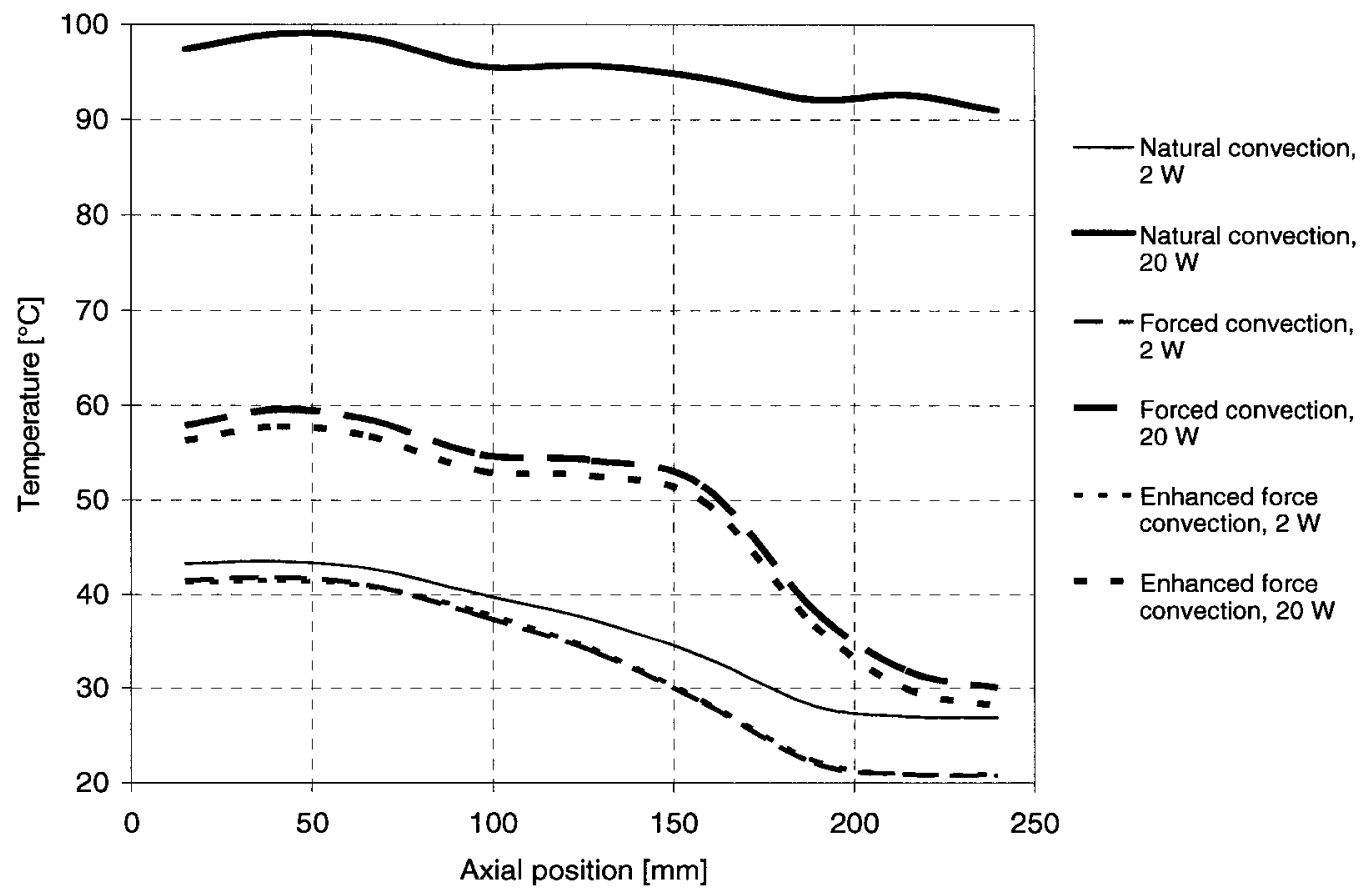

Figure 3.92 Nickel foam heat pipe axial temperature profile regarding cooling approach (insulated, gravity assisted vertical) [sample(s): HP062400] 


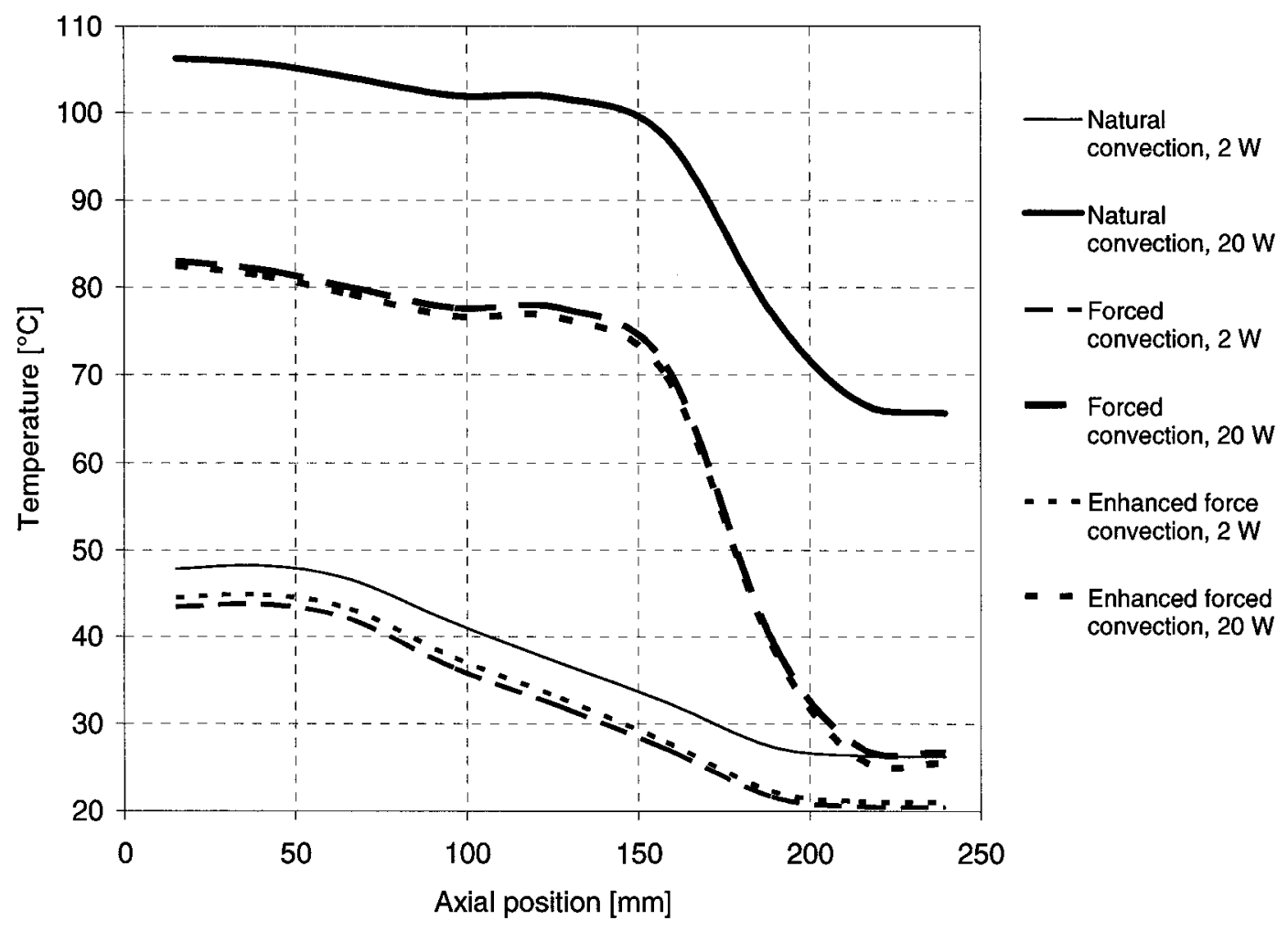

Figure 3.93 Nickel foam heat pipe axial temperature profile regarding cooling approach (insulated, against gravity vertical) [sample(s): HP062400]

Figures 3.91 to 3.93 illustrate the axial temperature profile for the nickel sample regarding cooling approaches, and at horizontal, gravity assisted, and against gravity vertical orientations respectively.

These three figures show that at any orientation the curves are divided into three groups; high temperature group composed of only one curve, natural convection high power, medium temperature group composing of two curves, forced convection high power and enhanced forced convection high power, and the low temperature group composing of all three low power curves. From these it can be concluded that the nickel foam wick sample has a very low cooling sensitivity at lower power, and in any orientation. At high power it is only sensitive to natural and forced convections. 


\subsection{Heat Pipe Axial Temperature Profile regarding Orientation}

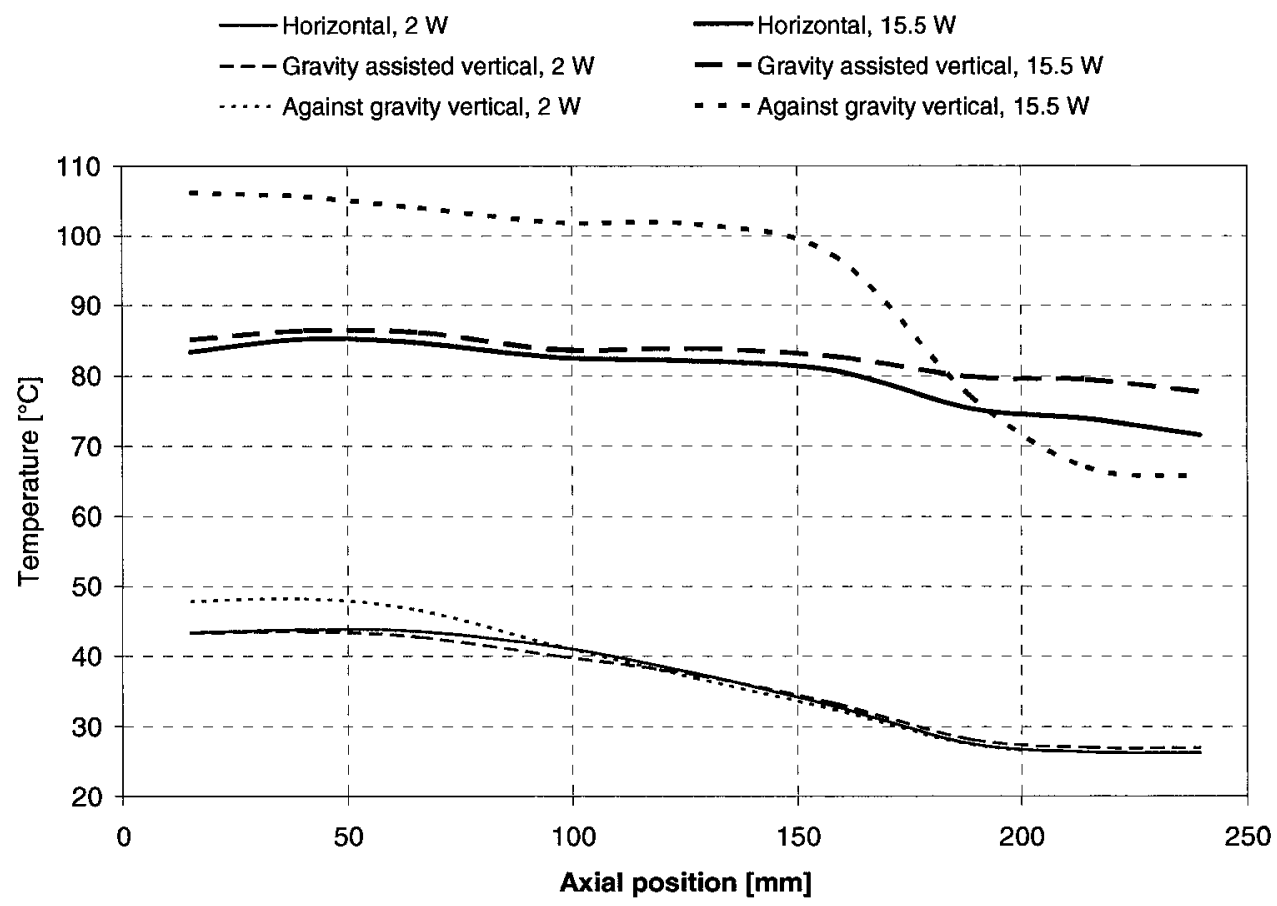

Figure 3.94 Nickel foam heat pipe axial temperature profile regarding orientation

(insulated, natural convection) [sample(s): HP062400]

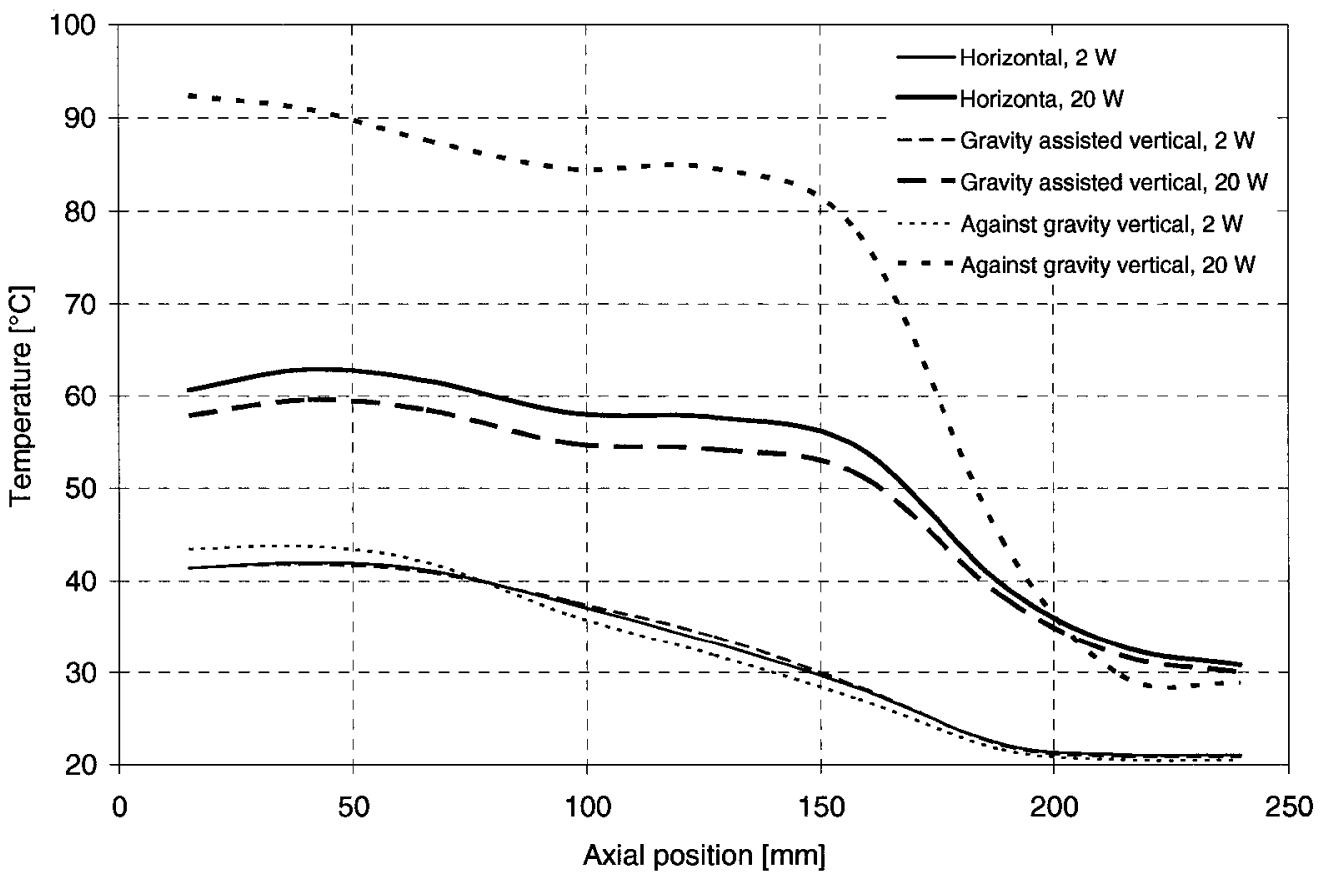

Figure 3.95 Nickel foam heat pipe axial temperature profile regarding orientation

(insulated, forced convection) [sample(s): HP062400] 


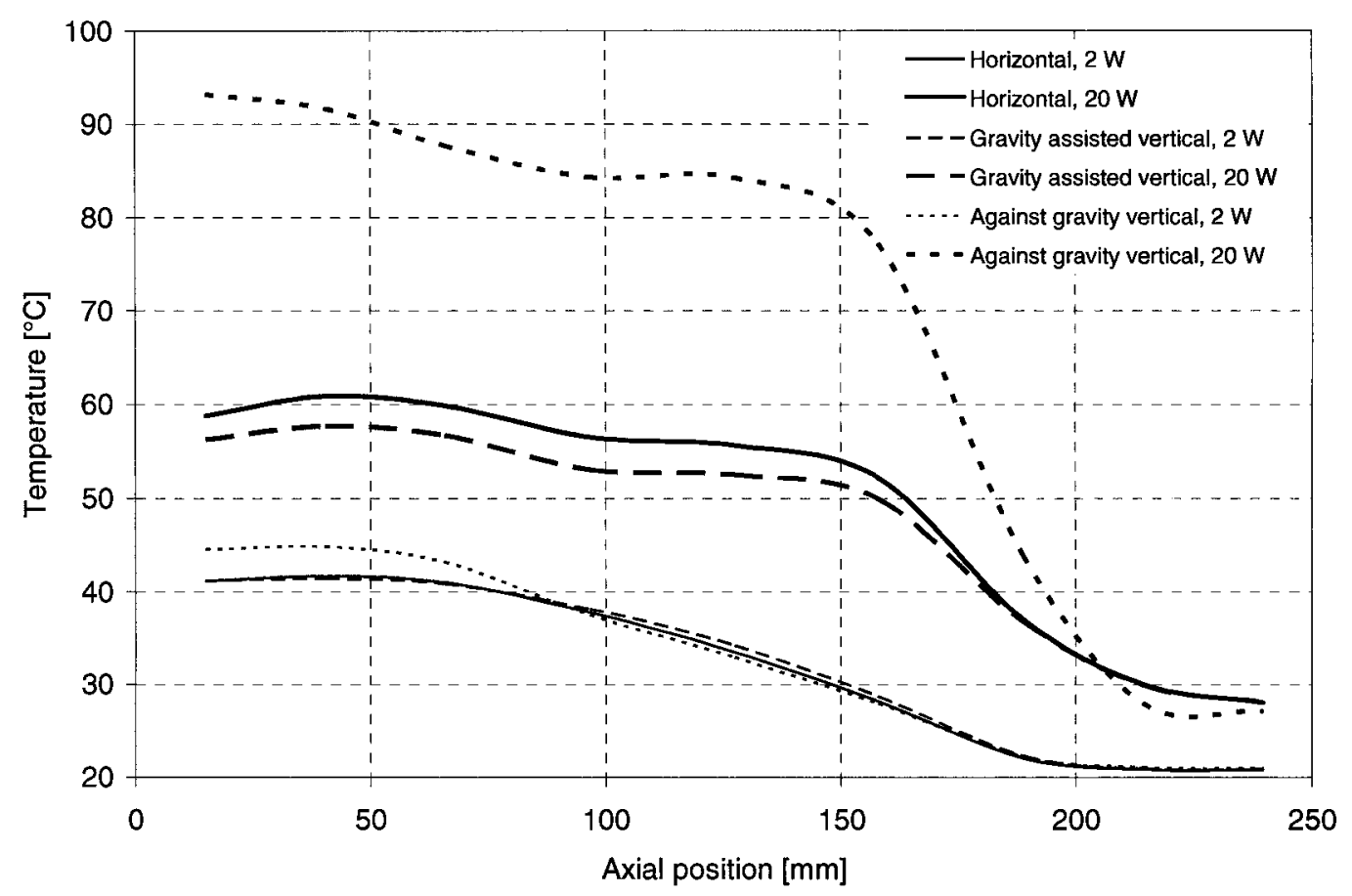

Figure 3.96 Nickel foam heat pipe axial temperature profile regarding orientation

(insulated, enhanced forced convection) [sample(s): HP062400]

Figures 3.94 to 3.96 illustrate the axial temperature profile for the nickel sample regarding orientation, natural, forced, and enhanced forced convection cooling approaches respectively. In these three figures, the curves can also be divided into three groups; the high temperature group composed of only one curve, against gravity vertical with high power; the medium temperature group composed of two curves, horizontal orientation high power and gravity assisted vertical orientation high power; and the low temperature group composed of all the three low power curves. From these it can be concluded that the nickel foam wick sample has a very low sensitivity to orientation at lower power levels with any cooling approach. At high power input, it is only sensitive to the orientation of against gravity vertical. There is a difference between the natural and forced convections, but the strength of the forced convection does not affect the results much. 


\subsubsection{Evaporator-Condenser Temperature Difference Characteristics}

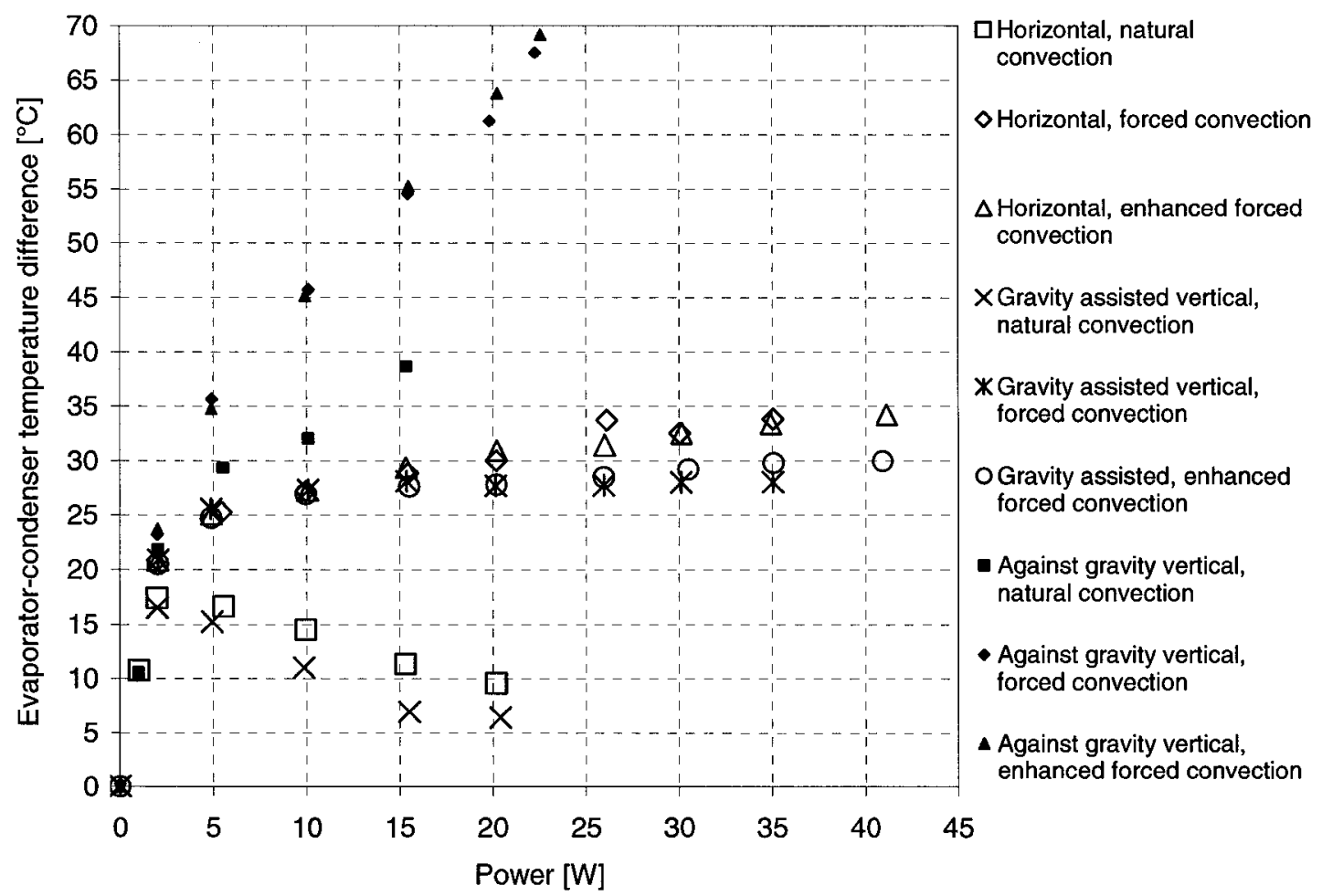

Figure 3.97 Nickel foam heat pipe evaporator-condenser temperature difference (insulated) [sample(s): HP062400]

Figure 3.97 illustrates the evaporator-condenser temperature difference under nine conditions. Like the copper mesh wick counterparts, the nickel foam wick sample evaporator-condenser temperature difference distribution can also be classified into three groups according to either orientation or cooling approach. The group of against gravity vertical orientation shows the largest values. Compared to the copper mesh wick counterparts, the value of the nickel sample in against gravity orientation, and with natural convection is far lower. The middle range group is composed of two scatter point curves at gravity assisted orientation, and with normal forced and enhanced forced convections, and two horizontal with normal forced and enhanced forced convections. The third group is composed of two curves with natural convections at either gravity 
assisted and against gravity orientations. This curve tends to move opposite to the other two groups; it decreases with the increasing power. This also makes the nickel samples distinct from the copper counterparts under the same circumstances. This may also be due to the unique nickel foam wick characteristics distinguishing it from the copper counterparts.

\subsubsection{Axial Thermal Resistance and Effective Conductivity}

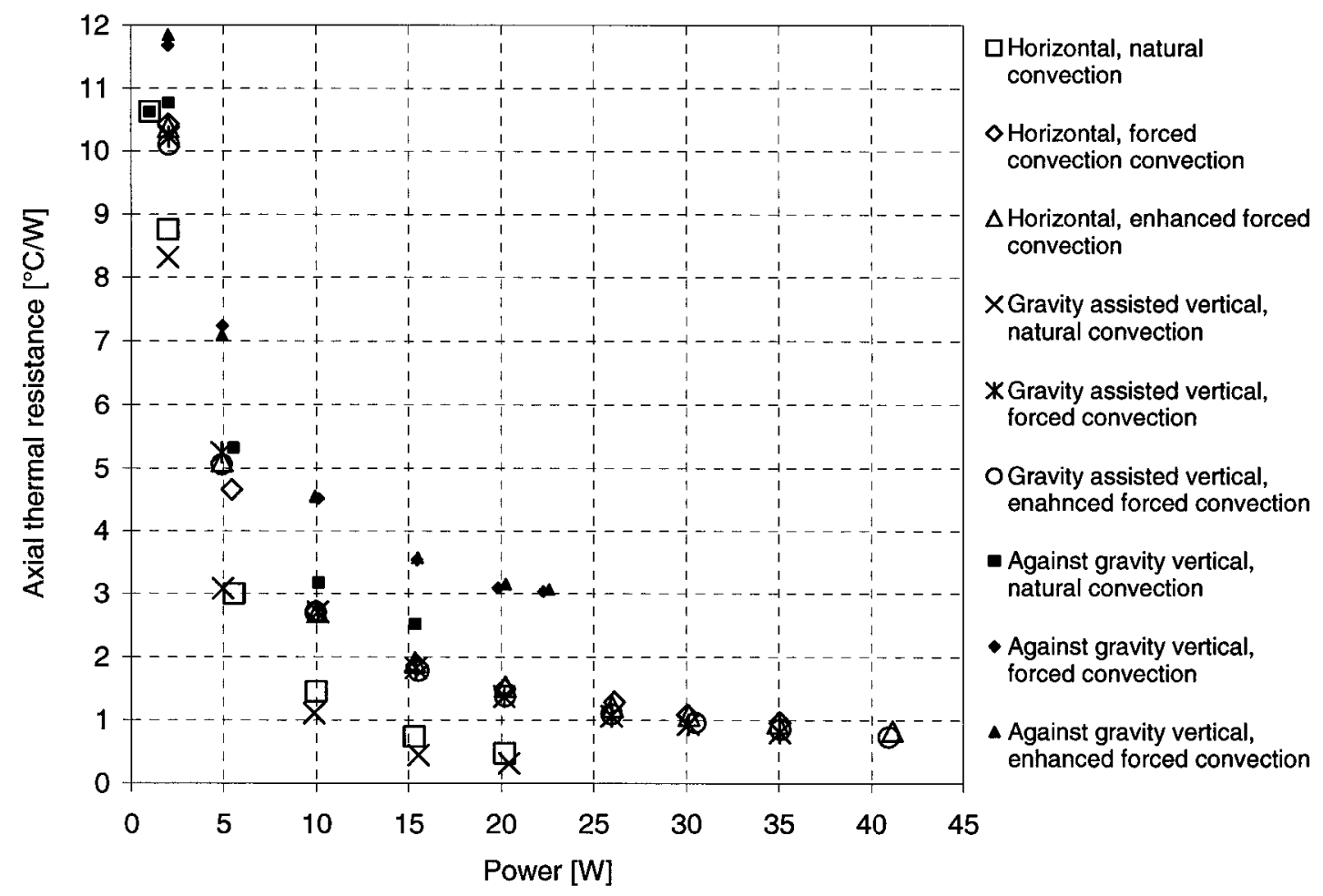

Figure 3.98 Nickel foam heat pipe axial thermal resistance (insulated) [sample(s): HP062400]

Figure 3.98 illustrates the axial thermal resistance for the nickel foam sample under the same circumstances. From the figure it can be seen that all the values decrease with increasing power. This is consistent with its copper counterparts.

Due to the fact that the nickel sample has a bigger diameter and a longer adiabatic length, it will not be comparable with its copper counterparts only by axial thermal 
resistance. Effective axial thermal conductivity makes it comparable with other test samples. The following content illustrates comparisons between the nickel sample and its copper counterparts by means of effective axial thermal conductivity.

Figure 3.99 illustrates the effective axial thermal conductivities under the same circumstances. From this figure it can be seen that the nickel foam heat pipe effective axial thermal conductivities are far more distinct from those of other test samples in either horizontal or gravity assisted orientations.

Figures 3.99 to 3.102 illustrate comparisons in the effective thermal conductivity of nickel samples with copper counterparts in horizontal orientations and with three cooling approaches. From the figures it can be seen that with any cooling approach, there is a same tendency that the nickel sample value is lower than other test samples but has an ascending moving tendency with power level increasing. While other test samples possess the opposite moving trends. This is due to two factors; first the nickel sample has a larger diameter and a tube wall thickness. At the relative low power level, its heat pipe effect is offset by the large percentage copper. Its performance is more similar to a conductance device. However with the increasing power, the fluid phase activity dominates, hence it begins to work as a real heat pipe.

In Figures 3.103 to 3.105 , it is clear that, with gravity assisted vertical orientation the nickel sample tends to have lower effective axial thermal conductivity, with any cooling approach. 


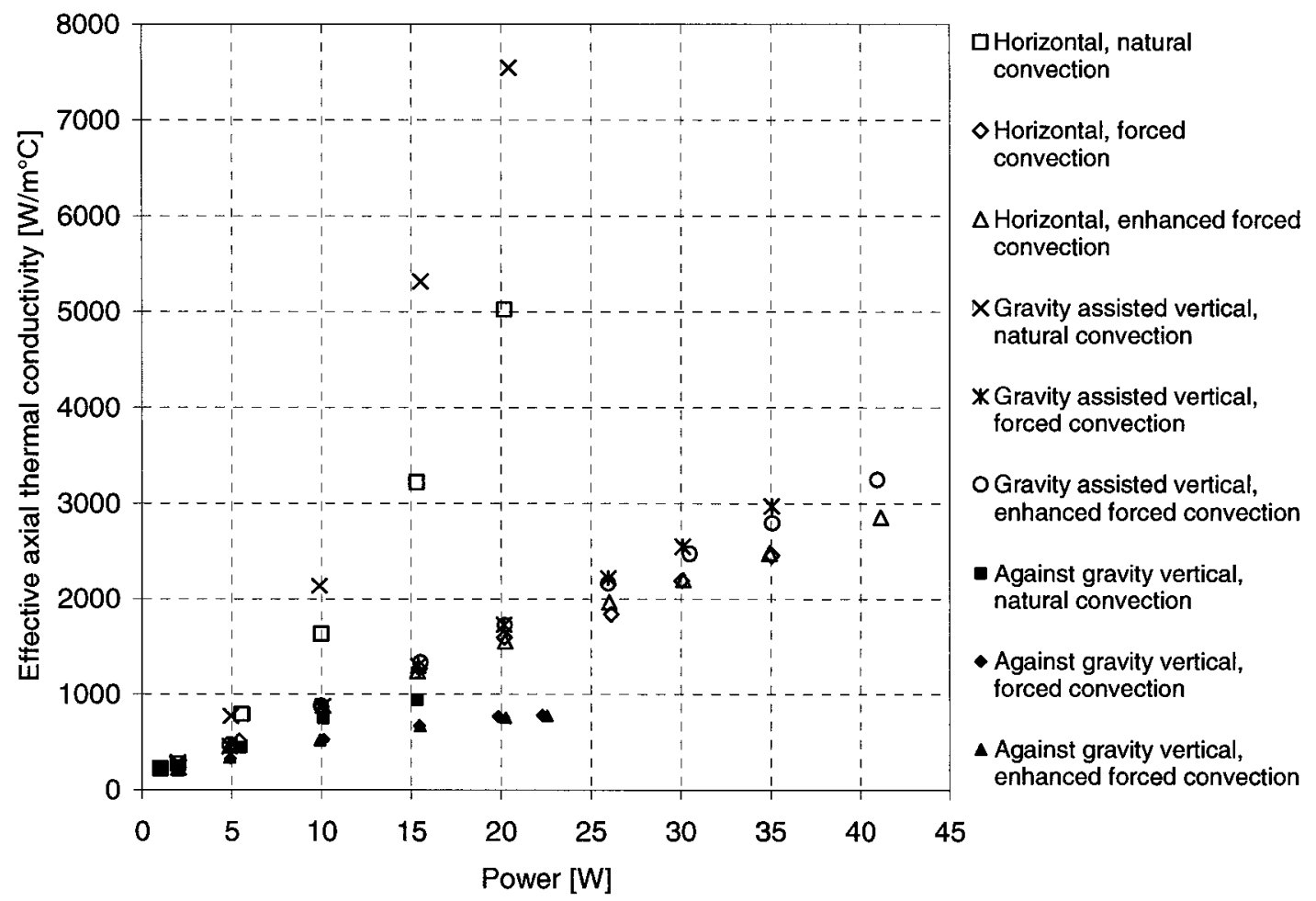

Figure 3.99 Nickel foam heat pipe effective axial thermal conductivity (insulated) [sample(s): HP062400]

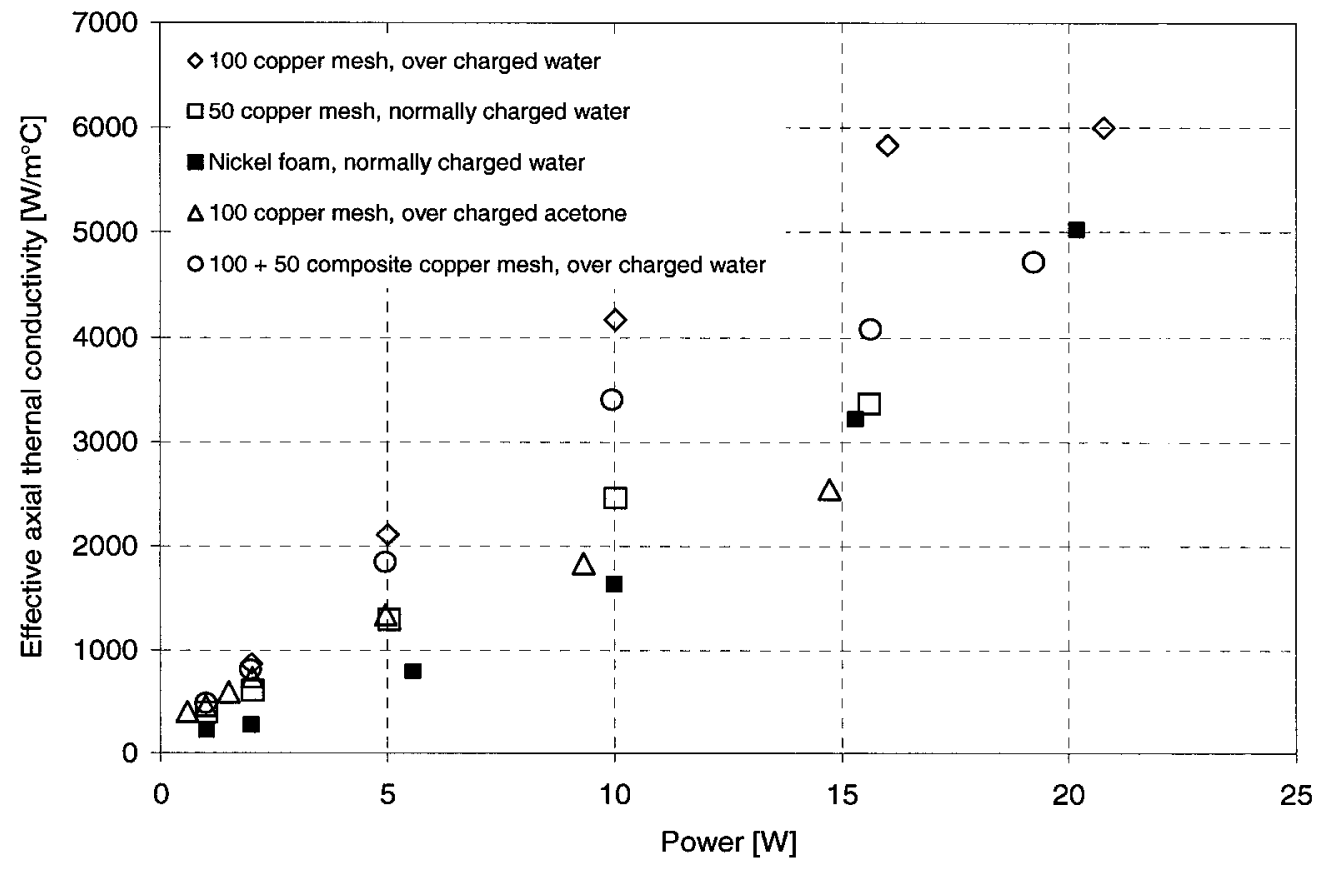

Figure 3.100 Comparison of nickel foam heat pipe effective axial thermal

conductivity with others (insulated, horizontal, natural convection) [sample(s): HP052502, HP071200, HP062400, HP071402, HP071301] 


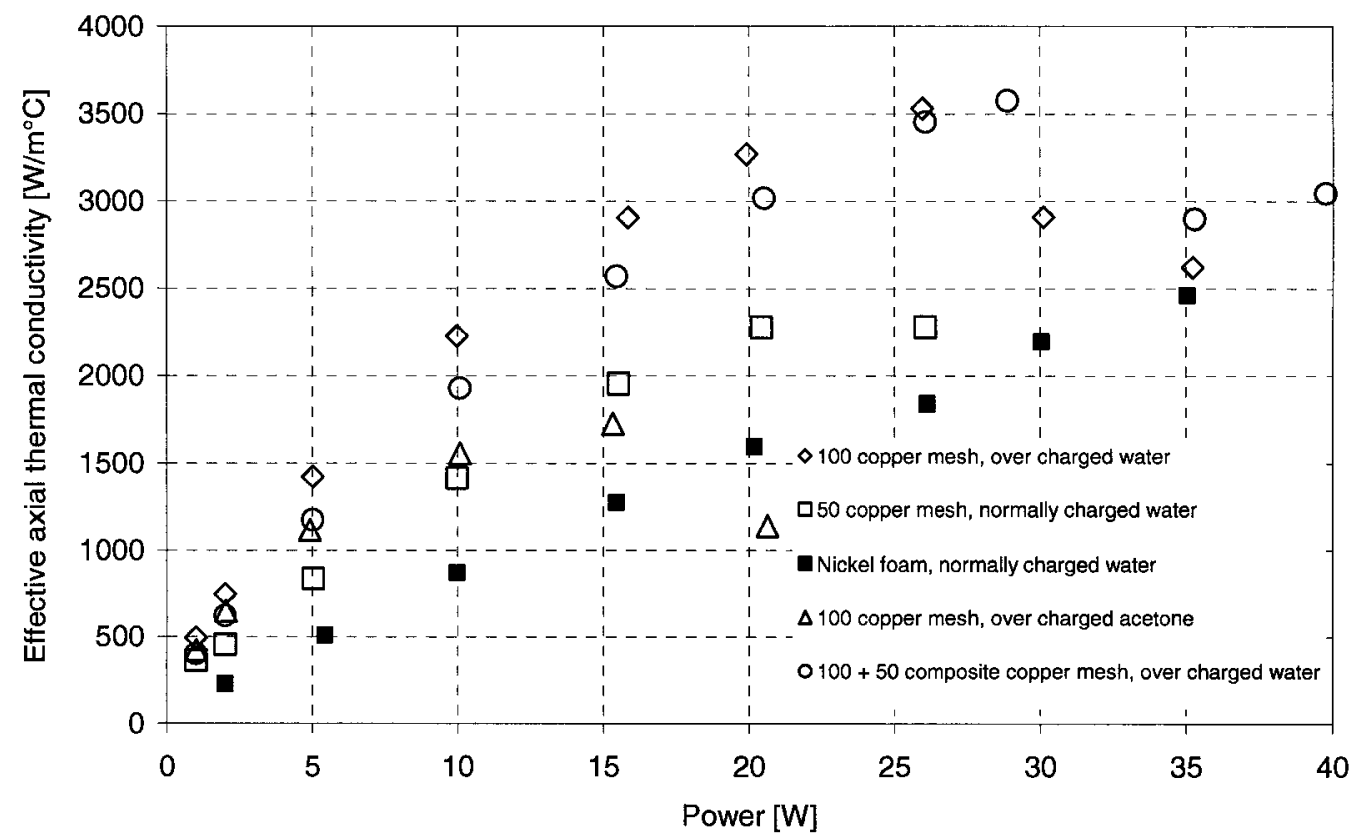

Figure 3.101 Comparison of nickel foam heat pipe effective axial thermal

conductivity with others (insulated, horizontal, forced convection) [sample(s): HP051701, HP071200, HP062400, HP071402, HP071301]

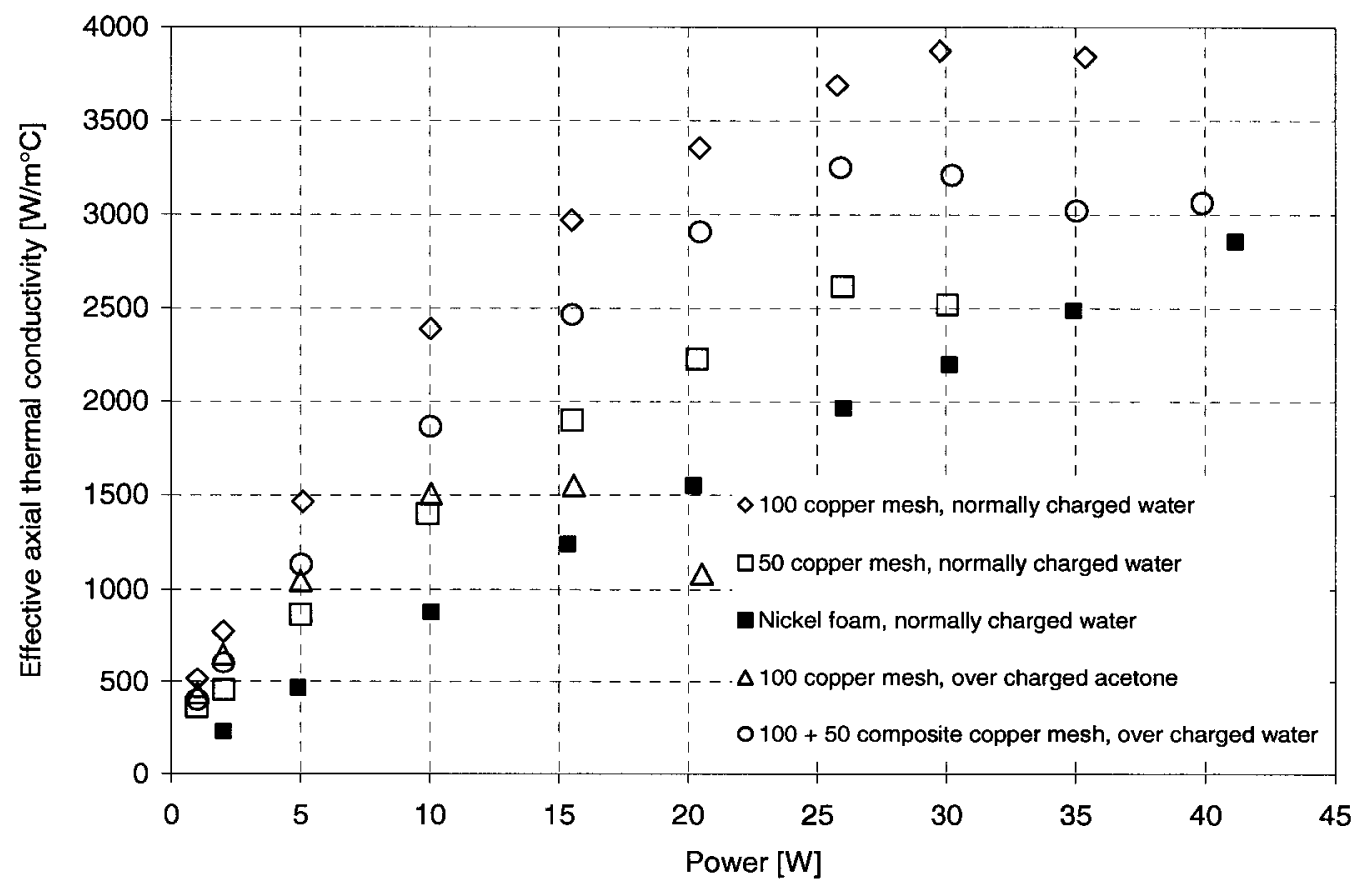

Figure 3.102 Comparison of nickel foam heat pipe effective axial thermal

conductivity with others (insulated, horizontal, enhanced forced convection) [sample(s): HP051701, HP071200, HP062400, HP071402, HP071301] 


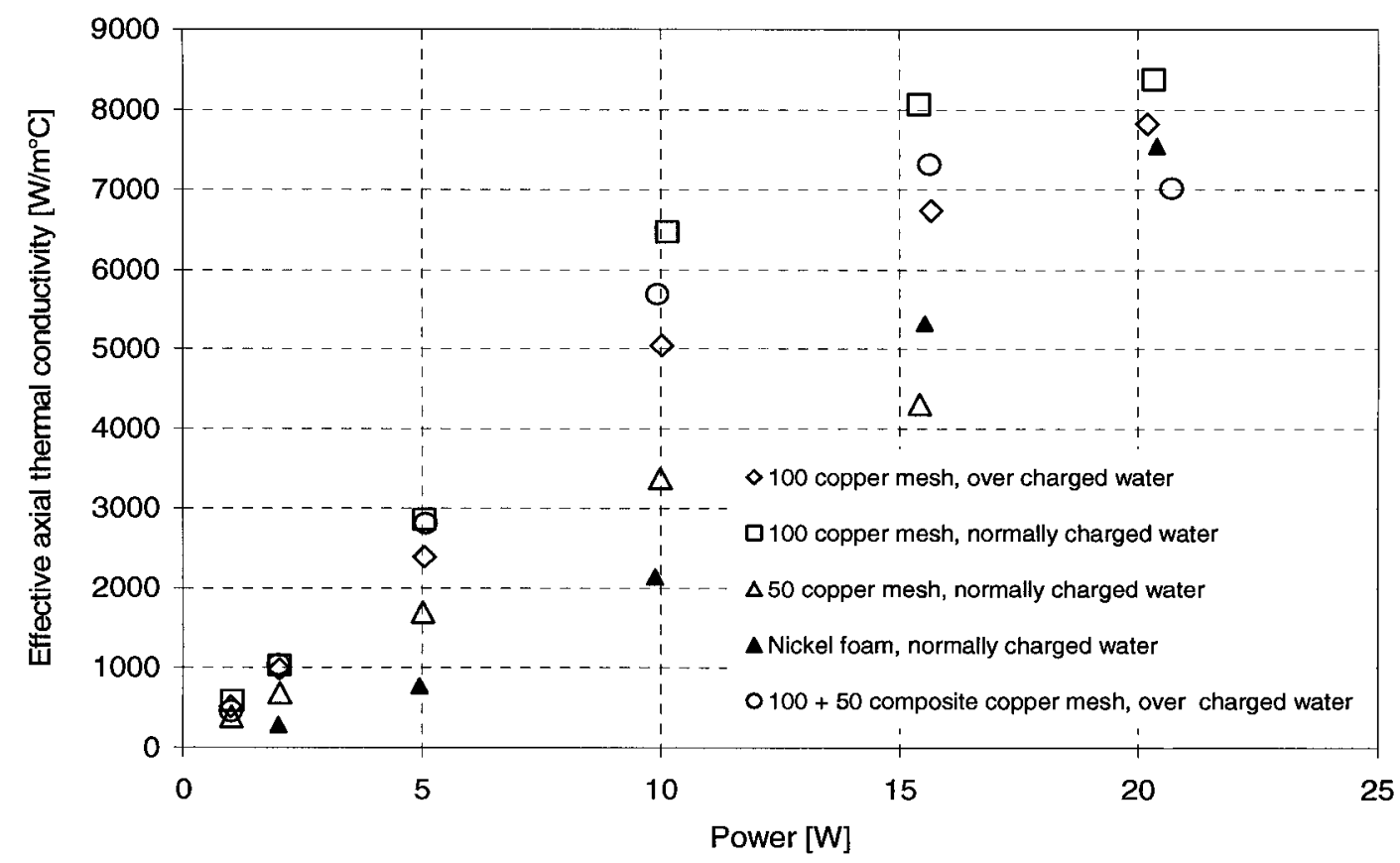

Figure 3.103 Comparison of nickel foam heat pipe effective axial thermal conductivity with others (insulated, gravity assisted vertical, natural convection) [sample(s): HP052502, HP051701, HP071200, HP062400, HP071301]

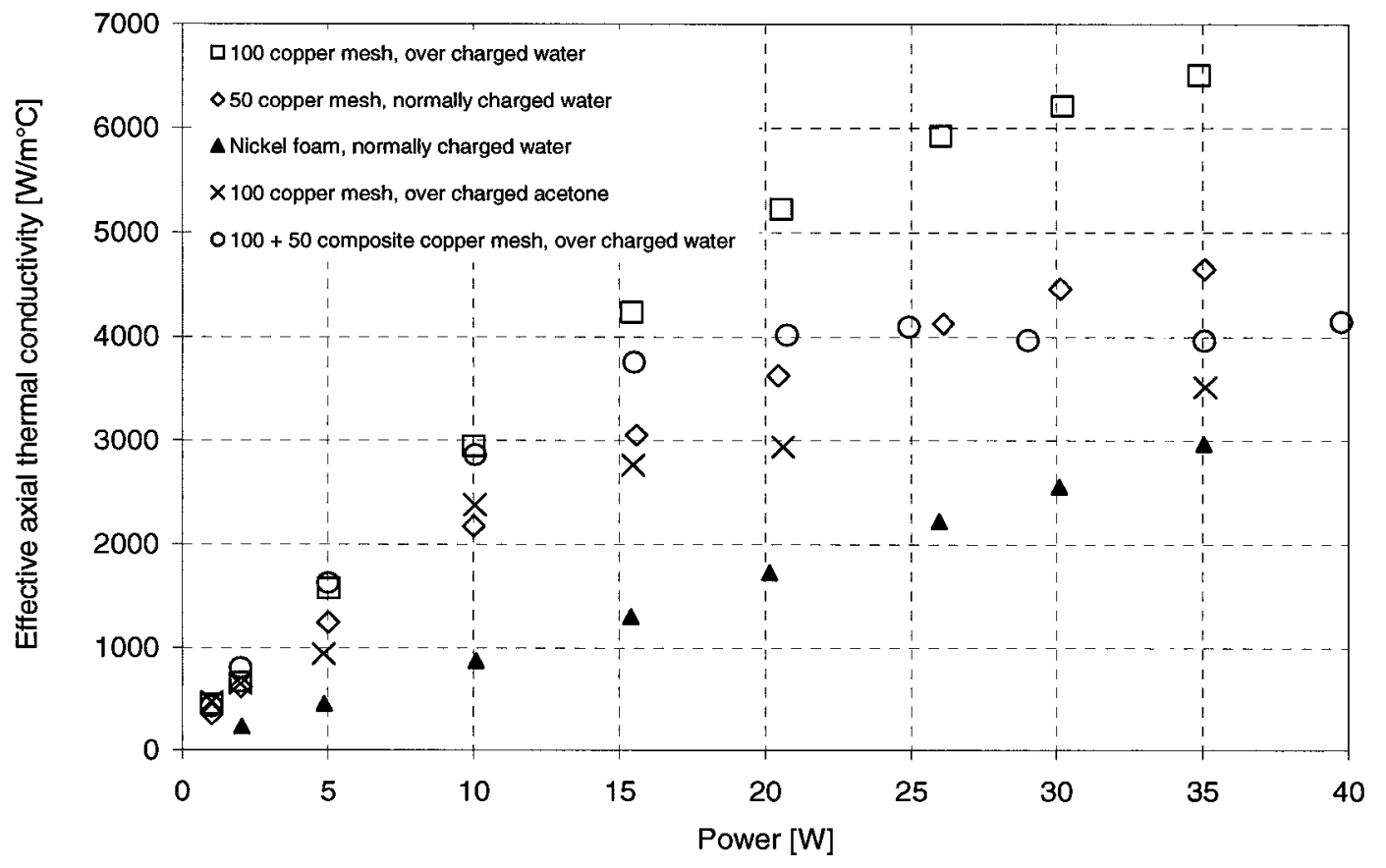

Figure 3.104 Comparison of nickel foam heat pipe effective axial thermal

conductivity with others (insulated, gravity assisted vertical, forced convection) [sample(s): HP052502, HP071200, HP062400, HP071402, HP071301] 


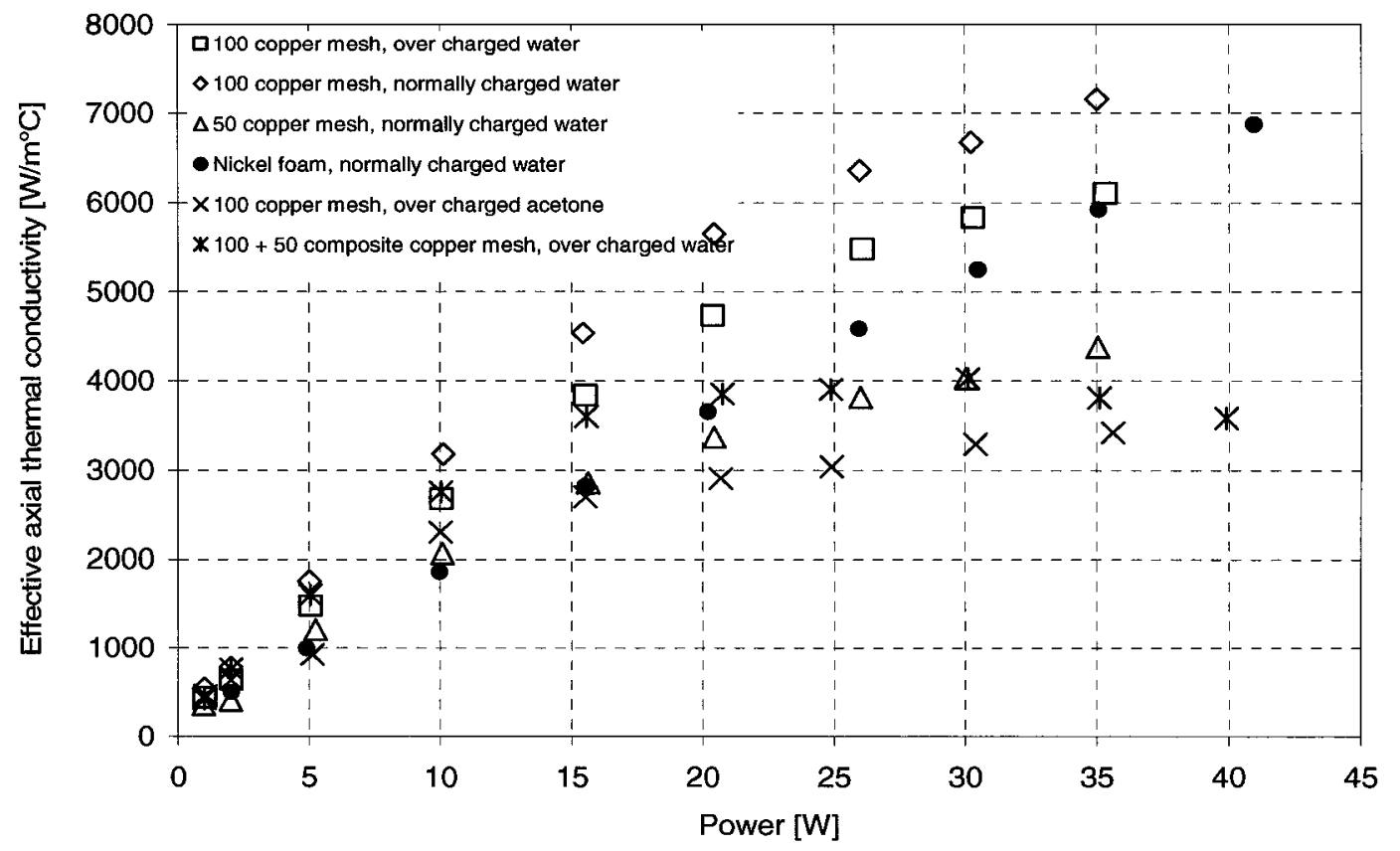

Figure 3.105 Comparison of nickel foam heat pipe effective axial thermal

conductivity with others (insulated, gravity assisted vertical, enhanced forced convection) [sample(s): HP052502, HP071200, HP071200, HP062400, HP071402, HP071301]

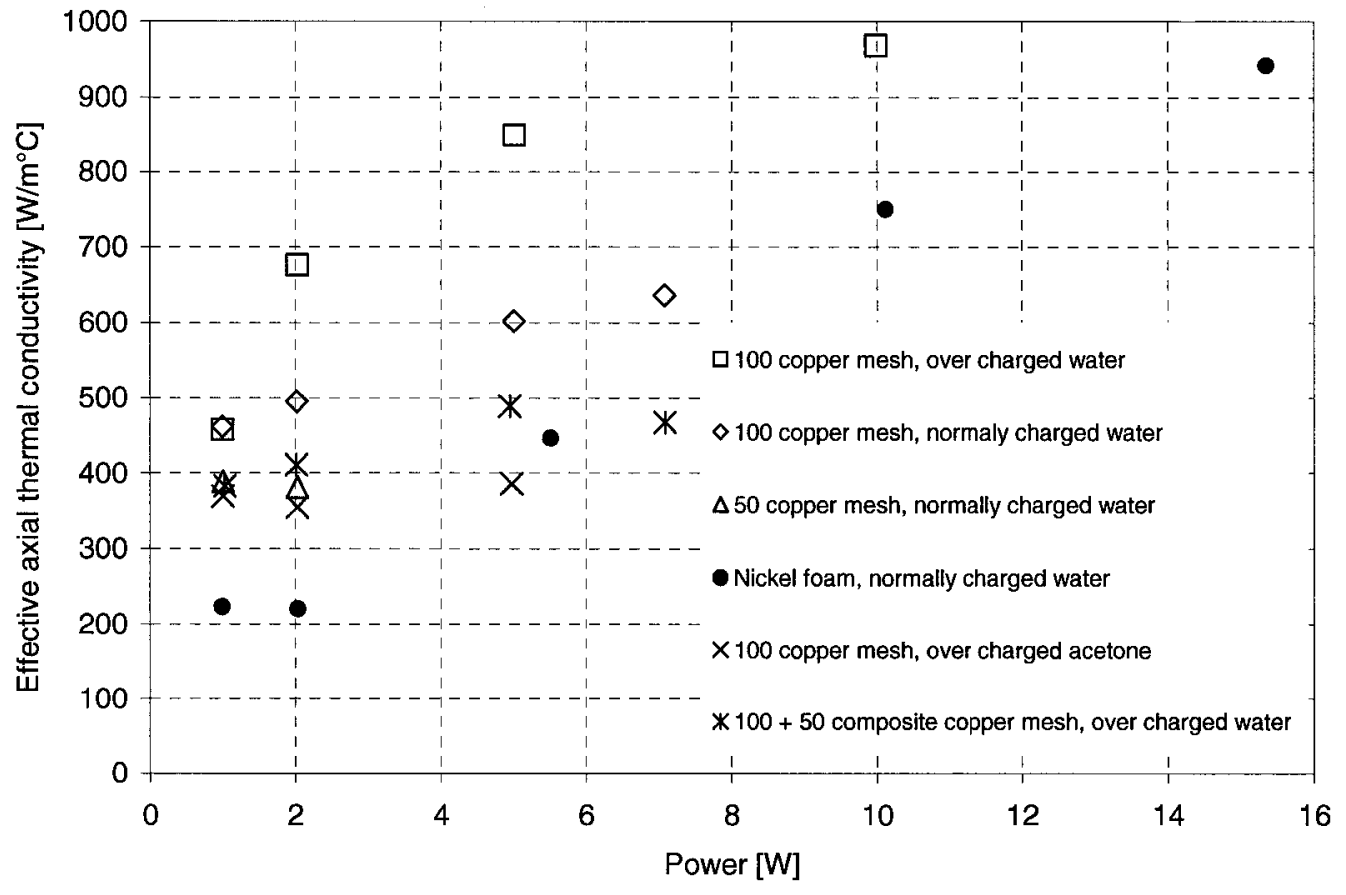

Figure 3.106 Comparison of nickel foam heat pipe effective axial thermal conductivity with others (insulated, against gravity vertical, natural convection) [sample(s): HP052502, HP071200, HP071200, HP062400, HP071402, HP071301] 


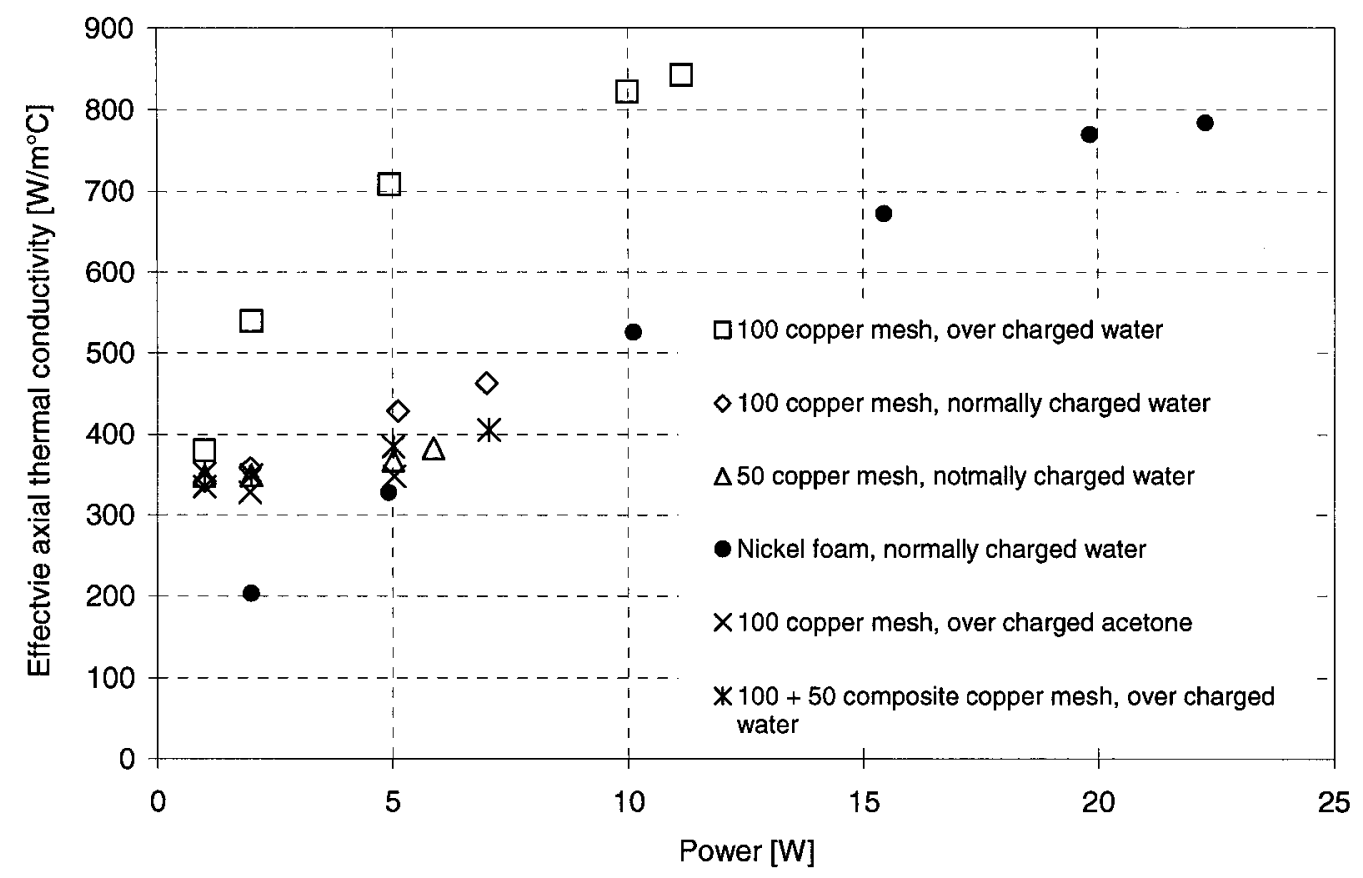

Figure 3.107 Comparison of nickel foam heat pipe effective axial thermal conductivity with others (insulated, against gravity vertical, forced convection) [sample(s): HP052502, HP071200, HP071200, HP062400, HP071402, HP071301]

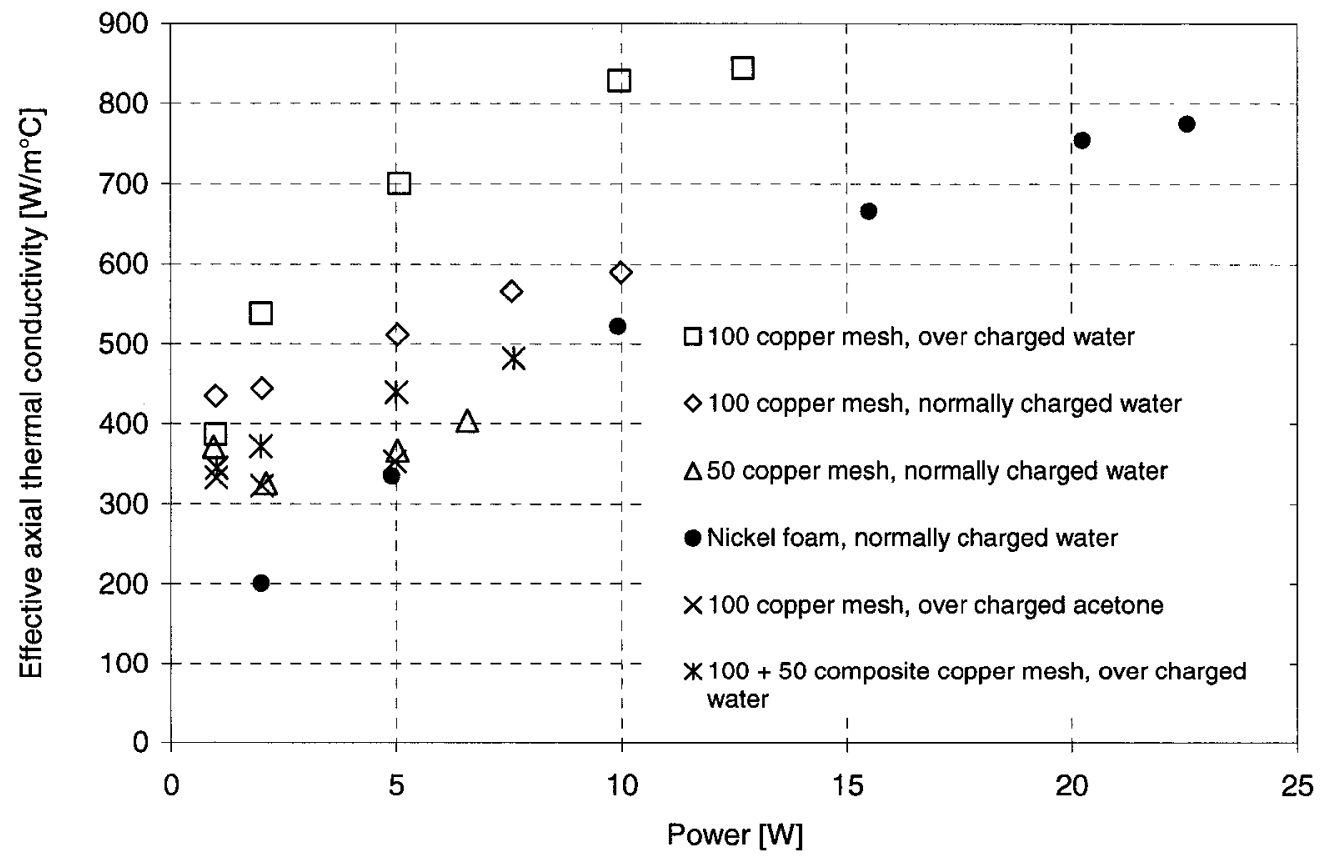

Figure 3.108 Comparison of nickel foam heat pipe effective axial thermal

conductivity with others (insulated, against gravity vertical, enhanced forced convection) [sample(s): HP052502, HP071200, HP071200, HP062400, HP071402, HP071301] 
From Figures 3.106 to 3.108 , we can see that compared with copper counterparts, at same power levels, the nickel sample performed worse in terms of axial effective thermal conductivity. It has a wider working power range than the copper counterparts at orientations of against gravity, with respect to the same evaporator temperature constraint.

Reviewing all the experimental results about the nickel sample, we find that the nickel sample performed worse than its counterparts in terms of axial effective thermal conductivity under all circumstances. These are mainly related to such factors as that of the wick structure as well as material thermal conductivity. The thermal conductivity for nickel is $91 \mathrm{~W} / \mathrm{mK}$; while for copper it is $380 \mathrm{~W} / \mathrm{mK}$ that is over 4 times greater than that of nickel material. This difference would result in a great difference in the wick effective thermal conductivity and is believed to be the main reason there is a reduced resulting performance of the nickel sample. Another factor is the wick structure. The nickel foam and the copper screen wire mesh have different structures. The information on the wick structure of the nickel foam we used is very limited. This causes difficulties in comparing the nickel foam and the copper wire screen meshes in the wick structure.

\subsubsection{Conclusion of Heat Pipe Testing}

Experiment 3 is the core part of this heat pipe investigation. During this process, a total of five heat pipe samples were tested, and each of them experienced three orientations, and three cooling approaches at each orientation. Based on the results and related analyses, we draw the following conclusions:

1. Heat pipe heat transfer capacity is several times greater than that of the same dimensioned metal device at similar temperature constraints. 
2. Heat pipe heat transfer effectiveness, explained in forms of effective axial thermal conductivity, is up to 20 times higher than its base material, copper

3. Heat pipe characteristics depend on intrinsic configurations, i.e., wick structures, working fluids, amount of working fluid. The comprehensive performance of a heat pipe depends on the synergistic effect of those factors.

4. Heat pipe performances vary with external situations, i.e., condensation conditions, gravitational force. Forced convection cooling helps improve effectiveness but decrease efficiency. Gravity assisted orientation helps little in heat transfer capacity, and helps more in efficiency; against gravity orientation decreases much in both effectiveness and efficiency, and even causes some samples to cease functioning.

5. Dry-out occur in the cases of the fine mesh acetone sample and the composite mesh water sample, the former one experienced an un-recoverable dry-out, the latter one went through a self-recoverable dry-out.

\subsection{Experiment 4}

\section{Verification of Wind Effect on the Thermocouples on the Condenser}

As will be illustrated in Chapter 4, the actual measured results match the theoretical prediction very well in natural convection cooling cases. However, some discrepancies were observed between the actual and theoretical results. This is particularly evident in some forced or enhanced forced convection cases. The actual measurements were lower than predictions. Such phenomenon occurred in many heat pipe cases, and is repeatable. 
It was suspected the thermocouples on the condenser measured lower temperatures due to the influence from the cooling wind rather than the heat pipe itself.

As introduced in the early part of this chapter, the temperature is measured by thermocouples on the heat pipe external surface. The thermocouples on the heater, the evaporator, and the adiabatic section were wrapped with insulators; the condenser was left exposed to the ambient. This would not affect in the case of natural convection cooling. However, the problem is that whether these thermocouples may have been affected by the wind from the fan attached on the cooling sink at the condenser. From Figure 3.3 it can be seen that a pair of medium fibreboard supports are located at either side of the condenser, perpendicular to the slots of the sink. With such a structure, the wind from the sink is not supposed to blow directly on the thermocouples of the condenser. It is assumed that the condenser temperature measured is accurate. To verify this assumption and clarify the suspicions mentioned, further analysis is required for the investigation of the differences between the actual measurements and theoretical predictions. It is necessary to investigate the wind effect on the thermocouples.

\subsubsection{Experimental Objectives}

This experiment is designed to verify the wind effect from the sink on the thermocouples attached on the heat pipe condenser, specifically,

1. Investigating whether the cooling wind from the sink slots affects the thermocouple measurements.

2. If the thermocouples are affected, then to what extent and under what circumstances

3. Validate all related measurements 


\section{Section 1: Comparison of insulated vs. non-insulated thermocouples}

This section is one of the experiments verifying the wind effect on the thermocouples.

The purpose is to compare the thermocouple measurements with, and without insulation under the circumstance of forced convective cooling on the condenser.

Figure 3.109 illustrates the experiment setup and the thermocouple distribution along the test setup. From the figure it can be seen that at either side of the condenser, two groups of thermocouples are respectively fixed on the condenser surface and the copper base. For each group, one thermocouple is wrapped with insulator (TC203 and TC205), and the other one is not wrapped (TC204 and TC206), instead exposing to the ambient. With the same procedure, to compare between the insulation and non-insulation cases, therefore to clarify the wind effect on the thermocouple measurements.

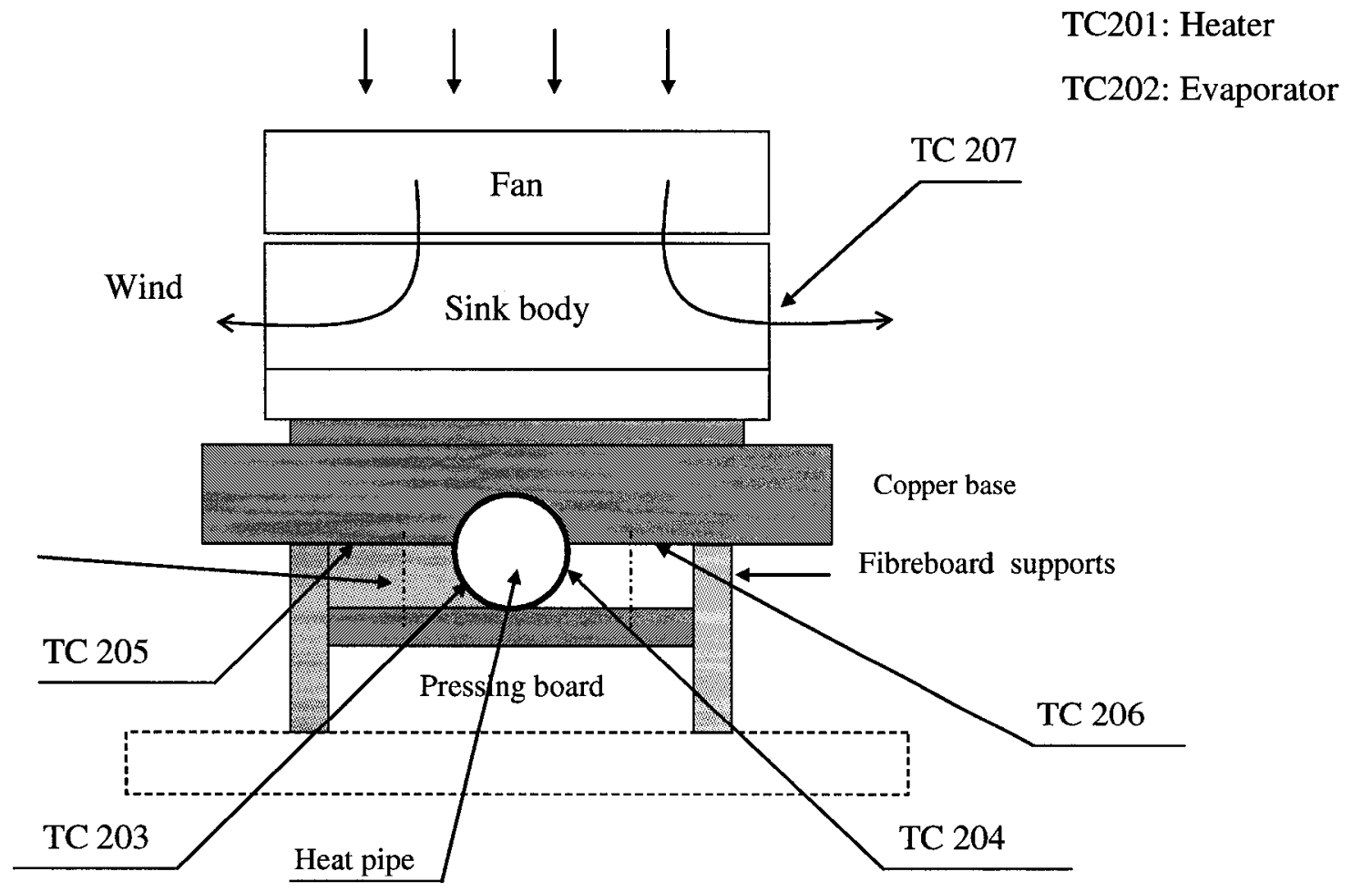

Figure 3.109 Setup and thermocouple distribution for the wind effect verification experiment, insulted vs. non-insulated 


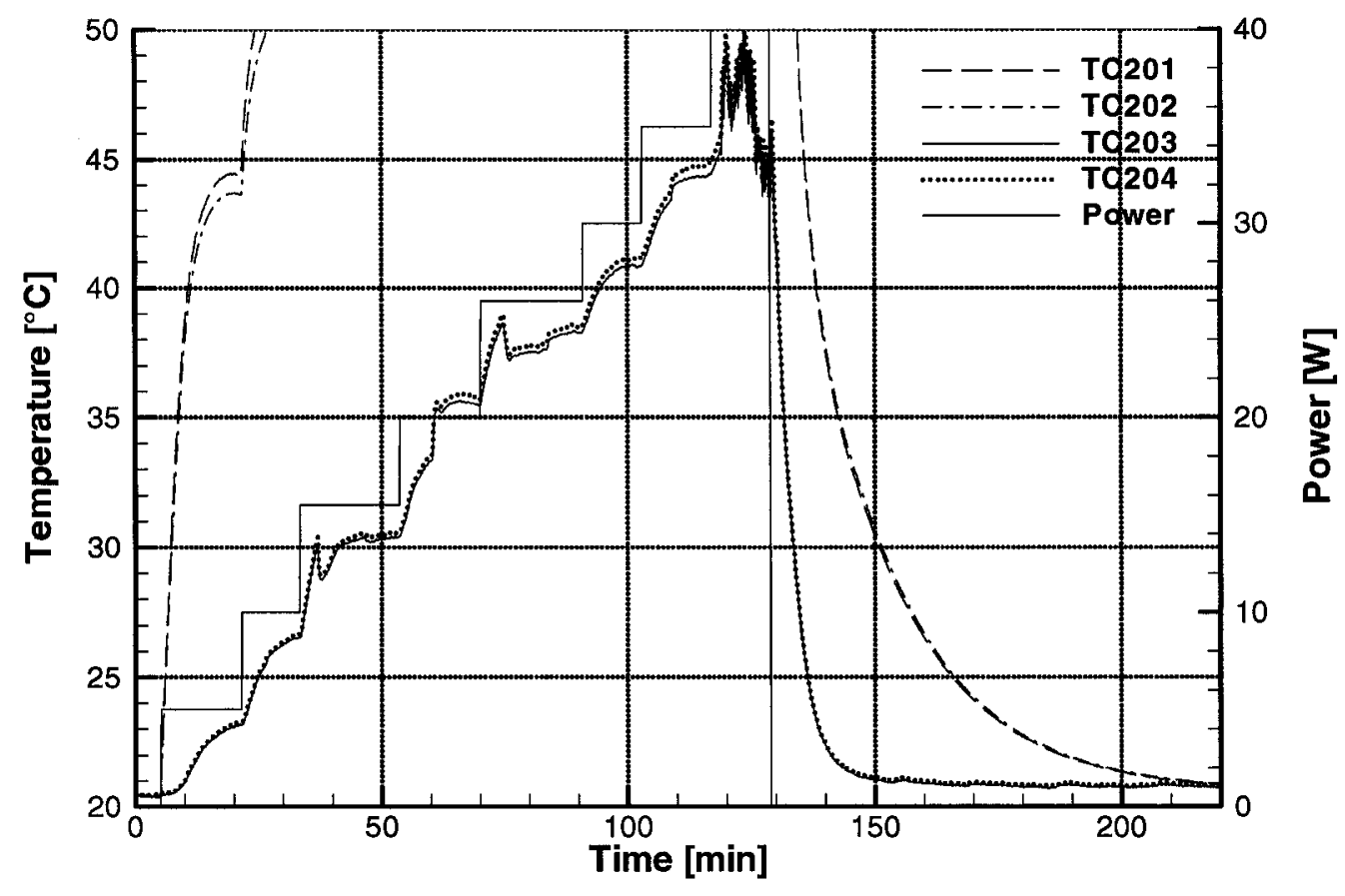

Figure 3.110 Comparison of thermocouple measurements with, and without insulation (horizontal, forced convection) [sample(s): HP071301]

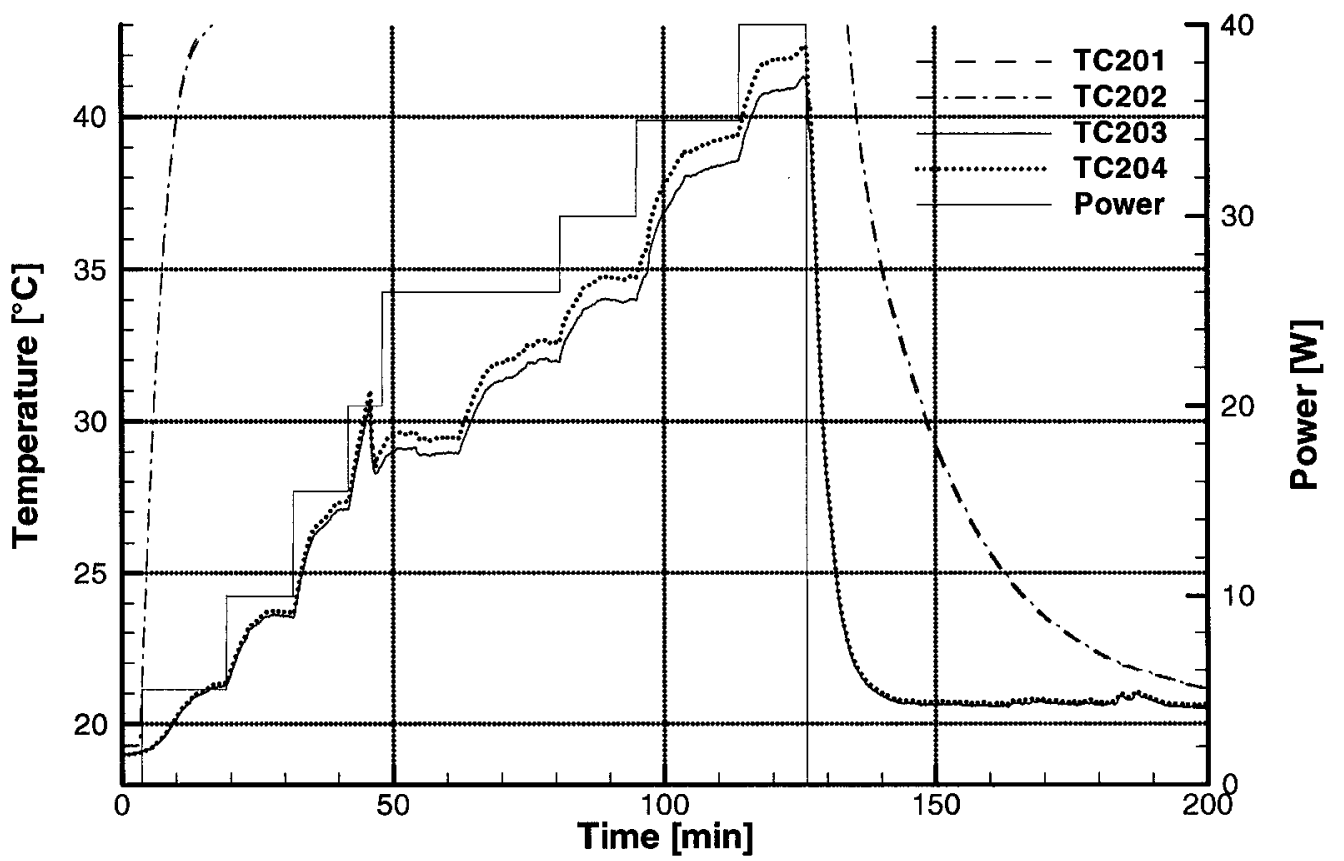

Figure 3.111 Comparison of thermocouple measurements with, and without insulation (horizontal, enhanced forced convection) [sample(s): HP071301] 
The experimental procedure is similar to those of the heat pipe tests as described in Experiment 3. In this experiment, we just need to do forced and enhanced forced convections with increasing power input tests. This gives stable power curves, telling the difference between the two groups of thermocouples.

Figures 3.110 and 3.111 show the curves obtained from the above procedure. From these curves it can be seen that both insulated and non-insulated temperature curves move synchronously, diverging with increases to power input. The maximum difference between the insulated and non-insulated thermocouples is about $0.5^{\circ} \mathrm{C}$ in the normal forced convection case and $1{ }^{\circ} \mathrm{C}$ in the enhanced forced convection case. It should be noted that opposite to our prediction, the temperatures with insulation are lower than those without insulation. An explanation for this would be that the insulation material not only acts as an insulator protecting the heat pipe from ambient but also helps the thermocouples contact the surface more firmly. This would ensure the measurement is more accurate. The following conclusions can be made:

1. The wind effect is far lower than the heat pipe dissipation.

2. The temperatures measured without insulation are at most 0.5 or $1^{\circ} \mathrm{C}$ higher than the real temperature of the condenser surface in the conditions of normal forced and enhanced forced convection.

\section{Section 2: Experiments with Wholly Enclosed Condenser}

This section was conducted under such a condition that the whole condenser, including the attached thermocouples was sealed with insulator. This was to ensure all the thermocouples are protected from the environment. Based on this, we can verify the conclusion from the proceeding section. 
Figure 3.112 shows the setup for this experiment. This setup adds an enclosure on the one described in the preceding section thus sealing the whole condenser space underneath the sink. Sealing the condenser protects all the thermocouples on the condenser from the environment. The thermocouples are arranged in the same way as the preceding section. The procedure is also the same as in the preceding section.

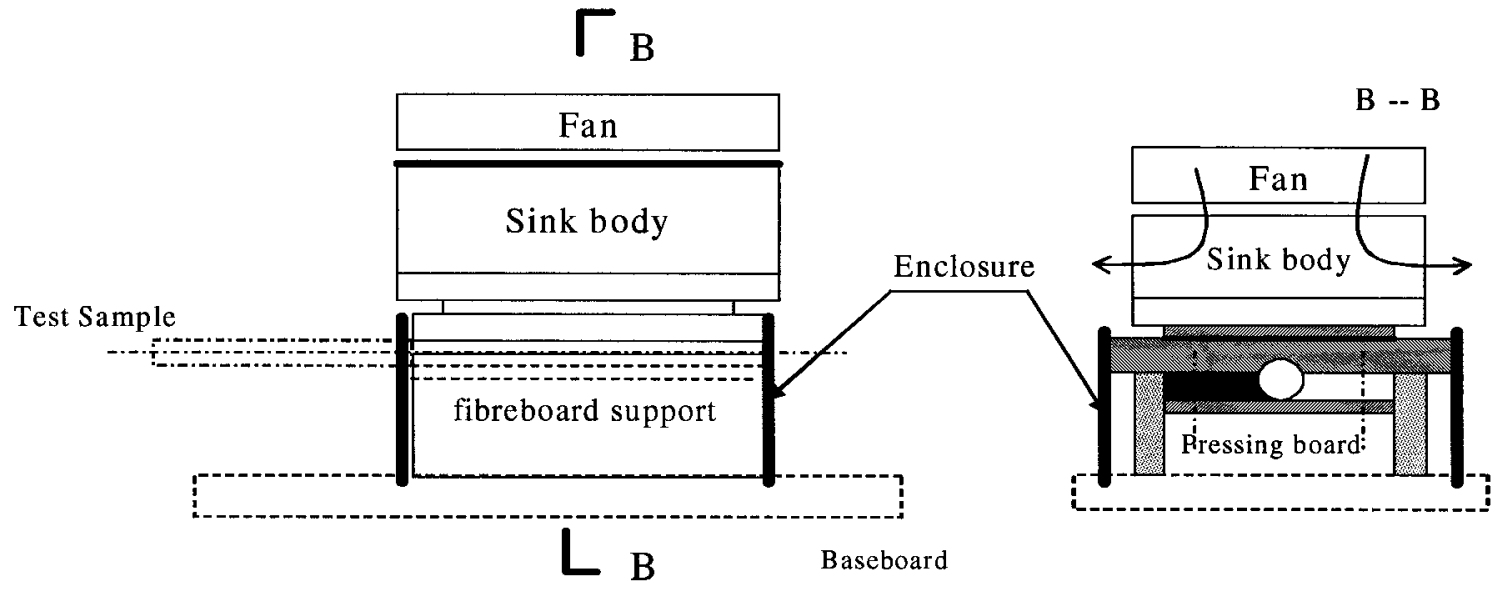

Figure 3.112 The setup for the experiment of verifying wind effect on the condenser thermocouples with wholly enclosed condenser

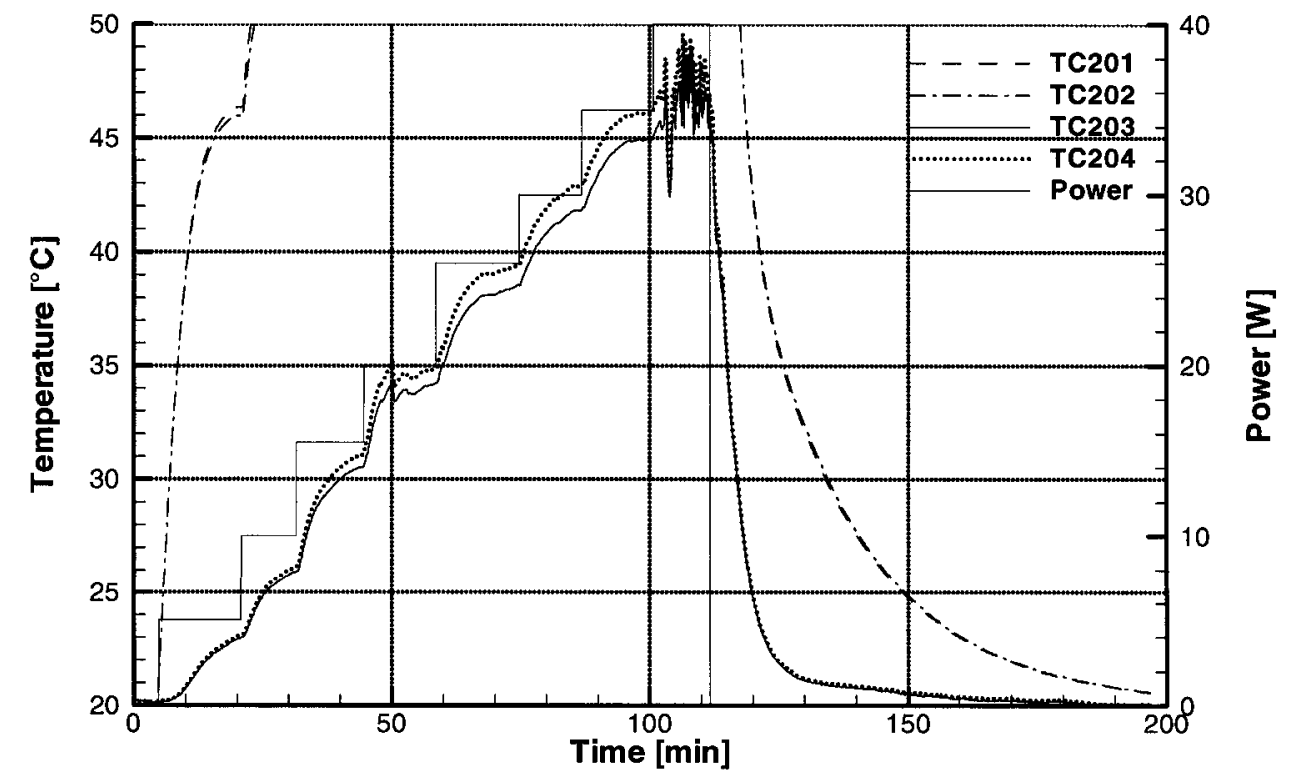

Figure 3.113 Comparison of thermocouple measurements with, and without insulation under the condition of wholly enclosing the condenser (horizontal, forced convection) [sample(s): HP071301] 


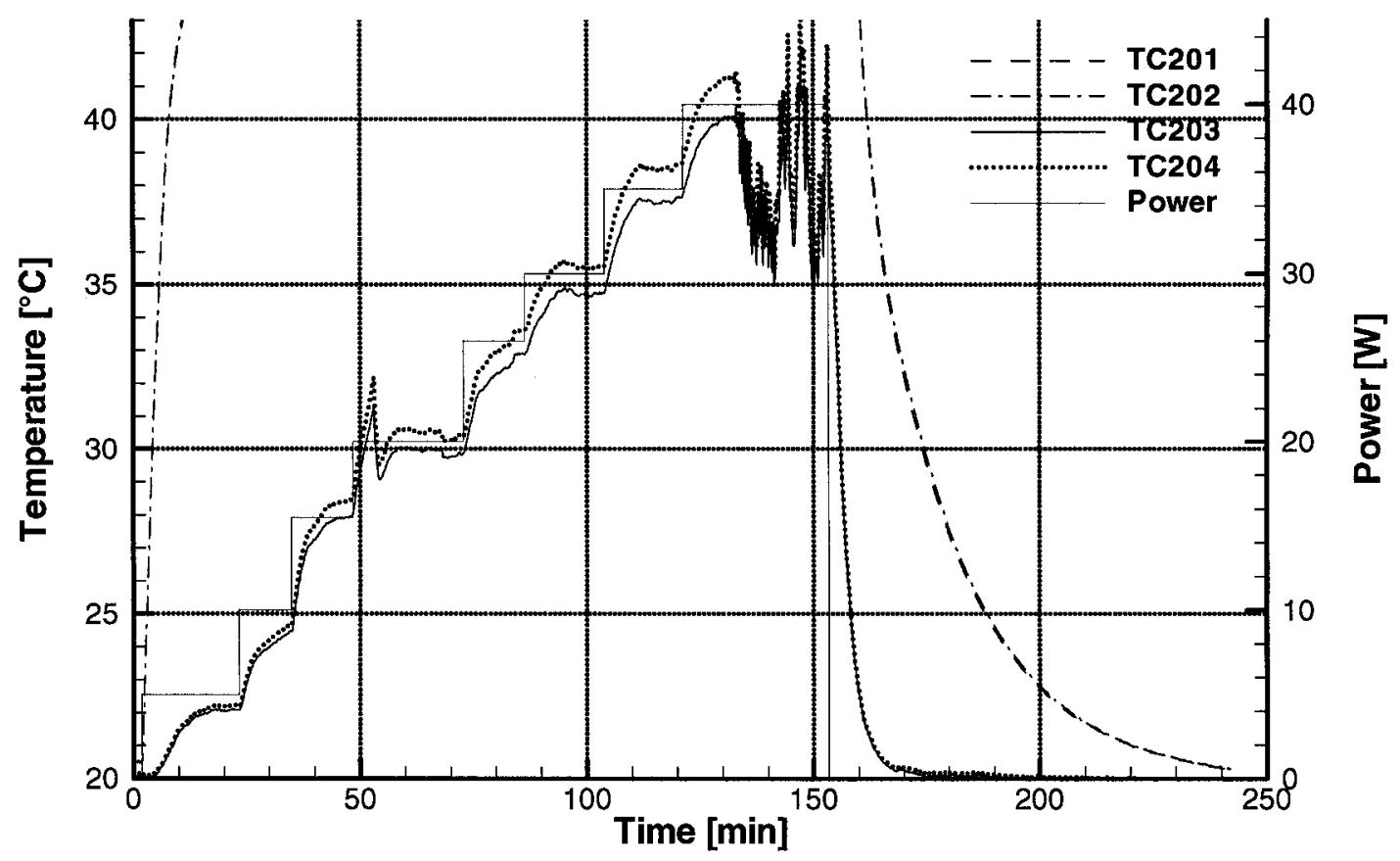

Figure 3.114 Comparison of thermocouple measurements with, and without insulation under the condition of wholly enclosing the condenser (horizontal, enhanced forced convection) [sample(s): HP071301]

Figures 3.113 and 3.114 illustrate the temperature curves obtained from the experiment under normal forced convection and enhanced forced convection conditions respectively. These curves show similar trends as that in the preceding section. Both insulated and non-insulated temperature curves move synchronously, and with the same tendency, diverging with increasing power input. The maximum difference between the insulated and non-insulated thermocouples is about $1{ }^{\circ} \mathrm{C}$ in the normal forced convection and the enhanced forced convection cases. Also, the temperatures with insulation are lower than those without insulation as observed in the preceding section.

Such results lead to the following conclusions: 
1. The temperatures measured without insulation are at most $1^{\circ} \mathrm{C}$ higher than the real temperature of the condenser surface in the situations of normal forced and enhanced forces convections on the condenser.

2. There is little difference in temperature between the two cases, normal forced convection and enhanced forced convection conditions when the whole condenser is enclosed.

3. There is little difference in the stable power temperature between the two conditions; enclosed and non-enclosed condenser.

\section{Section 3: Experiment with Blowing the Condenser Thermocouples}

The two preceding sections illustrate that the wind from the sink slots brings little influence on the condenser thermocouples. This section will apply additional blowing to the condenser underneath the sink to investigate the difference between this case and the ones without direct blowing.

Figure 3.115 shows the setup for this experiment. Based on the same setup and thermocouple distribution in Section 1, a small fan was located facing the condenser underneath in the axial direction. During the whole process of the experiment, this small fan directly blows on the condenser including the thermocouples.

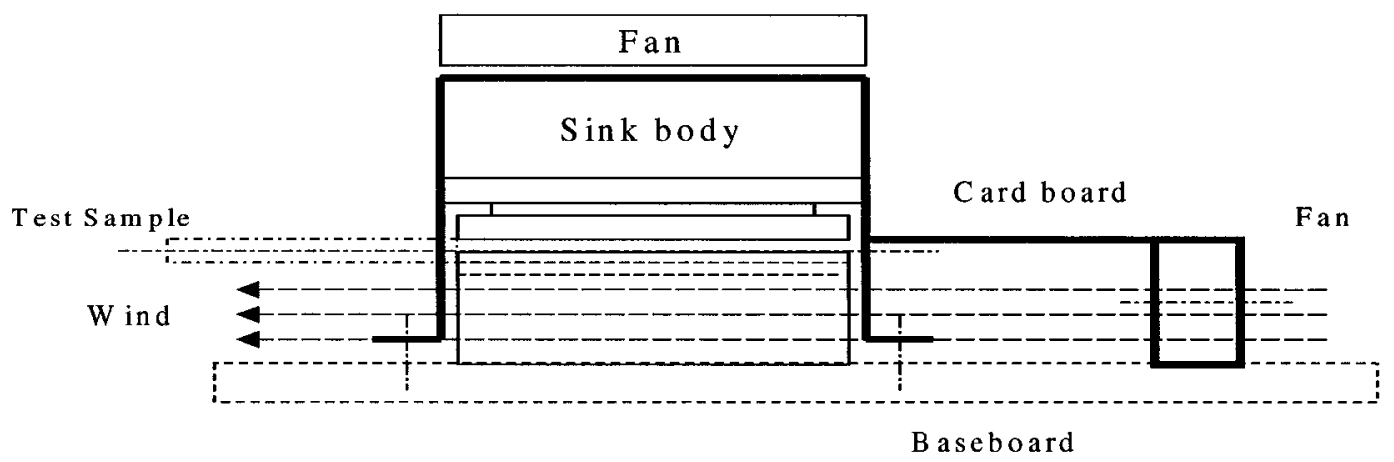

Figure 3.115 Setup for the experiment with directly blowing condenser 


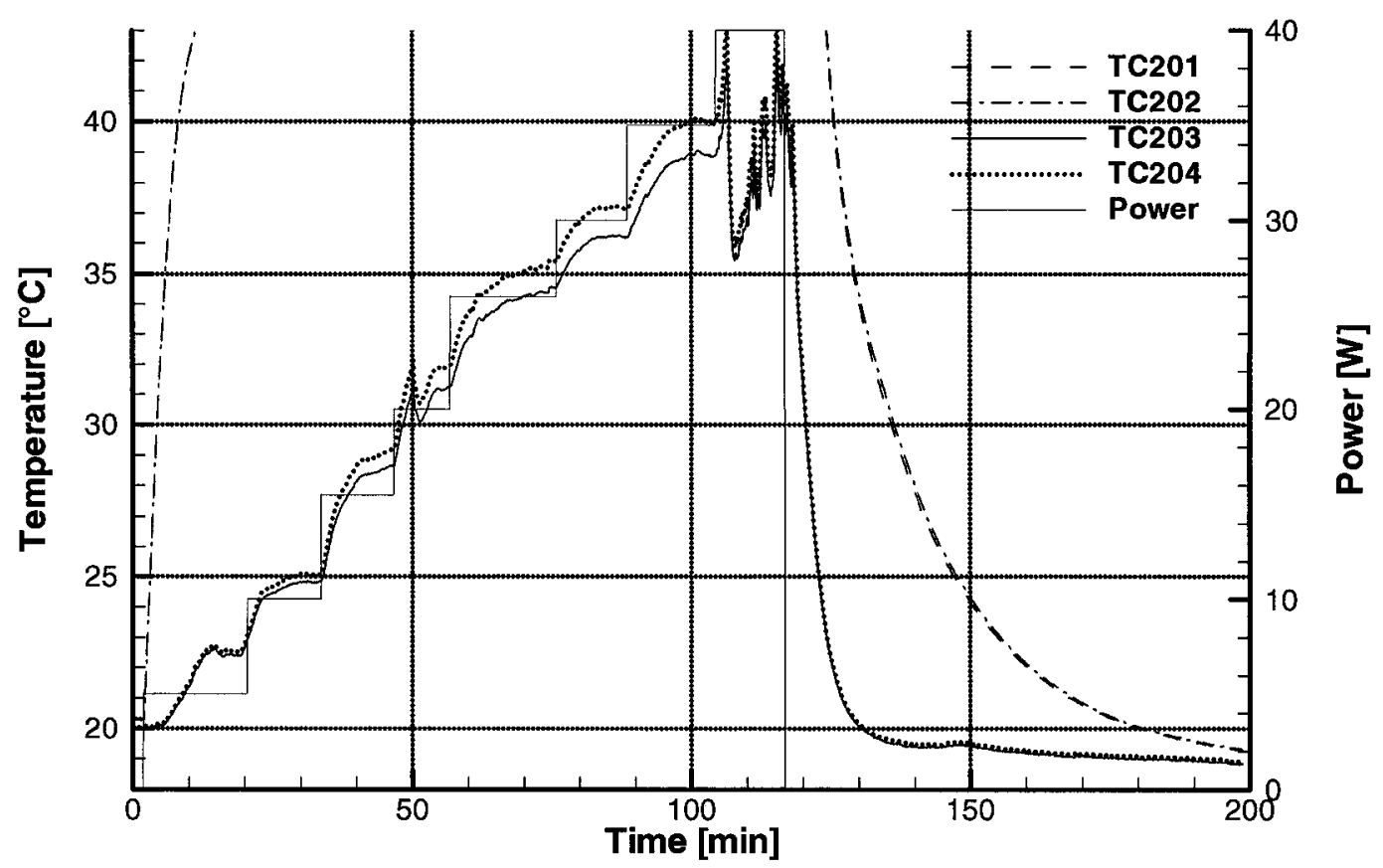

Figure 3.116 Comparison of thermocouple measurements with, and without insulation under the condition of directly blowing the condenser (horizontal, forced convection) [sample: HP071301]

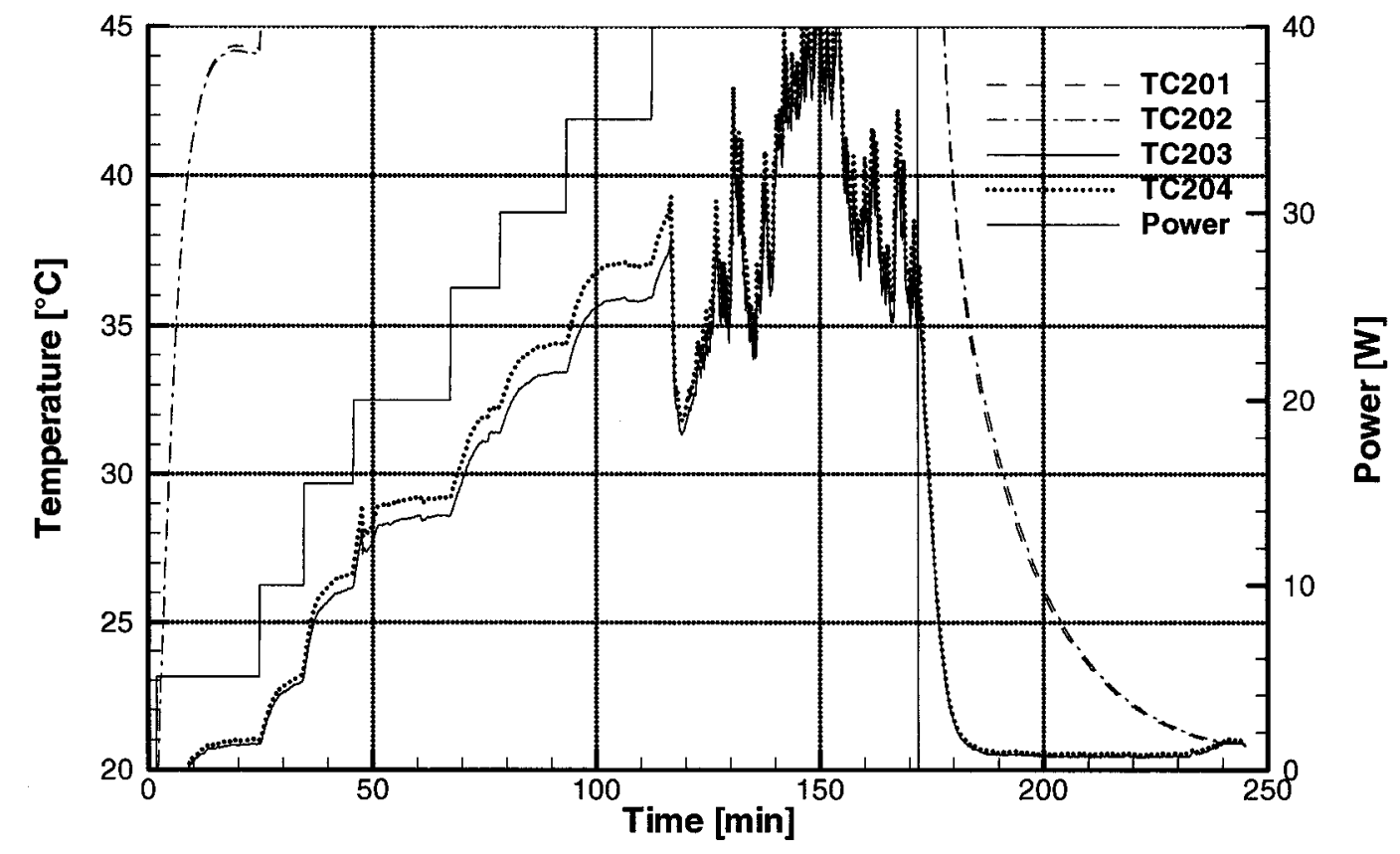

Figure 3.117 Comparison of thermocouple measurements with, and without insulation under the condition of directly blowing the condenser (horizontal, enhanced convection) [sample: HP071301] 
This experiment also follows the same procedures as those in the preceding sections. Figures 3.116 and 3.117 illustrate the temperature curves obtained. These curves also show the similar tendency as that in the preceding section. Both insulated and noninsulated temperature curves move synchronously, and with the same tendency, diverging with increasing power input. The maximum difference between the insulated and noninsulated thermocouples is about $1^{\circ} \mathrm{C}$ in the normal forced convection and the enhanced forced convection cases. The temperatures with insulation are lower than those without insulation as observed in the preceding section.

Such results lead to the following conclusions:

1. The temperatures measured without insulation are at most $1^{\circ} \mathrm{C}$ higher than the real temperature of the condenser surface in the conditions of normal forced and enhanced forced convection on the condenser.

2. There is little difference in temperature between the two conditions, normal forced convection and enhanced forced convection, when the whole condenser is enclosed.

3. There is little difference in the power stable temperature between the two conditions, enclosed and non-enclosed condenser.

4. Direct blowing on the condenser thermocouples causes the temperature to be about $5^{\circ} \mathrm{C}$ lower than the cases without direct blowing for the same corresponding power inputs.

\subsubsection{Conclusion of Wind Effect Investigation}

Based on the above results and deductions, we draw the following conclusions: 
1. A pressed insulator can both protect thermocouples from the environment, and helps the thermocouples contact the measured surface firmly, thus ensuring more accurate temperature measurement.

2. In any conditions, exposing to the ambient, enclosed, and directly exposing to the blowing. Little differences in temperature measurement were found for both insulated and non-insulated cases.

3. Any external convective effect on the condenser thermocouples; natural, normal forced, and enhanced forced convection is so small that it can be ignored compared with the heat pipe dissipating capacity.

4. The measurements in Experiment 3 are valid.

\subsection{Discussion on the Heat Pipe Characteristics}

\subsubsection{Startup and Shutdown}

Unlike the conductance devices, a heat pipe will not start to work as a heat pipe until an internal phase change occurs. For any given heat pipe, the factor affecting the startup and shutdown are the fluid working temperatures at either evaporation or condensation section, and a heat load upon the evaporator that need to be sufficient to maintain the evaporation temperature. This means that heat pipes would not work under all circumstances.

Different working fluids have different startup temperatures. The working fluids applied in our case are water and acetone. Each one has different evaporating points under a certain pressure at which the heat pipe start up. 


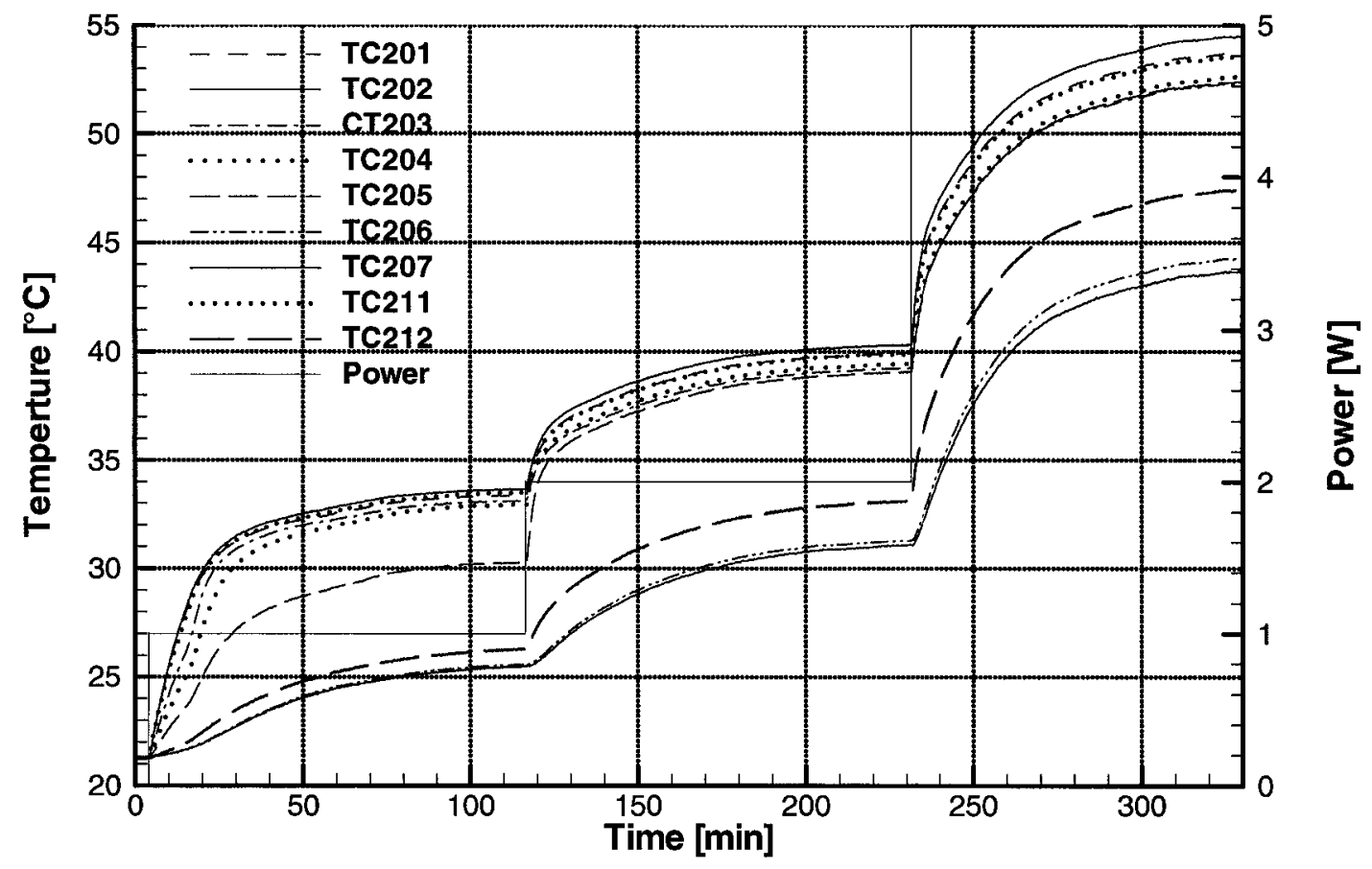

Figure 3.118 Copper water heat pipe startup (insulated, horizontal, natural convection) [sample(s): HP051701]

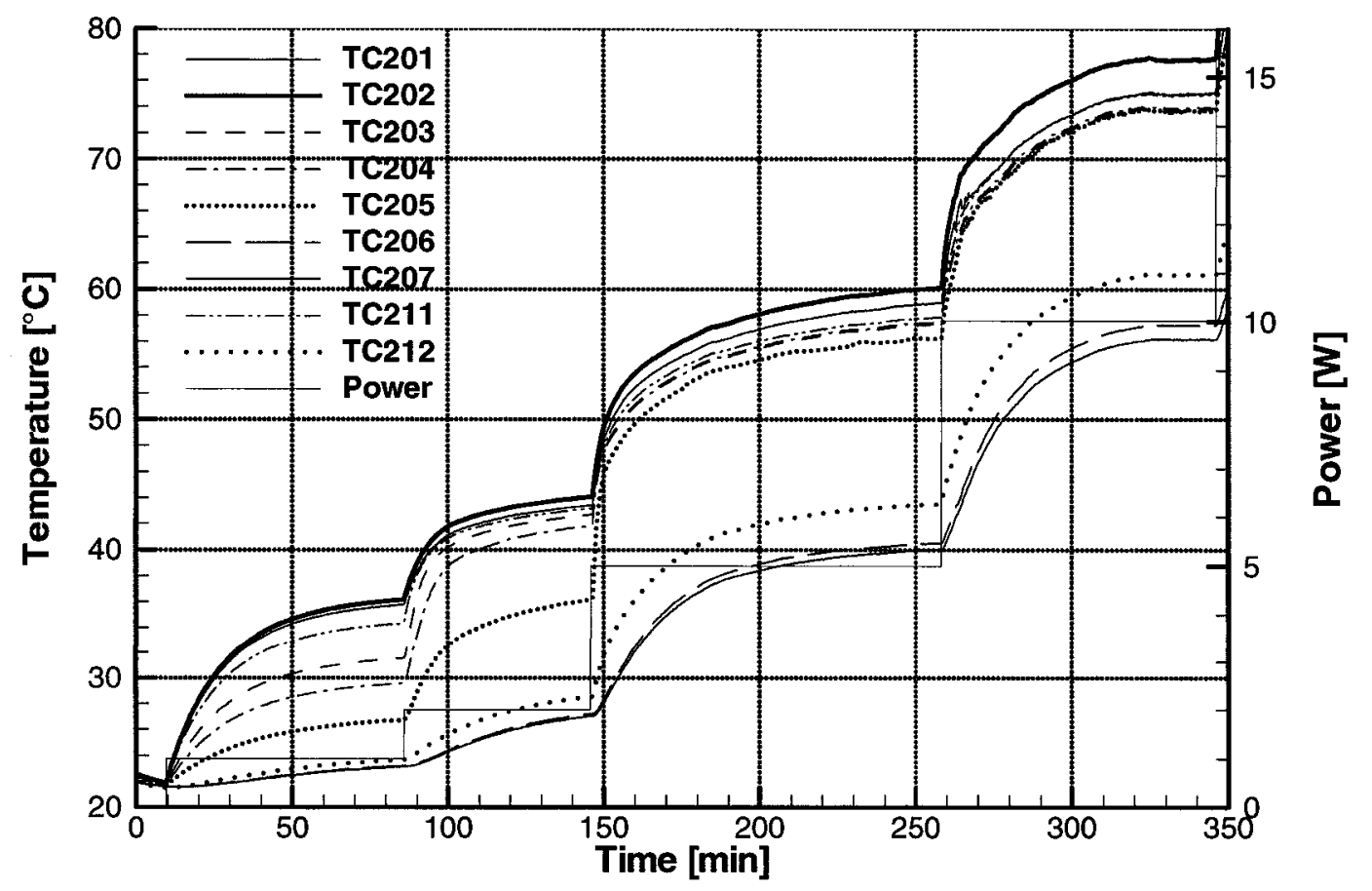

Figure 3.119 Copper water heat pipe startup (insulated, horizontal, natural convection) [sample(s):HP071200] 


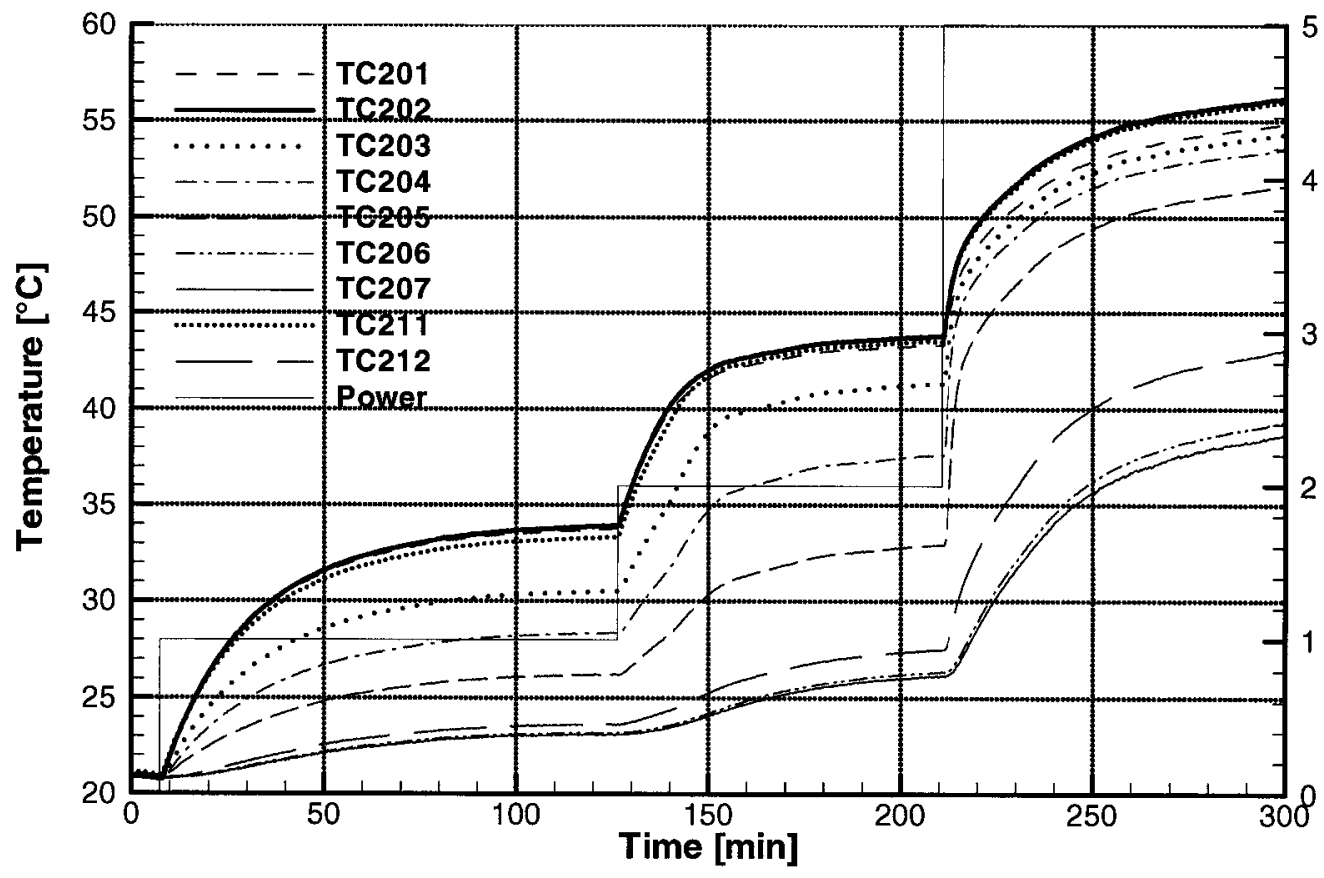

$\frac{\sum}{\vdots}$

Figure 3.120 Nickel-copper water heat pipe startup (insulated, horizontal, natural convection) [sample(s):HP062400]

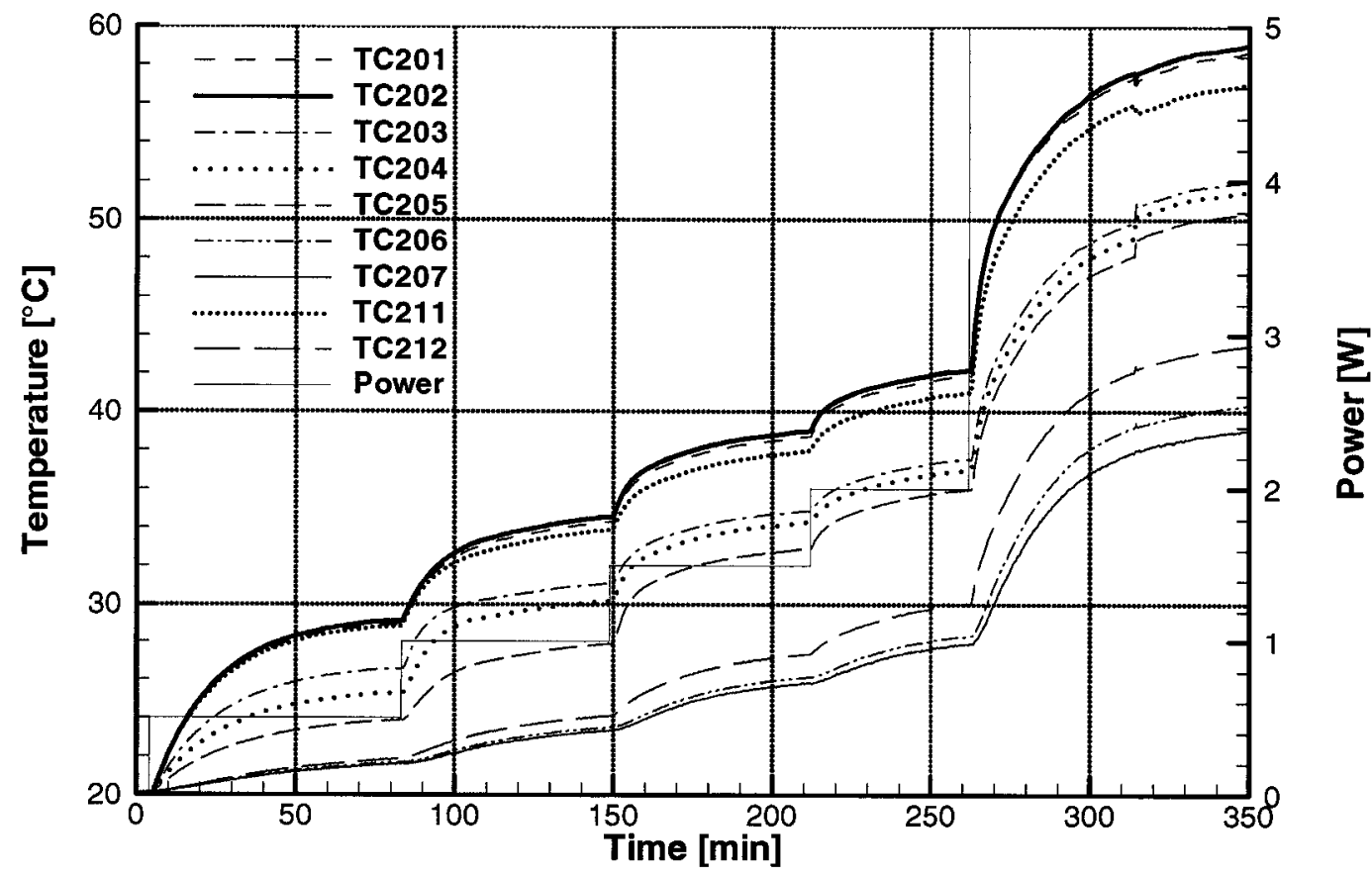

Figure 3.121 Copper acetone heat pipe startup (insulated, horizontal, natural convection) [sample(s):HP071402] 


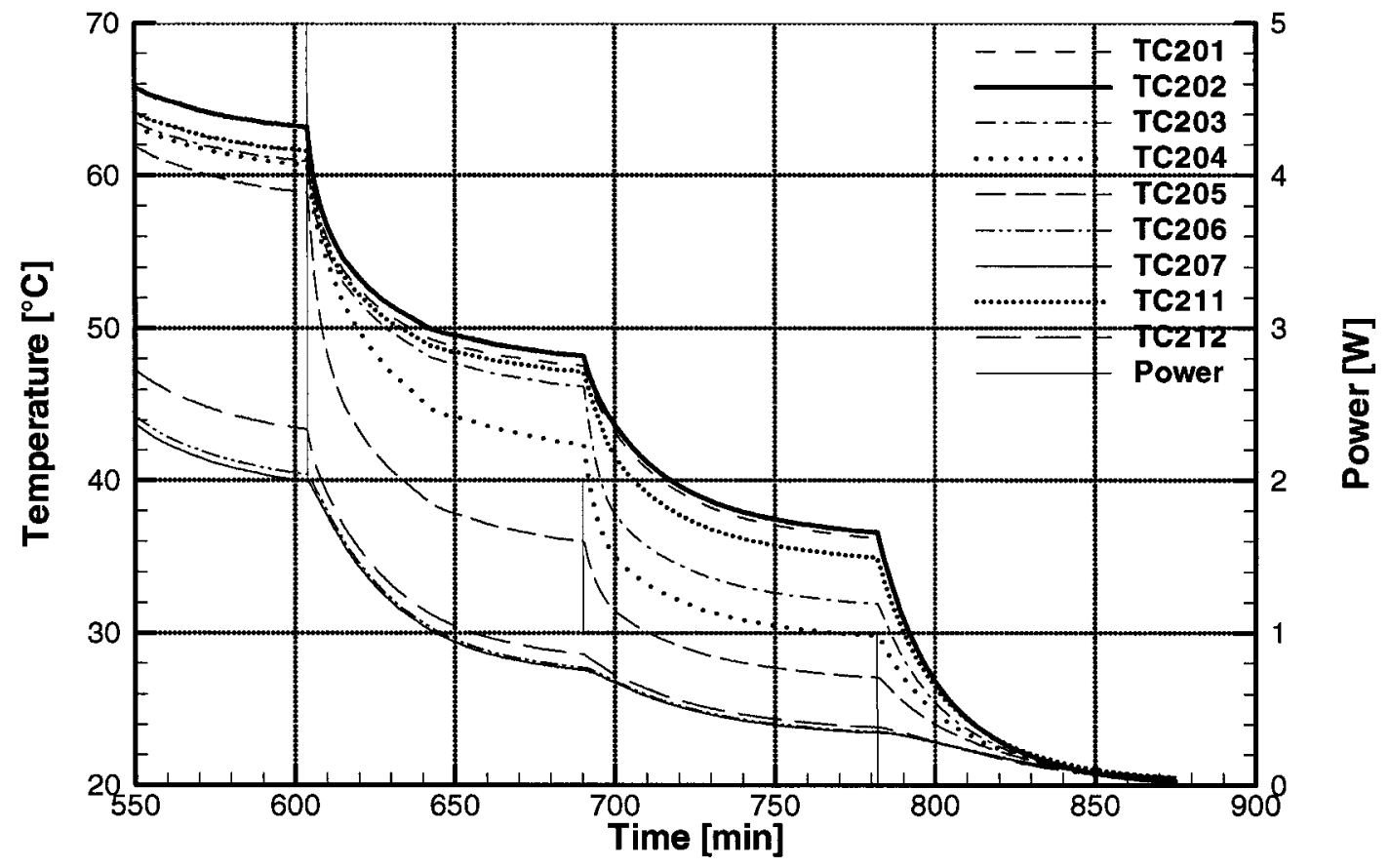

Figure 3.122 Copper water heat pipe shutdown (insulated, horizontal, natural convection) [sample(s):HP071200]

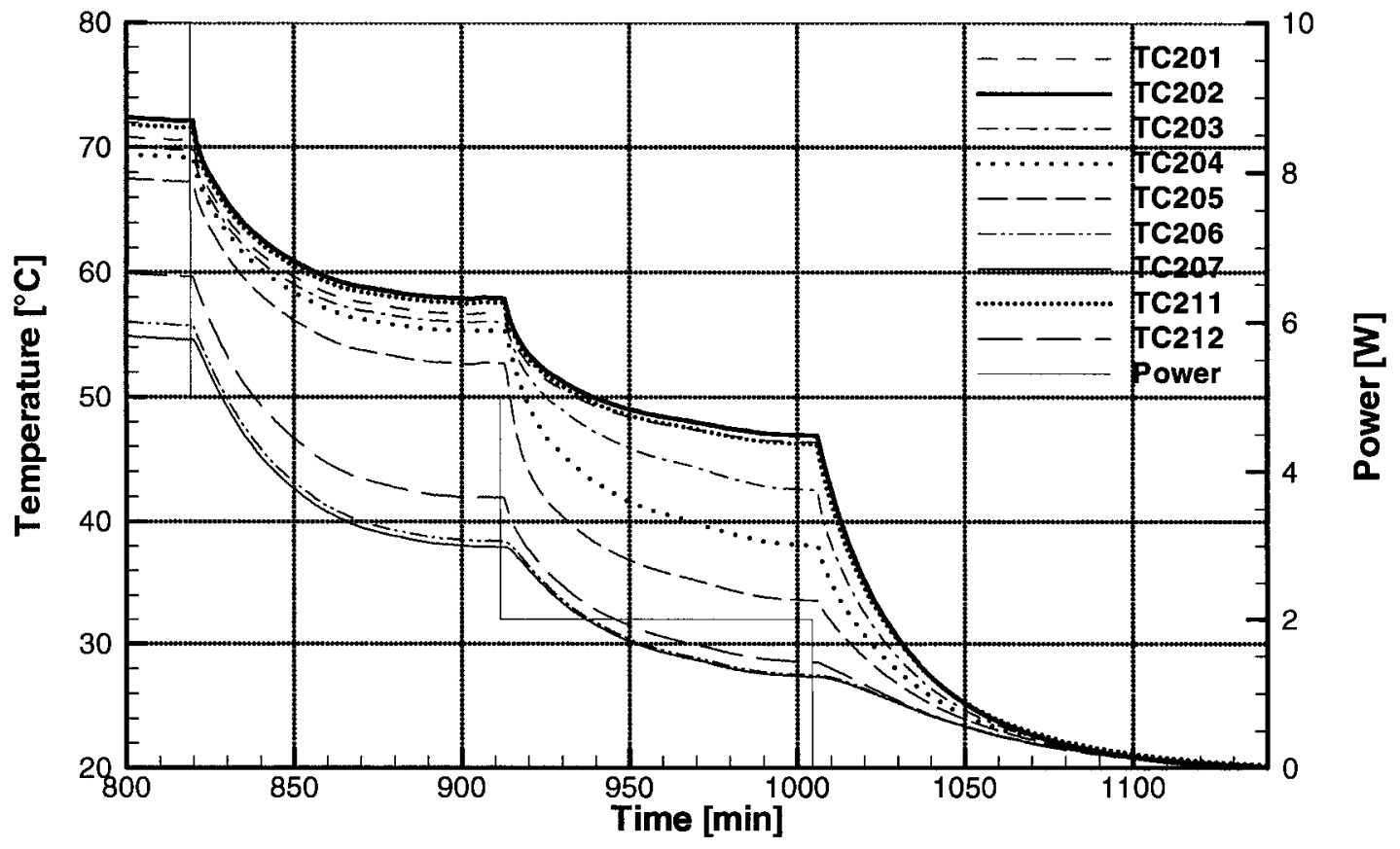

Figure 3.123 Nickel-copper water heat pipe shutdown (insulated, horizontal, natural convection) [sample(s):HP062400] 


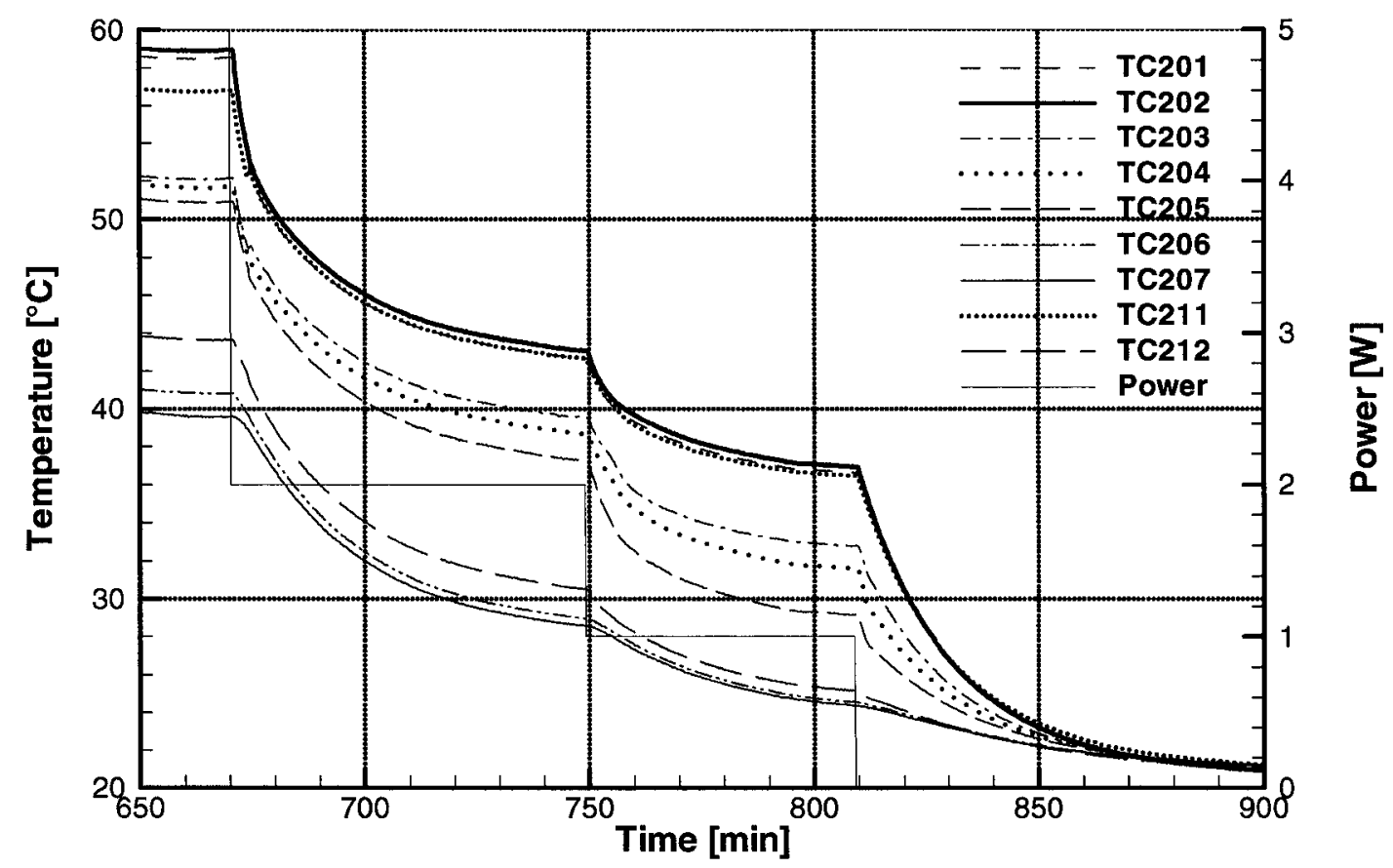

Figure 3.124 Copper acetone heat pipe shutdown (insulated, horizontal, natural convection) [sample(s):HP071402]

Figures 3.118 to 3.124 illustrate the processes of startup and shutdown for water and acetone heat pipes respectively. From these figures, it can be seen that in the startup process, with increasing power, the three adiabatic temperature curves tend to converge. While the other two section temperature curves tend to diverge, thus keeping a low temperature difference between the evaporation and condensation sections. In the shutdown process, the opposite processes occur. This is one of the features distinguishing heat pipes from normal solid metal heat transfer devices. It is the working fluid that by phase change maintains the low temperature gradient between two sections, realizing the heat pipe functionality. 


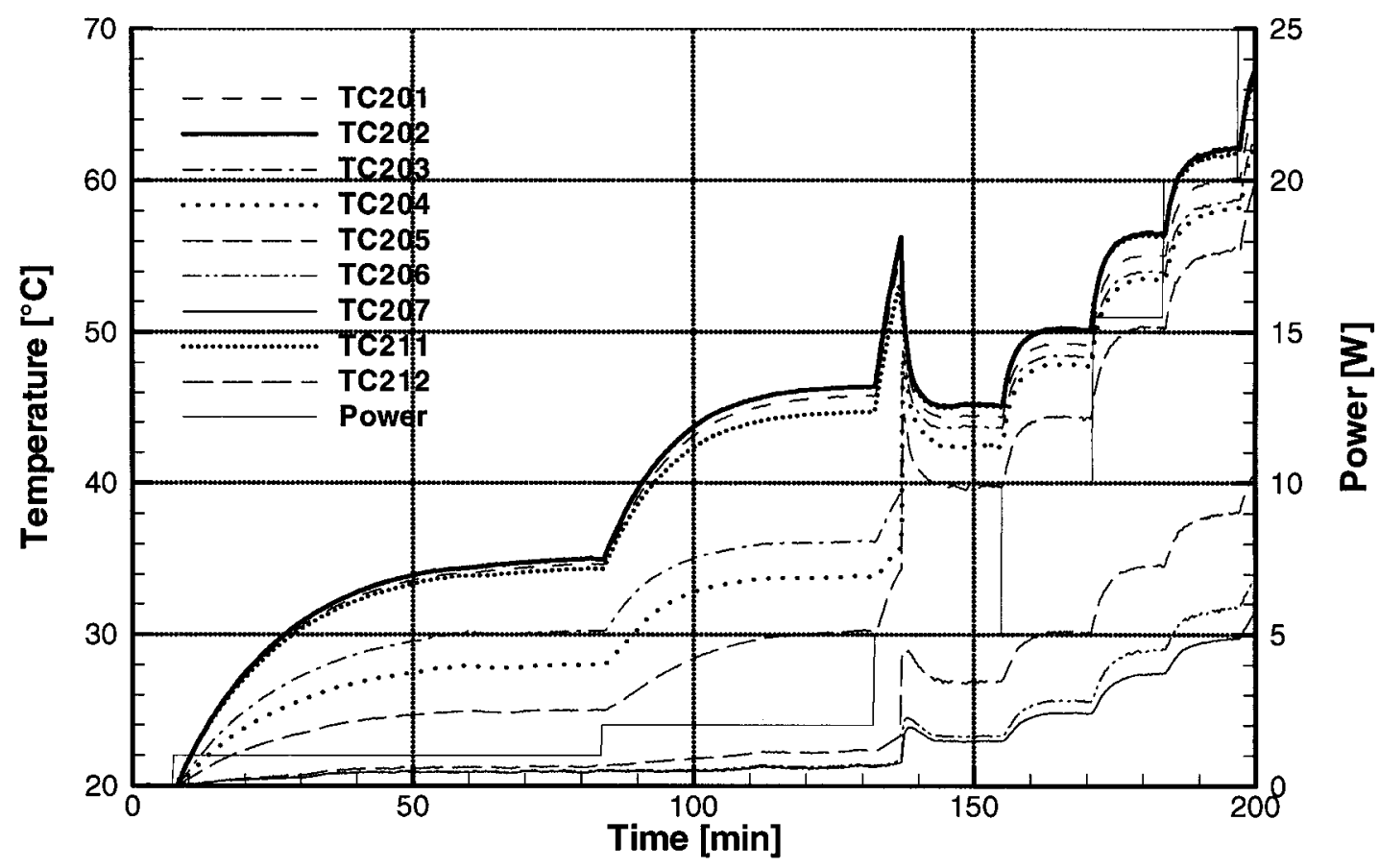

Figure 3.125 Copper water heat pipe setup (insulated, gravity assisted vertical, enhanced force convection) [sample(s):HP071200]

Figure 3.125 illustrates the startup phenomenon. The testing sample is a coarse copper mesh wick with normally charged water heat pipe. In gravity assisted vertical orientation, and with enhanced forced convection cooling. From this figure it can be seen that the testing sample does not start up until the higher power applies. The evaporator temperatures sharply drop and at the same moment the adiabatic section and condenser temperatures quickly move toward the evaporator temperature curves. Hence the evaporator-adiabatic-condenser temperature differences become smaller while the adiabatic temperatures converge together. This case provides a very good example of heat pipe startup. At the lower power levels, the temperature is not high enough so the testing sample just performs as a copper tube. When the power is enough to raise the evaporation temperature, the working fluid is activated, and the vapour rushed from the 
evaporator toward the condenser through the adiabatic section. During this process, the evaporator temperature drops due to the heat dissipation by the fluid. Also, the other two sections temperatures jump. After this process, the phase change begins; hence the test sample starts functioning as a heat pipe.

In Figure 3.125, the phenomenon of abrupt startup could be related to the wick priming process that is discussed in Chapter 1. For a newly charged heat pipe, if some gases or foreign matters were not completely eliminated from the materials including the container tube, the wick and the fluid. These gases or foreign matters would be enclosed with the wick and working fluid after the evacuation, fluid charging, and final sealing. Parts of these enclosed gases or foreign matters would be trapped in the wick. At a medium temperature these trapped gases expands or the gasifyable foreign matters generate gases. These expended gases in the wick could partially sever fluid flow in the wick, hindering the phase changing circulation. This is one of the possible reasons for the abrupt stratup phenomenon occurred in our experiments, and is termed "de-priming" in our work. When the temperature rises up to a certain degree, the gases in the wick quickly expend and finally explode, thus the fluid immediately refills the vacancy, and completes the capillary pressure pumped flow. Large amount of fluid is transported to the evaporator, and evaporates there. This suddenly generated vapour quickly flows through the adiabatic section to the condenser. Thus the temperatures near evaporator suddenly drop, and meanwhile the temperatures near the condenser suddenly jump, this is the phenomenon that is described in Figure 3.125. 


\subsubsection{Dry-out}

Dry-out is one of the phenomena affecting heat pipe working performance. In the testing experiments, dry-out only occurred in a few cases. The following figures illustrate such phenomena.

Figures 3.126 and 3.127 illustrate a dry-out which occurred to the acetone heat pipe testing. Figure 3.126 illustrates the whole process of dry-out. In Figure 3.126 a great evaporator temperature jump is observed at high power input level. During the evaporator temperature jump, temperature differences between the three sections become much larger; this is especially true in the evaporator-adiabatic section. While the temperatures in the adiabatic section are still converging, the condenser temperatures are maintained as usual. This indicates that the working fluid is unable to reach the evaporator area. Instead, it just circulates between the adiabatic section and the condenser, transferring heat from the adiabatic section to the condenser and neglecting the previous evaporator. With the constant heat load and lack of help in heat dissipation, the evaporator temperature is beyond control, as shown in Figure 3.126. Dry-out was finally stopped by decreasing the heat load to a lower power level and after that the heat pipe recovered its functionality. Figure 3.127 indicates the axial temperature profile in this dry-out case. In the figure we can see that the curve at $20.5 \mathrm{~W}$ is distinct from others with regard to the evaporator temperature distribution where the temperature is apart from its counterparts. Note that all the curves keep the normal adiabatic and condensation temperature distributions including dry-out. Similar dry-outs occurred in the same acetone test sample with the horizontal orientation and enhanced forced convection cooling. 


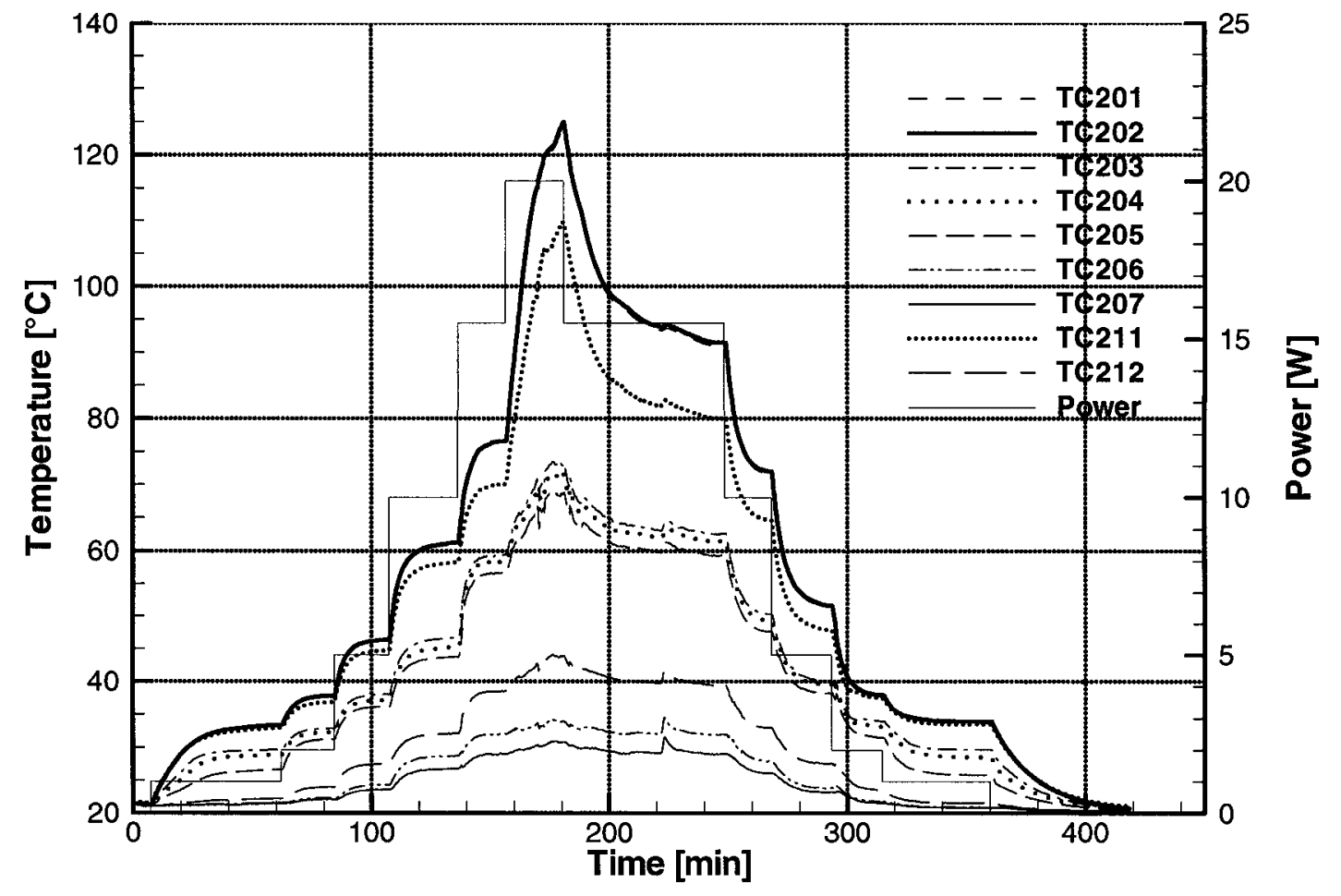

Figure 3.126 Copper acetone heat pipe dry-out (insulated, horizontal, forced convection) [sample(s):HP071402]

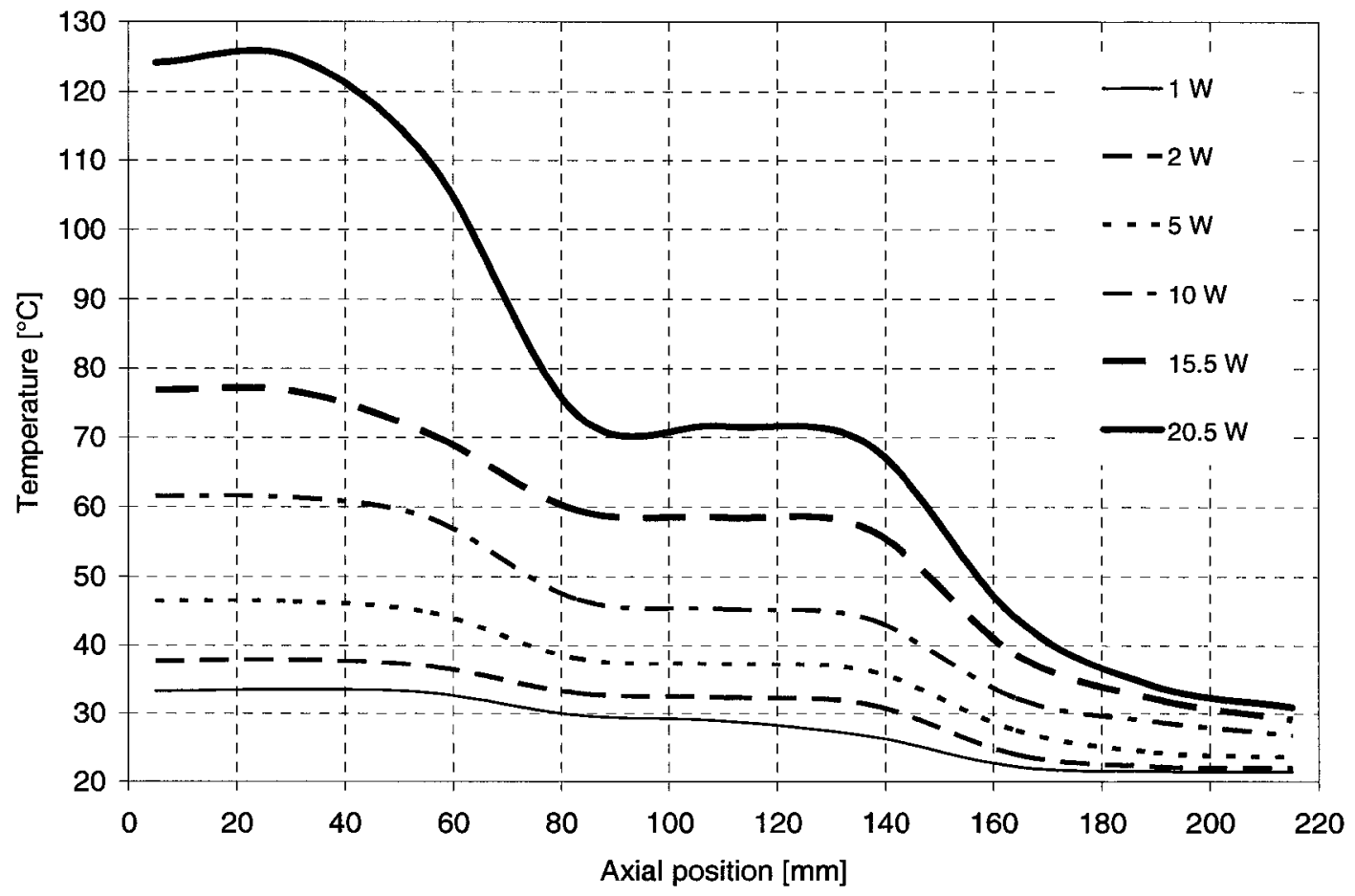

Figure 3.127 Copper acetone heat pipe dry-out axial temperature profile (insulated, horizontal, forced convection) [sample(s):HP071402] 


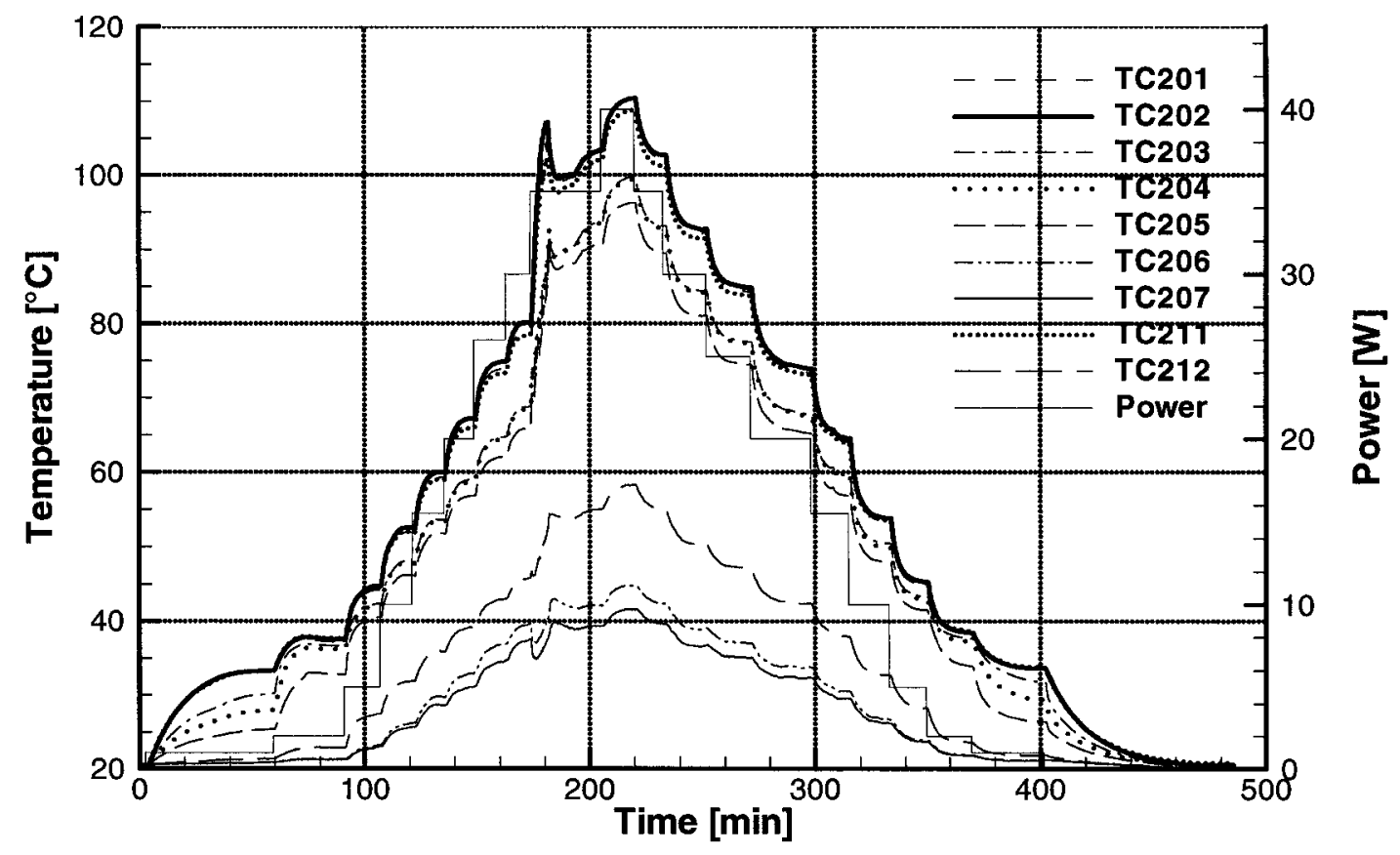

Figure 3.128 Copper water heat pipe dry-out (insulated, horizontal, forced convection) [sample(s):HP071301]

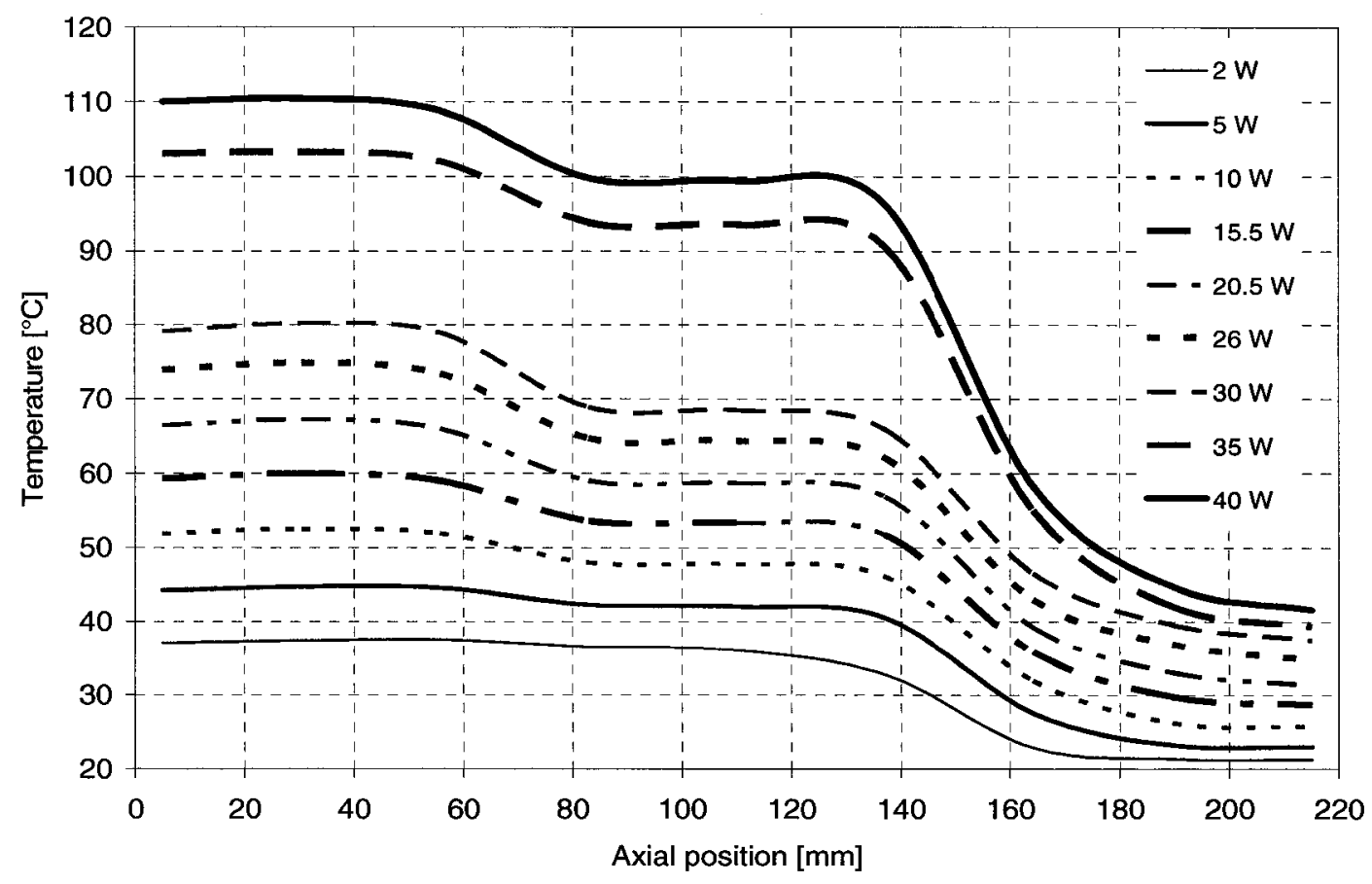

Figure 3.129 Copper water heat pipe dry-out axial temperature profile (insulated, horizontal, forced convection) [sample(s):HP071301] 
Figures 3.128 and 3.129 illustrate a dry-out which occurred in the composite copper, water heat pipe. In this case, dry-out was finally self-recovered. Figure 3.128 illustrates the whole process in which it can be seen that by entering $40 \mathrm{~W}$ all of the temperature curves climb. This is especially evident in the evaporator temperatures following the heater temperature. They tend to depart from the adiabatic temperatures while the adiabatic temperatures (TC205) tend to lag behind the other two (TC203 and TC204). This shows a recoverable dry-out, and if the process continued, a real dry-out would have definitely occurred. Fortunately, the heater temperature reached its zenith, and consequently the evaporator temperature ceased rising. Thus, the internal fluid was still able to reach the entire wick, and ensure the testing sample performed properly. Figure 3.129 shows such recoverable dry-out axial temperature profile. From the figure it can be seen that the high power dry-out curves are far away from others but the evaporator temperature distribution is still flat and do not have any tendency to jump. It was this flat evaporator temperature characteristic that ensured a uniform evaporator temperature, thus avoiding the partially local temperature jump that could cause partial dry-out and finally result in a real dry-out. This case illustrates the advantage of the composite mesh wick structure.

\subsection{Chapter Closure}

The experimental results have been analyzed qualitatively. Generally, results are consistent with our predictions. However, there are still some unexpected results, and some phenomena need further study. The next chapter includes quantitative analyses on several of these issues. 


\section{Chapter 4}

\section{Mathematical Modeling and Experimental Verification}

Chapter 3 analyzed the experimental results. This chapter introduces a one-dimensional mathematical model for heat pipes. The experimental results are compared with the onedimensional mathematical model predictions.

\subsection{Thermal Network and Temperature Analyses}

A heat pipe normally works in a thermal network. The heat pipe itself can be treated as a thermal network. Heat pipe performance, especially its temperature characteristic, is associated with these thermal networks characteristics. To analyze heat pipe temperature characteristics we first need to investigate the heat pipe structure in terms of a thermal network.

\subsubsection{Heat Pipe Thermal Network}

In this chapter the network analysis scope is confined within the heat pipe itself, from the external surface of the evaporation section through the adiabatic section to the external surface of the condensation section. Figure 4.1 is a schematic showing the thermal network. 


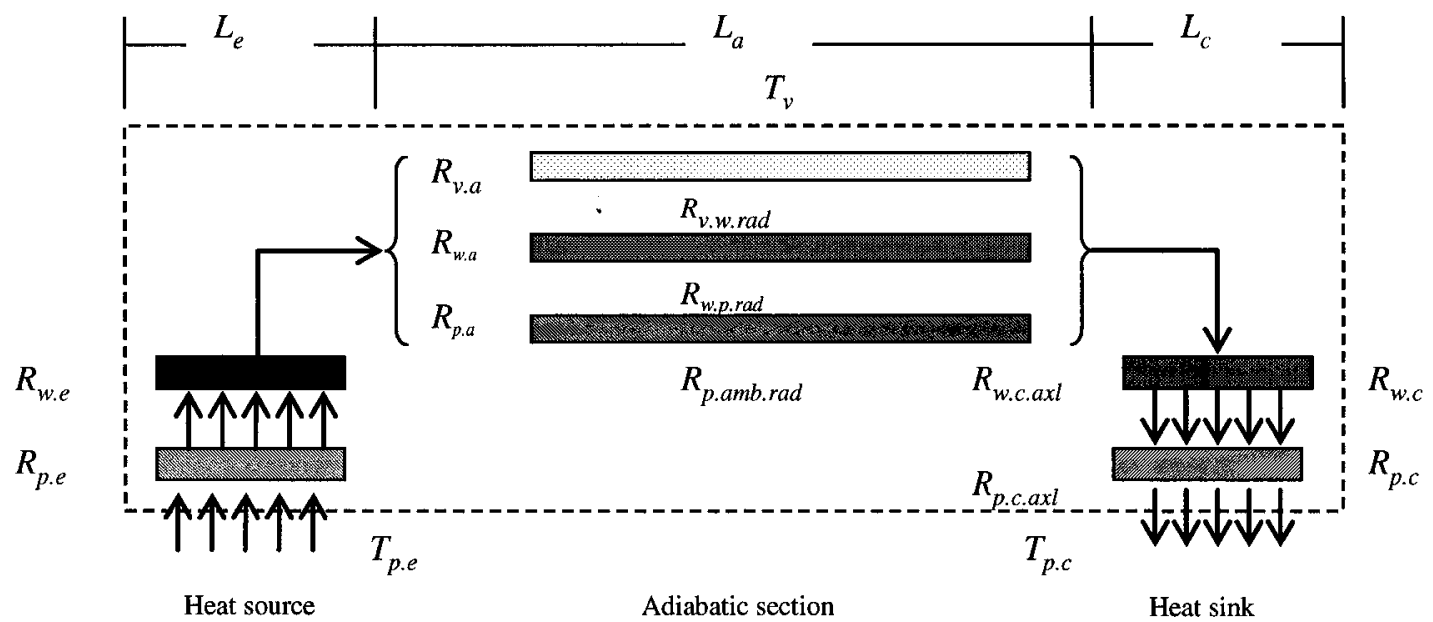

Figure 4.1 Schematics of heat pipe thermal resistance network

From Figure 4.1 it can be seen that the heat pipe is divided into three sections in the axial direction; the evaporation, adiabatic, and condensation sections. In the evaporation section, there are three thermal resistance parts; pipe wall resistance $\left(R_{p . e}\right)$, and wick resistance $\left(R_{w . e}\right)$. For the heat flux entering the evaporator, these three resistances act in series in the radial direction. In the adiabatic section, there are three portions of thermal resistance; wall resistance $\left(R_{p \cdot a}\right)$, wick resistance $\left(R_{w \cdot a}\right)$, and vapour flow resistance $\left(R_{v \cdot a}\right)$. In the adiabatic section, the heat flux takes an axial direction, while in the condensation section the thermal resistances act in series as those in the evaporation section. Because some of the resistances are magnitudes lower compared with others, they can be considered negligible (Peterson, 1994), and the heat pipe thermal resistance network can be simplified, as shown in Figure 4.2 

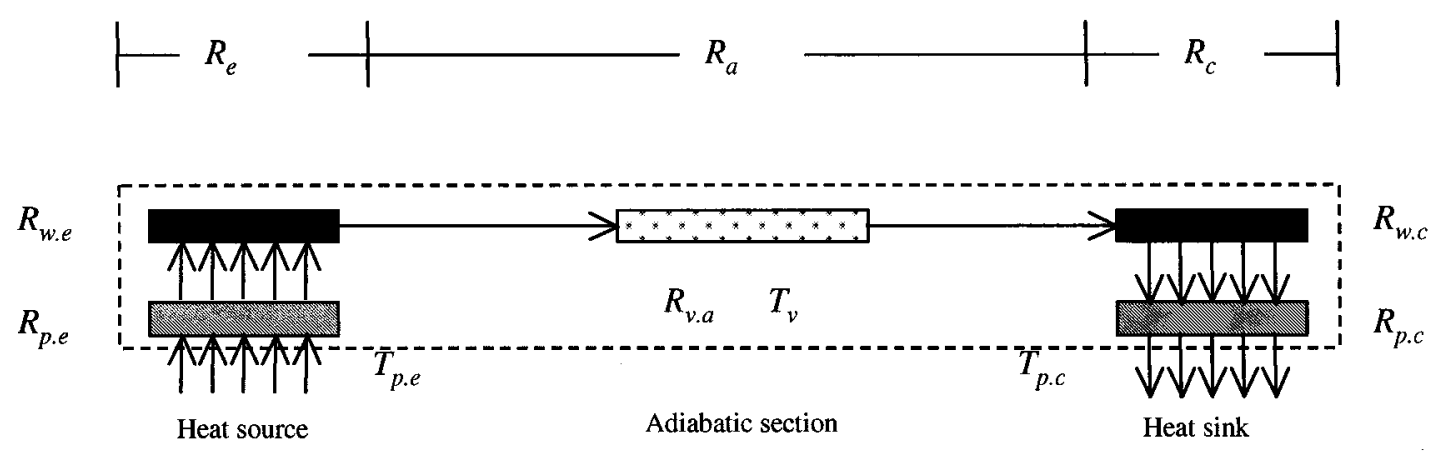

Figure 4.2 Simplified equivalent heat pipe thermal resistance network

Where, the parameters can be obtained through the following equations.

$$
\begin{aligned}
R_{p . e} & =\frac{r_{o} \cdot t_{p}}{2 \cdot L_{e} \cdot k_{p}} \\
R_{w . e} & =\frac{r_{o}^{2} \cdot t_{w}}{2 \cdot L_{e} \cdot r_{i} \cdot k_{e . e}} \\
k_{e . e} & =\frac{k_{l} \cdot\left[k_{l}+k_{w}-\left(1-\varepsilon_{\text {mesh }}\right) \cdot\left(k_{l}-k_{w}\right)\right]}{\left(k_{l}+k_{w}\right)+\left(1-\varepsilon_{m e s h}\right) \cdot\left(k_{l}-k_{w}\right)} \\
\varepsilon_{m e s h} & =1-\frac{1}{4} \cdot \pi \cdot S \cdot N_{m e s h} \cdot d_{w} \\
R_{v . a} & =\frac{\pi \cdot r_{o}^{2} \cdot F_{v} \cdot\left(\frac{1}{6} \cdot L_{e}+L_{a}+\frac{1}{6} \cdot L_{c}\right) \cdot T_{v}}{\rho_{v} \cdot \lambda} \\
F_{v}= & \frac{f_{v} \operatorname{Re} \cdot \mu_{\mathrm{v}}}{2 \cdot A_{v} \cdot r_{h . v}^{2} \cdot \rho_{v} \cdot \lambda} \\
R_{w . c} & =\frac{r_{o}^{2} \cdot t_{w}}{2 \cdot L_{c} \cdot r_{i} \cdot k_{e . c}} \\
R_{p . c}= & \frac{r_{o} \cdot t_{p}}{2 \cdot L_{c} \cdot k_{p}}
\end{aligned}
$$

Among these parameters, five thermal resistance elements are arranged in series. Thus the whole heat pipe thermal resistance can be expressed as,

$$
R_{H P . p}=R_{p . e}+R_{w . e}+R_{v, a}+R_{w . c}+R_{p . c}
$$


Consequently, the total heat pipe heat transfer coefficient is,

$$
U_{H P . p}=\frac{1}{R_{H P . p}}
$$

\subsubsection{Heat Pipe Thermal Network and Temperature Analyses}

The heat transfer rate relationship gives,

$$
Q=A \cdot U_{H P . p} \cdot\left(T_{p . e}-T_{p . c}\right)
$$

This can be rearranged to obtain the predicted evaporator temperature as,

$$
T_{p . e}=\frac{Q}{A \cdot U_{H P . p}}+T_{p . c}
$$

In Equation (4.12), the condenser pipe wall temperature, $T_{p . c}$, can be obtained from the experimental measurement. The following figures illustrate the comparisons of predicted and measured evaporator temperatures.

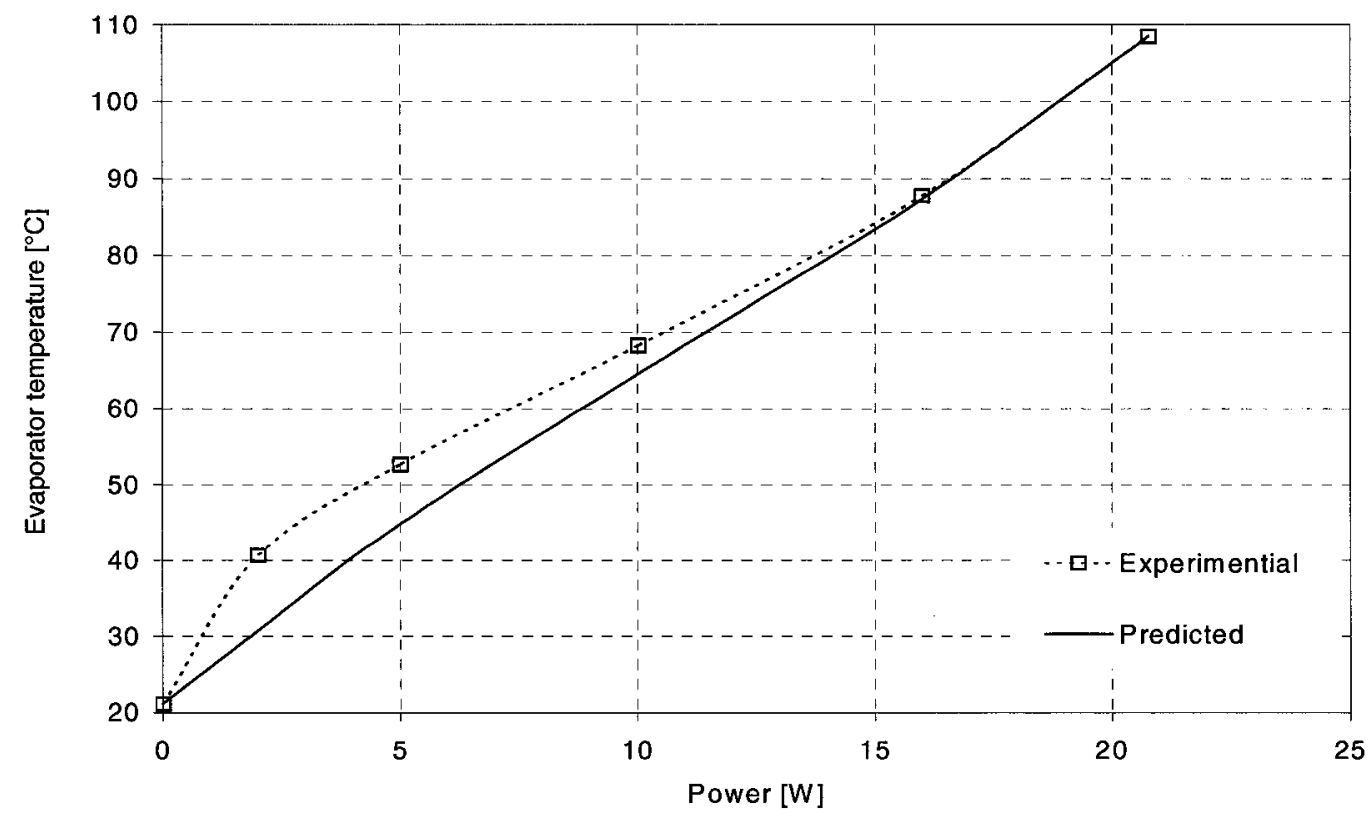

Figure 4.3 Comparison of predicted and measured evaporator temperature (100 mesh, water, insulated, horizontal, natural convection) [sample(s):HP052502] 
From Figure 4.3 we can see that at low power levels there is a discrepancy between the two curves. With increasing power, the two curves converge. Similar results were also obtained in other heat pipe samples under the same test conditions, including the one with fine copper mesh and normally charged water, the one with coarse copper mesh and normally charged water, and the one with composite copper mesh and over charged water. Among these test samples, the coarse and composite mesh samples show better performance with ascending power levels as shown in Figure 4.4. This could be related to the axial thermal resistance reduction due to the effective length decrease, which will be analyzed in Section 4.3 .

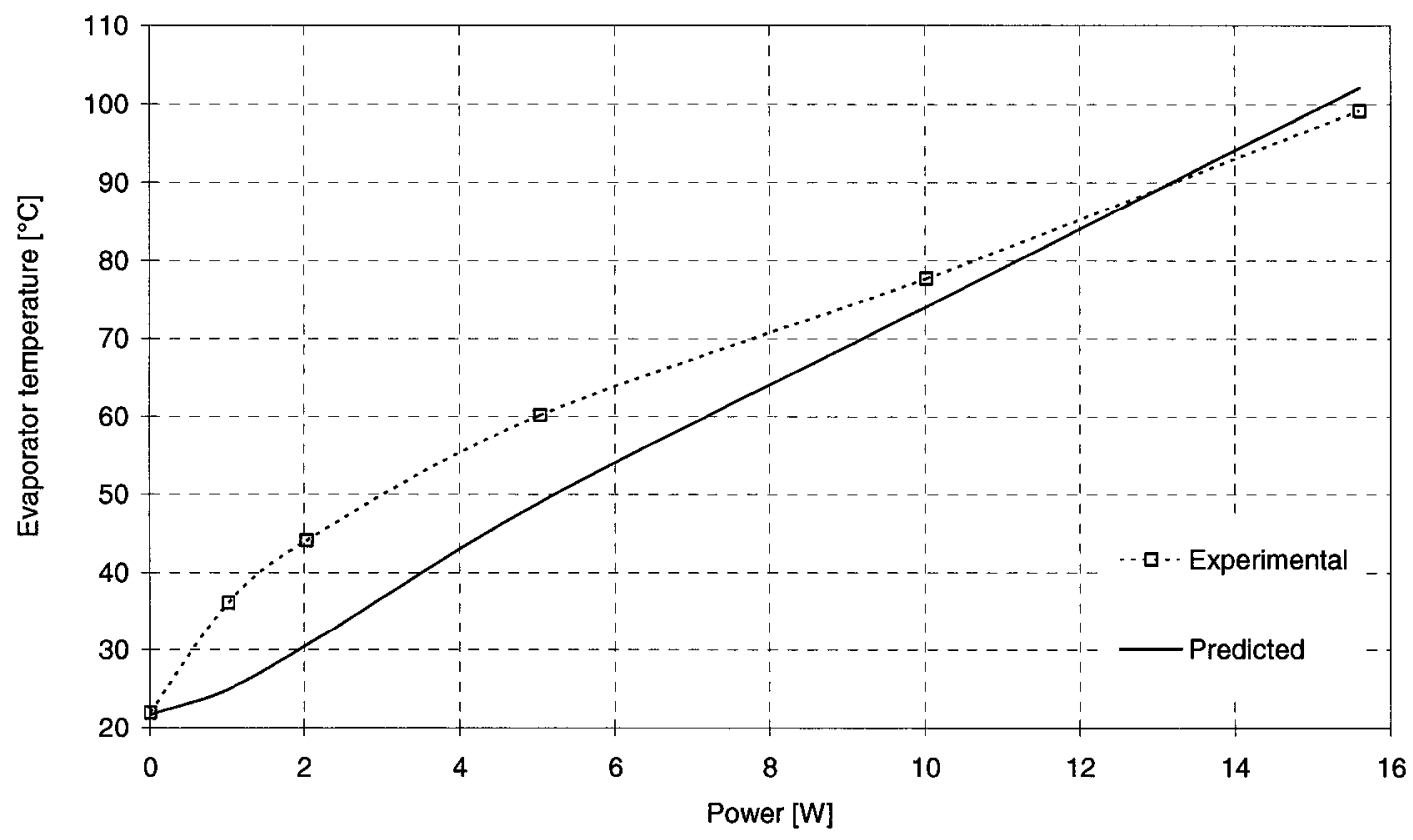

Figure 4.4 Comparison of predicted and measured evaporator temperatures (insulated, horizontal, natural convection) [sample(s):HP071200]

The fine mesh over charged acetone test sample performs far different from the prediction under the same testing conditions, as illustrated in Figure 4.5. The heat pipe meets the prediction at $6 \mathrm{~W}$ and $60^{\circ} \mathrm{C}$. However, after that it performs better than the prediction and tends to diverge from the prediction. Such tendencies were also observed 
under other testing conditions for the same test sample. Further exploration will be made in Section 4.3

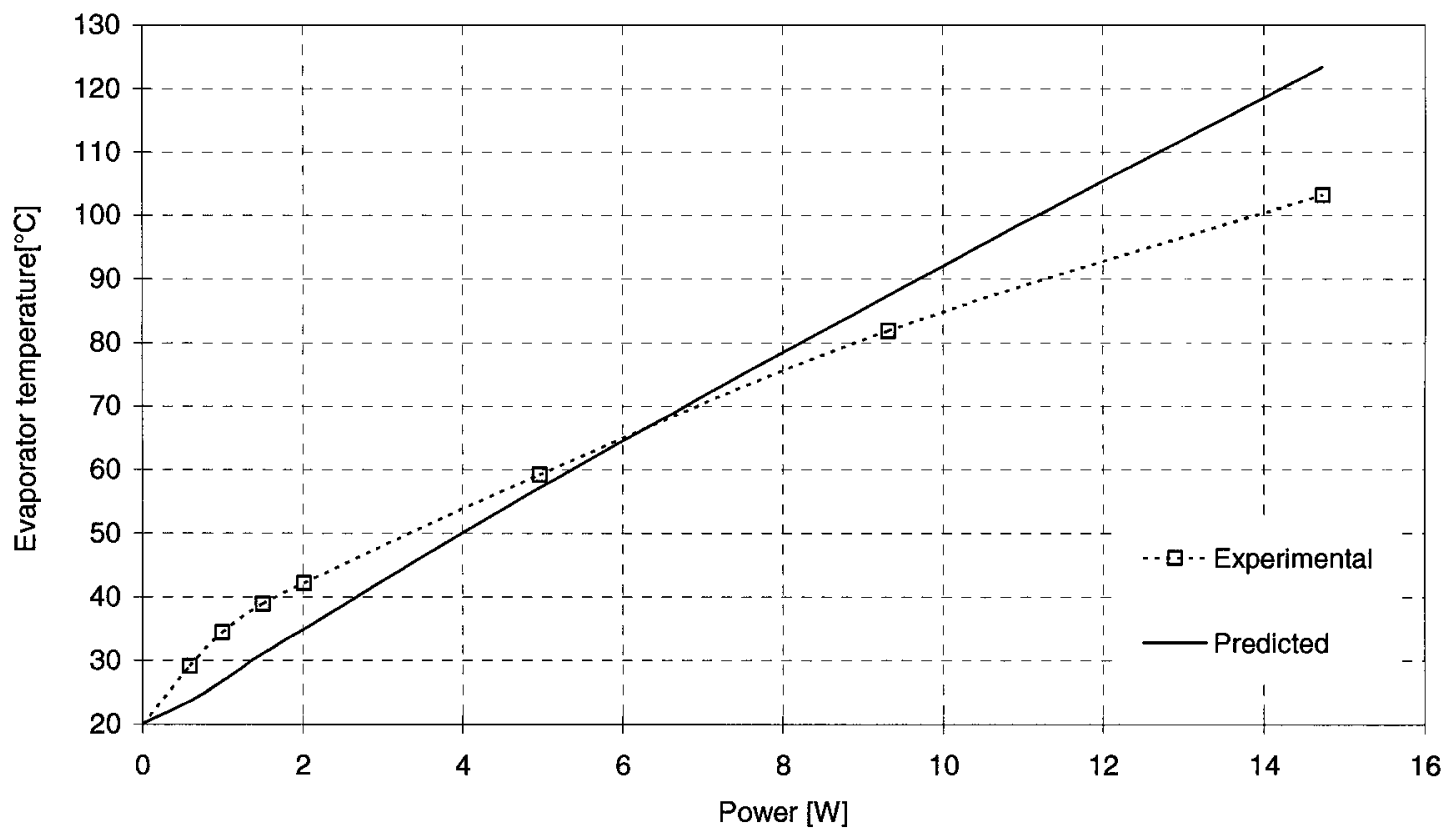

Figure 4.5 Comparison of predicted and measured evaporator temperatures (insulated, horizontal, natural convection) [sample(s):HP071402]

For the nickel foam wick test sample, as its wick structure is different from copper wire mesh counterparts, the effective thermal conductivity used in the preceding cases is no longer valid. In the nickel foam case, two effective thermal conductivity models for the wick were applied. They are as follows:

The first one is the "packed sphere" model (Chi, 1976).

For the evaporator section, the effective wick thermal conductivity is,

$$
k_{e . e}=\frac{k_{l} \cdot\left[2 \cdot k_{l}+k_{w}-2 \cdot\left(1-\varepsilon_{\text {foam }}\right) \cdot\left(k_{l}-k_{w}\right)\right]}{\left(2 \cdot k_{l}+k_{w}\right)+\left(1-\varepsilon_{f o a m}\right) \cdot\left(k_{l}-k_{w}\right)}
$$

For the condenser section, it takes the same form in the effective thermal conductivity as its evaporator counterpart, 


$$
k_{e . c}=\frac{k_{l} \cdot\left[2 \cdot k_{l}+k_{w}-2 \cdot\left(1-\varepsilon_{\text {foam }}\right) \cdot\left(k_{l}-k_{w}\right)\right]}{\left(2 \cdot k_{l}+k_{w}\right)+\left(1-\varepsilon_{\text {foam }}\right) \cdot\left(k_{l}-k_{w}\right)}
$$

The other one is a "porous metal" model (Dunn and Reay, 1978). By this model, the effective thermal conductivities for the evaporator and condenser sections take the following forms respectively,

$$
\begin{aligned}
& k_{e . e}=k_{w} \cdot\left[\frac{2+\frac{k_{l}}{k_{w}}-2 \cdot \varepsilon_{\text {foam }} \cdot\left(1-\frac{k_{l}}{k_{w}}\right)}{2+\frac{k_{l}}{k_{w}}+\varepsilon_{\text {foam }} \cdot\left(1-\frac{k_{l}}{k_{w}}\right)}\right] \\
& k_{e . c}=k_{w} \cdot\left[\frac{2+\frac{k_{l}}{k_{w}}-2 \cdot \varepsilon_{\text {foam }} \cdot\left(1-\frac{k_{l}}{k_{w}}\right)}{2+\frac{k_{l}}{k_{w}}+\varepsilon_{\text {foam }} \cdot\left(1-\frac{k_{l}}{k_{w}}\right)}\right]
\end{aligned}
$$

Substituting these in Equation (4.12) gives the predicted evaporator temperatures for the nickel foam heat pipe test sample.

Figure 4.6 shows the comparison of the predicted and measured temperatures. From the figure we can see that with increasing power the actual curve tends to converge toward the curve based on the Dunn \& Reay model. In the experiment, due to the heat source power limit, the nickel foam heat pipe did reach its optimum working power but the curve shows a strong trend of converging toward the curve based on the Dunn \& Reay model. The curve based on the Chi model has a larger slope and goes further apart from the experimental curve after intersection. These were also observed in other cases for the nickel foam heat pipe. This comparison indicates that the Dunn \& Reay model is more suitable for the sintered nickel foam case, and will be applied in later analyses. 


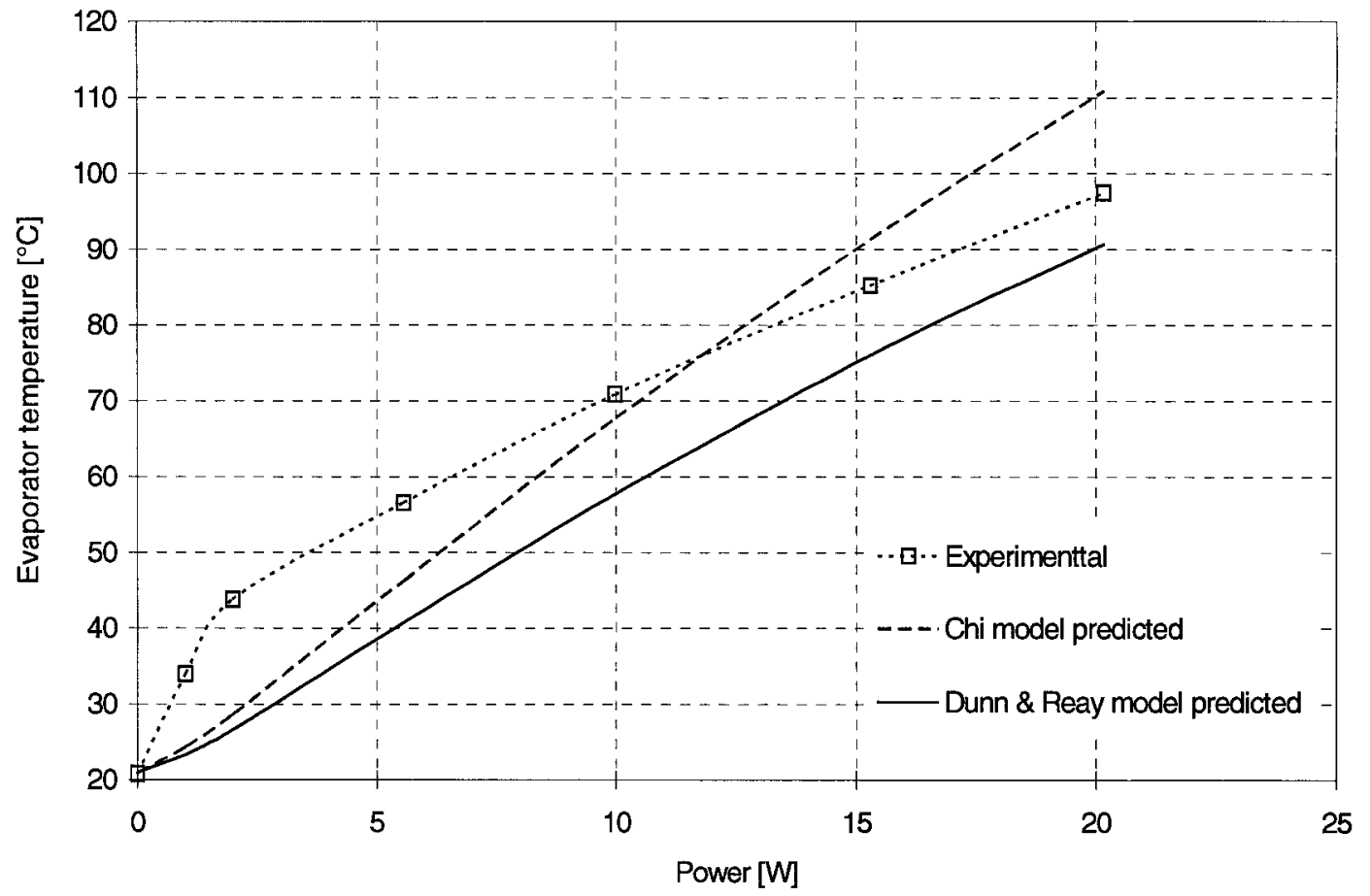

Figure 4.6 Comparison of predicted and measured evaporator temperatures (insulated, horizontal, natural convection) [sample(s):HP062400]

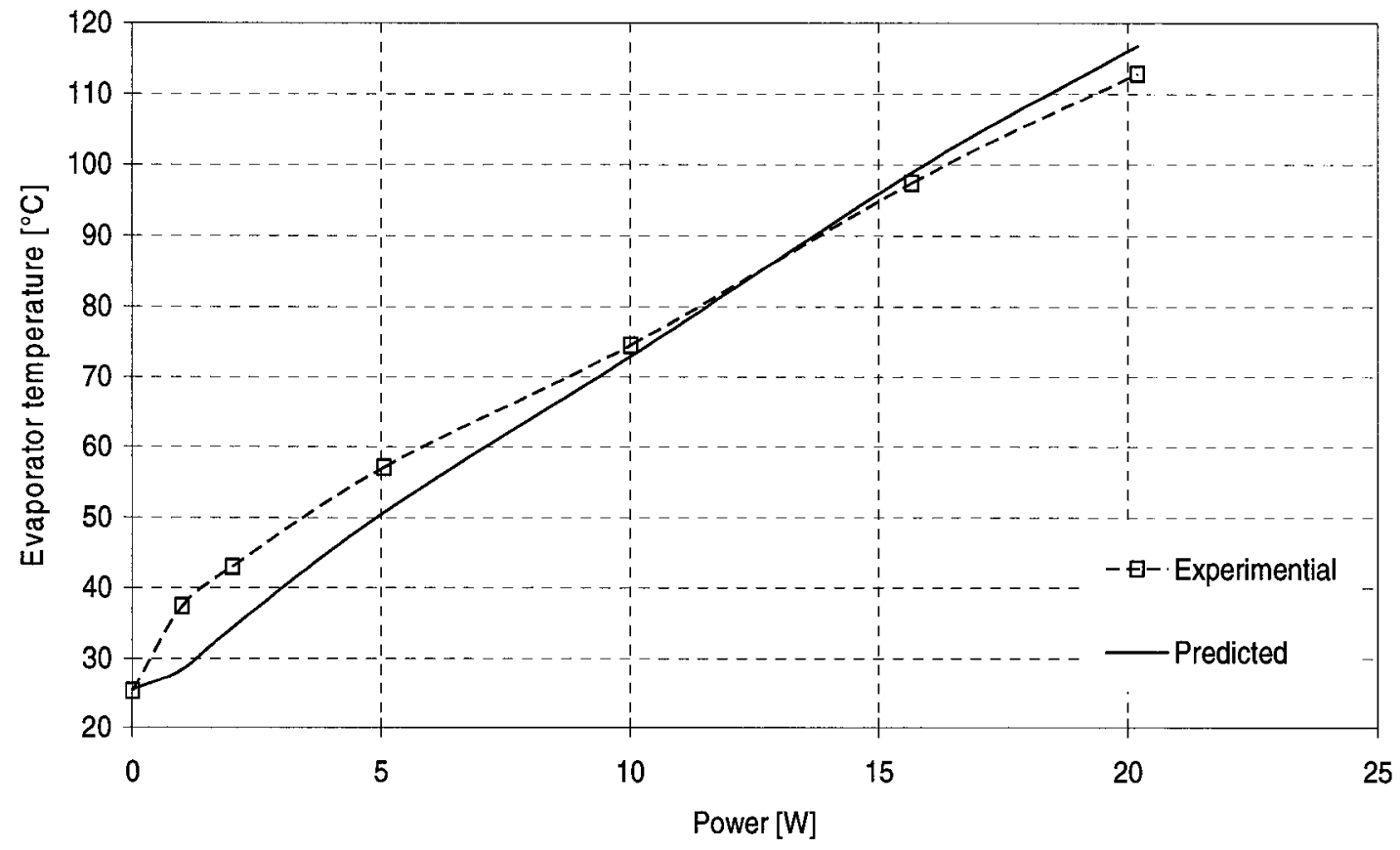

Figure 4.7 Comparison of predicted and measured evaporator temperatures (insulated, gravity assisted vertical, natural convection) [sample(s):HP052502] 
Figure 4.7 shows the comparison for the fine mesh water heat pipe test sample at a gravity assisted vertical orientation under natural convection cooling conditions. From the figure we can see that at low power levels the heat pipe behaves as it does in the horizontal orientation. With increasing power, the actual curve crosses the theoretical curve, and then tends to diverge. Such phenomena were observed in all other tested heat pipes in the same testing conditions. Among the test samples, the acetone sample shows more distinctly this tendency, and the nickel foam sample does not touch the theoretical curve, but gets closer. The reason could be related to the axial thermal resistance reduction, which will be analyzed in Section 4.3.

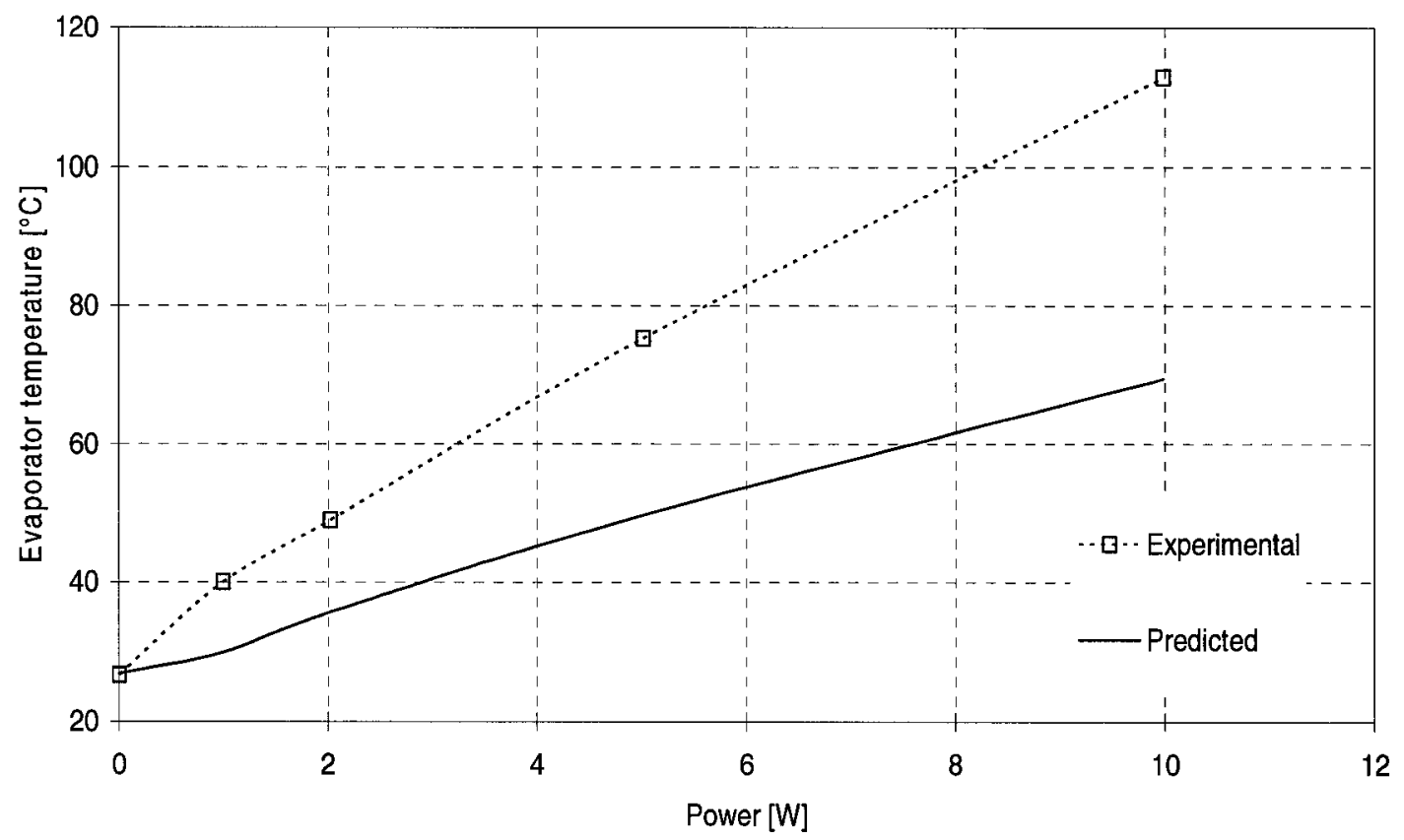

Figure 4.8 Comparison of predicted and measured evaporator temperatures (insulated, against gravity vertical, natural convection) [sample(s):HP052502] 


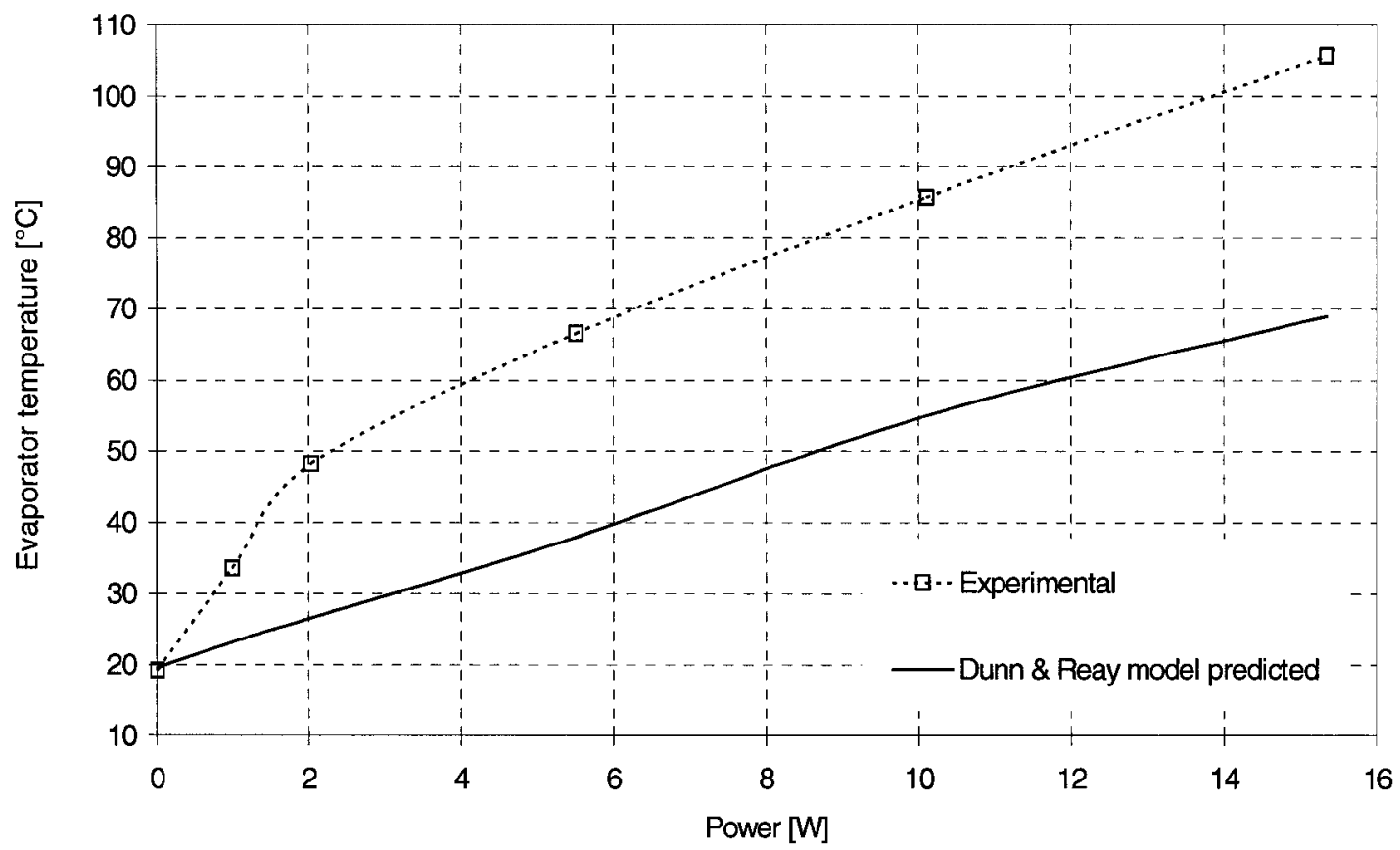

Figure 4.9 Comparison of predicted and measured evaporator temperatures (insulated, against gravity vertical, natural convection) [sample(s):HP062400]

For an against gravity vertical orientation, all the heat pipe samples perform worse than the theoretical prediction. Figures 4.8 and 4.9 show such comparison results for the fine mesh water heat pipe and the nickel foam water heat pipe samples respectively, under the same testing condition; against gravity orientation, and with natural convection cooling.

From Figure 4.8 we can see that at against gravity vertical orientation, the actual result curve diverges from the theoretical curve in a straight line with a larger slope. Similar observations are also found in other test samples, and the acetone sample has a larger slope. Some heat pipes could cease functioning in this orientation, depending on other factors such as the wick, working fluid, and other working conditions, e.g., varying cooling approaches. The nickel foam sample behaves differently from the copper mesh 
counterparts under the same working conditions. From Figure 4.9 we can see that its actual result curve goes almost in parallel to the theoretical curve. This indicates that unlike other samples, in this case the nickel foam heat pipe just gets offset from the theoretical prediction rather than ceasing functionality. The reason for the offset in this case will be analyzed in Section 4.3.

Besides natural convection cooling, forced and enhanced forced convection cooling approaches were also applied to all the test samples in all three orientations. With forced and enhanced convection, all the test samples reached higher heat transfer capacity compared to natural convection cooling. However, in all the water based test samples, discrepancies existed when comparing with theoretical predicted evaporator temperatures.

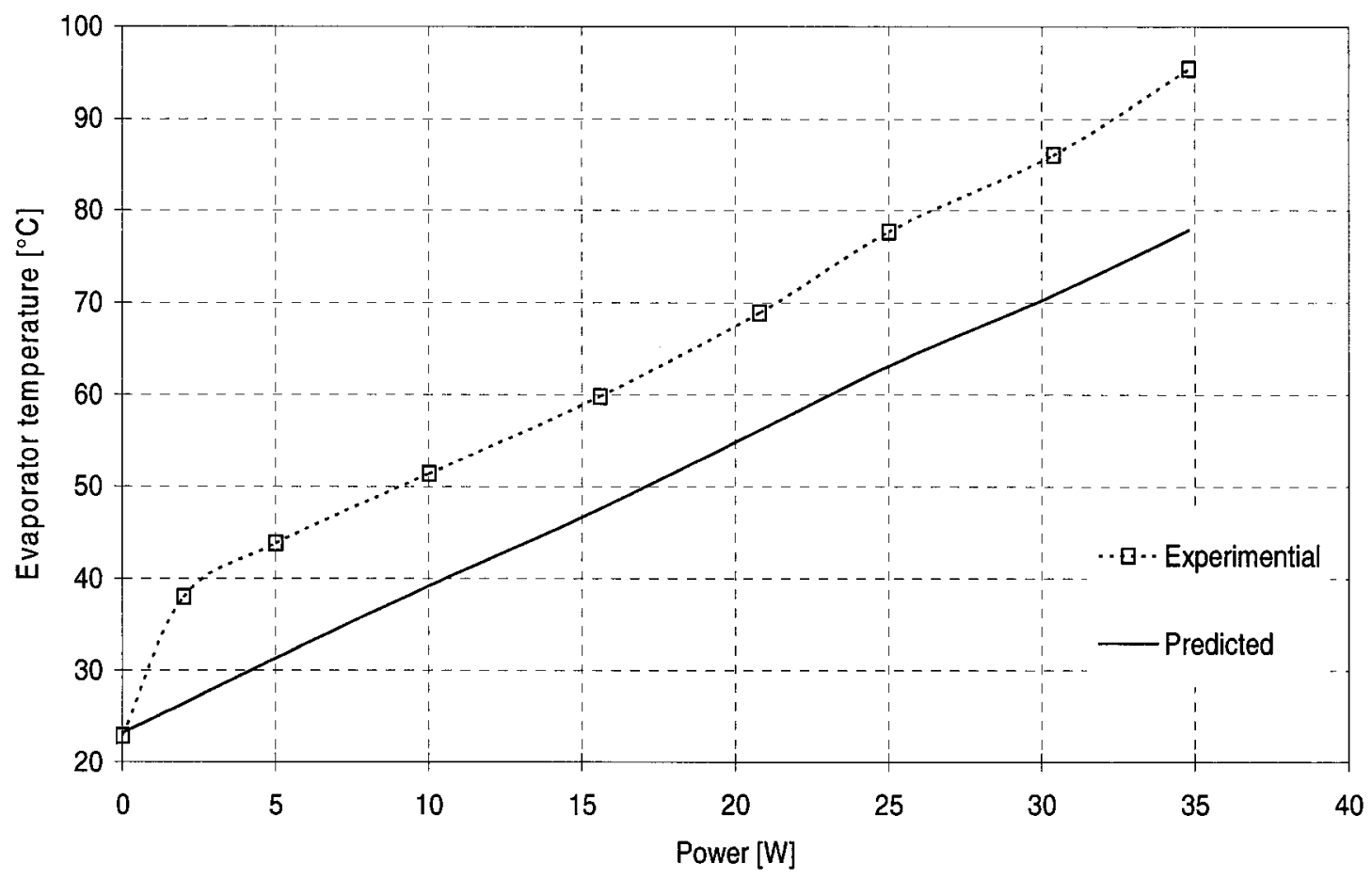

Figure 4.10 Comparison of predicted and measured evaporator temperatures (insulated, horizontal, forced convection) [sample(s):HP052502] 


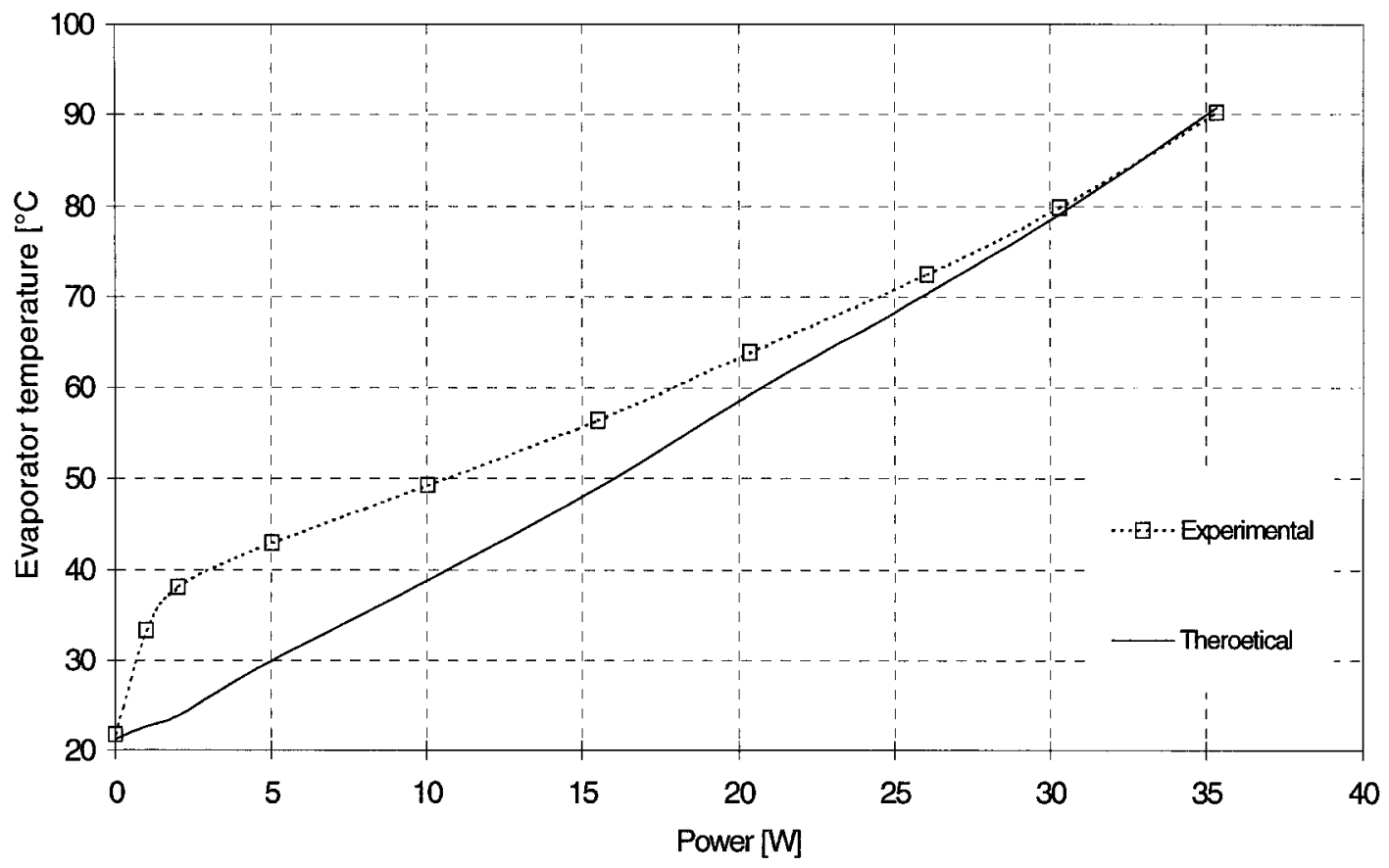

Figure 4.11 Comparison of predicted and measured evaporator temperatures (insulated, gravity assisted vertical, enhanced forced convection) [sample(s):HP052502]

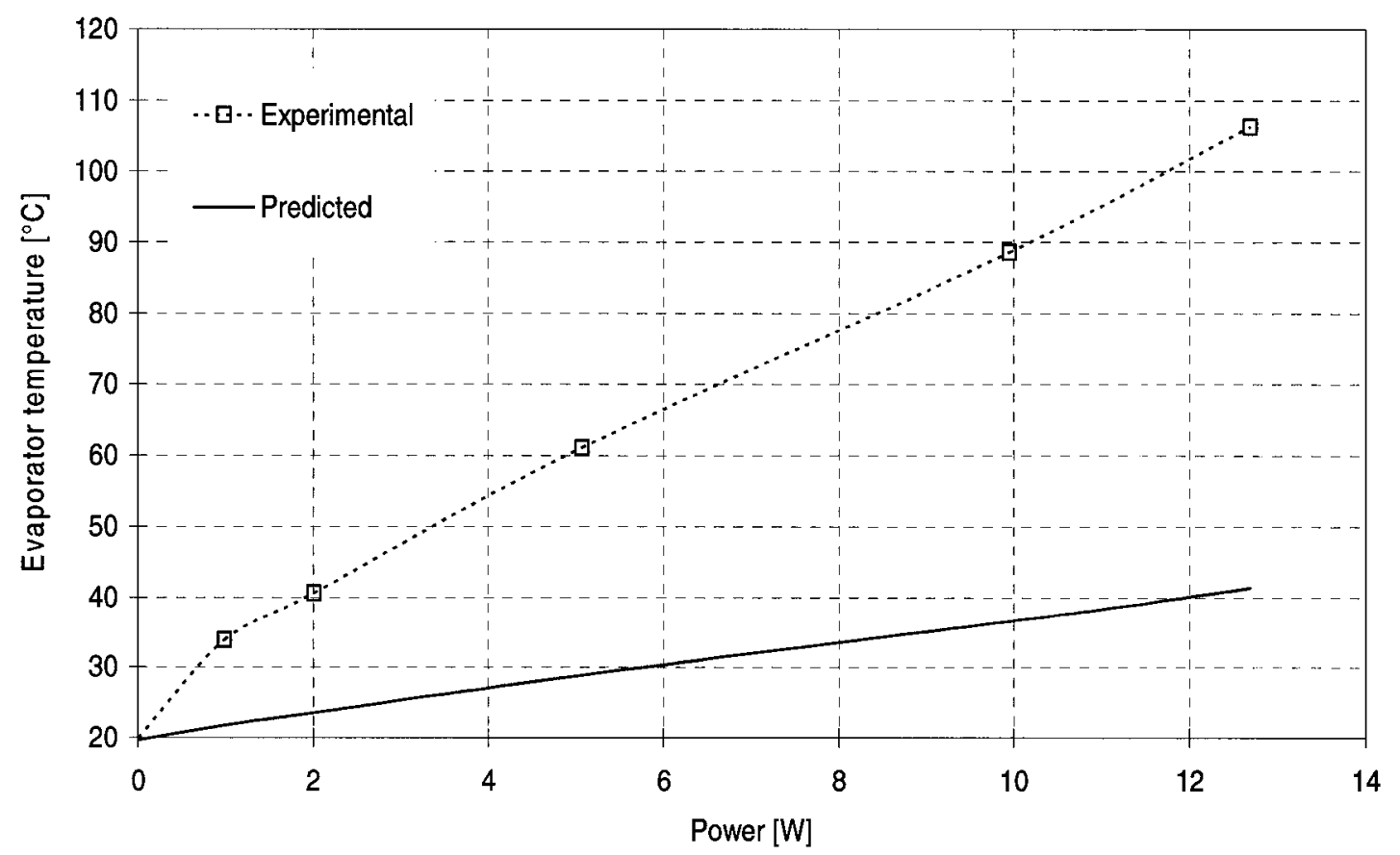

Figure 4.12 Comparison of predicted and measured evaporator temperatures

(insulated, against gravity vertical, enhanced forced convection) [sample(s):HP052502] 
Figure 4.10 shows that with forced convection cooling on the condenser, the actual measured evaporator temperatures have about $15^{\circ} \mathrm{C}$ offset from the predicted values, and the two curves go in parallel. Such phenomenon occurred to all water based heat pipe test samples in a horizontal orientation. At gravity assisted vertical orientations, such a difference is reduced with increasing power, as shown in Figure 4.11. In the against gravity orientations, this difference increases as shown in Figure 4.12. Also, similar results were observed for the nickel foam case.

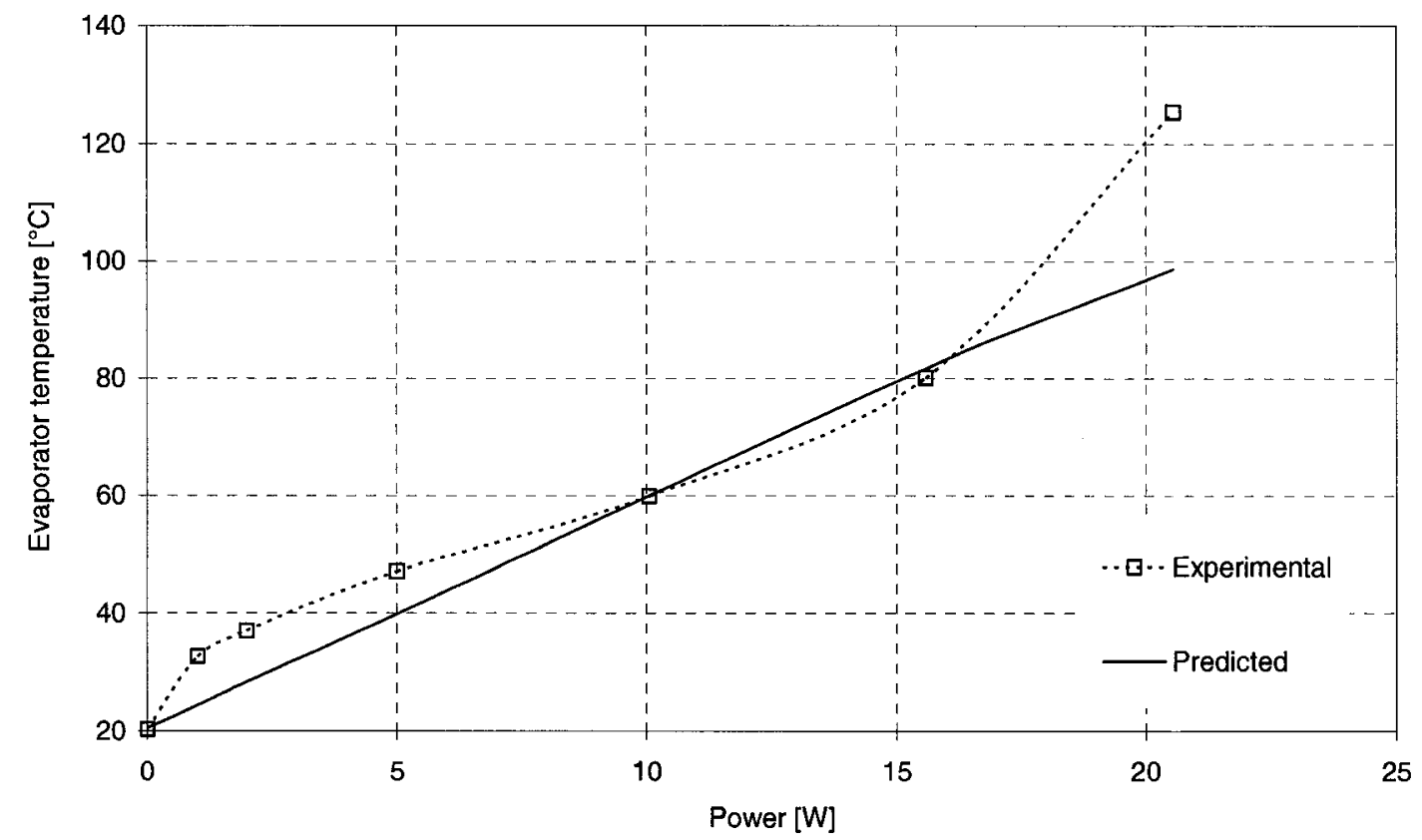

Figure 4.13 Comparison of predicted and measured evaporator temperatures (100 copper mesh, acetone; insulated, horizontal, enhanced forced convection) [sample(s):HP071402]

The only exception occurred to the acetone sample. Figure 4.13 shows the comparison of the experimental and predicted evaporator temperatures of the acetone test sample under enhanced forced convection cooling conditions. Below $15 \mathrm{~W}$, the two curves match very well. When power is increased to a value over $15 \mathrm{~W}$, the experimental curves starts to diverge from the theoretical curve. Hence, dry-out occurs. Referring to 
the natural convection case with the same acetone test sample shown in Figure 4.5, we can see that the experimental curve gets lower than the theoretical curve.

This offset was suspected due to the wind effect on the condenser. The wind from the sink slots could cause a lower temperature on the thermocouples attached on the condenser. Thus, the measured condenser temperature could be lower than the actual temperature on the condenser. Applying the lower condenser temperature in Equation (4.12) would definitely lead to lower evaporator temperatures. However, the effect of airflow from the cooling fan on the thermocouples measuring condensation zone temperatures was studied in Experiment 4 as described in Chapter 3, Section 3.5. It was shown that this effect was negligible in our cases.

\subsection{Heat Transfer Capacity Analyses}

The preceding section analyzed the heat pipe in terms of temperature characteristics. As a heat transfer device, another main characteristic is its heat transfer capacity. This section will focus on this issue.

A heat pipe distinguishes itself from other heat transfer devices by factors such as two phase heat transfer process. This two-phase process realizes heat transfer with a lower temperature gradient, thus significantly increases heat transfer capacity under a certain temperature limit. However this capacity is not unlimited, it is constrained.

There are many factors affecting the heat pipe capacity. To explore the limit to the heat pipe capacity, we need to first analyze the heat pipe intrinsic structure and mechanism. The main factor increasing the heat pipe capacity can be due to the two-phase process. 
On the other hand, the heat transfer capacity depends on the continuity of two-phase process.

The factors affecting the status of the working fluid and its vapour can be summarized as following; liquid capillarity, vapour flow, vapour-liquid flow interface, and boiling. As will be illustrated later, the capillary limit is the smallest among the four factors; a heat pipe capacity limit would be mainly determined by the capillary limit. This section mainly analyzes the issue of capillary limit.

\subsubsection{Capillary Effect on Heat Transfer Capacity}

Capillarity is a unique characteristic that distinguishes heat pipes from other heat transfer devices. It is the capillary force that drives the condensed working fluid back to the evaporator to compensate for the fluid consumed due to evaporation, thus realizing the phase change cycle. At a certain working temperature, the capillary force depends on the wick condition including material, structure, surface treatment, and the property of the working fluid as well. With the working temperature varying, these factors would also vary. This temperature change comes from the heat load laid upon the evaporator. Therefore it can be said that the capillary force in the heat pipe would change with the amount of heat to be transferred. Specific analyses are illustrated below.

For a certain heat pipe at a certain working temperature, the effective capillary pressure at the evaporator is determined by factors such as the wick capillary radius and its orientation, and can be expressed as (Chi, 1976),

$$
P_{p m}=\frac{2 \cdot \sigma}{r_{c}}-\rho_{l} \cdot g \cdot d_{v} \cdot \cos \psi-\rho_{l} \cdot g \cdot L_{p} \cdot \sin \psi
$$


where, $\psi$ is the heat pipe inclination measured from horizontal position (refer to Figures 3.30 to 3.32 ), and its relationship with the heat pipe working orientation is defined as:

$$
\begin{aligned}
& 0>\psi>\pi \text { : against gravity, } \\
& \pi>\psi>2 \pi \text { : gravity assisted, }
\end{aligned}
$$

$r_{c}$ is the wick capillary radius and can be calculated as below:

for wire screen wick:

$$
r_{c}=\frac{1}{2 \cdot N_{\text {mesh }}}
$$

for the foam wick, $r_{c}=2.5 \times 10^{-4} \mathrm{~m}$

Capillary heat transfer factor (Chi, 1976),

$$
(Q L)_{c, \max }=\frac{P_{p m}}{F_{l}+F_{v}}
$$

Where, the fractional coefficient for liquid flow, $F_{l}$, can be obtained from the equation,

$$
F_{l}=\frac{\mu_{l}}{K_{m e s h} \cdot A_{w} \cdot \rho_{l} \cdot \lambda}
$$

and the wick permeability, $K_{m e s h}$, can be obtained from the below,

For copper wire screen mesh,

$$
K_{m e s h}=\frac{d_{w}^{2} \cdot \varepsilon_{m e s h} 3}{122 \cdot\left(1-\varepsilon_{m e s h}\right)^{2}}
$$

For the nickel foam,

$$
K_{m e s h}=\frac{r_{s}^{2} \cdot \varepsilon_{f o a m} 3}{37.5 \cdot\left(1-\varepsilon_{\text {foam }}\right)^{2}}
$$

Where the wick cross sectional area, $A_{w}$, can be obtained from the below, 


$$
A_{w}=\pi \cdot \frac{\left(d_{i}^{2}-d_{v}^{2}\right)}{4}
$$

The fractional coefficient for vapour flow, $F_{v}$, can be obtained from the equation,

$$
F_{v}=\frac{f_{v} R_{e} \cdot \mu_{v}}{2 \cdot A_{v} \cdot r_{h . v}^{2} \cdot \rho_{v} \cdot \lambda}
$$

Here, the hydraulic radius for vapour flow, $r_{h . r}$, can be obtained from the blow,

$$
r_{h . v}=\frac{d_{v}}{2}
$$

the vapour core cross sectional area, $A_{v}$, can be obtained from the below,

$$
A_{v}=\pi \cdot r_{h . v}^{2}
$$

the drag coefficient, $f_{v} R e_{v}$, for circular vapour flow passage takes value of $16(\mathrm{Chi}, 1976)$ Thus obtain the maximum capillary transfer, i.e., capacity heat transfer limit,

$$
Q_{c . \max }=\frac{(Q L)_{c, \max }}{L_{e f f}}
$$

Where, the heat pipe effective length, $L_{e f f}$, can be calculated as below (Chi, 1976),

$$
L_{e f f}=\frac{1}{2} \cdot L_{e}+L_{a}+\frac{1}{2} \cdot L_{c}
$$

Figure 4.14 illustrates the comparison of predicted capillary heat transfer limit as a function of adiabatic temperature for different test samples. From this figure we can see that the nickel foam test sample has a far greater capillary limit than its copper wire screen wick counterparts. Also, this figure indicates the fact that the capillary limit of the nickel foam test sample is far beyond the heat source limit of $40 \mathrm{~W}$ applied in the experiments. In other words, this nickel foam heat pipe did not start to work at its optimum working power range. This supports the conjecture made in Section 4.1.2. 


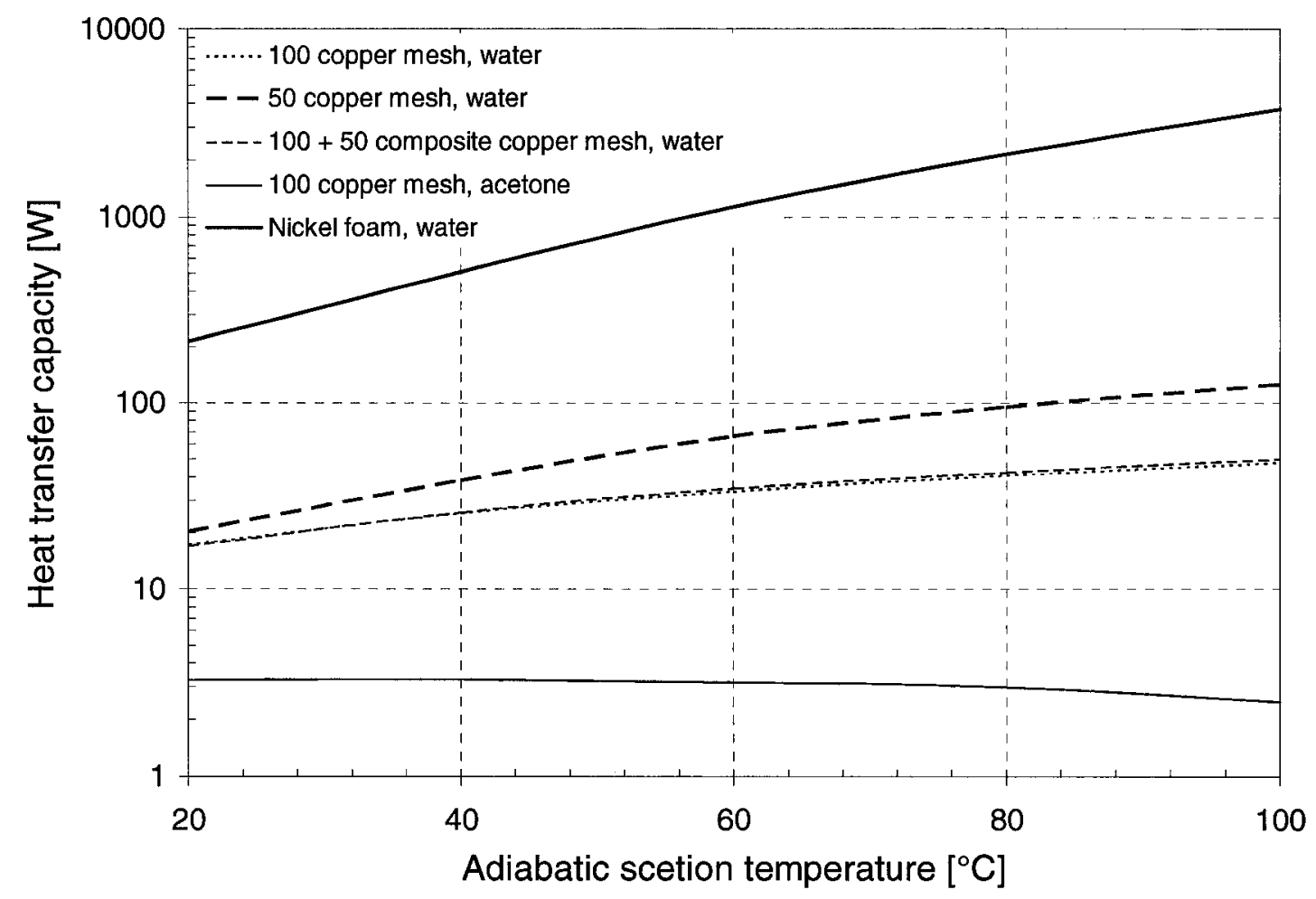

Figure 4.14 Comparison of heat pipe capillary limits (horizontal)

Figure 4.15 illustrates the comparison of predicted capillary heat transfer limit between the copper wire screen wick test samples. From this figure we can see that the fine and composite mesh water based test samples can reach $50 \mathrm{~W}$ in capillary limit, and the coarse mesh water test sample has a $125 \mathrm{~W}$ potential limit. These are consistent with the experimental results, i.e., at horizontal and gravity assisted vertical orientations all those water based test samples can normally work at $40 \mathrm{~W}$ under forced convection cooling assist. Here in the figure the only exception that is not consistent with the experimental results is the acetone case. The predicted capability limit is only about $4 \mathrm{~W}$; this prediction is not consistent with actual results. In fact the acetone test sample can normally work at about $15 \mathrm{~W}$ at horizontal and gravity assisted vertical orientations with forced convection. This again introduces hysteresis, and will be explored in Section 4.3. 


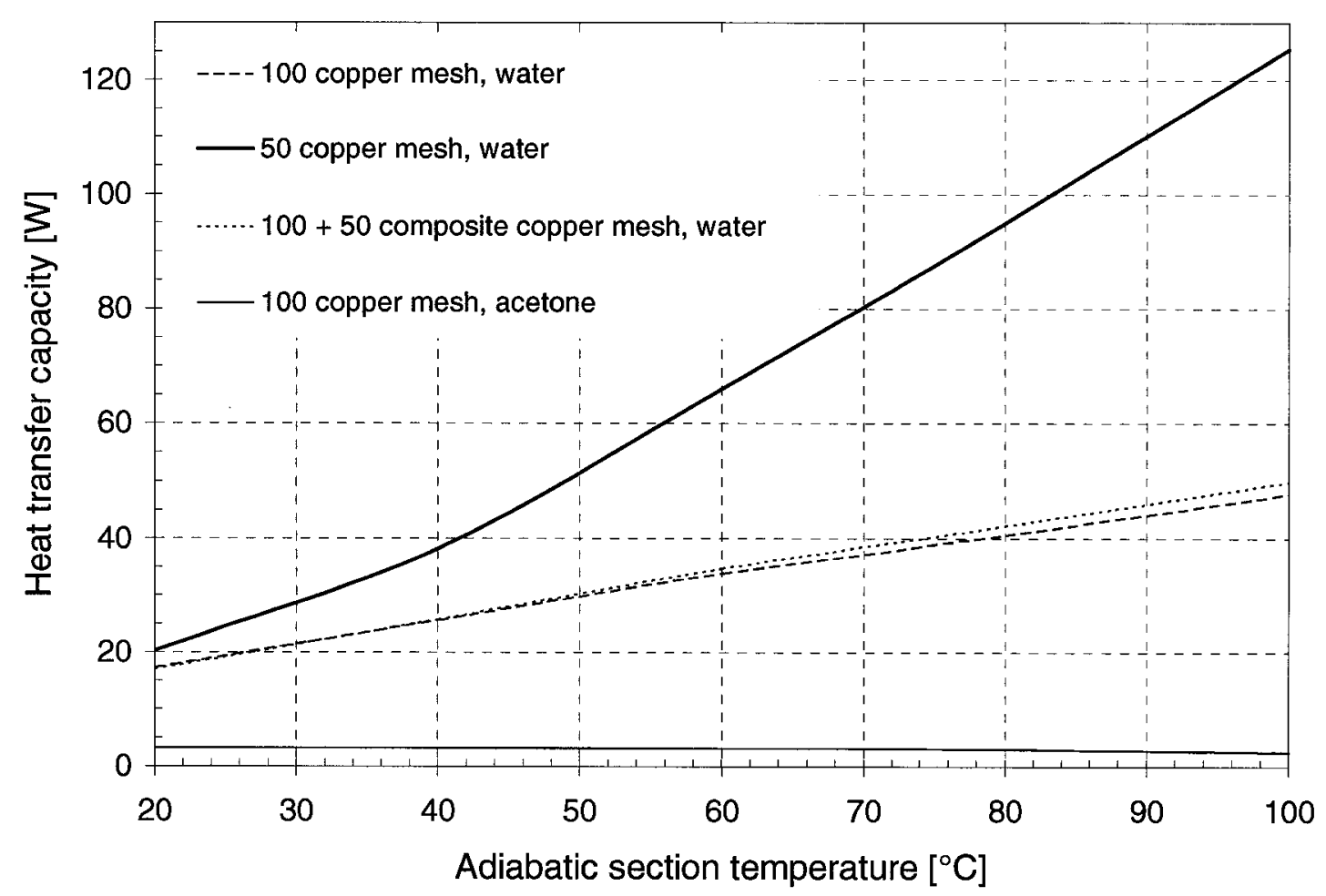

Figure 4.15 Comparison of heat pipe capillary limits (horizontal)

It is necessary to analyze the orientation effect on capillary limit. Figure 4.16 illustrates the predicted capillary limits at gravity assisted vertical orientation among all the test samples, and Figure 4.17 illustrates the predicted capillary limits at gravity assisted vertical orientation among the copper wire screen wick test samples. From these two figures, we can see that with gravity assist all the test samples get a significantly improved capillary limit. Especially note that the capillary limit of the acetone test sample improves up to nearly $20 \mathrm{~W}$. This is consistent with the experimental results in the gravity assisted cases. 


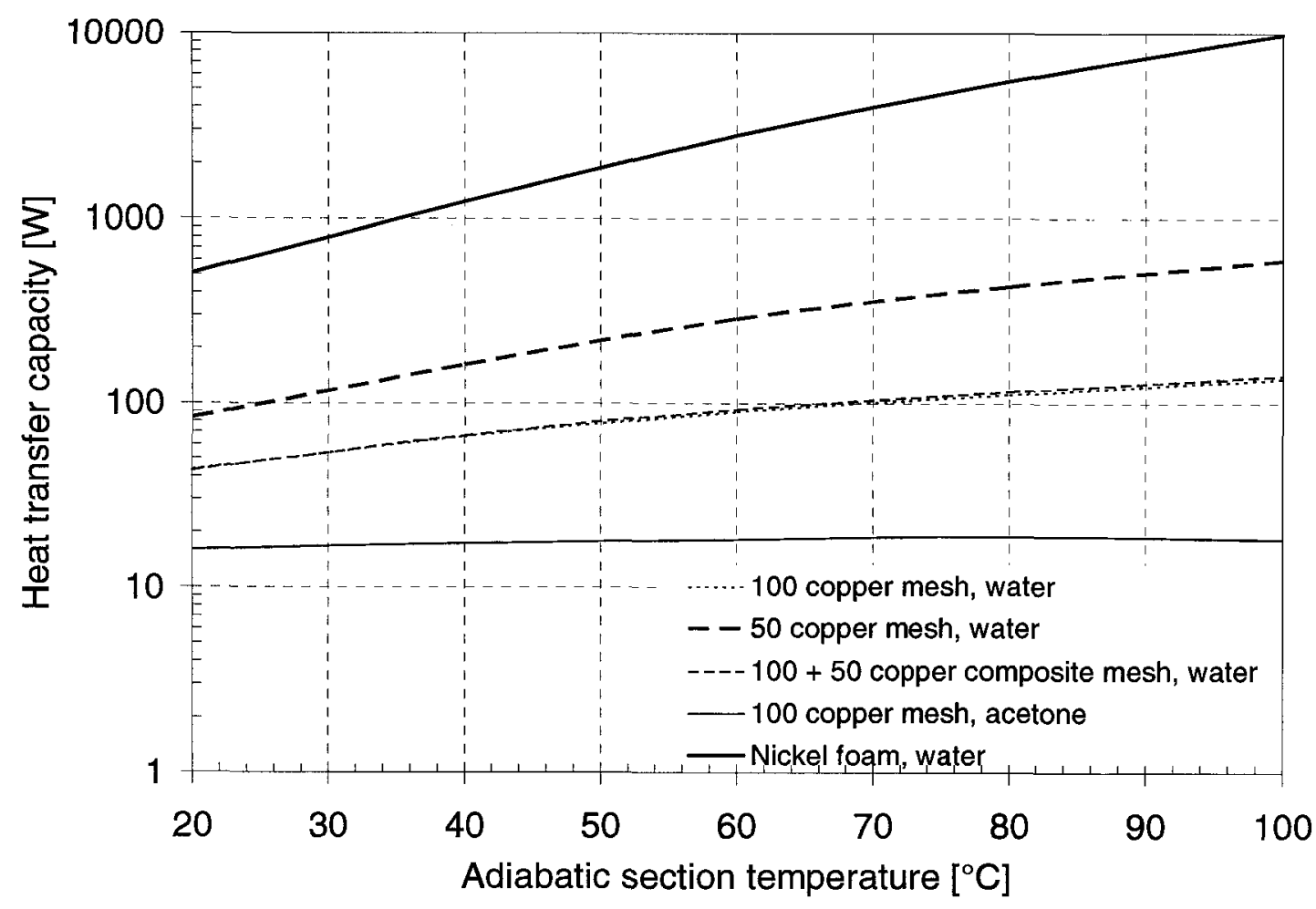

Figure 4.16 Comparison of heat pipe capillary limit (gravity assisted vertical)

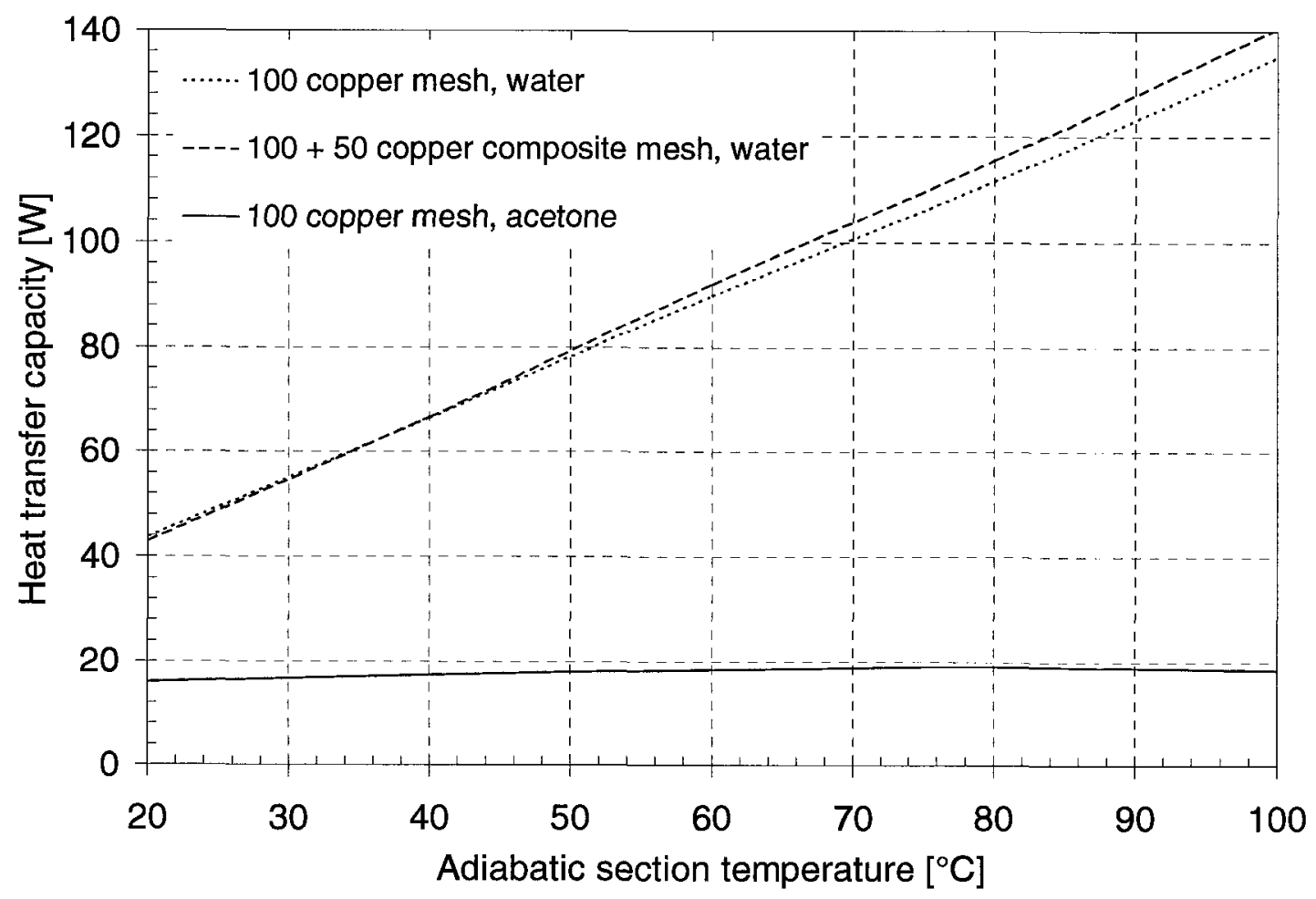

Figure 4.17 Comparison of heat pipe capillary limit (gravity assisted vertical) 


\subsubsection{Vapour Flow Effect on Heat Transfer Capacity}

The second factor affecting the heat pipe transfer capacity relates to the vapour flow state. The vapour flows from the evaporator to the condenser where it discharges heat and condenses to liquid fluid. If this vapour flow delivers enough mass of the working fluid, the continuity of the phase change can be ensured. Otherwise, the condenser would not receive enough mass to compensate for the liquid loss due to the preceding condensed liquid being sucked back to the evaporator. Thus, the phase change circulation ceases.

This factor causing the limit to the vapour flow relates to the sonic flow, and is termed sonic limit. The specific mechanism is analyzed by Chi (1976). The mathematical calculation is adapted from Chi (1976), as shown below,

$$
Q_{s . \max }=A_{v} \cdot \rho_{v} \cdot \lambda \cdot\left[\frac{\gamma_{v} \cdot R_{v} \cdot T_{v}}{2 \cdot\left(\gamma_{v}+1\right)}\right]^{1 / 2}
$$

where, water vapour specific heat ratio: $\gamma_{\nu}=1.33$

water molecular weight: $M=18$

$$
R_{v}=\frac{8.314 \times 10^{3}}{M} \cdot \frac{\mathrm{J}}{\mathrm{kg} \cdot \mathrm{mol} \cdot \mathrm{K}}
$$

Here, vapour temperature: $T_{v}=293 \mathrm{~K}$

\subsubsection{Flow Interface Effect on Heat Transfer Capacity}

Besides the vapour flow, the other main flow factor affecting the heat pipe transfer stems from the interface between the vapour and the liquid. Both move in an opposite direction, thus causing a shear force at the interface. The vapour velocity in a heat pipe is generally much higher than the liquid velocity (Chi, 1976). The shear force tends to tear the liquid 
from the wick surface and is proportional to the vapour momentum and the wick pore surface. With the heat load increasing this shear force would increase. When such force reaches a certain value, it will be able to hinder the liquid flow, thus the heat pipe reaches its transfer limit due to such shear force between the two opposite moving flows. This limit is termed entrainment limit. A specific calculation process is provided as below (Chi, 1976).

$$
Q_{e \cdot \max }=A_{v} \cdot \lambda \cdot\left(\frac{\sigma \cdot \rho_{v}}{2 \cdot r_{h . s}}\right)^{1 / 2}
$$

where $r_{h . s}$ is hydraulic radius of wick at vapour-wick interface,

for copper wire screen mesh wick

$$
r_{h . s}=r_{c}-\frac{d_{w}}{2}
$$

for the nickel foam wick sample,

$$
r_{h . s}=r_{c}
$$

\subsubsection{Boiling Effect on Heat Transfer Capacity}

Evaporation is required for a heat pipe to function, the evaporation process intakes heat by phase change. However, in a heat pipe, the wick structure can provide an interface for boiling which can cause vapour bubbles in the wick. These vapour bubbles could cause hot spots and obstruct the liquid circulation in the wick. Hence there is a radial heat flux limit for the evaporation, and such limit is termed boiling limit (Chi, 1976).

The boiling limit can be calculated with the following equation, 


$$
Q_{b . \max }=\frac{2 \cdot \pi \cdot L_{e} \cdot k_{e . e} \cdot T_{v}}{\lambda \cdot \rho_{v} \cdot \ln \left(\frac{r_{i}}{r_{h . v}}\right)} \cdot\left(\frac{2 \cdot \sigma}{r_{n}}\right)
$$

where, water nucleation radii: $r_{n}=2.54 \times 10^{-7} \mathrm{~m}(\mathrm{Chi}, 1976)$

For copper wire screen mesh wick, $k_{e . e}=\frac{k_{l} \cdot\left[k_{l}+k_{w}-\left(1-\varepsilon_{\text {mesh }}\right) \cdot\left(k_{l}-k_{w}\right)\right]}{\left(k_{l}+k_{w}\right)+\left(1-\varepsilon_{m e s h}\right) \cdot\left(k_{l}-k_{w}\right)}$

For nickel foam wick

$$
k_{e . e}=k_{w} \cdot\left[\frac{2+\frac{k_{l}}{k_{w}}-2 \cdot \varepsilon_{\text {foam }} \cdot\left(1-\frac{k_{l}}{k_{w}}\right)}{2+\frac{k_{l}}{k_{w}}+\varepsilon_{f o a m} \cdot\left(1-\frac{k_{l}}{k_{w}}\right)}\right]
$$

Figures 4.18 to 4.21 illustrate the comparisons among predicted capillary, sonic, entrainment, and boiling limits for different test samples. Comparing these figures we can see that all three limits ascend with an increasing adiabatic temperature, but the boiling limit moves in an opposite direction. The copper wire screen wick test samples are limited by the capillary limit. Note that, for the acetone test sample, its capillary limit is very low, and the boiling limit reaches only a few watts at high temperatures. This is a main reason for its propensity to experience dry-out. Also note that, the acetone test sample has a very high sonic limit compared with its other limits. 


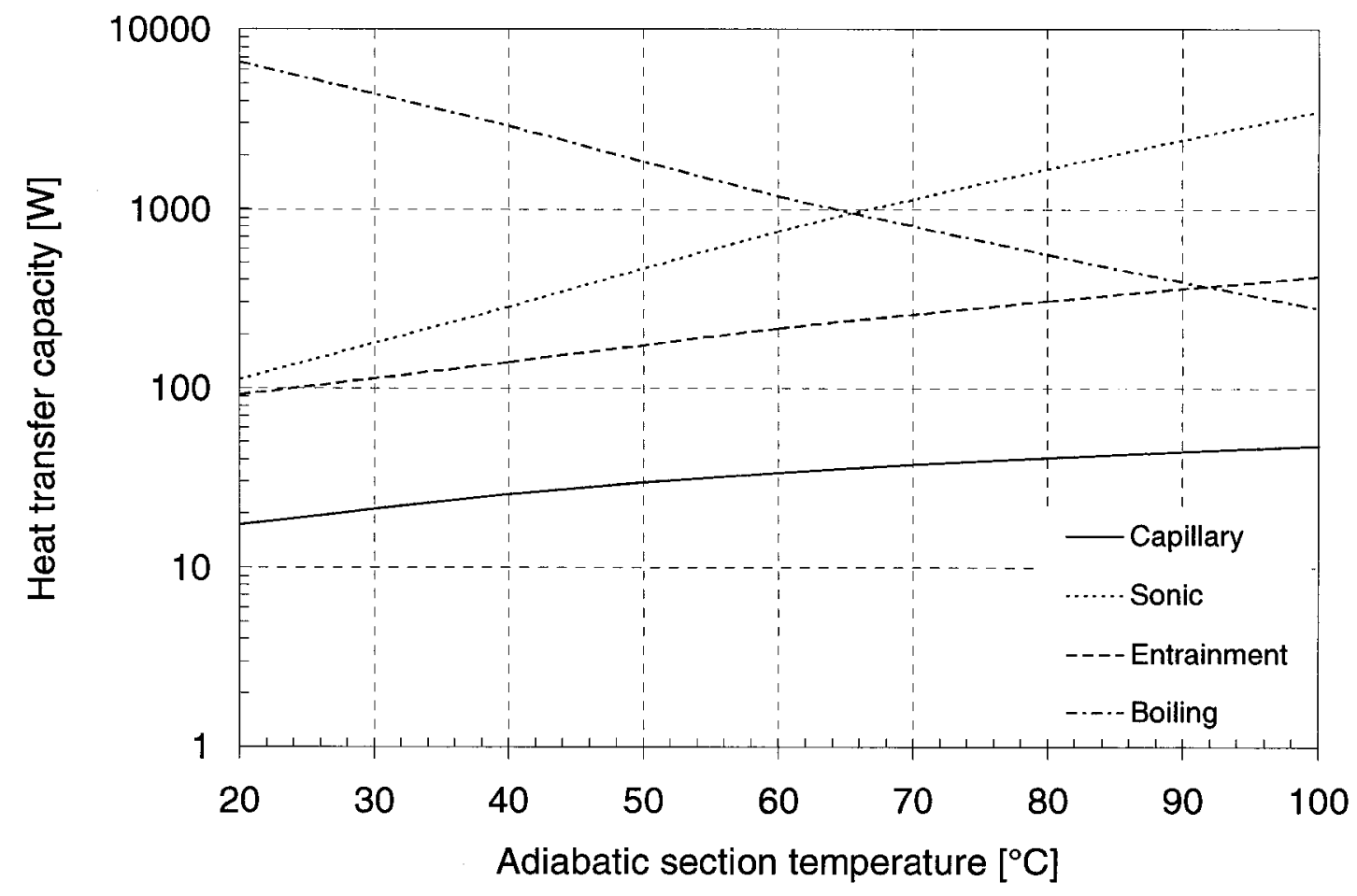

Figure 4.18 Comparison of predicted heat transfer limits (100 copper mesh, water)

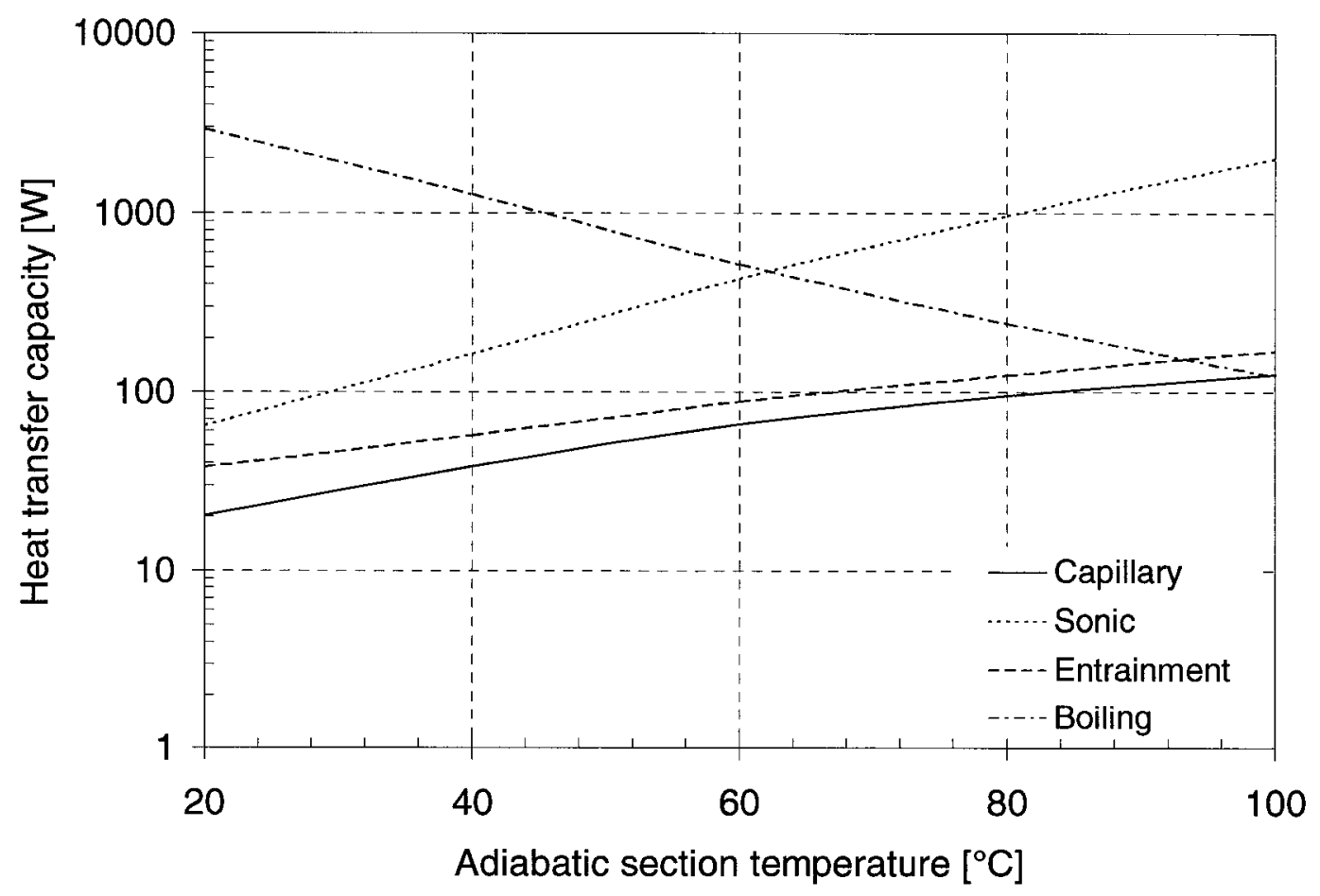

Figure 4.19 Comparison of predicted heat transfer limits (50 copper mesh, water) 


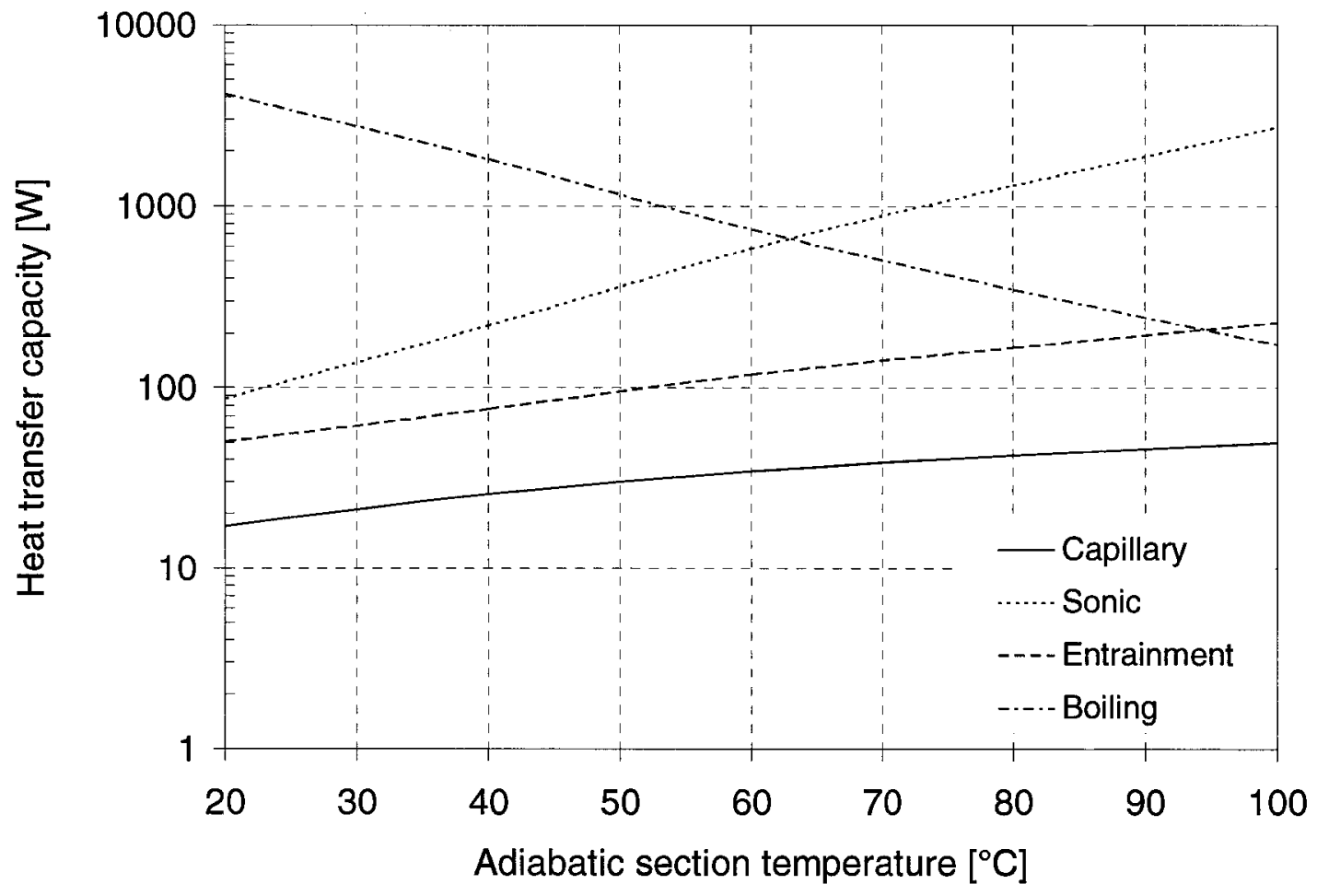

Figure 4.20 comparison of predicted heart transfer limits $(100+50$ composite copper mesh, water)

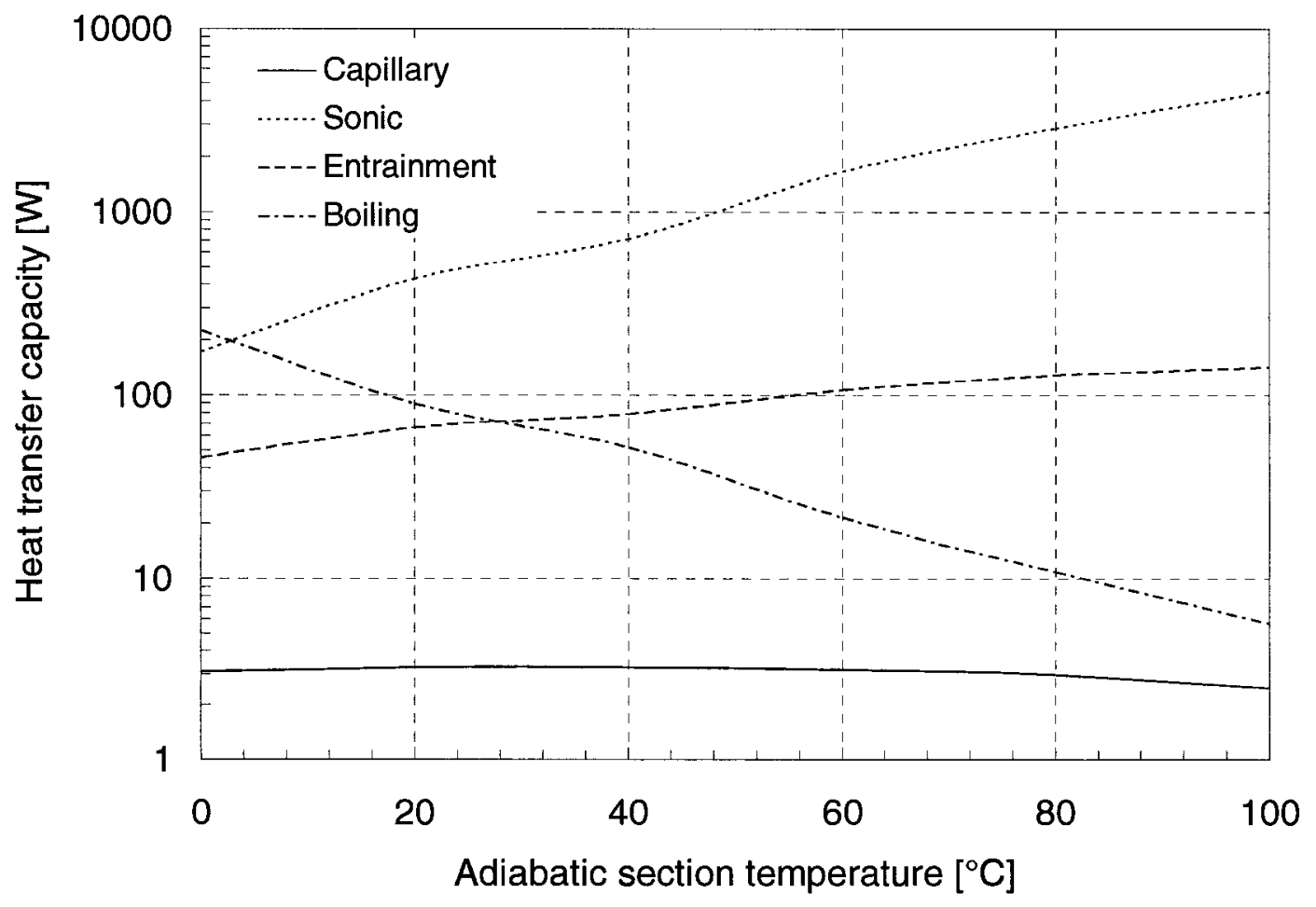

Figure 4.21 Comparison of predicted heat transfer limits (100 copper mesh, acetone) 


\subsection{Discussion on Heat Pipe Effective Length}

\subsubsection{Introduction}

When the experimental results and model predictions were compared, some discrepancies were observed. Some of these questions are explained in Chapter 3 and in early parts of this chapter. However, some of these explanations are only based on speculation. To prove these speculations, we need theoretical verification. Figure 4.22 shows the sectional differentiation for a heat pipe. Unlike traditional heat pipe section differentiation, in this diagram, the heat pipe is divided into five sections instead of three. Two transition sections are added. This is consistent with actual axial temperature profiles obtained from practical experiments.

Reviewing the experimental results, we find that, for the same heat pipe test sample these two transitional sections change under different circumstances. For instance, under natural convection cooling conditions the transition sections tend to be flat. With the increasing evaporator-condenser temperature gradient, the temperature profiles at transition sections have greater slopes, and the transition lengths tend to be longer. Meanwhile, the other three sections become shorter. The transition section temperature profile slope change would consequently result in the entire heat pipe axial temperature gradient change. This ensures that the other three sections keep an isothermal axial temperature profile. This phenomenon is commonly observed in heat pipe testing. It was estimated that this transitional section length change could result in changes in the effective length of the heat pipe, which is defined by Equation (4.26). Based on this assumption, analyses will be conducted. 


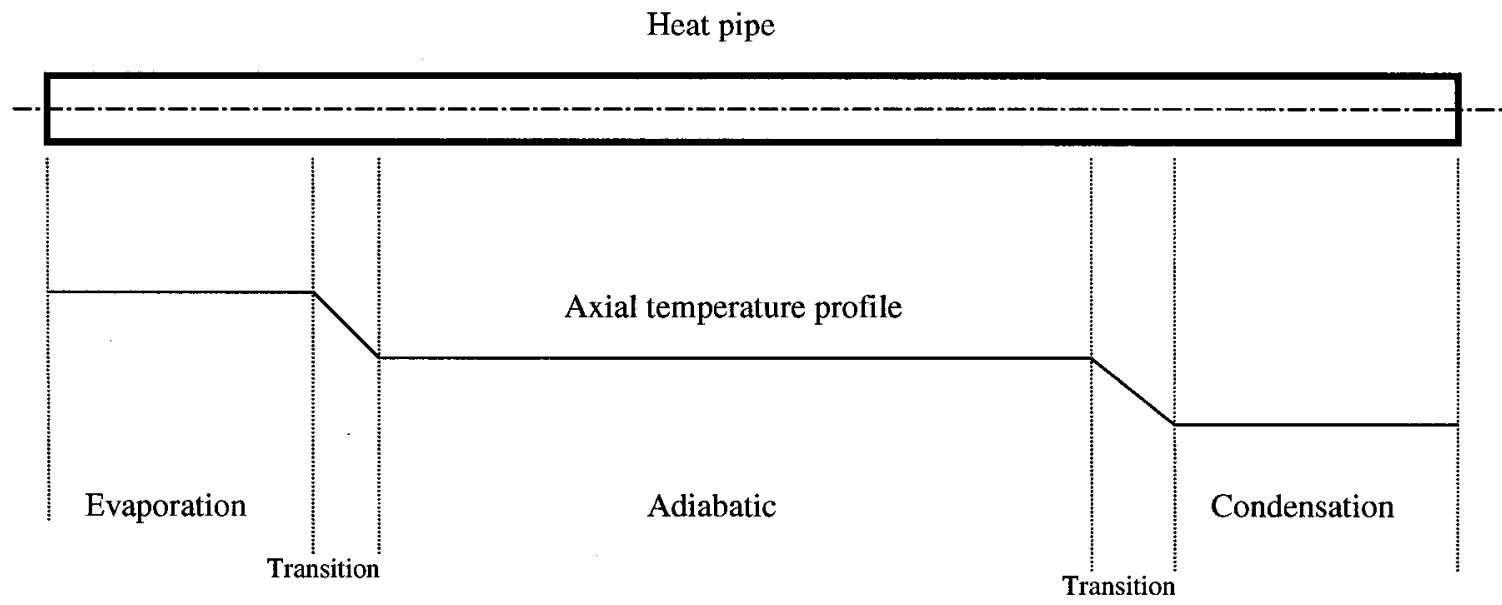

Figure 4.22 Heat pipe axial section differentiations

\subsubsection{Comparing Analyses}

First note that, with forced convection cooling, the actual evaporator temperature for the water case is higher than predictions but is lower for acetone. Based on these two cases, we can assume that for the heat pipe with water charged, the effective length decreases with the evaporator-condenser temperature difference. For the acetone case, its effective length would not be sensitive to the axial temperature gradient. Based on such assumptions, we take a shorter effective length for the water based heat pipe test sample. For instance we take the temperature measured at the edge of the condenser near the adiabatic section, and substitute this temperature values into Equation (4.12), thus obtaining corrected evaporator temperature predictions.

Figures 4.23 and 4.24 illustrate the results. In these figures, the experimental curves converge toward the corrected curve with increasing power. These results provide evidence for the assumption about the reduction of effective length. 


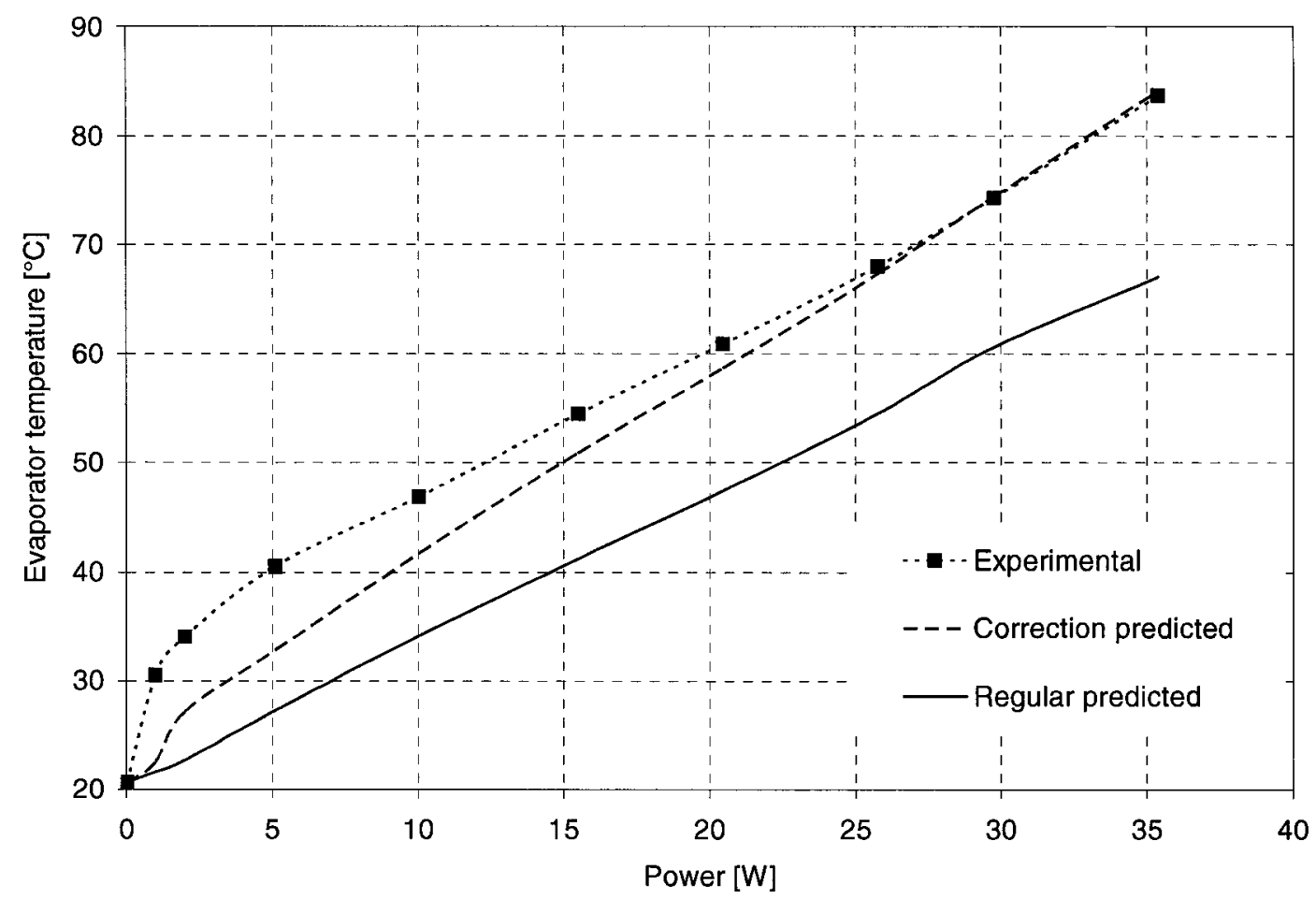

Figure 4.23 Comparison of evaporator temperature with correction (insulated, horizontal, enhanced forced convection) [sample(s):HP051701]

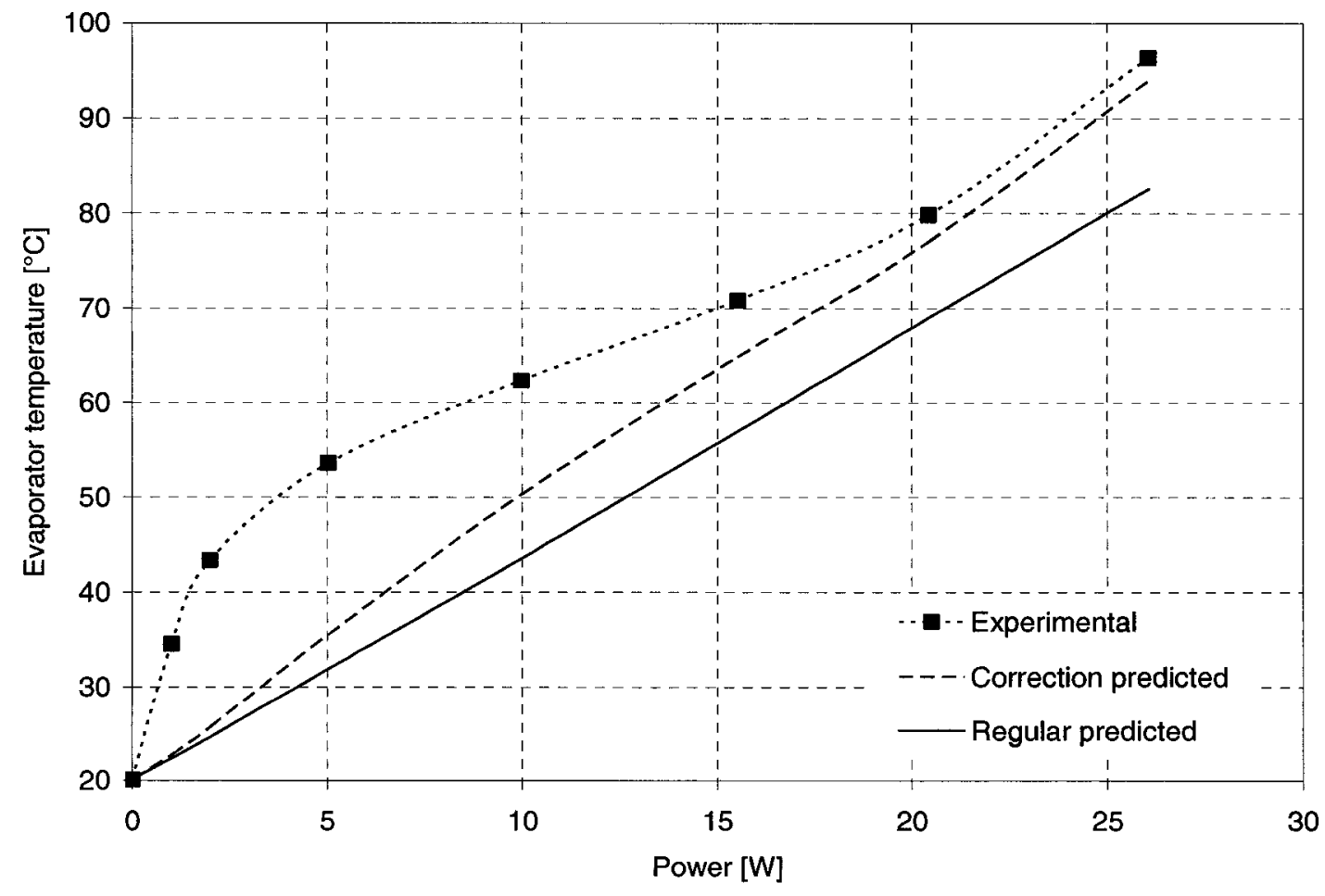

Figure 4.24 Comparison of evaporator temperature with correction (insulated, horizontal, forced convection) [sample(s):HP071200] 
Figures 4.25 to 4.28 illustrate the relationship between the effective length and the heat pipe capillary limit. These figures were obtained by theoretical calculations. From these figures we can note a general observation that the heat transfer capillary limit increases with decreasing heat pipe effective length.

Figures 4.25 to 4.27 show this relationship for the fine copper mesh water heat pipe, acetone heat pipe, and the nickel foam heat pipe at against gravity vertical orientation. From these figures we can see that each heat pipe test sample has a critical value for the effective length, when the effective length is over this value the capillary starts to transfer from negative to positive values. The physics meaning for the negative value here is that, the heat pipe would not work in an orientation of against gravity for a certain working length; rather, it would work in an opposite orientation providing the capacity with an equivalent of the negative value that shown here.

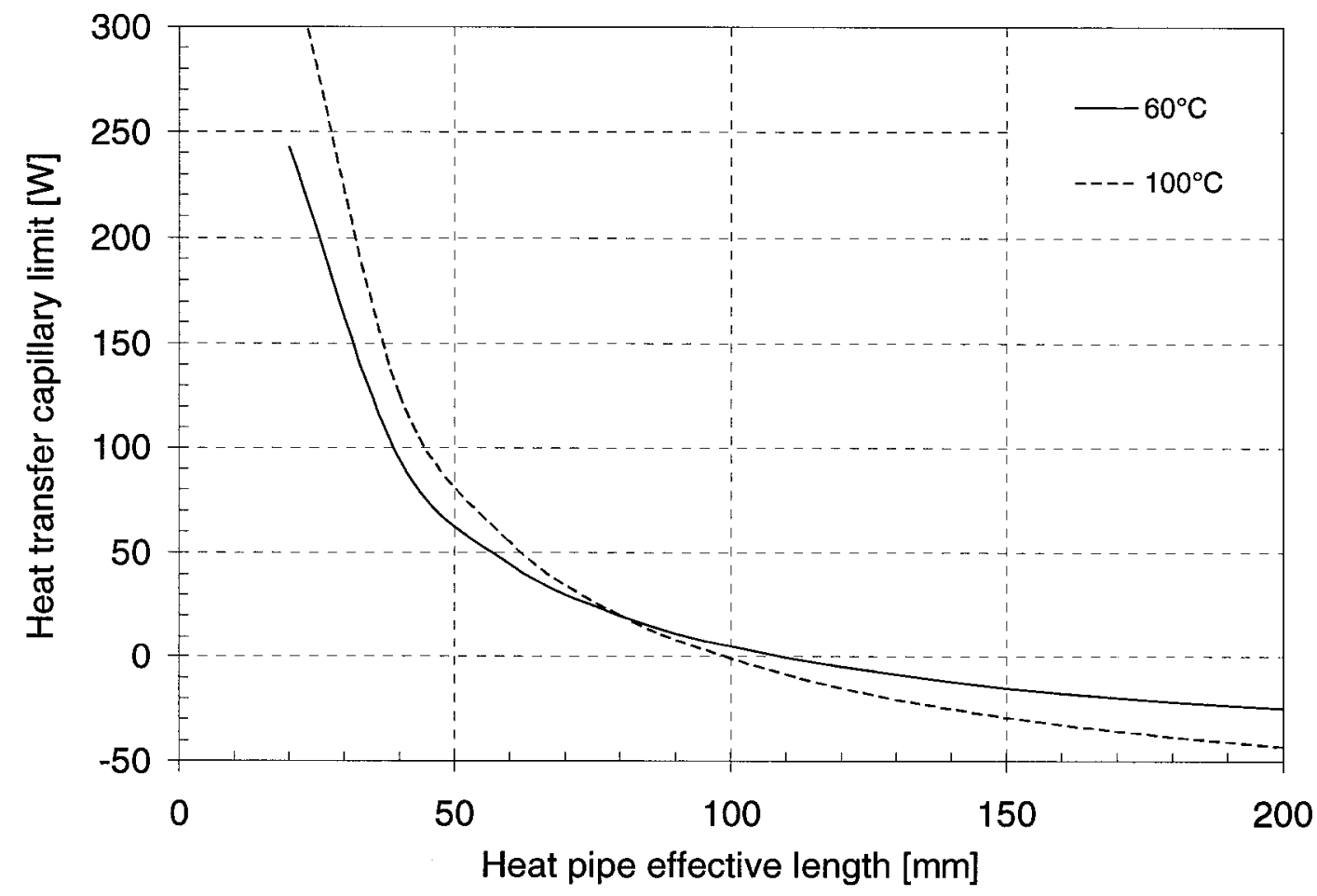

Figure 4.25 Capillary limit as a function of heat pipe effective length (against gravity vertical) [100 copper mesh water heat pipe] 


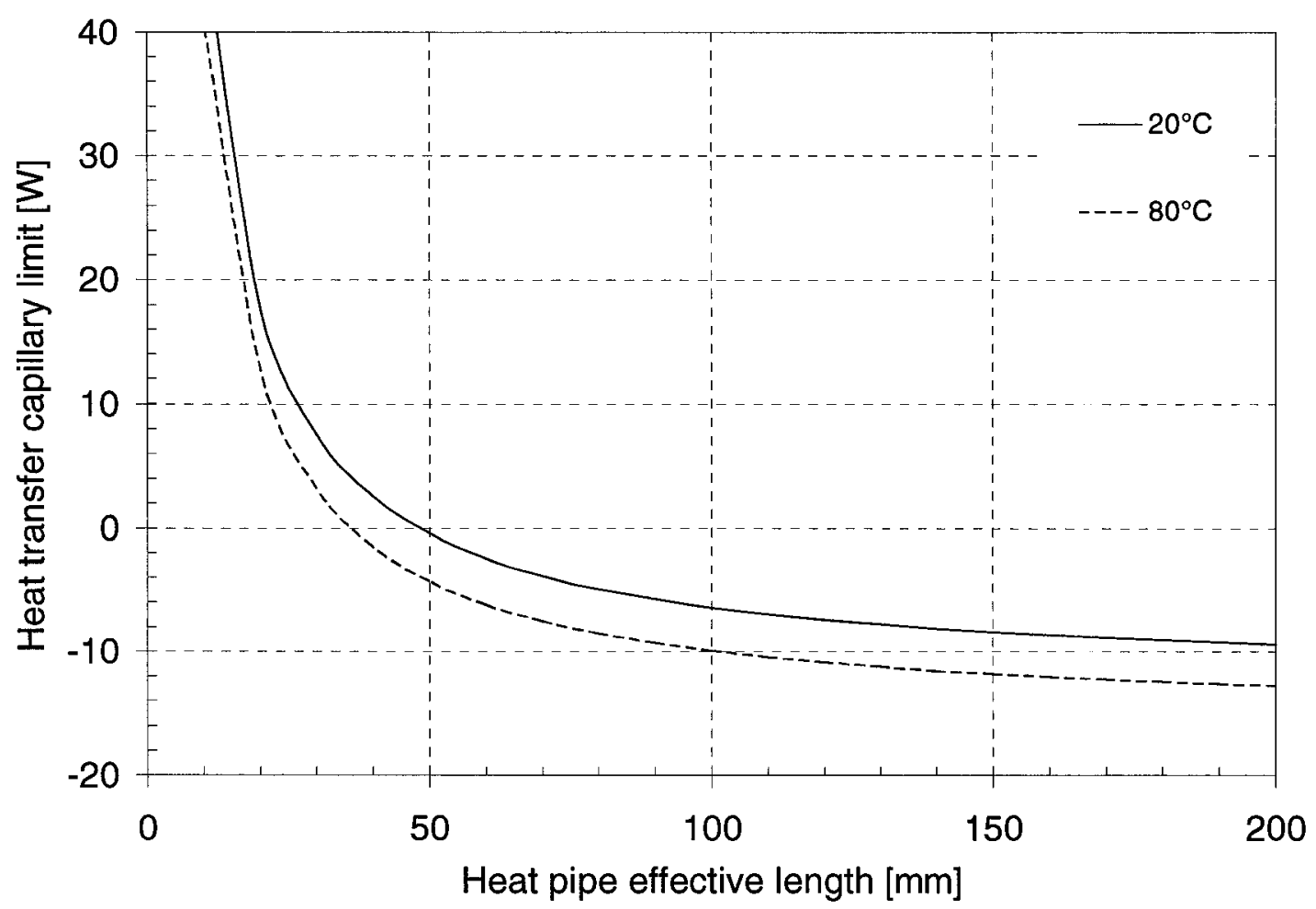

Figure 4.26 Capillary limit as a function of heat pipe effective length (against gravity vertical) [100 copper mesh acetone heat pipe]

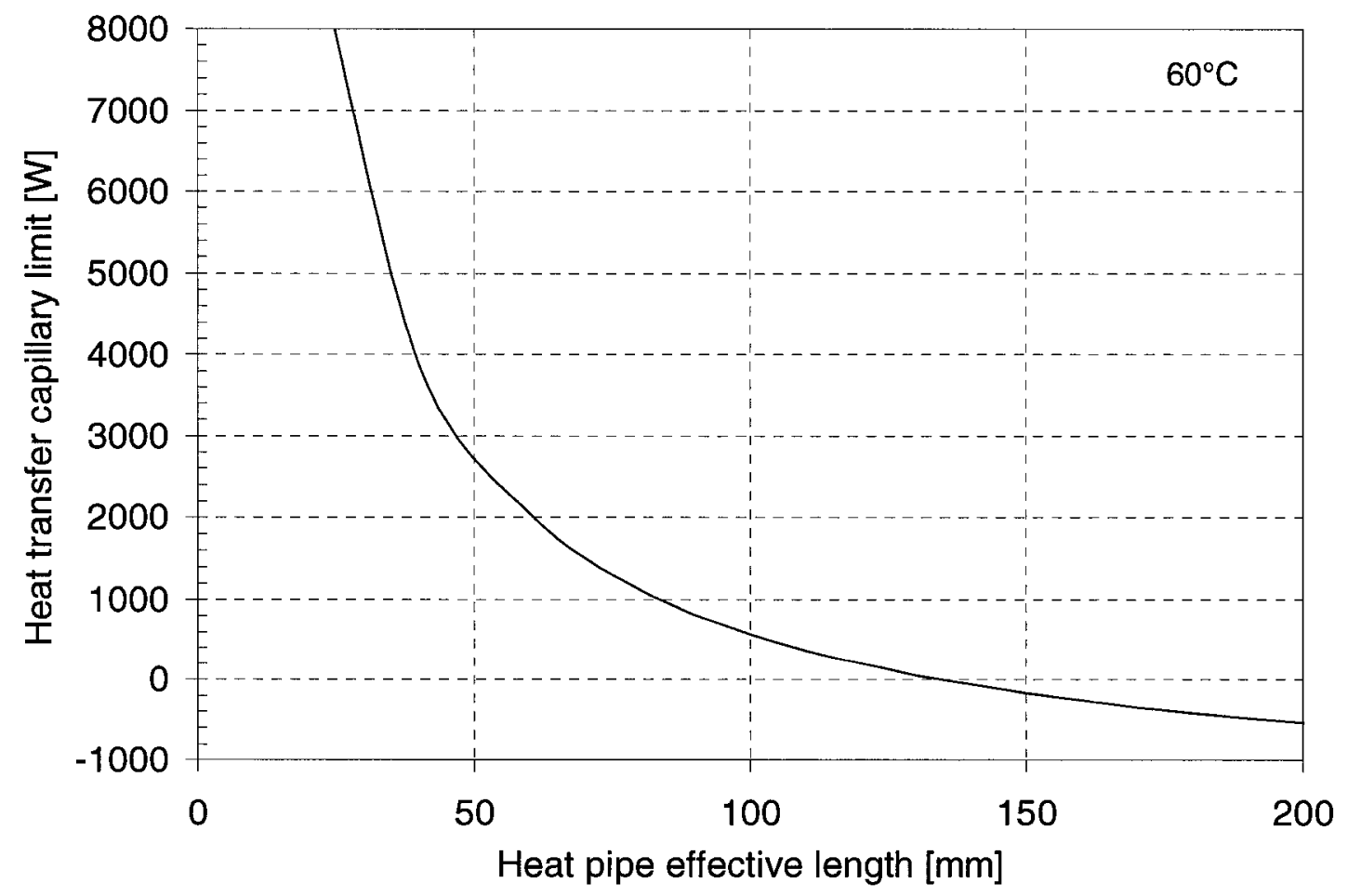

Figure 4.27 Capillary limit as a function of heat pipe effective length (against gravity vertical) [nickel foam water heat pipe] 


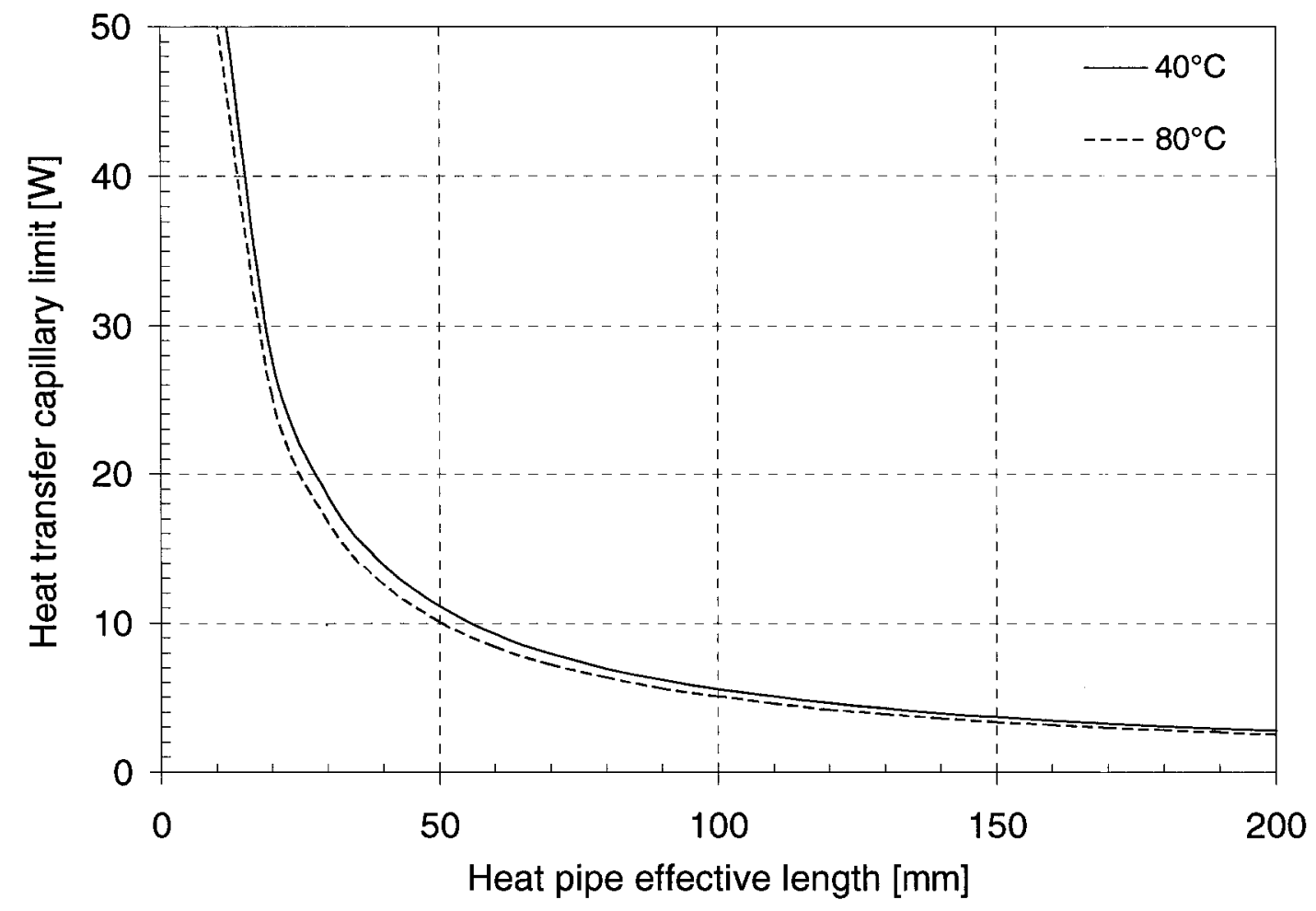

Figure 4.28 Capillary limit as a function of heat pipe effective length (horizontal)

[100copper mesh acetone heat pipe]

Figure 4.28 shows this relationship for the acetone case in a horizontal orientation. From this figure we can see that when the effective length is short enough $(30 \mathrm{~mm})$, its capillary limit can extend to over $15 \mathrm{~W}$. This is the actual power level at which the acetone heat pipe works in testing processes even though its theoretical capillary limit is estimated as only about $4 \mathrm{~W}$. Here, note that this $4 \mathrm{~W}$ limit is obtained based on a constant effective length assumption. The effective length concept will be further analyzed in the next section.

\subsubsection{Analysis of Effective Length Concept}

Section 4.3.1 illustrates the fact that the effective length concept is consistent with experimental results; the evaporator temperature discrepancy in forced convection 
cooling cases, and the inconsistence of acetone heat pipe capillary limit. In this section we will try to obtain theoretical explanations for such effects, and predict other related phenomena.

\subsubsection{Definition of Effective Length}

To further analyze the effective length, we need first to clarify the concept of the heat pipe effective length. The current heat pipe effective length is believed to be the distance between the centre points of the evaporator and the condenser of a heat pipe. This length is supposed be constant. Due to the fact illustrated in this section, it is shown that the actual effective length of a heat pipe would vary with circumstances. Thus, we introduce the following concepts; the geometric effective length, and the effective length.

The geometric effective length is defined as the distance between the centre points of the evaporator and the condenser. This length stays constant for a certain heat pipe configuration. The effective length would vary with the working conditions for a certain heat pipe configuration. While the geometric effective length is an absolute space distance, it would not change with working conditions. With such two concepts, we can make further analyses on this issue.

\subsubsection{Mechanism of Effective Length}

Reviewing the analyses in Section 4.2.1 on the issue of heat pipe capacity limits, it is clear that the heat transfer capacity is limited by factors such as capillary, sonic, entrainment, and boiling limits. This result not only shows the values of these capacity limits, but also indicates a more important fact that a heat pipe performance varies under 
certain circumstances. Among these four factors, the capillary limit is believed to be the most critical one affecting the heat pipe performance.

Also reviewing the results obtained in Section 4.3.2, it is noticed that the capillary limit increases with decreasing effective length. This fact gives a hint that the effective length relates to the capillary factor. Based on the results mentioned above, this section will undertake analyses of the effective length.

As defined in the preceding section, the geometric effective length relates to evaporation and condensation. It also relates the factors affecting these processes. Factors affecting these two processes are temperatures, pressures, wick material prosperities, wick structures, wick dimension and the properties of the working fluid. The synergy of these factors would determine the evaporation and condensation states, and consequently determine the distance between these two processes. Specific analyses are provided below.

Reviewing the analysis of capillary limit in Section 4.2.1, and adopting Equation (4.25), give the following equations,

$$
L_{\text {eff } \text { max }}=\frac{(Q L)_{c . \text { max }}}{Q_{c . \text { max }}}
$$

the term, $L_{\text {eff. } \max }$, can be treated as the equivalent effective length limit. This means the heat pipe would keep functioning within this length, and would cease functioning beyond this length.

Modifying this relationship, and substituting into Equation (4.17), we obtain the following equation, 


$$
L_{e f f}=\frac{\frac{2 \cdot \sigma}{r_{c}}-\rho_{l} \cdot g \cdot d_{v} \cdot \cos \psi-\rho_{l} \cdot g \cdot L_{p} \cdot \sin \psi}{Q_{c} \cdot\left(F_{l}+F_{v}\right)}
$$

Here the term, $L_{e f f}$, is the effective length that really contributes to the heat pipe functionality. $L_{p}$ is the entire pipe length, which involves a total axial capillary pressure difference. It is neither the effective length nor the geometric effective length. At a horizontal position, this length would not bring any capillary pressure affects to the heat pipe function, so it can be ignored. However at any inclined orientation, this length would affect the capillary function. At a gravity assisted orientation, if the total length of a heat pipe is longer than its effective length, the extra part of the wick would affect the performance of the effective length part, depending on the condenser conditions. For instance, if the extra part in the condenser has a low temperature, the fluid in this part of the wick would provide an upward force to the fluid in the effective length of the wick, thus the gravity assist would be offset. In an against gravity orientation, if the total length of a heat pipe is shorter than its effective length in an against gravity orientation, then the heat pipe would still function. This is discussed in Section 4.3.1.

In Equation (4.25b), two properties relate to temperature; surface tension, $\sigma$, and liquid density, $\rho_{l}$. And another two items, $F_{l}$ and $F_{v}$ contain temperature related elements; vapour density, $\rho_{v}$, liquid viscosity, $\mu_{l}$, vapour viscosity, $\mu_{v}$, and the fluid latent heat, $\lambda$. All of these elements are functions of temperature. Applying differentiation to Equation (4.25b) regarding the temperature related elements, with respect to temperature yields, 
$\frac{d\left(L_{e f f}\right)}{d T}=\frac{\left.\left(F_{l}+F_{v}\right) \cdot\left[\frac{2}{r_{c}} \cdot \frac{d \sigma}{d T}-g \cdot\left(d_{v} \cdot \cos \psi+L_{p} \cdot \sin \psi\right\rangle\right) \cdot \frac{d \rho_{l}}{d T}\right]-\left[\frac{2 \cdot \sigma}{r_{c}}-g \cdot\left(d_{v} \cdot \cos \psi+L_{p} \cdot \sin \psi\right\rangle \cdot \rho\right] \cdot\left[\frac{d\left(F_{l}\right)}{d T}+\frac{d\left(F_{v}\right)}{d T}\right]}{Q \cdot\left(F_{l}+F_{v}\right)^{2}}$

where,

$$
\frac{d\left(F_{l}\right)}{d T}=\left(\frac{1}{K_{m e s h} \cdot A_{w}}\right) \cdot \frac{\left(\rho_{l} \cdot \lambda\right) \cdot \frac{d \mu_{l}}{d T}-\mu_{l} \cdot\left(\lambda \cdot \frac{d \rho_{l}}{d T}+\rho_{l} \cdot \frac{d \lambda}{d T}\right)}{\left(\rho_{l} \cdot \lambda\right)^{2}}
$$

and,

$$
\frac{d\left(F_{v}\right)}{d T}=\left(\frac{f_{v} R_{e}}{2 \cdot A_{w} \cdot r_{h . v}^{2}}\right) \cdot \frac{\left(\rho_{v} \cdot \lambda\right) \cdot \frac{d \mu_{v}}{d T}-\mu_{l} \cdot\left(\lambda \frac{d \rho_{v}}{d T}+\rho_{v} \cdot \frac{d \lambda}{d T}\right)}{\left(\rho_{v} \cdot \lambda\right)^{2}}
$$

Equation (4.33) shows that in a mathematical sense, the effective length changes with respect to temperature. In this equation, the six temperature related elements have their own physical properties, and these properties vary with configurations. To analyze a specific heat pipe, we need to apply the physics properties. Table 4.1 shows that the water and acetone physics properties change with respect to temperature for the six items in Equations (4.33), (4.34), and (4.35). Because these values do not vary linearly, this table just shows an average change values for each $20^{\circ} \mathrm{C}$ interval. This is an approximate approach to the rates of physical properties changing with respect to temperature.

For simplicity, we can approximately treat these physical property change rates from Table 4.1, as their differentiation with respect to temperature. We can then substitute the corresponding values from Table 4.1 into Equations (4.33) to yield the curves illustrating the effective length change as a function of temperature, as shown in Figures 4.29 to 4.33 . From these figures we can see that according to Equation (4.33), the effective length change rate with respect to temperature would change with heat pipe configurations. 
Table 4.1 Working fluid property change rate per unit temperature

Water

Acetone

$\begin{array}{cc}\begin{array}{c}\text { Temperature } \\ \text { range }\left[{ }^{\circ} \mathbf{C}\right]\end{array} & \begin{array}{c}\text { Latent heat, } \boldsymbol{\lambda} \\ \text { [kJ/kg] }\end{array} \\ 20 \text { to } 40 & 2448 \\ 40 \text { to } 60 & 2402 \\ 60 \text { to } 80 & 2359 \\ 80 \text { to } 100 & 2309\end{array}$

$\begin{array}{cc}\begin{array}{c}\text { Temperature } \\ \text { range }\left[{ }^{\circ} \mathbf{C}\right]\end{array} & \begin{array}{c}\text { Liquid density, } \rho_{\mathbf{l}}, \\ {\left[\mathbf{k g} / \mathbf{m}^{\mathbf{3}}\right]}\end{array} \\ 20 \text { to } 40 & 998.0 \\ 40 \text { to } 60 & 992.1 \\ 60 \text { to } 80 & 983.3 \\ 80 \text { to } 100 & 972.0 \\ \text { Temperature } & \text { Vapour density, } \boldsymbol{\rho}_{\mathbf{v}} \\ \text { range }\left[{ }^{\circ} \mathbf{C}\right] & {\left[\mathbf{k g} / \mathbf{m}^{3} \text { ] }\right.} \\ 20 \text { to } 40 & 0.02 \\ 40 \text { to } 60 & 0.05 \\ 60 \text { to } 80 & 0.13 \\ 80 \text { to } 100 & 0.29\end{array}$

$\begin{array}{cc}\begin{array}{c}\text { Temperature } \\ \text { range }\left[{ }^{\circ} \mathbf{C}\right]\end{array} & \begin{array}{c}\text { Liquid viscosity, } \boldsymbol{\mu}_{\mathbf{I}} \\ {\left[\mathbf{1 0}^{-\mathbf{3}} \mathbf{\mathrm { kg }} / \mathbf{m} \cdot \mathbf{s}\right]}\end{array} \\ 20 \text { to } 40 & 1.0 \\ 40 \text { to } 60 & 0.65 \\ 60 \text { to } 80 & 0.47 \\ 80 \text { to } 100 & 0.36\end{array}$

$\begin{array}{cc}\begin{array}{c}\text { Temperature } \\ \text { range }\left[{ }^{\circ} \mathbf{C}\right]\end{array} & \begin{array}{c}\text { Vapour viscosity, } \boldsymbol{\mu}_{\mathrm{v}} \\ {\left[\mathbf{1 0}^{-5} \mathbf{k g} / \mathbf{m} \cdot \mathbf{s}\right]}\end{array} \\ 20 \text { to } 40 & 0.96 \\ 40 \text { to } 60 & 1.04 \\ 60 \text { to } 80 & 1.12 \\ 80 \text { to } 100 & 1.19 \\ \text { Temperature } & \begin{array}{c}\text { Liquid surface } \\ \text { tension, } \boldsymbol{\sigma}\end{array} \\ \text { range }\left[{ }^{\circ} \mathbf{C}\right] & {\left[\mathbf{1 0}^{-2} \mathbf{N} / \mathbf{m}\right]} \\ 20 \text { to } 40 & 7.28 \\ 40 \text { to } 60 & 7.00 \\ 60 \text { to } 80 & 6.66 \\ 80 \text { to } 100 & 6.26\end{array}$

(Source: adapted from Petson, 1994)

$\Delta \lambda / \Delta \mathbf{T}$
$\left[\mathbf{k J} / \mathbf{k g}{ }^{\circ} \mathbf{C}\right]$
-2.3
-2.15
-2.5
-2.55

$\Delta \rho_{1} / \Delta T$

$\left[\mathrm{kg} / \mathrm{m}^{3}{ }^{\circ} \mathbf{C}\right]$

$-0.295$

$-0.44$

$-0.565$

$-0.7$

$\Delta \boldsymbol{\rho}_{\mathbf{v}} / \Delta \mathbf{~ T}$
$\left[\mathbf{k g} / \mathbf{m}^{3}{ }^{\circ} \mathbf{C}\right]$
+0.0015
+0.004
+0.008
+0.0155

$\Delta \mu_{1} / \Delta T$

$\left[10^{-3} \mathrm{~kg} / \mathrm{m} \cdot \mathrm{s} \cdot{ }^{\circ} \mathrm{C}\right]$

$-0.00175$

$-0.0009$

$-0.00055$

$-0.0004$

$\Delta \mu_{\mathrm{v}} / \Delta \mathrm{T}$

$\left[10^{-5} \mathrm{~kg} / \mathrm{m} \cdot \mathrm{s} \cdot{ }^{\circ} \mathrm{C}\right]$

$+0.004$

$+0.004$

$+0.0035$

$+0.004$
$\Delta \sigma / \Delta T$
$\left[10^{-2} \mathrm{~N} / \mathrm{m}^{\circ} \mathrm{C}\right]$
$-0.014$

$-0.017$

$-0.02$

$-0.0185$
Latent heat, $\lambda$ $[\mathrm{kJ} / \mathrm{kg}]$

552

536

517

495

Liquid density, $\rho_{l}$, $\left[\mathbf{k g} / \mathbf{m}^{3}\right]$

790.0

768.0

744.0

$719 ; .0$

Vapour density, $\rho_{v}$ $\left[\mathbf{k g} / \mathbf{m}^{3}\right]$ 0.64

1.05

2.37

4.30

Liquid viscosity, $\mu_{l}$

$\left[10^{-3} \mathrm{~kg} / \mathrm{m} \cdot \mathrm{s}\right]$

0.323

0.269

0.226

0.192

Vapour viscosity, $\mu_{v}$

$\left[10^{-5} \mathrm{~kg} / \mathrm{m} \cdot \mathrm{s}\right]$

0.82

0.86

0.90

0.95

Liquid surface tension, $\sigma$ $\left[10^{-2} \mathrm{~N} / \mathrm{m}\right]$

2.37

2.12

1.86

1.62

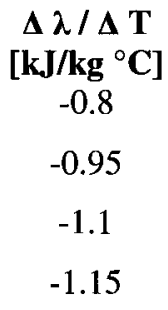

$\Delta \rho_{1} / \Delta T$

$\left[\mathrm{kg} / \mathrm{m}^{3}{ }^{\circ} \mathrm{C}\right.$ ]

$-1.1$

$-1.2$

$-1.25$

$-1.47$

$$
\begin{gathered}
\Delta \rho_{\mathrm{v}} / \Delta \mathrm{T} \\
{\left[\mathrm{kg} / \mathbf{m}^{3}{ }^{\circ} \mathbf{C}\right]} \\
+0.0205 \\
+0.066 \\
+0.0965 \\
+0.132
\end{gathered}
$$

$\Delta \mu_{1} / \Delta T$

$\left[10^{-3} \mathrm{~kg} / \mathrm{m} \cdot \mathrm{s}^{\cdot}{ }^{\circ} \mathrm{C}\right]$

$-0.00027$

$-0.000215$

$-0.00017$

$-0.00011$

$\Delta \mu_{\mathrm{v}} / \Delta \mathrm{T}$

$\left[10^{-5} \mathrm{~kg} / \mathrm{m} \cdot \mathrm{s}^{\circ}{ }^{\circ} \mathrm{C}\right]$

$+0.002$

$+0.002$

$+0.0025$

$+0.0015$

$\Delta \sigma / \Delta T$

$\left[10^{-2} \mathrm{~N} / \mathrm{m}^{\circ} \mathrm{C}\right]$

$-0.0125$

$-0.013$

$-0.012$

$-0.014$ 
Figure 4.29 shows that for the fine copper mesh water heat pipe configuration, at a certain power input, an increasing rate of the effective length decreases with an ascending temperature. Above $60^{\circ} \mathrm{C}$, the effective length starts to decrease with increasing temperature. For a certain temperature level, with the increasing power input, the span of the effective length change tends to be small. This curve description indicates that, the heat pipe effective length would become longer at a low working temperature with a certain power input. With the increasing working temperature, its effective length would reduce, and become even shorter than the designed working length, i.e., the geometric length. But with power input increasing, the effective length change rate would become small. Thus, the effective length converges to the designed working length. As discussed in the analyses of experimental results included in Section 4.1, at low power levels and small axial temperature gradients there are big discrepancies between the actual results and the theoretical predictions. With increasing power input, the discrepancies become smaller. However, in forced and enhanced forced convection cooling conditions, i.e., with greater axial temperature gradient, the discrepancies still exist even at higher power levels. These facts are consistent with the effect of effective length change. This consistency exactly matches the simulation results illustrated in Figure 4.29. Similar consistencies also occur in the case of coarse copper mesh water, composite copper mesh water, and nickel foam water heat pipes respectively, refer to Figures 4.30, 4.32, and 4.33.

The only exception occurred in the case of fine copper mesh acetone heat pipe. In Figure 4.31 we can see that for the fine copper mesh acetone heat pipe, its effective length changes under all circumstances. According to the effective length, modeling its real condensation point would move toward the evaporator. That is, the real condensation 
point would leave the supposed condensation point for the evaporator. Thus the temperature on the supposed condensation point is lower than that of the real condensation point. But the condensation temperature measurement point still stays on the supposed condensation point. This would result in a lower measured condensation temperature. Substituting this lower measured condensation temperature into Equation (4.12) would consequently yield a lower predicted evaporator temperature. However, in Figure 4.5 it can be seen that, before reaching $6 \mathrm{~W}$, the actual evaporator temperatures are higher than the predicted results. This is consistent with the effect of effective length change. While after $6 \mathrm{~W}$, the actual evaporator temperature curve intersects and then diverges from the predicted evaporator temperature curve. This brings an inconsistency with the effect of effective length change.

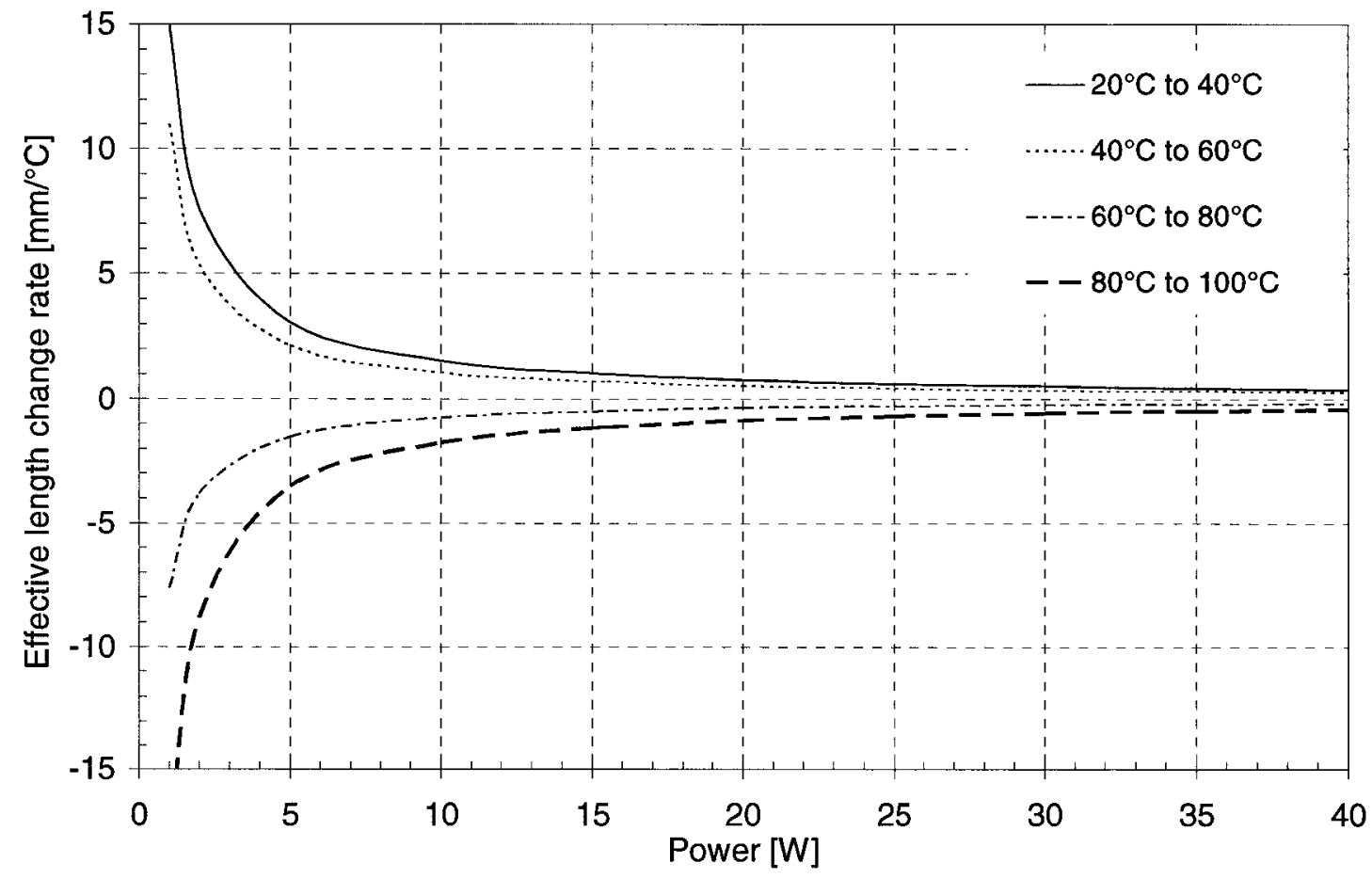

Figure 4.29 Simulation of heat pipe effective length change rate as functions of temperature and power (100 copper mesh, water; horizontal] 


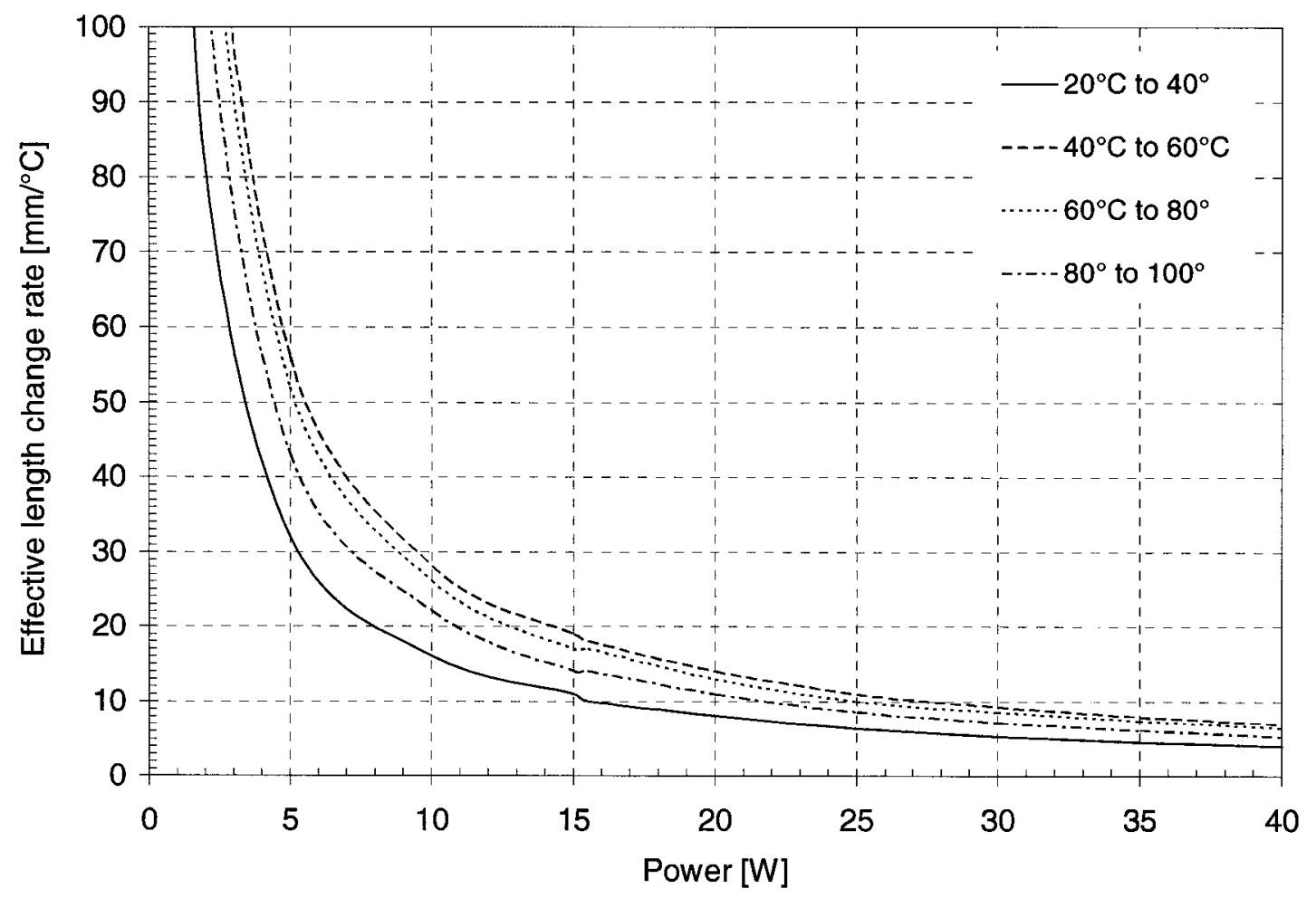

Figure 4.30 Simulation of heat pipe effective length change rate as functions of temperature and power [50 copper mesh water, horizontal]

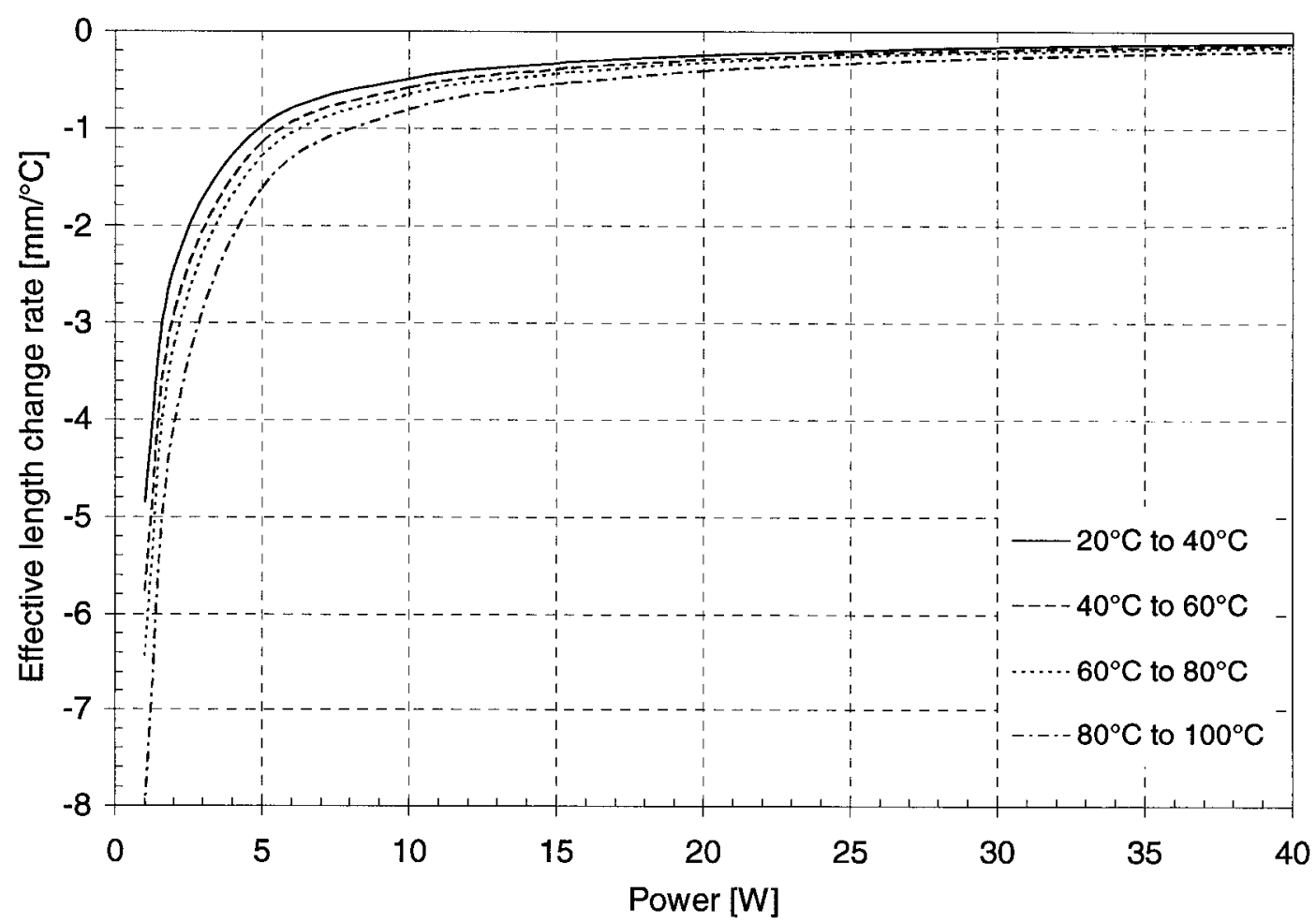

Figure 4.31 Simulation of heat pipe effective length change rate as functions of temperature and power [100 copper mesh acetone, horizontal] 


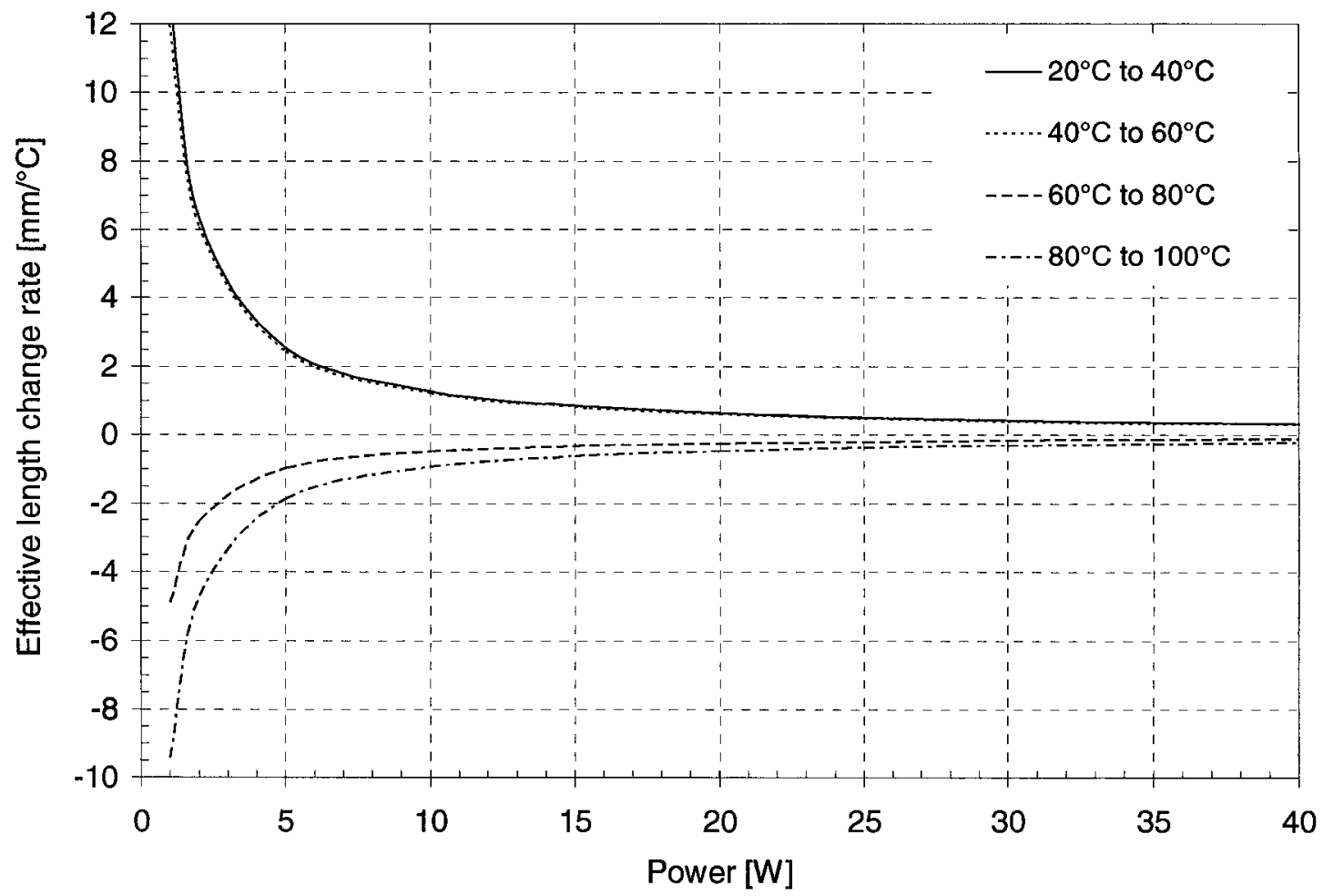

Figure 4.32 Simulation of heat pipe effective length change rate as functions of temperature and power [composite copper mesh water, horizontal]

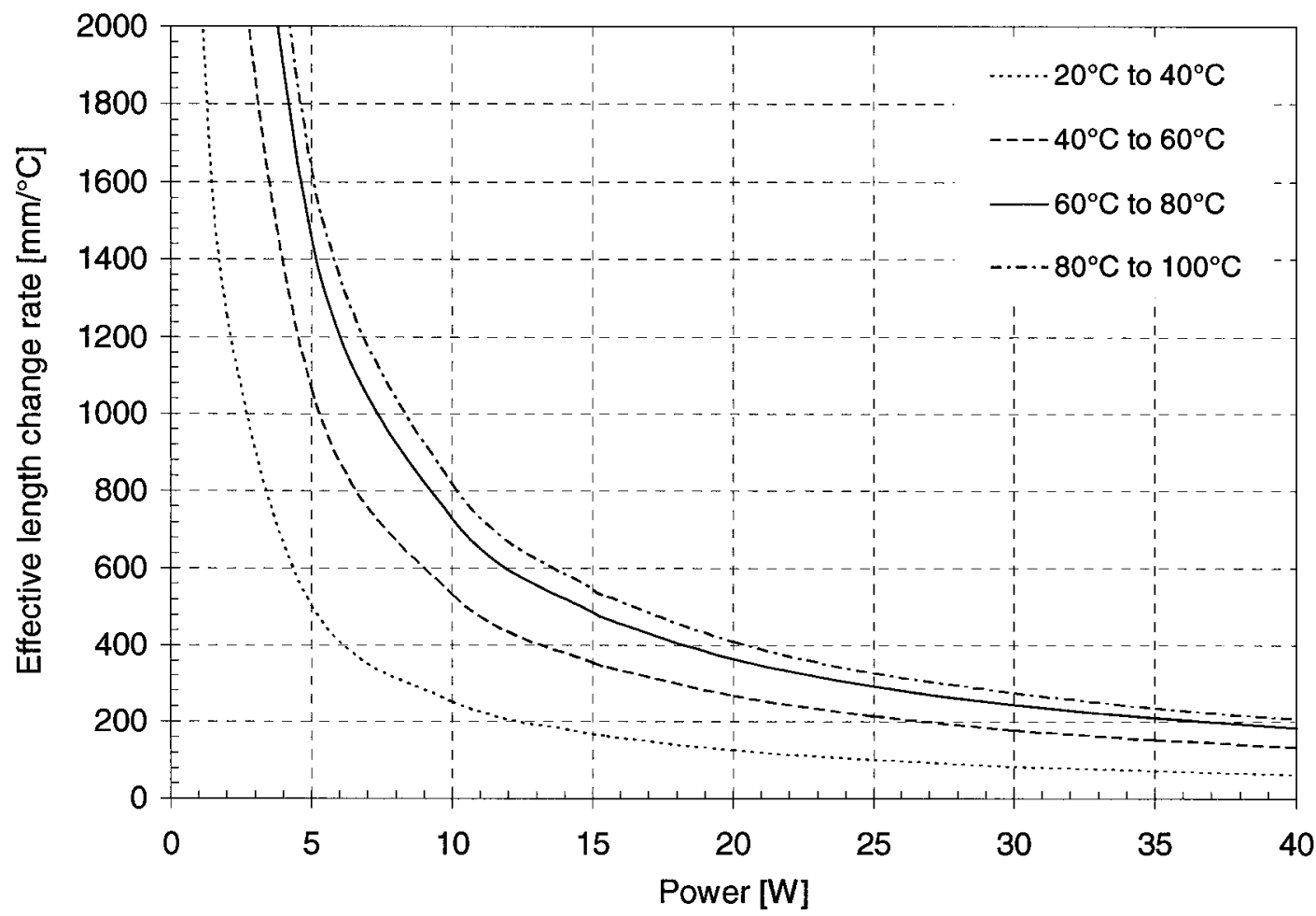

Figure 4.33 Simulation of heat pipe effective length change rate as functions of temperature and power [nickel foam water, horizontal] 
Also, we can see from Figures 4.29 to 4.33 that the effective length change rates vary at different temperature levels. By using the high temperature values, and subtracting those of low temperature, a trend of effective length change rate with large temperature intervals will occur. Figures 4.34 to 4.38 illustrate such a change in trend with larger temperature intervals for the five configurations analyzed above.

All the results except for the acetone case are exactly consistent with the deduction from the effective length concept in Section 4.3.1. They are also consistent with the analyses in Section 4.3.2. Reviewing the analyses above, the effective length is proven by both experiment results and theoretical analysis. This effect does not seem to be an occasional phenomenon.

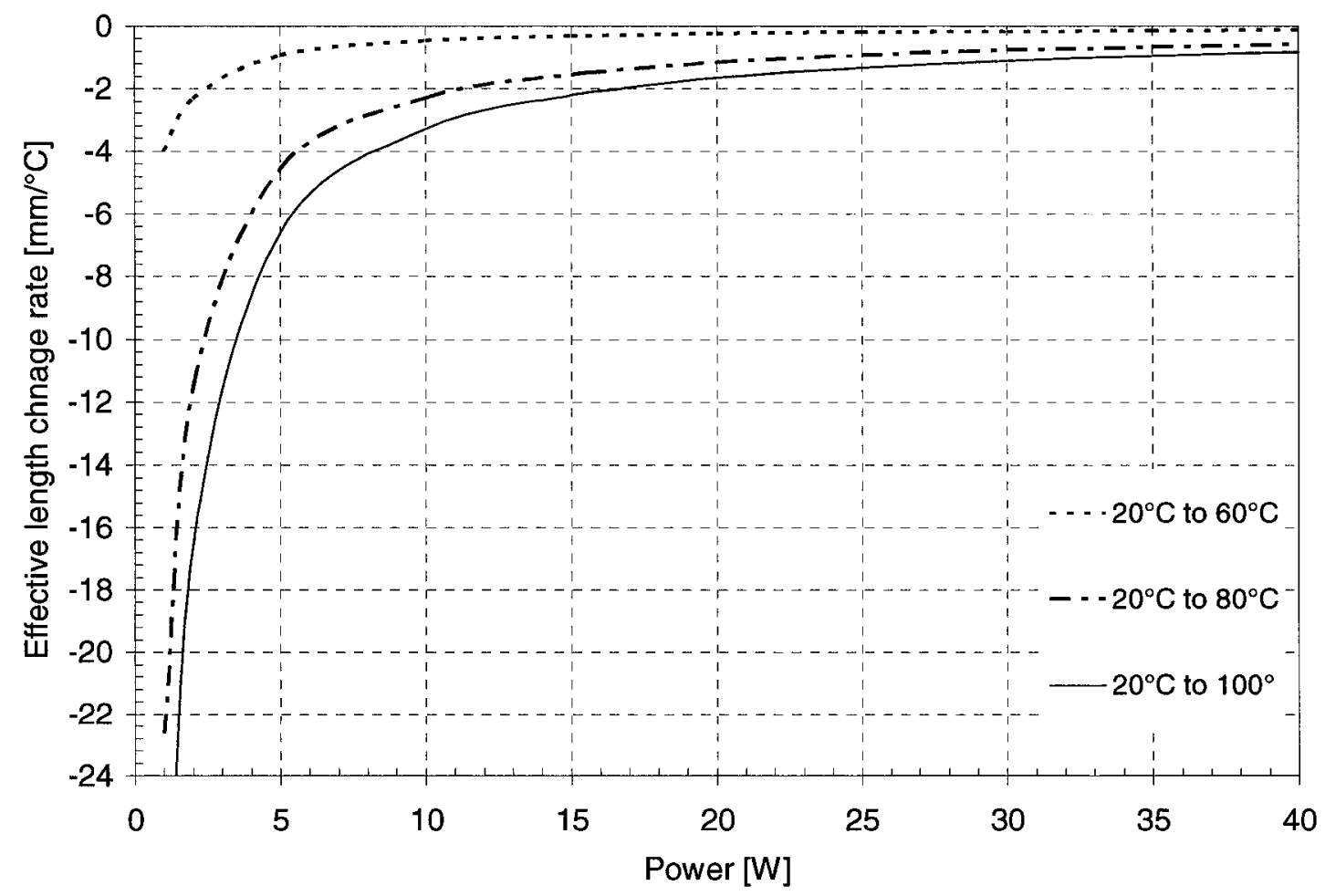

Figure 4.34 Simulation of heat pipe effective length change rate as functions of temperature and power [100 copper mesh water, horizontal] 


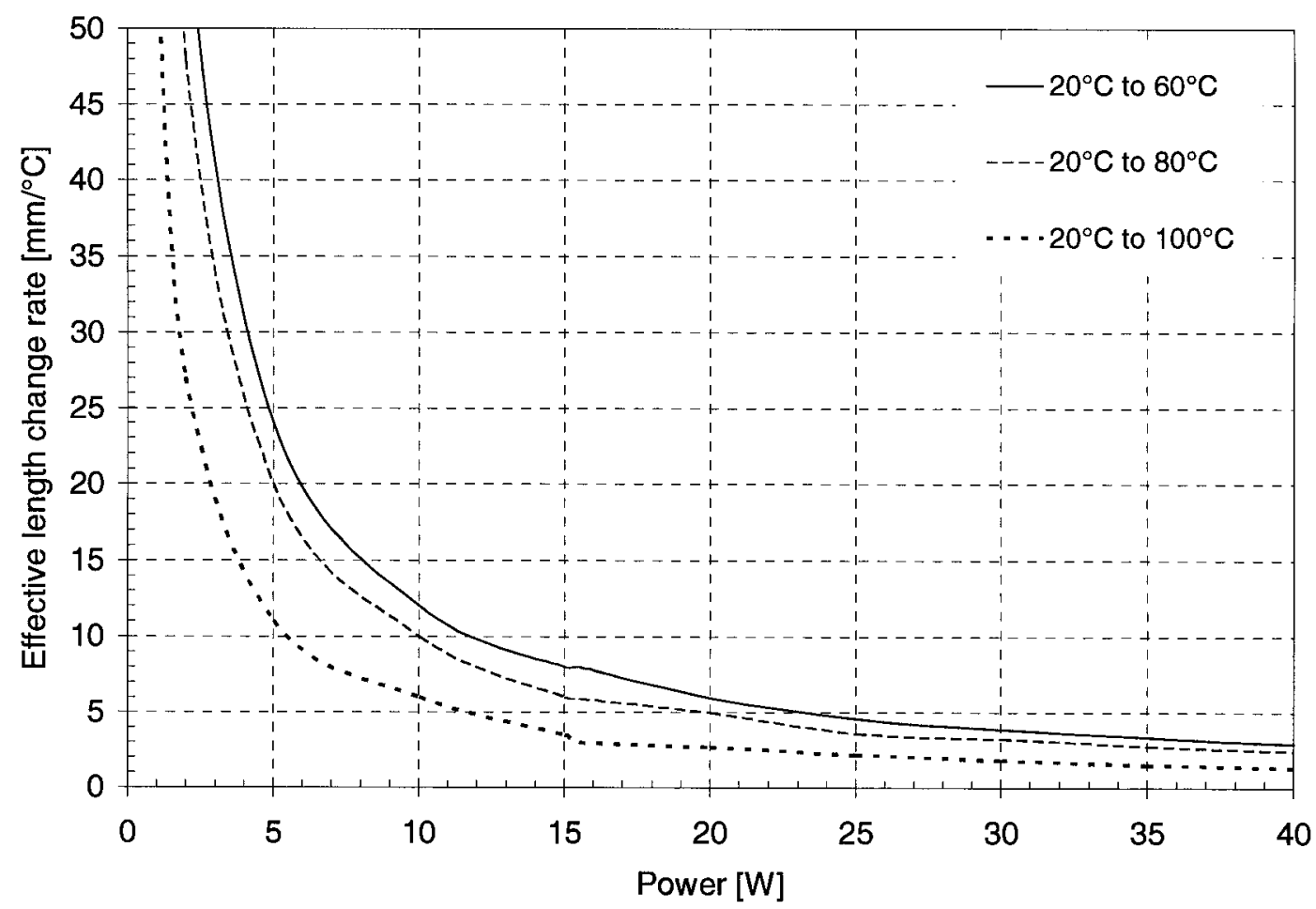

Figure 4.35 Simulation of heat pipe effective length change rate as functions of temperature and power [50 copper mesh water, horizontal]

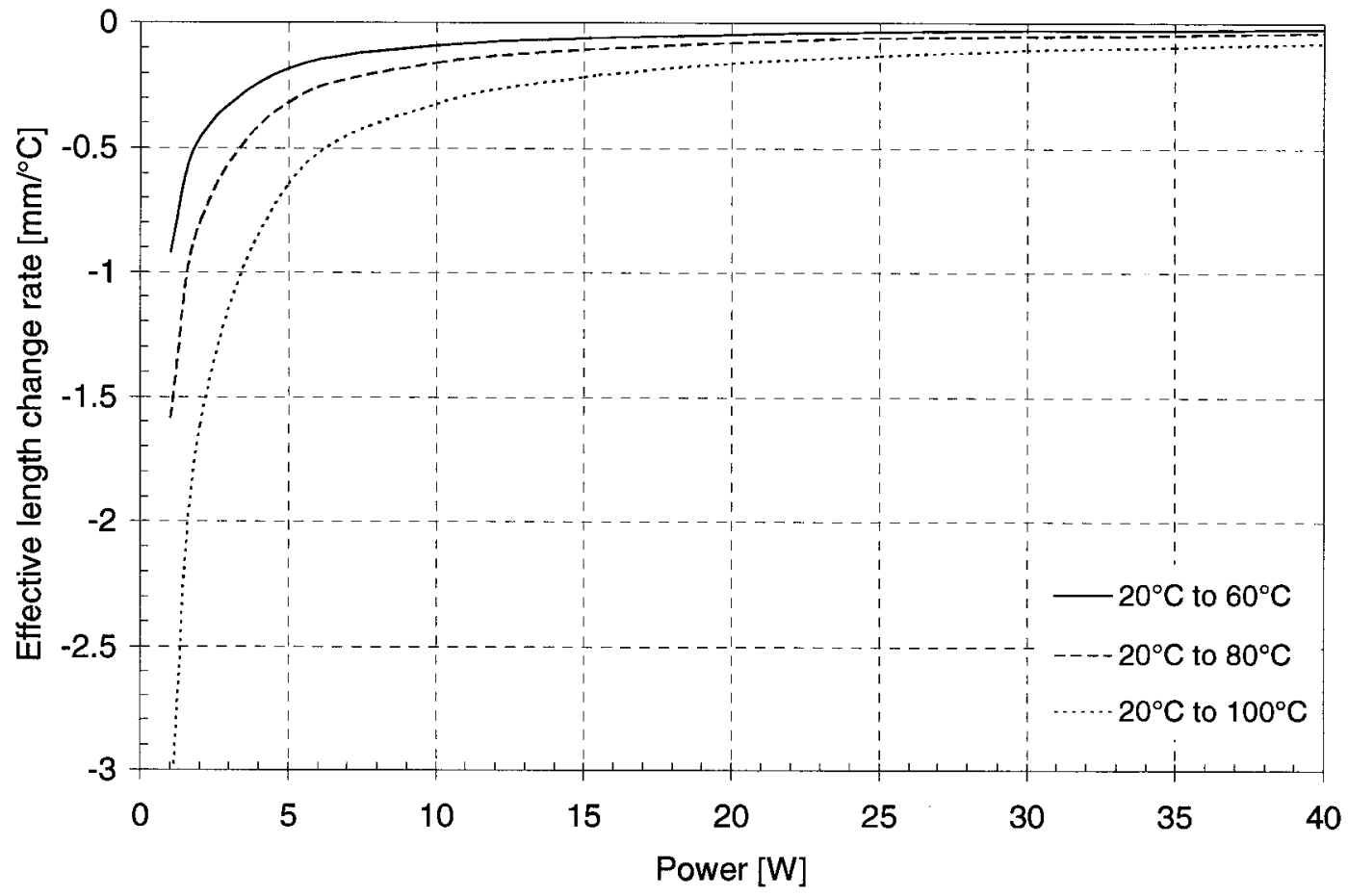

Figure 4.36 Simulation of heat pipe effective length change rate as functions of temperature and power [100 copper mesh acetone, horizontal] 


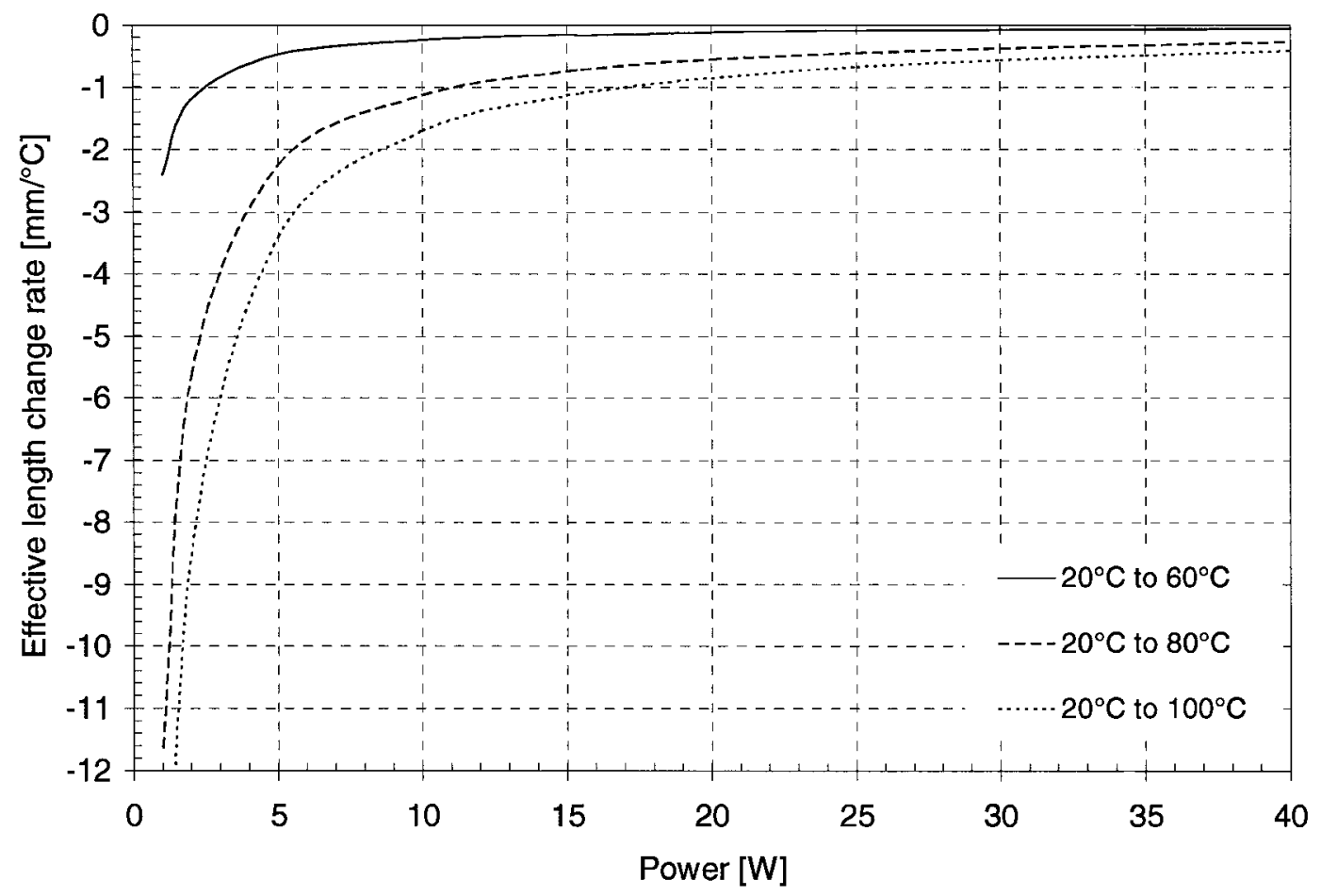

Figure 4.37 Simulation of heat pipe effective length change rate as functions of temperature and power [composite copper mesh water, horizontal]

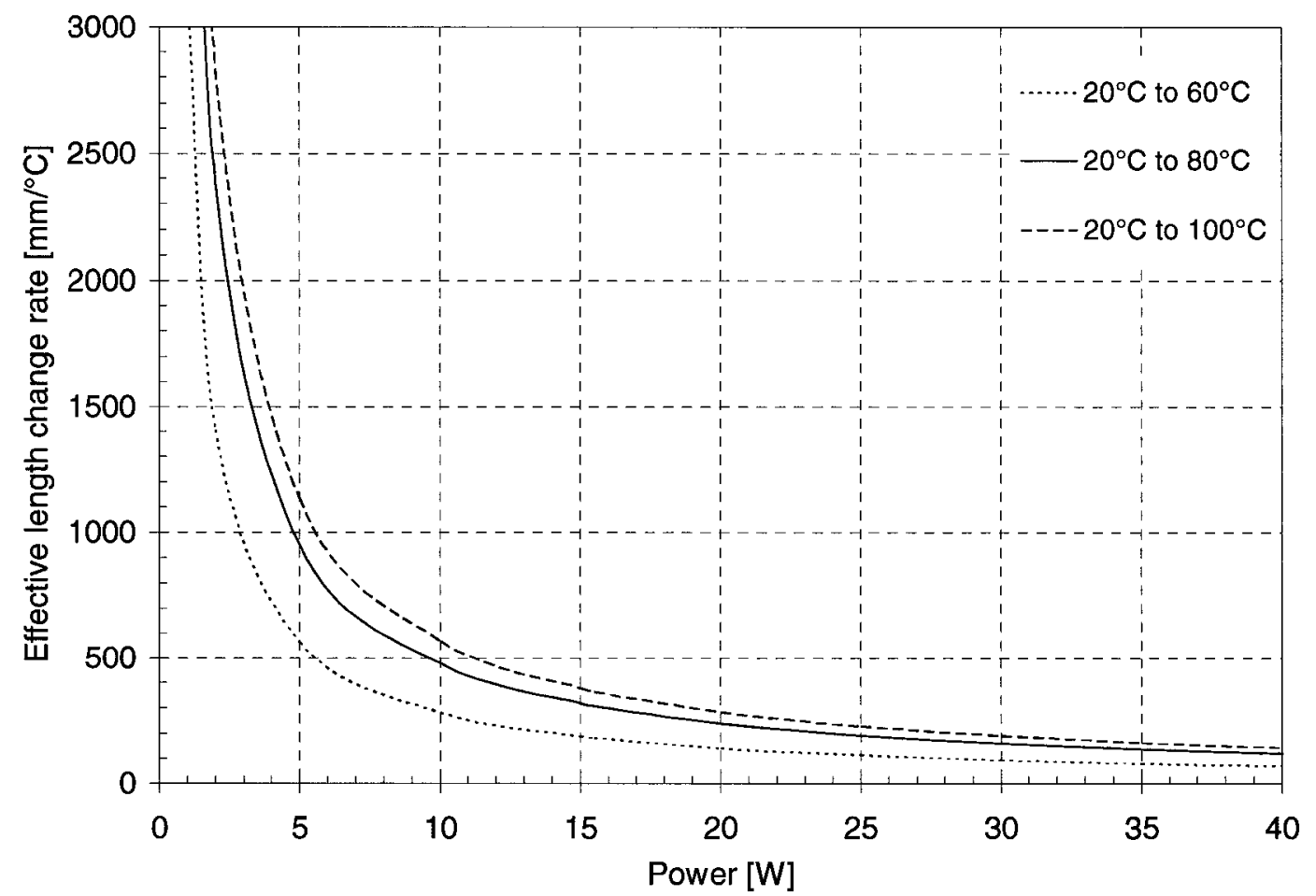

Figure 4.38 Simulation of heat pipe effective length change rate as functions of temperature and power [nickel foam water, horizontal] 
It should be noted that there are many factors affecting the effective length. This section explores the reason for the effective length with respect to the capillary limit. Equation (4.33) displays the effective length change rate with respect to temperature, rather than the effective length change. The results illustrated in Figure 4.29 to 4.38 only attempt to show the trend of the effective length change regarding Equations (4.33, (4.34), and (4.35), rather than to provide exact change values. Also, the values of physical property change rates substituted in Equations (4.33), (4.34) and (4.35) are adopted from existing physical property data with a large temperature interval of $20^{\circ} \mathrm{C}$. There is more space in this area to explore. Further investigations on the mechanism of the effective length are beyond the scope of this thesis.

\subsection{Chapter Closure}

This chapter quantitatively analyzed the experimental results discussed in Chapter 3. It verifies the heat pipe performance by applying theoretical approaches. To explain some unexpected results presented in Chapter 3 , this chapter analyzes the effective length concept by combining theoretical and experimental results. It also establishes a mathematical model for the effective length and its rate of change. 


\section{Chapter 5}

\section{Conclusions and Recommendations}

\subsection{Summary and Conclusion}

\section{Heat Pipe Fabrication}

According to the initial designs, a series of copper heat pipes were manufactured. The main configuration was $1 / 4$ " outer diameter copper tube. Wick structures included two layers of 100 mesh cooper wire screen, the two layers of 50 mesh copper wire screen, and the composite copper wire screen mesh compound with one layer of 100 and one layer of 50 mesh copper wire screens; the total net lengths were $220 \mathrm{~mm}$; working fluids are pure water and acetone. Besides these, three more copper tube configurations with outer diameter $3 / 8^{\prime \prime}$ and total net lengths of $240 \mathrm{~mm}$ were made. One was sintered nickel foam wick and pure water as working fluid. The other one was 100 mesh copper wire screen and pure water as working fluid. The final sample was a wickless thermosyphon. Among these three, the nickel foam heat pipes was tested and showed performance characteristics consistent with the theoretical predictions.

The copper mesh wick was manually inserted into the copper tube with the help of an aluminium mandrel. The experimental results showed that the insertion of mesh roll wick was effective. The sealing was done using argon-arc welding, and the heat pipe experimental performance indicated that this sealing technique was effective. The soldering method is not recommended for the copper tube heat pipe crimp-pinching sealing. 


\section{Evacuation and Fluid Charging Process Analyses}

In the fabrication procedure, all the heat pipes were evacuated with the two-stage rotary vane vacuum pump system. In the process, the ultimate vacuum gauge reading obtained was $2 \times 10^{-3}$ Torr or lower, and corresponding time was one hour with 400 to $500^{\circ} \mathrm{C}$ degassing. The estimated ultimate vacuum in the wick section of the heat pipe assembly was $4 \times 10^{-3}$ Torr. The heat pipe assemblies showed very stable static vacuum performance. The majority of the assemblies experienced pressure rises of $5 \times 10^{-4}$ Torr, at the $4 \times 10^{-3}$ Torr base vacuum, after 60 hours static vacuum test, after three cycles of dynamic vacuum baking at 400 to $500^{\circ} \mathrm{C}$ for one minute, no rise in pressure were observed. The above condition included a stainless steel ferrule fitting connection with the SWAGELOK SS4BK Bellows-Sealed Valve sealing. These evacuation results were proven in the later testing to be effective for water and acetone heat pipe configurations.

A series of theoretical analyses were employed to analyze vacuum system gas flow ranges, and the heat pipe assembly gas loads in the evacuation process. Also, based on the theoretical analyses, a series of solutions to the copper materials degassing and false vacuum phenomena were applied to the practical work, and were proven to be effective for this system.

The fluid charging process is making use of the atmospheric back pressure with cooling assist on the heat pipe assembly. The fluid was pre-purified prior to filling in the charging flask. This charging method was proven to be effective. The heat pipes normally worked as expected. But it was suspected that the "de-priming" phenomenon might be related with this charging method. Further investigations are recommended. 


\section{Heat Pipe Performance Testing}

In this thesis work, the performance testing mainly consisted of steady-state operating tests. Six heat pipe configurations were employed. The 100 copper mesh over charged water, the 100 copper mesh normally charged water, the 50 copper mesh normally charged water, the 50 and 100 composite copper mesh over charged water, the 100 copper mesh acetone, and the nickel foam normally charged water. Three non-heat pipe copper tube configurations were also tested for comparison: the copper tube, the copper tube with 100 copper mesh, and the copper tube with 100 copper mesh and water. All these pipe configurations were tested with insulation and three orientations: horizontal, gravity assisted vertical, and against gravity vertical. These heat pipes were also cooled in three different approaches: natural, forced, and enhanced forced convections.

The experimental results indicated that compared with the non-heat pipe copper tube configurations, for the same evaporator temperature constraint, the heat pipe improved the heat transfer capacity up to 400 percent and 730 percent. Also compared with the nonheat pipe copper tube configurations, the heat pipe significantly improved the axial thermal conductivities in the range from 260 percent to 1300 percent, depending on the testing conditions.

The testing was conducted with a copper based heating film as a heat source, and an aluminium finned sink. And the testing was started from a lower power input, e.g., 0.5 and $1 \mathrm{~W}$ for acetone and water heat pipes, respectively. The heat pipes started from 1 and $2 \mathrm{~W}$ with the characterization of adiabatic temperature curves converging. For every heat pipe testing sample, the ultimate evaporator temperature and power input were respectively set 
at 100 to $120^{\circ} \mathrm{C}$ and 35 to $40 \mathrm{~W}$, depending on the insulation resistance to heat and the heating element voltage limit.

One testing item was the heat pipe cooling effect, comparing the heater surface temperature with different heat pipe configurations under different circumstances. The experimental results show that at the same orientations, forced convective air cooling significantly improved the heat pipe cooling effects, and resulted in lower evaporator temperatures, and greater evaporator-condenser temperature difference. These results were consistent with predictions. Compared to the forced convection, the enhanced forced convective air cooling contributes a little to the heat pipe cooling effects. This was mainly due to the high thermal resistance caused by the turbulent airflow on the condenser finned sink. This indicates that compared to the natural convection, forced convection greatly improved heat pipe cooling effect. However, further increasing the cooling air mass flow rate did not contribute to the heat pipe cooling effect. Also as predicted, the gravity assisted vertical orientation improves the heat pipe cooling effect, and against gravity vertical orientation worsens the heat pipe cooling effect. In some cases, it led to the heat pipe "depriming".

Axial thermal conductibility is a comprehensive indicator for the heat pipe performance evaluation. Based on the experimental results, axial thermal conductivity values were compared among the different testing configurations. The comparative results showed that at the same orientations, natural convective air cooling resulted in the best axial thermal conductivity. This was due to the smaller axial temperature gradient, even though the natural convection did not lead to a better cooling effect than the forced and enhanced forced convection. With the same cooling condition, gravity assisted vertical 
orientation helped to improve axial thermal conductivity, and against gravity vertical brought an adverse contribution to the axial thermal conductivity.

Axial temperature profile was a useful method for observing the heat pipe working status. Compared with the non-heat pipe copper tube configurations, a heat pipe axial temperature profile had three portions: the evaporation, adiabatic, and condensation sections. The essential value of the axial temperature was to reflect the heat pipe working temperature distribution along the axial direction. This value can be used to investigate the status of the heat pipe effective working sections, e.g., assess the actual effective length.

A heat pipe configuration determines the heat pipe characteristics to a great extent. One important factor is the wick structure. In this experimental investigation, four types of wick were involved, as introduced in the early part of this section. The actual experimental results indicated that fine mesh wick had better capillarity but paid a penalty in the permeability. The coarse mesh wick showed mean performances on these issues. The composite mesh wick combined the merits of the two types mesh. These were proven in the experiments, and are consistent with predictions. Being a different metal material, the nickel foam wick is not comparable with the copper counterparts, but it did show a dominant merit in the capillarity, and the axial thermal conductivity. In this work, two types of working fluids were involved: water and acetone. Comparative investigations were conducted between these two heat pipe configurations. Experimental results showed that the water based heat pipe configurations had better performance than the acetone sample, This was apparent for the heat transfer capacity limit, cooling effect, against gravity, high temperature working stability. The acetone heat pipe configuration showed merits in the low startup temperature, and sensitivity to the heating temperature compared with the water 
counterparts. The amount of charged working fluid to some extent affected the heat pipe performance. Take the water heat pipe configurations for example; the range of fluid over charge rate was from 7 percent to 58 percent. Among these heat pipes, the normally charged samples showed to be more sensitive to the heating temperature than the over charged samples. The over charged samples possessed a better stability and robustness than the normally charged counterparts. The nickel foam heat pipe test sample was slightly under charged at -8 percent, and also showed a good performance. There were two over charged heat pipe failures with estimated over charge rate of 208 percent and 310 percent. The acetone heat pipe had an 80 percent over charge rate and performed normally. These results indicated that the fluid charging range had a wide validity. Typically, the margin could be from -10 percent to 100 percent. There was a critical over charge rate: about 100 percent. Generally, within this charge range, under charged heat pipes had a better sensitivity and transition characteristics; over charged heat pipes had a better stability and robustness. Determination the rate of charge depends on the specific applications.

\section{Theoretical Analyses}

The theoretical analyses were mainly used to verify the effectiveness of the manufacturing process by comparing the experimental results and the theoretical predictions. The theoretical analyses were conducted on the issues of thermal network and heat transfer capacities.

The thermal network analyses mainly applied existing heat pipe thermal resistance models to analyze the heat pipe evaporator temperatures. In this way, they were used to compare the experimental and predicted evaporator temperatures; and to verify the effects 
of our initial designs and heat pipe fabrication method. The analysis results showed that for the water testing samples at the horizontal orientation with natural convective air cooling, and in the normal working state, the actual evaporator temperatures were consistent with the theoretical predictions. The exceptions were those at horizontal orientation with forced and enhanced forced convective air cooling; and those at vertical orientations either gravity assisted or against gravity; as well as the acetone testing sample under all circumstances. All the copper mesh water heat pipe test samples had higher evaporator temperatures than the predictions. At the gravity assisted vertical orientation, the actual evaporator temperatures tended to be lower than the predictions. The inverse results occurred to the vertical orientation of against gravity. For the acetone heat pipe test sample, at the horizontal orientation with natural convective air cooling, the actual evaporator temperature tended to be lower than the predictions. Similar to the water heat pipe test samples, with forced and enhanced forced convective air cooling, the actual evaporator temperatures tended to be higher than that with natural air cooling condition. All of these discrepancies were shown to be related to the heat pipe effective length changes.

The heat transfer capacity analyses mainly focused on four transfer limits: the capillary, sonic, entrainment, and boiling limits. The viscosity limit increases greatly with increasing working temperature. It therefore does not affect the overall performance, thus can be ignored. Among the heat transfer capacity limits, the capillary limit was the smallest, and was used for comparison with the experimental results. The comparison results showed that, for the water heat pipes, at horizontal and gravity assisted vertical orientations; and for the acetone heat pipe at the gravity assisted vertical orientation, the heat transfer capacities were within the predictions. The exceptions occurred in the case of 
the water heat pipe at against gravity vertical orientation, and the acetone heat pipe at the orientations of horizontal and against gravity. In these cases, the heat transfer capacities exceeded the theoretical predictions. These exceptions are also shown to be related to the heat pipe effective length changes.

The consistencies between experimental results and theoretical predictions indicate that the heat pipes made with our method performed properly. The effective length related discrepancies between the experimental results and theoretical predictions supported these conclusions. Mathematical models were established describing the heat pipe effective length and the effective length change rate.

\subsection{Contributions}

The contributions made during this thesis work are summarized as follow:

\section{Heat Pipe Manufacturing at Canadian University}

This thesis work established the heat pipe manufacturing techniques at Carleton University. It also finishes the initial heat pipe fabrication process, and probes an entire set of heat pipe fabrication procedures. Thus, it lays the foundation for future research and development such as loop heat pipe. Also these results are valuable reference for heat pipe industrial mass productions.

\section{Theoretical Analysis on Heat Pipe Evacuation Process}

This thesis applies existing vacuum technology theorem to analyze the evacuation process in the heat pipe fabrication. This is a good application to combine multi-discipline 
knowledge. Thesis work explored the approach to the theoretical analyses in the heat pipe evacuation process, which seems to be ignored but is important in the heat pipe manufacturing procedure. This will benefit the evacuation process control in the heat pipe industrial productions.

\section{Experimental Data}

This thesis work conducted experimental investigations on the heat pipe steady-state performance. It acquired large amount of temperature data based on over 1000 hours of experiments. This data covered different wick structure, working fluids, and testing conditions. It is a helpful reference for current and future applications.

\section{Research of Heat Pipe Effective Length}

This thesis work established a set of mathematical models to link the heat pipe effective length variation to the physical prosperities. The concept of heat pipe effective length is used in the trial and error process in heat pipe design phase, and this length has been supposed to be constant. In this thesis work, the mathematical models were used to explain the discrepancies between the experimental results and the theoretical predictions. The factor of effective length variation has been recommended to be considered in heat pipe practices.

\subsection{Recommendations for Future Work}

There are certain areas that can be further investigated and improved, and are summarized as follows. 


\section{The Improvement of Vacuum and Charging System}

The existing vacuum and fluid charging system was proved to be suitable for the heat pipe evacuation and working fluid charging. This system is sufficient for the initial fabrication, and is easy to operate as well as easy to maintain. However, this system possesses weakness which needs to be improved. First, this vacuum system does not have an air release valve. Each time, to stop the vacuum pump, it is required to release the entire vacuum to prevent the back suck. This causes inconvenience to the operator, and cannot conduct static vacuum test without detaching the assembly, neither. Also frequent detaching would accelerate the components wear and tear, thus decreasing the reliability. Second, this system directly uses atmospheric back pressure charging approach without a fluid re-purifying function. The charged fluid could still contain some gases. Also, due to the piping capillary effect, it is difficult to control the amount of charged fluid. Third, this system has a long piping (a large ratio of length to diameter). This greatly reduces the conductance to the gas flow.

Based on the above analyses, it is recommended that an air release valve between the vacuum pump and Valve 1 be added. Referring to Figure 2.4, this valve will either prevents the back suction or allow the conduct of the static vacuum test without detaching the assembly. It is also recommended that a funnel-shaped reservoir be added between Valve 1, 2 and 3. Referring to Figure 2.4, the required amount of fluid can be first charged into this reservoir, where the fluid can be conducted degassing or re-purifying processes. With a funnel shape, the reservoir can ensure all the fluid flow into the heat pipe assembly. It would therefore be easier to control the amount of fluid being charged. Another 
recommendation is to increase the diameter or shorten the length of the pipeline, thus improving the conductance to the gas flow, and therefore reduce the vacuum process time.

\section{Improvement of Sealing Method}

The existing sealing method is to apply pinching tool to lock the sealing ream, and then apply argon-arc welding. This method has the chance for the assembly of losing vacuum during the pinching and welding processes. To solve this problem, a simultaneous sealing method is a better choice. For example, the electrical-resistance welding technique, which can complete the sealing with one tool, combining crimping and welding, thus reduce the chance of leaking.

\section{Improvement of Testing Data Acquisition Function}

The existing data acquisition system plays a very important role in the experiments. The voltage changed from time to time in the testing process, and affected the experiments. We recommend to measure and record the voltage variation during the testing process, thus providing the power variation reference for the temperature variation. This can be conducted by adding sensors connecting the voltmeter and the data acquisition system.

\section{Power Supply System Stability Control}

As discussed in the preceding section, due to the power change, the heat pipe working conditions would also change. This would cause systematic errors. To avoid this problem, it is recommended to apply power stabilisation control, to ensure the power input is stable, and thus provide assurance for the heat pipe testing. This would increase the data validity. 


\section{Appendix}

\section{Appendix I: The Specifications of the Testing Elements}

\section{Table I.1 “1/4” Heater assembly}

\begin{tabular}{ll}
\hline Base & \\
\hline Material & Copper plate \\
Dimension (in) & $2 \times 2 \times 1 / 4$ \\
Form & Semi-circular groove with diameter of $1 / 4$ " on one side \\
\hline Tightening Element & Aluminium plate \\
\hline Material & $2 \times 1 \frac{11 / 2 \times 1 / 8}{}$ \\
Dimension (in) & Flat with 4 through holes for screws \\
Form & \\
\hline Heating Element & OMEGA KH 202/10 Flexible Heater (Kapton) \\
\hline Material & $2 \times 2 \times 0.010$ \\
Dimension (in) & -200 to 200 \\
Operating temperature $\left({ }^{\circ} \mathrm{C}\right)$ & 115 \\
Voltage $(\mathrm{V})$ & 10 \\
Watt Density (W/in $\left.{ }^{2}\right)$ & \\
\hline Adhesive & OMEGE OB-101-2 Epoxy \\
\hline Material & -55 to 105 \\
Service temperature $\left({ }^{\circ} \mathrm{C}\right)$ & 1.047 \\
Thermal conductivity $(\mathrm{W} / \mathrm{mK})$ & \\
\hline Thermal Compound & "Wakefield Engineering" 120 Series Thermal Joint Compound \\
\hline Material & -40 to 200 \\
Service temperature $\left({ }^{\circ} \mathrm{C}\right)$ & 0.735 \\
Thermal conductivity $(\mathrm{W} / \mathrm{mK})$ & \\
\hline
\end{tabular}

Table I.2 "1/1/4" Cooling sink assembly

\begin{tabular}{ll}
\hline Base & \\
\hline Material & Copper plate \\
Dimension (in) & $2 \times 2 \times 1 / 4$ \\
Form & Semi-circle groove with diameter of $1 / 4 "$ on one side \\
\hline Tightening Element & \\
\hline Material & Aluminium plate \\
Dimension (in) & $2 \times 11 / 2 \times 1 / 8$ \\
Form & Flat with 4 through holes for screws \\
\hline Finned Sink & \\
\hline Material & Aluminium and Copper embedded \\
Dimension (mm) & $80 \times 61 \times 40$ \\
Fin number & 22 \\
Fin thickness (mm) & 1 \\
Fin space (mm) & 1.5 \\
\hline Fan & \\
\hline Dimension (mm) & $60 \times 60 \times 25$ \\
\hline Air flow (m $/ \mathrm{Min})$ & 0.6 to 0.9 (estimated) \\
\hline Thermal Compound & \\
\hline Material & "Wakefield Engineering" 120 Series Thermal Joint Compound \\
Service temperature $\left({ }^{\circ} \mathrm{C}\right)$ & -40 to 200 \\
Thermal conductivity $(\mathrm{W} / \mathrm{mK})$ & 0.735 \\
\hline
\end{tabular}


Table I.3 Test sample [061800]

\begin{tabular}{ll}
\hline Envelope & Copper tube \\
& O.D. $1 / 4^{\prime \prime}(6.35 \mathrm{~mm})$ \\
Material & I.D. $3 / 16^{\prime \prime}(4.76 \mathrm{~mm})$ \\
Dimension & Thickness: $1 / 32^{\prime \prime}$ \\
& Working length: $220 \mathrm{~mm}$ \\
\hline Wick & \\
\hline Material & Copper wire screen \#100/in $(3937 \mathrm{mesh} / \mathrm{m})$ \\
Dimension (mm) & $220 \times 26 \times 0.23$ \\
Number of Layer & 2 \\
\hline Charged Fluid & N/A \\
\hline Seal & Crimped \\
\hline Internal Condition & Atmospheric air \\
\hline
\end{tabular}

TableI.4 Test sample [062300]

\begin{tabular}{ll}
\hline Envelope & \\
\hline & Copper tube \\
Material & O.D. 1/4" $(6.35 \mathrm{~mm})$ \\
Dimension & I.D. $3 / 16^{\prime \prime}(4.76 \mathrm{~mm})$ \\
& Thickness: $1 / 32^{\prime \prime}$ \\
& Working length: $220 \mathrm{~mm}$ \\
\hline Wick & N/A \\
\hline Charged Fluid & N/A \\
\hline Seal & Crimped \\
\hline Internal Condition & Atmospheric air \\
\hline
\end{tabular}

Table I.5 Test sample [051001]

\begin{tabular}{ll}
\hline Envelope & \\
\hline & Copper tube \\
Material & O.D. 1/4" $(6.35 \mathrm{~mm})$ \\
Dimension & I.D. $3 / 16^{\prime \prime}(4.76 \mathrm{~mm})$ \\
& Thickness: $1 / 32 "$ \\
& Working length: $220 \mathrm{~mm}$ \\
\hline Wick & \\
\hline Material & Copper wire screen \#100/in $(3937 \mathrm{mesh} / \mathrm{m})$ \\
Dimension (mm) & $220 \times 26 \times 0.23$ \\
Number of layer & 2 \\
\hline Charged fluid & \\
\hline Material & Distilled water \\
\hline Amount & $1.6 \mathrm{~g}$ \\
\hline Seal & Crimped, and argon-arc welded \\
\hline Internal condition & \\
\hline
\end{tabular}


Table I.6 Test sample [sample(s):HP052502]

\begin{tabular}{|c|c|}
\hline \multicolumn{2}{|l|}{ Envelope } \\
\hline $\begin{array}{l}\text { Material } \\
\text { Dimension }\end{array}$ & $\begin{array}{l}\text { Copper tube } \\
\text { O.D. } 1 / 4^{\prime \prime}(6.35 \mathrm{~mm}) \\
\text { I.D. } 3 / 16^{\prime \prime}(4.7625 \mathrm{~mm}) \\
\text { Thickness: } 1 / 32^{\prime \prime} \\
\text { Working Length: } 220 \mathrm{~mm}\end{array}$ \\
\hline \multicolumn{2}{|l|}{ Wick } \\
\hline $\begin{array}{l}\text { Material } \\
\text { Dimension (mm) } \\
\text { Number of Layer }\end{array}$ & $\begin{array}{l}\text { Copper wire screen \#100/in }(3937 \mathrm{mesh} / \mathrm{m}) \\
220 \times 26 \times 0.23 \\
2\end{array}$ \\
\hline \multicolumn{2}{|l|}{ Working fluid } \\
\hline $\begin{array}{l}\text { Material } \\
\text { Required amount (cc.) } \\
\text { Actually charged (cc.) } \\
\text { Over charge rate }(\%) \\
\end{array}$ & $\begin{array}{l}\text { R.O. water } \\
0.82 \\
1.3 \text { at } 20^{\circ} \mathrm{C} \\
58.08 \\
\end{array}$ \\
\hline \multicolumn{2}{|l|}{ Seal } \\
\hline & Crimped, and argon-arc welded \\
\hline \multicolumn{2}{|l|}{ Internal condition } \\
\hline & Vacuum (estimated about 30 Torr at $20^{\circ} \mathrm{C}$ ) \\
\hline
\end{tabular}

Table I.7 Test sample [sample(s):HP051701]

\begin{tabular}{ll}
\hline Envelope & \\
\hline & Copper tube \\
Material & O.D. 1/4" $(6.35 \mathrm{~mm})$ \\
Dimension & I.D. $3 / 16^{\prime \prime}(4.76 \mathrm{~mm})$ \\
& Thickness: $1 / 32^{\prime \prime}$ \\
& Working Length: $220 \mathrm{~mm}$ \\
\hline Wick & Copper wire screen \#100/in $(3937$ mesh $/ \mathrm{m})$ \\
\hline Material & $220 \times 26 \times 0.23$ \\
Dimension (mm) & 2 \\
Number of layer & \\
\hline Working fluid & R.O. water \\
\hline Material & 0.82 \\
Required amount $(\mathrm{cc})$. & 0.9 at $20^{\circ} \mathrm{C}$ \\
Actually charged (cc.) & 9.44 \\
Over charge rate $(\%)$ & \\
\hline Seal & Crimped, and argon-arc welded \\
\hline
\end{tabular}

\footnotetext{
${ }^{1}$ R.O. water: reverse osmosis processed pure water
} 
Table I.8 Test Sample [sample(s):HP071200]

\begin{tabular}{ll}
\hline Envelope & Copper tube \\
& O.D. 1/4" $(6.35 \mathrm{~mm})$ \\
Material & I.D. $3 / 16^{\prime \prime}(4.76 \mathrm{~mm})$ \\
Dimension & Thickness: $1 / 32^{\prime \prime}$ \\
& Working length: $220 \mathrm{~mm}$ \\
\hline Wick & Copper wire screen \#50/in $(1968.50 \mathrm{mesh} / \mathrm{m})$ \\
\hline Material & $220 \times 25 \times 0.46$ \\
Dimension (mm) & 2 \\
Number of layer & \\
\hline Working fluid & R.O. water \\
\hline Material & 1.59 \\
Required amount $(\mathrm{cc})$. & 1.7 at $20^{\circ} \mathrm{C}$ \\
Actually charged (cc.) & 6.84 \\
Over charge rate $(\%)$ & \\
\hline Seal & Crimped, and argon-arc welded \\
\hline
\end{tabular}

Table I.9 Test Sample [sample(s):HP062400]

\begin{tabular}{ll}
\hline Envelope & Copper tube \\
& O.D. 3/8" $(9.526 \mathrm{~mm})$ \\
Material & I.D. $5 / 16^{\prime \prime}(7.9375 \mathrm{~mm})$ \\
Dimension & Thickness: $1 / 16^{\prime \prime}$ \\
& Working length: $240 \mathrm{~mm}$ \\
\hline Wick & Nickel Melamine Foam \\
\hline Material & $240 \times 21 \times 1.96$ \\
Dimension (mm) & 1 \\
Number of layer & \\
\hline Working fluid & R.O. water \\
\hline Material & 6.37085 \\
Required amount (cc.) & 6.3 at $20^{\circ} \mathrm{C}$ \\
Actually charged (cc.) & -8.69 \\
\hline Over charge rate $(\%)$ & \\
\hline Seal & Crimped, and argon-arc welded \\
\hline Internal condition & Vacuum (estimated about 30 Torr at $\left.20^{\circ} \mathrm{C}\right)$ \\
\hline
\end{tabular}


Table I.10 Test sample [sample(s):HP071402]

\begin{tabular}{ll}
\hline Envelope & Copper tube \\
& O.D. $1 / 4^{\prime \prime}(6.35 \mathrm{~mm})$ \\
Material & I.D. $3 / 16^{\prime \prime}(4.7625 \mathrm{~mm})$ \\
Dimension & Thickness: $1 / 32^{\prime \prime}$ \\
& Working length: $220 \mathrm{~mm}$ \\
\hline Wick & \\
\hline Material & Copper wire screen \#100/in $(3937 \mathrm{mesh} / \mathrm{m})$ \\
Dimension (mm) & $220 \times 26 \times 0.2286$ \\
Number of layer & 2 \\
\hline Working fluid & \\
\hline Material & Acetone \\
Required amount (cc.) & 0.822344608 cc. \\
Actually charged (cc.) & $1.4778 \mathrm{cc}$. at $20^{\circ} \mathrm{C}$ \\
Over charge rate $(\%)$ & $79.7056836 \%$ \\
\hline Seal & \\
\hline & Crimped, and argon-arc welded \\
\hline Internal condition & Vacuum (estimated about 30 Torr at $\left.20^{\circ} \mathrm{C}\right)$ \\
\hline
\end{tabular}

Table I.11 Test sample [sample(s):HP071301]

\begin{tabular}{ll}
\hline Envelope & Copper tube \\
\hline & O.D. 1/4" $(6.35 \mathrm{~mm})$ \\
Material & I.D. 3/16" $(4.7625 \mathrm{~mm})$ \\
Dimension & Thickness: $1 / 32^{\prime \prime}$ \\
& Working Length: $220 \mathrm{~mm}$ \\
\hline Wicks (composite) & \\
\hline Mesh 1 (contacting the envelope) & \\
\hline Material & Copper wire screen \#50/in $(1968.50 \mathrm{mesh} / \mathrm{m})$ \\
Dimension (mm) & $220 \times 25 \times 0.46$ \\
Number of layer & 2 \\
\hline Mesh 2 (contacting the mesh 1) & \\
\hline Material & Copper wire screen \#100/in $(3937 \mathrm{mesh} / \mathrm{m})$ \\
Dimension (mm) & $220 \times 26 \times 0.2286$ \\
Number of layer & 2 \\
\hline Material & R.O. water \\
Required amount (cc.) & $1.3 \mathrm{cc}$. \\
Actually charged (cc.) & $1.7 \mathrm{cc}$ at $20^{\circ} \mathrm{C}$ \\
Over charge rate (\%) & 30.77 \\
\hline Seal & Crimped, and argon-arc welded \\
\hline
\end{tabular}




\section{Appendix II: The Experimental Result Recordings}

Table II.1 Voltage-Power Relationship of the Kapton Heater Film

\begin{tabular}{|l|l|l|l|l|l|}
\hline Power $(\mathbf{W})$ & Voltage $(\mathbf{V})$ & Current $(\mathbf{A})$ & Power $(\mathbf{W})$ & Voltage $(\mathbf{V})$ & Current $(\mathbf{A})$ \\
\hline 4.50 & 15.0 & 0.03 & 16.023 & 76.3 & 0.21 \\
\hline 1.00 & 20.0 & 0.05 & 17.05 & 77.5 & 0.22 \\
\hline 1.50 & 25.0 & 0.06 & 18.018 & 81.9 & 0.22 \\
\hline 2.00 & 28.6 & 0.07 & 19.067 & 82.9 & 0.23 \\
\hline 3.01 & 33.4 & 0.09 & 20.01 & 87.0 & 0.23 \\
\hline 4.00 & 40.0 & 0.10 & 21.00 & 87.5 & 0.24 \\
\hline 5.00 & 41.6 & 0.12 & 22.08 & 92.0 & 0.24 \\
\hline 6.01 & 46.2 & 0.13 & 23.00 & 92.0 & 0.25 \\
\hline 7.07 & 50.5 & 0.14 & 24.00 & 96.0 & 0.25 \\
\hline 8.04 & 53.6 & 0.15 & 25.038 & 96.3 & 0.26 \\
\hline 9.01 & 56.3 & 0.16 & 26.00 & 100.0 & 0.26 \\
\hline 10.00 & 62.5 & 0.16 & 27.135 & 100.5 & 0.27 \\
\hline 11.02 & 64.8 & 0.17 & 27.945 & 103.5 & 0.27 \\
\hline 12.01 & 6.7 & 0.18 & 29.036 & 103.7 & 0.28 \\
\hline 13.07 & 68.8 & 0.19 & 30.016 & 107.2 & 0.28 \\
\hline 14.00 & 70.0 & 0.20 & 35.01 & 116.7 & 0.30 \\
\hline 15.00 & 75.0 & 0.20 & 39.968 & 124.9 & 0.32 \\
\hline
\end{tabular}

Table II.2 Heat pipe testing recording [sample(s):HP051701]

\begin{tabular}{|c|c|c|c|c|c|}
\hline \multirow{2}{*}{ Ultimate Value } & \multirow{2}{*}{$\begin{array}{l}\text { Cooling } \\
\text { condition }\end{array}$} & \multicolumn{4}{|c|}{ Orientation condition } \\
\hline & & $\psi=0^{\circ}$ & $\psi=-90^{\circ}$ & $\psi=+90^{\circ}$ & Notes \\
\hline Power [W] & \multirow{2}{*}{ Natural convection } & 20.056 & 20.332 & 7.616 & \\
\hline Heater temperature $\left[{ }^{\circ} \mathrm{C}\right]$ & & 110.6 & 111.8 & 1.2 .45 & \\
\hline Power $[\mathrm{W}]$ & \multirow{2}{*}{ Forced convection } & 29.079 & 35.04 & 6.994 & \\
\hline Heater temperature $\left[{ }^{\circ} \mathrm{C}\right]$ & & 101 & 78.3 & 101 & \\
\hline Power [W] & \multirow{2}{*}{$\begin{array}{l}\text { Enhanced forced } \\
\text { convection }\end{array}$} & 35.37 & 35.04 & 9.984 & \\
\hline Heater temperature $\left[{ }^{\circ} \mathrm{C}\right]$ & & 91 & 76.6 & 113.7 & \\
\hline
\end{tabular}

Table II.3 Heat pipe testing recording [sample(s):HP052502]

\begin{tabular}{|c|c|c|c|c|c|}
\hline \multirow{2}{*}{ Ultimate Value } & \multirow{2}{*}{$\begin{array}{l}\text { Cooling } \\
\text { condition }\end{array}$} & \multicolumn{4}{|c|}{ Orientation condition } \\
\hline & & $\psi=0^{\circ}$ & $\psi=-90^{\circ}$ & $\psi=+90^{\circ}$ & Notes \\
\hline Power [W] & \multirow{2}{*}{ Natural convection } & 20.769 & 20.194 & 10.048 & \\
\hline Heater temperature $\left[{ }^{\circ} \mathrm{C}\right]$ & & 109.75 & 117 & 113.7 & \\
\hline Power [W] & \multirow{2}{*}{ Forced convection } & 34.8 & 34.92 & 11.135 & \\
\hline Heater temperature $\left[{ }^{\circ} \mathrm{C}\right]$ & & 98.8 & 102.8 & 98.1 & \\
\hline Power [W] & \multirow{2}{*}{$\begin{array}{l}\text { Enhanced forced } \\
\text { convection }\end{array}$} & 34.65 & 35.34 & 12.69 & \\
\hline Heater temperature $\left[{ }^{\circ} \mathrm{C}\right]$ & & 92.4 & 98 & 107.3 & \\
\hline
\end{tabular}


Table II.4 Heat pipe testing recording [sample(s):HP07120]

\begin{tabular}{|c|c|c|c|c|c|}
\hline \multirow{2}{*}{ Ultimate Value } & \multirow{2}{*}{$\begin{array}{l}\text { Cooling } \\
\text { condition }\end{array}$} & \multicolumn{4}{|c|}{ Orientation condition } \\
\hline & & $\psi=0^{\circ}$ & $\psi=-90^{\circ}$ & $\psi=+90^{\circ}$ & Notes \\
\hline Power [W] & \multirow{2}{*}{ Natural convection } & 15.6 & 15.42 & 5.005 & \\
\hline Heater temperature $\left[{ }^{\circ} \mathrm{C}\right]$ & & 101 & 95 & 95 & \\
\hline Power [W] & \multirow{2}{*}{ Forced convection } & 26.052 & 35.04 & 5.868 & \\
\hline Heater temperature $\left[{ }^{\circ} \mathrm{C}\right]$ & & 99.8 & 92 & 98.9 & \\
\hline Power [W] & \multirow{2}{*}{$\begin{array}{l}\text { Enhanced forced } \\
\text { convection }\end{array}$} & 30.044 & 35.04 & 6.578 & \\
\hline Heater temperature $\left[{ }^{\circ} \mathrm{C}\right]$ & & 99.5 & 86.9 & 102.35 & \\
\hline
\end{tabular}

Table II.5 Heat pipe testing recording [sample(s):HP062400]

\begin{tabular}{|c|c|c|c|c|c|}
\hline \multirow{2}{*}{ Ultimate Value } & \multirow{2}{*}{$\begin{array}{c}\text { Cooling } \\
\text { condition }\end{array}$} & \multicolumn{4}{|c|}{ Orientation condition } \\
\hline & & $\psi=0^{\circ}$ & $\psi=-90^{\circ}$ & $\psi=+90^{\circ}$ & Notes \\
\hline Power [W] & \multirow{2}{*}{ Natural convection } & 20.171 & 20.401 & 15.34 & \\
\hline Heater temperature $\left[{ }^{\circ} \mathrm{C}\right]$ & & 100.9 & 102.5 & 107.8 & \\
\hline Power [W] & \multirow{2}{*}{ Forced convection } & 35.04 & 35.04 & 22.272 & \\
\hline Heater temperature $\left[{ }^{\circ} \mathrm{C}\right]$ & & \begin{tabular}{|l|}
77.7 \\
\end{tabular} & 7.76 & 102 & \\
\hline Power [W] & \multirow{2}{*}{$\begin{array}{l}\text { Enhanced forced } \\
\text { convection }\end{array}$} & 41.085 & 40.953 & 22.56 & \\
\hline Heater temperature $\left[{ }^{\circ} \mathrm{C}\right]$ & & 82 & 78.44 & 101.32 & \\
\hline
\end{tabular}

Table II.6 Heat pipe testing recording [sample(s):HP071402]

\begin{tabular}{|c|c|c|c|c|c|}
\hline \multirow{2}{*}{ Ultimate Value } & \multirow{2}{*}{$\begin{array}{c}\text { Cooling } \\
\text { condition }\end{array}$} & \multicolumn{4}{|c|}{ Orientation condition } \\
\hline & & $\psi=0^{\circ}$ & $\psi=-90^{\circ}$ & $\psi=+90^{\circ}$ & Notes \\
\hline Power $[\mathrm{W}]$ & \multirow{2}{*}{ Natural convection } & 15.5 & 15.5 & 4.972 & \\
\hline Heater temperature $\left[{ }^{\circ} \mathrm{C}\right]$ & & 105.167 & 103.94 & 99.148 & \\
\hline Power [W] & \multirow{2}{*}{ Forced convection } & 20.631 & 35.1 & 5.038 & \\
\hline Heater temperature $\left[{ }^{\circ} \mathrm{C}\right]$ & & 126.12 & 107.1 & 96.35 & \\
\hline Power [W] & \multirow{2}{*}{$\begin{array}{l}\text { Enhanced forced } \\
\text { convection }\end{array}$} & 20.539 & 35.61 & 4.972 & \\
\hline Heater temperature $\left[{ }^{\circ} \mathrm{C}\right]$ & & 126.5 & 103.8 & 92.92 & \\
\hline
\end{tabular}

Table II.7 Heat pipe testing recording [sample(s):HP071301]

\begin{tabular}{|c|c|c|c|c|c|}
\hline \multirow{2}{*}{ Ultimate Value } & \multirow{2}{*}{$\begin{array}{l}\text { Cooling } \\
\text { condition }\end{array}$} & \multicolumn{4}{|c|}{ Orientation condition } \\
\hline & & $\psi=0^{\circ}$ & $\psi=-90^{\circ}$ & $\psi=+90^{\circ}$ & Notes \\
\hline Power [W] & \multirow{2}{*}{ Natural convection } & 19.228 & 20.7 & 7.085 & \\
\hline Heater temperature $\left[{ }^{\circ} \mathrm{C}\right]$ & & 107.865 & 105.33 & 115.675 & \\
\hline Power [W] & \multirow{2}{*}{ Forced convection } & 39.744 & 39.744 & 7.046 & \\
\hline Heater temperature $\left[{ }^{\circ} \mathrm{C}\right]$ & & 117.473 & 100.82 & 111.42 & \\
\hline Power [W] & \multirow{2}{*}{$\begin{array}{l}\text { Enhanced forced } \\
\text { convection }\end{array}$} & 39.872 & 39.904 & 7.602 & \\
\hline Heater temperature $\left[{ }^{\circ} \mathrm{C}\right]$ & & 112.8 & 102.087 & 103.313 & \\
\hline
\end{tabular}




\section{References}

Bai, L. (2004). The FEM analysis of a heat pipe, MASc Thesis, Carleton University, Ottawa, Ontario.

Basiulis, A. and Eallonardo, C. M. (1973), Heat-Pipe System for Space Shuttle Traveling Wave Tube Amplifier, AIAA $8^{\text {th }}$ Thermaophysics Conference, Palm Springs, Calif.

Basiulis, A. (1982), Design, Fabrication, and Test of Liquid-Metal Heat-Pipe Sandwich Panels, The $3^{\text {rd }}$ AIAA/ASME joint Thermopgysics, Fluids, Plasma \& Heat Transfer Conference, St. Louis, MO.

Berman, A. (1992), Vacuum Engineering Calculations, Formulas, and Solved Exercises, Academic Press, Inc. San Diego

Chi, S. W. (1976), Heat Pipe Theory and Practice: A Sourcebook. McGraw-Hill, New York.

Camarda, C. J. (1978), Aerothermal Test of a Heat-Pipe-Cooled Leading Edge at Mach 7 , NASA Technical Paper, Langley Research Center, Hampton, Virginia.

Cao, Y. (2000), An Innovative Turbine Blade Cooling Technology \& Micro/Miniature Heat Pipe for Turbine Blades, AFOSR Research, Florida International University.

Carey, Van P. (1992). Liquid-Vapor Phase-Change Phenomena: An Introduction to the Thermophysics of Vaporization and Condensation Processes in Heat Transfer Equipment, Hemisphere Publishing Corporation, Washington.

Diele, K. and Jaeckel, R. (1966), Leybold Vacuum Handbook, Pergamon Press, Oxford.

Dunn, P. D. and Reay, D. A. (1978). Heat Pipes, $2^{\text {nd }}$ ed. Pergamon Press, Oxford.

Dushman, S. (1962), Scientific Foundations of Vacuum Technique, Johm Wiley \& Sons, Inc. New York.

Faghri, A. (1994), Performance Characteristics of An Annular Heat Pipe, Wright State University, Dayton, Ohio.

Glass, D.E.; Merrigan, M. A.; Sena, J. Tom, and Reid, R. S. (1998), Fabrication and Testing of a Leading-Edge-Shaped Heat Pipe, NASA Research Report, Hampton, Virginia 
Holman, J.P. (1997). Heat Transfer, $8^{\text {th }}$ ed. McGraw-Hill, New York.

Ivanovskii, M. N.; Sorokin, V.P. and Yagodkin, I.V. (1982), The Physical Principles of Heat Pipes, Clarendon Press, Oxford.

Kaviany, M. (1991) Principles of Heat transfer in Porous Media, Spinger-Verlag, New York.

Lock, G. S. H. (1992). The Tubular Thermosyphon: Variations on a Theme, Oxford University Press, Oxford.

Lock, G. S. H. (1994). Latent Heat Transfer: An Introduction to Fundamentals, Oxford University Press, Oxford.

MacDonald, E. (2004), Experimental and Numerical Investigation of Passive TwoPhase Heat Transfer Devices for Space Applications, MASc Thesis, Carleton University, Ottawa, Ontario.

Marmur, A. (1992), Penetration and displacement in capillary Systems, Technion-Israel Institute of Technology, Haifa, Israel.

Myers, D. (1999), Surfaces, Interfaces, and Colloids: Principles and Applications, John Wiley \& Sons, New York.

Peterson, G. P. (1994), An Introduction to Heat Pipes: Modeling, Testing, and Applications, John Wilek \& Sons, Inc. New York.

Powell, R. L. (1976), Thermocouple Thermometry, Boulder, Colorado.

Savage, C. J. and Aalders, B.G.M. Noordwijk, J.P. Brussels, Belgium, and Munzel, W.D. (1979), Heat Pipe Experiment on ASTRO-8(ii), The AIAA $14^{\text {th }}$ Thermaophysics Conference, Orlando, Fla.

Schrader, M. E. (1992), High- and Medium-Energy Surfaces: Ultrahigh Vacuum Approach, Hebrew University of Jerusalem, Jerusalem, Israel.

Ward, T. L.; Ortega, Joseph K. E. Martin and Kroliczek, E. J. (1979), Development of a High Performance Thermal Structure Heat Pipe, The AIAA $14^{\text {th }}$ Thermaophysics Conference, Orlando, Fla. 